Prepared for the Missouri River Recovery-Integrated Science Program

U.S. Army Corps of Engineers, Yankton, South Dakota

\title{
Channel Morphodynamics in Four Reaches of the Lower Missouri River, 2006-07
}

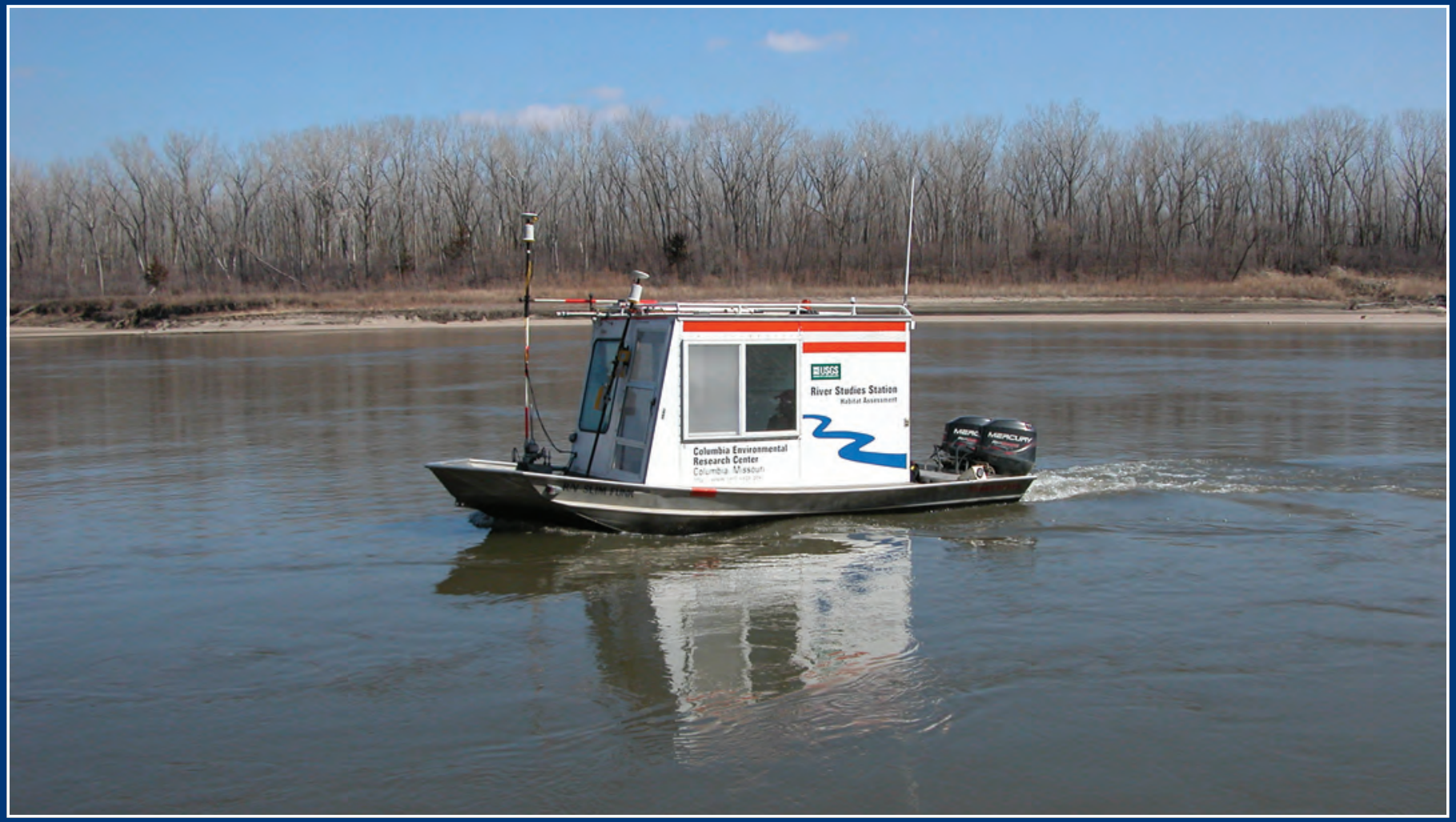

Scientific Investigations Report 2009-5074 
Cover photograph. The research vessel Slim Funk using Real Time Kinematic GPS positioning to measure the depth, elevation, velocity, and substrate in the Little Sioux reach of the Missouri River in early March 2006. Photo by Matt Smith, contracted to the USGS by Arctic Slope Regional Corporation. 


\section{Channel Morphodynamics in Four Reaches of the Lower Missouri River, 2006-07}

By Caroline M. Elliott, Joanna M. Reuter, and Robert B. Jacobson

Prepared for the Missouri River Recovery-Integrated Science Program U.S. Army Corps of Engineers, Yankton, South Dakota

Scientific Investigations Report 2009-5074 


\section{U.S. Department of the Interior \\ KEN SALAZAR, Secretary \\ U.S. Geological Survey \\ Suzette M. Kimball, Acting Director}

U.S. Geological Survey, Reston, Virginia: 2009

For more information on the USGS - the Federal source for science about the Earth, its natural and living resources, natural hazards, and the environment, visit http://www.usgs.gov or call 1-888-ASK-USGS

For an overview of USGS information products, including maps, imagery, and publications, visit http://www.usgs.gov/pubprod

To order this and other USGS information products, visit http://store.usgs.gov

Any use of trade, product, or firm names is for descriptive purposes only and does not imply endorsement by the U.S. Government.

Although this report is in the public domain, permission must be secured from the individual copyright owners to reproduce any copyrighted materials contained within this report.

Suggested citation:

Elliott, C.M., Reuter, J. R., and Jacobson, R.B., 2009, Channel morphodynamics in four reaches of the Lower Missouri River, 2006-07: U.S. Geological Survey Scientific Investigations Report 2009-5074, 258 p. 


\section{Contents}

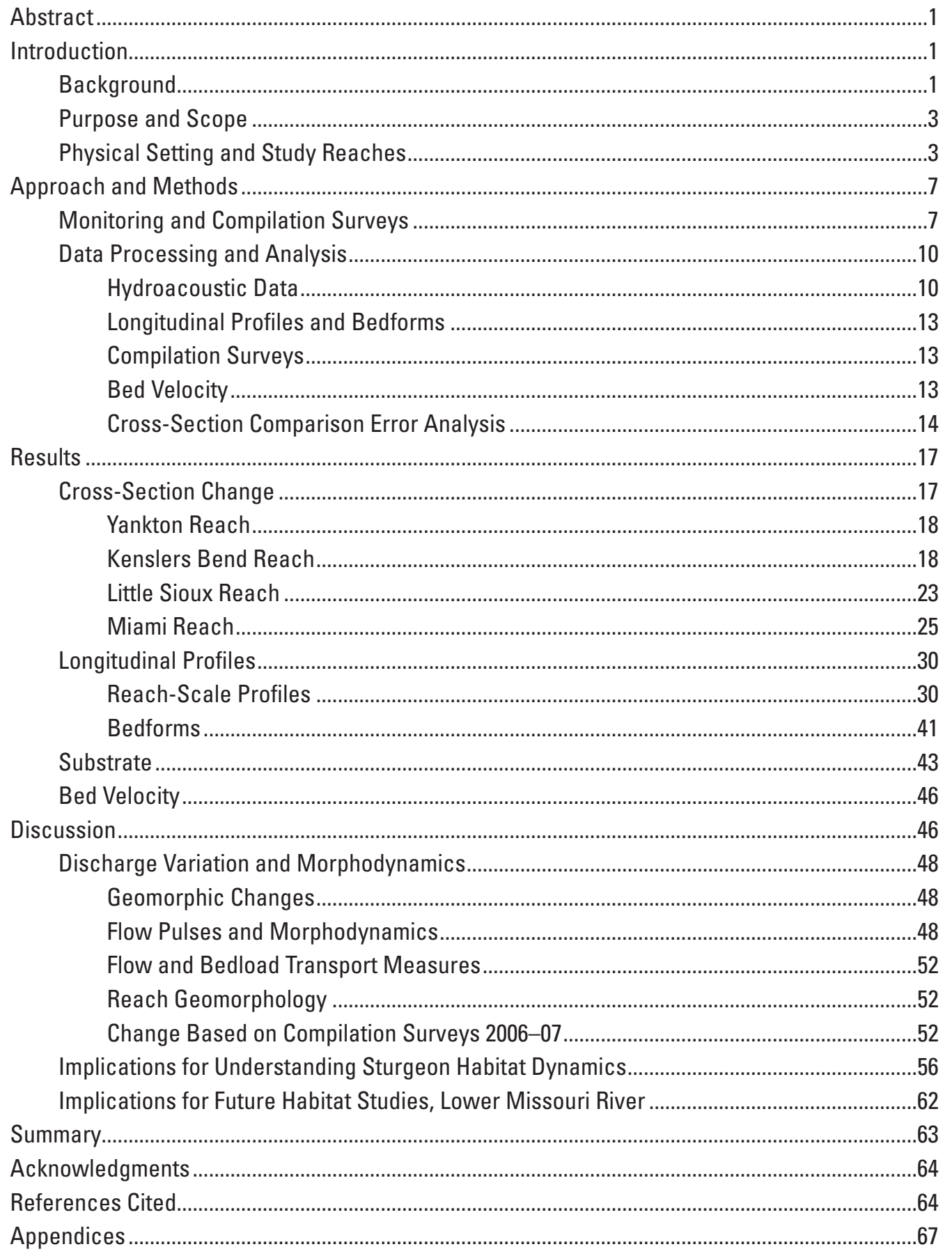




\section{Figures}

1-5. Maps showing:

1. Lower Missouri River with the four study reaches, Gavins Point Dam, major tributaries, and gages indicated

2. Aerial orthophotograph of the Yankton reach on the South Dakota-Nebraska border with June 2007 bathymetry, and randomly selected monitoring transects ....4

3. Aerial orthophotograph of the Kenslers Bend reach on the South DakotaNebraska border with June 2007 bathymetry, and randomly selected monitoring transects

4. Aerial orthophotograph of the Little Sioux reach on the Nebraska-lowa border with June 2007 bathymetry, and randomly selected monitoring transects...

5. Aerial orthophotograph of the Miami reach in Missouri with June 2007 bathymetry, and randomly selected monitoring transects

6. Graph showing hydrographs and survey dates for 2006-07 at Gavins Point Dam, Nebr.,

Sioux City, lowa., Decatur, Nebr., and Waverly, Mo.

7. Map showing aerial orthophotograph of the Yankton reach with compilation survey transects, randomly selected survey transects, and long profile lines indicated...

8-23. Graphs showing:

8. Repeat cross sections at Yankton and the Little Sioux reaches used in cross section change error analysis.

9. Typical survey cross sections in the Yankton reach ..............................................14

10. Deposition, erosion, and net change for the Yankton reach......................................17

11. Typical survey cross sections in the Kenslers Bend reach ......................................18

12. Deposition, erosion, and net change for the Kenslers Bend reach ..........................19

13. Typical survey cross sections in the Little Sioux reach .............................................23

14. Deposition, erosion, and net change for the Little Sioux reach ................................24

15. Typical survey cross sections for the Miami reach....................................................30

16. Deposition, erosion, and net change for the Miami reach ..........................................36

17. Longitudinal profiles for the Yankton reach ...........................................................38

18. Longitudinal profiles for Kenslers Bend reach .......................................................38

19. Long and short profiles for each survey date in the Little Sioux reach.....................39

20. Long and short profiles for each survey date in the Miami reach ............................40

21. Compiled short and long longitudinal profiles for 2006 and 2007 in the Little Sioux reach

22. Compiled short and long longitudinal profiles for 2006 and 2007 in the Miami reach.

23. Bedform dimensions and one-dimensional sediment flux calculated from bedforms in the four reaches

24-26. Maps showing:

24. Example side-scan sonar survey with bed features indicated..................................45

25. Side-scan sonar and bedforms in the Yankton reach ..............................................46

26. Side-scan sonar and bedforms in the Kenslers Bend reach ...................................47

27-33. Graphs showing:

27. Average bed velocity and discharge in all reaches

28. Reach averaged deposition, erosion, and net change for the Yankton, Kenslers Bend, Little Sioux, and Miami reaches 
29. Average deposition and erosion in the four reaches with corresponding surveys and hydrographs

30. Percent of cross-section area with deposition, erosion, or net change for the four monitoring reaches

31. Typical cross sections at the same scale for the four reaches.................................55

32. Histograms of depths at median annual flow in the four reaches ...........................56

33. Longitudinal profiles at median or near-median annual flow for the four reaches..57

34-37. Maps showing:

34. Changes in elevation based on 2006 and 2007 compilation surveys in the Yankton reach.

35. Changes in elevation based on 2006 and 2007 compilation surveys in the Kenslers Bend reach

36. Changes in elevation based on 2006 and 2007 compilation surveys in the Little Sioux reach

37. Changes in elevation based on 2006 and 2007 compilation surveys in the Miami reach

38-39. Graphs showing:

38. Changes in elevation from the 2006 to 2007 compilation surveys in the four reaches

39. Areas of deposition, erosion, and net change for the four reaches from 2006 and 2007 compilation surveys

\section{Tables}

1. Reach characteristics of the four study reaches...........................................................

2. Survey reaches with survey dates and discharges....................................................12

3. Error values from repeat cross sections at each site ................................................13

4. Cross-section comparison values for the Yankton reach, 2006-07................................15

5. Cross-section comparison values for the Kenslers Bend reach, 2006-07 ......................20

6. Cross-section comparison values for the Little Sioux reach, 2006-07 ............................26

7. Cross-section comparison values for the Miami reach, 2006-07...................................31

8. Bedform dimensions and dune movement calculations for each reach and

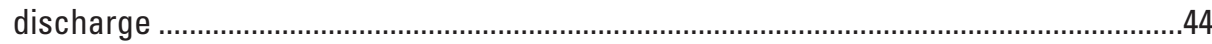

9. Bed velocity from acoustic Doppler current profiler data ................................................49

10. Cross-section erosion and deposition for the four reaches.............................................5

11. Change in grid area from compilation surveys, 2006-07.................................................54 


\section{Conversion Factors, Abbreviations, and Datums}

\begin{tabular}{|c|c|c|}
\hline Multiply & By & To obtain \\
\hline \multicolumn{3}{|c|}{ Length } \\
\hline mile (mi) & 1.609 & kilometer $(\mathrm{km})$ \\
\hline \multicolumn{3}{|c|}{ Flow rate } \\
\hline foot per second $(\mathrm{ft} / \mathrm{s})$ & 0.3048 & meter per second $(\mathrm{m} / \mathrm{s})$ \\
\hline \multicolumn{3}{|c|}{ Mass } \\
\hline ton per year (ton/yr) & 0.9072 & megagram per year $(\mathrm{Mg} / \mathrm{yr})$ \\
\hline \multicolumn{3}{|c|}{ Speed } \\
\hline knot $(\mathrm{kn})$ & 0.51444 & meter/second $(\mathrm{m} / \mathrm{s})$ \\
\hline \multicolumn{3}{|c|}{ Length } \\
\hline centimeter $(\mathrm{cm})$ & 0.3937 & inch (in.) \\
\hline meter $(\mathrm{m})$ & 3.281 & foot $(\mathrm{ft})$ \\
\hline kilometer $(\mathrm{km})$ & 0.6214 & mile (mi) \\
\hline \multicolumn{3}{|c|}{ Area } \\
\hline square meter $\left(\mathrm{m}^{2}\right)$ & 0.0002471 & acre \\
\hline square kilometer $\left(\mathrm{km}^{2}\right)$ & 247.1 & acre \\
\hline square meter $\left(\mathrm{m}^{2}\right)$ & 10.76 & square foot $\left(\mathrm{ft}^{2}\right)$ \\
\hline square kilometer $\left(\mathrm{km}^{2}\right)$ & 0.3861 & square mile $\left(\mathrm{mi}^{2}\right)$ \\
\hline \multicolumn{3}{|c|}{ Volume } \\
\hline cubic meter $\left(\mathrm{m}^{3}\right)$ & 35.31 & cubic foot $\left(\mathrm{ft}^{3}\right)$ \\
\hline cubic meter $\left(\mathrm{m}^{3}\right)$ & 0.0008107 & acre-foot (acre-ft) \\
\hline \multicolumn{3}{|l|}{ cubic kilometer $\left(\mathrm{km}^{3}\right)$} \\
\hline \multicolumn{3}{|c|}{ Flow rate } \\
\hline cubic meter per second $\left(\mathrm{m}^{3} / \mathrm{s}\right)$ & 70.07 & acre-foot per day (acre-ft/d) \\
\hline cubic meter per second $\left(\mathrm{m}^{3} / \mathrm{s}\right)$ & 35.31 & cubic foot per second $\left(\mathrm{ft}^{3} / \mathrm{s}\right)$ \\
\hline meter per second $(\mathrm{m} / \mathrm{s})$ & 3.281 & foot per second $(\mathrm{ft} / \mathrm{s})$ \\
\hline meter per hour $(\mathrm{m} / \mathrm{hr})$ & 3.281 & foot per hour (ft/hr) \\
\hline \multicolumn{3}{|c|}{ Mass } \\
\hline megagram per year (Mg/yr) & 1.102 & ton per year (ton/yr) \\
\hline metric ton per year & 1.102 & ton per year (ton/yr) \\
\hline
\end{tabular}

Distances along the Missouri River are given in river miles upstream from the junction with the Mississippi River at St. Louis, Missouri.

Temperature in degrees Celsius $\left({ }^{\circ} \mathrm{C}\right)$ may be converted to degrees Fahrenheit $\left({ }^{\circ} \mathrm{F}\right)$ as follows:

$$
{ }^{\circ} \mathrm{F}=\left(1.8 x^{\circ} \mathrm{C}\right)+32
$$

Vertical coordinate information is referenced to the North American Vertical Datum of 1988 (NAVD 88).

Horizontal coordinate information is referenced to the World Geodetic System of 1984 (WGS 84). 


\title{
Channel Morphodynamics in Four Reaches of the Lower Missouri River, 2006-07
}

\author{
By Caroline M. Elliott, Joanna M. Reuter, and Robert B. Jacobson
}

\section{Abstract}

Channel morphodynamics in response to flow modifications from Gavins Point Dam are examined in four reaches of the Lower Missouri River. Measures include changes in channel morphology and indicators of sediment transport in four 6 kilometer long reaches located downstream from Gavins Point Dam, near Yankton, South Dakota, Kenslers Bend, Nebraska, Little Sioux, Iowa, and Miami, Missouri. Each of the four reaches was divided into 300 transects with a 20-meter spacing and surveyed during the summer in 2006 and 2007. A subset of 30 transects was randomly selected and surveyed 7-10 times in 2006-07 over a wide range of discharges including managed and natural flow events. Hydroacoustic mapping used a survey-grade echosounder and a Real Time Kinematic Global Positioning System to evaluate channel change. Acoustic Doppler current profiler measurements were used to evaluate bed-sediment velocity. Results indicate varying amounts of deposition, erosion, net change, and sediment transport in the four Lower Missouri River reaches. The Yankton reach was the most stable over monthly and annual time-frames. The Kenslers Bend and Little Sioux reaches exhibited substantial amounts of deposition and erosion, although net change was generally low in both reaches. Total, or gross geomorphic change was greatest in the Kenslers Bend reach. The Miami reach exhibited varying rates of deposition and erosion, and low net change. The Yankton, Kenslers Bend, and Miami reaches experienced net erosion during the time period that bracketed the managed May 2006 spring rise event from Gavins Point Dam.

\section{Introduction}

This report addresses the potential for pulsed flow modifications (spring rises) to alter channel morphodynamics in the Lower Missouri River (fig. 1). Channel morphodynamics refers to the change in channel morphology characteristics associated with erosion and deposition of sediment (that is, from geomorphic processes). Channel morphodynamics are an important component of aquatic habitat dynamics because morphodynamics involve changing the geometry and substrate characteristics that determine how water and energy are distributed in a reach, thereby creating aquatic habitat.

In this report we emphasize understanding of dynamics of habitat required by the pallid sturgeon (Scaphirhynchus albus) for spawning. We assessed sediment transport and channel morphodynamics at four river reaches over 2 years, 2006-07. During the spring of 2006, a pulsed flow modification was implemented on the Lower Missouri River. While no flow modification was implemented during 2007, three of the four reaches experienced substantial flow variation that allowed us to assess channel morphodynamics arising from sediment transport.

\section{Background}

The Missouri River drains 1,371,000 square kilometers $\left(\mathrm{km}^{2}\right)$ of North America and has the Nation's largest reservoir system with 91 cubic kilometers $\left(\mathrm{km}^{3}\right)$ of storage (Galat and others, 2005; Jacobson and others, 2009). The mainstem system of reservoirs impounds the runoff from 53 percent of the drainage basin (fig. 1). The reservoir system has substantially altered the flow regime, including reducing intra-annual flow variability, generally decreasing spring pulses, and generally increasing summer low flows. The intensity of hydrologic alteration diminishes downstream from the dams as tributaries enter the Missouri River. The 590 kilometers (km) downstream from the Kansas River confluence (at Kansas City, Missouri) retains substantial intra-annual variability including spring-summer flow pulses (Galat and Lipkin, 2000; Jacobson and Heuser, 2002; Pegg and others, 2003; Jacobson and others, 2009).

The sediment load of the river also has decreased as a result of trapping by the reservoir system. The sediment load has decreased from 326 million megagrams per year $(\mathrm{Mg} / \mathrm{y}$, or 326 million metric tons per year) to 55 million $\mathrm{Mg} / \mathrm{y}$ as measured at Hermann, Missouri, from the pre-dam to post-dam time period (Jacobson and others, 2009a). Downstream from Gavins Point Dam at present, the suspended-sediment load increases from 0.24 million $\mathrm{Mg} / \mathrm{y}$ at Yankton, South Dakota; 7.3 million $\mathrm{Mg} / \mathrm{y}$ at Sioux City, Iowa; 18.6 million $\mathrm{Mg} / \mathrm{y}$ at Omaha, Nebraska; and 41.9 million Mg/y at Kansas City, Missouri (Jacobson and others, 2009a). The ratio of post-dam 
$98^{\circ}$

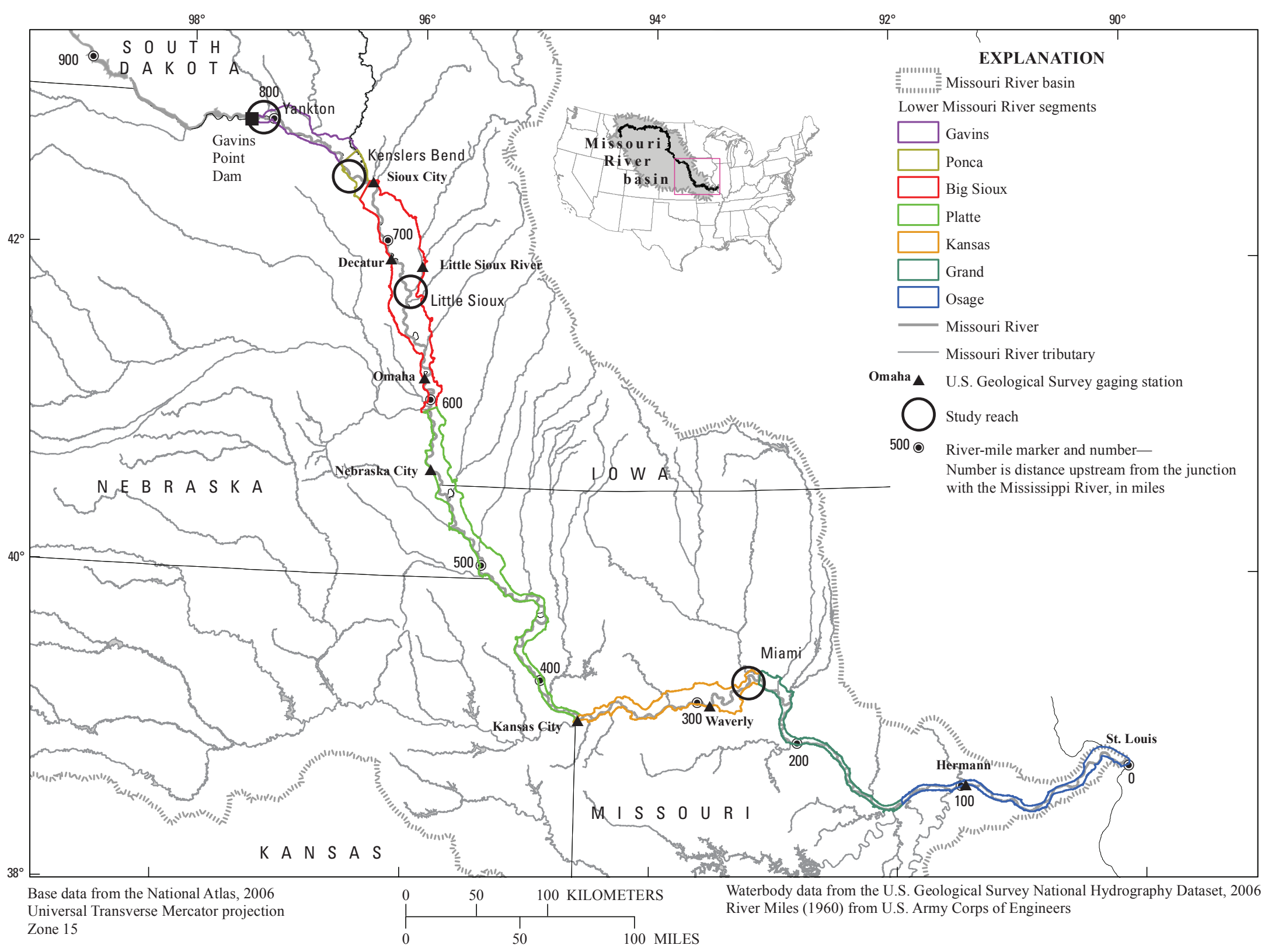

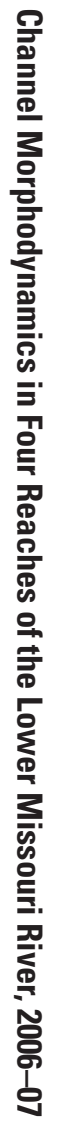

Figure 1. Lower Missouri River with the four study reaches, Gavins Point Dam, major tributaries, and gages indicated. 
to pre-dam suspended sediment levels varies longitudinally in the Lower Missouri River from 0.2 percent at Yankton, South Dakota, to 12.5 and 14.1 percent at Omaha, Nebraska, and Kansas City, Missouri, respectively, to 16.9 percent at Hermann, Missouri (Jacobson and others, 2009a).

From St. Louis, Missouri to Sioux City, Iowa, the Lower Missouri River has been engineered for bank stabilization and navigation. Wing dikes and revetments now stabilize 1,200 $\mathrm{km}$ of riverbanks. Dikes and other navigation structures focus the flow in the thalweg to maintain a narrow, swift, and selfdredging navigation channel in what was historically a shallow river characterized by interspersed braided, anabranched, and single-channel reaches. River engineering resulted in the loss of as much as $400 \mathrm{~km}^{2}$ of river-corridor habitats (Funk and Robinson, 1974; Hesse and Sheets, 1993; National Research Council, 2002; U.S. Army Corps of Engineers, 2004; Galat and others, 2005).

Declines of native species have been attributed to changes in flow regime, sediment regime, and channel morphology on the Lower Missouri River (National Research Council, 2002; U.S. Fish and Wildlife Service, 2003). Biological effects include the general decline of native fish species populations, commercial fish catches (Hesse, 1987; Pflieger and Grace, 1987; Hesse and others, 1989; Hesse and Sheets, 1993; Galat and others, 2005) and sandbar nesting birds (U.S. Fish and Wildlife Service, 2000a). In 2000, the U.S. Fish and Wildlife Service issued a Biological Opinion, subsequently amended in 2003, that indicated management of the reservoir, and bank stabilization and navigation projects threatened three listed species, the pallid sturgeon (Scaphirhynchus albus), interior least tern (Sternula antillarum athalassos) and the piping plover (Charadrius melodus) (U.S. Fish and Wildlife Service, 2000a, 2003). Among other remedies, the Biological Opinion required changes in flow regime and channel morphology to provide habitat for reproduction and survival of these species. Specifically, the Biological Opinion called for naturalization of the flow regime to:

1. Build sandbars in $95 \mathrm{~km}$ of the Lower Missouri River downstream from Gavins Point Dam, to support nesting of the least tern and piping plover;

2. Connect the main channel to the flood plain seasonally, to augment nutrient and energy exchange and to provide fish access to overbank habitats;

3. Maintain nursery habitat for larval and juvenile pallid sturgeon by achieving seasonal low flows in late summer;

4. Provide an environmental spawning cue for the pallid sturgeon through some combination of discharge and discharge-related variables like temperature, turbidity, and water velocity; and

5. Provide access to spawning habitat and/or "conditioning" of spawning habitat for pallid sturgeon by flushing fine sediment from coarse substrate.
This report specifically addresses item 5 in the list: the extent to which flow modification can alter spawning habitat by increasing sediment transport, especially flow modifications that may result in flushing fine sediment from coarse substrate. The specific question is included in a study design that addresses general aspects of sediment transport and morphodynamics associated with discharge variation on a multiyear time-frame. Measuring sediment transport in a large sand-bedded river is difficult, and direct and indirect methods produce varying results (Gaeuman and Jacobson, 2007). Also, modeling sediment transport in aquatic habitat assessments presents some fundamental challenges (Jacobson and others, 2009). Hydrodynamic habitat simulation studies typically use models that assume a fixed bed and lack the ability to model sediment transport and channel evolution. For low-flow studies or studies on rivers with immobile beds, this is a minor problem. For studies that attempt to simulate the ecological effects of flows capable of transporting bed material, this has been a substantial limitation because the models do not account for changing channel boundary conditions (geometry and flow resistance). Understanding of morphodynamics gained in this study will provide guidance on errors associated with fixed-bed assumptions in hydrodynamic modeling of the Lower Missouri River.

\section{Purpose and Scope}

We assessed channel morphodynamics through repeated measurements of channel morphology, substrate, and measures of sediment transport in four reaches of the Lower Missouri River. These reaches are located near Yankton, South Dakota, Kenslers Bend, Nebraska, Little Sioux, Iowa, and Miami, Missouri. The specific objective of this project to evaluate sediment transport over a range of discharges to assess the potential role of flow modifications and natural flow events in modifying habitats thought to be important in life history of the endangered pallid sturgeon. Changes in cross-sectional shape and size, substrate, acoustic bed velocity, bedload-transport rates, and velocity structures were monitored over a range of discharges to address sensitivity of erosion and deposition to flow variation arising from one manipulated flow release and several natural events. Patterns in scour and fill were analyzed between survey dates for each reach and among the four reaches along the Lower Missouri River.

To communicate effectively with stakeholders, managers, and other scientists working on the Lower Missouri River, we have used a mix of U.S. customary units and International System of Units (SI) units of measure. For locations along the river, the customary units of river mile (RM) are used. Reachscale hydraulic variables - velocity and depth - are in SI units of meters per second and meters.

\section{Physical Setting and Study Reaches}

The four study reaches are all located on the Lower Missouri River below Gavins Point Dam, which is situated 


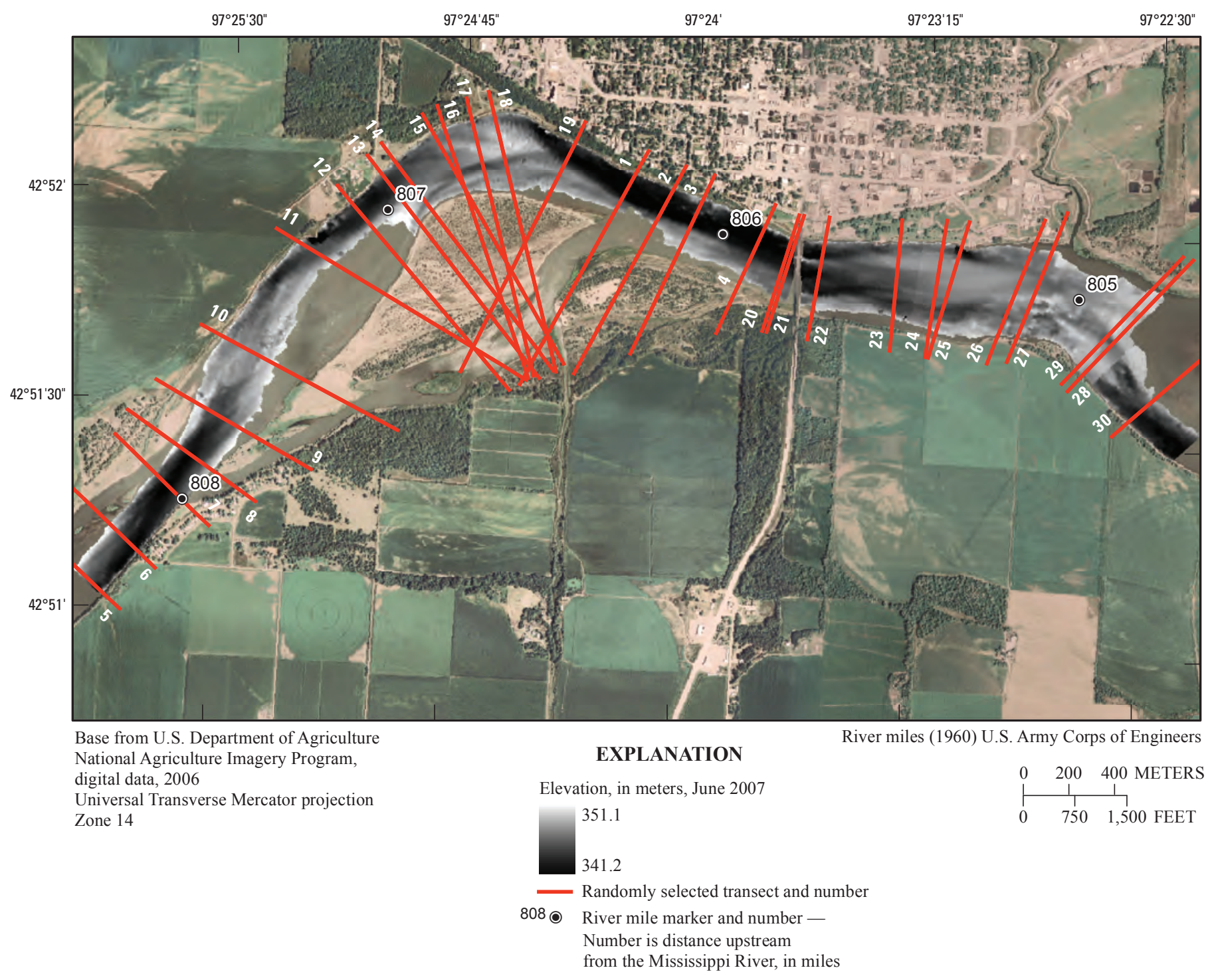

Figure 2. Yankton reach on the South Dakota-Nebraska border with June 2007 bathymetry, and randomly selected monitoring transects.

at RM 811 (fig. 1, table 1). These reaches were chosen based on telemetry data from sturgeon locations in 2005 and the presence of coarse substrate in the Yankton and Kenslers Bend reaches. Each reach is approximately 6-km long.

The upstream reach site is located on the NebraskaSouth Dakota border adjacent to Yankton, South Dakota (RM 808.3-804.5; fig. 2). This reach is located $5 \mathrm{~km}$ downstream from Gavins Point Dam, and has a substantially altered flow regime because of proximity to the dam (Jacobson and Galat, 2006; Jacobson, 2008). The Yankton reach retains some of the natural geomorphology of the Missouri River that existed before channelization (Elliott and Jacobson, 2006). There is a large island and side channel in the upstream portion of the reach, and the average bankfull width is 436 meters $(\mathrm{m})$. Much of the exposed bank in this reach is heavily armored (71 percent, table 1) with coarse bank revetment (angular boulders and rip-rap) to prevent bank erosion. There are no in-channel engineering structures present in this reach.
The Kenslers Bend reach (RM 748.2-743.9, fig. 3) is located on the Nebraska-South Dakota border between Ponca State Park and Sioux City, Iowa. This segment of river is channelized with engineering structures but does not support commercial navigation. The average bankfull width is $243 \mathrm{~m}$. Nearly half (44 percent) of the banks in this reach are directly protected with bank revetment, and there are 33 spur, or wing dikes in this reach that generally occur in dike fields on the inside of bends opposite revetted banks. Wing or spur dikes are rock structures oriented perpendicular to slightly downstream from the general direction of flow. For $1.3 \mathrm{~km}$ downstream from river mile 745.5 the right bank is composed of bedrock ( 9 percent of the bank in the reach).

The Little Sioux Reach (RM 673.6-669.8) is located on the Nebraska-Iowa border just upstream of the confluence with the Little Sioux River (fig. 4). This reach is within the portion of the Lower Missouri River channelized for navigation. The average bankfull width in this reach is $223 \mathrm{~m}$. The banks in 


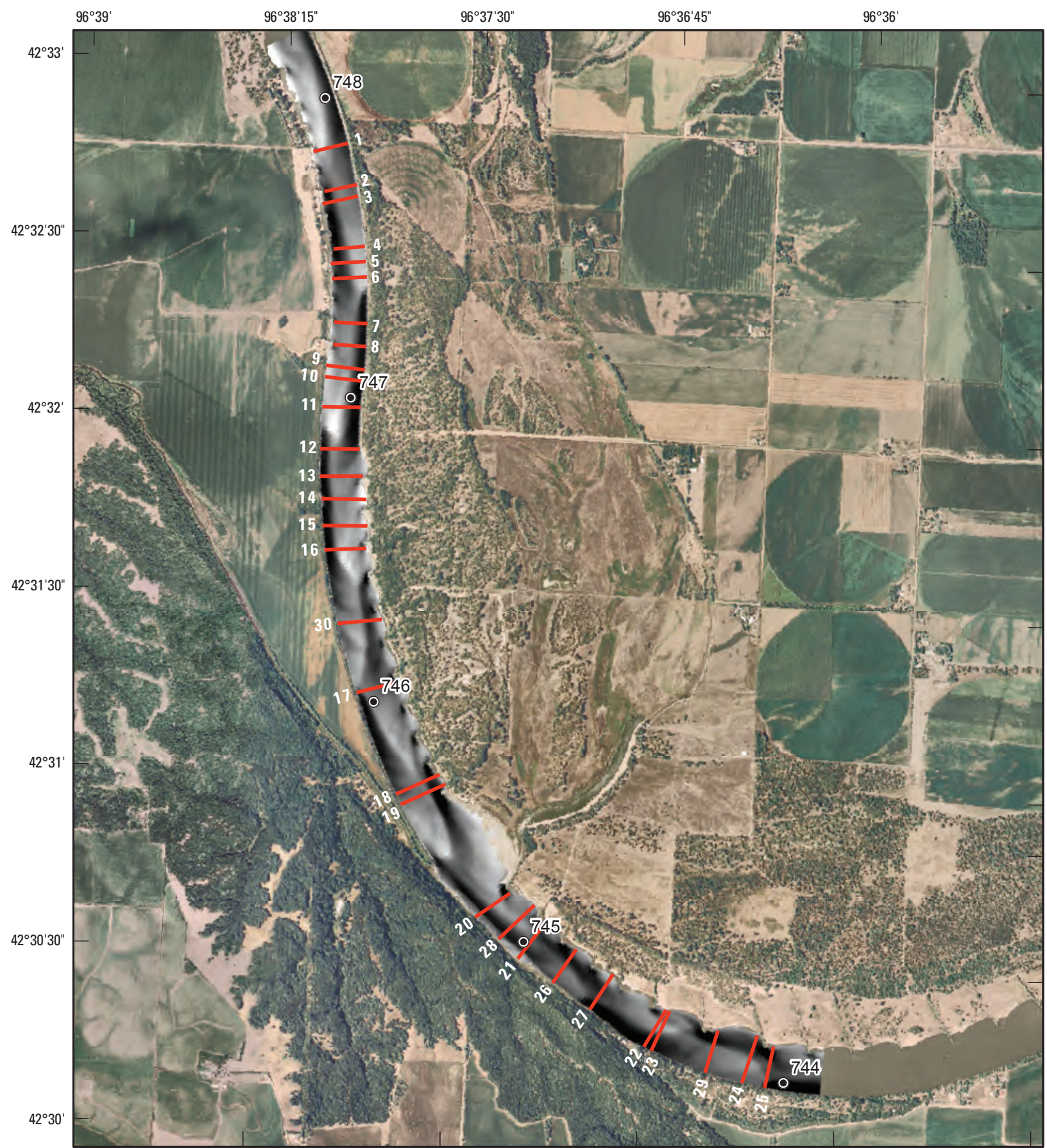

Base from U.S. Department of Agriculture National Agriculture Imagery Program, digital data, 2006

Universal Transverse Mercator projection Zone 14

\section{EXPLANATION}

Elevation, in meters, July 2007

331.0

320.4

Randomly selected transect and number

River miles (1960) U.S. Army Corps of Engineers 0 200400 METERS $\begin{array}{lll}1 & \\ 0 & 750 & 1,500 \\ & \text { FEET }\end{array}$

Figure 3. Kenslers Bend reach on the South Dakota-Nebraska border with June 2007 bathymetry, and randomly selected monitoring transects. 


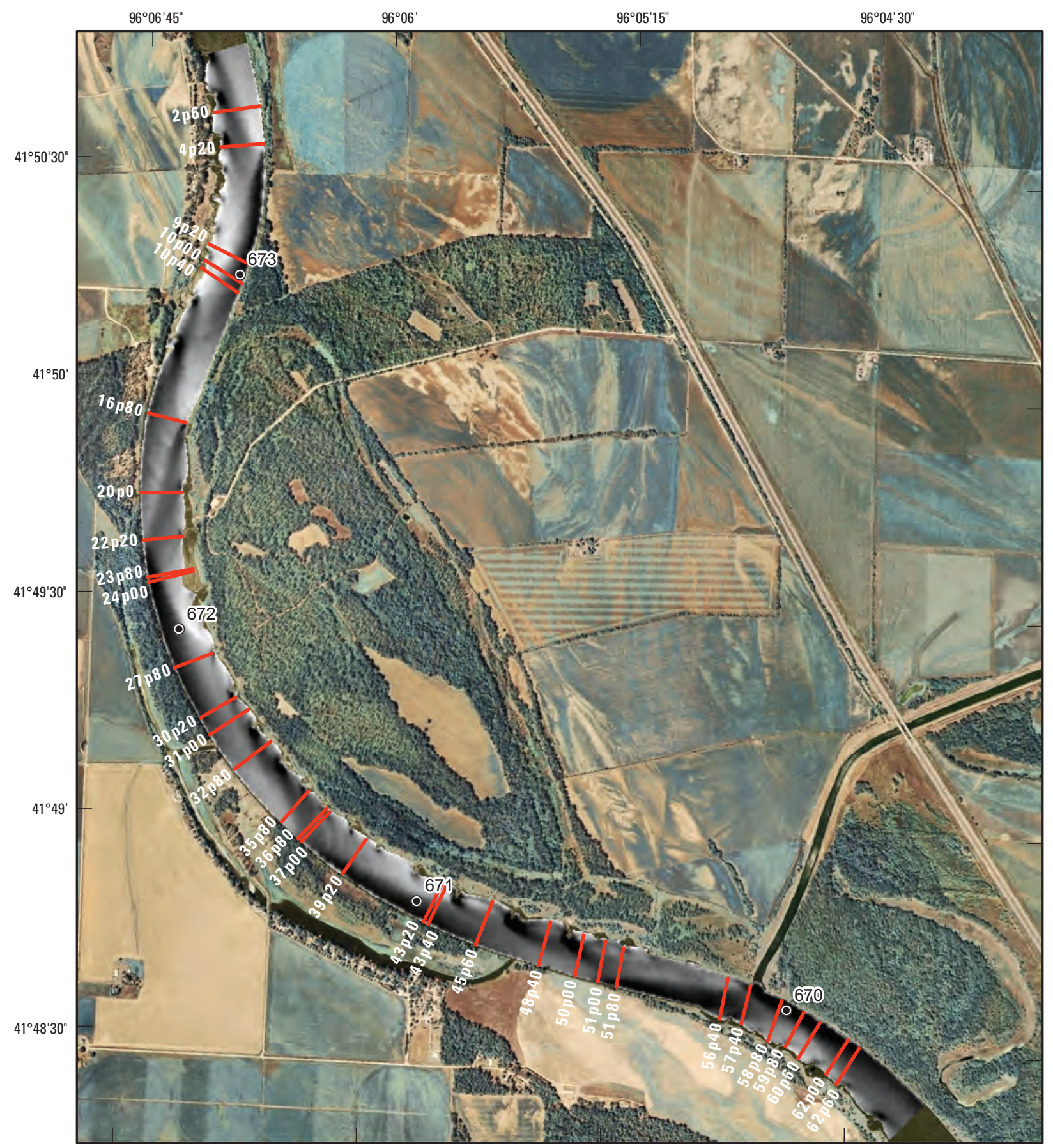

Base from U.S. Department of Agriculture National Agriculture Imagery Program, digital data, 2006

Universal Transverse Mercator projection Zone 14
EXPLANATION

Elevation, in meters, July 2008

305.15

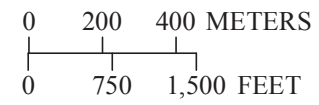

297.2

Randomly selected transect and number

670 River mile marker and number -

Number is distance upstream

from the Mississippi River, in miles

Figure 4. Little Sioux reach on the Nebraska-lowa border with June 2007 bathymetry, and randomly selected monitoring transects. 
this reach are revetted on the outside of bends and have wingdike structures on the inside of bends. A little over half (56 percent) of the banks in this reach are directly protected with revetment. The opposite bank from revetment is kept in place by 36 rock dikes, all of them spur, or wing-dike style dikes (table 1).

The Miami reach (RM 263.7-259.6, fig. 5) is located in Missouri, in the channelized portion of the Lower Missouri River. Because of tributary inputs and distance from the dam (fig. 6), this part of the Missouri River has a more naturalized flow regime (Jacobson and Galat, 2006; Jacobson, 2008). The average bankfull width in this reach is $345 \mathrm{~m}$. There is some bedrock interaction with the channel on the right descending bank near the Miami Bridge (about 4 percent of the bankline in the reach). This reach has an engineered channel that includes bank revetment ( 23 percent) and several types of dike features. In addition to wing dikes, the reach has L-head dikes, which are wing dikes with extensions oriented parallel to flow, and are generally longer than spur dikes. There are 4.8 dikes per $\mathrm{km}$ in this reach, 16 wing dikes, and $16 \mathrm{~L}$-head dikes (table 1).

The time period that encompasses this study was a period of drought in the Missouri River Basin, and the releases from Gavins Point Dam, including the $710 \mathrm{~m}^{3} / \mathrm{s}$ spring rise (fig. 6) in May 2006, were small compared to those occurring in wetter periods (U.S. Army Corps of Engineers, 2006b). A high-flow event from Gavins Point Dam in 1997 peaked at $1,985 \mathrm{~m}^{3} / \mathrm{s}$ and releases exceeded $1,600 \mathrm{~m}^{3} / \mathrm{s}$ for over 200 days. The 1997 high flows created large sandbars and substantially changed the geomorphology of the 59-mile reach below Gavins Point Dam (Elliott and Jacobson, 2006). The effects of regional storm events and tributary inputs increase downstream from the dam and produce natural flow events, such as the high flows in the Miami reach in 2007 (fig 6).

\section{Approach and Methods}

Changes in channel morphology, substrate, and acoustic velocity were monitored over a range of discharges in a 2-year period. The monitoring period included a manipulated flow pulse (spring rise) in May 2006 as well as some unanticipated natural flow pulses (fig. 6).

\section{Monitoring and Compilation Surveys}

In each of the four 6-km study reaches, the channel was divided into 300 transects with 20-m spacing (fig. 7). All of these transects were surveyed in the summers of 2006 and 2007 as a compilation survey for hydrodynamic models. A subset of 30-33 transects at each site was randomly selected at the beginning of the project, and the same set of transects was resurveyed 3-5 times each year in 2006-07 (table 2). All surveys used a Real Time Kinematic Global Positioning System (RTK-GPS) and hydroacoustic mapping protocols established by the U.S. Geological Survey, Columbia Environmental Research Center (USGS CERC) (Elliott and others, 2004; Reuter and others, 2008). Transects were drawn perpendicular to the channel for each reach and subsequently resurveyed using Hypack navigation and survey software (Hypack, Inc., Middletown, Connecticut, fig. 7).

Random selection of transects was essential for calculating unbiased estimates of geomorphic change. These transects were used to evaluate change in the cross-section shape and area, substrate conditions, and in velocity structure. Selection of a subset of the full set of transects also was necessary to allow completion of surveys during transient flow events; surveys of randomized transects were generally completed during 1 day. There were seven to ten surveys per site over the 2006-07 study period (table 2). Surveys were coordinated with the planned flow modification in May 2006 (U.S. Army Corps of Engineers, 2006a; Jacobson and Galat, 2008). In the two downstream reaches, the planned flow modification was small relative to natural variation, particularly in the Miami reach where a nonmanaged high-flow event occurred in AprilMay 2007 (fig. 6). Efforts were made to survey this reach at the highest possible discharge consistent with boat safety.

Aquatic surveys of monitoring transects in all reaches used RTK-GPS positioning, a survey grade echosounder, a bed-material classifying system, and a 1,200 kilohertz (kHz) Workhorse Rio Grande acoustic Doppler current profiler (ADCP, Teledyne RD Instruments, Poway, California). A survey grade RTK-GPS with real-time base station corrections which provide sub-decimeter horizontal and vertical accuracy was used. Simultaneous collection of RTK-GPS-controlled ADCP data provided current-velocity data and bed-velocity measurements (Gaeuman and Jacobson, 2005, 2006).

A survey-grade $200 \mathrm{kHz}$ InnerSpace (InnerSpace 456, InnerSpace Technology, Inc., Karlstadt, New Jersey) echosounder with an 8 degree transducer was used by USGS Nebraska Water Resources Division crews in the Yankton and Kenslers Bend reaches in 2006-07. A USGS Iowa Water Resources Division crew assisted with surveys in the Yankton and Kenslers Bend reaches in 2006, using an 8 degree transducer with a $200 \mathrm{kHz}$ Bathy 500-MF echosounder (Ocean Data Equipment Corp., Providence, Rhode Island). In the Little Sioux and Miami reaches USGS CERC crews used a $210 \mathrm{kHz}$ survey-grade Odom EchoTrac echosounder with an 8 degree transducer (Odom Hydrographic Systems Inc., Baton Rouge, Louisiana). Crews used consistent setup parameters and logging for these instruments following USGS CERC habitat mapping protocols (Reuter and others, 2008). Soundings were logged using laptop computers and Hypack navigation with a sampling rate of 5 hertz $(\mathrm{Hz})$ for monitoring transects. Boat speeds were kept below 1.5 meters per second (m/s, 3 knots) where possible, and did not exceed $2.5 \mathrm{~m} / \mathrm{s}$ (5 knots). Depth data were not collected in areas the boat could not navigate, generally areas shallower than 0.6 m. Careful boat operation, RTK-GPS, and navigation software allowed planned transects to be driven accurately within $3 \mathrm{~m}$, and in many cases within $1.5 \mathrm{~m}$ of the planned survey 


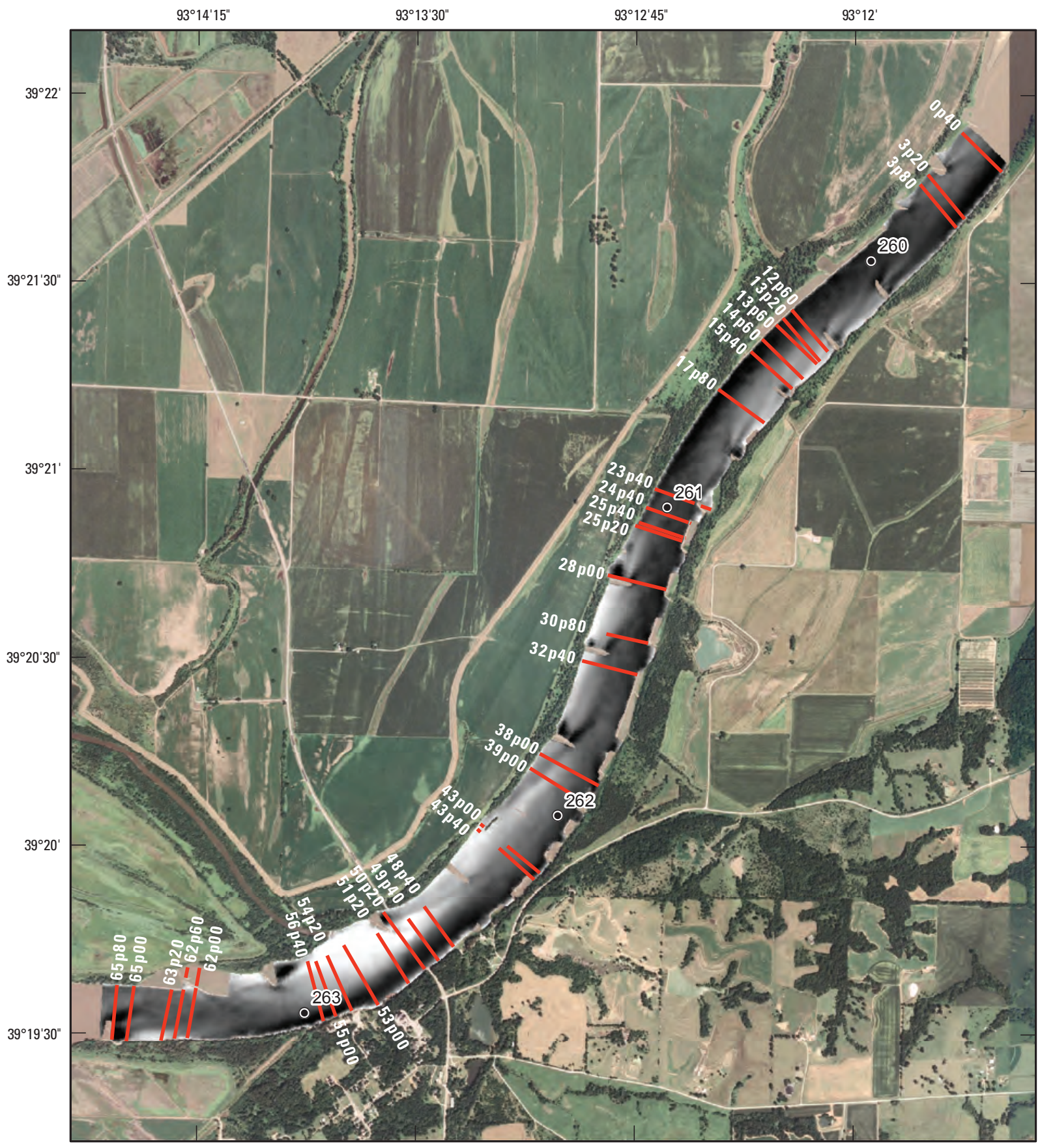

Base from U.S. Department of Agriculture National Agriculture Imagery Program, digital data, 2006

Universal Transverse Mercator projection Zone 15

\section{EXPLANATION}

Elevation, in meters, August 2007

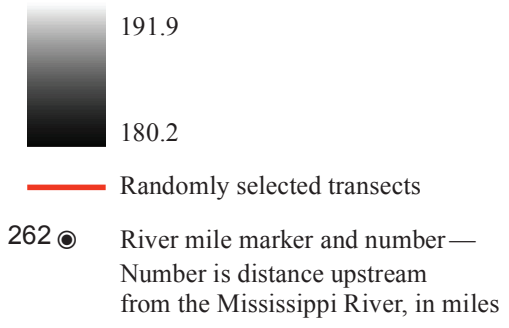

Figure 5. Miami reach in Missouri with June 2007 bathymetry, and randomly selected monitoring transects. 


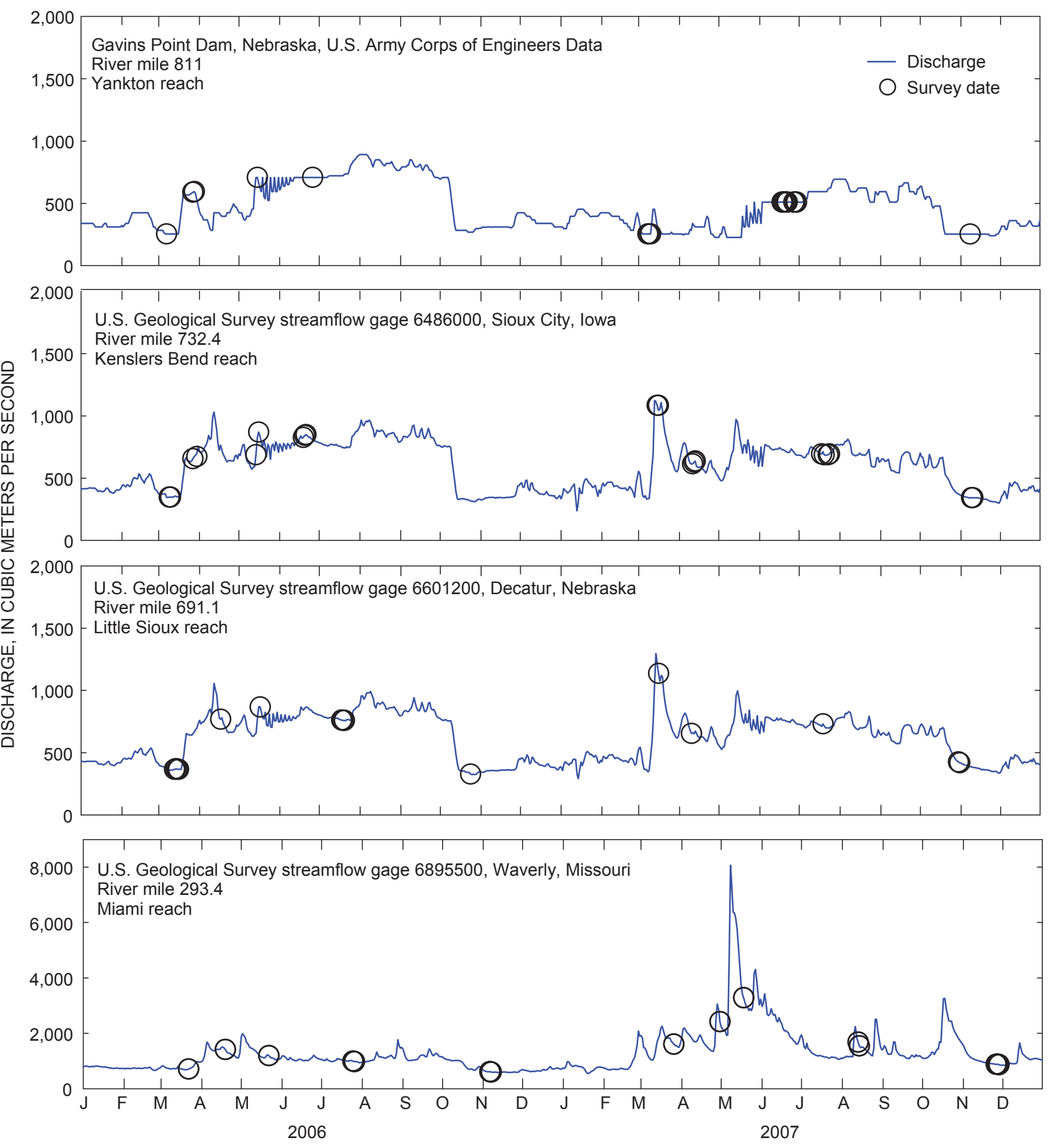

Figure 6. Hydrographs and survey dates for 2006-07 at Gavins Point Dam, Nebr., Sioux City, lowa., Decatur, Nebr., and Waverly, Mo.

lines. Elevation control was established at each site before the first survey was conducted, and the same benchmarks were used throughout the project. At Yankton and Kenslers Bend sites, however, slight differences in the base coordinate units entered in the benchmarks between the 2006 and 2007 surveys resulted in an offset between the lines surveyed in 2006 and 2007. Consequently, at the Yankton site, the 2006 and 2007 surveys can only be compared within years. At the Kenslers Bend site the 2006 surveys and March 2007 surveys can be compared, and the rest of the 2007 surveys can be compared with one another.

Terrestrial RTK-GPS surveys were conducted to extend the hydroacoustic surveys from the water's edge to the top of the bank. These surveys were conducted in the spring and fall low-flow surveys in the Yankton and Kenslers Bend reaches. Due to high flows in the spring in the Little Sioux and Miami reaches, ground surveys only complement the fall low-flow survey in 2007 . 
Longitudinal profiles were collected using the surveygrade echosounders during each survey based on the approximate thalweg location as it existed in 2006 (fig. 7). The same longitudinal profile through the entire reach was resurveyed for each site on each survey date in 2006-07 in the Little Sioux and Miami sites. A second, $500 \mathrm{~m}$ long profile that included at least one channel cross-over was resurveyed after 2.5-3 hours to quantify bedform changes and dimensions. A faster data logging rate $(10 \mathrm{~Hz})$ was used on the longitudinal profiles to acquire higher-resolution data, and boat speed was kept at a minimum, usually slightly higher than current velocity in the thalweg to allow steering. Longitudinal profiles were collected for bedform differencing calculations to develop estimates for one-dimensional bedload transport in the two downstream (Little Sioux and Miami) reaches for 2006 and 2007 (Simons and others, 1965).

Side-scan sonar surveys were performed to provide higher-resolution information on substrate and other habitat features in each of the four reaches in 2007. Side-scan sonar produces a high-resolution image of the bed based on hydroacoustic returns. A $900 \mathrm{kHz}$ towfish (Marine Sonic Technology, Ltd. White Marsh, Virginia) was lowered below the boat using a powered winch. The side-scan was deployed to produce an image of the bed along the boat track, with a total swath width of $40 \mathrm{~m}$. Multiple longitudinal transects were driven at approximately $30-\mathrm{m}$ intervals to produce images covering the full extent of the reaches. Differential GPS positioning was used to approximate the location of the towfish, the precise position of which varies based on the amount of cable deployed. Side-scan allows for interpretation of bottom material and identification of additional habitat features such as large woody debris.

\section{Data Processing and Analysis}

Hydroacoustic and survey data for all four reaches were processed and analyzed by USGS CERC. Data were analyzed using automated scripts where possible for consistency and efficiency. All data are maintained and archived by USGS CERC.

\section{Hydroacoustic Data}

Thirty-four surveys in 2006-07 (table 2) over the four study reaches were completed and processed to obtain watersurface elevation, depth, bed elevation, velocity, and bedvelocity data. Hydroacoustic data processing methods are presented in more detail in Reuter and others (2008). The eight compilation surveys (of all 300 transects) were processed and interpolated into 5-m grids for reach visualization and area change calculations. All data are projected in Universal Transverse Mercator Zone 14 North (Yankton, Kenslers Bend) or 15 North (Little Sioux, Miami) with the World Geodetic System of 1984 datum.

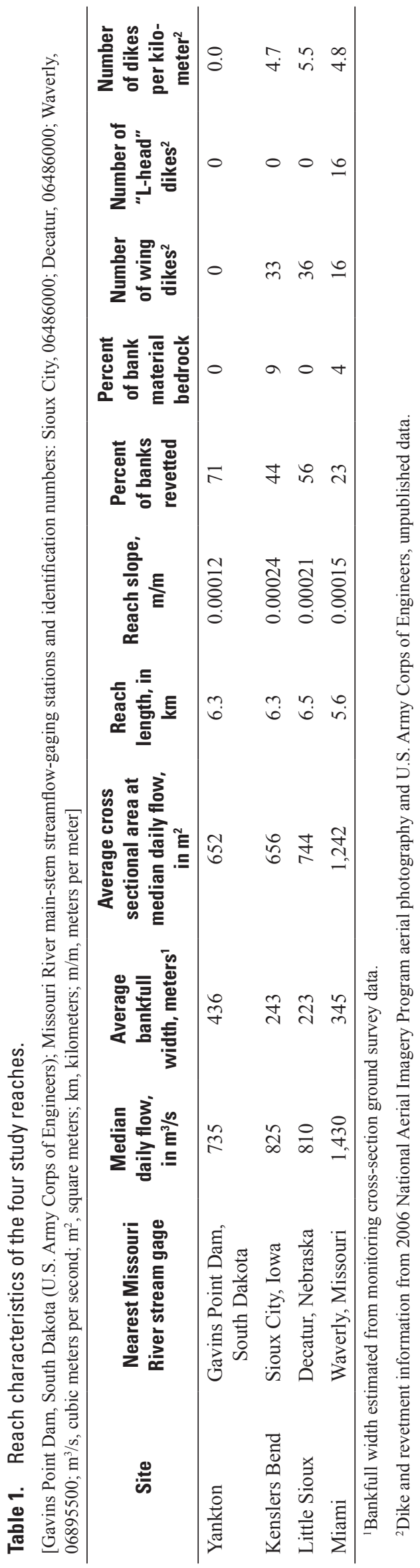


Depth and water-surface elevation data from the echosounder and RTK-GPS for each survey were processed using Hypack software, exported as text files and converted to ArcMap shapefiles (version 9.2, ESRI, Redlands, California). Water surface elevations were checked and adjusted in regions where RTK-GPS quality was degraded, such as under bridges or near the bank where tree cover can block satellite signals or cause multipath errors. Depth was subtracted from watersurface elevation to provide bed elevation.

Bed-elevation points for the randomly selected transects were processed to compare changes between each survey over the 2006-07 period using a custom Python (Python Software Foundation, Hampton, New Hampshire) script. The script projects bed elevations for each transect to the planned line and calculates erosion and deposition areas for $0.25-\mathrm{m}$ increments along the transect between each survey date pair in a spreadsheet. Geomorphic change was calculated for the area common to each pair of survey dates.
Transects that were surveyed multiple times in a day, (typically during reciprocal discharge measurements) also were analyzed to determine survey replicability and acceptable offline data parameters. For the four reaches, the absolute value of the distance offline was low (table 3, 1.04-1.83 m). However, near the banks where obstacles and shallow areas frequently occur and boat navigation is more difficult, data points frequently were further offline than data collected in the middle of transects. To include more near-bank data, a threshold of $3 \mathrm{~m}$ was chosen and scripts were run to include cross-section data for analysis that is within a 3-m distance from planned survey lines.

To allow comparisons between survey reaches spaced over hundreds of river miles with varying degrees of channel engineering, surveys were compared to cross-section areas at median-annual flow. The median-flow area was determined for every cross section, and the average cross-section area for each reach at median-annual flow was then calculated.

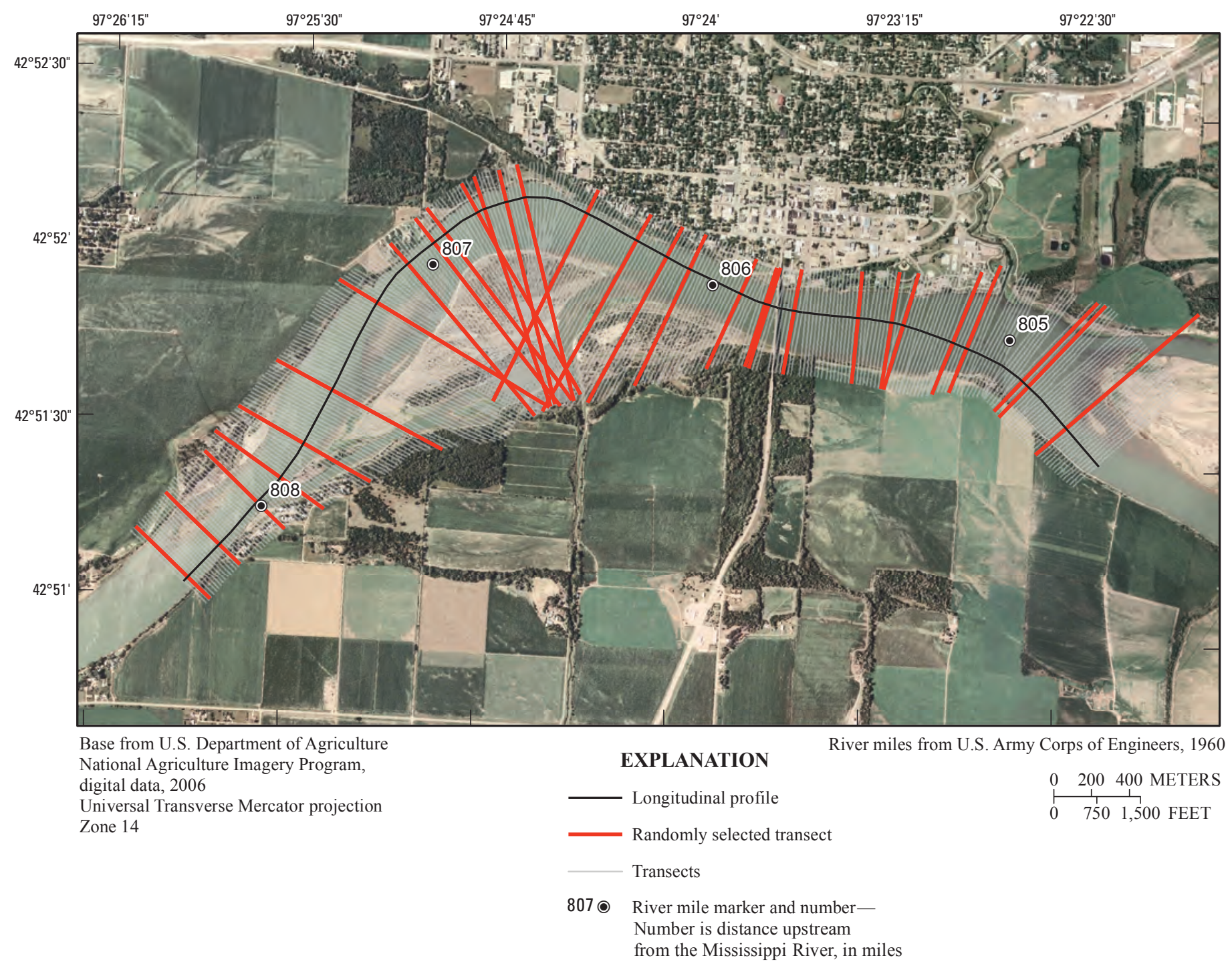

Figure 7. Yankton reach with compilation survey transects, randomly selected survey transects, and long profile lines indicated. 
Table 2. Survey reaches with survey dates and discharges.

\begin{tabular}{|c|c|c|c|c|}
\hline Site & Survey date & Survey crew/vessel & $\begin{array}{l}\text { Discharge (daily average } \\
\left.\qquad \mathrm{m}^{3} / \mathrm{s}\right)\end{array}$ & Gage $^{1}$ \\
\hline \multicolumn{5}{|c|}{2006} \\
\hline \multirow[t]{4}{*}{ Yankton } & $\begin{array}{c}03 / 07 / 2006,03 / 08 / 2006 \\
03 / 10 / 2006\end{array}$ & NE WSC & 245 & Gavins Point Dam \\
\hline & 03/27/2006-03/29/2006 & NE, IA WSC & $590-595$ & Gavins Point Dam \\
\hline & $05 / 15 / 2006$ & NE, IA WSC & 710 & Gavins Point Dam \\
\hline & $06 / 26 / 2006-06 / 28 / 2006$ & NE, IA WSC & 170 & Gavins Point Dam \\
\hline \multirow[t]{4}{*}{ Kenslers Bend } & 03/9/2006, 03/10/2006 & NE WSC & 350 & Sioux City \\
\hline & $03 / 27 / 2006,03 / 30 / 2006$ & IA WSC & $660-675$ & Sioux City \\
\hline & $\begin{array}{c}05 / 14 / 2006,05 / 16 / 2006 \\
05 / 25 / 2006\end{array}$ & NE, IA WSC & $690-870$ & Sioux City \\
\hline & $06 / 19 / 2006-06 / 22 / 2006$ & NE, IA WSC & $825-850$ & Sioux City \\
\hline \multirow[t]{5}{*}{ Little Sioux } & 03/13/2006-03/16/2006 & CERC, R.V. Slim Funk & $365-370$ & Decatur \\
\hline & $04 / 17 / 2006$ & CERC, R.V. Slim Funk & 770 & Decatur \\
\hline & $05 / 17 / 2006$ & CERC, R.V. Slim Funk & 865 & Decatur \\
\hline & 07/18/2006-07/20/2006 & CERC, R.V. Slim Funk & 760 & Decatur \\
\hline & $10 / 24 / 2006$ & CERC, R.V. Slim Funk & 330 & Decatur \\
\hline \multirow[t]{5}{*}{ Miami } & $03 / 23 / 2006$ & CERC, R.V. Lucien Brush & 730 & Waverly \\
\hline & 04/19/2006 & CERC, R.V. Lucien Brush & 1,410 & Waverly \\
\hline & $05 / 22 / 2006$ & CERC, R.V. Lucien Brush & 1,190 & Waverly \\
\hline & 07/25/2006-07/26/2006 & CERC, R.V. Lucien Brush & $975-990$ & Waverly \\
\hline & $11 / 06 / 2006$ & CERC, R.V. Lucien Brush & 600 & Waverly \\
\hline \multicolumn{5}{|c|}{2007} \\
\hline \multirow[t]{3}{*}{ Yankton } & $\begin{array}{c}03 / 08 / 2007,03 / 09 / 2007 \\
03 / 13 / 2007,03 / 14 / 2007\end{array}$ & NE WSC & $255-455$ & Gavins Point Dam \\
\hline & 06/18/2007-06/29/2007 & NE WSC & 510 & Gavins Point Dam \\
\hline & $11 / 08 / 2007$ & NE WSC & 255 & Gavins Point Dam \\
\hline \multirow[t]{4}{*}{ Kenslers Bend } & 03/15/2007, 03/16/2007 & NE WSC & 1,085 & Sioux City \\
\hline & 04/11/2007-04/13/2007 & NE WSC & $615-640$ & Sioux City \\
\hline & 07/18/2007-07/25/2007 & NE WSC & $690-715$ & Sioux City \\
\hline & 11/09/2007, 11/10/2007 & NE WSC & 340 & Sioux City \\
\hline \multirow[t]{4}{*}{ Little Sioux } & 03/16/2007 & CERC, R.V. Slim Funk & 1,140 & Decatur \\
\hline & 04/10/2007 & CERC, R.V. Slim Funk & 655 & Decatur \\
\hline & 07/19/2007, 07/20/2007 & CERC, R.V. Lucien Brush & $715-730$ & Decatur \\
\hline & $10 / 30 / 2007,10 / 31 / 2007$ & CERC, R.V. Slim Funk & $420-425$ & Decatur \\
\hline \multirow[t]{5}{*}{ Miami } & $03 / 26 / 2007$ & $\begin{array}{c}\text { CERC, R.V. Funk w/ Brush } \\
\text { equipment }\end{array}$ & 1,605 & Waverly \\
\hline & $04 / 30 / 2007$ & CERC, R.V. Lucien Brush & 2,420 & Waverly \\
\hline & 05/18/2007 & CERC, R.V. Lucien Brush & 3,285 & Waverly \\
\hline & 08/13/2007-08/15/2007 & CERC, R.V. Lucien Brush & $1,490-1680$ & Waverly \\
\hline & $11 / 26 / 2007-11 / 28 / 2007$ & CERC, R.V. Lucien Brush & $865-880$ & Waverly \\
\hline
\end{tabular}


Table 3. Error values from repeat cross sections at each site.

\begin{tabular}{lccccc}
\hline Site & $\begin{array}{c}\text { Average apparent } \\
\text { deposition (square } \\
\text { meters) }\end{array}$ & $\begin{array}{c}\text { Average apparent } \\
\text { erosion (square } \\
\text { meters) }\end{array}$ & $\begin{array}{c}\text { Average appar- } \\
\text { ent total change } \\
\text { (square meters) }\end{array}$ & $\begin{array}{c}\text { Percent of index } \\
\text { cross section } \\
\text { area' }\end{array}$ & $\begin{array}{c}\text { Average absolute } \\
\text { value of survey dis- } \\
\text { tance off planned } \\
\text { lines, (meters) }\end{array}$ \\
\hline Yankton & 2.3 & -3.8 & 6.1 & 0.9 & 1.83 \\
Kenslers Bend & 7.8 & -17.3 & 25.2 & 3.8 & 1.82 \\
Little Sioux & 8.8 & -13.2 & 22.0 & 3.0 & 1.04 \\
Miami & 21.4 & -24.7 & 46.2 & 3.7 & 1.32 \\
\hline
\end{tabular}

${ }^{1}$ Index cross-section area is calculated from the average median-flow cross-section area.

The reach-average median-flow cross-sectional area, or index cross-sectional area is used to normalize comparisons of geomorphic change among reaches.

\section{Longitudinal Profiles and Bedforms}

Paired longitudinal profiles from the echosounder and RTK-GPS were processed in Hypack to remove erroneous pings related to spurious echoes from fish, turbulence, or poor bottom conditions. These profiles were then analyzed to determine average bedform dimensions and to measure dune movement, which has been demonstrated to be a reliable method for measuring bedload transport in large sand-bedded rivers such as the Missouri (Gaeuman and Jacobson, 2007). Bedform movement rates were calculated by methods developed by the National Center for Earth-surface Dynamics (McElroy, 2006). In the Little Sioux and Miami reaches characteristic bedform calculations were made for the 500-m long region where the paired longitudinal profiles overlap. This calculation was conducted between the longitudinal thalweg profile and the shorter longitudinal thalweg profile driven 2-3 hours later. Characteristic bedform dimensions were calculated for the Yankton and Kenslers Bend reaches from longitudinal profiles, but paired profiles were not available for measurement of dune movement.

\section{Compilation Surveys}

The water-surface and bed-elevation data from the echosounder and RTK-GPS for the compilation surveys were processed in a similar manner as the monitoring surveys to create point data in ArcMap. The data then were interpolated using krigging, blanked to a 5-m grid, and stored in the ESRI grid format (ESRI, Redlands, California) (Reuter and others, 2008). Elevation and depth grids were produced for volumetric change calculations between the summer 2006 and summer 2007 surveys. Calculations were performed in ArcMap using the map calculator function.

\section{Bed Velocity}

Data obtained with an ADCP were processed in Winriver 10.06 software (Teledyne RD Instruments, Poway, California) and exported with bottom-track and GPS positioning to allow for calculation of bed velocities (Gaeuman and Jacobson, 2005 , 2006, 2007). Bed-velocity calculations were performed with a Python script that calculates the difference between the bottom-track and GPS-based boat positions to determine bed velocity for each ensemble. These values also were averaged across a cross section by projecting the values on the navigation lines and taking a length-weighted average to determine an average bed velocity for each cross section in each survey.
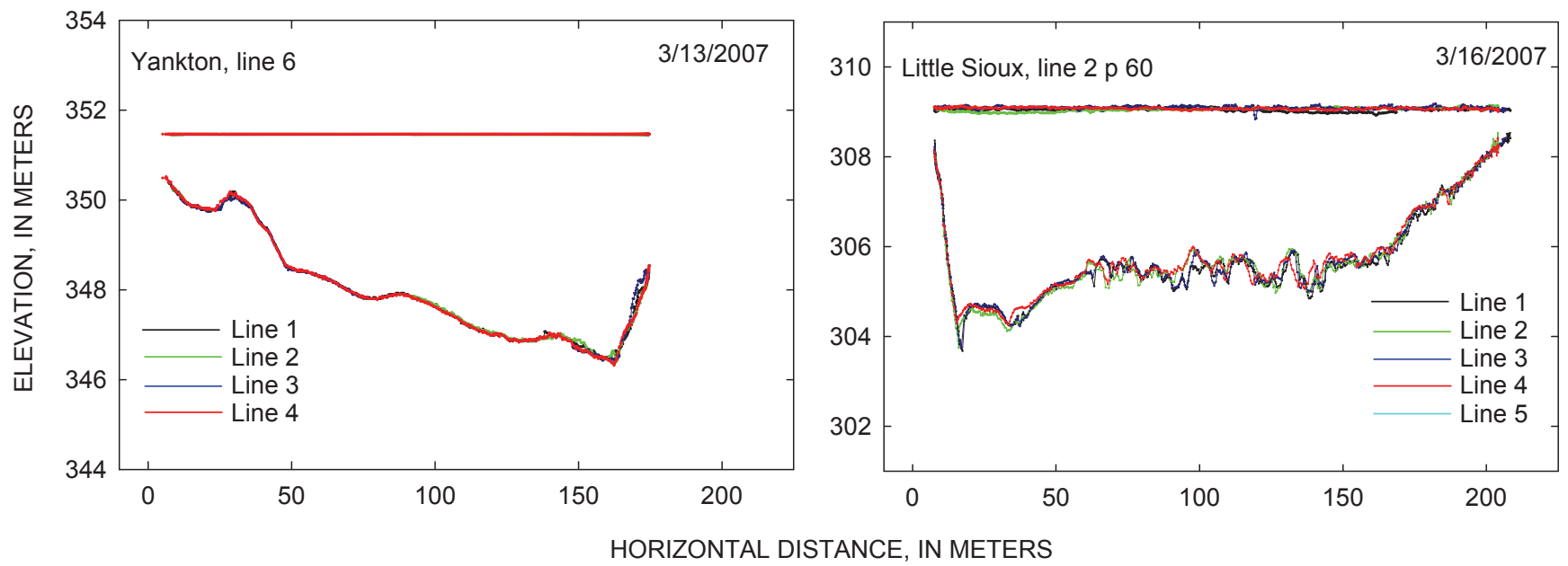

Figure 8. Repeat cross sections at Yankton and the Little Sioux reaches used in cross section change error analysis. 


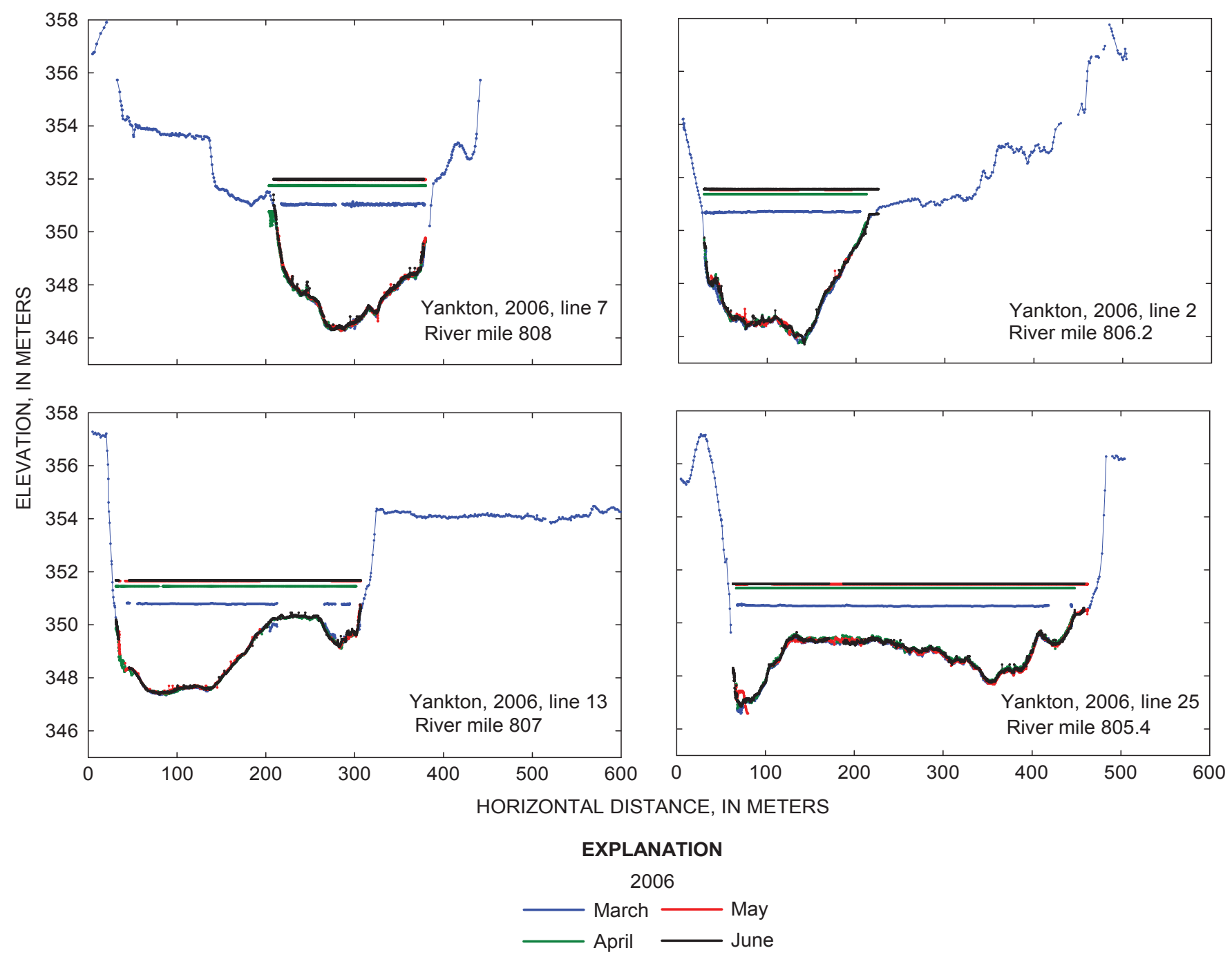

Figure 9. Typical survey cross sections in the Yankton reach.

\section{Cross-Section Comparison Error Analysis}

Lines were driven as closely as possible to the planned lines using Hypack navigation software. The absolute value of the average distance offline for all of the surveys was $1.43 \mathrm{~m}$ (fig. 8, table 3), well below the goal of driving lines to within 3 $\mathrm{m}$ of the planned survey lines. Several sets of repeat cross sections were analyzed at each site for multiple dates to evaluate the error caused by boat navigation. At least four repeat cross sections were driven in succession and compared for differences in erosion and deposition quantities (fig. 8). The range in apparent deposition and erosion calculated from repeat surveys varied for each reach and were smallest at Yankton and largest at Miami (table 3). Variation in the apparent deposition and erosion ranged 2.3- $-24.7 \mathrm{~m}^{2}$ (square meters). Variation in the average apparent total change was $6.1-46.2 \mathrm{~m}^{2}, 0.9$ to 3.8 percent of index cross-sectional areas. These values represent the minimum detectible geomorphic change based on the precision of boat driving and background bottom variation related to bedform amplitudes, to dune movement, and the orientation of the transect across dune fields. Therefore, for each site only changes above these minimum thresholds were considered detectible. For example, for cross sections at Miami, the average median-flow cross-sectional area is $1,242 \mathrm{~m}^{2}$; the minimum detectable change $\left(46.2 \mathrm{~m}^{2}\right)$ is approximately 3.7 percent of the total cross-sectional area (table 3). Minimum detectible areas were lowest at Yankton because of fewer large sand dunes and less overall topographic variation in the bed. At sites with larger dunes, such as the Kenslers Bend, Little Sioux, and Miami reaches (for example, fig. 8), a slight change in boat navigation could represent the difference between a dune crest and trough, and, therefore, more variation in area change detection is possible for repeat transects. 
Table 4. Cross-section comparison values for the Yankton reach, 2006-07.

\begin{tabular}{|c|c|c|c|c|c|c|c|c|c|c|c|c|c|}
\hline \multirow[b]{2}{*}{$\begin{array}{l}\text { River } \\
\text { mile }\end{array}$} & \multirow[b]{2}{*}{$\begin{array}{c}\text { Line } \\
\text { number }\end{array}$} & \multicolumn{3}{|c|}{ March 2006 to March II 2006} & \multicolumn{3}{|c|}{ March II 2006 to May 2006} & \multicolumn{3}{|c|}{ May 2006 to June 2006} & \multicolumn{3}{|c|}{ March 2006 to June 2006} \\
\hline & & $\begin{array}{l}\text { Deposi- } \\
\text { tion area } \\
\text { (square } \\
\text { meters) }\end{array}$ & $\begin{array}{l}\text { Erosion } \\
\text { area } \\
\text { (square } \\
\text { meters) }\end{array}$ & $\begin{array}{c}\text { Net } \\
\text { change } \\
\text { (square } \\
\text { meters) }\end{array}$ & $\begin{array}{l}\text { Deposi- } \\
\text { tion area } \\
\text { (square } \\
\text { meters) }\end{array}$ & $\begin{array}{l}\text { Erosion } \\
\text { area, } \\
\text { (square } \\
\text { meters) }\end{array}$ & $\begin{array}{c}\text { Net } \\
\text { change } \\
\text { (square } \\
\text { meters) }\end{array}$ & $\begin{array}{l}\text { Deposi- } \\
\text { tion area } \\
\text { (square } \\
\text { meters) }\end{array}$ & $\begin{array}{l}\text { Erosion } \\
\text { area } \\
\text { (square } \\
\text { meters) }\end{array}$ & $\begin{array}{c}\text { Net } \\
\text { change } \\
\text { (square } \\
\text { meters) }\end{array}$ & $\begin{array}{c}\text { Deposi- } \\
\text { tion area } \\
\text { (square } \\
\text { meters) }\end{array}$ & $\begin{array}{l}\text { Erosion } \\
\text { area } \\
\text { (square } \\
\text { meters) }\end{array}$ & $\begin{array}{c}\text { Net } \\
\text { change } \\
\text { (square } \\
\text { meters) }\end{array}$ \\
\hline 808.3 & 5 & 3.6 & -3.4 & 0.2 & 1.2 & -0.2 & 0.9 & 0.0 & 0.0 & 0.0 & 3.4 & -0.9 & 2.5 \\
\hline 808.2 & 6 & 3.8 & -0.6 & 3.1 & 4.7 & -0.3 & 4.4 & 0.8 & -2.7 & -1.9 & 10.1 & -0.4 & 9.7 \\
\hline 808 & 7 & 4.7 & -2.2 & 2.5 & 7.8 & -1.1 & 6.8 & 4.2 & -2.5 & 1.7 & 11.1 & -0.6 & 10.5 \\
\hline 807.9 & 8 & 7.8 & -0.7 & 7.2 & 3.4 & -2.1 & 1.3 & 1.6 & -4.9 & -3.3 & 6.6 & -1.7 & 4.9 \\
\hline 807.7 & 9 & 4.5 & -2.1 & 2.5 & 3.0 & -4.2 & -1.2 & 9.7 & -0.1 & 9.6 & 9.8 & -0.1 & 9.7 \\
\hline 807.5 & 10 & 10.7 & -6.5 & 4.2 & 0.1 & -0.7 & -0.6 & 1.2 & 0.0 & 1.2 & 9.6 & -4.3 & 5.3 \\
\hline 807.2 & 11 & 2.6 & -5.3 & -2.7 & 3.4 & -0.8 & 2.6 & 0.4 & -2.1 & -1.7 & 5.6 & -3.5 & 2.1 \\
\hline 807.1 & 12 & 1.0 & -4.5 & -3.5 & 3.5 & -0.3 & 3.2 & 1.7 & -1.3 & 0.4 & 4.1 & -1.4 & 2.7 \\
\hline 807 & 13 & 5.1 & -3.5 & 1.6 & 5.4 & -1.6 & 3.8 & 2.2 & -3.2 & -1.0 & 6.8 & -3.2 & 3.5 \\
\hline 806.9 & 14 & 4.3 & -3.4 & 0.9 & 5.3 & -3.0 & 2.3 & 6.1 & -6.4 & -0.2 & 5.5 & -3.7 & 1.8 \\
\hline 806.8 & 15 & 2.6 & -4.5 & -1.9 & 21.0 & -0.3 & 20.7 & 1.6 & -11.1 & -9.6 & 9.6 & -0.8 & 8.8 \\
\hline 806.8 & 16 & 1.1 & -13.7 & -12.7 & 14.5 & -0.4 & 14.0 & 5.6 & -2.7 & 2.9 & 8.9 & -2.8 & 6.0 \\
\hline 806.7 & 17 & 4.3 & -2.2 & 2.0 & 6.8 & -0.7 & 6.1 & 0.3 & -10.1 & -9.8 & 1.8 & -3.5 & -1.7 \\
\hline 806.6 & 18 & 18.9 & -1.3 & 17.6 & 2.7 & -7.5 & -4.8 & 7.6 & -5.2 & 2.4 & 18.4 & -1.3 & 17.2 \\
\hline 806.5 & 19 & 13.0 & -3.4 & 9.6 & 7.1 & -1.2 & 5.9 & 2.2 & -5.8 & -3.6 & 7.9 & -2.8 & 5.1 \\
\hline 806.3 & 1 & 7.5 & -1.0 & 6.4 & 11.8 & -0.4 & 11.5 & 3.9 & -4.7 & -0.8 & 18.6 & -0.4 & 18.2 \\
\hline 806.2 & 2 & 14.7 & -2.1 & 12.6 & 6.1 & -3.6 & 2.5 & 4.2 & -4.8 & -0.6 & 17.2 & -1.2 & 15.9 \\
\hline 806.1 & 3 & 18.5 & -2.5 & 16.0 & 2.5 & -19.3 & -16.8 & 17.0 & -0.5 & 16.5 & 15.3 & -2.7 & 12.6 \\
\hline 805.9 & 4 & 11.7 & -3.3 & 8.4 & 3.3 & -11.8 & -8.6 & 18.1 & -2.1 & 16.0 & 18.6 & -2.8 & 15.8 \\
\hline 805.8 & 20 & 10.2 & -3.0 & 7.2 & 3.3 & -16.4 & -13.2 & 23.1 & -4.3 & 18.7 & 16.3 & -2.7 & 13.7 \\
\hline 805.8 & 21 & 11.8 & -4.9 & 6.8 & 2.7 & -21.0 & -18.3 & 24.5 & -0.5 & 24.0 & 16.2 & -2.5 & 13.7 \\
\hline 805.7 & 22 & 12.0 & -2.0 & 10.0 & 2.2 & -14.2 & -12.0 & 12.7 & -4.3 & 8.3 & 10.2 & -2.9 & 7.3 \\
\hline 805.5 & 23 & 0.7 & -0.2 & 0.5 & -no data- & -no data- & -no data- & -no data- & -no data- & -no data- & 0.6 & -0.5 & 0.2 \\
\hline 805.4 & 24 & 28.8 & -0.8 & 28.0 & 1.5 & -30.9 & -29.4 & 23.2 & -1.8 & 21.4 & 21.5 & -1.3 & 20.1 \\
\hline 805.4 & 25 & 34.1 & -0.5 & 33.6 & 5.0 & -28.2 & -23.2 & 16.8 & -7.3 & 9.6 & 22.3 & -1.6 & 20.6 \\
\hline 805.2 & 26 & 25.2 & -4.3 & 21.0 & 3.7 & -28.2 & -24.6 & 26.8 & -3.4 & 23.4 & 23.3 & -3.8 & 19.5 \\
\hline 805.1 & 27 & 38.7 & -2.6 & 36.1 & 2.8 & -17.5 & -14.7 & 6.2 & -10.6 & -4.3 & 16.5 & -6.3 & 10.2 \\
\hline 804.9 & 28 & -no data- & -no data- & -no data- & -no data- & -no data- & -no data- & 5.2 & 0.0 & 5.2 & -no data- & -no data- & -no data- \\
\hline $\begin{array}{l}804.8 \\
804.6\end{array}$ & $\begin{array}{l}29 \\
30\end{array}$ & $\begin{array}{l}\text {-no data- } \\
\text {-no data- }\end{array}$ & $\begin{array}{l}\text {-no data- } \\
\text {-no data- }\end{array}$ & $\begin{array}{l}\text {-no data- } \\
\text {-no data- }\end{array}$ & $\begin{array}{l}\text {-no data- } \\
\text {-no data- }\end{array}$ & $\begin{array}{l}\text {-no data- } \\
\text {-no data- }\end{array}$ & $\begin{array}{l}\text {-no data- } \\
\text {-no data- }\end{array}$ & $\begin{array}{c}\text {-no data- } \\
0.2\end{array}$ & $\begin{array}{l}\text {-no data- } \\
-0.2\end{array}$ & -no data- & $\begin{array}{l}\text {-no data- } \\
\text {-no data- }\end{array}$ & $\begin{array}{l}\text {-no data- } \\
\text {-no data- }\end{array}$ & $\begin{array}{l}\text {-no data- } \\
\text {-no data- }\end{array}$ \\
\hline \multicolumn{2}{|c|}{ Total area } & 302.1 & -84.6 & 217.4 & 134.7 & -216.0 & -81.3 & 227.1 & -102.8 & 124.2 & 315.7 & -59.7 & 256.0 \\
\hline \multicolumn{2}{|c|}{$\begin{array}{l}\text { Number of cross } \\
\text { sections }\end{array}$} & 27 & 27 & 27 & 26 & 26 & 26 & 28 & 28 & 28 & 27 & 27 & 27 \\
\hline \multicolumn{2}{|l|}{ Average } & 11.2 & -3.1 & 8.1 & 5.2 & -8.3 & -3.1 & 8.1 & -3.7 & 4.4 & 11.7 & -2.2 & 9.5 \\
\hline
\end{tabular}


Table 4. Cross-section comparison values for the Yankton reach, 2006-07.-Continued

\begin{tabular}{|c|c|c|c|c|c|c|c|c|c|c|}
\hline \multirow[b]{2}{*}{$\begin{array}{l}\text { River } \\
\text { mile }\end{array}$} & \multirow[b]{2}{*}{$\begin{array}{c}\text { Line } \\
\text { number }\end{array}$} & \multicolumn{3}{|c|}{ March 2007 to June 2007} & \multicolumn{3}{|c|}{ June 2007 to November 2007} & \multicolumn{3}{|c|}{ March 2007 to November 2007} \\
\hline & & $\begin{array}{c}\text { Deposi- } \\
\text { tion area } \\
\text { (square } \\
\text { meters) }\end{array}$ & $\begin{array}{l}\text { Erosion } \\
\text { area } \\
\text { (square } \\
\text { meters) }\end{array}$ & $\begin{array}{c}\text { Net } \\
\text { change } \\
\text { (square } \\
\text { meters) }\end{array}$ & $\begin{array}{c}\text { Deposi- } \\
\text { tion area } \\
\text { (square } \\
\text { meters) }\end{array}$ & $\begin{array}{l}\text { Erosion } \\
\text { area } \\
\text { (square } \\
\text { meters) }\end{array}$ & $\begin{array}{c}\text { Net } \\
\text { change } \\
\text { (square } \\
\text { meters) }\end{array}$ & $\begin{array}{c}\text { Deposi- } \\
\text { tion area } \\
\text { (square } \\
\text { meters) }\end{array}$ & $\begin{array}{c}\text { Erosion } \\
\text { area } \\
\text { (square } \\
\text { meters) }\end{array}$ & $\begin{array}{c}\text { Net } \\
\text { change } \\
\text { (square } \\
\text { meters) }\end{array}$ \\
\hline 808.3 & 5 & 0.7 & -18.6 & -18.0 & 3.9 & -11.3 & -7.4 & 0.1 & -20.9 & -20.8 \\
\hline 808.2 & 6 & 0.5 & -20.9 & -20.4 & 6.8 & -6.3 & 0.5 & 0.7 & -20.5 & -19.8 \\
\hline 808 & 7 & -no data- & -no data- & -no data- & -no data- & -no data- & -no data- & -no data- & -no data- & -no data- \\
\hline 807.9 & 8 & 0.6 & -7.3 & -6.8 & 4.5 & -7.6 & -3.1 & 0.4 & -11.6 & -11.2 \\
\hline 807.7 & 9 & 3.0 & -7.2 & -4.3 & 2.3 & -8.5 & -6.1 & 1.1 & -10.8 & -9.8 \\
\hline 807.5 & 10 & 4.8 & -22.5 & -17.6 & 11.2 & -16.9 & -5.7 & 0.2 & -23.5 & -23.3 \\
\hline 807.2 & 11 & 7.7 & -3.4 & 4.3 & 0.1 & -26.7 & -26.6 & 0.1 & -22.4 & -22.3 \\
\hline 807.1 & 12 & 3.4 & -4.6 & -1.2 & 0.8 & -13.4 & -12.6 & 0.7 & -15.1 & -14.4 \\
\hline 807 & 13 & 8.5 & -0.9 & 7.7 & 0.0 & -24.1 & -24.1 & 0.2 & -15.9 & -15.7 \\
\hline 806.9 & 14 & 17.1 & -0.3 & 16.9 & 3.3 & -19.7 & -16.4 & 6.5 & -6.7 & -0.2 \\
\hline 806.8 & 15 & 3.7 & -5.4 & -1.7 & 0.5 & -23.4 & -23.0 & 0.3 & -24.9 & -24.7 \\
\hline 806.8 & 16 & 0.4 & -5.1 & -4.8 & 0.5 & -6.0 & -5.5 & 0.0 & -10.3 & -10.3 \\
\hline 806.7 & 17 & 0.3 & -15.9 & -15.6 & 1.5 & -6.8 & -5.3 & 0.1 & -18.5 & -18.5 \\
\hline 806.6 & 18 & 1.0 & -9.3 & -8.3 & 2.8 & -6.6 & -3.9 & 0.2 & -8.6 & -8.4 \\
\hline 806.5 & 19 & 4.7 & -23.0 & -18.3 & 12.3 & -9.7 & 2.6 & 10.1 & -29.4 & -19.4 \\
\hline 806.3 & 1 & 1.0 & -16.7 & -15.6 & 11.1 & -7.7 & 3.4 & 5.7 & -18.1 & -12.3 \\
\hline 806.2 & 2 & 1.9 & -22.8 & -20.9 & 3.5 & -7.5 & -4.0 & 1.4 & -26.3 & -24.9 \\
\hline 806.1 & 3 & -no data- & -no data- & -no data- & 0.0 & -1.4 & -1.4 & -no data- & -no data- & -no data- \\
\hline 805.9 & 4 & -no data- & -no data- & -no data- & 0.0 & -0.4 & -0.4 & -no data- & -no data- & -no data- \\
\hline 805.8 & 20 & -no data- & -no data- & -no data- & -no data- & -no data- & -no data- & -no data- & -no data- & -no data- \\
\hline 805.8 & 21 & -no data- & -no data- & -no data- & 0.0 & -0.3 & -0.3 & -no data- & -no data- & -no data- \\
\hline 805.7 & 22 & -no data- & -no data- & -no data- & 0.0 & -0.9 & -0.9 & -no data- & -no data- & -no data- \\
\hline 805.5 & 23 & 2.8 & -12.6 & -9.7 & 0.6 & -20.8 & -20.2 & 0.6 & -29.0 & -28.4 \\
\hline 805.4 & 24 & 13.1 & -3.2 & 9.9 & 0.0 & -39.4 & -39.4 & 0.3 & -30.5 & -30.2 \\
\hline 805.4 & 25 & 17.3 & -10.3 & 7.0 & 3.6 & -41.4 & -37.8 & 0.6 & -31.5 & -30.9 \\
\hline 805.2 & 26 & 7.9 & -28.7 & -20.8 & 8.3 & -21.9 & -13.6 & 1.1 & -38.1 & -36.9 \\
\hline 805.1 & 27 & 0.1 & -47.7 & -47.6 & 30.6 & -1.2 & 29.4 & 3.8 & -18.0 & -14.3 \\
\hline 804.9 & 28 & -no data- & -no data- & -no data- & -no data- & -no data- & -no data- & -no data- & -no data- & -no data- \\
\hline 804.8 & 29 & 0.2 & -1.6 & -1.5 & 0.0 & -4.5 & -4.5 & 0.0 & -5.7 & -5.7 \\
\hline 804.6 & 30 & 0.3 & -11.5 & -11.2 & 2.2 & -5.7 & -3.5 & 0.8 & -15.5 & -14.7 \\
\hline \multicolumn{2}{|c|}{ Total area } & 100.9 & -299.3 & -198.4 & 110.4 & -340.2 & -229.8 & 34.8 & -451.9 & -417.1 \\
\hline \multicolumn{2}{|c|}{$\begin{array}{l}\text { Number of cross } \\
\text { sections }\end{array}$} & 23 & 23 & 23 & 23 & 27 & 27 & 23 & 23 & 23 \\
\hline \multicolumn{2}{|l|}{ Average } & 4.4 & -13.0 & -8.6 & 4.8 & -12.6 & -8.5 & 1.5 & -19.6 & -18.1 \\
\hline
\end{tabular}




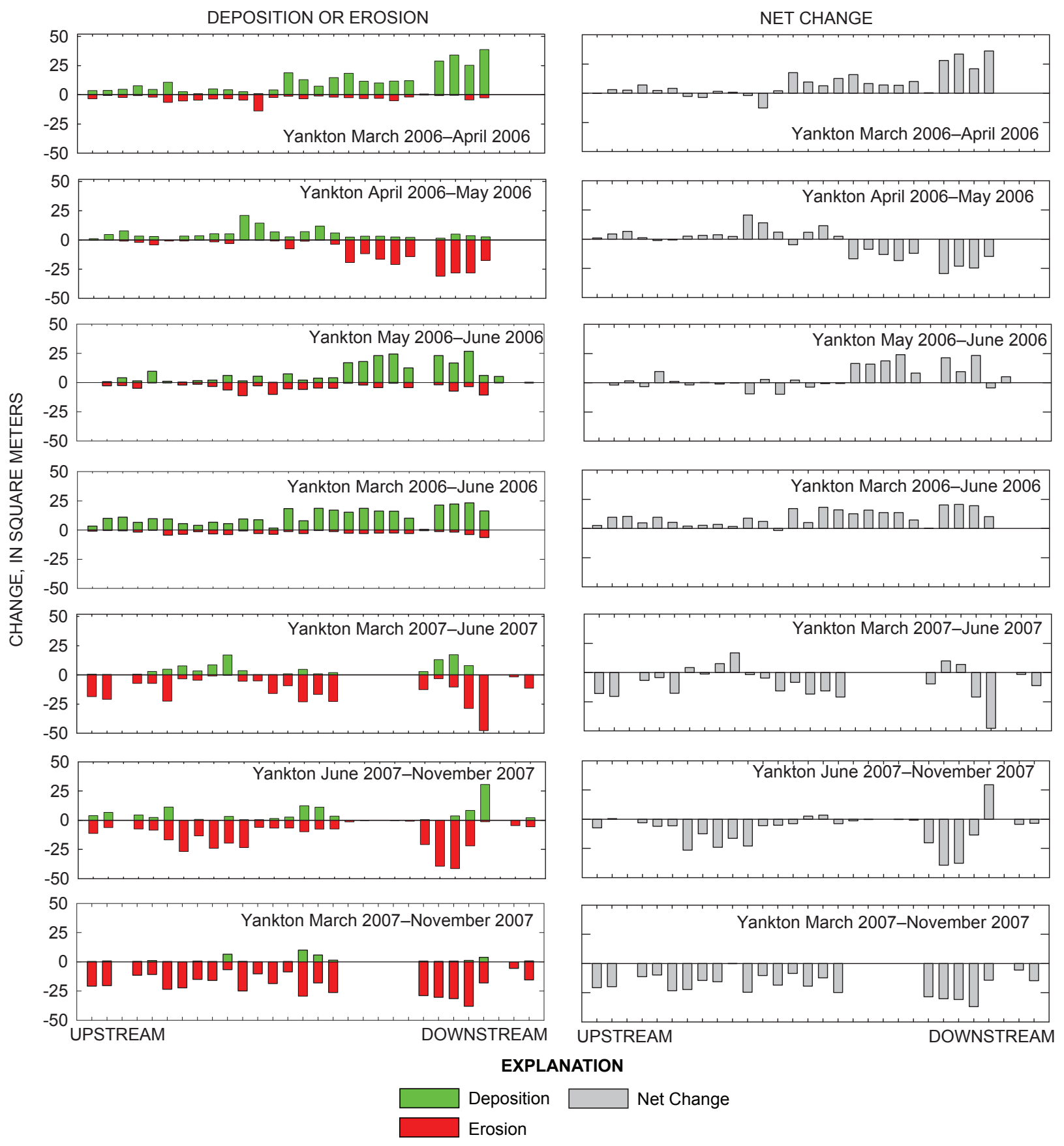

Figure 10. Deposition, erosion, and net change for the Yankton reach.

\section{Results}

The following section presents results of monitoring the four reaches over 2006-07. Monitoring results for cross-section change, longitudinal profiles, bedforms, substrate, and bed velocity are presented for each reach. There are many possible combinations of dates to compare for the four sites and we have chosen to present the bulk of the results in the form of figures and tables and highlight the main trends in the text.

\section{Cross-Section Change}

Change for the purposes of this analysis is only calculated for the common area between a pair of cross-section surveys. Ground survey points were not collected for every survey date and are not as dense as boat survey points, therefore, change calculations do not include edges of cross sections; field observations indicated that bank erosion was negligible during the course of this study. 

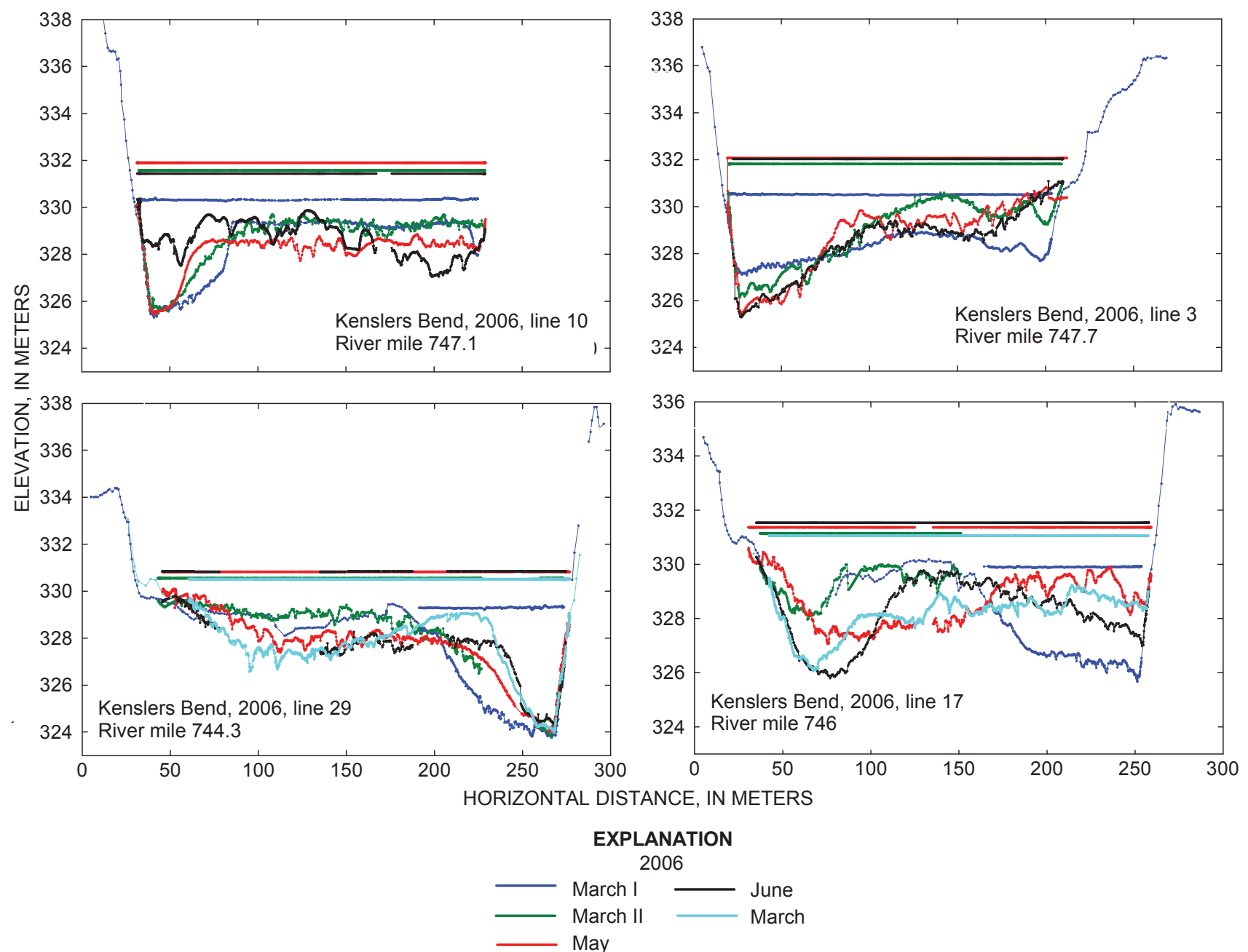

Figure 11. Typical survey cross sections in the Kenslers Bend reach.

\section{Yankton Reach}

Thirty cross sections were surveyed in the Yankton reach three times each in 2006 and 2007 (figs. 9, 10, appendix 1). The planned lines for these surveys were slightly different in 2006 than 2007, therefore, the cross-section comparisons and areas were calculated accordingly (table 4). Net cross-section change (fig. 10, table 4) was minimal, generally less than 3 percent compared to the index cross-section area for the study period. Typical cross sections (fig. 9) showed relatively little change in cross-section form and structure during the survey period. Magnitudes of erosion and deposition were often below the range of change detection based on error analysis (tables 3, 4).

There was a small amount of net erosion in the April 2006-May 2006 time period which encompasses the May 12 spring rise event from Gavins Point Dam (fig. 10, table 4). Magnitudes of erosion and deposition were highest in the cross sections located downstream from river mile 806.5, including the region adjacent to the Yankton Bridge at Highway 81 . This was also the narrowest portion of the Yankton monitoring reach.

\section{Kenslers Bend Reach}

In the Kenslers Bend reach, 30 cross sections were surveyed four times each in 2006 and 2007 (appendix 2, figs. 11, 12, table 5). Morphodynamic changes in the Kenslers Bend reach were much more variable and of a higher magnitude than the Yankton reach. The average net change for all cross sections was -8.5-8.5 percent of the index cross-section area. Typical cross sections show the types of scour and fill that occurred in this reach (fig. 11). Changes in bed elevation were substantial compared to the Yankton reach.

Overall trends were net deposition of $36.9 \mathrm{~m}^{2}$ on average for each cross section in 2006 and a nearly opposite trend of net erosion averaging $-36.1 \mathrm{~m}^{2}$ per cross section, in 2007 (table 5). Some individual cross sections had net erosion in 2006 and deposition in 2007, opposite of overall reach trends (fig. 12, table 5). March 2006-April 2006 was a time period of net deposition in all but one cross section, followed by a time period of net erosion from April 2006-May 2006, in most cross sections, which includes the May pulsed flow from Gavins Point Dam. 
DEPOSITION OR EROSION

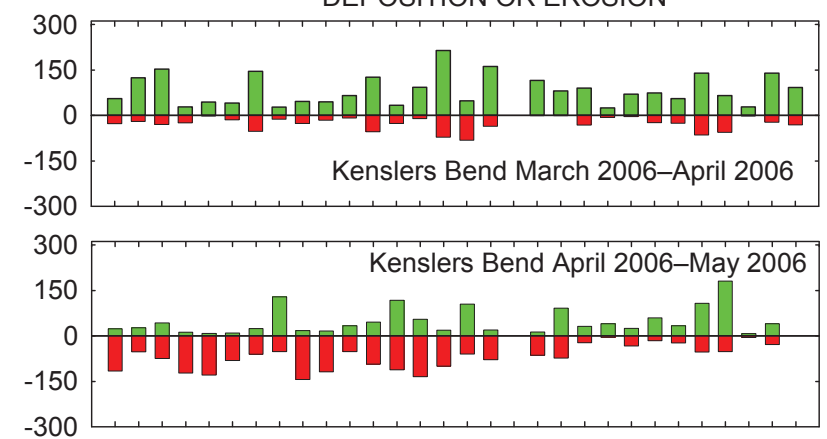

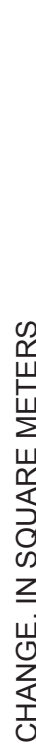

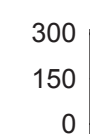
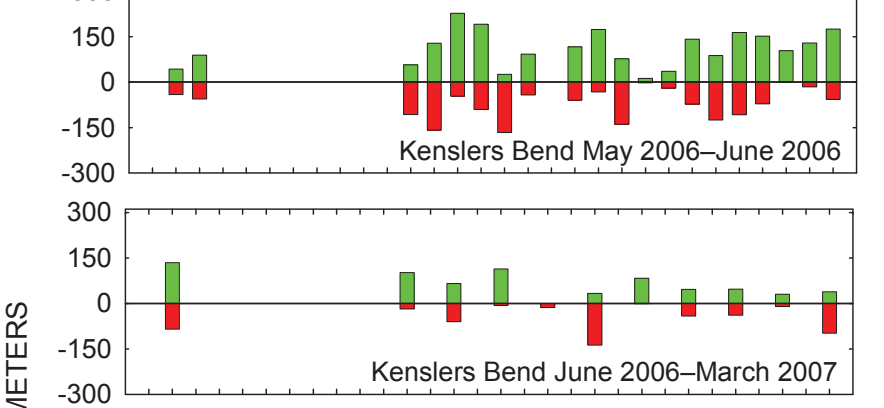

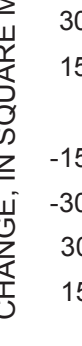

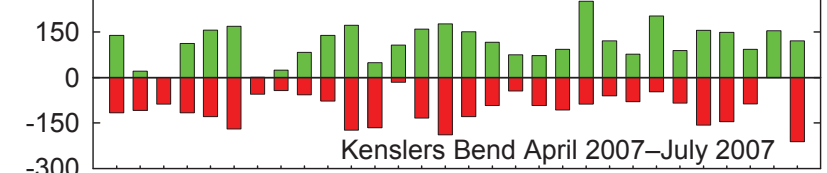

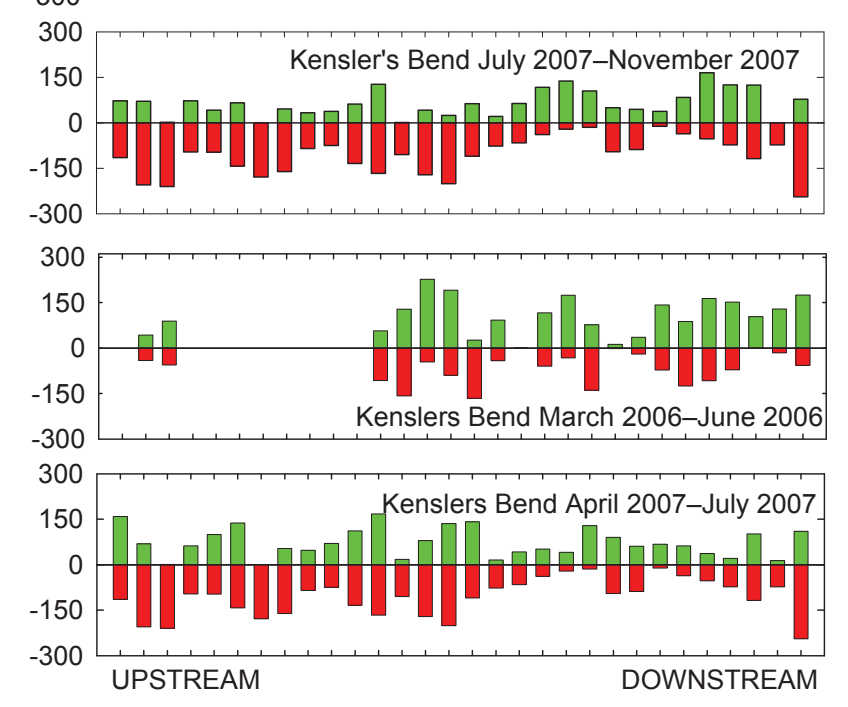

NET CHANGE
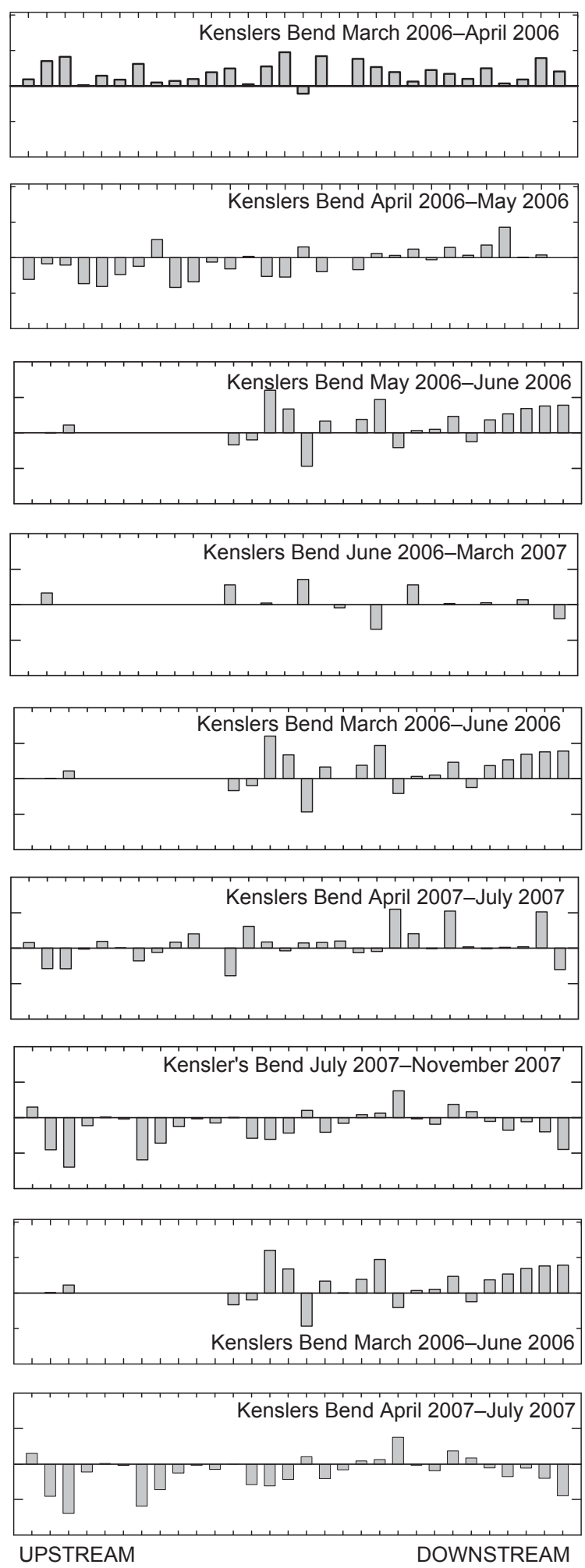

\section{EXPLANATION}

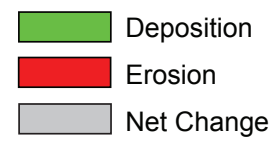

Figure 12. Deposition, erosion, and net change for the Kenslers Bend reach. 
Table 5. Cross section comparison values for the Kenslers Bend reach, 2006-07.

\begin{tabular}{|c|c|c|c|c|c|c|c|c|c|c|}
\hline \multirow[b]{2}{*}{ River mile } & \multirow[b]{2}{*}{$\begin{array}{c}\text { Line } \\
\text { number }\end{array}$} & \multicolumn{3}{|c|}{ March 2006 to March II 2006} & \multicolumn{3}{|c|}{ March II 2006 to May 2006} & \multicolumn{3}{|c|}{ May 2006 to June 2006} \\
\hline & & $\begin{array}{c}\text { Deposi- } \\
\text { tion area } \\
\text { (square } \\
\text { meters) }\end{array}$ & $\begin{array}{c}\text { Erosion } \\
\text { area } \\
\text { (square } \\
\text { meters) }\end{array}$ & $\begin{array}{l}\text { Net change } \\
\text { (square } \\
\text { meters) }\end{array}$ & $\begin{array}{c}\text { Deposi- } \\
\text { tion area } \\
\text { (square } \\
\text { meters) }\end{array}$ & $\begin{array}{c}\text { Erosion } \\
\text { area, } \\
\text { (square } \\
\text { meters) }\end{array}$ & $\begin{array}{l}\text { Net change } \\
\text { (square } \\
\text { meters) }\end{array}$ & $\begin{array}{c}\text { Deposi- } \\
\text { tion area } \\
\text { (square } \\
\text { meters) }\end{array}$ & $\begin{array}{l}\text { Erosion } \\
\text { area } \\
\text { (square } \\
\text { meters) }\end{array}$ & $\begin{array}{c}\text { Net change } \\
\text { (square } \\
\text { meters) }\end{array}$ \\
\hline 747.8 & 1 & 55.5 & -27.0 & 28.5 & 23.8 & -115.6 & -91.7 & -no data- & -no data- & -no data- \\
\hline 747.7 & 2 & 124.3 & -19.4 & 104.9 & 27.1 & -52.0 & -25.0 & 0.3 & -73.4 & -73.0 \\
\hline 747.7 & 3 & 153.0 & -29.1 & 123.9 & 42.9 & -74.0 & -31.0 & 8.7 & -67.8 & -59.1 \\
\hline 747.5 & 4 & 28.4 & -24.6 & 3.8 & 12.2 & -121.7 & -109.5 & -no data- & -no data- & -no data- \\
\hline 747.4 & 5 & 44.6 & -0.9 & 43.7 & 8.2 & -129.1 & -120.8 & -no data- & -no data- & -no data- \\
\hline 747.4 & 6 & 41.3 & -14.3 & 27.0 & 9.7 & -80.9 & -71.1 & -no data- & -no data- & -no data- \\
\hline 747.2 & 7 & 145.8 & -52.4 & 93.5 & 24.3 & -60.6 & -36.2 & -no data- & -no data- & -no data- \\
\hline 747.2 & 8 & 27.3 & -12.1 & 15.2 & 128.8 & -51.6 & 77.2 & -no data- & -no data- & -no data- \\
\hline 747.1 & 9 & 47.0 & -25.9 & 21.1 & 18.3 & -143.6 & -125.3 & -no data- & -no data- & -no data- \\
\hline 747.1 & 10 & 44.9 & -15.9 & 29.0 & 16.3 & -117.7 & -101.4 & -no data- & -no data- & -no data- \\
\hline 747 & 11 & 66.2 & -8.0 & 58.2 & 33.8 & -51.7 & -17.9 & -no data- & -no data- & -no data- \\
\hline 746.8 & 12 & 127.2 & -53.0 & 74.2 & 46.3 & -93.0 & -46.7 & 5.4 & -85.8 & -80.5 \\
\hline 746.7 & 13 & 33.8 & -25.9 & 7.9 & 118.3 & -111.9 & 6.3 & 55.2 & -47.5 & 7.7 \\
\hline 746.7 & 14 & 92.9 & -9.8 & 83.1 & 55.2 & -133.9 & -78.7 & 118.8 & -84.9 & 33.9 \\
\hline 746.6 & 15 & 214.6 & -72.0 & 142.6 & 19.1 & -100.0 & -80.9 & 193.7 & -155.3 & 38.3 \\
\hline 746.5 & 16 & 48.7 & -81.4 & -32.6 & 105.2 & -59.3 & 45.8 & 21.9 & -140.3 & -118.4 \\
\hline 746.3 & 30 & 162.1 & -35.8 & 126.3 & 19.7 & -78.3 & -58.6 & 148.9 & -60.5 & 88.4 \\
\hline 746 & 17 & -no data- & -no data- & -no data- & -no data- & -no data- & -no data- & 14.1 & 0.0 & 14.1 \\
\hline 745.7 & 18 & 115.2 & -0.1 & 115.1 & 13.5 & -63.5 & -50.0 & 13.8 & -90.1 & -76.2 \\
\hline 745.7 & 19 & 80.9 & -0.4 & 80.4 & 91.5 & -73.4 & 18.1 & 35.6 & -96.1 & -60.5 \\
\hline 745.2 & 20 & 90.5 & -31.7 & 58.8 & 31.9 & -22.1 & 9.8 & 15.2 & -4.1 & 11.1 \\
\hline 745.1 & 28 & 25.1 & -6.0 & 19.1 & 40.2 & -4.0 & 36.2 & 0.7 & -40.6 & -39.9 \\
\hline 745 & 21 & 70.9 & -3.3 & 67.6 & 25.3 & -33.5 & -8.2 & 5.5 & -46.0 & -40.6 \\
\hline 744.8 & 26 & 74.8 & -23.5 & 51.3 & 59.7 & -16.0 & 43.8 & 33.4 & -25.2 & 8.3 \\
\hline 744.7 & 27 & 55.2 & -25.4 & 29.8 & 34.2 & -23.1 & 11.1 & 15.7 & -98.1 & -82.4 \\
\hline 744.5 & 22 & 139.7 & -64.7 & 75.0 & 107.3 & -52.9 & 54.4 & 15.1 & -29.9 & -14.8 \\
\hline 744.4 & 23 & 66.2 & -55.5 & 10.7 & 180.9 & -51.7 & 129.2 & 9.6 & -26.1 & -16.5 \\
\hline 744.3 & 29 & 28.6 & -1.3 & 27.3 & 7.8 & -5.5 & 2.2 & 38.0 & -3.3 & 34.7 \\
\hline 744.1 & 24 & 139.9 & -22.0 & 117.9 & 40.1 & -28.5 & 11.6 & 8.5 & -65.3 & -56.8 \\
\hline 744.1 & 25 & 92.4 & -31.1 & 61.3 & -no data- & -no data- & -no data- & -no data- & -no data- & -no data- \\
\hline Total area & & $2,436.8$ & -772.4 & $1,664.5$ & $1,341.6$ & $-1,949.3$ & -607.6 & 758.2 & $-1,240.4$ & -482.2 \\
\hline $\begin{array}{l}\text { Number of cr } \\
\text { sections }\end{array}$ & & 29 & 29 & 29 & 28 & 28 & 28 & 20 & 20 & 20 \\
\hline Average & & 84.0 & -26.6 & 57.4 & 47.9 & -69.6 & -21.7 & 37.9 & -62.0 & -24.1 \\
\hline
\end{tabular}


Table 5. Cross section comparison values for the Kenslers Bend reach, 2006-07.-Continued

\begin{tabular}{|c|c|c|c|c|c|c|c|c|c|c|}
\hline \multirow[b]{2}{*}{ River mile } & \multirow[b]{2}{*}{$\begin{array}{c}\text { Line } \\
\text { number }\end{array}$} & \multicolumn{3}{|c|}{ June 2006 to March 2007} & \multicolumn{3}{|c|}{ March 2006 to June 2006} & \multicolumn{3}{|c|}{ April 2007 to July 2007} \\
\hline & & $\begin{array}{c}\text { Deposi- } \\
\text { tion area } \\
\text { (square } \\
\text { meters) }\end{array}$ & $\begin{array}{c}\text { Erosion } \\
\text { area } \\
\text { (square } \\
\text { meters) } \\
\end{array}$ & $\begin{array}{c}\begin{array}{c}\text { Net } \\
\text { change }\end{array} \\
\text { (square } \\
\text { meters) } \\
\end{array}$ & $\begin{array}{c}\text { Deposi- } \\
\text { tion area } \\
\text { (square } \\
\text { meters) } \\
\end{array}$ & $\begin{array}{c}\text { Erosion } \\
\text { area } \\
\text { (square } \\
\text { meters) }\end{array}$ & $\begin{array}{c}\text { Net change } \\
\text { (square } \\
\text { meters) }\end{array}$ & $\begin{array}{c}\text { Deposi- } \\
\text { tion area } \\
\text { (square } \\
\text { meters) } \\
\end{array}$ & $\begin{array}{l}\text { Erosion } \\
\text { area, } \\
\text { (square } \\
\text { meters) } \\
\end{array}$ & $\begin{array}{c}\text { Net change } \\
\text { (square } \\
\text { meters) }\end{array}$ \\
\hline 747.8 & 1 & -no data- & -no data- & -no data- & -no data- & -no data- & -no data- & 139.2 & -116.6 & 22.6 \\
\hline 747.7 & 2 & 135.0 & -84.6 & 50.3 & 42.9 & -41.6 & 1.3 & 21.1 & -108.2 & -87.1 \\
\hline 747.7 & 3 & -no data- & -no data- & -no data- & 89.1 & -55.6 & 33.5 & 0.0 & -87.4 & -87.4 \\
\hline 747.5 & 4 & -no data- & -no data- & -no data- & -no data- & -no data- & -no data- & 112.2 & -116.3 & -4.1 \\
\hline 747.4 & 5 & -no data- & -no data- & -no data- & -no data- & -no data- & -no data- & 157.5 & -129.1 & 28.4 \\
\hline 747.4 & 6 & -no data- & -no data- & -no data- & -no data- & -no data- & -no data- & 169.9 & -169.8 & 0.1 \\
\hline 747.2 & 7 & -no data- & -no data- & -no data- & -no data- & -no data- & -no data- & 0.8 & -54.5 & -53.7 \\
\hline 747.2 & 8 & -no data- & -no data- & -no data- & -no data- & -no data- & -no data- & 24.8 & -43.1 & -18.4 \\
\hline 747.1 & 9 & -no data- & -no data- & -no data- & -no data- & -no data- & -no data- & 82.7 & -57.4 & 25.3 \\
\hline 747.1 & 10 & -no data- & -no data- & -no data- & -no data- & -no data- & -no data- & 139.0 & -78.2 & 60.8 \\
\hline 747 & 11 & -no data- & -no data- & -no data- & -no data- & -no data- & -no data- & 173.3 & -173.6 & -0.3 \\
\hline 746.8 & 12 & 101.7 & -18.2 & 83.5 & 57.3 & -107.0 & -49.7 & 48.9 & -165.7 & -116.8 \\
\hline 746.7 & 13 & -no data- & -no data- & -no data- & 128.8 & -158.3 & -29.6 & 107.0 & -15.7 & 91.3 \\
\hline 746.7 & 14 & 66.1 & -59.2 & 6.8 & 226.5 & -46.4 & 180.2 & 160.3 & -134.0 & 26.3 \\
\hline 746.6 & 15 & -no data- & -no data- & -no data- & 190.7 & -89.7 & 101.0 & 177.7 & -189.2 & -11.5 \\
\hline 746.5 & 16 & 113.8 & -6.9 & 106.9 & 26.0 & -166.3 & -140.3 & 151.1 & -129.5 & 21.6 \\
\hline 746.3 & 30 & -no data- & -no data- & -no data- & 92.2 & -42.1 & 50.1 & 116.1 & -92.4 & 23.8 \\
\hline 746 & 17 & 0.0 & -13.3 & -13.3 & 0.1 & 0.0 & 0.1 & 74.4 & -44.3 & 30.1 \\
\hline 745.7 & 18 & -no data- & -no data- & -no data- & 116.1 & -59.3 & 56.8 & 72.4 & -92.1 & -19.6 \\
\hline 745.7 & 19 & 33.1 & -136.9 & -103.8 & 173.9 & -32.7 & 141.2 & 92.8 & -106.5 & -13.7 \\
\hline 745.2 & 20 & -no data- & -no data- & -no data- & 77.1 & -138.8 & -61.6 & 252.7 & -87.7 & 164.9 \\
\hline 745.1 & 28 & 83.6 & -0.3 & 83.3 & 12.0 & -1.8 & 10.2 & 121.5 & -60.5 & 61.0 \\
\hline 745 & 21 & -no data- & -no data- & -no data- & 35.3 & -19.7 & 15.6 & 77.4 & -79.2 & -1.8 \\
\hline 744.8 & 26 & 46.9 & -41.5 & 5.4 & 142.2 & -72.3 & 69.9 & 203.7 & -46.5 & 157.1 \\
\hline 744.7 & 27 & -no data- & -no data- & -no data- & 88.0 & -125.4 & -37.4 & 89.5 & -84.6 & 4.8 \\
\hline 744.5 & 22 & 47.4 & -38.7 & 8.7 & 163.4 & -107.7 & 55.7 & 155.5 & -157.2 & -1.7 \\
\hline 744.4 & 23 & -no data- & -no data- & -no data- & 151.9 & -71.7 & 80.2 & 149.3 & -146.2 & 3.1 \\
\hline 744.3 & 29 & 30.8 & -10.0 & 20.8 & 103.6 & 0.0 & 103.6 & 93.4 & -87.1 & 6.3 \\
\hline 744.1 & 24 & -no data- & -no data- & -no data- & 129.4 & -15.6 & 113.8 & 154.4 & 0.0 & 154.4 \\
\hline 744.1 & 25 & 39.2 & -97.7 & -58.5 & 174.4 & -57.1 & 117.4 & 120.8 & -211.2 & -90.4 \\
\hline \multicolumn{2}{|l|}{ Total area } & 697.4 & -507.3 & 190.1 & 2221.0 & -1409.1 & 811.9 & $3,439.2$ & $-3,063.8$ & 375.5 \\
\hline \multicolumn{2}{|c|}{$\begin{array}{l}\text { Number of cross } \\
\text { sections }\end{array}$} & 11 & 11 & 11 & 22 & 22 & 22 & 30 & 30 & 30 \\
\hline \multicolumn{2}{|l|}{ Average } & 63.4 & -46.1 & 17.3 & 101.0 & -64.0 & 36.9 & 114.6 & -102.1 & 12.5 \\
\hline
\end{tabular}


Table 5. Cross section comparison values for the Kenslers Bend reach, 2006-07.-Continued

\begin{tabular}{|c|c|c|c|c|c|c|c|c|c|c|}
\hline \multirow[b]{2}{*}{ River mile } & \multirow[b]{2}{*}{$\begin{array}{c}\text { Line } \\
\text { number }\end{array}$} & \multicolumn{3}{|c|}{ July 2007 to November 2007} & \multicolumn{3}{|c|}{ April 2007 to November 2007} & \multicolumn{3}{|c|}{ March 2006 to March 2007} \\
\hline & & $\begin{array}{c}\text { Deposi- } \\
\text { tion area } \\
\text { (square } \\
\text { meters) }\end{array}$ & $\begin{array}{c}\text { Erosion } \\
\text { area } \\
\text { (square } \\
\text { meters) }\end{array}$ & $\begin{array}{l}\text { Net change } \\
\text { (square } \\
\text { meters) }\end{array}$ & $\begin{array}{c}\text { Deposi- } \\
\text { tion area } \\
\text { (square } \\
\text { meters) }\end{array}$ & $\begin{array}{l}\text { Erosion } \\
\text { area } \\
\text { (square } \\
\text { meters) }\end{array}$ & $\begin{array}{l}\text { Net change } \\
\text { (square } \\
\text { meters) }\end{array}$ & $\begin{array}{l}\text { Deposi- } \\
\text { tion area } \\
\text { (square } \\
\text { meters) }\end{array}$ & $\begin{array}{l}\text { Erosion } \\
\text { area } \\
\text { (square } \\
\text { meters) }\end{array}$ & $\begin{array}{c}\text { Net change } \\
\text { (square } \\
\text { meters) }\end{array}$ \\
\hline 747.8 & 1 & 72.1 & -50.0 & 22.2 & 159.0 & -114.5 & 44.6 & 131.8 & -144.9 & -13.1 \\
\hline 747.7 & 2 & 70.8 & -111.8 & -41.0 & 68.4 & -204.9 & -136.5 & 117.5 & -61.8 & 55.7 \\
\hline 747.7 & 3 & 2.0 & -124.4 & -122.3 & 0.1 & -209.7 & -209.5 & -no data- & -no data- & -no data- \\
\hline 747.5 & 4 & 72.6 & -102.6 & -30.1 & 62.2 & -96.3 & -34.1 & 103.3 & -128.6 & -25.3 \\
\hline 747.4 & 5 & 41.7 & -67.9 & -26.2 & 99.4 & -97.1 & 2.2 & -no data- & -no data- & -no data- \\
\hline 747.4 & 6 & 66.0 & -72.4 & -6.5 & 137.3 & -142.9 & -5.7 & 167.1 & -149.7 & 17.3 \\
\hline 747.2 & 7 & 0.0 & -139.5 & -139.5 & 0.0 & -179.2 & -179.2 & -no data- & -no data- & -no data- \\
\hline 747.2 & 8 & 46.4 & -136.0 & -89.6 & 53.1 & -161.2 & -108.1 & 91.7 & -32.5 & 59.2 \\
\hline 747.1 & 9 & 33.3 & -96.3 & -62.9 & 47.3 & -85.1 & -37.9 & -no data- & -no data- & -no data- \\
\hline 747.1 & 10 & 38.1 & -103.7 & -65.6 & 69.7 & -75.0 & -5.3 & 128.7 & -98.8 & 29.9 \\
\hline 747 & 11 & 61.8 & -84.4 & -22.6 & 111.2 & -134.4 & -23.1 & -no data- & -no data- & -no data- \\
\hline 746.8 & 12 & 126.8 & -51.9 & 74.8 & 167.3 & -166.6 & 0.7 & 119.2 & -97.9 & 21.3 \\
\hline 746.7 & 13 & 1.5 & -195.8 & -194.3 & 17.6 & -105.4 & -87.8 & -no data- & -no data- & -no data- \\
\hline 746.7 & 14 & 42.2 & -160.6 & -118.4 & 79.6 & -171.8 & -92.2 & 187.2 & -36.2 & 151.0 \\
\hline 746.6 & 15 & 24.7 & -78.4 & -53.7 & 136.0 & -201.2 & -65.2 & -no data- & -no data- & -no data- \\
\hline 746.5 & 16 & 62.8 & -59.6 & 3.2 & 140.8 & -110.0 & 30.8 & 44.2 & -94.9 & -50.7 \\
\hline 746.3 & 30 & 21.0 & -181.0 & -160.0 & 15.4 & -77.1 & -61.6 & -no data- & -no data- & -no data- \\
\hline 746 & 17 & 63.9 & -118.0 & -54.0 & 41.8 & -66.0 & -24.2 & 0.0 & -0.1 & -0.1 \\
\hline 745.7 & 18 & 117.4 & -85.6 & 31.8 & 51.6 & -39.1 & 12.5 & -no data- & -no data- & -no data- \\
\hline 745.7 & 19 & 138.4 & -106.3 & 32.1 & 40.2 & -21.3 & 19.0 & 123.5 & -54.1 & 69.4 \\
\hline 745.2 & 20 & 104.9 & -146.5 & -41.7 & 128.1 & -14.9 & 113.2 & -no data- & -no data- & -no data- \\
\hline 745.1 & 28 & 50.0 & -155.6 & -105.7 & 90.4 & -95.4 & -5.0 & 96.1 & 0.0 & 96.0 \\
\hline 745 & 21 & 44.5 & -78.8 & -34.3 & 60.1 & -88.2 & -28.1 & -no data- & -no data- & -no data- \\
\hline 744.8 & 26 & 38.0 & -139.3 & -101.3 & 67.5 & -11.8 & 55.7 & 152.9 & -137.9 & 15.0 \\
\hline 744.7 & 27 & 83.7 & -52.5 & 31.2 & 61.6 & -36.2 & 25.5 & -no data- & -no data- & -no data- \\
\hline 744.5 & 22 & 165.5 & -181.0 & -15.5 & 36.7 & -53.5 & -16.8 & 131.8 & -82.8 & 49.0 \\
\hline 744.4 & 23 & 125.5 & -192.7 & -67.2 & 20.1 & -73.0 & -52.9 & -no data- & -no data- & -no data- \\
\hline 744.3 & 29 & 124.2 & -147.4 & -23.2 & 101.1 & -118.3 & -17.1 & 136.0 & 0.0 & 136.0 \\
\hline 744.1 & 24 & 0.0 & -256.6 & -256.6 & 12.9 & -73.5 & -60.7 & -no data- & -no data- & -no data- \\
\hline 744.1 & 25 & 77.7 & -122.1 & -44.4 & 109.9 & -244.7 & -134.8 & 116.7 & -123.5 & -6.8 \\
\hline Total area & & $1,917.3$ & $-3,598.7$ & $-1,681.4$ & $2,186.6$ & $-3,268.3$ & $-1,081.7$ & $1,847.7$ & $-1,243.8$ & 603.9 \\
\hline $\begin{array}{l}\text { Number of cr } \\
\text { sections }\end{array}$ & & 30 & 30 & 30 & 30 & 30 & 30 & 16 & 16 & 16 \\
\hline Average & & 63.9 & -120.0 & -56.0 & 72.9 & -108.9 & -36.1 & 115.5 & -77.7 & 37.7 \\
\hline
\end{tabular}



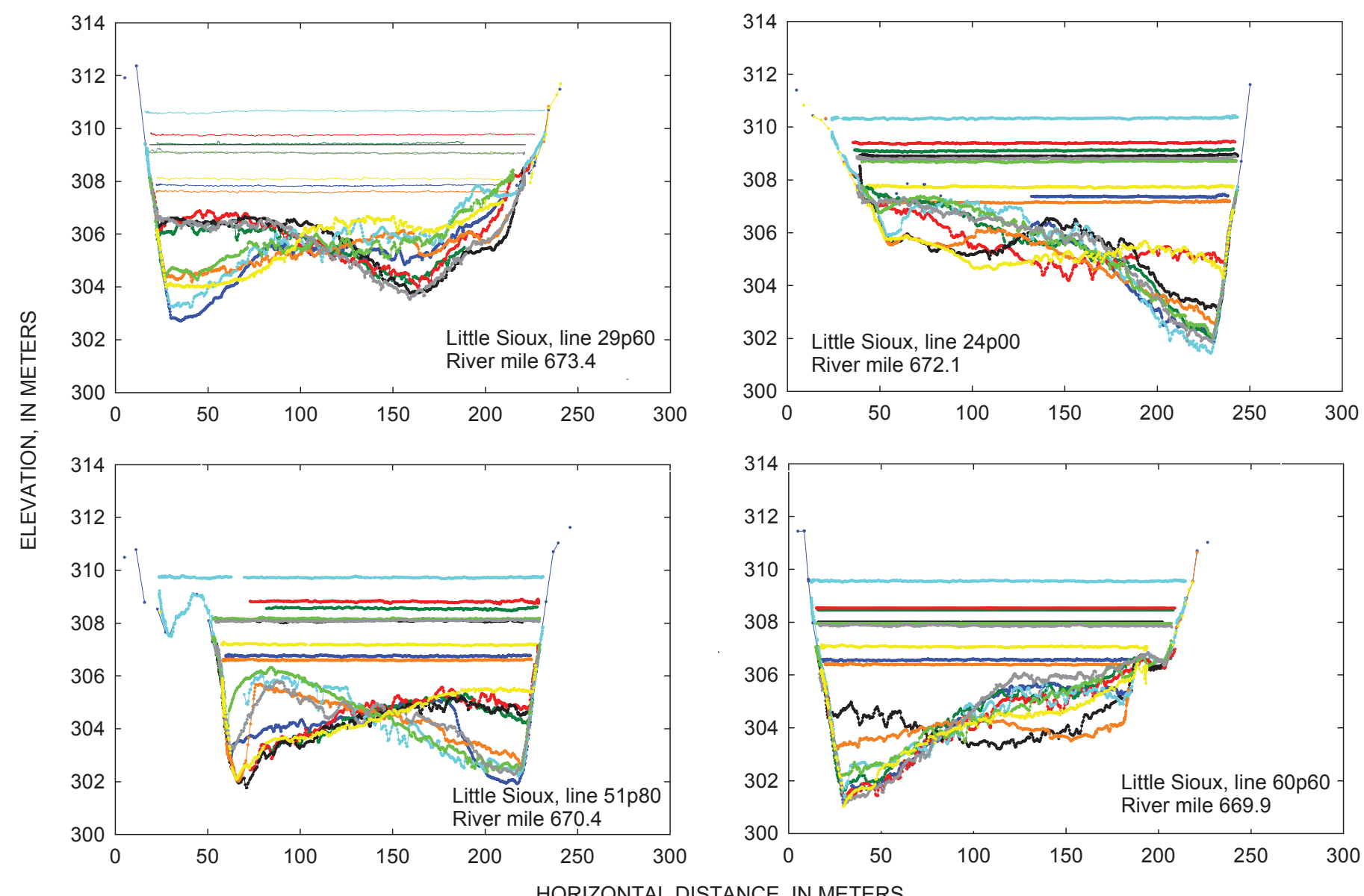

HORIZONTAL DISTANCE, IN METERS

EXPLANATION

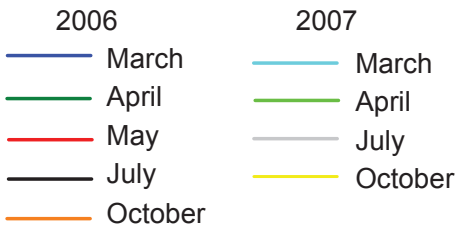

Figure 13. Typical survey cross sections in the Little Sioux reach.

In the Kenslers Bend reach the most channel change occurred in where bars scoured and became open channel or channel became bars. This is likely from bar migration through the reach. This style of channel change occurred in over half of the Kenslers Bend cross sections.

\section{Little Sioux Reach}

Thirty-three transects were surveyed at the Little Sioux reach five times in 2006 and four times in 2007 resulting in 16 comparisons (appendix 3, figs. 13, 14, table 6). Typical cross sections from the Little Sioux reach showed changes in the channel similar to that seen in the Kenslers Bend reach (fig. 13). Although there were large magnitudes of deposition and erosion, between -12.41-13.6 percent of the index crosssectional area, net change was low through the reach (-4.1-3.3 percent). In the Sioux reach, overall trends of erosion and deposition tended to be similar in adjacent cross sections reflecting change on a larger scale, such as mid-channel bars moving through the system.

The overall trend in the Sioux reach in 2006 was mixed with deposition and erosion occurring on all cross sections on an individual basis, but net erosion and deposition varying by cross-section location (fig. 14, table 6). In 2007 there was higher-magnitude deposition and erosion, and a measurable net change on a cross-section by cross-section basis, but reach average net change was $-2.3 \mathrm{~m}^{2}$ (fig. 14, table 6). April 2006 to May 2006 was the time period that included the 2006 spring rise event; the reach had approximately equal amounts of erosion and deposition (fig. 14, table 10). During the winter from October 2006 to March 2007, net cross-section change was positive in nearly every cross section, but nearly all cross 
A.

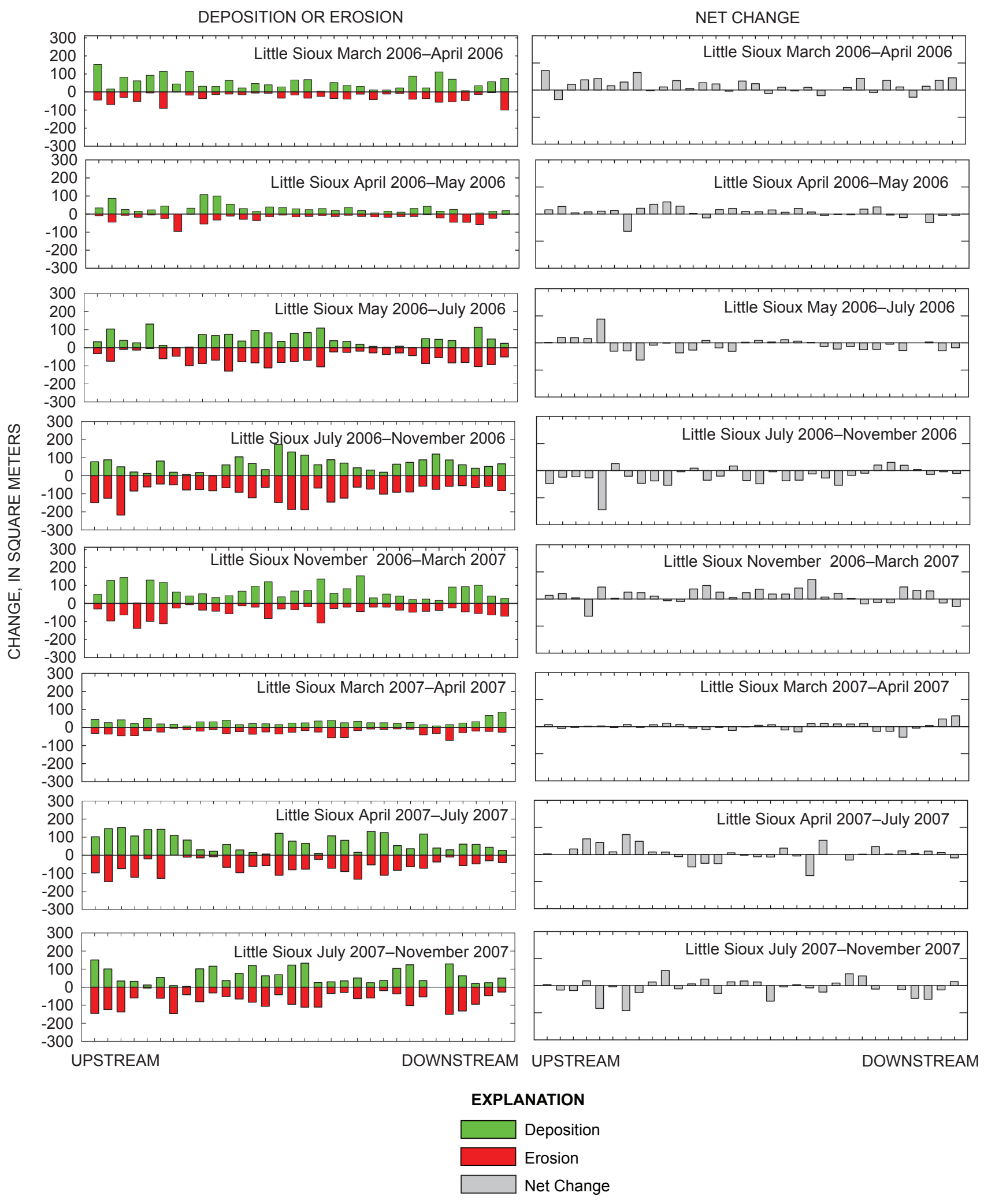

Figure 14. Deposition, erosion, and net change for the Little Sioux reach. 
B.

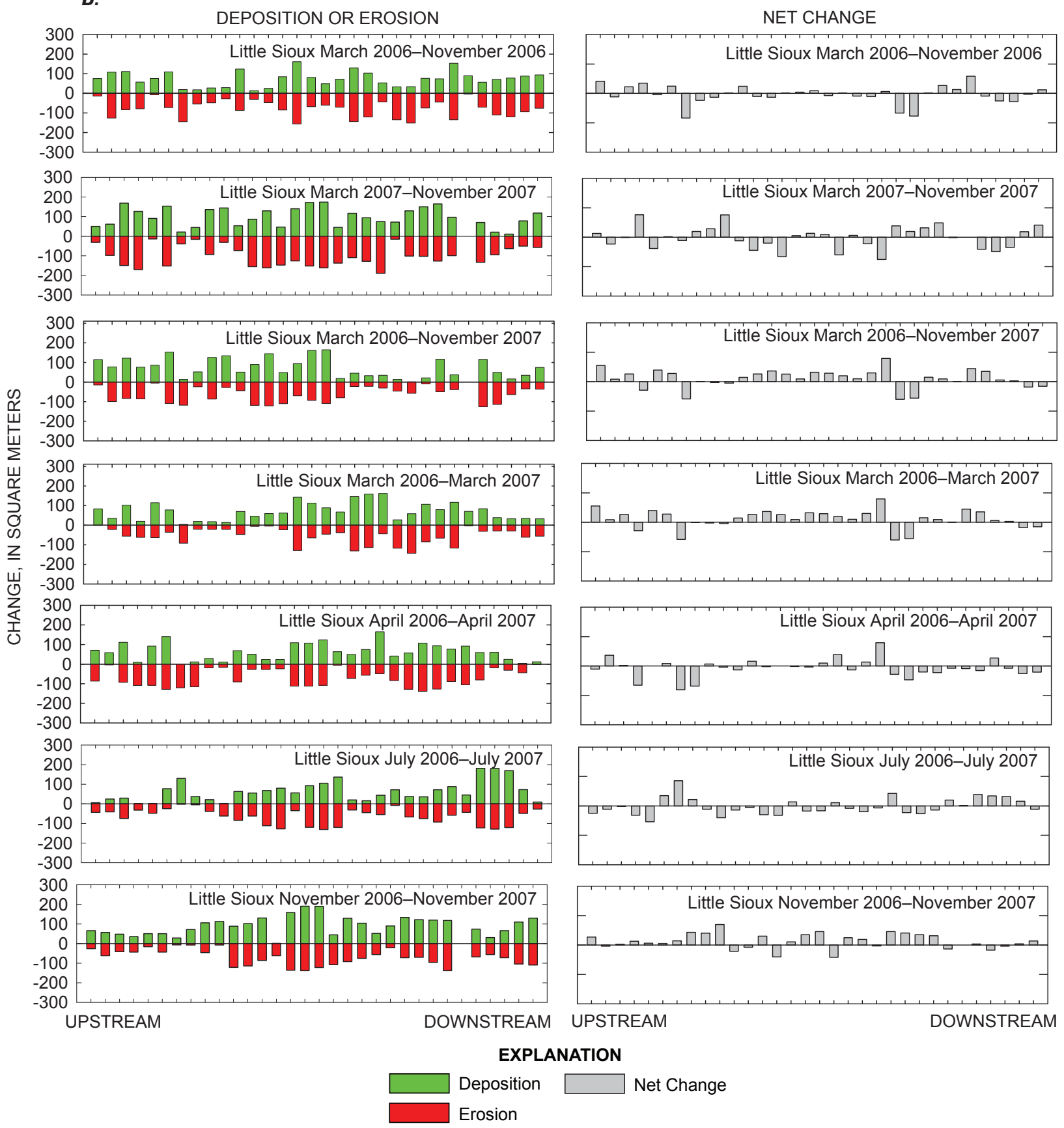

Figure 14. Deposition, erosion, and net change for the Little Sioux reach.-Continued

sections had erosion as well as deposition. In general, this reach experienced erosion and deposition at every cross section during most time periods, but the overall net change was low (fig. 14).

\section{Miami Reach}

Thirty-three transects were surveyed at the Miami reach five times in 2006 and five times in 2007 (appendix 4, figs. 15, 16, table 7). Erosion and deposition magnitudes ranged from
$-9.25-8.56$ percent of the index cross-section area $\left(1,242 \mathrm{~m}^{2}\right)$, but net change was between -6.1 and 5.2 percent.

In 2006, nearly all cross sections showed erosion, and the net result was erosion for the entire reach (fig 16 ). In 2007, the net result for the reach was erosion, but there was less erosion and more deposition on an individual cross-section basis (table 7). From May 2006 to July 2006, there were high rates of erosion, particularly from river miles $262.2-261.0$, a bend that had a large amount of net erosion. From the late summer to fall (July 2006 to October 2006), the same region 
Table 6. Cross section comparison values for the Little Sioux reach, 2006-07.

\begin{tabular}{|c|c|c|c|c|c|c|c|c|c|c|c|c|c|}
\hline \multirow[b]{2}{*}{ River mile } & \multirow[b]{2}{*}{$\begin{array}{c}\text { Line } \\
\text { number }\end{array}$} & \multicolumn{3}{|c|}{ March 2006 to April 2006} & \multicolumn{3}{|c|}{ April 2006 to May 2006} & \multicolumn{3}{|c|}{ May 2006 to July 2006} & \multicolumn{3}{|c|}{ July 2006 to November 2006} \\
\hline & & $\begin{array}{c}\text { Deposi- } \\
\text { tion area } \\
\text { (square } \\
\text { meters) }\end{array}$ & $\begin{array}{l}\text { Erosion } \\
\text { area } \\
\text { (square } \\
\text { meters) }\end{array}$ & $\begin{array}{c}\text { Net } \\
\text { change } \\
\text { (square } \\
\text { meters) }\end{array}$ & $\begin{array}{c}\text { Deposi- } \\
\text { tion area } \\
\text { (square } \\
\text { meters) }\end{array}$ & $\begin{array}{l}\text { Erosion } \\
\text { area, } \\
\text { (square } \\
\text { meters) }\end{array}$ & $\begin{array}{c}\text { Net } \\
\text { change } \\
\text { (square } \\
\text { meters) }\end{array}$ & $\begin{array}{c}\text { Deposi- } \\
\text { tion area } \\
\text { (square } \\
\text { meters) }\end{array}$ & $\begin{array}{l}\text { Erosion } \\
\text { area } \\
\text { (square } \\
\text { meters) }\end{array}$ & $\begin{array}{c}\text { Net } \\
\text { change } \\
\text { (square } \\
\text { meters) }\end{array}$ & $\begin{array}{c}\text { Deposi- } \\
\text { tion area } \\
\text { (square } \\
\text { meters) }\end{array}$ & $\begin{array}{c}\text { Erosion } \\
\text { area } \\
\text { (square } \\
\text { meters) }\end{array}$ & $\begin{array}{c}\text { Net } \\
\text { change } \\
\text { (square } \\
\text { meters) }\end{array}$ \\
\hline 673.4 & $2 \mathrm{p} 60$ & 152.9 & -44.4 & 108.5 & 33.6 & -9.8 & 23.8 & 34.1 & -32.4 & 1.7 & 77.6 & -149.5 & -71.9 \\
\hline 673.4 & $4 \mathrm{p} 20$ & 17.2 & -70.0 & -52.9 & 86.5 & -44.8 & 41.8 & 104.7 & -74.3 & 30.4 & 88.2 & -124.5 & -36.3 \\
\hline 673.0 & $10 \mathrm{p} 00$ & 82.3 & -50.5 & 31.8 & 26.0 & -19.0 & 7.0 & 42.3 & -12.8 & 29.6 & 50.1 & -84.0 & -33.9 \\
\hline 673.0 & $10 \mathrm{p} 40$ & 61.6 & -4.9 & 56.8 & 16.3 & -4.3 & 12.1 & 27.9 & -3.5 & 24.4 & 21.0 & -61.1 & -40.1 \\
\hline 673.0 & 9 p20 & 92.8 & -29.8 & 63.0 & 23.5 & -7.4 & 16.2 & 131.7 & 0.0 & 131.7 & 13.5 & -230.3 & -216.8 \\
\hline 672.6 & $16 \mathrm{p} 80$ & 113.8 & -90.0 & 23.9 & 44.1 & -24.2 & 19.9 & 14.3 & -59.5 & -45.2 & 82.0 & -43.0 & 39.0 \\
\hline 672.4 & $20 \mathrm{p} 20$ & 45.4 & -0.8 & 44.6 & 0.7 & -95.4 & -94.7 & 1.3 & -45.7 & -44.4 & 19.6 & -50.2 & -30.5 \\
\hline 672.3 & $22 \mathrm{p} 20$ & 114.4 & -17.0 & 97.4 & 33.1 & -0.8 & 32.3 & 4.1 & -99.1 & -95.1 & 7.7 & -78.0 & -70.2 \\
\hline 672.2 & $23 \mathrm{p} 80$ & 32.1 & -36.2 & -4.1 & 108.6 & -54.9 & 53.8 & 74.2 & -86.4 & -12.2 & 18.6 & -76.0 & -57.5 \\
\hline 672.1 & $24 \mathrm{p} 00$ & 31.0 & -14.0 & 17.0 & 100.2 & -32.9 & 67.3 & 67.7 & -68.0 & -0.3 & 1.5 & -82.9 & -81.4 \\
\hline 671.9 & $27 \mathrm{p} 80$ & 64.6 & -12.2 & 52.4 & 54.8 & -10.4 & 44.4 & 74.4 & -129.1 & -54.7 & 60.1 & -65.8 & -5.7 \\
\hline 671.8 & 30 p20 & 22.5 & -14.9 & 7.7 & 31.0 & -28.9 & 2.1 & 38.0 & -77.5 & -39.5 & 105.2 & -91.2 & 14.0 \\
\hline 671.7 & $31 \mathrm{p} 00$ & 46.6 & -6.2 & 40.4 & 14.8 & -35.9 & -21.1 & 97.2 & -82.9 & 14.3 & 68.3 & -121.9 & -53.6 \\
\hline 671.6 & $32 \mathrm{p} 80$ & 40.4 & -6.6 & 33.9 & 39.9 & -14.4 & 25.5 & 82.9 & -110.9 & -28.0 & 34.0 & -64.0 & -30.0 \\
\hline 671.4 & $35 \mathrm{p} 80$ & 28.5 & -34.1 & -5.6 & 37.2 & -5.2 & 31.9 & 35.3 & -81.4 & -46.1 & 175.0 & -148.8 & 26.2 \\
\hline 671.3 & $36 \mathrm{p} 80$ & 66.8 & -16.6 & 50.2 & 29.6 & -15.3 & 14.3 & 80.2 & -76.3 & 3.8 & 131.9 & -186.7 & -54.7 \\
\hline 671.3 & $37 \mathrm{p} 00$ & 69.1 & -34.3 & 34.8 & 25.2 & -12.3 & 12.8 & 84.3 & -69.4 & 14.9 & 114.9 & -188.0 & -73.1 \\
\hline 671.2 & 39 p20 & 5.6 & -24.4 & -18.9 & 30.8 & -8.0 & 22.8 & 110.1 & -105.3 & 4.8 & 61.3 & -67.7 & -6.4 \\
\hline 671.0 & $43 \mathrm{p} 20$ & 53.4 & -37.9 & 15.5 & 21.0 & -11.2 & 9.8 & 39.8 & -21.8 & 18.0 & 89.1 & -145.2 & -56.1 \\
\hline 670.9 & $43 p 40$ & 36.5 & -41.6 & -5.0 & 37.2 & -5.4 & 31.8 & 35.2 & -25.2 & 9.9 & 70.5 & -123.6 & -53.1 \\
\hline 670.8 & 45 p60 & 31.1 & -15.9 & 15.2 & 19.4 & -7.8 & 11.7 & 20.0 & -18.1 & 1.9 & 44.1 & -62.4 & -18.3 \\
\hline 670.6 & $48 \mathrm{p} 40$ & 11.4 & -42.4 & -31.0 & 6.8 & -15.4 & -8.6 & 8.5 & -27.8 & -19.3 & 31.9 & -73.1 & -41.3 \\
\hline 670.6 & $50 \mathrm{p} 00$ & 11.7 & -11.0 & 0.7 & 16.7 & -17.8 & -1.1 & 2.8 & -36.8 & -34.0 & 19.6 & -101.0 & -81.4 \\
\hline 670.5 & $51 \mathrm{p} 00$ & 22.9 & -8.6 & 14.4 & 10.5 & -13.3 & -2.8 & 8.9 & -28.1 & -19.3 & 64.7 & -90.4 & -25.7 \\
\hline 670.4 & $51 \mathrm{p} 80$ & 87.8 & -23.1 & 64.6 & 31.8 & -4.5 & 27.3 & 1.5 & -39.0 & -37.5 & 74.6 & -88.6 & -13.9 \\
\hline 670.3 & $56 \mathrm{p} 40$ & 22.5 & -36.8 & -14.3 & 42.4 & -2.8 & 39.6 & 50.6 & -86.9 & -36.3 & 88.3 & -57.3 & 31.0 \\
\hline 670.2 & $57 \mathrm{p} 40$ & 110.5 & -56.7 & 53.8 & 16.2 & -21.5 & -5.3 & 47.2 & -54.1 & -6.9 & 120.3 & -74.7 & 45.6 \\
\hline 670.1 & $58 \mathrm{p} 80$ & 70.7 & -53.7 & 17.0 & 26.3 & -45.2 & -18.9 & 40.6 & -82.5 & -41.9 & 87.7 & -57.8 & 29.9 \\
\hline 670.0 & $59 \mathrm{p} 80$ & 7.7 & -47.7 & -40.0 & -no data- & -no data- & -no data- & -no data- & -no data- & -no data- & 61.6 & -55.2 & 6.4 \\
\hline 669.9 & 60 p60 & 34.5 & -13.4 & 21.1 & 6.3 & -52.7 & -46.4 & 113.8 & -108.3 & 5.5 & 41.9 & -63.1 & -21.2 \\
\hline 669.8 & $62 \mathrm{p} 00$ & 57.1 & -3.3 & 53.8 & 15.6 & -23.9 & -8.4 & 49.5 & -93.0 & -43.5 & 51.7 & -57.9 & -6.2 \\
\hline 669.8 & $62 \mathrm{p} 60$ & 76.3 & -8.3 & 68.0 & 18.7 & -25.2 & -6.5 & 25.1 & -51.9 & -26.8 & 66.6 & -82.0 & -15.5 \\
\hline \multicolumn{2}{|l|}{ Total area } & $1,721.8$ & -907.3 & 814.5 & $1,005.1$ & -987.0 & 18.2 & $1,548.1$ & $-1,888.1$ & -340.0 & $2,043.0$ & $-3,045.8$ & $-1,002.8$ \\
\hline \multicolumn{2}{|c|}{$\begin{array}{l}\text { Number of cross sec- } \\
\text { tions }\end{array}$} & 32 & 32 & 32 & 31 & 31 & 31 & 31 & 31 & 31 & 32 & 32 & 32 \\
\hline \multicolumn{2}{|l|}{ Average } & 53.8 & -28.4 & 25.5 & 32.4 & -31.8 & 0.6 & 49.9 & -60.9 & -11.0 & 63.8 & -95.2 & -31.3 \\
\hline
\end{tabular}


Table 6. Cross section comparison values for the Little Sioux reach, 2006-07.-Continued

\begin{tabular}{|c|c|c|c|c|c|c|c|c|c|c|c|c|c|}
\hline \multirow[b]{2}{*}{$\begin{array}{l}\text { River } \\
\text { mile }\end{array}$} & \multirow[b]{2}{*}{$\begin{array}{l}\text { Line } \\
\text { number }\end{array}$} & \multicolumn{3}{|c|}{ November 2006 to March 2007} & \multicolumn{3}{|c|}{ March 2007 to April 2007} & \multicolumn{3}{|c|}{ April 2007 to July 2007} & \multicolumn{3}{|c|}{ July 2007 to November } \\
\hline & & $\begin{array}{c}\text { Deposi- } \\
\text { tion area } \\
\text { (square } \\
\text { meters) }\end{array}$ & $\begin{array}{c}\text { Erosion } \\
\text { area } \\
\text { (square } \\
\text { meters) }\end{array}$ & $\begin{array}{l}\text { Net } \\
\text { change } \\
\text { (square } \\
\text { meters) }\end{array}$ & $\begin{array}{c}\text { Deposi- } \\
\text { tion area } \\
\text { (square } \\
\text { meters) }\end{array}$ & $\begin{array}{l}\text { Erosion } \\
\text { area, } \\
\text { (square } \\
\text { meters) }\end{array}$ & $\begin{array}{c}\text { Net } \\
\text { change } \\
\text { (square } \\
\text { meters) }\end{array}$ & $\begin{array}{c}\text { Deposi- } \\
\text { tion area } \\
\text { (square } \\
\text { meters) }\end{array}$ & $\begin{array}{c}\text { Erosion } \\
\text { area } \\
\text { (square } \\
\text { meters) }\end{array}$ & $\begin{array}{c}\text { Net } \\
\text { change } \\
\text { (square } \\
\text { meters) }\end{array}$ & $\begin{array}{c}\text { Deposi- } \\
\text { tion area } \\
\text { (square } \\
\text { meters) }\end{array}$ & $\begin{array}{l}\text { Erosion } \\
\text { area } \\
\text { (square } \\
\text { meters) }\end{array}$ & $\begin{array}{c}\text { Net } \\
\text { change } \\
\text { (square } \\
\text { meters) }\end{array}$ \\
\hline 673.4 & $2 \mathrm{p} 60$ & 50.6 & -30.2 & 20.4 & 43.8 & -33.3 & 10.5 & 101.4 & -98.2 & 3.1 & 151.1 & -145.2 & 5.9 \\
\hline 673.4 & $4 \mathrm{p} 20$ & 127.7 & -97.4 & 30.2 & 26.5 & -37.0 & -10.6 & 146.8 & -147.2 & -0.5 & 100.2 & -124.4 & -24.2 \\
\hline 673.0 & $10 \mathrm{p} 00$ & 143.7 & -137.8 & 5.9 & 41.8 & -45.9 & -4.1 & 152.5 & -122.7 & 29.8 & 33.9 & -60.7 & -26.8 \\
\hline 673.0 & $10 \mathrm{p} 40$ & 3.8 & -98.9 & -95.1 & 21.1 & -19.1 & 1.9 & 106.2 & -20.5 & 85.8 & 31.8 & -5.0 & 26.8 \\
\hline 673.0 & 9 p20 & 129.6 & -63.7 & 66.0 & 50.3 & -46.8 & 3.5 & 140.9 & -74.9 & 66.0 & 11.3 & -137.0 & -125.7 \\
\hline 672.6 & $16 \mathrm{p} 80$ & 116.9 & -112.1 & 4.7 & 19.6 & -24.9 & -5.3 & 142.4 & -128.6 & 13.8 & 54.3 & -60.4 & -6.0 \\
\hline 672.4 & $20 \mathrm{p} 20$ & 63.2 & -25.5 & 37.7 & 17.2 & -5.7 & 11.5 & 110.0 & 0.0 & 110.0 & 8.1 & -145.9 & -137.8 \\
\hline 672.3 & $22 \mathrm{p} 20$ & 41.7 & -6.6 & 35.1 & 8.3 & -12.7 & -4.4 & 83.6 & -11.4 & 72.2 & 3.7 & -41.9 & -38.2 \\
\hline 672.2 & $23 \mathrm{p} 80$ & 54.0 & -37.4 & 16.6 & 31.0 & -20.7 & 10.3 & 29.1 & -15.7 & 13.3 & 101.4 & -81.2 & 20.2 \\
\hline 672.1 & $24 \mathrm{p} 00$ & 33.5 & -42.9 & -9.4 & 30.6 & -12.0 & 18.6 & 21.2 & -9.2 & 12.1 & 116.0 & -32.8 & 83.2 \\
\hline 671.9 & $27 \mathrm{p} 80$ & 42.7 & -57.0 & -14.2 & 40.7 & -30.0 & 10.7 & 59.1 & -71.1 & -12.0 & 35.6 & -52.5 & -16.9 \\
\hline 671.8 & 30 p20 & 68.5 & -12.9 & 55.6 & 15.4 & -23.5 & -8.1 & 28.6 & -97.5 & -68.9 & 75.9 & -66.3 & 9.7 \\
\hline 671.7 & $31 \mathrm{p} 00$ & 95.4 & -20.5 & 74.9 & 21.2 & -38.2 & -17.1 & 14.2 & -63.6 & -49.4 & 120.3 & -83.9 & 36.4 \\
\hline 671.6 & $32 \mathrm{p} 80$ & 120.7 & -82.8 & 37.9 & 20.3 & -25.1 & -4.8 & 6.2 & -57.6 & -51.4 & 62.7 & -105.8 & -43.0 \\
\hline 671.4 & $35 \mathrm{p} 80$ & 37.3 & -29.9 & 7.5 & 15.4 & -36.6 & -21.2 & 120.0 & -111.9 & 8.2 & 68.4 & -47.2 & 21.1 \\
\hline 671.3 & $36 \mathrm{p} 80$ & 69.5 & -34.9 & 34.6 & 24.7 & -27.1 & -2.5 & 76.9 & -81.3 & -4.4 & 121.7 & -95.8 & 25.9 \\
\hline 671.3 & $37 \mathrm{p} 00$ & 71.9 & -18.0 & 53.9 & 25.3 & -18.3 & 7.0 & 64.9 & -77.9 & -13.1 & 132.1 & -111.9 & 20.2 \\
\hline 671.2 & 39 p20 & 135.4 & -108.0 & 27.4 & 35.9 & -25.8 & 10.1 & 9.4 & -24.4 & -15.0 & 25.5 & -110.8 & -85.3 \\
\hline 671.0 & 43 p20 & 55.8 & -28.2 & 27.6 & 39.0 & -57.6 & -18.6 & 107.1 & -72.4 & 34.7 & 29.0 & -36.0 & -6.9 \\
\hline 670.9 & $43 p 40$ & 82.1 & -20.6 & 61.5 & 26.3 & -55.9 & -29.6 & 81.9 & -90.6 & -8.7 & 34.5 & -29.5 & 5.0 \\
\hline 670.8 & $45 \mathrm{p} 60$ & 152.9 & -44.9 & 108.1 & 34.1 & -17.7 & 16.4 & 15.6 & -132.8 & -117.2 & 51.1 & -63.4 & -12.3 \\
\hline 670.6 & $48 \mathrm{p} 40$ & 30.8 & -20.3 & 10.4 & 26.1 & -9.2 & 16.9 & 131.9 & -54.2 & 77.6 & 24.3 & -60.1 & -35.8 \\
\hline 670.6 & $50 \mathrm{p} 00$ & 52.1 & -20.1 & 31.9 & 25.8 & -11.0 & 14.8 & 124.6 & -124.9 & -0.3 & 36.6 & -21.9 & 14.6 \\
\hline 670.5 & $51 \mathrm{p} 00$ & 41.0 & -37.3 & 3.7 & 22.2 & -8.3 & 13.9 & 52.5 & -84.3 & -31.7 & 103.7 & -37.5 & 66.2 \\
\hline 670.4 & $51 \mathrm{p} 80$ & 21.8 & -48.4 & -26.6 & 27.9 & -9.9 & 17.9 & 35.7 & -34.5 & 1.1 & 123.7 & -69.5 & 54.3 \\
\hline 670.3 & $56 \mathrm{p} 40$ & 24.8 & -43.7 & -18.9 & 14.4 & -41.2 & -26.8 & 116.3 & -72.5 & 43.8 & 35.2 & -54.3 & -19.0 \\
\hline 670.2 & $57 \mathrm{p} 40$ & 17.5 & -38.3 & -20.9 & 8.6 & -34.3 & -25.8 & 39.5 & -37.8 & 1.8 & -no data- & -no data- & -no data- \\
\hline 670.1 & $58 \mathrm{p} 80$ & 91.0 & -24.0 & 67.0 & 15.9 & -74.5 & -58.6 & 28.8 & -9.3 & 19.5 & 128.4 & -150.9 & -22.5 \\
\hline 670.0 & $59 \mathrm{p} 80$ & 93.2 & -45.8 & 47.4 & 24.4 & -32.7 & -8.2 & 60.2 & -54.9 & 5.3 & 62.9 & -132.7 & -69.9 \\
\hline 669.9 & 60p60 & 101.0 & -55.5 & 45.5 & 31.4 & -25.5 & 5.9 & 59.2 & -41.4 & 17.8 & 19.3 & -94.5 & -75.3 \\
\hline 669.8 & $62 \mathrm{p} 00$ & 41.2 & -63.5 & -22.3 & 65.2 & -23.3 & 41.9 & 43.5 & -33.2 & 10.4 & 24.0 & -47.8 & -23.7 \\
\hline 669.8 & 62 p60 & 28.5 & -70.1 & -41.6 & 84.7 & -25.2 & 59.6 & 26.6 & -46.2 & -19.6 & 50.0 & -27.3 & 22.7 \\
\hline \multicolumn{2}{|c|}{ Total area } & $2,239.7$ & $-1,577.0$ & 662.7 & 935.1 & -909.1 & 26.0 & $2,336.8$ & $-2,102.7$ & 234.1 & $1,976.9$ & $-2,334.0$ & -357.1 \\
\hline \multicolumn{2}{|c|}{$\begin{array}{l}\text { Number of cross } \\
\text { sections }\end{array}$} & 32 & 32 & 32 & 32 & 32 & 32 & 32 & 32 & 32 & 31 & 31 & 31 \\
\hline \multicolumn{2}{|l|}{ Average } & 70.0 & -49.3 & 20.7 & 29.2 & -28.4 & 0.8 & 73.0 & -65.7 & 7.3 & 63.8 & -75.3 & -11.5 \\
\hline
\end{tabular}


Table 6. Cross section comparison values for the Little Sioux reach, 2006-07.-Continued

\begin{tabular}{|c|c|c|c|c|c|c|c|c|c|c|}
\hline \multirow[b]{2}{*}{$\begin{array}{c}\text { River } \\
\text { mile }\end{array}$} & \multirow[b]{2}{*}{$\begin{array}{c}\text { Line } \\
\text { number }\end{array}$} & \multicolumn{3}{|c|}{ March 2006 to October 2006} & \multicolumn{3}{|c|}{ March 2007 to October 2007} & \multicolumn{3}{|c|}{ March 2006 to October 2007} \\
\hline & & $\begin{array}{c}\text { Deposi- } \\
\text { tion area } \\
\text { (square } \\
\text { meters) }\end{array}$ & $\begin{array}{l}\text { Erosion } \\
\text { area } \\
\text { (square } \\
\text { meters) }\end{array}$ & $\begin{array}{c}\text { Net } \\
\text { change } \\
\text { (square } \\
\text { meters) }\end{array}$ & $\begin{array}{l}\text { Deposi- } \\
\text { tion area } \\
\text { (square } \\
\text { meters) }\end{array}$ & $\begin{array}{c}\text { Erosion } \\
\text { area, } \\
\text { (square } \\
\text { meters) }\end{array}$ & $\begin{array}{c}\text { Net } \\
\text { change } \\
\text { (square } \\
\text { meters) }\end{array}$ & $\begin{array}{c}\text { Deposi- } \\
\text { tion area } \\
\text { (square } \\
\text { meters) }\end{array}$ & $\begin{array}{l}\text { Erosion } \\
\text { area } \\
\text { (square } \\
\text { meters) }\end{array}$ & $\begin{array}{c}\text { Net } \\
\text { change } \\
\text { (square } \\
\text { meters) }\end{array}$ \\
\hline 673.4 & $2 \mathrm{p} 60$ & 75.4 & -13.3 & 62.0 & 50.6 & -31.0 & 19.6 & 115.8 & -13.7 & 102.1 \\
\hline 673.4 & $4 \mathrm{p} 20$ & 108.6 & -125.6 & -17.0 & 62.3 & -97.2 & -35.0 & 77.8 & -99.2 & -21.4 \\
\hline 673.0 & $10 \mathrm{p} 00$ & 111.6 & -77.9 & 33.7 & 169.2 & -170.3 & -1.1 & 122.6 & -84.0 & 38.6 \\
\hline 673.0 & $10 \mathrm{p} 40$ & 58.4 & -5.7 & 52.6 & 127.4 & -12.9 & 114.5 & 76.1 & -4.0 & 72.1 \\
\hline 673.0 & $9 \mathrm{p} 20$ & 76.6 & -82.6 & -6.0 & 91.7 & -148.6 & -56.8 & 86.9 & -83.7 & 3.2 \\
\hline 672.6 & $16 \mathrm{p} 80$ & 110.1 & -72.7 & 37.4 & 154.1 & -151.6 & 2.5 & 153.4 & -108.6 & 44.8 \\
\hline 672.4 & 20 p20 & 19.6 & -144.5 & -124.9 & 22.2 & -38.5 & -16.3 & 14.0 & -117.6 & -103.6 \\
\hline 672.3 & $22 \mathrm{p} 20$ & 18.4 & -54.0 & -35.6 & 45.2 & -15.7 & 29.6 & 52.8 & -23.9 & 28.9 \\
\hline 672.2 & $23 \mathrm{p} 80$ & 27.2 & -47.2 & -20.0 & 136.5 & -92.8 & 43.8 & 126.4 & -86.0 & 40.4 \\
\hline 672.1 & $24 \mathrm{p} 00$ & 30.2 & -27.7 & 2.5 & 144.5 & -30.6 & 113.9 & 134.9 & -27.8 & 107.1 \\
\hline 671.9 & $27 \mathrm{p} 80$ & 124.7 & -88.5 & 36.2 & 54.3 & -72.6 & -18.3 & 52.0 & -48.3 & 3.7 \\
\hline 671.8 & 30 p20 & 14.4 & -30.2 & -15.8 & 87.3 & -154.4 & -67.1 & 90.8 & -118.3 & -27.5 \\
\hline 671.7 & $31 \mathrm{p} 00$ & 26.3 & -46.2 & -19.9 & 130.1 & -160.1 & -30.1 & 145.3 & -120.3 & 25.0 \\
\hline 671.6 & 32 p80 & 85.4 & -84.1 & 1.3 & 47.6 & -146.8 & -99.1 & 49.5 & -109.6 & -60.1 \\
\hline 671.4 & $35 \mathrm{p} 80$ & 161.8 & -155.2 & 6.5 & 140.3 & -132.2 & 8.1 & 94.9 & -73.0 & 21.9 \\
\hline 671.3 & $36 \mathrm{p} 80$ & 81.8 & -67.8 & 14.0 & 172.1 & -151.6 & 20.5 & 162.2 & -92.0 & 70.1 \\
\hline 671.3 & $37 \mathrm{p} 00$ & 49.4 & -59.9 & -10.5 & 174.8 & -160.6 & 14.1 & 165.5 & -108.2 & 57.3 \\
\hline 671.2 & 39 p20 & 72.1 & -69.8 & 2.3 & 46.0 & -136.3 & -90.2 & 19.5 & -80.0 & -60.5 \\
\hline 671.0 & 43 p20 & 130.5 & -143.4 & -12.9 & 117.5 & -108.3 & 9.3 & 46.3 & -22.3 & 24.0 \\
\hline 670.9 & $43 p 40$ & 103.8 & -120.2 & -16.4 & 94.4 & -127.4 & -32.9 & 33.1 & -21.2 & 11.9 \\
\hline 670.8 & $45 \mathrm{p} 60$ & 53.6 & -43.3 & 10.4 & 75.7 & -188.7 & -113.0 & 35.7 & -30.3 & 5.3 \\
\hline 670.6 & $48 \mathrm{p} 40$ & 34.0 & -134.1 & -100.1 & 72.8 & -14.5 & 58.3 & 13.7 & -44.9 & -31.2 \\
\hline 670.6 & $50 \mathrm{p} 00$ & 35.0 & -150.5 & -115.5 & 129.7 & -100.5 & 29.2 & 1.9 & -56.6 & -54.7 \\
\hline 670.5 & $51 \mathrm{p} 00$ & 76.9 & -73.7 & 3.2 & 150.4 & -102.0 & 48.4 & 21.9 & -8.1 & 13.8 \\
\hline 670.4 & $51 \mathrm{p} 80$ & 74.9 & -34.4 & 40.5 & 165.1 & -91.8 & 73.3 & 117.0 & -30.0 & 87.0 \\
\hline 670.3 & $56 \mathrm{p} 40$ & 154.1 & -134.1 & 20.0 & 97.0 & -99.0 & -2.0 & 37.2 & -37.8 & -0.6 \\
\hline 670.2 & $57 \mathrm{p} 40$ & 90.9 & -3.5 & 87.4 & -no data- & -no data- & -no data- & -no data- & -no data- & -no data- \\
\hline 670.1 & $58 \mathrm{p} 80$ & 56.8 & -70.3 & -13.5 & 70.8 & -132.3 & -61.6 & 116.1 & -124.4 & -8.3 \\
\hline 670.0 & 59 p80 & 71.7 & -110.0 & -38.3 & 21.0 & -94.0 & -72.9 & 50.1 & -113.7 & -63.6 \\
\hline 669.9 & 60 p60 & 78.8 & -119.9 & -41.1 & 11.6 & -63.1 & -51.5 & 16.4 & -63.5 & -47.1 \\
\hline 669.8 & $62 \mathrm{p} 00$ & 88.8 & -93.0 & -4.2 & 78.5 & -50.3 & 28.2 & 35.9 & -34.2 & 1.7 \\
\hline 669.8 & $62 \mathrm{p} 60$ & 94.6 & -75.5 & 19.1 & 118.8 & -56.5 & 62.3 & 75.3 & -35.5 & 39.8 \\
\hline \multicolumn{2}{|l|}{ Total area } & $2,396.5$ & $-2,558.7$ & -162.3 & $3,059.8$ & $-3,132.4$ & -72.6 & $2,340.8$ & $-2,020.7$ & 320.2 \\
\hline \multicolumn{2}{|c|}{$\begin{array}{l}\text { Number of cross } \\
\text { sections }\end{array}$} & 32 & 32 & 32 & 31 & 31 & 31 & 31 & 31 & 31 \\
\hline \multicolumn{2}{|l|}{ Average } & 74.9 & -80.0 & -5.1 & 98.7 & -101.0 & -2.3 & 75.5 & -65.2 & 10.3 \\
\hline
\end{tabular}


Table 6. Cross section comparison values for the Little Sioux reach, 2006-07.-Continued

\begin{tabular}{|c|c|c|c|c|c|c|c|c|c|c|c|c|c|}
\hline \multirow[b]{2}{*}{$\begin{array}{l}\text { River } \\
\text { mile }\end{array}$} & \multirow[b]{2}{*}{$\begin{array}{c}\text { Line } \\
\text { number }\end{array}$} & \multicolumn{3}{|c|}{ March 2006 to March 2007} & \multicolumn{3}{|c|}{ April 2006 to April 2007} & \multicolumn{3}{|c|}{ July 2006 to July 2007} & \multicolumn{3}{|c|}{ October 2006 to October 2007} \\
\hline & & $\begin{array}{c}\text { Deposi- } \\
\text { tion area } \\
\text { (square } \\
\text { meters) }\end{array}$ & $\begin{array}{c}\text { Erosion } \\
\text { area } \\
\text { (square } \\
\text { meters) }\end{array}$ & $\begin{array}{c}\text { Net } \\
\text { change } \\
\text { (square } \\
\text { meters) }\end{array}$ & $\begin{array}{c}\text { Deposi- } \\
\text { tion area } \\
\text { (square } \\
\text { meters) }\end{array}$ & $\begin{array}{c}\text { Erosion } \\
\text { area, } \\
\text { (square } \\
\text { meters) }\end{array}$ & $\begin{array}{c}\text { Net } \\
\text { change } \\
\text { (square } \\
\text { meters) }\end{array}$ & $\begin{array}{c}\text { Deposi- } \\
\text { tion area } \\
\text { (square } \\
\text { meters) }\end{array}$ & $\begin{array}{c}\text { Erosion } \\
\text { area } \\
\text { (square } \\
\text { meters) }\end{array}$ & $\begin{array}{c}\text { Net } \\
\text { change } \\
\text { (square } \\
\text { meters) }\end{array}$ & $\begin{array}{c}\text { Deposi- } \\
\text { tion area } \\
\text { (square } \\
\text { meters) }\end{array}$ & $\begin{array}{c}\text { Erosion } \\
\text { area } \\
\text { (square } \\
\text { meters) }\end{array}$ & $\begin{array}{c}\text { Net } \\
\text { change } \\
\text { (square } \\
\text { meters) }\end{array}$ \\
\hline 673.4 & $2 \mathrm{p} 60$ & 83.7 & -1.2 & 82.5 & 70.6 & -86.2 & -15.6 & 5.8 & -43.5 & -37.7 & 65.8 & -25.7 & 40.0 \\
\hline 673.4 & $4 \mathrm{p} 20$ & 35.6 & -22.4 & 13.2 & 59.1 & -3.6 & 55.5 & 24.0 & -41.1 & -17.1 & 56.7 & -62.3 & -5.6 \\
\hline 673.0 & $10 \mathrm{p} 00$ & 101.6 & -61.9 & 39.7 & 112.0 & -108.2 & 3.8 & 29.3 & -32.2 & -3.0 & 47.8 & -43.9 & 3.9 \\
\hline 673.0 & $10 \mathrm{p} 40$ & 20.7 & -63.2 & -42.4 & 10.2 & -107.5 & -97.3 & 0.1 & -48.1 & -48.0 & 35.5 & -16.5 & 18.9 \\
\hline 673.0 & $9 \mathrm{p} 20$ & 115.2 & -55.2 & 59.9 & 92.7 & -92.2 & 0.5 & 0.9 & -82.2 & -81.4 & 50.8 & -41.3 & 9.6 \\
\hline 672.6 & $16 \mathrm{p} 80$ & 77.9 & -35.7 & 42.2 & 140.1 & -126.9 & 13.2 & 77.2 & -24.9 & 52.3 & 50.8 & -43.6 & 7.2 \\
\hline 672.4 & 20p20 & 4.3 & -91.5 & -87.2 & 0.0 & -120.5 & -120.5 & 130.2 & -1.6 & 128.6 & 28.3 & -6.9 & 21.4 \\
\hline 672.3 & $22 \mathrm{p} 20$ & 20.0 & -20.6 & -0.6 & 11.2 & -113.6 & -102.4 & 37.4 & -4.8 & 32.6 & 71.9 & -7.4 & 64.5 \\
\hline 672.2 & $23 \mathrm{p} 80$ & 18.0 & -21.4 & -3.4 & 28.9 & -17.9 & 11.0 & 21.3 & -38.6 & -17.2 & 106.2 & -45.8 & 60.3 \\
\hline 672.1 & $24 \mathrm{p} 00$ & 14.1 & -20.9 & -6.8 & 11.1 & -16.3 & -5.1 & 2.1 & -62.2 & -60.1 & 112.1 & -7.6 & 104.5 \\
\hline 671.9 & $27 \mathrm{p} 80$ & 70.4 & -48.4 & 22.0 & 69.4 & -89.2 & -19.8 & 62.9 & -84.3 & -21.4 & 88.3 & -120.8 & -32.5 \\
\hline 671.8 & 30 p20 & 46.0 & -6.2 & 39.8 & 50.6 & -26.5 & 24.0 & 54.5 & -61.9 & -7.5 & 102.3 & -114.0 & -11.7 \\
\hline 671.7 & $31 \mathrm{p} 00$ & 59.6 & -4.6 & 55.0 & 24.2 & -26.8 & -2.5 & 67.4 & -112.5 & -45.1 & 131.0 & -86.2 & 44.9 \\
\hline 671.6 & $32 \mathrm{p} 80$ & 61.8 & -22.6 & 39.2 & 24.4 & -23.8 & 0.5 & 79.6 & -128.0 & -48.3 & 1.0 & -62.4 & -61.4 \\
\hline 671.4 & $35 \mathrm{p} 80$ & 143.3 & -129.2 & 14.0 & 110.2 & -111.9 & -1.7 & 55.8 & -35.1 & 20.6 & 159.0 & -143.5 & 15.5 \\
\hline 671.3 & $36 \mathrm{p} 80$ & 112.8 & -64.2 & 48.6 & 107.2 & -111.7 & -4.5 & 92.3 & -119.3 & -27.0 & 190.7 & -138.0 & 52.7 \\
\hline 671.3 & $37 \mathrm{p} 00$ & 89.4 & -45.9 & 43.5 & 123.4 & -107.9 & 15.5 & 105.5 & -130.7 & -25.3 & 189.9 & -121.9 & 68.1 \\
\hline 671.2 & 39 p20 & 68.0 & -38.2 & 29.8 & 63.7 & -4.9 & 58.7 & 136.6 & -120.5 & 16.1 & 44.6 & -107.4 & -62.8 \\
\hline 671.0 & $43 \mathrm{p} 20$ & 145.7 & -131.1 & 14.6 & 49.8 & -69.1 & -19.2 & 19.4 & -31.6 & -12.3 & 128.9 & -92.0 & 36.9 \\
\hline 670.9 & $43 \mathrm{p} 40$ & 158.9 & -113.7 & 45.2 & 74.6 & -54.0 & 20.6 & 15.6 & -45.3 & -29.8 & 103.6 & -75.2 & 28.4 \\
\hline 670.8 & 45 p60 & 161.8 & -43.4 & 118.5 & 165.7 & -45.9 & 119.7 & 43.6 & -54.7 & -11.1 & 51.7 & -56.8 & -5.0 \\
\hline 670.6 & $48 \mathrm{p} 40$ & 27.6 & -117.3 & -89.7 & 42.0 & -83.8 & -41.8 & 71.9 & -8.2 & 63.7 & 89.7 & -20.9 & 68.8 \\
\hline 670.6 & $50 \mathrm{p} 00$ & 58.8 & -142.5 & -83.7 & 57.4 & -127.2 & -69.8 & 37.6 & -72.6 & -35.0 & 133.5 & -72.3 & 61.1 \\
\hline 670.5 & $51 \mathrm{p} 00$ & 107.1 & -84.3 & 22.8 & 107.9 & -138.0 & -30.1 & 35.9 & -75.7 & -39.8 & 122.1 & -69.8 & 52.3 \\
\hline 670.4 & $51 \mathrm{p} 80$ & 79.5 & -65.6 & 13.9 & 94.2 & -127.0 & -32.8 & 71.8 & -93.2 & -21.5 & 119.4 & -72.7 & 46.7 \\
\hline 670.3 & $56 \mathrm{p} 40$ & 117.6 & -116.2 & 1.4 & 77.0 & -88.1 & -11.1 & 87.4 & -58.0 & 29.4 & 117.6 & -138.1 & -20.5 \\
\hline 670.2 & $57 \mathrm{p} 40$ & 70.6 & -3.8 & 66.8 & 92.6 & -105.3 & -12.7 & 45.2 & -43.9 & 1.3 & -no data- & -no data- & -no data- \\
\hline 670.1 & $58 \mathrm{p} 80$ & 84.1 & -31.1 & 52.9 & 59.7 & -82.2 & -22.5 & 180.9 & -123.1 & 57.8 & 74.0 & -68.6 & 5.4 \\
\hline 670.0 & $59 \mathrm{p} 80$ & 38.7 & -29.5 & 9.1 & 60.4 & -19.5 & 40.9 & 180.4 & -129.6 & 50.8 & 30.7 & -56.6 & -26.0 \\
\hline 669.9 & 60 p60 & 33.3 & -28.9 & 4.4 & 25.0 & -35.9 & -10.8 & 169.5 & -121.6 & 47.9 & 66.2 & -72.3 & -6.2 \\
\hline 669.8 & $62 \mathrm{p} 00$ & 34.5 & -60.9 & -26.5 & 4.0 & -42.4 & -38.4 & 72.8 & -48.9 & 23.9 & 110.2 & -104.3 & 5.9 \\
\hline 669.8 & $62 \mathrm{p} 60$ & 33.2 & -55.7 & -22.5 & 12.6 & -43.5 & -30.9 & 9.6 & -26.7 & -17.2 & 129.6 & -108.9 & 20.8 \\
\hline \multicolumn{2}{|l|}{ Total area } & $2,293.7$ & $-1,777.3$ & 516.4 & $2,032.2$ & $-2,357.6$ & -325.4 & $2,024.4$ & $-2,105.0$ & -80.7 & $2,809.8$ & $-2,203.8$ & 606.0 \\
\hline \multicolumn{2}{|c|}{$\begin{array}{l}\text { Number of cross } \\
\text { sections }\end{array}$} & 32 & 32 & 32 & 32 & 32 & 32 & 32 & 32 & 32 & 31 & 31 & 31 \\
\hline \multicolumn{2}{|l|}{ Average } & 71.7 & -55.5 & 16.1 & 63.5 & -73.7 & -10.2 & 63.3 & -65.8 & -2.5 & 90.6 & -71.1 & 19.5 \\
\hline
\end{tabular}



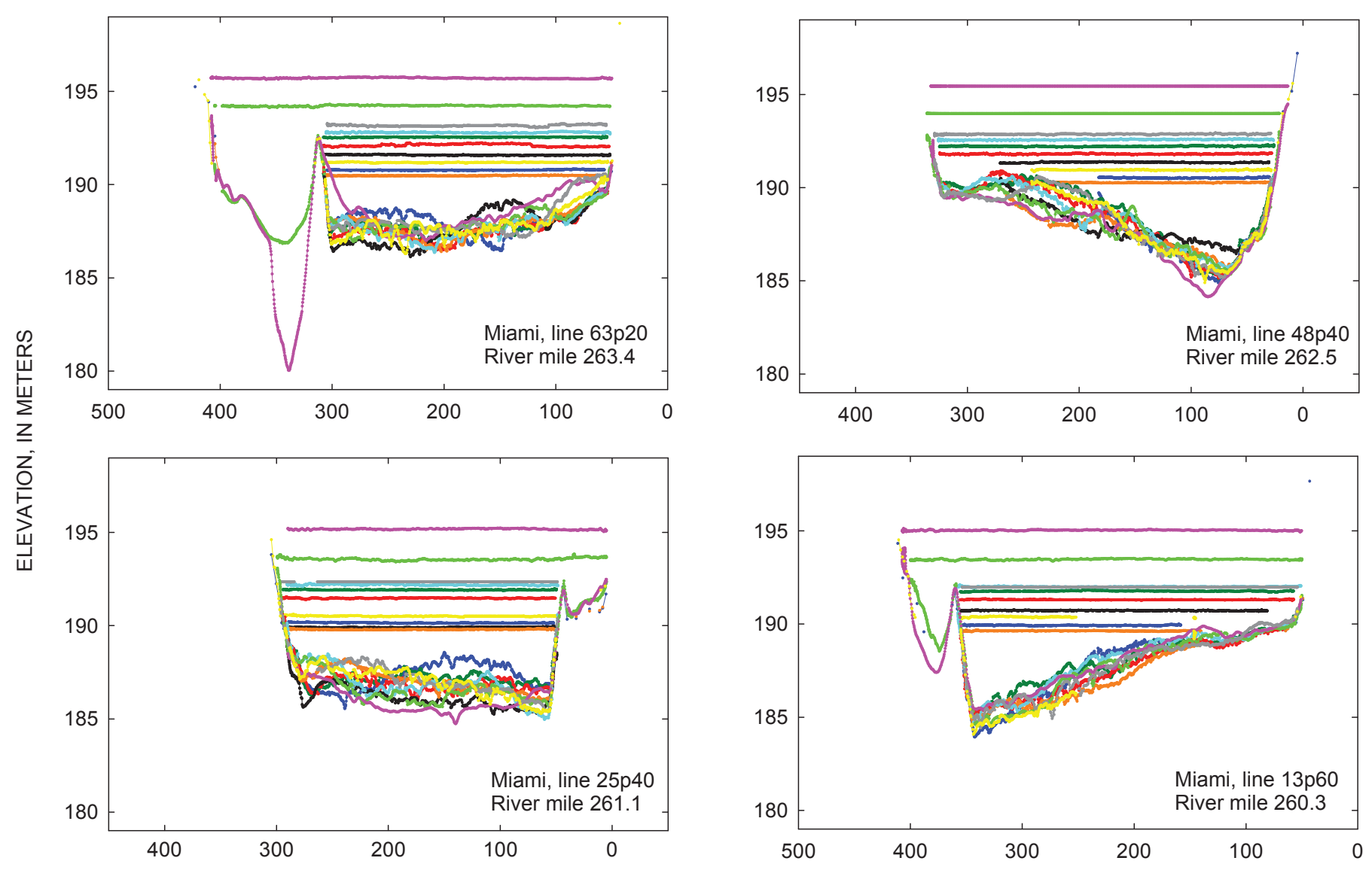

HORIZONTAL DISTANCE, IN METERS

EXPLANATION

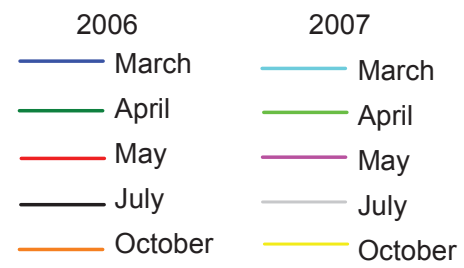

Figure 15. Typical survey cross sections for the Miami reach.

(RM 262.2-261.0) experienced high deposition, and the reach experienced net overall deposition.

In general, the region around the Miami bridge (fig. 16) was more stable than the rest of the reach and scour and fill was highest downstream from wing dikes. Cross sections in the Miami reach did not have erosion and deposition occur on individual cross sections as was seen in the Little Sioux reach. Instead, there was generally a trend for a given cross section towards deposition or erosion for any pair of dates (fig. 16).

\section{Longitudinal Profiles}

Longitudinal profiles were assessed for reach-scale comparisons and characteristic bedform calculations. These profiles were surveyed along planned lines in what was estimated to be the thalweg of each reach based on channel plan form in March 2006. The same line was driven for each subsequent survey.

\section{Reach-Scale Profiles}

Longitudinal thalweg profiles were not collected for every survey in the Yankton and Kenslers Bend reaches. The longitudinal profiles surveyed in June 2007 show a thalweg trace and characteristic bedforms for the Yankton reach (fig. 17). The July 2007 survey at Kenslers Bend demonstrates characteristic bedforms in the reach (fig. 18). Gravel and sand dunes were present on portions of the bed in both of these reaches. 
Table 7. Cross section comparison values for the Miami reach, 2006-07.

\begin{tabular}{|c|c|c|c|c|c|c|c|c|c|c|c|c|c|}
\hline \multirow[b]{2}{*}{$\begin{array}{l}\text { River } \\
\text { mile }\end{array}$} & \multirow[b]{2}{*}{$\begin{array}{c}\text { Line } \\
\text { number }\end{array}$} & \multicolumn{3}{|c|}{ March 2006 to April 2006} & \multicolumn{3}{|c|}{ April 2006 to May 2006} & \multicolumn{3}{|c|}{ May 2006 to July 2006} & \multicolumn{3}{|c|}{ July 2006 to November 2006} \\
\hline & & $\begin{array}{c}\text { Deposi- } \\
\text { tion area } \\
\text { (square } \\
\text { meters) }\end{array}$ & $\begin{array}{c}\text { Erosion } \\
\text { area } \\
\text { (square } \\
\text { meters) }\end{array}$ & $\begin{array}{c}\text { Net } \\
\text { change } \\
\text { (square } \\
\text { meters) }\end{array}$ & $\begin{array}{c}\text { Deposi- } \\
\text { tion area } \\
\text { (square } \\
\text { meters) }\end{array}$ & $\begin{array}{l}\text { Erosion } \\
\text { area, } \\
\text { (square } \\
\text { meters) }\end{array}$ & $\begin{array}{c}\text { Net } \\
\text { change } \\
\text { (square } \\
\text { meters) }\end{array}$ & $\begin{array}{c}\text { Deposi- } \\
\text { tion area } \\
\text { (square } \\
\text { meters) }\end{array}$ & $\begin{array}{l}\text { Erosion } \\
\text { area } \\
\text { (square } \\
\text { meters) }\end{array}$ & $\begin{array}{c}\text { Net } \\
\text { change } \\
\text { (square } \\
\text { meters) }\end{array}$ & $\begin{array}{c}\text { Deposi- } \\
\text { tion area } \\
\text { (square } \\
\text { meters) }\end{array}$ & $\begin{array}{l}\text { Erosion } \\
\text { area } \\
\text { (square } \\
\text { meters) }\end{array}$ & $\begin{array}{c}\text { Net } \\
\text { change } \\
\text { (square } \\
\text { meters) }\end{array}$ \\
\hline 259.5 & $0 \mathrm{p} 40$ & 46.7 & -25.0 & 21.7 & 40.1 & -27.1 & 13.0 & 24.6 & -37.3 & -12.6 & 33.7 & -64.0 & -30.3 \\
\hline 259.7 & $3 \mathrm{p} 20$ & 57.8 & -4.3 & 53.5 & 44.6 & -22.5 & 22.1 & 27.6 & -47.4 & -19.8 & 19.1 & -70.4 & -51.3 \\
\hline 259.7 & $3 p 80$ & 69.4 & -7.1 & 62.3 & 55.4 & -38.2 & 17.2 & 12.3 & -64.5 & -52.2 & 22.6 & -76.5 & -53.9 \\
\hline 260.3 & $12 \mathrm{p} 60$ & 69.3 & -37.8 & 31.5 & 6.4 & -72.9 & -66.5 & 13.9 & -64.0 & -50.1 & 18.5 & -22.6 & -4.1 \\
\hline 260.3 & 13 p20 & 81.3 & -31.9 & 49.4 & 2.5 & -91.1 & -88.6 & 28.4 & -37.0 & -8.5 & 5.5 & -51.5 & -46.0 \\
\hline 260.3 & $13 \mathrm{p} 60$ & 86.5 & -44.7 & 41.7 & 6.5 & -100.5 & -94.1 & 17.2 & -35.5 & -18.2 & 8.1 & -50.3 & -42.2 \\
\hline 260.4 & 14 p60 & 55.4 & -59.7 & -4.3 & 9.9 & -74.4 & -64.5 & 23.2 & -44.6 & -21.4 & 13.0 & -42.4 & -29.4 \\
\hline 260.5 & $15 \mathrm{p} 40$ & 64.8 & -44.8 & 20.0 & 15.2 & -76.8 & -61.6 & 13.9 & -35.6 & -21.7 & 6.8 & -44.2 & -37.3 \\
\hline 260.6 & $17 \mathrm{p} 80$ & 55.5 & -43.2 & 12.3 & 51.0 & -48.8 & 2.3 & 27.0 & -47.7 & -20.6 & 17.5 & -44.4 & -27.0 \\
\hline 261.0 & $23 \mathrm{p} 40$ & 23.5 & -33.1 & -9.6 & 11.9 & -74.8 & -62.9 & 0.0 & -192.2 & -192.2 & 210.8 & 0.0 & 210.8 \\
\hline 261.0 & $24 p 40$ & 28.7 & -58.4 & -29.7 & 9.7 & -75.7 & -66.0 & 0.0 & -177.2 & -177.2 & 233.3 & 0.0 & 233.3 \\
\hline 261.1 & $25 \mathrm{p} 20$ & 42.2 & -76.1 & -33.8 & 10.8 & -61.9 & -51.2 & 0.0 & -194.4 & -194.4 & 224.1 & 0.0 & 224.1 \\
\hline 261.1 & $25 \mathrm{p} 40$ & 41.8 & -84.1 & -42.4 & 18.3 & -72.2 & -53.9 & 1.3 & -189.4 & -188.1 & 219.2 & -0.1 & 219.0 \\
\hline 261.2 & $28 \mathrm{p} 00$ & 20.6 & -64.8 & -44.1 & 35.0 & -45.3 & -10.3 & 0.0 & -189.3 & -189.3 & 227.8 & -4.7 & 223.1 \\
\hline 261.4 & 30 p80 & 32.1 & -55.4 & -23.3 & 33.0 & -27.5 & 5.6 & 0.1 & -197.9 & -197.7 & 186.2 & -1.5 & 184.8 \\
\hline 261.5 & $32 \mathrm{p} 40$ & 16.1 & -40.5 & -24.4 & 26.6 & -29.0 & -2.5 & 0.0 & -180.4 & -180.4 & 253.8 & -21.4 & 232.4 \\
\hline 261.9 & $38 \mathrm{p} 00$ & 73.8 & -4.2 & 69.6 & -no data- & -no data- & -no data- & -no data- & -no data- & -no data- & 79.2 & -66.9 & 12.2 \\
\hline 261.9 & $39 \mathrm{p} 00$ & -no data- & -no data- & -no data- & -no data- & -no data- & -no data- & -no data- & -no data- & -no data- & -no data- & -no data- & -no data- \\
\hline 262.2 & 43p00 & 42.9 & -12.2 & 30.8 & 10.5 & -58.9 & -48.4 & 6.2 & -293.0 & -286.7 & 234.4 & -21.1 & 213.3 \\
\hline 262.2 & 43 p40 & 83.2 & -6.2 & 77.0 & 25.8 & -64.6 & -38.8 & 13.5 & -262.6 & -249.1 & 241.2 & -51.1 & 190.1 \\
\hline 262.5 & $48 \mathrm{p} 40$ & 66.5 & -1.9 & 64.6 & 3.2 & -40.4 & -37.2 & 78.0 & -31.3 & 46.7 & 16.6 & -56.7 & -40.2 \\
\hline 262.6 & 49p40 & 42.3 & -10.5 & 31.7 & 31.5 & -4.2 & 27.3 & 44.1 & -16.8 & 27.3 & 0.3 & -78.2 & -77.9 \\
\hline 262.6 & $50 \mathrm{p} 20$ & 52.9 & -24.0 & 28.8 & 46.9 & -9.8 & 37.0 & 10.1 & -15.4 & -5.3 & 0.7 & -87.4 & -86.7 \\
\hline 262.7 & $51 \mathrm{p} 20$ & -no data- & -no data- & -no data- & -no data- & -no data- & -no data- & -no data- & -no data- & -no data- & -no data- & -no data- & -no data- \\
\hline 262.8 & $53 \mathrm{p} 00$ & 110.2 & 0.0 & 110.2 & 18.6 & -33.3 & -14.8 & 4.3 & -88.9 & -84.6 & 9.7 & -132.2 & -122.6 \\
\hline 262.9 & $54 \mathrm{p} 20$ & 77.7 & -1.3 & 76.4 & 10.3 & -61.9 & -51.6 & 2.8 & -71.5 & -68.7 & 46.9 & -86.1 & -39.2 \\
\hline 262.9 & $55 \mathrm{p} 00$ & 62.9 & -11.6 & 51.4 & 20.0 & -51.8 & -31.8 & 19.6 & -65.8 & -46.2 & 32.0 & -72.5 & -40.5 \\
\hline 263.0 & $55 \mathrm{p} 60$ & 58.9 & -37.4 & 21.5 & 44.2 & -18.3 & 25.9 & 29.4 & -90.7 & -61.3 & 34.8 & -79.2 & -44.4 \\
\hline 263.3 & $62 \mathrm{p} 00$ & 30.3 & -40.5 & -10.2 & 29.8 & -55.2 & -25.4 & 51.1 & -66.7 & -15.6 & 13.8 & -35.6 & -21.9 \\
\hline 263.4 & $62 \mathrm{p} 60$ & 35.3 & -47.3 & -12.0 & 17.2 & -65.0 & -47.8 & 72.8 & -77.9 & -5.1 & 72.6 & -46.4 & 26.2 \\
\hline 263.4 & $63 \mathrm{p} 20$ & 38.6 & -83.4 & -44.8 & 14.4 & -58.9 & -44.4 & 85.6 & -62.6 & 23.0 & 105.9 & -78.2 & 27.8 \\
\hline 263.5 & $65 \mathrm{p} 00$ & 36.3 & -39.8 & -3.6 & 66.8 & -63.7 & 3.1 & 49.2 & -17.2 & 32.0 & 103.7 & -96.2 & 7.5 \\
\hline 263.6 & $65 \mathrm{p} 80$ & 196.6 & -35.9 & 160.7 & 76.4 & -58.2 & 18.1 & 90.1 & -110.9 & -20.8 & 45.1 & -172.8 & -127.7 \\
\hline Total area & & $1,800.2$ & $-1,067.0$ & 733.1 & 792.6 & $-1,583.3$ & -168.7 & 746.4 & $-3,005.3$ & $-2,258.9$ & $2,736.8$ & $-1,654.8$ & $1,082.1$ \\
\hline $\begin{array}{l}\text { Number of } \\
\text { sections }\end{array}$ & cross & 31 & 31 & 31 & 30 & 30 & 30 & 30 & 30 & 30 & 31 & 31 & 31 \\
\hline Average & & 58.1 & -34.4 & 23.6 & 26.4 & -52.8 & -5.6 & 24.9 & -100.2 & -75.3 & 88.3 & -53.4 & 34.9 \\
\hline
\end{tabular}


Table 7. Cross section comparison values for the Miami reach, 2006-07.-Continued

\begin{tabular}{|c|c|c|c|c|c|c|c|c|c|c|c|c|c|}
\hline \multirow[b]{2}{*}{ River mile } & \multirow[b]{2}{*}{$\begin{array}{l}\text { Line } \\
\text { number }\end{array}$} & \multicolumn{3}{|c|}{ March 2007 to April 2007} & \multicolumn{3}{|c|}{ April 2007 to May 2007} & \multicolumn{3}{|c|}{ May 2007 to August 2007} & \multicolumn{3}{|c|}{ August 2007 to November 2007} \\
\hline & & $\begin{array}{c}\text { Deposi- } \\
\text { tion area } \\
\text { (square } \\
\text { meters) }\end{array}$ & $\begin{array}{c}\text { Erosion } \\
\text { area } \\
\text { (square } \\
\text { meters) }\end{array}$ & $\begin{array}{c}\text { Net } \\
\text { change } \\
\text { (square } \\
\text { meters) }\end{array}$ & $\begin{array}{l}\text { Deposi- } \\
\text { tion area } \\
\text { (square } \\
\text { meters) }\end{array}$ & $\begin{array}{c}\text { Erosion } \\
\text { area, } \\
\text { (square } \\
\text { meters) }\end{array}$ & $\begin{array}{c}\text { Net } \\
\text { change } \\
\text { (square } \\
\text { meters) }\end{array}$ & $\begin{array}{c}\text { Deposi- } \\
\text { tion area } \\
\text { (square } \\
\text { meters) }\end{array}$ & $\begin{array}{c}\text { Erosion } \\
\text { area } \\
\text { (square } \\
\text { meters) }\end{array}$ & $\begin{array}{c}\text { Net } \\
\text { change } \\
\text { (square } \\
\text { meters) }\end{array}$ & $\begin{array}{c}\text { Deposi- } \\
\text { tion area } \\
\text { (square } \\
\text { meters) }\end{array}$ & $\begin{array}{c}\text { Erosion } \\
\text { area } \\
\text { (square } \\
\text { meters) }\end{array}$ & $\begin{array}{c}\text { Net } \\
\text { change } \\
\text { (square } \\
\text { meters) }\end{array}$ \\
\hline 259.5 & $0 \mathrm{p} 40$ & 4.0 & -100.8 & -96.8 & 67.1 & -24.6 & 42.4 & 27.6 & -25.2 & 2.4 & 41.0 & -16.5 & 24.4 \\
\hline 259.7 & $3 \mathrm{p} 20$ & 19.6 & -63.1 & -43.5 & 3.5 & -156.5 & -153.0 & 140.1 & -5.4 & 134.7 & 56.9 & -11.7 & 45.2 \\
\hline 259.7 & $3 \mathrm{p} 80$ & 8.9 & -87.4 & -78.6 & 33.6 & -124.2 & -90.5 & -no data- & -no data- & -no data- & -no data- & -no data- & -no data- \\
\hline 260.3 & $12 \mathrm{p} 60$ & 13.9 & -53.8 & -40.0 & 60.0 & -5.8 & 54.2 & 5.6 & -75.0 & -69.4 & 1.6 & -33.5 & -32.0 \\
\hline 260.3 & $13 \mathrm{p} 20$ & 16.4 & -91.6 & -75.2 & 62.3 & -28.4 & 33.9 & 13.2 & -63.6 & -50.4 & 21.9 & -50.3 & -28.4 \\
\hline 260.3 & $13 \mathrm{p} 60$ & 8.0 & -87.4 & -79.4 & 82.8 & -7.8 & 75.0 & 2.4 & -73.3 & -71.0 & 2.7 & -57.5 & -54.9 \\
\hline 260.4 & $14 p 60$ & 35.2 & -46.2 & -11.0 & 23.0 & -56.4 & -33.4 & 58.8 & -28.4 & 30.5 & 13.6 & -61.9 & -48.4 \\
\hline 260.5 & $15 \mathrm{p} 40$ & 30.8 & -85.2 & -54.4 & 30.3 & -105.1 & -74.8 & 133.5 & -16.5 & 117.0 & 10.6 & -121.8 & -111.2 \\
\hline 260.6 & $17 \mathrm{p} 80$ & 34.1 & -44.0 & -9.9 & 8.1 & -141.7 & -133.6 & 234.2 & -1.3 & 232.9 & 2.8 & -102.5 & -99.7 \\
\hline 261.0 & $23 \mathrm{p} 40$ & 30.5 & -86.7 & -56.2 & 45.3 & -94.2 & -48.8 & 203.6 & -10.3 & 193.3 & 28.1 & -73.5 & -45.4 \\
\hline 261.0 & $24 p 40$ & 29.2 & -83.0 & -53.8 & -no data- & -no data- & -no data- & -no data- & -no data- & -no data- & 21.2 & -57.7 & -36.5 \\
\hline 261.1 & $25 \mathrm{p} 20$ & 25.0 & -122.2 & -97.2 & 15.5 & -153.8 & -138.4 & 246.4 & -12.8 & 233.6 & 37.3 & -27.7 & 9.6 \\
\hline 261.1 & $25 \mathrm{p} 40$ & 20.4 & -97.0 & -76.6 & 13.3 & -140.9 & -127.6 & 264.7 & -14.5 & 250.2 & 23.8 & -48.1 & -24.4 \\
\hline 261.2 & $28 \mathrm{p} 00$ & 13.1 & -85.4 & -72.3 & 241.2 & -82.2 & 159.0 & 28.7 & -133.3 & -104.7 & 70.2 & -83.5 & -13.4 \\
\hline 261.4 & $30 \mathrm{p} 80$ & 67.7 & -108.7 & -41.0 & 106.5 & -48.5 & 58.0 & 50.5 & -108.2 & -57.6 & 47.4 & -26.6 & 20.8 \\
\hline 261.5 & $32 \mathrm{p} 40$ & 36.5 & -90.4 & -53.9 & 62.7 & -46.8 & 15.9 & 188.7 & -133.3 & 55.4 & 74.1 & -62.2 & 11.9 \\
\hline 261.9 & $38 \mathrm{p} 00$ & 85.0 & -17.6 & 67.4 & 32.3 & -78.3 & -46.0 & 26.0 & -27.3 & -1.3 & 34.4 & -8.8 & 25.5 \\
\hline 261.9 & $39 \mathrm{p} 00$ & 18.6 & -106.4 & -87.9 & 107.6 & -0.2 & 107.4 & 24.8 & -29.0 & -4.3 & 32.0 & -23.5 & 8.5 \\
\hline 262.2 & $43 \mathrm{p} 00$ & 34.9 & -58.9 & -24.0 & 56.3 & -69.8 & -13.5 & 37.7 & -63.4 & -25.7 & 8.0 & -55.8 & -47.8 \\
\hline 262.2 & $43 p 40$ & 35.5 & -60.8 & -25.3 & 72.5 & -65.2 & 7.3 & 58.5 & -94.7 & -36.2 & 22.3 & -62.1 & -39.7 \\
\hline 262.5 & $48 \mathrm{p} 40$ & 8.5 & -45.2 & -36.7 & 11.8 & -98.1 & -86.3 & 77.0 & -4.1 & 72.9 & 18.1 & -11.0 & 7.2 \\
\hline 262.6 & $49 p 40$ & -no data- & -no data- & -no data- & 2.1 & -150.9 & -148.8 & 76.9 & 0.0 & 76.9 & 27.5 & -8.6 & 18.9 \\
\hline 262.6 & $50 \mathrm{p} 20$ & 8.7 & -36.6 & -27.9 & 2.3 & -92.5 & -90.2 & 62.0 & -2.1 & 59.9 & 13.1 & -20.4 & -7.3 \\
\hline 262.7 & $51 \mathrm{p} 20$ & 24.5 & -64.5 & -40.0 & 18.9 & -61.5 & -42.6 & 75.8 & -0.4 & 75.5 & 0.1 & -84.9 & -84.8 \\
\hline 262.8 & $53 \mathrm{p} 00$ & 7.0 & -122.4 & -115.4 & 3.1 & -104.3 & -101.2 & 108.5 & -5.2 & 103.3 & 4.2 & -47.2 & -43.1 \\
\hline 262.9 & $54 \mathrm{p} 20$ & 31.5 & -44.9 & -13.4 & 19.5 & -33.3 & -13.9 & 80.2 & -6.3 & 74.0 & 7.6 & -90.5 & -82.8 \\
\hline 262.9 & $55 \mathrm{p} 00$ & 11.9 & -74.1 & -62.1 & -no data- & -no data- & -no data- & -no data- & -no data- & -no data- & 1.6 & -106.8 & -105.1 \\
\hline 263.0 & $55 \mathrm{p} 60$ & 39.9 & -36.2 & 3.7 & 28.2 & -54.1 & -25.9 & 136.0 & -7.7 & 128.3 & 5.3 & -88.0 & -82.7 \\
\hline 263.3 & $62 \mathrm{p} 00$ & 26.7 & -65.2 & -38.5 & 144.3 & -10.8 & 133.5 & 21.4 & -77.9 & -56.5 & 22.8 & -38.6 & -15.8 \\
\hline 263.4 & $62 \mathrm{p} 60$ & 32.9 & -29.7 & 3.2 & 150.5 & -5.3 & 145.2 & 37.2 & -125.2 & -88.0 & 31.7 & -48.0 & -16.3 \\
\hline 263.4 & $63 \mathrm{p} 20$ & 82.3 & -21.7 & 60.6 & 155.6 & -26.4 & 129.3 & 34.2 & -137.7 & -103.5 & 28.2 & -89.5 & -61.3 \\
\hline 263.5 & $65 \mathrm{p} 00$ & 27.7 & -66.8 & -39.2 & 52.9 & -88.4 & -35.6 & 156.5 & -52.8 & 103.7 & 22.1 & -27.2 & -5.1 \\
\hline 263.6 & $65 \mathrm{p} 80$ & 19.6 & -125.9 & -106.3 & 254.2 & -81.2 & 173.0 & 126.6 & -374.1 & -247.4 & 59.4 & -138.0 & -78.6 \\
\hline Total area & & 888.6 & $-2,309.9$ & $-1,421.3$ & $1,967.2$ & $-2,237.4$ & -270.2 & $2,737.5$ & $-1,708.9$ & $1,028.6$ & 791.7 & $-1,784.0$ & -992.3 \\
\hline $\begin{array}{l}\text { Number of c } \\
\text { sections }\end{array}$ & cross & 32 & 32 & 32 & 31 & 31 & 31 & 30 & 30 & 30.0 & 32.0 & 32.0 & 32.0 \\
\hline Average & & 27.8 & -72.2 & -44.4 & 63.5 & -72.2 & -8.7 & 91.3 & -57.0 & 34.3 & 24.7 & -55.8 & -31.0 \\
\hline
\end{tabular}


Table 7. Cross section comparison values for the Miami reach, 2006-07.-Continued

\begin{tabular}{|c|c|c|c|c|c|c|c|c|c|c|}
\hline \multirow[b]{2}{*}{ River mile } & \multirow[b]{2}{*}{$\begin{array}{c}\text { Line } \\
\text { number }\end{array}$} & \multicolumn{3}{|c|}{ November 2006 to March 2007} & \multicolumn{3}{|c|}{ March 2006 to November 2006} & \multicolumn{3}{|c|}{ March 2007 to November 2007} \\
\hline & & $\begin{array}{c}\text { Deposi- } \\
\text { tion area } \\
\text { (square } \\
\text { meters) }\end{array}$ & $\begin{array}{l}\text { Erosion } \\
\text { area } \\
\text { (square } \\
\text { meters) }\end{array}$ & $\begin{array}{c}\text { Net } \\
\text { change } \\
\text { (square } \\
\text { meters) }\end{array}$ & $\begin{array}{c}\text { Deposi- } \\
\text { tion area } \\
\text { (square } \\
\text { meters) }\end{array}$ & $\begin{array}{c}\text { Erosion } \\
\text { area, } \\
\text { (square } \\
\text { meters) }\end{array}$ & $\begin{array}{c}\text { Net } \\
\text { change } \\
\text { (square } \\
\text { meters) }\end{array}$ & $\begin{array}{c}\text { Deposi- } \\
\text { tion area } \\
\text { (square } \\
\text { meters) }\end{array}$ & $\begin{array}{c}\text { Erosion } \\
\text { area } \\
\text { (square } \\
\text { meters) }\end{array}$ & $\begin{array}{c}\text { Net change } \\
\text { (square } \\
\text { meters) }\end{array}$ \\
\hline 259.5 & $0 \mathrm{p} 40$ & 42.7 & -32.4 & 10.2 & 40.1 & -29.4 & 10.7 & 20.6 & -48.1 & -27.5 \\
\hline 259.7 & $3 \mathrm{p} 20$ & 51.0 & -31.2 & 19.9 & 44.6 & -33.1 & 11.6 & 41.8 & -58.4 & -16.5 \\
\hline 259.7 & $3 \mathrm{p} 80$ & 51.6 & -38.4 & 13.2 & 55.4 & -53.3 & 2.1 & 47.4 & -29.4 & 18.1 \\
\hline 260.3 & $12 \mathrm{p} 60$ & 114.1 & -15.5 & 98.6 & 6.4 & -126.4 & -120.0 & 1.2 & -53.2 & -52.0 \\
\hline 260.3 & $13 \mathrm{p} 20$ & 143.1 & -2.2 & 140.9 & 2.5 & -137.2 & -134.8 & 2.3 & -122.5 & -120.2 \\
\hline 260.3 & $13 p 60$ & 155.2 & -6.3 & 148.8 & 6.5 & -149.2 & -142.8 & 0.8 & -82.9 & -82.0 \\
\hline 260.4 & $14 \mathrm{p} 60$ & 118.3 & -27.6 & 90.8 & 9.9 & -151.1 & -141.1 & 11.3 & -73.6 & -62.3 \\
\hline 260.5 & $15 \mathrm{p} 40$ & 131.5 & -4.6 & 126.9 & 15.2 & -114.9 & -99.7 & 10.0 & -133.4 & -123.4 \\
\hline 260.6 & $17 \mathrm{p} 80$ & 55.2 & -31.5 & 23.7 & 51.0 & -105.5 & -54.4 & 45.3 & -55.5 & -10.2 \\
\hline 261.0 & $23 \mathrm{p} 40$ & 44.3 & -109.2 & -64.9 & 11.9 & -73.3 & -61.5 & 72.0 & -29.2 & 42.8 \\
\hline 261.0 & $24 \mathrm{p} 40$ & 20.4 & -121.0 & -100.6 & 9.7 & -103.7 & -94.0 & 52.1 & -27.7 & 24.5 \\
\hline 261.1 & $25 \mathrm{p} 20$ & 43.8 & -82.8 & -39.0 & 10.8 & -139.6 & -128.8 & 51.1 & -43.5 & 7.6 \\
\hline 261.1 & $25 \mathrm{p} 40$ & 46.9 & -85.9 & -39.1 & 18.3 & -155.5 & -137.1 & 73.9 & -47.3 & 26.6 \\
\hline 261.2 & $28 \mathrm{p} 00$ & 131.7 & -105.7 & 25.9 & 35.0 & -110.8 & -75.8 & 44.2 & -75.6 & -31.3 \\
\hline 261.4 & 30 p80 & 78.5 & -49.8 & 28.8 & 33.0 & -100.0 & -67.0 & 74.7 & -94.5 & -19.8 \\
\hline 261.5 & $32 \mathrm{p} 40$ & 110.3 & -152.6 & -42.4 & 26.6 & -92.2 & -65.6 & 68.1 & -38.8 & 29.3 \\
\hline 261.9 & $38 \mathrm{p} 00$ & 94.5 & -77.9 & 16.6 & 0.0 & -92.6 & -92.6 & 94.4 & -48.7 & 45.7 \\
\hline 261.9 & 39 p00 & 135.3 & -83.7 & 51.6 & -no data- & -no data- & -no data- & 63.2 & -39.4 & 23.8 \\
\hline 262.2 & $43 \mathrm{p} 00$ & 117.2 & -7.7 & 109.4 & 10.5 & -100.2 & -89.7 & 4.3 & -115.4 & -111.0 \\
\hline 262.2 & $43 p 40$ & 98.9 & -9.6 & 89.2 & 25.8 & -56.3 & -30.5 & 5.6 & -99.5 & -93.9 \\
\hline 262.5 & $48 \mathrm{p} 40$ & 36.7 & -12.3 & 24.4 & 3.2 & -14.2 & -11.0 & 3.9 & -46.9 & -42.9 \\
\hline 262.6 & $49 \mathrm{p} 40$ & -no data- & -no data- & -no data- & 31.5 & -31.2 & 0.3 & -no data- & -no data- & -no data- \\
\hline 262.6 & $50 \mathrm{p} 20$ & 54.9 & -1.0 & 54.0 & 46.9 & -59.2 & -12.4 & 0.2 & -65.8 & -65.5 \\
\hline 262.7 & $51 \mathrm{p} 20$ & 110.9 & -12.7 & 98.1 & 0.0 & -82.2 & -82.2 & 14.7 & -106.7 & -92.0 \\
\hline 262.8 & $53 \mathrm{p} 00$ & 194.1 & -4.7 & 189.4 & 18.6 & -119.2 & -100.6 & 3.1 & -159.6 & -156.4 \\
\hline 262.9 & $54 \mathrm{p} 20$ & 87.9 & -54.5 & 33.4 & 10.3 & -160.2 & -149.9 & 13.1 & -49.3 & -36.2 \\
\hline 262.9 & $55 \mathrm{p} 00$ & 76.6 & -39.6 & 37.0 & 20.0 & -161.8 & -141.8 & 15.2 & -66.6 & -51.4 \\
\hline 263.0 & $55 \mathrm{p} 60$ & 58.5 & -64.4 & -6.0 & 44.2 & -150.3 & -106.1 & 40.6 & -17.2 & 23.4 \\
\hline 263.3 & $62 \mathrm{p} 00$ & 81.6 & -52.7 & 28.9 & 29.8 & -141.2 & -111.4 & 45.0 & -22.4 & 22.6 \\
\hline 263.4 & $62 \mathrm{p} 60$ & 17.5 & -52.6 & -35.1 & 17.2 & -99.8 & -82.6 & 75.8 & -31.7 & 44.1 \\
\hline 263.4 & $63 \mathrm{p} 20$ & 23.5 & -44.6 & -21.0 & 14.4 & -87.1 & -72.7 & 75.9 & -51.4 & 24.5 \\
\hline 263.5 & $65 \mathrm{p} 00$ & 74.3 & -119.4 & -45.1 & 66.8 & -46.2 & 20.5 & 60.0 & -36.1 & 23.9 \\
\hline 263.6 & $65 \mathrm{p} 80$ & 316.8 & -94.0 & 222.9 & 76.4 & -62.4 & 14.0 & 21.9 & -281.4 & -259.5 \\
\hline \multicolumn{2}{|l|}{ Total area } & $2,917.6$ & $-1,628.1$ & $1,289.5$ & 792.6 & $-3,139.4$ & $-2,346.8$ & $1,154.0$ & $-2,251.2$ & -522.4 \\
\hline \multicolumn{2}{|c|}{$\begin{array}{l}\text { Number of cross } \\
\text { sections }\end{array}$} & 32 & 32 & 32 & 32 & 32 & 32 & 32 & 32 & 32 \\
\hline \multicolumn{2}{|l|}{ Average } & 91.2 & $\begin{array}{l}-50.9 \\
\end{array}$ & 40.3 & 24.8 & -98.1 & -73.3 & 36.1 & -70.4 & -16.3 \\
\hline
\end{tabular}


Table 7. Cross section comparison values for the Miami reach, 2006-07.-Continued

\begin{tabular}{|c|c|c|c|c|c|c|c|c|c|c|}
\hline \multirow[b]{2}{*}{ River mile } & \multirow[b]{2}{*}{$\begin{array}{c}\text { Line } \\
\text { number }\end{array}$} & \multicolumn{3}{|c|}{ March 2006 to November 2007} & \multicolumn{3}{|c|}{ March 2006 to March 2007} & \multicolumn{3}{|c|}{ April 2006 to April 2007} \\
\hline & & $\begin{array}{c}\text { Deposi- } \\
\text { tion area } \\
\text { (square } \\
\text { meters) }\end{array}$ & $\begin{array}{l}\text { Erosion } \\
\text { area } \\
\text { (square } \\
\text { meters) } \\
\end{array}$ & $\begin{array}{l}\text { Net change } \\
\text { (square } \\
\text { meters) }\end{array}$ & $\begin{array}{c}\text { Deposi- } \\
\text { tion area } \\
\text { (square } \\
\text { meters) }\end{array}$ & $\begin{array}{c}\text { Erosion } \\
\text { area } \\
\text { (square } \\
\text { meters) }\end{array}$ & $\begin{array}{c}\text { Net } \\
\text { change } \\
\text { (square } \\
\text { meters) } \\
\end{array}$ & $\begin{array}{c}\text { Deposi- } \\
\text { tion area } \\
\text { (square } \\
\text { meters) }\end{array}$ & $\begin{array}{l}\text { Erosion } \\
\text { area, } \\
\text { (square } \\
\text { meters) }\end{array}$ & $\begin{array}{c}\text { Net } \\
\text { change } \\
\text { (square } \\
\text { meters) }\end{array}$ \\
\hline 259.5 & 0p40 & 21.9 & -47.3 & -25.5 & 35.1 & -33.1 & 2.0 & 1.5 & -118.0 & -116.4 \\
\hline 259.7 & $3 \mathrm{p} 20$ & 68.9 & -60.9 & 8.0 & 61.0 & -36.5 & 24.6 & 18.3 & -90.7 & -72.4 \\
\hline 259.7 & $3 p 80$ & 40.9 & -61.8 & -21.0 & 31.5 & -44.8 & -13.3 & 3.8 & -158.1 & -154.3 \\
\hline 260.3 & $12 \mathrm{p} 60$ & 12.6 & -36.9 & -24.3 & 51.6 & -42.2 & 9.4 & 8.7 & -70.7 & -62.1 \\
\hline 260.3 & $13 \mathrm{p} 20$ & 16.1 & -89.0 & -72.9 & 73.6 & -26.3 & 47.3 & 23.6 & -101.0 & -77.4 \\
\hline 260.3 & $13 \mathrm{p} 60$ & 9.2 & -40.5 & -31.3 & 55.5 & -19.3 & 36.2 & 16.5 & -101.5 & -84.9 \\
\hline 260.4 & $14 \mathrm{p} 60$ & 19.2 & -110.2 & -91.0 & 51.7 & -80.5 & -28.8 & 38.1 & -73.6 & -35.5 \\
\hline 260.5 & $15 \mathrm{p} 40$ & 25.0 & -122.2 & -97.2 & 66.3 & -40.0 & 26.3 & 46.5 & -94.6 & -48.2 \\
\hline 260.6 & $17 \mathrm{p} 80$ & 91.4 & -111.0 & -19.6 & 53.7 & -63.0 & -9.3 & 32.2 & -63.8 & -31.6 \\
\hline 261.0 & $23 \mathrm{p} 40$ & 55.8 & -131.5 & -75.7 & 47.2 & -165.7 & -118.5 & 16.9 & -182.0 & -165.1 \\
\hline 261.0 & $24 \mathrm{p} 40$ & 50.5 & -166.3 & -115.8 & 62.6 & -202.9 & -140.3 & 17.9 & -182.3 & -164.4 \\
\hline 261.1 & $25 \mathrm{p} 20$ & 72.6 & -159.2 & -86.6 & 63.2 & -157.4 & -94.3 & 22.2 & -179.8 & -157.6 \\
\hline 261.1 & $25 \mathrm{p} 40$ & 90.1 & -167.5 & -77.4 & 79.8 & -183.8 & -104.1 & 40.6 & -178.8 & -138.2 \\
\hline 261.2 & $28 \mathrm{p} 00$ & 35.4 & -73.6 & -38.2 & 54.3 & -60.7 & -6.4 & 36.2 & -71.2 & -35.0 \\
\hline 261.4 & 30 p80 & 43.3 & -65.0 & -21.7 & 106.9 & -108.8 & -1.9 & 63.1 & -82.7 & -19.6 \\
\hline 261.5 & $32 \mathrm{p} 40$ & 55.2 & -43.2 & 12.0 & 67.8 & -85.0 & -17.2 & 64.6 & -111.3 & -46.7 \\
\hline 261.9 & $38 \mathrm{p} 00$ & 102.6 & -65.7 & 36.9 & 71.6 & -80.4 & -8.8 & 37.8 & -48.8 & -11.0 \\
\hline 261.9 & $39 \mathrm{p} 00$ & -no data- & -no data- & -no data- & -no data- & -no data- & -no data- & -no data- & -no data- & -no data- \\
\hline 262.2 & $43 \mathrm{p} 00$ & 11.3 & -104.2 & -92.9 & 63.0 & -44.8 & 18.2 & 30.0 & -66.6 & -36.6 \\
\hline 262.2 & $43 p 40$ & 46.4 & -72.1 & -25.7 & 93.7 & -25.5 & 68.2 & 55.3 & -89.4 & -34.1 \\
\hline 262.5 & $48 \mathrm{p} 40$ & 24.3 & -8.9 & 15.4 & 64.9 & -6.4 & 58.5 & 20.4 & -63.4 & -43.0 \\
\hline 262.6 & $49 p 40$ & 29.3 & -29.5 & -0.2 & -no data- & -no data- & -no data- & 27.9 & -13.5 & 14.4 \\
\hline 262.6 & $50 \mathrm{p} 20$ & 14.6 & -52.3 & -37.7 & 54.3 & -26.6 & 27.7 & 9.6 & -38.5 & -28.9 \\
\hline 262.7 & $51 \mathrm{p} 20$ & 6.3 & -66.4 & -60.1 & 68.3 & -36.4 & 31.9 & -no data- & -no data- & -no data- \\
\hline 262.8 & $53 \mathrm{p} 00$ & 3.1 & -81.8 & -78.7 & 82.2 & -4.4 & 77.8 & 1.7 & -149.5 & -147.9 \\
\hline 262.9 & $54 \mathrm{p} 20$ & 30.2 & -116.1 & -85.9 & 30.3 & -80.1 & -49.7 & 18.9 & -158.5 & -139.6 \\
\hline 262.9 & $55 \mathrm{p} 00$ & 63.7 & -142.4 & -78.7 & 71.8 & -101.9 & -30.1 & 17.8 & -161.4 & -143.6 \\
\hline 263.0 & $55 \mathrm{p} 60$ & 74.5 & -115.3 & -40.9 & 67.2 & -131.5 & -64.3 & 37.7 & -119.8 & -82.1 \\
\hline 263.3 & $62 \mathrm{p} 00$ & 47.6 & -69.1 & -21.5 & 39.1 & -83.2 & -44.1 & 48.0 & -120.4 & -72.4 \\
\hline 263.4 & $62 \mathrm{p} 60$ & 48.5 & -78.1 & -29.6 & 44.1 & -118.0 & -73.8 & 35.1 & -93.7 & -58.6 \\
\hline 263.4 & 63 p20 & 64.9 & -99.9 & -35.0 & 50.2 & -109.7 & -59.5 & 71.8 & -25.9 & 45.9 \\
\hline 263.5 & $65 \mathrm{p} 00$ & 69.2 & -51.2 & 18.0 & 54.3 & -60.1 & -5.8 & 66.2 & -107.7 & -41.4 \\
\hline 263.6 & $65 \mathrm{p} 80$ & 93.3 & -99.5 & -6.3 & 307.6 & -54.4 & 253.2 & 96.0 & -109.9 & -13.8 \\
\hline \multicolumn{2}{|l|}{ Total area } & $1,438.1$ & $-2,739.0$ & $-1,300.9$ & $2,124.4$ & $-2,313.4$ & -189.0 & $1,025.1$ & $-3,227.1$ & $-2,202.0$ \\
\hline \multicolumn{2}{|c|}{$\begin{array}{l}\text { Number of cross sec- } \\
\text { tions }\end{array}$} & 32 & 32 & 32 & 31 & 31 & 31 & 31 & 31 & 31 \\
\hline \multicolumn{2}{|l|}{ Average } & 44.9 & -85.6 & -40.7 & 68.5 & -74.6 & -6.1 & 33.1 & -104.1 & -71.0 \\
\hline
\end{tabular}


Table 7. Cross section comparison values for the Miami reach, 2006-07.-Continued

\begin{tabular}{|c|c|c|c|c|c|c|c|c|c|c|}
\hline \multirow[b]{2}{*}{ River mile } & \multirow[b]{2}{*}{$\begin{array}{c}\text { Line } \\
\text { number }\end{array}$} & \multicolumn{3}{|c|}{ May 2006 to May 2007} & \multicolumn{3}{|c|}{ July 2006 to August 2007} & \multicolumn{3}{|c|}{ March 2006 to March 2007} \\
\hline & & $\begin{array}{c}\text { Deposi- } \\
\text { tion area } \\
\text { (square } \\
\text { meters) }\end{array}$ & $\begin{array}{l}\text { Erosion } \\
\text { area } \\
\text { (square } \\
\text { meters) }\end{array}$ & $\begin{array}{c}\text { Net change } \\
\text { (square } \\
\text { meters) }\end{array}$ & $\begin{array}{l}\text { Deposi- } \\
\text { tion area } \\
\text { (square } \\
\text { meters) }\end{array}$ & $\begin{array}{l}\text { Erosion } \\
\text { area } \\
\text { (square } \\
\text { meters) }\end{array}$ & $\begin{array}{l}\text { Net change } \\
\text { (square } \\
\text { meters) }\end{array}$ & $\begin{array}{c}\text { Deposi- } \\
\text { tion area } \\
\text { (square } \\
\text { meters) }\end{array}$ & $\begin{array}{l}\text { Erosion } \\
\text { area } \\
\text { (square } \\
\text { meters) }\end{array}$ & $\begin{array}{c}\text { Net } \\
\text { change } \\
\text { (square } \\
\text { meters) }\end{array}$ \\
\hline 259.5 & $0 \mathrm{p} 40$ & 38.7 & -125.7 & -87.0 & 31.8 & -103.7 & -71.9 & 24.5 & -41.7 & -17.2 \\
\hline 259.7 & $3 \mathrm{p} 20$ & 0.0 & -247.5 & -247.5 & 4.6 & -97.6 & -93.0 & 43.3 & -39.9 & 3.4 \\
\hline 259.7 & $3 \mathrm{p} 80$ & 0.7 & -262.7 & -262.1 & 0.0 & 0.0 & 0.0 & 23.5 & -22.9 & 0.6 \\
\hline 260.3 & $12 \mathrm{p} 60$ & 73.2 & -14.6 & 58.6 & 73.5 & -34.2 & 39.3 & 0.3 & -46.7 & -46.4 \\
\hline 260.3 & $13 \mathrm{p} 20$ & 49.9 & -4.8 & 45.1 & 51.1 & -47.9 & 3.2 & 59.4 & -38.7 & 20.8 \\
\hline 260.3 & $13 p 60$ & 84.4 & -0.3 & 84.1 & 59.4 & -28.1 & 31.3 & 2.6 & -41.2 & -38.6 \\
\hline 260.4 & $14 \mathrm{p} 60$ & 56.9 & -61.3 & -4.3 & 77.1 & -29.6 & 47.5 & 78.1 & -49.6 & 28.5 \\
\hline 260.5 & $15 \mathrm{p} 40$ & 42.2 & -103.6 & -61.4 & 105.5 & -28.2 & 77.3 & 72.6 & -69.1 & 3.5 \\
\hline 260.6 & $17 \mathrm{p} 80$ & 0.7 & -168.1 & -167.4 & 92.2 & -6.0 & 86.2 & 30.2 & -16.8 & 13.4 \\
\hline 261.0 & $23 \mathrm{p} 40$ & 26.3 & -177.3 & -151.0 & 234.5 & 0.0 & 234.5 & 54.4 & -76.6 & -22.1 \\
\hline 261.0 & $24 \mathrm{p} 40$ & -no data- & -no data- & -no data- & 193.6 & 0.0 & 193.6 & 10.6 & -86.7 & -76.1 \\
\hline 261.1 & $25 \mathrm{p} 20$ & 10.0 & -254.8 & -244.8 & 183.2 & 0.0 & 183.2 & 19.6 & -51.0 & -31.4 \\
\hline 261.1 & $25 \mathrm{p} 40$ & 12.6 & -224.6 & -212.0 & 205.1 & -2.1 & 203.0 & 39.2 & -51.5 & -12.3 \\
\hline 261.2 & $28 \mathrm{p} 00$ & 191.8 & -57.5 & 134.3 & 221.0 & -2.1 & 218.9 & 69.6 & -90.2 & -20.6 \\
\hline 261.4 & $30 \mathrm{p} 80$ & 96.0 & -63.2 & 32.8 & 172.9 & 0.0 & 172.9 & 59.5 & -50.8 & 8.7 \\
\hline 261.5 & $32 \mathrm{p} 40$ & 114.2 & -142.6 & -28.4 & 214.5 & -7.0 & 207.5 & 112.3 & -125.3 & -13.0 \\
\hline 261.9 & $38 \mathrm{p} 00$ & -no data- & -no data- & -no data- & 75.9 & -26.8 & 49.1 & 123.1 & -61.1 & 62.1 \\
\hline 261.9 & $39 \mathrm{p} 00$ & -no data- & -no data- & -no data- & -no data- & -no data- & -no data- & 105.7 & -30.4 & 75.3 \\
\hline 262.2 & $43 \mathrm{p} 00$ & 73.4 & -75.1 & -1.7 & 270.9 & -11.5 & 259.4 & 38.6 & -40.2 & -1.6 \\
\hline 262.2 & $43 p 40$ & 76.0 & -64.0 & 12.0 & 254.4 & -29.3 & 225.1 & 41.7 & -46.4 & -4.7 \\
\hline 262.5 & $48 \mathrm{p} 40$ & 0.9 & -93.0 & -92.1 & 28.9 & -94.8 & -65.9 & 15.8 & -34.4 & -18.5 \\
\hline 262.6 & $49 \mathrm{p} 40$ & 0.0 & -161.7 & -161.7 & 0.4 & -95.0 & -94.6 & 9.4 & -18.1 & -8.7 \\
\hline 262.6 & $50 \mathrm{p} 20$ & 0.0 & -156.2 & -156.2 & 11.2 & -102.1 & -91.0 & 10.1 & -21.6 & -11.6 \\
\hline 262.7 & $51 \mathrm{p} 20$ & -no data- & -no data- & -no data- & -no data- & -no data- & -no data- & 20.3 & -14.3 & 5.9 \\
\hline 262.8 & $53 \mathrm{p} 00$ & 2.3 & -236.5 & -234.3 & 14.9 & -61.3 & -46.4 & 46.3 & -13.3 & 33.0 \\
\hline 262.9 & $54 \mathrm{p} 20$ & 13.4 & -115.3 & -101.9 & 61.2 & -20.4 & 40.8 & 59.9 & -62.7 & -2.8 \\
\hline 262.9 & $55 \mathrm{p} 00$ & -no data- & -no data- & -no data- & 73.4 & -19.6 & 53.8 & 35.9 & -50.1 & -14.3 \\
\hline 263.0 & $55 \mathrm{p} 60$ & 25.9 & -159.9 & -133.9 & 72.5 & -16.8 & 55.7 & 59.4 & -42.0 & 17.4 \\
\hline 263.3 & $62 \mathrm{p} 00$ & 109.1 & -22.6 & 86.5 & 88.4 & -42.9 & 45.5 & 81.4 & -29.9 & 51.6 \\
\hline 263.4 & $62 \mathrm{p} 60$ & 146.4 & -12.0 & 134.4 & 131.5 & -80.0 & 51.5 & 42.7 & -33.7 & 9.0 \\
\hline 263.4 & $63 \mathrm{p} 20$ & 227.0 & -7.4 & 219.6 & 172.2 & -79.7 & 92.5 & 61.2 & -57.7 & 3.5 \\
\hline 263.5 & $65 \mathrm{p} 00$ & 37.2 & -117.2 & -80.1 & 40.9 & -49.2 & -8.3 & 55.1 & -76.3 & -21.2 \\
\hline 263.6 & $65 \mathrm{p} 80$ & 230.8 & -87.7 & 143.1 & 78.3 & -164.0 & -85.8 & 101.6 & -138.2 & -36.6 \\
\hline Total area & & $1,739.7$ & $-3,216.8$ & 369.6 & $3,295.2$ & $-1,280.1$ & $2,015.1$ & $1,608.0$ & $-1,669.1$ & -61.2 \\
\hline $\begin{array}{l}\text { Number of c } \\
\text { sections }\end{array}$ & cross & 28 & 28 & 28 & 31 & 31 & 31 & 33.0 & 33.0 & 33.0 \\
\hline Average & & 62.1 & -114.9 & 13.2 & 106.3 & -41.3 & 65.0 & 48.7 & -50.6 & -1.9 \\
\hline
\end{tabular}


A.

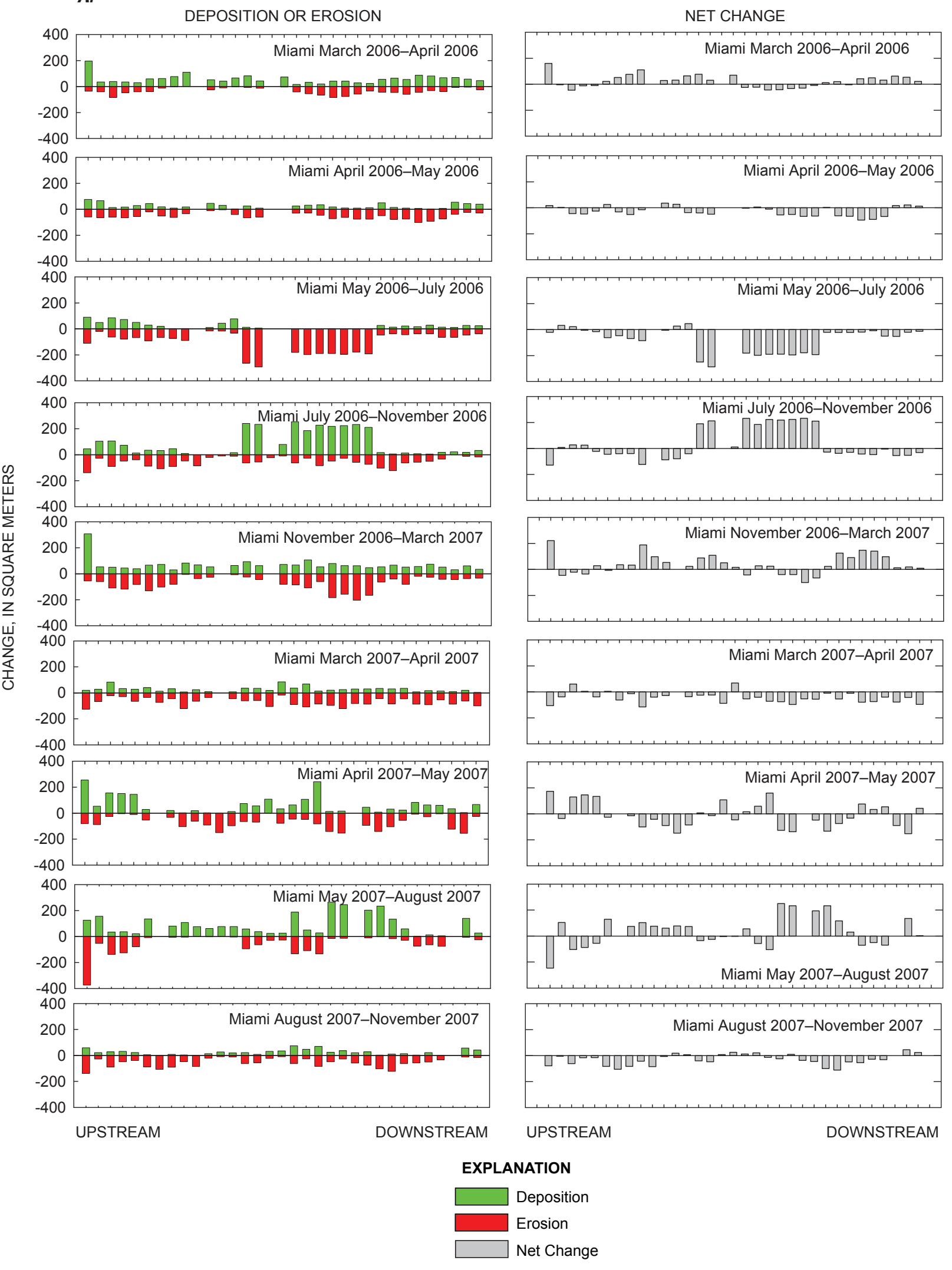

Figure 16. Deposition, erosion, and net change for the Miami reach. 
B.

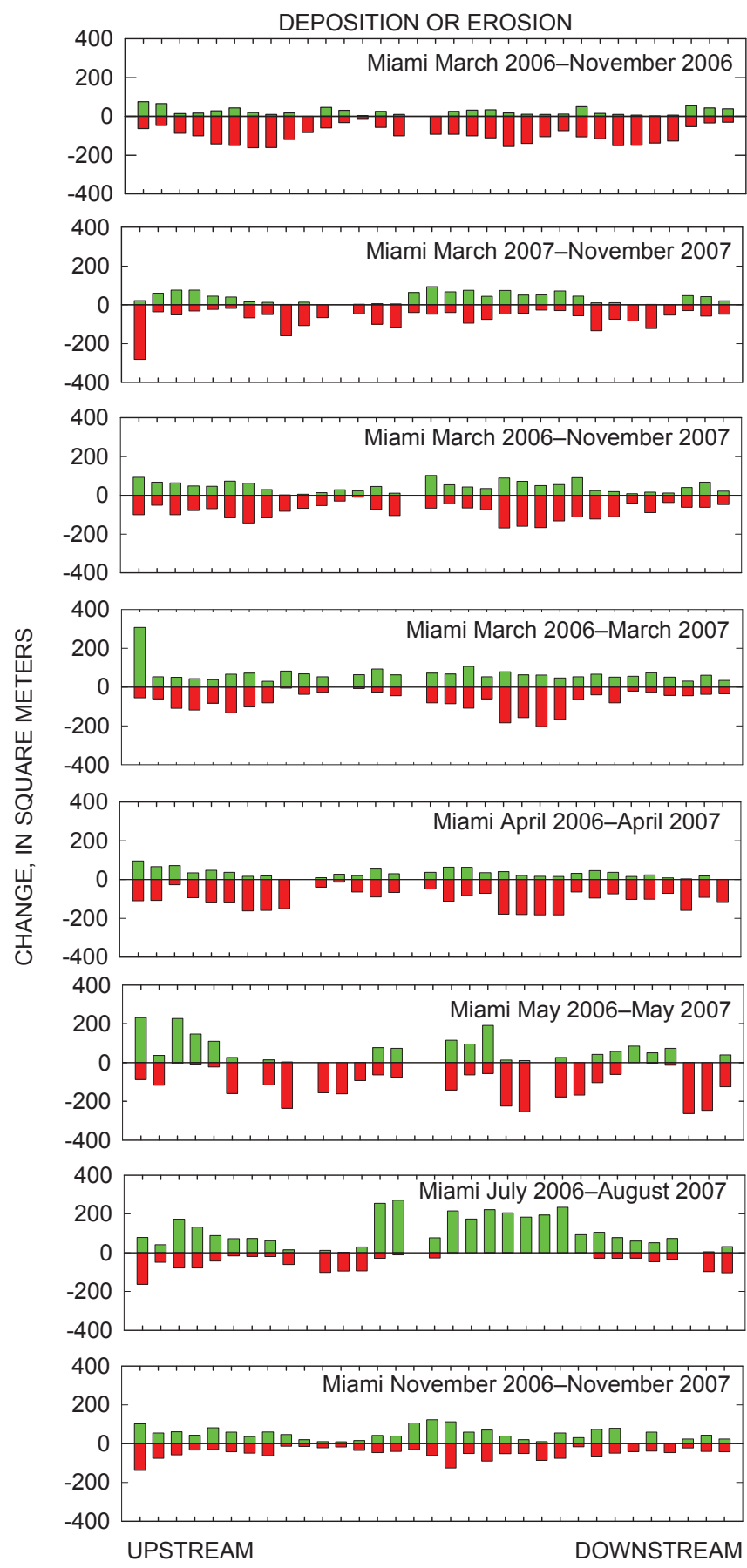

NET CHANGE
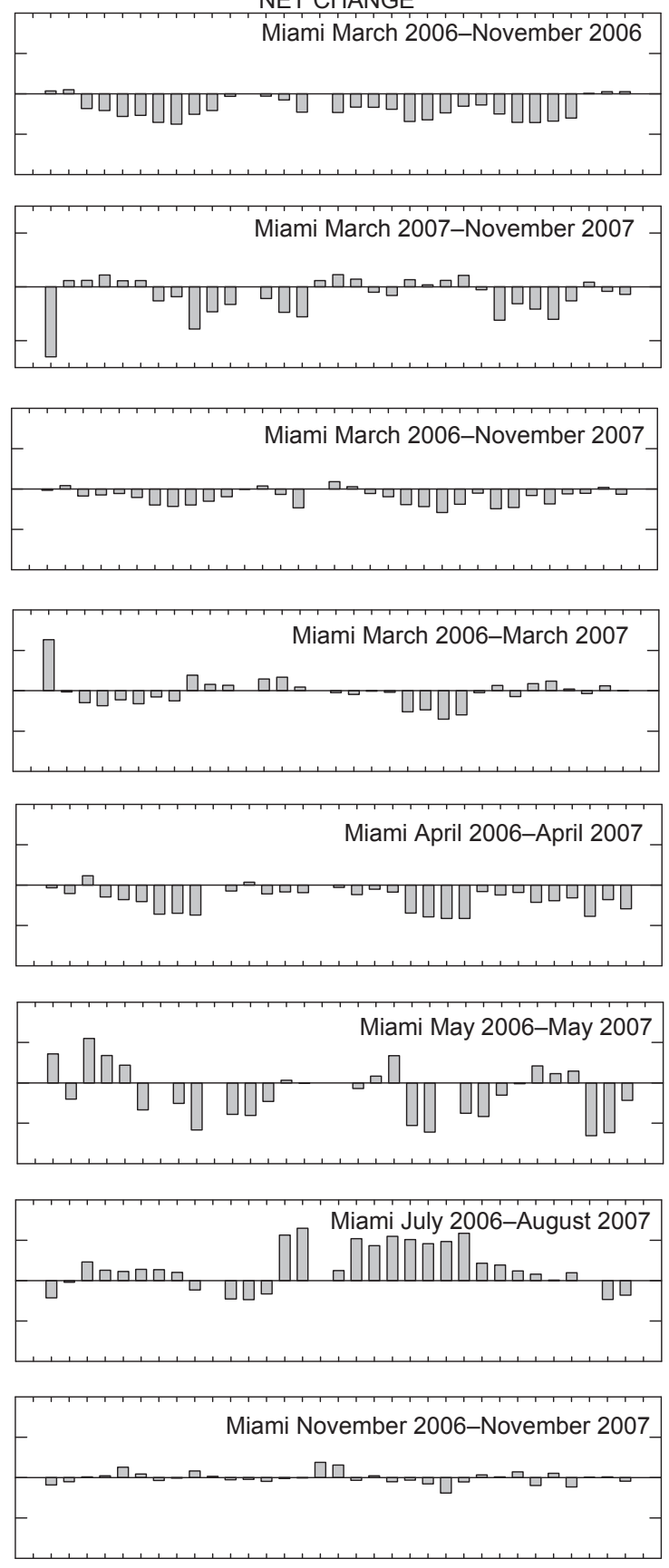

UPSTREAM

EXPLANATION

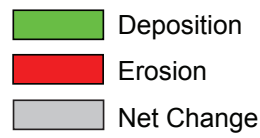

Figure 16. Deposition, erosion, and net change for the Miami reach.-Continued 

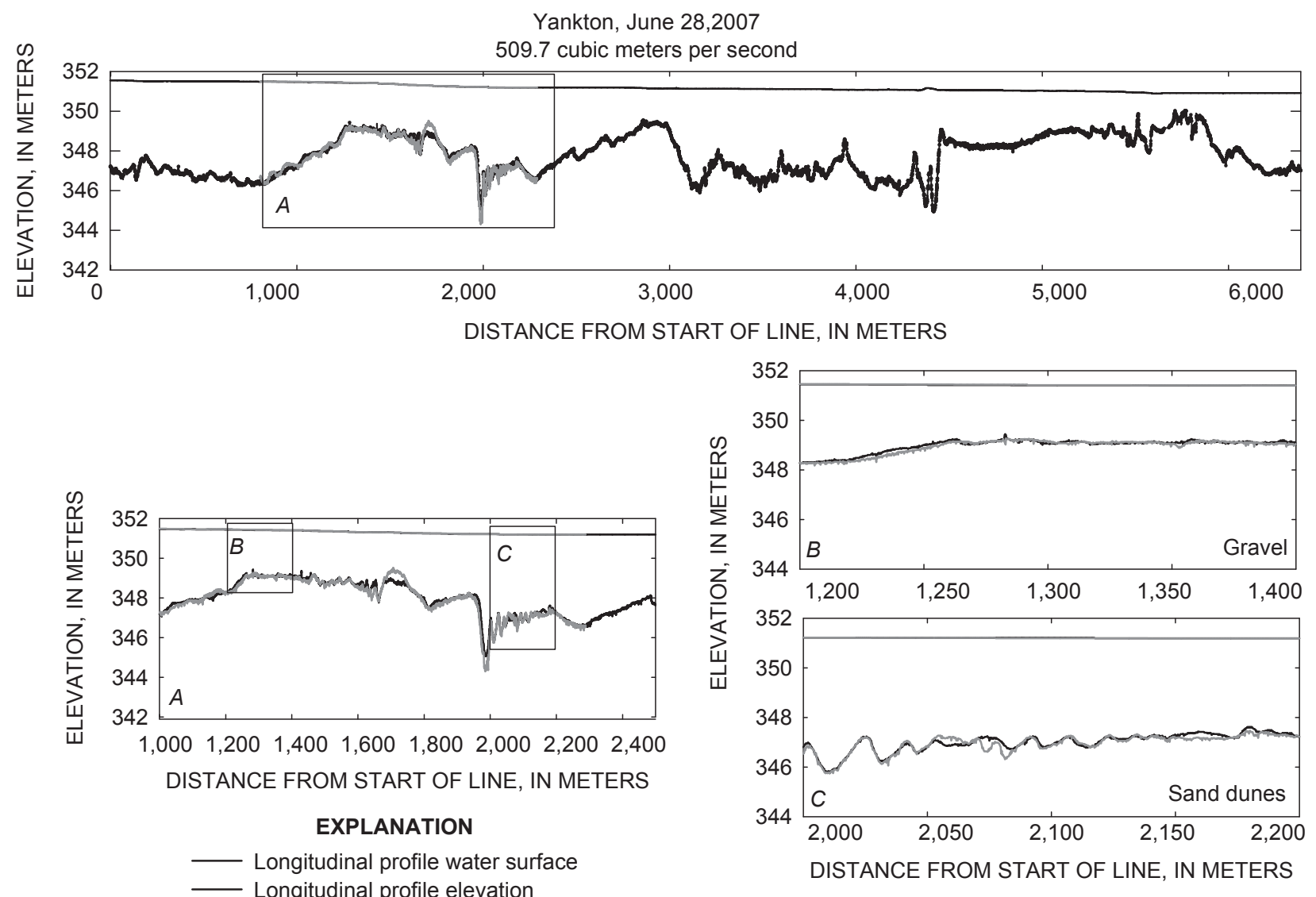

\section{EXPLANATION}

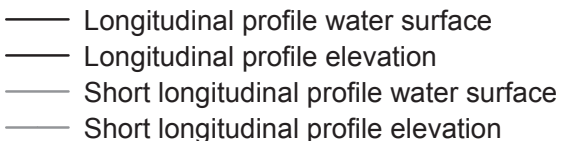

Figure 17. Longitudinal profiles for the Yankton reach.

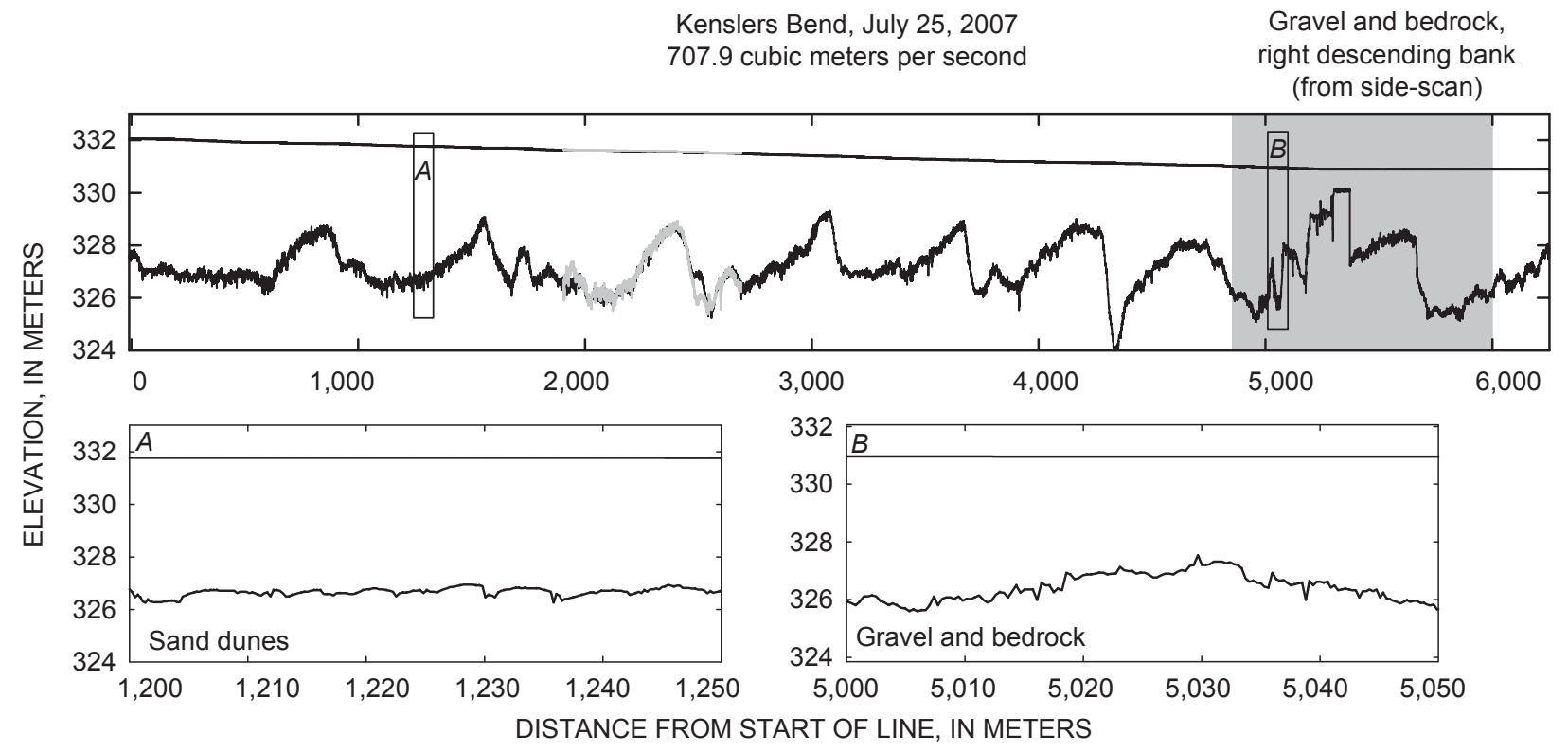

EXPLANATION

$\begin{array}{ll}- \text { Longitudinal profile water surface } & \text { Short longitudinal profile water surface } \\ - \text { Longitudinal profile elevation } & \text { Short longitudinal profile elevation }\end{array}$

Figure 18. Longitudinal profiles for Kenslers Bend reach. 
Little Sioux Reach, 2006 and 2007 longitudinal profiles
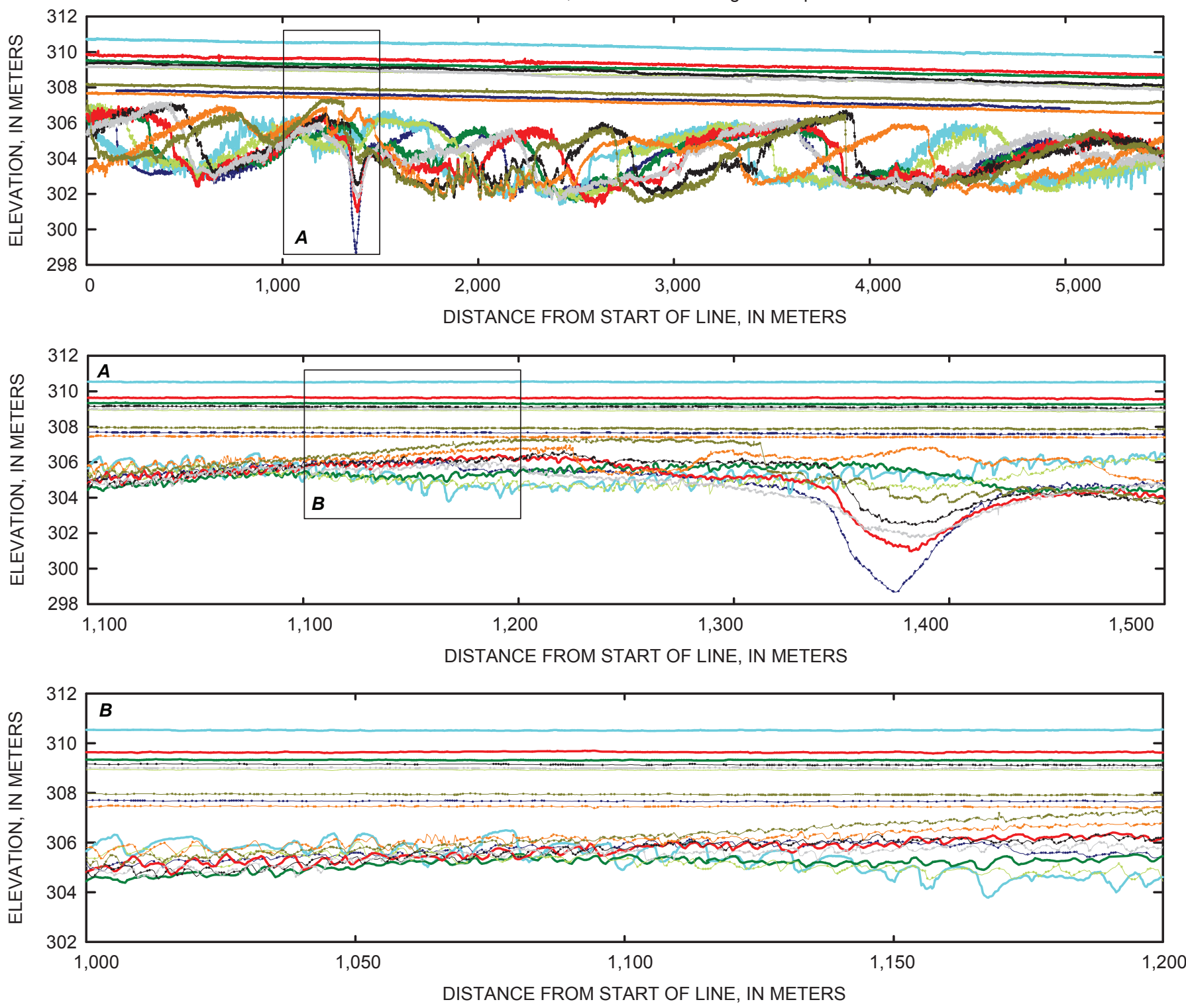

\section{EXPLANATION}

— March 2006, 371.0 cubic meters per second

— March 2007, 1,138.3 cubic meters per second

_ April 2006, 767.4 cubic meters per second

— April 2007, 654.1 cubic meters per second

_ May 2006, 866.4 cubic meters per second

— July 2006, 761.7 cubic meters per second

_ July 2007, 730.6 cubic meters per second

_ October 2006, 328.5 cubic meters per second

— November 2007, 419.1 cubic meters per second

Figure 19. Long and short profiles for each survey date in the Little Sioux reach. 

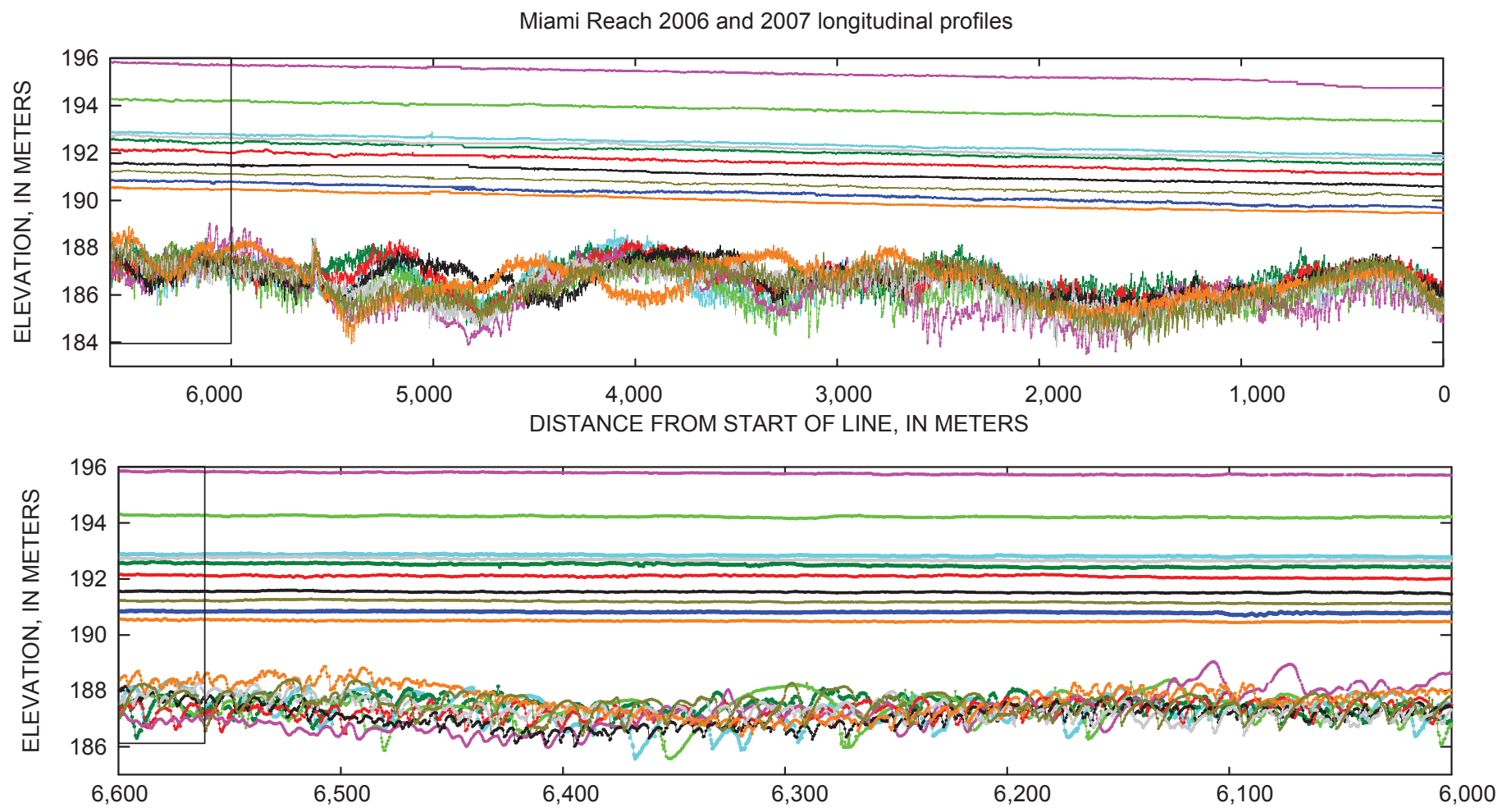

DISTANCE FROM START OF LINE, IN METERS

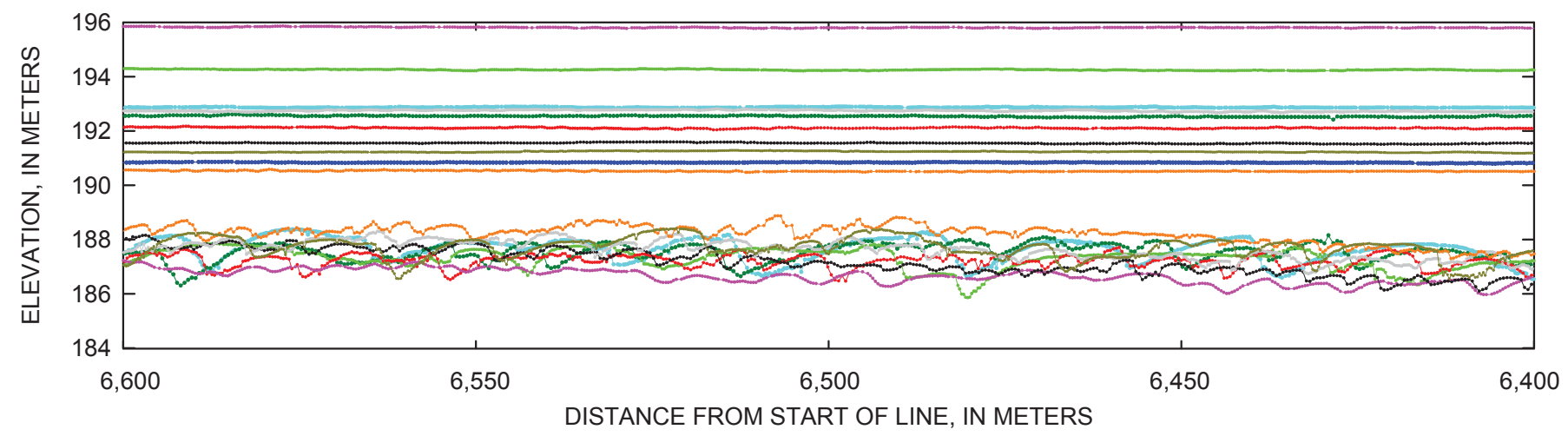

\section{EXPLANATION}

_ March 2006, 1,138.3 cubic meters per second

_- March 2007, 1,608.6 cubic meters per second

- April 2006, 1,410.2 cubic meters per second

_ April 2007, 2,418.3 cubic meters per second

— May 2006, 1,129.1 cubic meters per second

— May 2007, 3,284.4 cubic meters per second

— July 2006, 991.1 cubic meters per second

_ August 2007, 1,489.5 cubic meters per second

— November 2006, 608.8 cubic meters per second

— November 2007, 880.7 cubic meters per second

Figure 20. Long and short profiles for each survey date in the Miami reach. 
Little Sioux, 2006
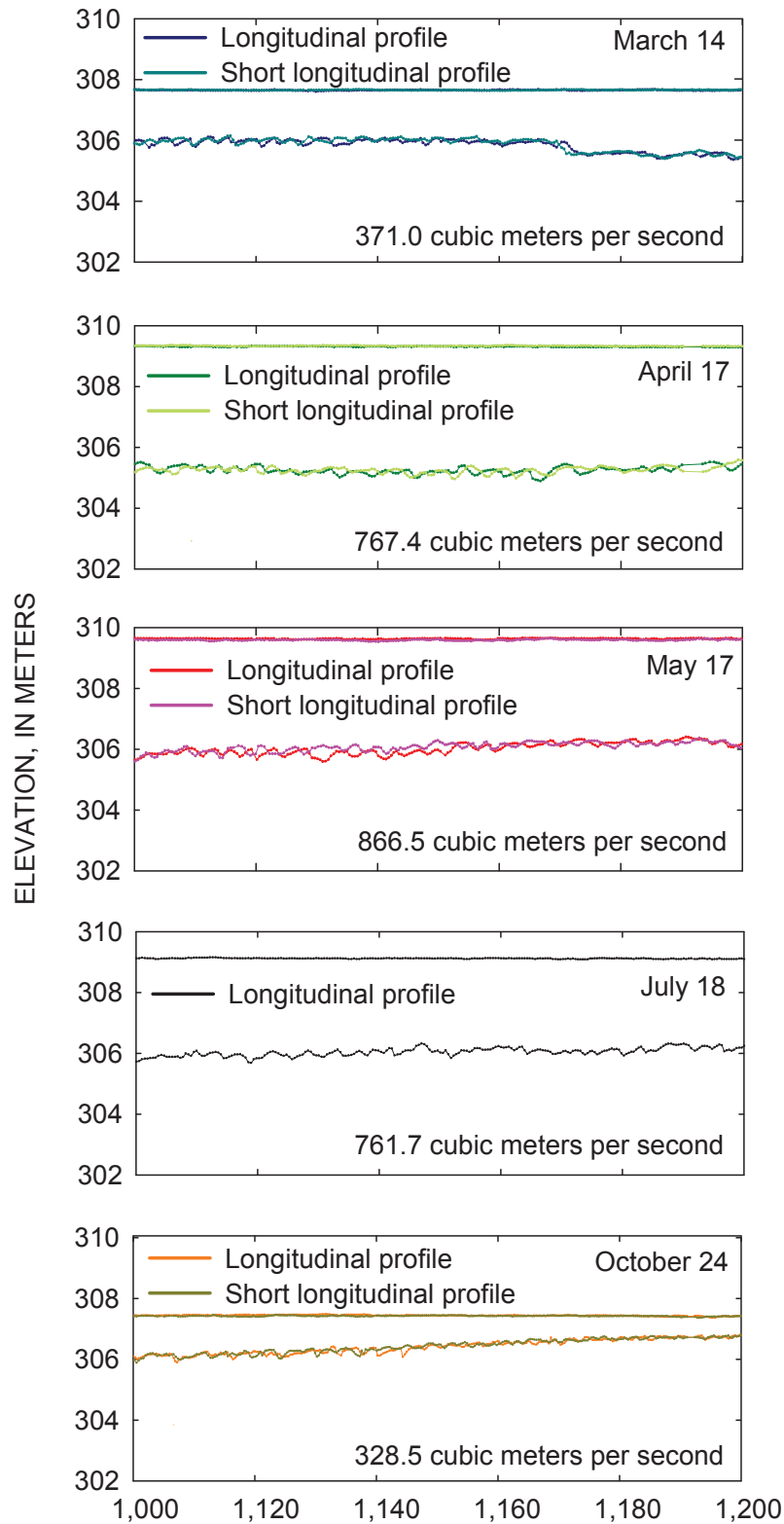

Little Sioux, 2007
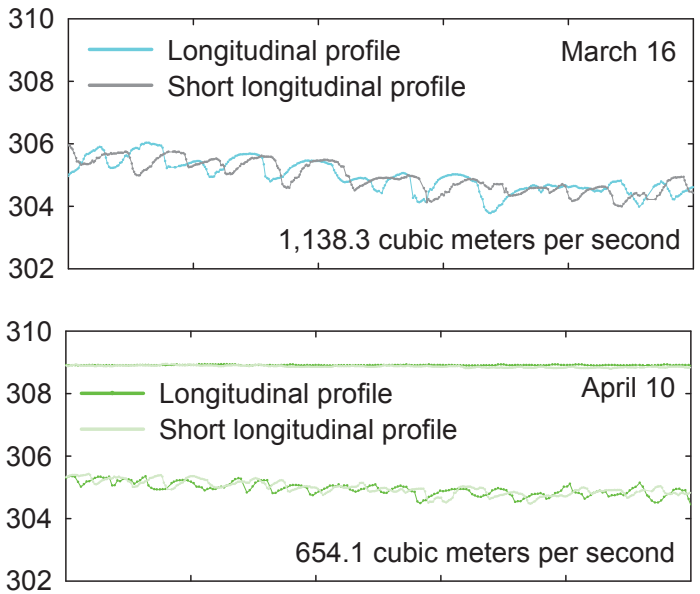
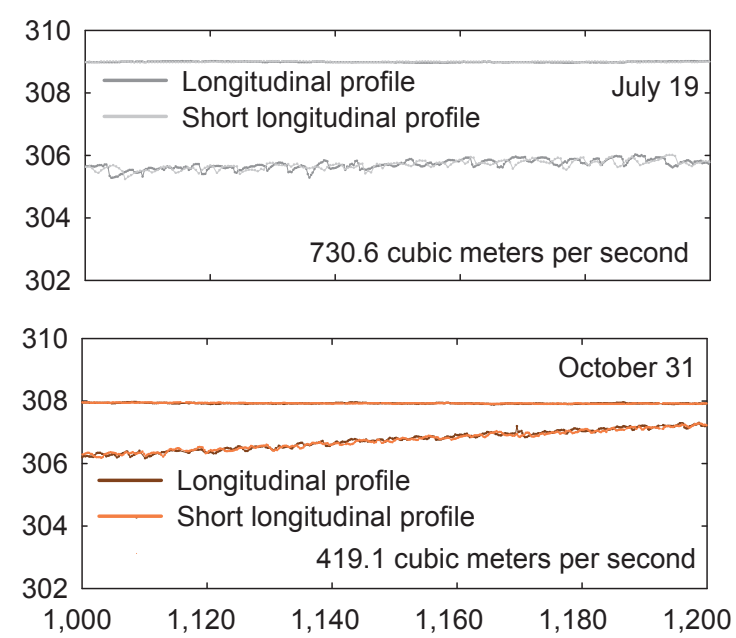

DISTANCE FROM START OF LINE, IN METERS

Figure 21. Compiled short and long longitudinal profiles for 2006 and 2007 in the Little Sioux reach.

Longitudinal thalweg profiles were surveyed in the Little Sioux and Miami reaches for every survey over the 2-year period (figs. 19 and 20). Short profiles in a 500-m long subsection of the longitudinal profile also were resampled 2-4 hours later to measure dune movement. The bed changed in elevation considerably over the surveys in the Little Sioux reach. Large-scale channel feature (sandbar) movement as well as smaller-scale (dune) movement occurred in this reach over the range of discharges surveyed. Thalweg elevation changes in the Little Sioux reach were in the range of 4-5 m. Thalweg bed elevation change was mostly at the dune scale, and the changes in elevations in the Miami reach were 2-4 m (fig. 20).

\section{Bedforms}

Changes in bedform size were observed in longitudinal profiles in all reaches with variation in discharge (table 8, figs. 21-23). Detailed sequences of bedform change over a large range of discharges were surveyed in the Little Sioux and Miami reaches (figs. 21-22). Characteristic dune length and height for all reaches generally increased with discharge (fig. 23).

Gravel bedforms in the Yankton reach tended to be fairly flat with small $<0.3 \mathrm{~m}$ fluctuations in height (fig. 17 C), or have gentle dune-forms that covered tens of meters in 

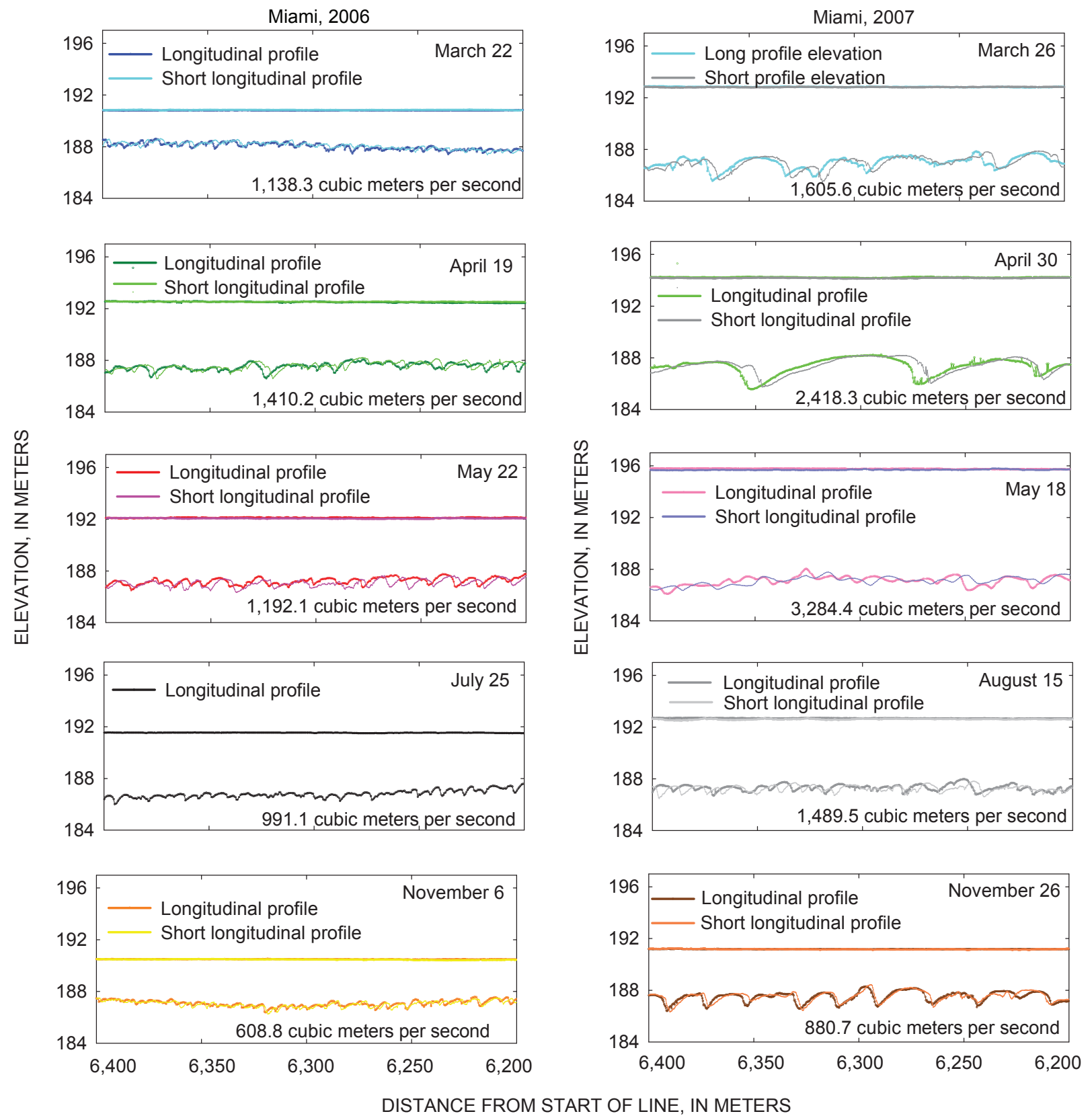

Figure 22. Compiled short and long longitudinal profiles for 2006 and 2007 in the Miami reach.

longitudinal distance and average amplitudes greater than 0.3 m (table 8; fig. 17 D). These low-relief bedforms were less steep than bedforms in the other three reaches with very low dune length to height ratios (fig. 23). Side-scan sonar indicated some locations in the Yankton reach where sand dunes cover bedrock, but these conditions were likely transient and were difficult to detect unless side-scan sonar imagery was collected the same date as a longitudinal profile. The Kenslers Bend reach was mostly sand bedded, although there is gravel and bedrock on the right bank near river mile 745.5. The lon- gitudinal profile follows portions of the channel thalweg that were sand bedded and gravel bedded (fig. 18).

In the Kenslers Bend reach characteristic bedform measurements were made in a sand-bedded portion of the longitudinal profile. Dunes for the entire study period ranged from $0.25-0.41 \mathrm{~m}$ in height to $1.8-5.4 \mathrm{~m}$ in length (table 8). Dune length to height ratios generally increased with discharge (fig. 23). Dunes were similar to dune sizes for comparable discharges in the Little Sioux Reach (fig. 23).

Characteristic dunes in the predominately sand-bedded Little Sioux reach ranged in size for all of the surveys from 

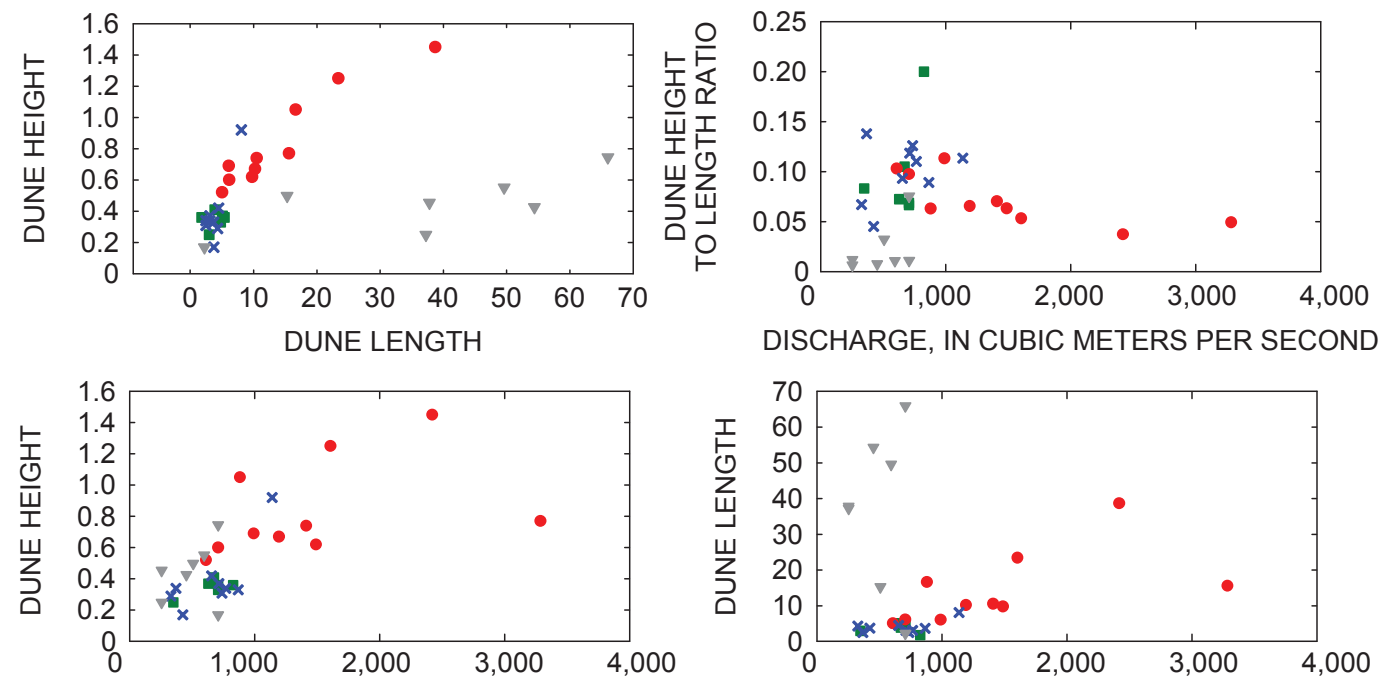

DISCHARGE, IN CUBIC METERS PER SECOND
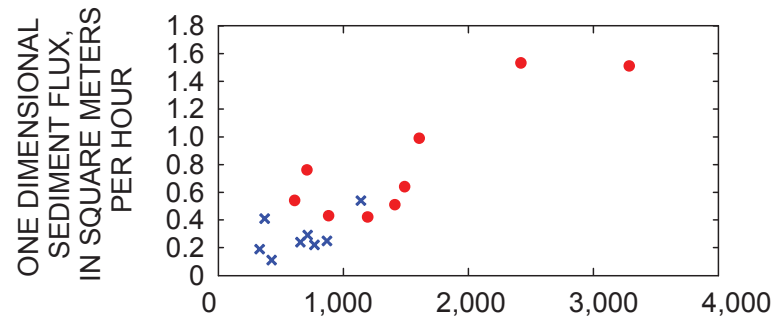

DISCHARGE, IN CUBIC METERS PER SECOND

\section{EXPLANATION}

$\checkmark$ Yankton

- Kenslers Bend

$\times \quad$ Little Sioux

- Miami

DISCHARGE, IN CUBIC METERS PER SECOND

Figure 23. Bedform dimensions and one-dimensional sediment flux calculated from bedforms in the four reaches.

0.17-0.92 m high (figs. 21, 23, table 8). Dune length varied from $2.46-8.1 \mathrm{~m}$. The largest bedforms occurred during the highest flow surveyed, March 16, 2007, at 1,140 m³.s. Bedform differencing between the long and short longitudinal profiles, a measure of sediment transport produced one-dimensional sediment fluxes ranging from $0.11-0.54 \mathrm{~m}^{2} / \mathrm{hr}$ (table 8 , fig. 23).

In the Miami reach, characteristic dunes varied in height for all of the surveys from $0.52-1.45 \mathrm{~m}$ (fig. 22, table 8). Dune length varied from 5.04-38.7 m. Bedform size increased with discharge, until the highest surveyed discharge, 3,280 $\mathrm{m}^{3} / \mathrm{s}$ measured on May 18, 2007 (figs. 22, 23). Dunes decreased in size considerably at this flow, and the mean bed elevation was also much lower than the two previous surveys that spring (figs. 20, 22-23). This change in bedforms occurred on the falling limb of the hydrograph, after a peak flow of $8,070 \mathrm{~m}^{3} / \mathrm{s}$ on May 8, 2007. During the 18-day period between the April 30 survey $\left(2,420 \mathrm{~m}^{3} / \mathrm{s}\right)$ and the May 18 survey there were 11 days with a flow above the April 30 flow $\left(3,280 \mathrm{~m}^{3} / \mathrm{s}\right)$. The May 18 survey thalweg bed elevation was 1-2 $\mathrm{m}$ below previously surveyed dune fields over much of the surveyed length (fig. 20). During this event the bed may have scoured to an armored level. In this case there may have not been sufficient sediment supply from upstream at this stage in the flood event to replenish the dunes in this reach.
Bedform differencing calculations resulted in onedimensional bed-material sediment flux values of 0.42 to 1.53 $\mathrm{m}^{2} / \mathrm{hr}$ in the Miami reach (table 8, fig. 23). One-dimensional bed-material sediment flux increased with discharge generally in the Little Sioux and Miami reaches, but this sediment transport measure decreased from the April 2007 to May 2007 surveys in the Miami reach.

\section{Substrate}

Side-scan sonar surveys conducted in all the reaches in 2007 allowed for visualization of substrate such as sand, gravel, and bank revetment rock, and other river habitat and biotic features including large woody debris and fish (fig. 24). In addition, the side-scan sonar surveys conducted in the Yankton and Kenslers Bend reaches coincided with surveys that allow for additional longitudinal profile interpretation. At Yankton, a region with a flat long profile with very small $(<0.2 \mathrm{~m})$ oscillations in height was validated by side scan as gravel (fig. 25). In the Kenslers Bend reach, the transition on the right descending bank near river mile 745 from sand bed to gravel was observed on side scan, and subtle differences in bedforms from jagged crests to more rounded crests were verified as gravel/bedrock and sand dunes, respectively (fig. 26). 
Table 8. Bedforms dimensions and dune movement calculations for each reach and discharge.

[Gavins Point Dam, South Dakota (U.S. Army Corps of Engineers); Missouri River main-stem streamflow-gaging stations and identification numbers: Sioux City, Iowa, 06486000; Decatur, Nebraska, 06601200; Waverly, Missouri 06895500]

\begin{tabular}{|c|c|c|c|c|c|c|c|c|}
\hline Site & Date & Gage & $\begin{array}{l}\text { Discharge, } \\
\text { (cubic } \\
\text { meters per } \\
\text { second) }\end{array}$ & $\begin{array}{l}\text { Water tempera- } \\
\text { ture (degrees } \\
\text { Celsius') }\end{array}$ & $\begin{array}{l}\text { Characteristic } \\
\text { dune height } \\
\text { (meters) }\end{array}$ & $\begin{array}{l}\text { Characteristic } \\
\text { dune length } \\
\text { (meters) }\end{array}$ & $\begin{array}{l}\text { Dune height } \\
\text { to length } \\
\text { ratio }\end{array}$ & $\begin{array}{c}\text { One-dimensional bed } \\
\text { material sediment flux } \\
\text { ('2square meters per hour) }\end{array}$ \\
\hline \multirow[t]{7}{*}{ Yankton } & $03 / 08 / 2006$ & Gavins Point Dam & 255 & 3 & 0.25 & 37.25 & 0.01 & -no data- \\
\hline & 03/28/2006 & Gavins Point Dam & 595 & 4 & 0.55 & 49.61 & 0.01 & -no data- \\
\hline & $05 / 15 / 2006$ & Gavins Point Dam & 708 & 15 & 0.75 & 66.00 & 0.01 & -no data- \\
\hline & $06 / 26 / 2006$ & Gavins Point Dam & 708 & 23 & 0.17 & 2.25 & 0.08 & -no data- \\
\hline & 03/09/2007 & Gavins Point Dam & 255 & 3 & 0.46 & 37.80 & 0.01 & -no data- \\
\hline & 03/13/2007 & Gavins Point Dam & 453 & 3 & 0.43 & 54.38 & 0.01 & -no data- \\
\hline & 06/28/2007 & Gavins Point Dam & 508 & 24 & 0.50 & 15.30 & 0.03 & -no data- \\
\hline \multirow[t]{6}{*}{ Kenslers Bend } & 03/10/2006 & Sioux City & 348 & 5 & 0.25 & 3.00 & 0.08 & -no data- \\
\hline & 03/30/2006 & Sioux City & 674 & 8 & 0.41 & 3.90 & 0.11 & -no data- \\
\hline & $05 / 15 / 2006$ & Sioux City & 708 & 10 & 0.33 & 4.80 & 0.07 & -no data- \\
\hline & 06/19/2006 & Sioux City & 827 & 23 & 0.36 & 1.80 & 0.20 & -no data- \\
\hline & $04 / 12 / 2007$ & Sioux City & 629 & 5 & 0.37 & 5.10 & 0.07 & -no data- \\
\hline & $07 / 25 / 2007$ & Sioux City & 708 & 27 & 0.36 & 5.40 & 0.07 & -no data- \\
\hline \multirow[t]{9}{*}{ Little Sioux } & $03 / 14 / 2006$ & Decatur & 368 & 4 & 0.34 & 2.46 & 0.14 & 0.41 \\
\hline & $04 / 17 / 2006$ & Decatur & 767 & 16 & 0.34 & 3.08 & 0.11 & 0.22 \\
\hline & 05/17/2006 & Decatur & 867 & 15 & 0.33 & 3.69 & 0.09 & 0.25 \\
\hline & 07/18/2006 & Decatur & 736 & 27 & 0.31 & 2.46 & 0.13 & -no data- \\
\hline & $10 / 24 / 2006$ & Decatur & 329 & 7 & 0.29 & 4.32 & 0.07 & 0.19 \\
\hline & 03/16/2007 & Decatur & 1,138 & 3 & 0.92 & 8.10 & 0.11 & 0.54 \\
\hline & $04 / 10 / 2007$ & Decatur & 654 & 6 & 0.42 & 4.50 & 0.09 & 0.24 \\
\hline & 07/19/2007 & Decatur & 714 & 28 & 0.37 & 3.12 & 0.12 & 0.29 \\
\hline & $10 / 31 / 2007$ & Decatur & 425 & 11 & 0.17 & 3.75 & 0.05 & 0.11 \\
\hline \multirow[t]{10}{*}{ Miami } & 03/22/2006 & Waverly & 708 & 6 & 0.60 & 6.15 & 0.10 & 0.76 \\
\hline & $04 / 19 / 2006$ & Waverly & 1,410 & 19 & 0.74 & 10.50 & 0.07 & 0.51 \\
\hline & $05 / 22 / 2006$ & Waverly & 1,192 & 20 & 0.67 & 10.20 & 0.07 & 0.42 \\
\hline & $07 / 25 / 2006$ & Waverly & 991 & 29 & 0.69 & 6.08 & 0.11 & -no data- \\
\hline & $11 / 06 / 2006$ & Waverly & 609 & 11 & 0.52 & 5.04 & 0.10 & 0.54 \\
\hline & 03/26/2007 & Waverly & 1,606 & 13 & 1.25 & 23.40 & 0.05 & 0.99 \\
\hline & 04/30/2007 & Waverly & 2,418 & 15 & 1.45 & 38.70 & 0.04 & 1.53 \\
\hline & 05/18/2007 & Waverly & 3,285 & 21 & 0.77 & 15.60 & 0.05 & 1.51 \\
\hline & $08 / 15 / 2007$ & Waverly & 1,490 & 30 & 0.62 & 9.77 & 0.06 & 0.64 \\
\hline & $11 / 26 / 2007$ & Waverly & 881 & 6 & 1.05 & 16.65 & 0.06 & 0.43 \\
\hline
\end{tabular}

${ }^{1}$ Temperature data from measurements made at the same time as bedform measurements using an Acoustic Doppler Current Profiler (ADCP).

${ }^{2}$ No data values- calculations are for sand-bed only; no calculations available for Yankton. For all other -no data- values bend long and short profile survey pairs were not available for calculations. 

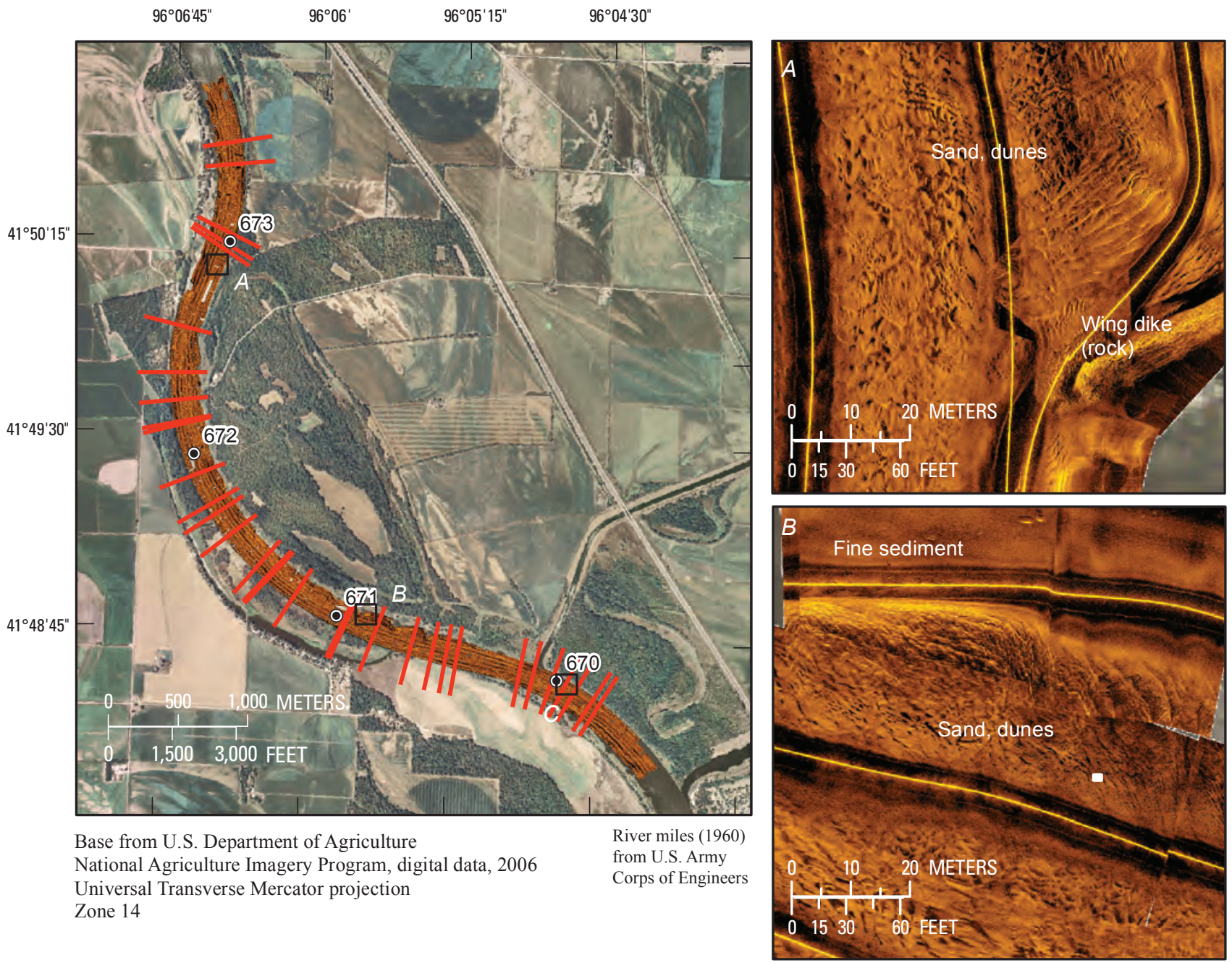

EXPLANATION

Randomly selected transects

- River mile

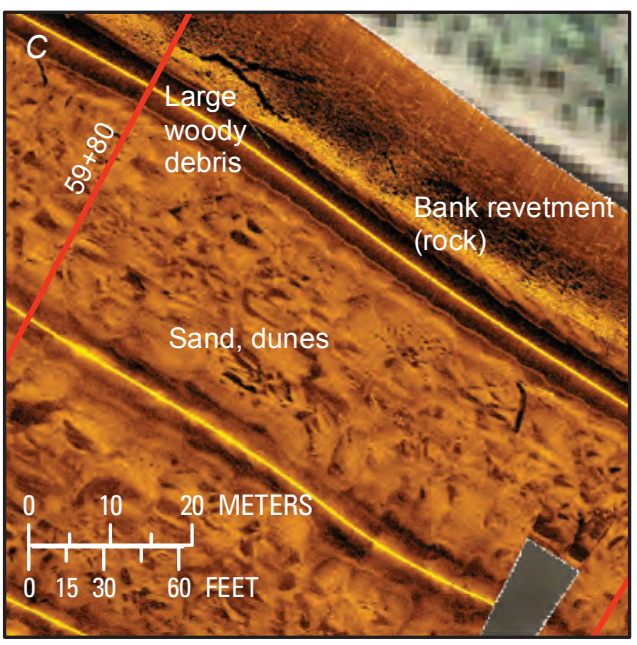

Figure 24. Side-scan sonar survey with bed features indicated. 


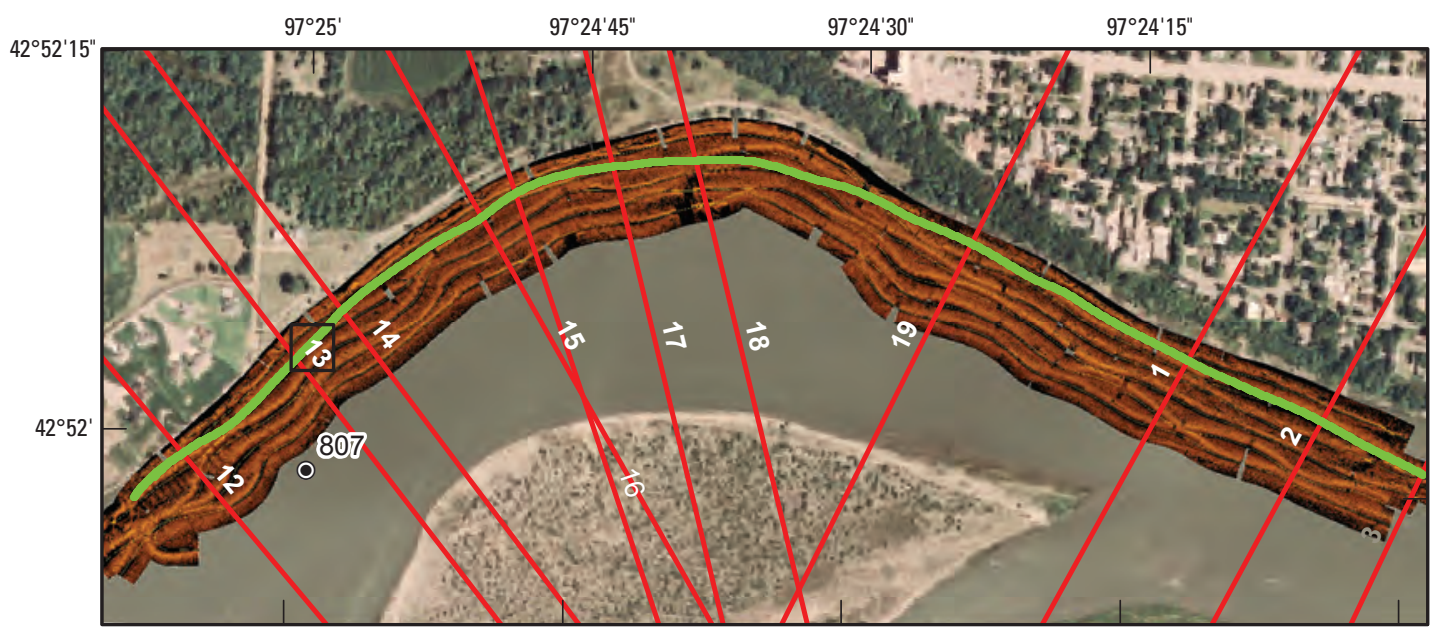

Base from U.S. Department of Agriculture

National Agriculture Imagery Program, digital data, 2006 Universal Transverse Mercator projection Zone 14

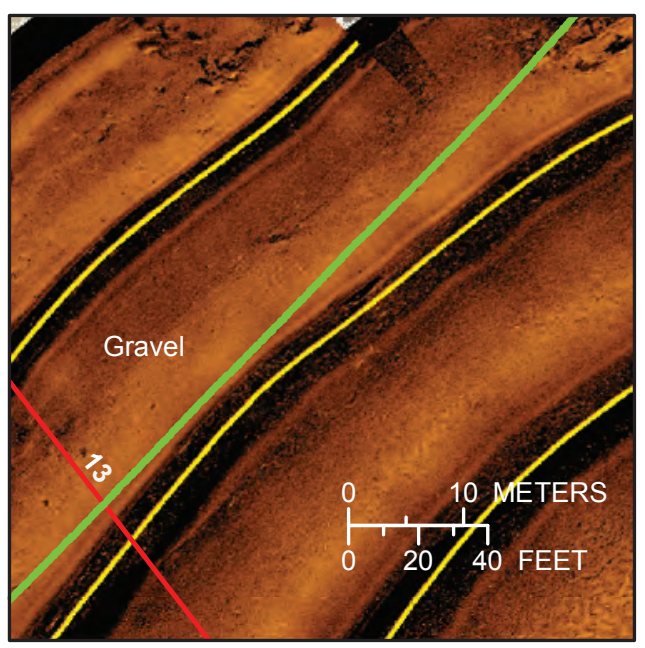

River miles from U.S. Army Corps of Engineers, 1960

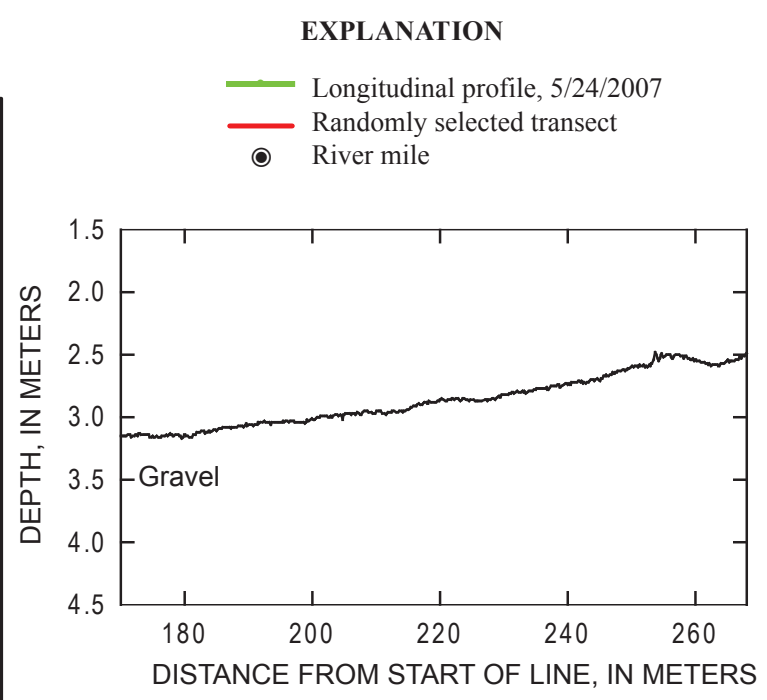

Figure 25. Side-scan sonar and bedforms in the Yankton reach.

\section{Bed Velocity}

In addition to bedform differencing, bed-velocity measurements using ADCP data were calculated as an additional measure of sediment transport. Bed velocity was measured by subtracting the bottom-tracking position produced by the ADCP from the position derived from the GPS over a time period. The ADCPs deployed for these transects utilize four beams, which widen through the water column with distance from the instrument. Each beam measures an independent region on the bed, which is spatially averaged by the ADCP. Nevertheless, spatially patchy distributions of sediment movement can introduce considerable variability in bed-velocity measurements. Potential errors in bed-velocity calculations are related to the assumption that bedload velocity is homogenous within the sampled area, and that noise introduced by suspended sediment is negligible (Rennie and others, 2002;
Rennie and Millar, 2004). Average bed-velocity magnitudes for an entire survey date are used to reduce measurement noise (table 9). Bed velocities generally increased with discharge in all the reaches (fig. 27). The highest average bed-velocity magnitude occurred in the Miami reach during a high flow on April 30, 2007. The highest surveyed discharge on May 18, 2007 , had a considerably lower bed-velocity magnitude, similar to the trend observed in bedform size and one-dimensional bed-material flux in the Miami reach from April to May in 2007.

\section{Discussion}

The following sections address sensitivity of reach morphodynamics to discharge variations, differences among the monitored reaches, and implications for understanding 

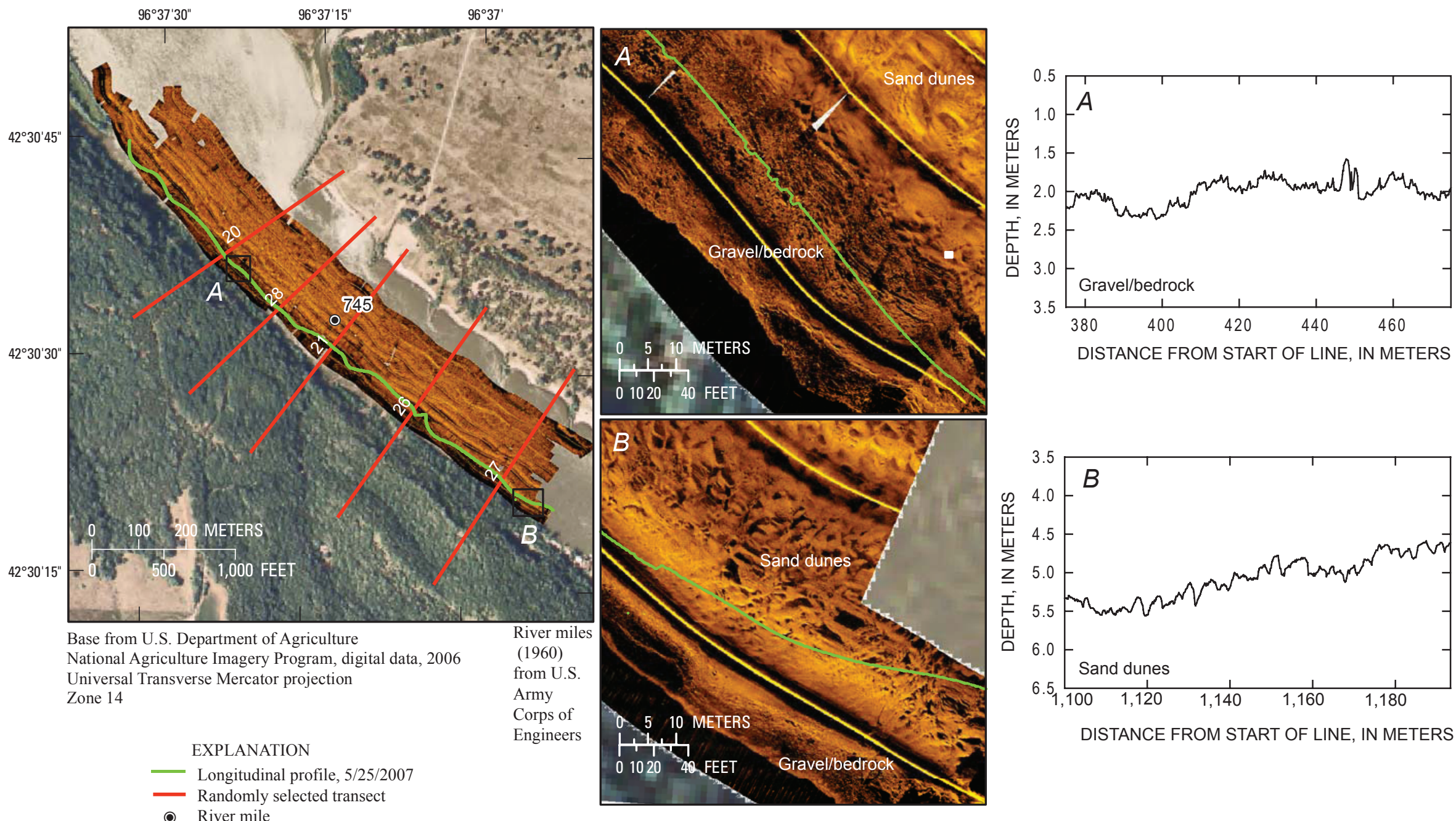

Figure 26. Side-scan sonar and bedforms in the Kenslers Bend reach 


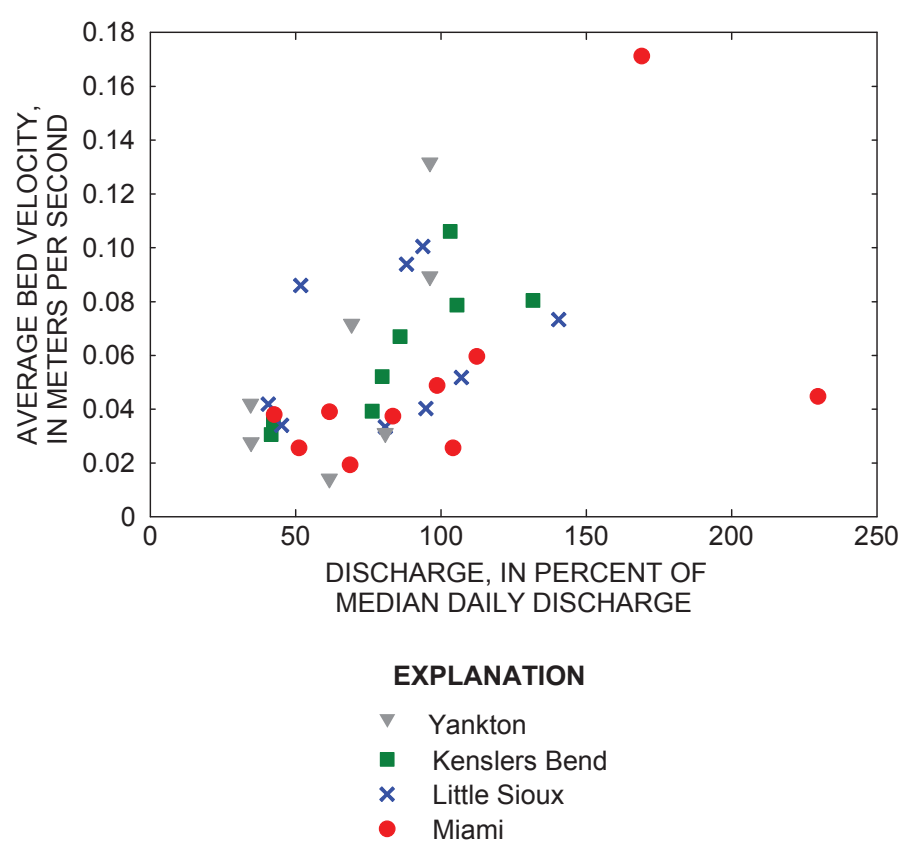

Figure 27. Average bed velocity and discharge in all reaches.

sturgeon habitat dynamics. Although the 2006 pulsed flow modification from Gavins Point Dam did not provide a distinct, isolated pulse, and the 2007 control year included substantial uncontrolled flow pulses, results from the four monitored reaches provide information on spatial variability and morphodynamic sensitivity to flow pulses.

\section{Discharge Variation and Morphodynamics}

Monitoring results provide several measures of how discharge variation affects sediment transport and channel morphology. Geomorphic changes address how the channel geometry changes at the cross section and reach scale. Resurveys of the randomized cross sections document change within and among years. Resurveys of the entire reach (compilation surveys) document total geomorphic change on an annual timeframe. These direct morphologic measures are complemented with indirect measures of bedload transport; bedform differencing and bed velocity.

\section{Geomorphic Changes}

For comparison purposes, the magnitudes of erosion and deposition were normalized by a percent of the average survey cross-section area at median annual flow or index crosssection area. For all possible date combinations, the Yankton reach had the least deposition, erosion, and net change (fig. 28, table 10). Percent total change in the Yankton reach was 2-3 percent of index cross-section area. The Kenslers Bend reach had the highest rates of deposition, erosion, and net change of the four reaches with total change rates from 14-33 percent of index cross-sectional area (table 10). The Little Sioux reach had fairly high rates of deposition and erosion, and moderately low total change rates of 8 to 26 percent of index cross-section area. The Miami reach had intermediate rates of deposition, erosion, and total change, 6 to 14 percent when compared to the other three reaches (table 10).

Average magnitudes of deposition and erosion in the Yankton reach were much lower than the other three reaches (fig. 28, table 10). This was probably because of several factors. First, this reach is located closest (approximately $5 \mathrm{RM}$ ) downstream from Gavins Point Dam, in a reach characterized by declining water-surface elevations and channel incision (U.S. Army Corps of Engineers, 2007b; Jacobson and others, 2009). Also, this reach has distinctly coarser substrate compared to the other three primarily sand-bedded reaches, ranging from gravel to cobble bottom overlain with patches of small dunes and rippled sand (fig. 24).

\section{Flow Pulses and Morphodynamics}

In March 2006 all the surveys took place before the planned manipulated flow pulse from Gavins Point Dam (U.S. Army Corps of Engineers, 2007a). The pulsed flow modification from Gavins Point Dam occurred during a 7-year period of drought in the basin (U.S. Army Corps of Engineers, 2006b) and was small when compared to high flow releases from the dam such as the 1997 flow event. The 2006 pulse was initiated from Gavins Point Dam on May 12 from a base discharge of $456 \mathrm{~m}^{3} / \mathrm{s}$. Discharge was increased to about $710 \mathrm{~m}^{3} / \mathrm{s}$ over 2 days, held constant for 2 days, and then dropped about $113 \mathrm{~m}^{3} / \mathrm{s}$ over 3 days. After May 19, discharges were cycled between 710 and $594 \mathrm{~m}^{3} / \mathrm{s}, 1$ day up and 2 days down until June 13 (U.S. Army Corps of Engineers, 2007a). All reaches were re-surveyed in April 2006 (figs. 6, 29, table 10); these resurveys generally captured increases from low winter flows to near navigation level flows. In all of the reaches there was net deposition from March 2006 to April 2006. Subsequently, from the April 2006 survey to May 2006 survey the Yankton, Kenslers Bend, and Miami reaches experienced net erosion, and the Little Sioux reach experienced no net change (figs. 28-29, table 10). Net erosion in the Yankton was small in the time period.

Discharges for the May 2006 surveys were greater than the April surveys at the three upstream reaches, and all four reaches had substantial flow variation (manipulated and nonmanipulated) between the two surveys. During the summer flows in 2006 the general trend was erosion in all reaches. Between the October/November 2006 and March 2007 surveys, deposition was dominant in the Little Sioux and Miami reaches. During the June 2006 to March 2007 time period, which includes late summer and winter flows in the Kenslers Bend reach, there was net deposition as well. There was no planned pulsed-flow modification from Gavins Point Dam in 2007. Nevertheless, there was substantial flow variation because of ramping of flows to support navigation, unplanned flow pulses out of the James, Vermillion, and Big Sioux Rivers, and the sustained high flow in the spring in the Miami 
Table 9. Bed velocity from acoustic Doppler current profiler data.

[Gavins Point Dam, South Dakota (U.S. Army Corps of Engineers); Missouri River main-stem streamflow-gaging stations and identification numbers: Sioux City, Iowa, 06486000; Decatur, Nebraska, 06601200; Waverly, Missouri 06895500]

\begin{tabular}{|c|c|c|c|c|c|c|}
\hline Site & Date & Gage & $\begin{array}{c}\text { Discharge } \\
\text { (cubic meters } \\
\text { per second) }\end{array}$ & $\begin{array}{c}\text { Percent } \\
\text { discharge of } \\
\text { median daily } \\
\text { discharge }\end{array}$ & $\begin{array}{l}\text { Mean bed } \\
\text { velocity mag- } \\
\text { nitude (meters } \\
\text { per second) }\end{array}$ & $\begin{array}{l}\text { Mean depth } \\
\text { (meters) }\end{array}$ \\
\hline \multirow[t]{7}{*}{ Yankton } & 20060307 & Gavins Point Dam & 255 & 35 & 0.03 & 2.40 \\
\hline & 20060328 & Gavins Point Dam & 595 & 81 & 0.03 & 2.70 \\
\hline & 20060515 & Gavins Point Dam & 708 & 96 & 0.13 & 2.76 \\
\hline & 20060627 & Gavins Point Dam & 708 & 96 & 0.09 & 2.61 \\
\hline & 20070314 & Gavins Point Dam & 453 & 62 & 0.01 & 2.40 \\
\hline & 20070626 & Gavins Point Dam & 510 & 69 & 0.07 & 2.62 \\
\hline & 20071108 & Gavins Point Dam & 254 & 34 & 0.04 & 2.06 \\
\hline \multirow[t]{8}{*}{ Kenslers Bend } & 20060309 & Sioux City & 348 & 42 & 0.04 & 2.26 \\
\hline & 20060330 & Sioux City & 657 & 80 & 0.05 & 2.92 \\
\hline & 20060516 & Sioux City & 869 & 105 & 0.08 & 3.33 \\
\hline & 20060620 & Sioux City & 850 & 103 & 0.11 & 3.45 \\
\hline & 20070315 & Sioux City & 1,085 & 132 & 0.08 & 2.94 \\
\hline & 20070411 & Sioux City & 629 & 76 & 0.04 & 2.55 \\
\hline & 20070620 & Sioux City & 708 & 86 & 0.07 & 3.20 \\
\hline & 20071110 & Sioux City & 343 & 42 & 0.03 & 2.09 \\
\hline \multirow[t]{9}{*}{ Little Sioux } & 20060316 & Decatur & 365 & 45 & 0.03 & 2.46 \\
\hline & 20060417 & Decatur & 767 & 95 & 0.04 & 3.93 \\
\hline & 20060517 & Decatur & 867 & 107 & 0.05 & 4.14 \\
\hline & 20060720 & Decatur & 759 & 94 & 0.10 & 3.62 \\
\hline & 20061024 & Decatur & 329 & 41 & 0.04 & 2.27 \\
\hline & 20070316 & Decatur & 1,138 & 141 & 0.07 & 4.74 \\
\hline & 20070410 & Decatur & 654 & 81 & 0.03 & 3.45 \\
\hline & 20070720 & Decatur & 714 & 88 & 0.09 & 3.53 \\
\hline & 20071031 & Decatur & 419 & 52 & 0.09 & 2.75 \\
\hline \multirow[t]{10}{*}{ Miami } & 20060323 & Waverly & 731 & 51 & 0.03 & 3.17 \\
\hline & 20060419 & Waverly & 1,410 & 99 & 0.05 & 4.38 \\
\hline & 20060522 & Waverly & 1,192 & 83 & 0.04 & 4.07 \\
\hline & 20060727 & Waverly & 983 & 69 & 0.02 & 3.44 \\
\hline & 20061106 & Waverly & 609 & 43 & 0.04 & 3.01 \\
\hline & 20070326 & Waverly & 1,606 & 112 & 0.06 & 4.66 \\
\hline & 20070430 & Waverly & 2,418 & 169 & 0.17 & 5.78 \\
\hline & 20070518 & Waverly & 3,285 & 230 & 0.04 & 7.49 \\
\hline & 20070814 & Waverly & 1,490 & 104 & 0.03 & 4.73 \\
\hline & 20071126 & Waverly & 881 & 62 & 0.04 & 3.54 \\
\hline
\end{tabular}


Table 10. Cross-section erosion and deposition for the four reaches.

$\left[\mathrm{m}^{2}\right.$, square meters; index cross-section area, average cross-section area at median annual flow]

\begin{tabular}{|c|c|c|c|c|c|c|c|c|}
\hline Site & Dates & $\begin{array}{l}\text { Number of } \\
\text { cross sec- } \\
\text { tions }\end{array}$ & $\begin{array}{l}\text { Average deposi- } \\
\text { tion area }\left(\mathrm{m}^{2}\right)\end{array}$ & $\begin{array}{c}\text { Average } \\
\text { erosion area } \\
\left(\mathbf{m}^{2}\right)\end{array}$ & $\begin{array}{l}\text { Average net } \\
\text { change }\left(\mathrm{m}^{2}\right)\end{array}$ & $\begin{array}{l}\text { Percent net } \\
\text { change of } \\
\text { index cross- } \\
\text { section area }\end{array}$ & $\begin{array}{c}\text { Average } \\
\text { total change } \\
\left(\mathrm{m}^{2}\right)\end{array}$ & $\begin{array}{l}\text { Percent total } \\
\text { change of } \\
\text { index cross- } \\
\text { section area }\end{array}$ \\
\hline \multicolumn{9}{|c|}{2006} \\
\hline \multirow[t]{3}{*}{ Yankton } & March-April & 27 & 11.2 & -3.1 & 8.1 & 1.2 & 14.3 & 2.2 \\
\hline & April-May & 28 & 4.8 & -7.7 & -2.9 & -0.4 & 12.5 & 1.9 \\
\hline & May-June & 30 & 7.6 & -3.4 & 4.1 & 0.6 & 11.0 & 1.7 \\
\hline Kenslers & March-April & 30 & 81.2 & -25.7 & 55.5 & 8.5 & 107.0 & 16.3 \\
\hline \multirow[t]{2}{*}{ Bend } & April-May & 29 & 46.3 & -67.2 & -21.0 & -3.2 & 113.5 & 17.3 \\
\hline & May-June & 21 & 36.1 & -59.1 & -23.0 & -3.5 & 95.2 & 14.5 \\
\hline Little & March-April & 33 & 52.2 & -27.5 & 24.7 & 3.3 & 79.7 & 10.7 \\
\hline \multirow[t]{3}{*}{ Sioux } & April-May & 32 & 31.4 & -30.8 & 0.6 & 0.1 & 62.3 & 8.4 \\
\hline & May-July & 32 & 58.1 & -59.0 & -0.9 & -0.1 & 117.1 & 15.7 \\
\hline & July-October & 33 & 61.9 & -92.3 & -30.4 & -4.1 & 154.2 & 20.7 \\
\hline \multirow[t]{4}{*}{ Miami } & March-April & 32 & 56.3 & -33.3 & 22.9 & 1.8 & 89.6 & 7.2 \\
\hline & April-May & 30 & 26.4 & -52.8 & -26.4 & -2.1 & 79.2 & 6.4 \\
\hline & May-July & 30 & 24.9 & -100.2 & -75.3 & -6.1 & 125.1 & 10.1 \\
\hline & July-November & 31 & 88.3 & -53.4 & 34.9 & 2.8 & 141.7 & 11.4 \\
\hline \multicolumn{9}{|c|}{2007} \\
\hline \multirow[t]{2}{*}{ Yankton } & March-June & 25 & 4.0 & -12.0 & -7.9 & -1.2 & 16.0 & 2.5 \\
\hline & June-November & 29 & 3.8 & -11.7 & -7.9 & -1.2 & 15.5 & 2.4 \\
\hline Kenslers & June 2006-January 2007 & 12 & 58.1 & -42.3 & 15.8 & 2.4 & 100.4 & 15.3 \\
\hline \multirow[t]{2}{*}{ Bend } & April-July & 30 & 114.6 & -102.1 & 12.5 & 1.9 & 216.8 & 33.1 \\
\hline & July-November & 30 & 63.9 & -120.0 & -56.0 & -8.5 & 183.9 & 28.0 \\
\hline Little & October 2006-March 2007 & 33 & 67.9 & -47.8 & 20.1 & 2.7 & 115.7 & 15.5 \\
\hline \multirow[t]{3}{*}{ Sioux } & March-April & 33 & 28.3 & -27.5 & 0.8 & 0.1 & 55.9 & 7.5 \\
\hline & April-July & 33 & 70.8 & -63.7 & 7.1 & 1.0 & 134.5 & 18.1 \\
\hline & July-October & 32 & 61.8 & -72.9 & -11.2 & -1.5 & 134.7 & 18.1 \\
\hline \multirow[t]{5}{*}{ Miami } & November 2006-March 2007 & 32 & 91.2 & -50.9 & 40.3 & 3.2 & 142.1 & 11.4 \\
\hline & March-April & 32 & 27.8 & -72.2 & -44.4 & -3.6 & 100.0 & 8.0 \\
\hline & April-June & 31 & 63.5 & -72.2 & -8.7 & -0.7 & 135.6 & 10.9 \\
\hline & June-August & 31 & 88.3 & -55.1 & 33.2 & 2.7 & 143.4 & 11.5 \\
\hline & August-November & 33 & 24.0 & -54.1 & -30.1 & -2.4 & 78.1 & 6.3 \\
\hline \multicolumn{9}{|c|}{ Annual } \\
\hline \multirow[t]{2}{*}{ Yankton } & March 2006-June 2006 & 27 & 11.7 & -2.2 & 9.5 & 1.5 & 13.9 & 2.1 \\
\hline & March 2007-November 2007 & 24 & 1.4 & -18.8 & -17.4 & -2.7 & 20.3 & 3.1 \\
\hline Kenslers & March 2006-June 2006 & 22 & 101.0 & -64.0 & 36.9 & 5.6 & 165.0 & 25.2 \\
\hline Bend & April 2007-November 2007 & 30 & 72.9 & -108.9 & -36.1 & -5.5 & 181.8 & 27.7 \\
\hline Little & March 2006-October 2006 & 33 & 72.6 & -77.5 & -4.9 & -0.7 & 150.2 & 20.2 \\
\hline \multirow[t]{2}{*}{ Sioux } & March 2007-October 2007 & 32 & 95.6 & -97.9 & -2.3 & -0.3 & 193.5 & 26.0 \\
\hline & March 2006-October 2007 & 32 & 73.2 & -63.1 & 10.0 & 1.3 & 136.3 & 18.3 \\
\hline \multirow[t]{3}{*}{ Miami } & March 2006-November 2006 & 32 & 24.8 & -98.1 & -73.3 & -5.9 & 122.9 & 9.9 \\
\hline & March 2007-November 2007 & 32 & 36.1 & -70.4 & -34.3 & -2.8 & 106.4 & 8.6 \\
\hline & March 2006-November 2007 & 32 & 44.9 & -85.6 & -40.7 & -3.3 & 130.5 & 10.5 \\
\hline \multicolumn{9}{|c|}{ Intra-annual } \\
\hline Yankton $^{1}$ & -no data- & -no data- & -no data- & -no data- & -no data- & -no data- & -no data- & -no data- \\
\hline Kenslers Bend & March 2006- March 2007 & 16 & 115.5 & -77.7 & 37.7 & 5.8 & 193.2 & 29.5 \\
\hline Little & March 2006- March 2007 & 33 & 69.5 & -53.9 & 15.6 & 2.1 & 123.4 & 16.6 \\
\hline \multirow[t]{3}{*}{ Sioux } & April 2006-April 2007 & 33 & 61.6 & -71.4 & -9.9 & -1.3 & 133.0 & 17.9 \\
\hline & June 2006-200707 & 33 & 61.3 & -63.8 & -2.4 & -0.3 & 125.1 & 16.8 \\
\hline & October 2006- October 2007 & 32 & 87.8 & -68.9 & 18.9 & 2.5 & 156.7 & 21.1 \\
\hline \multirow[t]{5}{*}{ Miami } & March 2006-March 2007 & 31 & 68.5 & -74.6 & -6.1 & -0.5 & 143.2 & 11.5 \\
\hline & April 2006-April 2007 & 32 & 32.0 & -100.8 & -68.8 & -5.5 & 132.9 & 10.7 \\
\hline & June 2006-June 2007 & 28 & 62.1 & -114.9 & -52.8 & -4.2 & 177.0 & 14.3 \\
\hline & July 2006-August 2007 & 31 & 106.3 & -41.3 & 65.0 & 5.2 & 147.6 & 11.9 \\
\hline & November 2006-November 2007 & 33 & 48.7 & -50.6 & -1.9 & -0.1 & 99.3 & 8.0 \\
\hline
\end{tabular}

\footnotetext{
${ }^{1}$ Because of changes in cross section data collection at Yankton, no intra-annual data are available for this reach.
} 

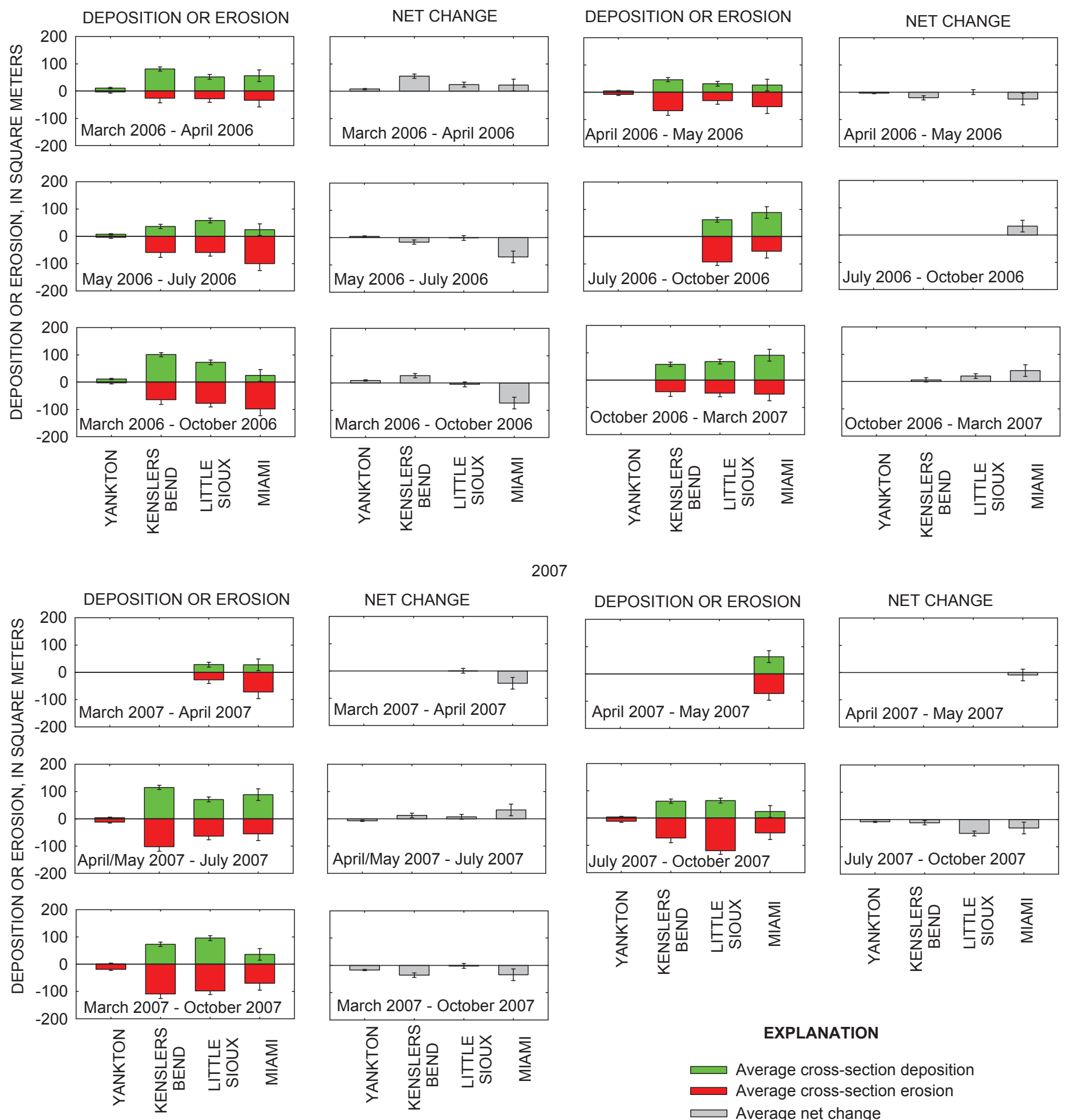

\section{EXPLANATION}

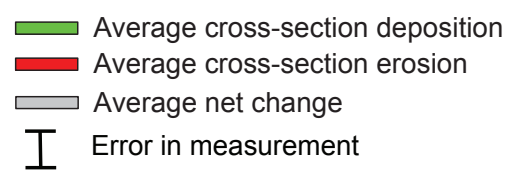

Figure 28. Reach averaged deposition, erosion, and net change for the Yankton, Kenslers Bend, Little Sioux, and Miami reaches. 
reach. During the 2007 summer months the general trend was net erosion (figs. 28-30). This was also a period associated with high rates of total geomorphic change in the Kenslers Bend and Little Sioux reaches. The "bump" down to fall flows appeared to be associated with erosion as well (fig. 30, table $10)$.

Pulsed flow magnitudes that were considered in design of the 2006 spring rise ranged $480-680 \mathrm{~m}^{3} / \mathrm{s}$ above a background navigation release of nominally $710 \mathrm{~m}^{3} / \mathrm{s}$ (Jacobson, 2008; Jacobson and Galat, 2008). While it is difficult to attribute morphodynamic effects to individual flow pulses monitored during these surveys, it is clear that flow pulses with magnitudes as little as $250 \mathrm{~m}^{3} / \mathrm{s}$ above background navigation flows were associated with measurable changes in the bed. In the spring in the two upstream reaches that are most likely to be affected by modified pulsed flows, flow pulses were associated with net erosion (fig. 30). This result supports the hypothesis that pulsed flow modifications are of a magnitude that can have significant influence on channel morphodynamics and perhaps on habitat quantity and quality.

\section{Flow and Bedload Transport Measures}

In addition to direct measures of geomorphic change, monitoring included assessment of one-dimensional bedload transport rates by bedform differencing (Simons and others, 1980) and bed velocities (Gaeuman and Jacobson, 2006). Onedimensional bedload transport rates measure bedload movement by dune displacement in the thalweg. As such, these are the highest rates of bedload transport within a reach and cannot be extrapolated to total or cross-sectional average bedload fluxes. However, the one-dimensional bedload transport data do provide a consistent index of bedload transport potential among reaches. The lowest rates measured are about $0.1 \mathrm{~m}^{2} / \mathrm{h}$ at about $370 \mathrm{~m}^{3} / \mathrm{s}$ and rates increase linearly to greater than 1.5 $\mathrm{m}^{2} / \mathrm{h}$ at 2,400-3,300 $\mathrm{m}^{3} / \mathrm{s}$ at Miami (fig. 23, table 8). The highest discharge for which one-dimensional rates were obtained $\left(3,280 \mathrm{~m}^{3} / \mathrm{s}\right)$ was $1.51 \mathrm{~m}^{2} / \mathrm{h}$ whereas, at a somewhat lower discharge $\left(2,420 \mathrm{~m}^{3} / \mathrm{s}\right)$ the rate was $1.53 \mathrm{~m}^{2} / \mathrm{h}$. Leveling off of the one-dimensional transport rate may represent increasing proportion of sand being transported in suspension rather than as bedload (Nittrouer and others, 2008).

Average bed velocities calculated for the reaches over the same discharges also increased linearly with discharge, although the relation seems to deteriorate at the highest discharges measured (fig. 27, table 8). Bed-velocity data are spatially very heterogeneous, but data averaged over hundreds of ADCP ensembles yield consistent results. Similar to onedimensional bedload transport rates, there may be thresholds above which bed velocities cannot be measured reliably.

These two methods confirm that for the sandbed Missouri River, bedload is moving at all discharges in the thalweg, and that transport increases linearly with increasing discharge. Hence, flow pulses of any size can be expected to increase bedload sediment transport, and therefore, have the potential to affect morphodynamics and habitats in the channel.

\section{Reach Geomorphology}

Representative cross sections for each reach show the average bankfull widths for each reach on the Lower Missouri River do not increase in a monotonically downstream manner (fig. 31), as expected on nonengineered large rivers (Leopold and Maddock, 1953). Analysis of depth maps created from the elevation grids and modeled water surfaces at median daily flow shows that mean depths increase downstream (fig. 32). The Yankton reach, because of the lack of channelization for navigation, is the widest of the four reaches with the shallowest mean depth. The Kenslers Bend and Sioux reaches are the narrowest reaches with the mean depths being greater in the Little Sioux reach (figs. 31-32). The constricted nature of the Kenslers Bend and Sioux reaches may explain the large magnitude of geomorphic changes in these reaches over the course of the year.

In the Kenslers Bend and Little Sioux reaches, the expected course of the thalweg as defined by the engineered channel planform was not always the deepest part of the channel (fig. 33). The Kenslers Bend longitudinal profile shows many bars that are located where the channel thalweg is expected based on channel engineering structures. The Little Sioux reach exhibits a similar pattern, although there are fewer and larger bars than the Kenslers Bend reach (fig. 33). The Miami longitudinal profile shows that the thalweg follows the expected pattern based on channel engineering structures.

Shifting in the thalweg and bar locations are associated with the large total geomorphic changes in these reaches. While it is not possible to associate change events with individual flow pulses, the amount of change associated with relatively small flow pulses indicates high sensitivity of channel morphodynamics to flow pulses. Peak flows experienced at streamflow gaging stations at Sioux City, Iowa, and Decatur, Nebraska, during 2006-07 were equaled or exceeded 12-19 percent of the time based on the post-regulation period of record at these gages, indicating that the discharges responsible for morphologic change were not extreme. The peak flows correspond roughly to pulses $350-620 \mathrm{~m}^{3} / \mathrm{s}$ above navigation discharges, within pulse magnitudes considered in design of spring pulsed flows (Jacobson and Galat, 2008).

\section{Change Based on Compilation Surveys 2006-07}

Change maps were calculated for the grids created from the 2006 and 2007 compilation surveys (figs. 34-37). Similar to trends detected in cross sections, the Yankton reach had the most stable total bathymetry between the 2 years (fig. 34 , figs. 38-39). There was some scour on bars, generally less than $1 \mathrm{~m}$ and some deposition near the thalweg in deeper areas, also usually less than $1 \mathrm{~m}$. This is substantially less morphodynamic change than between the two summer compilation surveys in the other reaches (table 11). Net change of Yankton was $86,928 \mathrm{~m}^{3}$, the highest net deposition among the four reaches; total geomorphic change (the sum of erosion and deposition), however, was lowest in this reach, at 404,724 $\mathrm{m}^{3}$. 

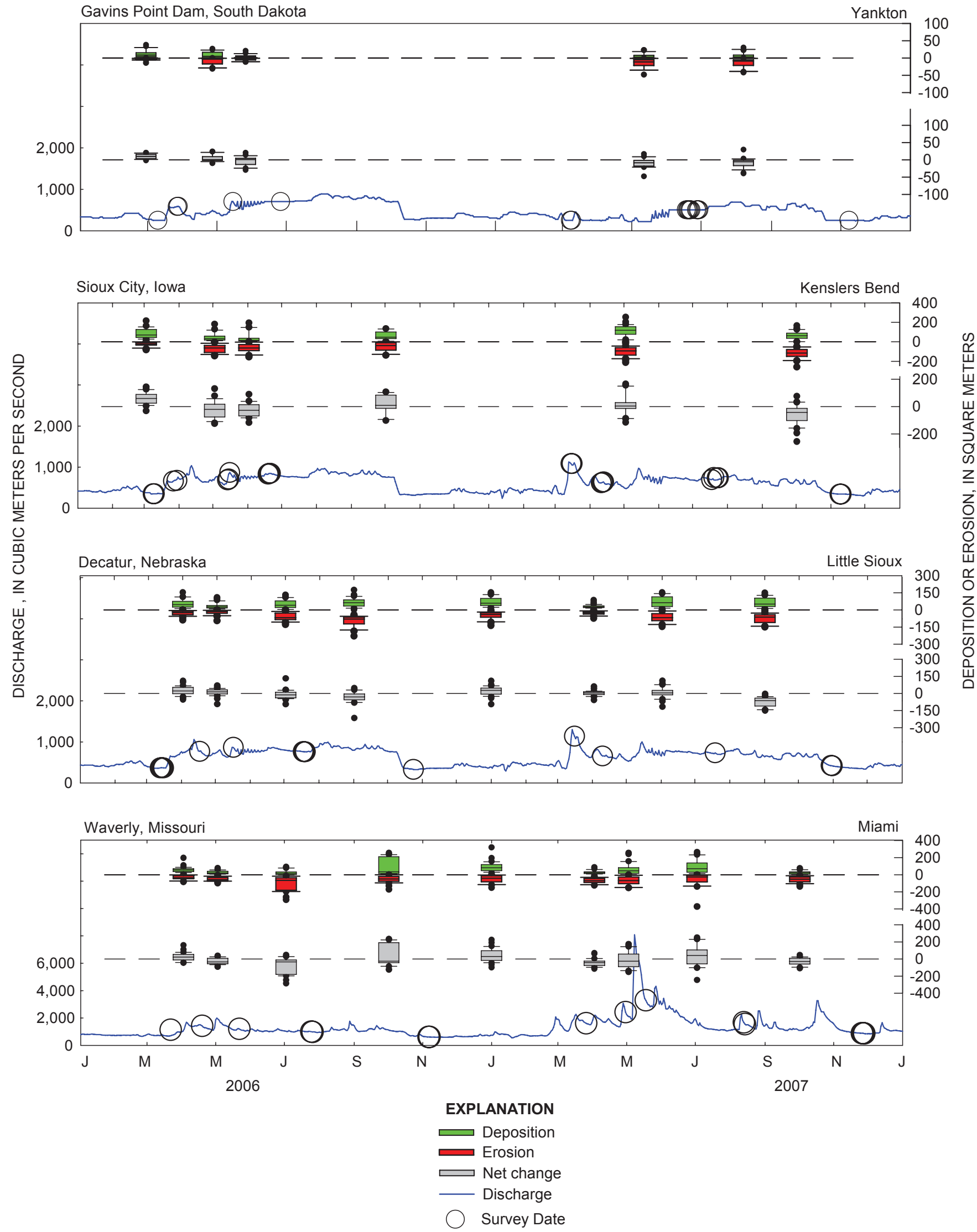

Figure 29. Average deposition and erosion in the four reaches with corresponding surveys and hydrographs. 


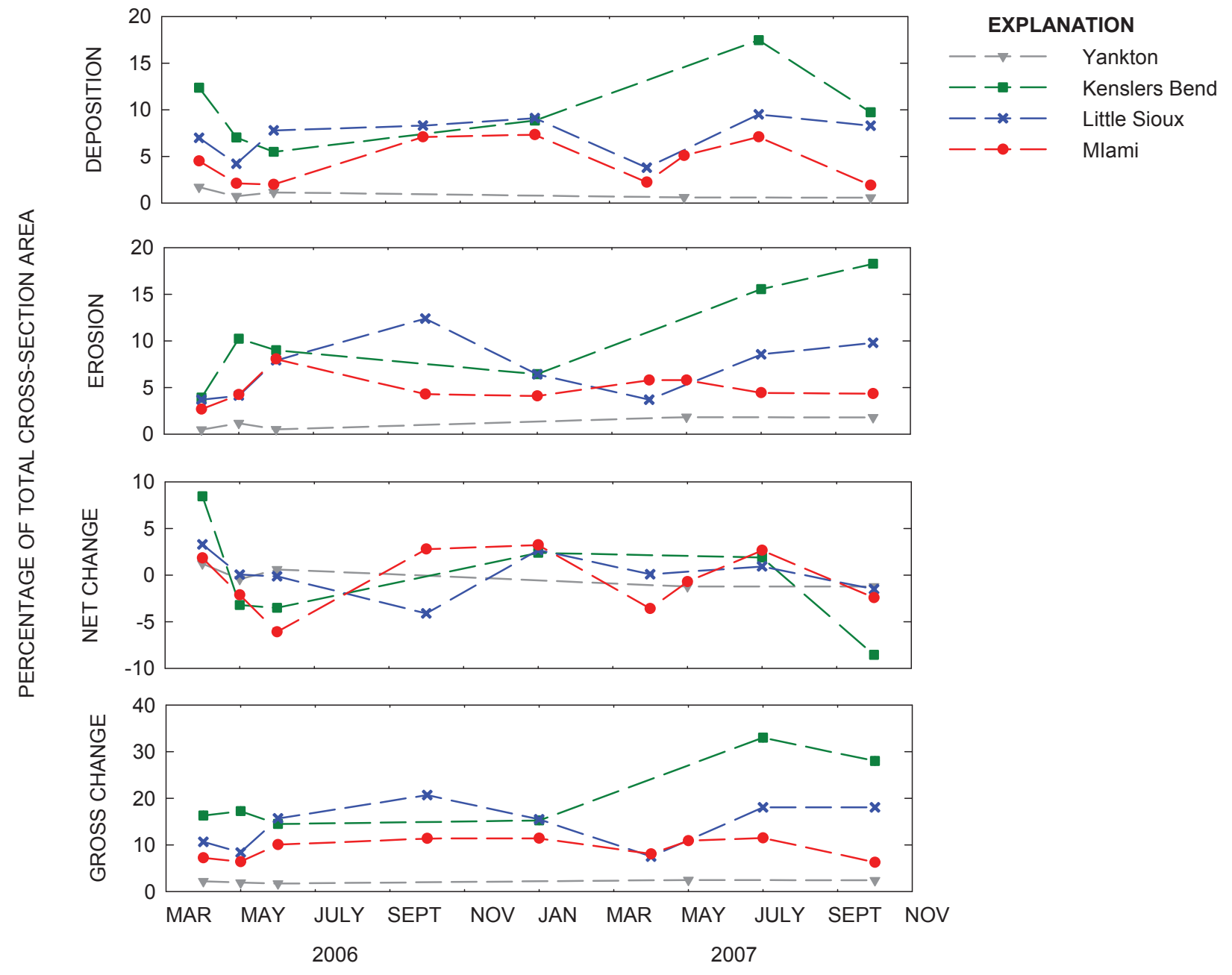

Figure 30. Percent of cross-section area with deposition, erosion, or net change for the four monitoring reaches.

In the Kenslers Bend reach, the channel thalweg and bar pattern reversed location in many transects from June 2006 to June 2007 because of migrating bars (fig. 35, Appendix B). Deposition and erosion magnitudes were as much as 6-7 $\mathrm{m}$ in some places. Magnitudes of erosion and deposition between the June 2006 to July 2007 compilation survey grids were the highest of all the survey reaches, but net deposition was $35,952 \mathrm{~m}^{3}$, less than that at Yankton (table 11, fig. 39). Total or gross change magnitudes were highest in this reach $(2,081,501$ $\mathrm{m}^{3}$ ) with nearly twice the volume of total change in the Sioux reach (table 11, fig. 39).
Channel position shifting similar to the Kenslers Bend reach occurred in the Little Sioux reach because of bar migration (fig. 36). Deposition and erosion magnitudes were also 3-4 $\mathrm{m}$ in some places in this reach (fig. 38). The total net change between the survey grids from the July 2006 to July 2007 surveys was $-134,200 \mathrm{~m}^{3}$, the highest magnitude net change of the four reaches (table 11, fig. 39). Total, or gross change was 1,065,998 $\mathrm{m}^{3}$ (table 11).

Trends in the Miami reach differed from the other three reaches. The locations of the thalweg and bars were fairly stable (fig. 37). However, deposition occurred on bars, in or near the thalweg in main channel areas, and inside dike fields

Table 11. Change in grid area from compilation surveys, 2006-07.

\begin{tabular}{lcccc}
\hline \multicolumn{1}{c}{ Reach } & $\begin{array}{c}\text { Deposition } \\
\text { (cubic meters) }\end{array}$ & $\begin{array}{c}\text { Erosion (cubic } \\
\text { meters) }\end{array}$ & $\begin{array}{c}\text { Net change } \\
\text { (cubic meters) }\end{array}$ & $\begin{array}{c}\text { Total change } \\
\text { (cubic meters) }\end{array}$ \\
\hline Yankton & 245,826 & $-158,898$ & 86,928 & 404,724 \\
Kenslers Bend & $1,058,726$ & $-1,022,775$ & 35,952 & $2,081,501$ \\
Little Sioux & 465,862 & $-600,137$ & $-134,200$ & $1,065,998$ \\
Miami & 407,124 & $-455,427$ & $-48,303$ & 862,551 \\
\hline
\end{tabular}



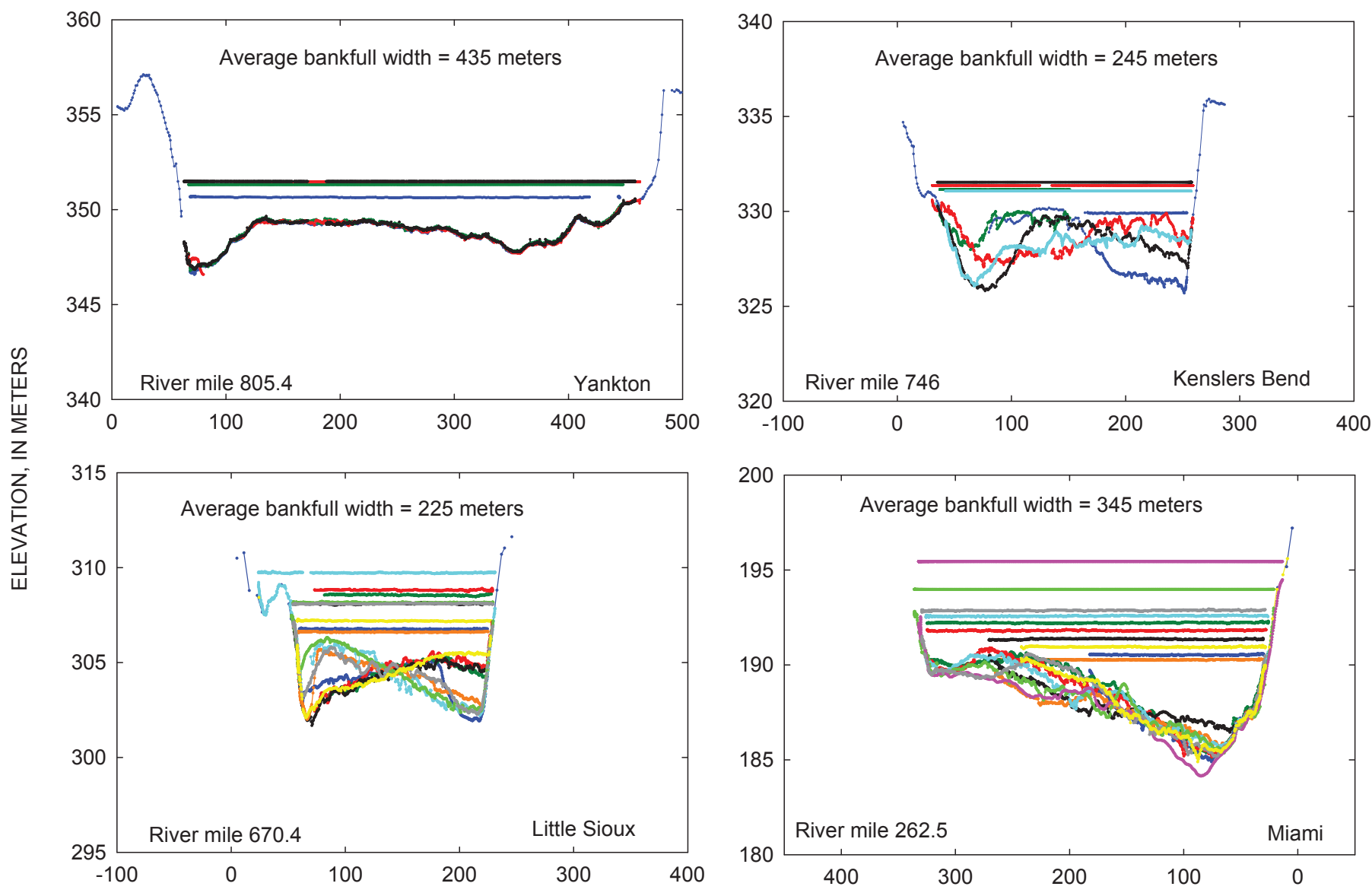

HORIZONTAL DISTANCE, IN METERS

\section{EXPLANATION}

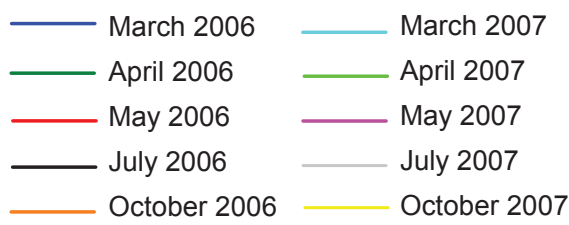

Figure 31. Typical cross sections at the same scale for the four reaches.

near the bank. Erosion occurred in scour holes downstream from dikes and in some thalweg areas. Deposition and erosion magnitudes were generally $2 \mathrm{~m}$ or less (fig. 38). The net change was $-48,303 \mathrm{~m}^{2}$ from the July 2006 to August 2007 surveys (table 11, fig. 39). Total or gross change in this reach for 2006-07 was $862,551 \mathrm{~m}^{3}$.

In the Yankton reach there was little change in the general structure of the topography, and the multithread channel pattern was similar over the 2 years. As an additional measure of morphodynamic change at the three downstream reaches, transects were classified as occurring in either cross-overs (sites where the thalweg is in the process of crossing to the opposite bank) or bends according to the location of the transects on the 2006 and 2007 compilation survey bathymetry (figs. 34-37). In the Kenslers Bend reach, 57 percent of the randomly selected transects were in cross-overs and 43 percent in bends in 2006. In 2007, however, 43 percent of the transects were located in bends and 57 percent were located in cross-overs, and one-half of the bends and cross-overs changed from one class to the other. In the Little Sioux Reach in 2006, randomly selected cross sections primarily were located at cross-overs rather than bends (63 to 38 percent). In 2007, this number changed with 56 percent of the transects in cross-overs and 44 percent in bends.

Based on the July 2006 and August 2007 compilation survey bathymetry, the bends at Miami were stable, and there were not any changes between the classification of cross sections as occurring on bends or cross-overs. More random cross sections fell in bends than cross-overs ( 66 percent to 38 percent). 

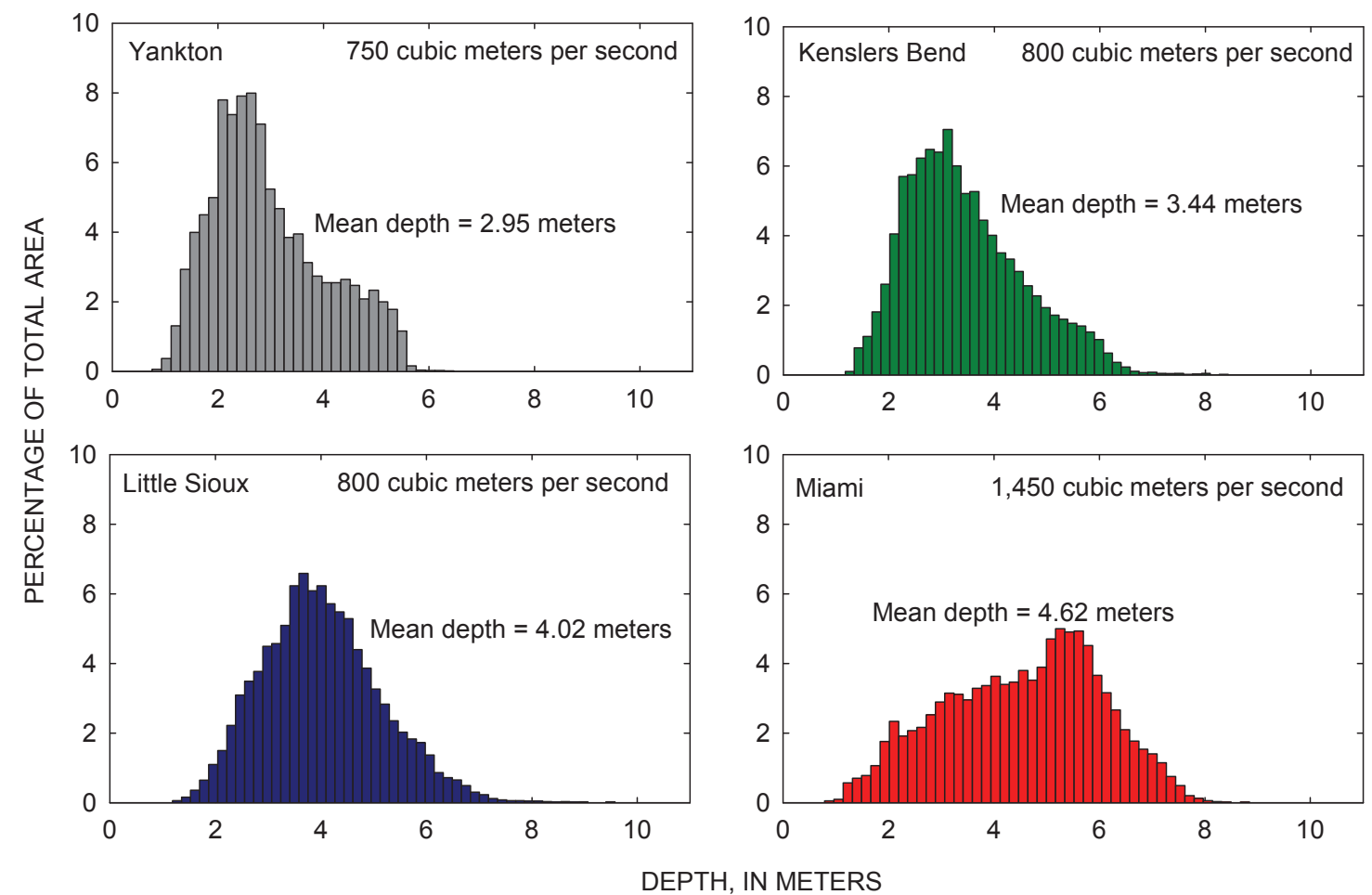

Figure 32. Depths at median annual flow in the four reaches.

\section{Implications for Understanding Sturgeon Habitat Dynamics}

This report provides unique documentation of morphodynamic changes on the Lower Missouri River. No other published datasets quantify geomorphic changes at the reach scale as many as five times per year and correlate changes with indicators of bedload transport. These data provide fundamental understanding of processes, rates, and scale of morphodynamic changes, which have a bearing on many aspects of habitat restoration on the river.

These data document substantial variation among river segments. The Yankton reach showed very small amounts of change, consistent with armoring of the bed and the high proportion of stabilized banks. Change was greatest in the Kenslers Bend and Little Sioux reaches where a narrow channel concentrates flow and sediment transport resulting in large vertical changes in the riverbed.

The downstream three reaches are characterized by substantial geomorphic change even during times of relatively small discharges. Expressed as a percentage of index cross-sectional area, 8 to 33 percent of the channel area was changed between successive surveys at Kenslers Bend, Little Sioux, and Miami (table 10). At Yankton, only 2 to 3 percent was changed (table 10). This morphodynamic change sets the background level of variability within which habitats are created, used, and assessed on this river. For example, assessment of habitat availability with hydrodynamic models that do not take morphodynamic change into account may incur substantial errors in predicting habitat availability within and among years (Jacobson and others, 2009b). Assessments of habitat restoration efforts also need to account for the background level of morphodynamic change to assure accurate measures of project performance.

Although these results document substantial intrareach and intrayear variability, they also confirm that on an annual basis erosion and deposition tend to cancel out to define an equilibrium channel morphology in the four reaches. In Yankton and Miami reaches, the equilibrium results in a balance of erosion and deposition such that channel features remain in the same location and same proportion from year to year. In the Kenslers Bend and Little Sioux reaches, the rough balance of erosion and deposition attests to a reach-scale equilibrium, but within the reaches channel features shift positions substantially. The implication is that habitat assessments based on static-bed hydrodynamic models may not be reliable for predicting habitat locations within a reach over time. Such models, however, will do better at predicting total habitat availability in the reach without specifying where it is located.

One of the primary questions addressed by this report is the extent to which flow pulses could "condition" spawning substrates by flushing them of fine sediment. Among all the reaches, the Yankton reach has the best prospects for natural gravel-cobble substrate thought to be required by sturgeon for spawning (Buckley and Kynard, 1981; Paragamian and others, 2001; Laustrup and others, 2007). The monitoring and sidescan data indicate that the substrate is mostly clean, having relatively small and discontinuous patches of sand overlying it. Flow pulses are capable of transporting the sand and flushing it from the underlying substrate; however, the large 

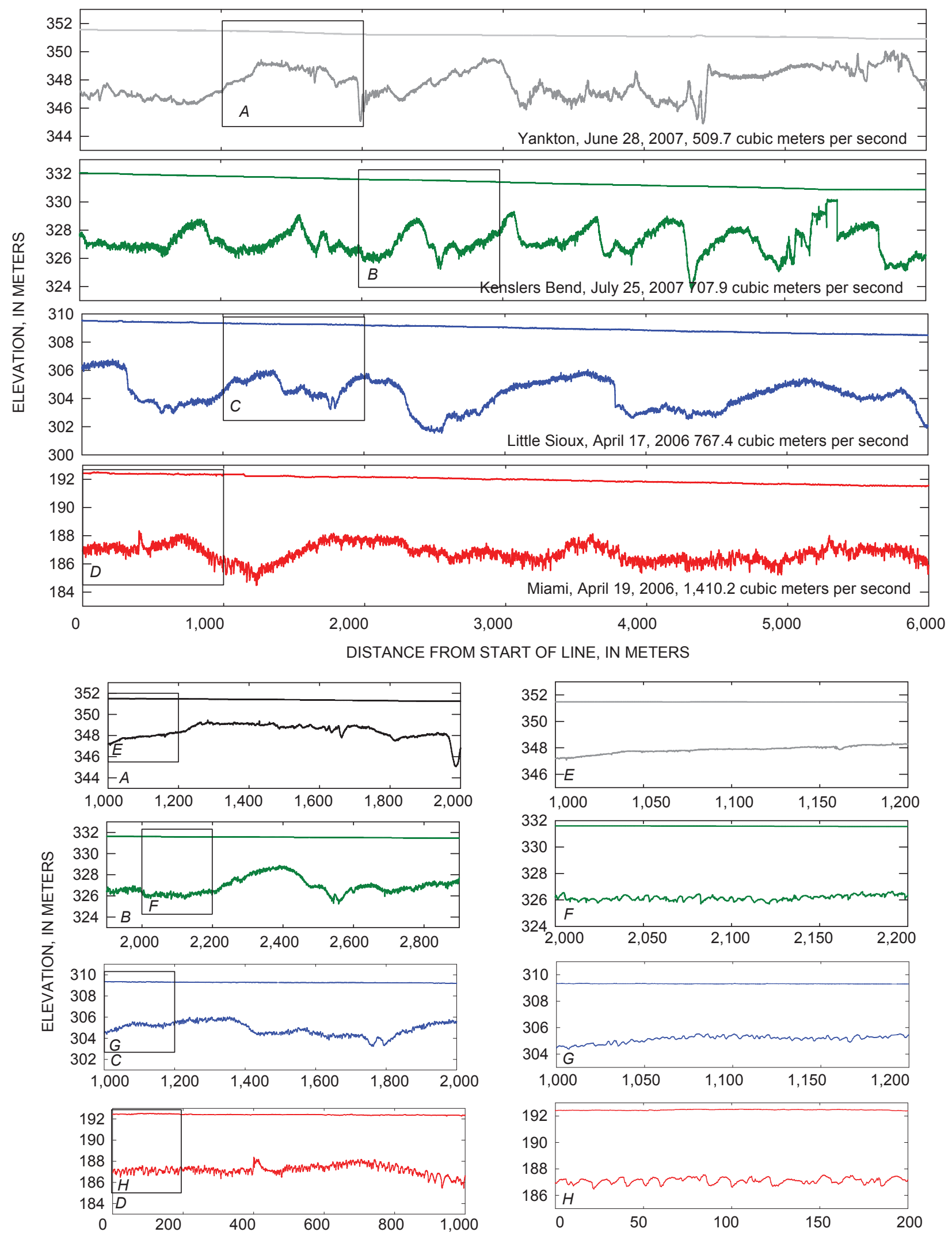

DISTANCE FROM START OF LINE, IN METERS

Figure 33. Longitudinal profiles at median or near-median annual flow for the four reaches. 


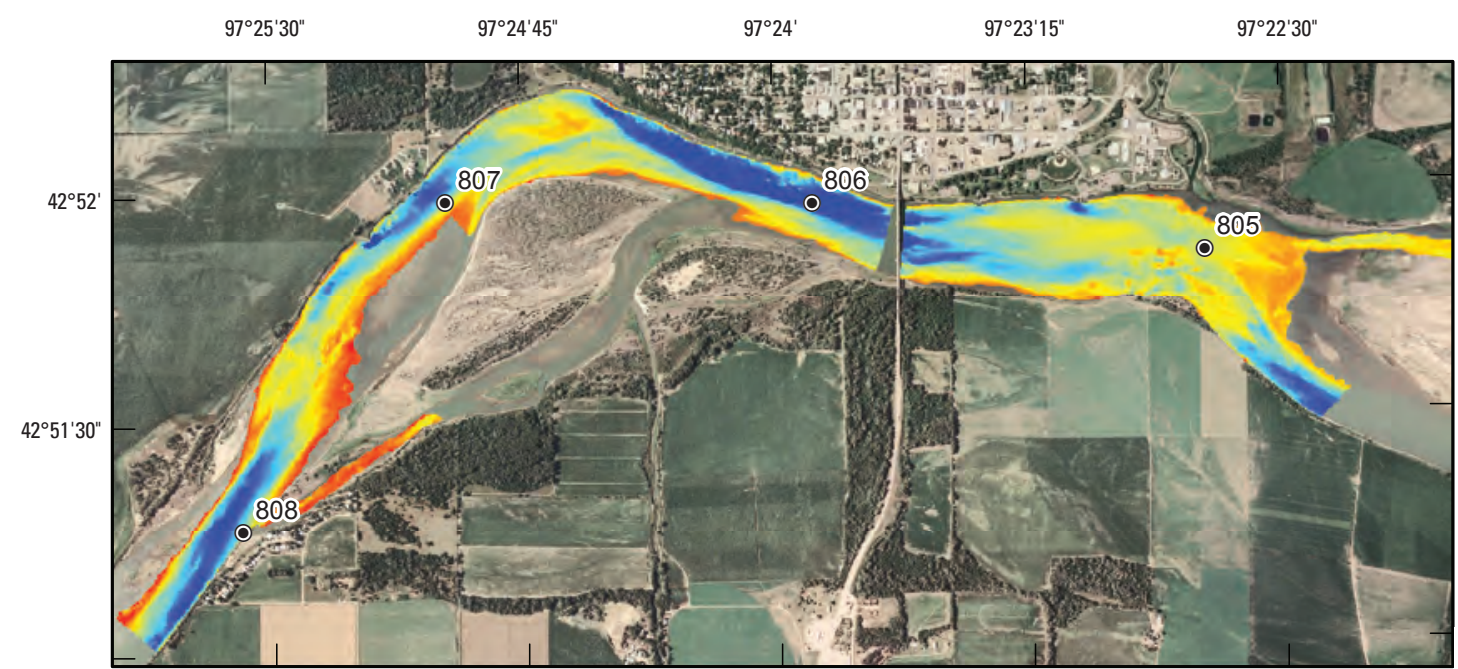

EXPLANATION
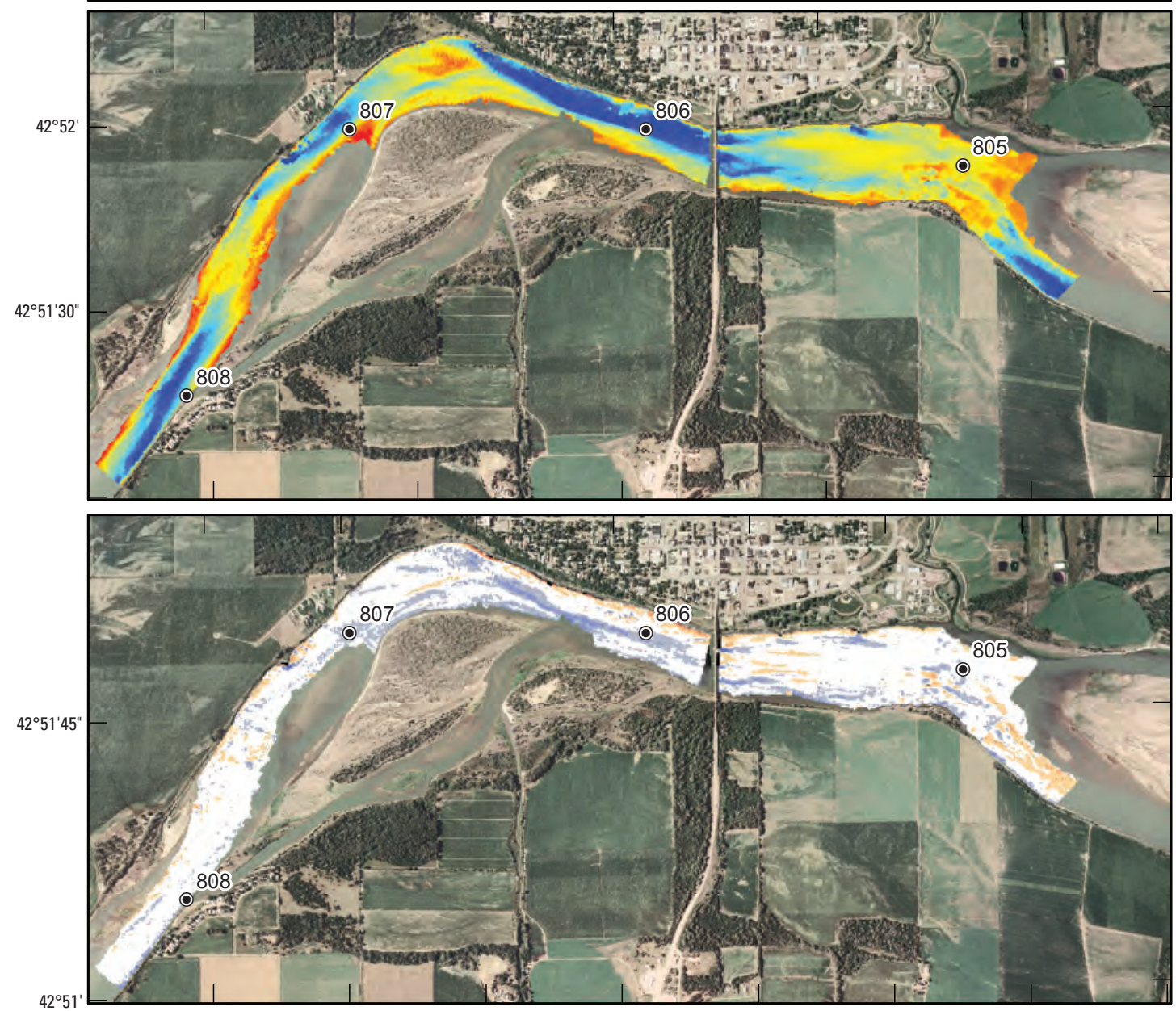

Elevation, in meters

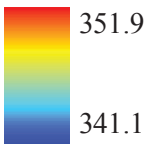

Change in elevation, 2006-07, in meters

\begin{tabular}{|l}
\hline$<-5$ to -4 \\
$<-4$ to -3 \\
$<-3$ to -2 Erosion \\
$<-2$ to -1 \\
$<-1$ to -0.3 \\
\hline$<-0.3$ to 0 No change \\
\hline$<0$ to 0.3 \\
\hline$<0.3$ to 1 \\
$<1$ to 2 Deposition \\
$<2$ to 3 \\
$<3$ to 4
\end{tabular}

805 o River mile marker and number- Number is distance upstream from the Mississippi River, in miles

Base from U.S. Department of Agriculture

National Agriculture Imagery Program, digital data, 2006

Universal Transverse Mercator projection Zone 15
River miles (1960) from U.S. Army Corps of Engineers $0 \quad 200400$ METERS

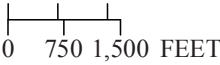

Figure 34. Changes in elevation based on 2006 and 2007 compilation surveys in the Yankton reach. 


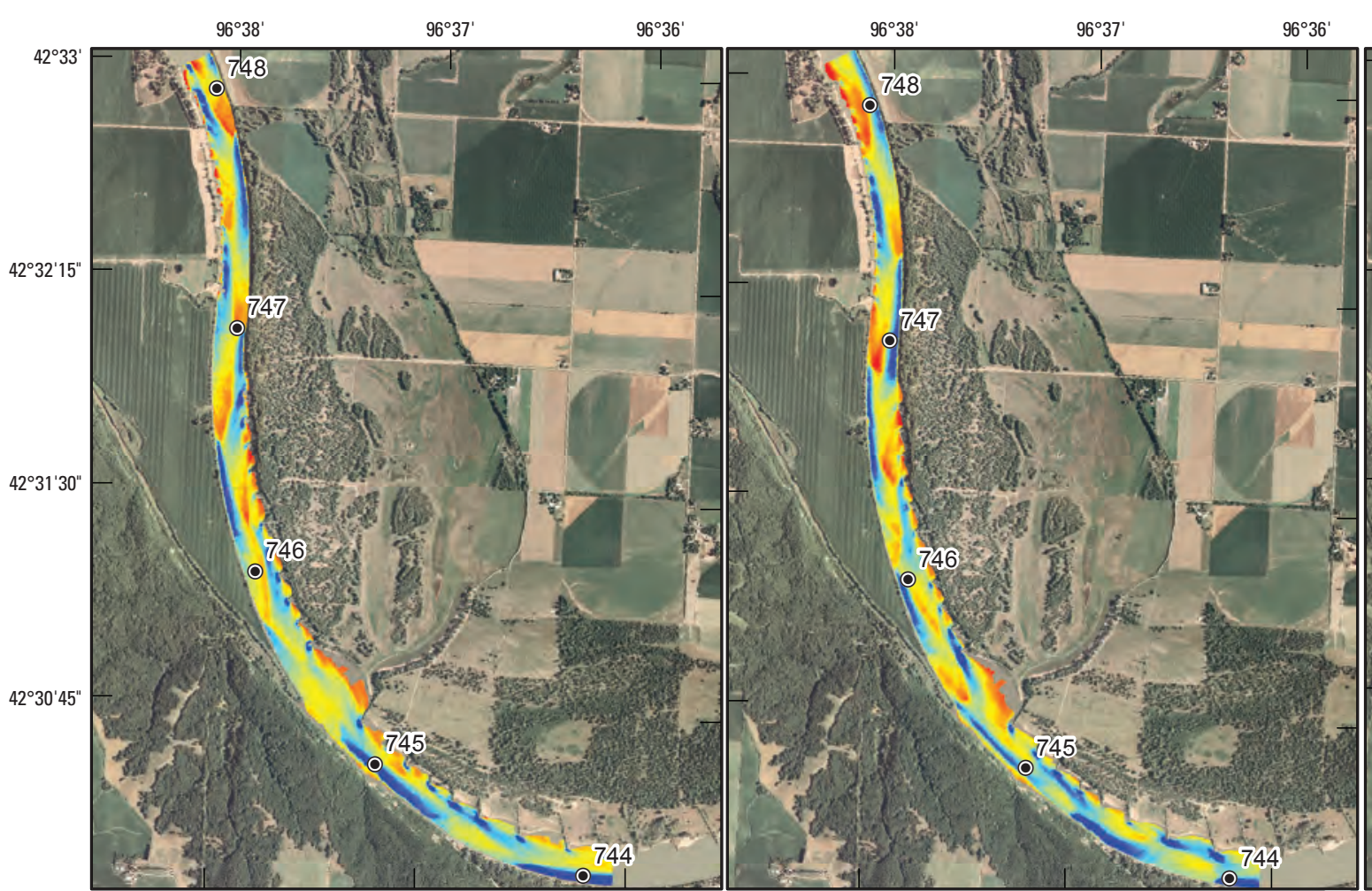

Base from U.S. Department of Agriculture National Agriculture Imagery Program, digital data, 2006 Universal Transverse Mercator projection Zone 14

\section{EXPLANATION}

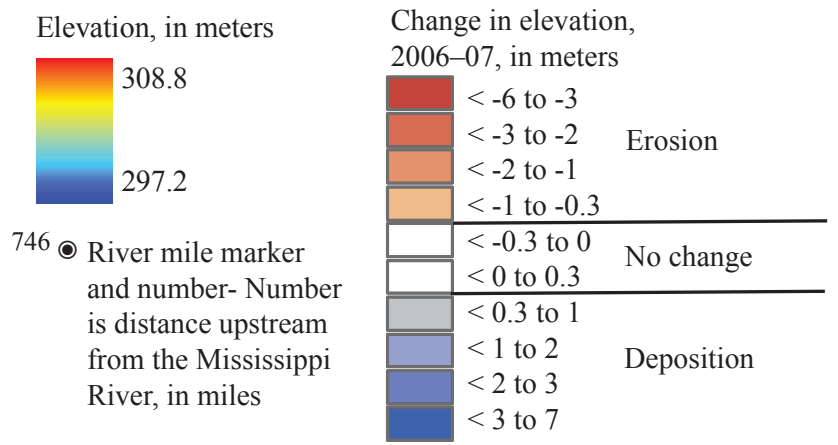

$\begin{array}{ll}96^{\circ} 38^{\prime} & 96^{\circ} 37\end{array}$ $96^{\circ} 36^{\prime}$

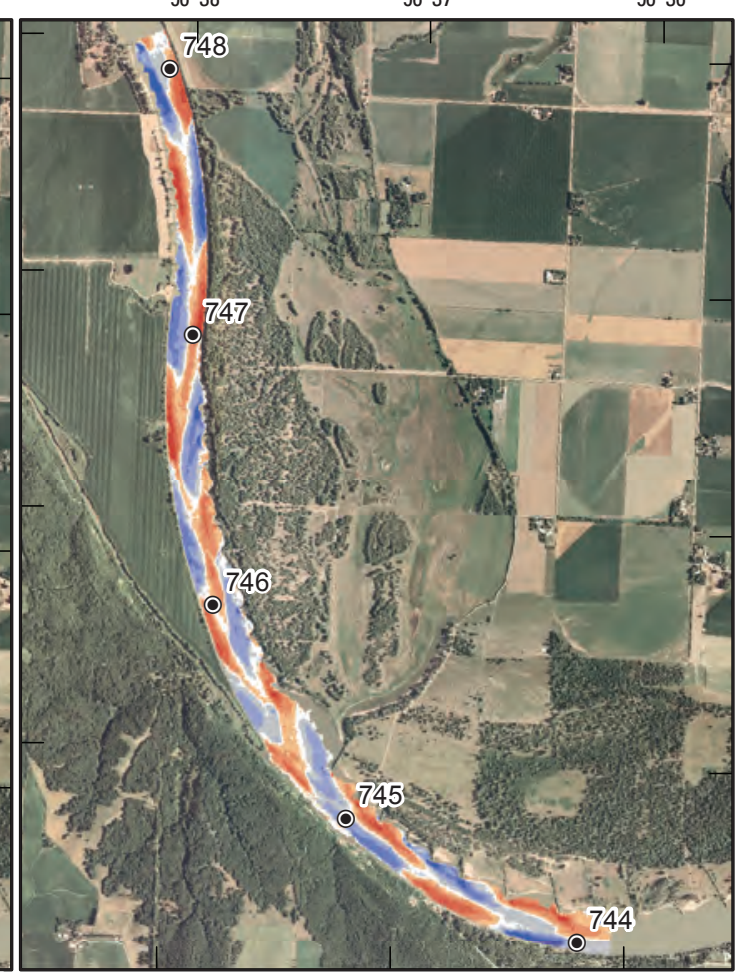

River miles (1960) from U.S. Army Corps of Engineers

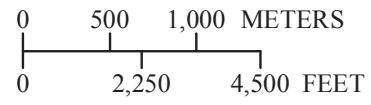

Figure 35. Changes in elevation based on 2006 and 2007 compilation surveys in the Kenslers Bend reach. 


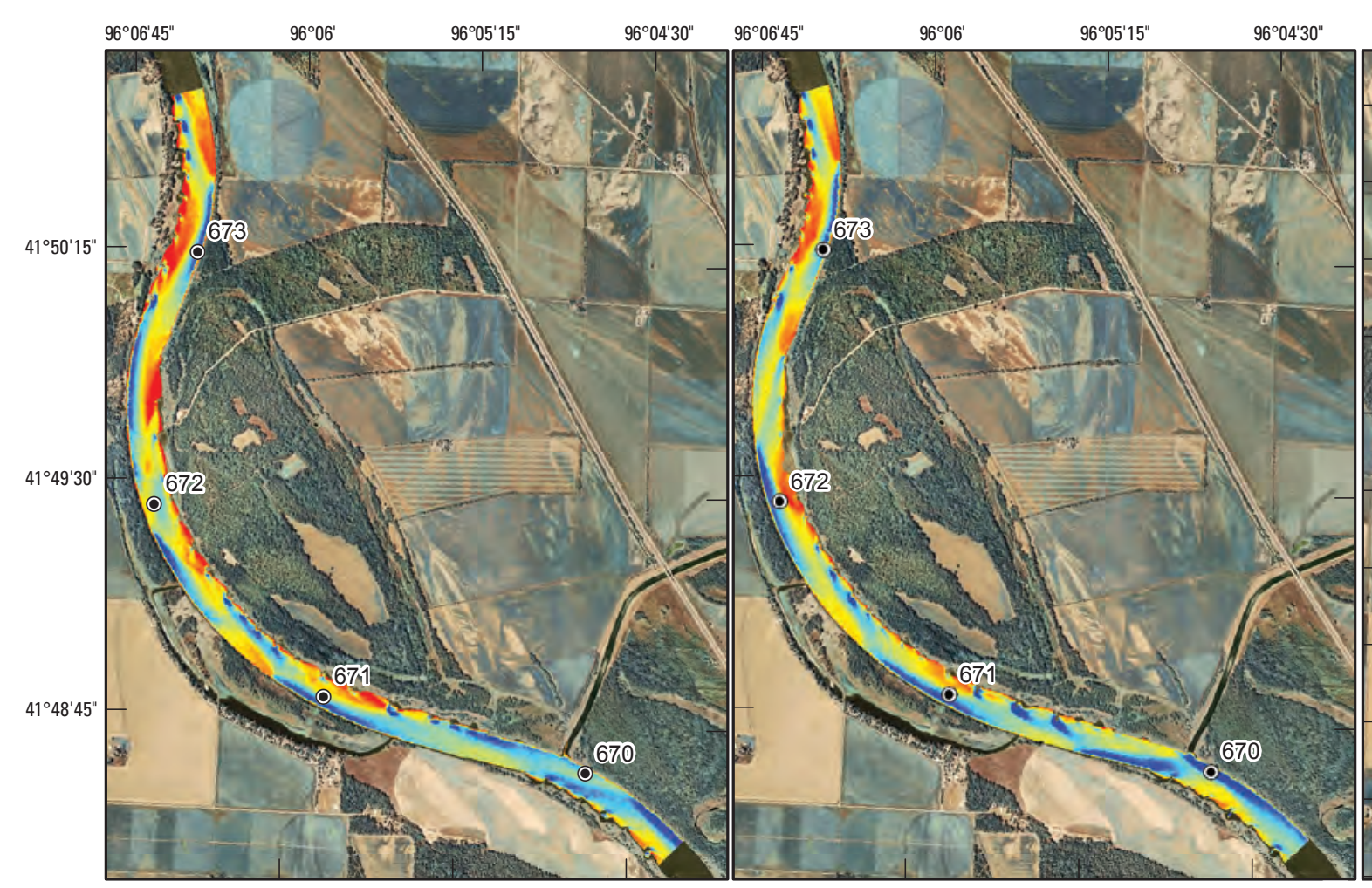

Base from U.S. Department of Agriculture National Agriculture Imagery Program, digital data, 2006 Universal Transverse Mercator projection Zone 14

\section{EXPLANATION}

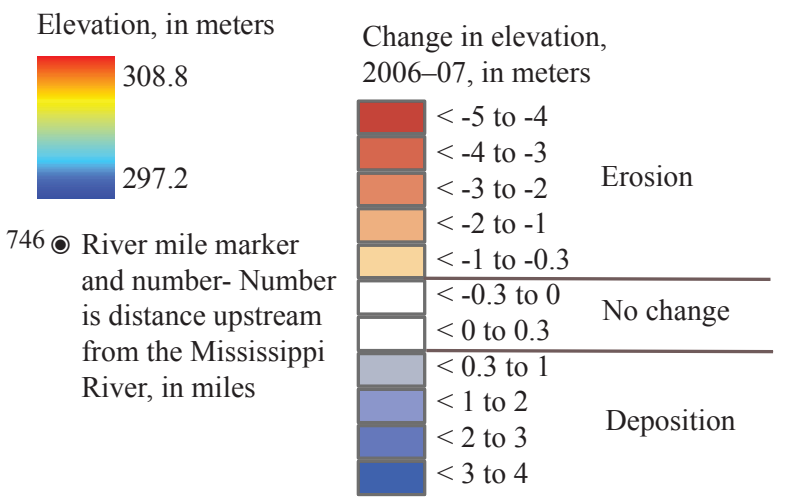

Figure 36. Changes in elevation based on 2006 and 2007 compilation surveys in the Little Sioux reach. 


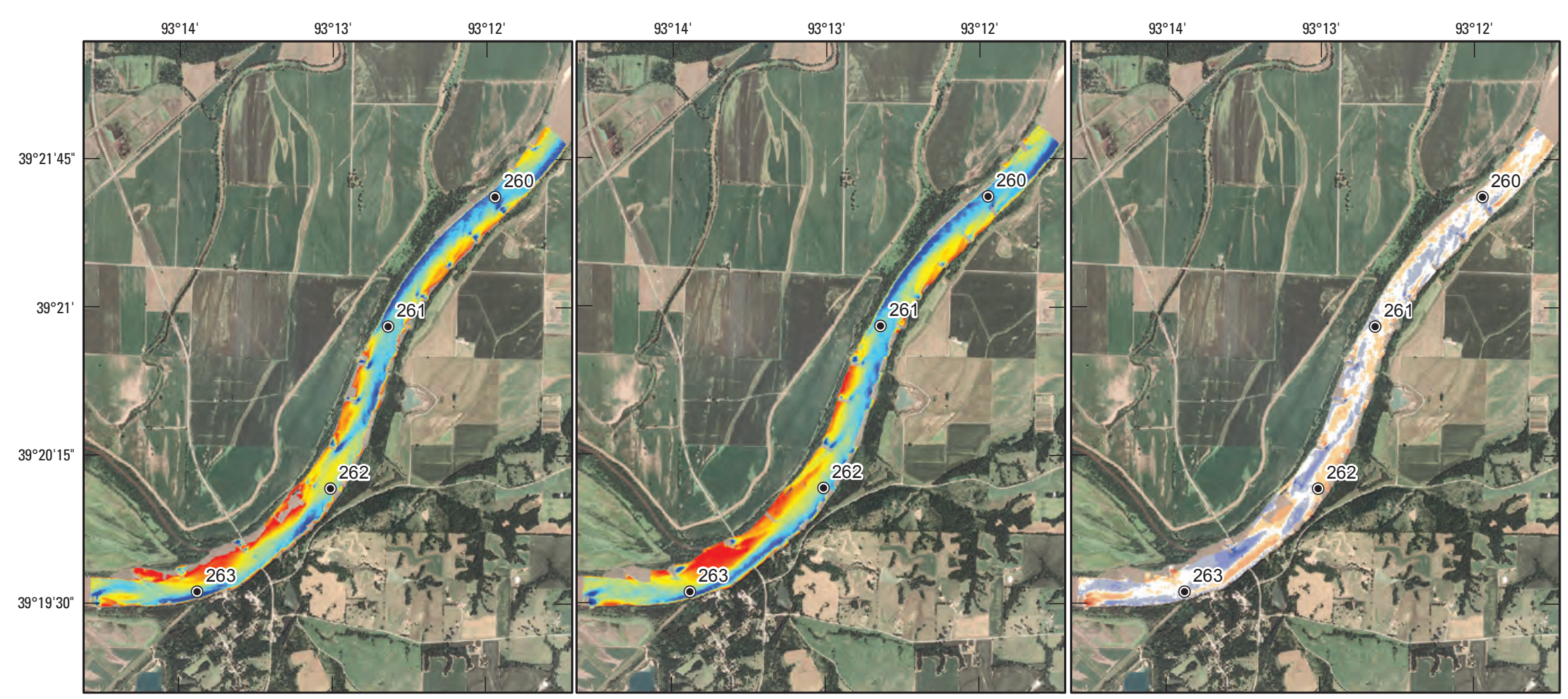

Base from U.S. Department of Agriculture National Agriculture Imagery Program, digital data, 2006

Universal Transverse Mercator projection

Zone 15

\section{EXPLANATION}

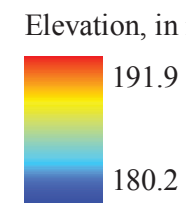

$263 \odot$ River mile marker and number- Number

is distance upstream from the Mississippi River, in miles
River miles (1960) from U.S. Army Corps of Engineers

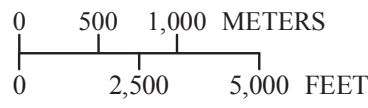

Change in elevation 2006-07, in meters

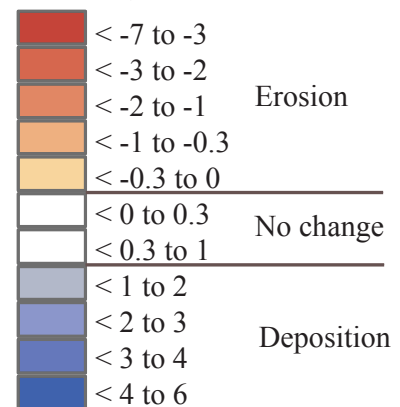

Figure 37. Changes in elevation based on 2006 and 2007 compilation surveys in the Miami reach. 

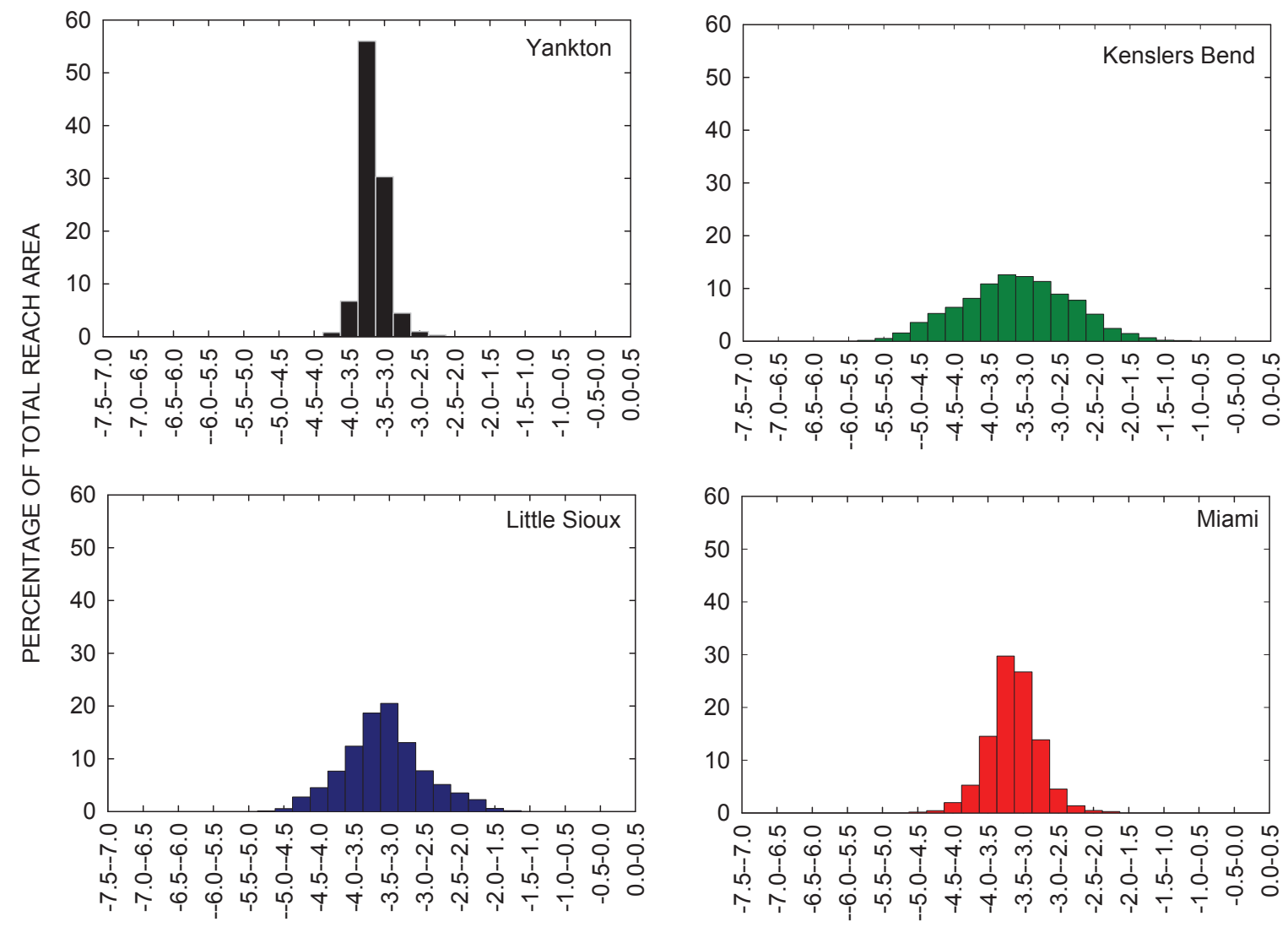

CHANGE, IN METERS

Figure 38. Changes in elevation from the 2006 to 2007 compilation surveys in the four reaches.

quantity of coarse substrate in the reach indicates that availability is probably not a limiting factor in sturgeon reproduction. In contrast, the downstream three reaches have sandbeds with hard, coarse substrate limited to areas along engineered rock structures or along bedrock banks. Flow pulses in these reaches are capable of transporting sediment and, therefore, have the potential to scour sand from hard substrate areas where hard substrate occurs at depth, although the areas would be smaller and have a patchier distribution compared to Yankton.

\section{Implications for Future Habitat Studies, Lower Missouri River}

Sediment transport and morphodynamic processes likely will continue to be important issues for restoration and management of the Lower Missouri River because they are fundamental to habitat dynamics for many species. In addition to a need to understand how flow pulses may transport sediment and alter sturgeon spawning habitats, understanding of sediment transport and morphodynamic processes is also fundamental to managing emergent sandbars and shallow, aquatic habitats.
The data and analysis presented here document that flows similar in magnitude to those under consideration for manipulated flow releases are capable of transporting sediment and altering habitats. Whether pulsed flows can "condition" spawning substrate at the right time, in the right place, and in patches of the right size for successful pallid sturgeon spawning remains unknown. Improved understanding of where and when sturgeon spawn will be necessary to address these questions. Emerging data from pallid sturgeon telemetry indicate that hatchery origin fish can spawn on riprap on revetted outside bends (A. DeLonay, unpub. data). These observations support the idea that availability of habitats with necessary substrate characteristics may not be limiting for sturgeon spawning, although whether observed spawning is optimal or successful has yet to be determined.

Understanding of sediment transport and morphodynamics also is important for restoring and managing emergent sandbar habitats and shallow, aquatic habitats. The former are important for nesting of the interior least tern and piping plover, and the latter has been assumed to be important for recruitment of sturgeon because it provides rearing habitat for larval and juvenile fish, and perhaps because it hosts aquatic insects and fishes that sturgeon prey upon (U.S. Fish and Wildlife Service, 2000b, 2003). Critical questions remain about which flows are responsible for creating and maintaining these 


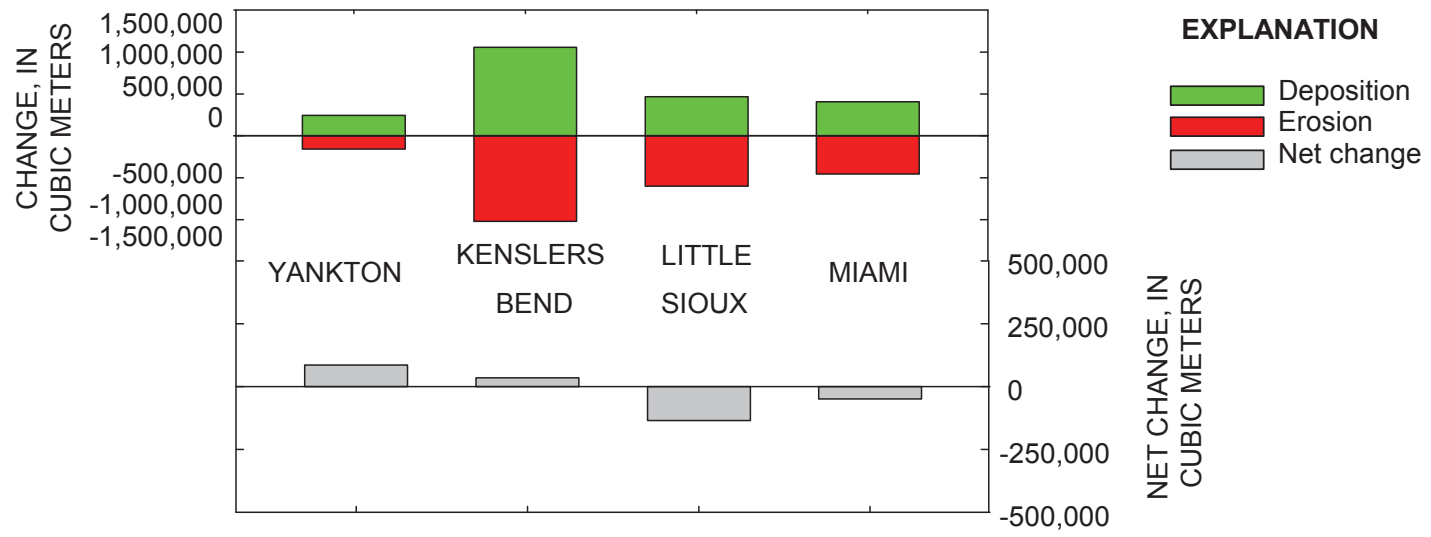

Figure 39. Areas of deposition, erosion, and net change for the four reaches from 2006 and 2007 compilation surveys.

habitats, and which flows are responsible for destroying habitat, and are effective flows manageable? Geomorphic surveys like those used in this study can be used to develop relations between flow and morphodynamic responses, but it will be necessary to use a sampling design that can isolate effects of individual flow events. The logistic requirements of bracketing surveys around individual flow events on a large, dynamic river would be challenging.

Finally, morphodynamics at the scale of sedimentary bedforms are of interest because of their role in altering flow resistance, and because bedforms define habitats at scales used by native fishes like pallid sturgeon. Improved understanding of how bedforms vary seasonally and with discharge is needed to improve parameter estimates for hydrodynamic models that serve as very useful tools in habitat assessments (Jacobson and others, 2009). Understanding of the spatial and temporal variation of bedforms in the Lower Missouri River has the potential to provide insight into flow refugia, fish migration pathways, food sources, and larval drift dynamics.

\section{Summary}

This report summarizes morphodynamic monitoring data collected in four reaches of the Lower Missouri River. The Yankton reach experienced the least amount of geomorphic change during the study period 2006-07. This is likely because of the proximity to the dam and relative coarseness of the bed. The substrate in the Yankton reach is predominately gravel with little sand on the bed. Furthermore, flows in the Yankton reach were the lowest of the four reaches. Channel morphological changes in the Yankton reach tend to be distributed uniformly, and, therefore, do not substantially alter habitats.

The Kenslers Bend and Little Sioux reaches both have a meandering thalweg within a relatively simple, engineered channel planform. Multiple bends and cross-overs occur within a bend in these reaches and sandbars appear to be migrating through them on annual timeframes. Magnitudes of deposition and erosion in both reaches are high relative to the other two reaches, yet net change is low in the Sioux reach and somewhat higher in the Kenslers Bend reach. Net change, deposition, and erosion as a proportion of index cross-section area are highest in the Kenslers Bend reach. Both reaches exhibit constricted cross sections and the Sioux reach is the narrowest and deepest of the four reaches.

The Miami reach exhibits a channel pattern that fits the channel engineering at the bend and cross-over scale. Scour and fill occurred mainly on bars or behind wing dikes in this reach. Sediment transport surrogates, including bed velocity, bedform geometry, and bedform differencing show an increasing trend with discharge with the exception of the highest discharge during the May 2007 survey when sediment supply may have been exhausted or sediment transport rates were too high for the methods used.

The time period that encompassed the pulsed-flow modification in May 2006 resulted in net erosion in three of the four reaches, although the magnitude of net erosion was low in the Yankton reaches. The Little Sioux reach had essentially no net change during the April-May time period in 2006. This was followed by a period dominated by erosion during the sustained summer high flows. From fall 2006 to March 2007 the general trend among sites was deposition. The spring 2007 trends are difficult to link to individual flow events because of timing of surveys in relation to high spring flows out of upstream tributaries such as the James, Vermillion, and Big Sioux Rivers; and the sustained high flow in the spring in the Miami reach.

The monitoring data indicate that flow pulses of the scale considered in design of the flow pulses (spring rises) on the Lower Missouri River are capable of transporting sediment in all four reaches, and may, therefore, have the potential to flush fine sediment from spawning substrate where hard substrate occurs at depth. Coarse, hard substrate thought to be required for spawning of sturgeon is abundant in the Yankton reach and may be altered by flow pulses, but because of its abundance, 
clean patches may not be a constraint on spawning. In the downstream three reaches, our data show that flow pulses are capable of transporting sandbed material, but it is not clear if transport would result in creating patches of sufficient size and quality to benefit pallid sturgeon reproduction.

\section{Acknowledgments}

We would like to thank the USGS Nebraska and Iowa Water Science Centers for data collection in the Yankton and Kenslers Bend reaches, specifically Michael Anderson and Brenda Woodward. Hal Johnson, of USGS CERC, and Matt Smith, and Chad Vishy of Arctic Slope Resources Corporation provided technical assistance in data collection and some data processing.

\section{References Cited}

Buckley, J., and Kynard, B., 1981, Spawning and rearing of shortnosed sturgeon from the Connecticut River: Progressive Fish Culturist, v. 43, no. 2, p. 74-76.

Elliott, C.M., and Jacobson, R.B., 2006, Geomorphic classification and assessment of channel dynamics in the Missouri National Recreational River, South Dakota and Nebraska: U.S. Geological Survey Scientific Investigations Report 2006-5313, 66 p.

Elliott, C.M., Jacobson, R.B., and DeLonay, A.J., 2004, Physical aquatic habitat assessment, Fort Randall segment of the Missouri River, Nebraska and South Dakota: U.S. Geological Survey Open-File Report 2004-1060, 34 p.

Funk, J.L., and Robinson, J.W., 1974, Changes in the channel of the lower Missouri River and effects on fish and wildlife: Jefferson City, Mo., Missouri Department of Conservation.

Gaeuman, D., and Jacobson, R.B., 2005, Aquatic habitat mapping with an acoustic Doppler current profiler-considerations for data quality: U.S. Geological Survey Open-File Report 2005-1163, 20 p.

Gaeuman, D., and Jacobson, R.B., 2006, Acoustic bed velocity and bed load dynamics in a large sand bed river: Journal of Geophysical Research-Earth Surface, v. 111, p. 1-14.

Gaeuman, D., and Jacobson, R.B., 2007, Field assessment of alternative bedload transport estimators: Journal of Hydraulic Engineering, v. 133, no. 12, p. 1319-1328.

Galat, D.L., Berry, C.R., Jr., Peters, E.J., and White, R.G., 2005, Missouri River basin, in Benke, A.C., and Cushing, C.E., eds., Rivers of North America: Oxford, Elsevier Academic Press, p. 427-480.
Galat, D.L., and Lipkin, R., 2000, Restoring ecological integrity of great rivers - Historical hydrographs aid in defining reference conditions for the Missouri River: Hydrobiologia, v. $422 / 423$, p. $29-48$.

Hesse, L.W., 1987, Taming the wild Missouri River-What has it cost?: Fisheries, v. 12, no. 22, p. 2-9.

Hesse, L.W., Schmulback, J.C., Carr, J.M., Keenlyne, K.D., Unkenholz, D.G., Robinson, J.W., and Mestl, G.E., eds., 1989, Missouri River fishery resources in relation to past, present, and future stresses, Canadian Special Publication of Fisheries and Aquatic Sciences, 353-371 p.

Hesse, L.W., and Sheets, W., 1993, The Missouri River hydrosystem: Fisheries, v. 18, no. 5, p. 5-14.

Jacobson, R.B., 2008, Analysis of pulsed flow modification alternatives, Lower Missouri River, 2005: U.S. Geological Survey Open-File Report 2008-1113, 14 p.

Jacobson, R.B., Blevins, D.W., and Bitner, C.J., 2009a, Sediment regime constraints on river restoration-An example from the Lower Missouri River, in James, L.A., Rathburn, S.L., and Whittecar, G.R., eds., Management and Restoration of Fluvial Systems with Broad Historical Changes and Human Impacts: Denver, Colo., Geological Society of America, v. 451, p. 1-22.

Jacobson, R.B., and Galat, D.L., 2006, Flow and form in rehabilitation of large-river ecosystems-An example from the Lower Missouri River: Geomorphology, v. 77, p. 249-269.

Jacobson, R.B., and Galat, D.L., 2008, Design of a naturalized flow regime on the Lower Missouri River: Ecohydrology, v. 1, no. 2, p. 80-104.

Jacobson, R.B., and Heuser, J., 2002, Visualization of flow alternatives, Lower Missouri River: U.S. Geological Survey Open-File Report 2002-122, no pagination

Jacobson, R.B., Johnson, H.E. III, and Dietsch, B.J., 2009b, Hydrodynamic simulations of physical aquatic habitat availability for pallid sturgeon in the Lower Missouri River, at Yankton, South Dakota, Kenslers Bend, Nebraska, Little Sioux, Iowa, and Miami, Missouri 2006-07: U.S. Geological Survey Scientific Investigations Report 2009-5058, $68 \mathrm{p}$.

Laustrup, M.S., Jacobson, R.B., and Simpkins, D.G., 2007, Distribution of potential spawning habitat for sturgeon in the Lower Missouri River, 2003-06: U.S. Geological Survey Open-File Report 2007-1192, 26 p.

Leopold, L.B., and Maddock, T., 1953, The hydraulic geometry of stream channels and some physiographic implications: U.S. Geological Survey Professional Paper 23257 p. 
McElroy, B., 2006, The calculator for topographic characteristics and sediment transport from sand bed surveys, The Stream Restoration Toolbox (1.1 ed.): Minneapolis, Minn., National Center for Earth-surface Dynamics.

National Research Council, 2002, The Missouri River ecosystem - exploring the prospects for recovery: Washington, D.C., National Academy Press, 176 p.

Nittrouer, J.A., Allison, M.A., and Campanella, R., 2008, Bedform transport rates for the lower most Mississippi River: Journal of Geophysical Research-Earth Surface, v. 113.

Paragamian, V.L., Kruse, G., and Wakkinen, V., 2001, Spawning habitat of Kootenai River white sturgeon, post-Libby Dam: North American Journal of Fisheries Management, v. 21, p. 22-33.

Pegg, M.A., Pierce, C.L., and Roy, A., 2003, Hydrological alteration along the Missouri River Basin-A time series approach: Aquatic Sciences, v. 65, p. 63-72.

Pflieger, W.L., and Grace, T.B., 1987, Changes in the fish fauna of the lower Missouri River, 1940-1983, in Matthews, W.J., and Heins, D.C., eds., Community and Evolutionary Ecology of North American Stream Fishes: Norman, Okla., University of Oklahoma Press, p. 166-177.

Rennie, C.D., and Millar, R.G., 2004, Measurement of the spatial distribution of fluvial bedload transport velocity in both sand and gravel: Earth Surface Processes and Landforms, v. 29, p. 1173-1193.

Rennie, C.D., Millar, R.G., and Church, M.A., 2002, Measurement of bedload velocity using an acoustic Doppler current profiler: Journal of Hydraulic Engineering-Asce, v. 128, p. 473-483.

Reuter, J.M., Jacobson, R.B., Elliott, C.M., Johnson, H.E., and DeLonay, A.J., 2008, Hydraulic and substrate maps of reaches used by sturgeon (genus Scaphirhynchus) in the Lower Missouri River, 2005-07: U.S. Geological Survey Data Series 386, 422 p.

Simons, D.B., Li, R.M., and Schall, J.D., 1980, Report regarding the safe yield of sand and gravel from the Russian River -Dry Creek System, Supplement to Evaluation Report: Aggregate Resources Management Study: Draft Environmental Impact Report
Simons, D.B., Richardson, E.V., and F., N.C., 1965, Bedload equation for ripples and dunes: U.S. Geological Survey Professional Paper 9 p.

U.S. Army Corps of Engineers, 2004, Summary Missouri River final environmental impact statement - master water Control Manual review and update: Portland, Oregon: U.S. Army Corps of Engineers 28 p.

U.S. Army Corps of Engineers, 2006a, Missouri River Mainstem system - 2005-2006 annual operating plan: U.S. Army Corps of Engineers Northwestern Division 28 p.

U.S. Army Corps of Engineers, 2006b, Missouri River Mainstem System 2006-2007 Annual Operating Plan: 89 p.

U.S. Army Corps of Engineers, 2007a, Missouri River Mainstem Reservoir System Summary of Actual 2006 Regulation Missouri River Basin: U.S. Army Corps of Engineers 77 p.

U.S. Army Corps of Engineers, 2007b, Missouri River stage trends: U.S. Army Corps of Engineers, Northwestern Division $43 \mathrm{p}$.

U.S. Fish and Wildlife Service, 2000a, Biological opinion on the operation of the Missouri River main stem reservoir system, operation and maintenance of the Missouri River Bank Stabilization and Navigation Project, and operation of the Kansas River Reservoir System: U.S. Fish and Wildlife Service., 296 p. http://www.nwd-mr.usace.army.mil/mmanual/opinion.html

U.S. Fish and Wildlife Service, 2000b, Biological opinion on the operation of the Missouri River main stem Reservoir system, operation and maintenance of the Missouri River bank stabilization and navigation project, and operation of the Kansas River reservoir system: U.S. Fish and Wildlife Service, $286 \mathrm{p}$.

U.S. Fish and Wildlife Service, 2003, Amendment to the 2000 biological opinion on the operation of the Missouri River main stem reservoir system, operation and maintenance of the Missouri River Bank Stabilization and Navigation Project, and operation of the Kansas River Reservoir System: U.S. Fish and Wildlife Service., 308 p. 

Appendix 1. Yankton Reach Cross Sections 


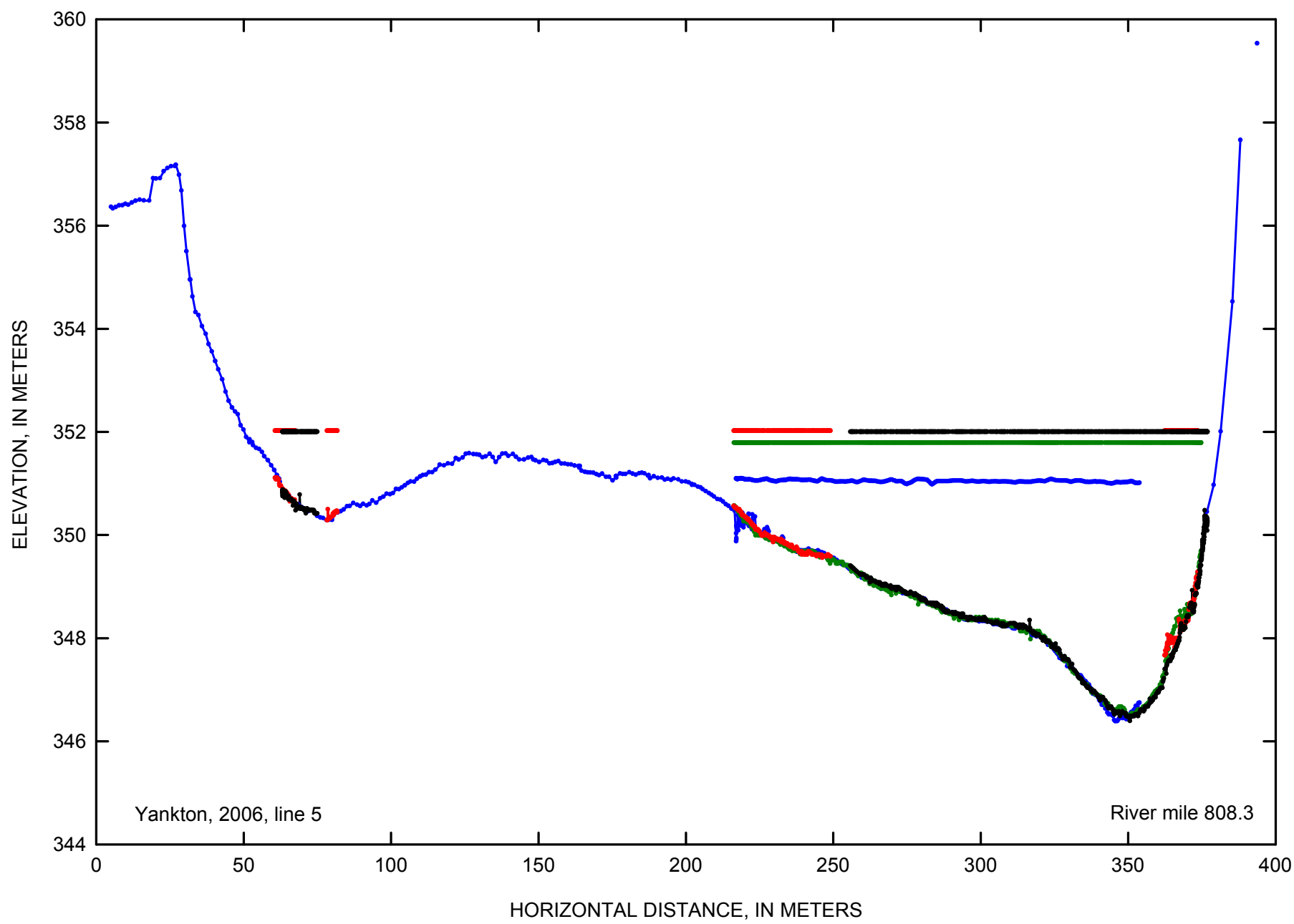

EXPLANATION

March 2006

April 2006

M May 2006
J June 2006

Figure 1-1. Yankton cross-section line 5 at Missouri River mile 808.3 in 2006. 


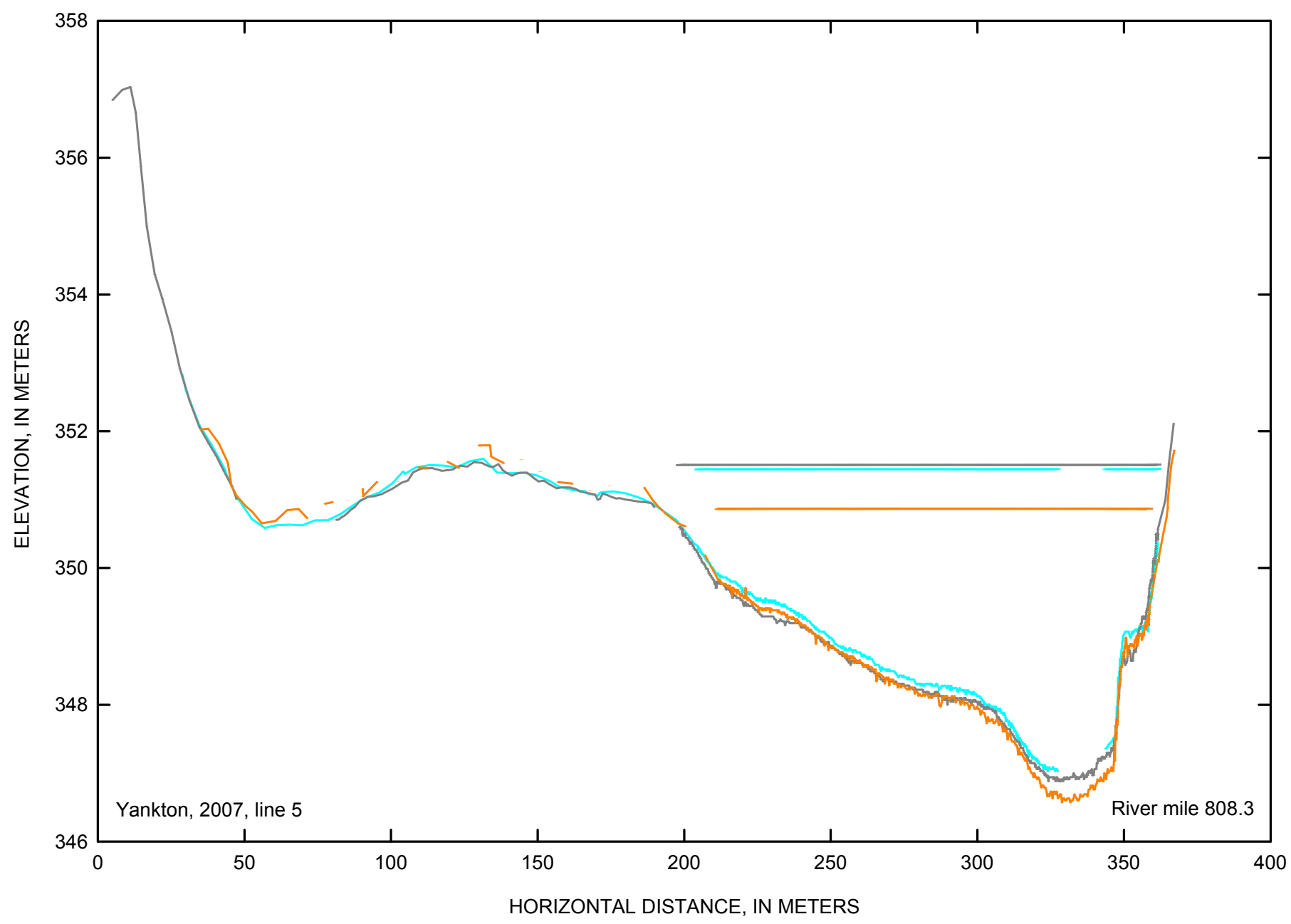

\section{EXPLANATION}

March 2007

June 2007

November 2007

Figure 1-2. Yankton cross-section line 5 at Missouri River mile 808.3 in 2007. 


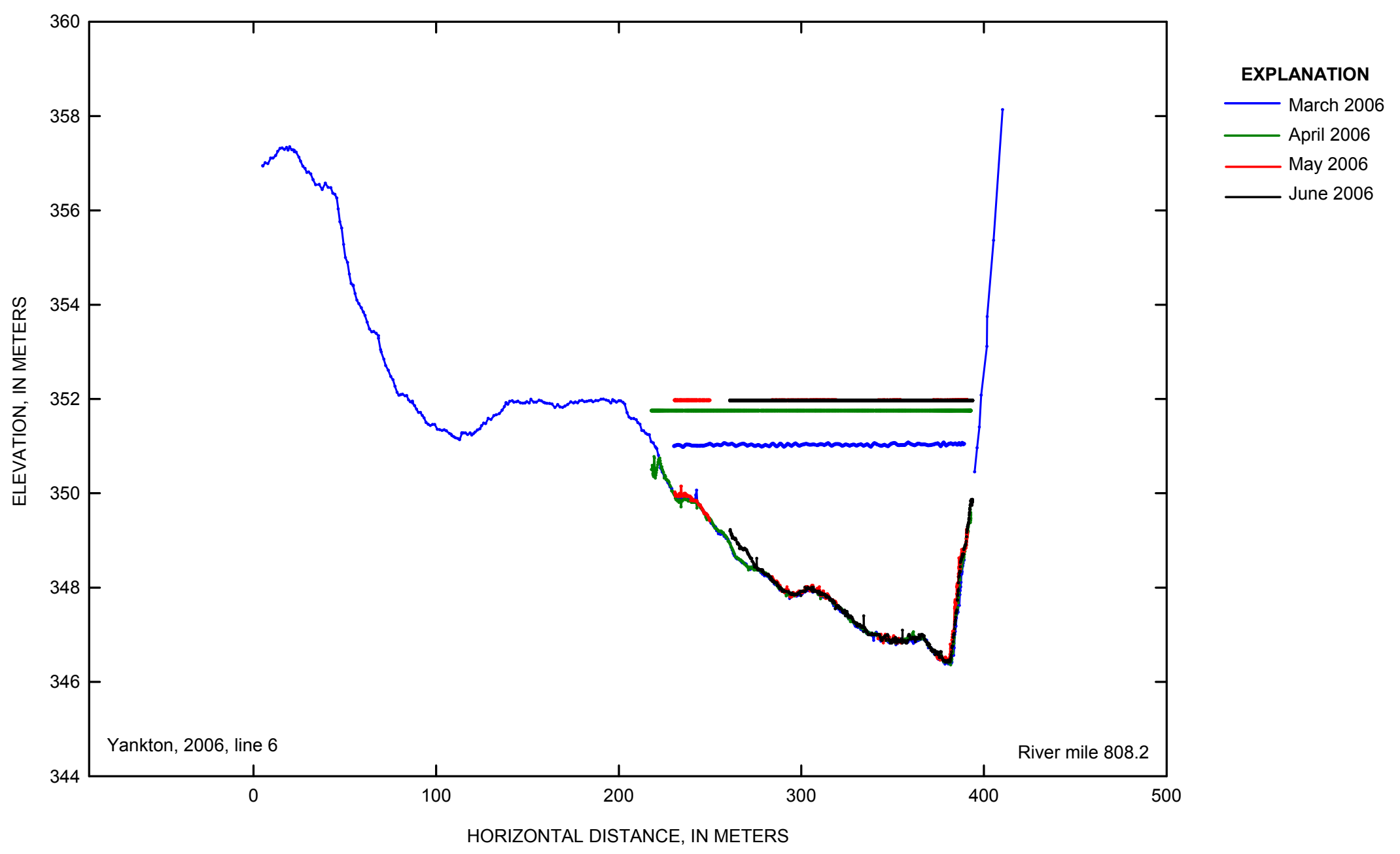

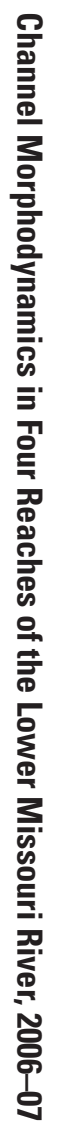

Figure 1-3. Yankton cross-section line 6 at Missouri River mile 808.2 in 2006. 


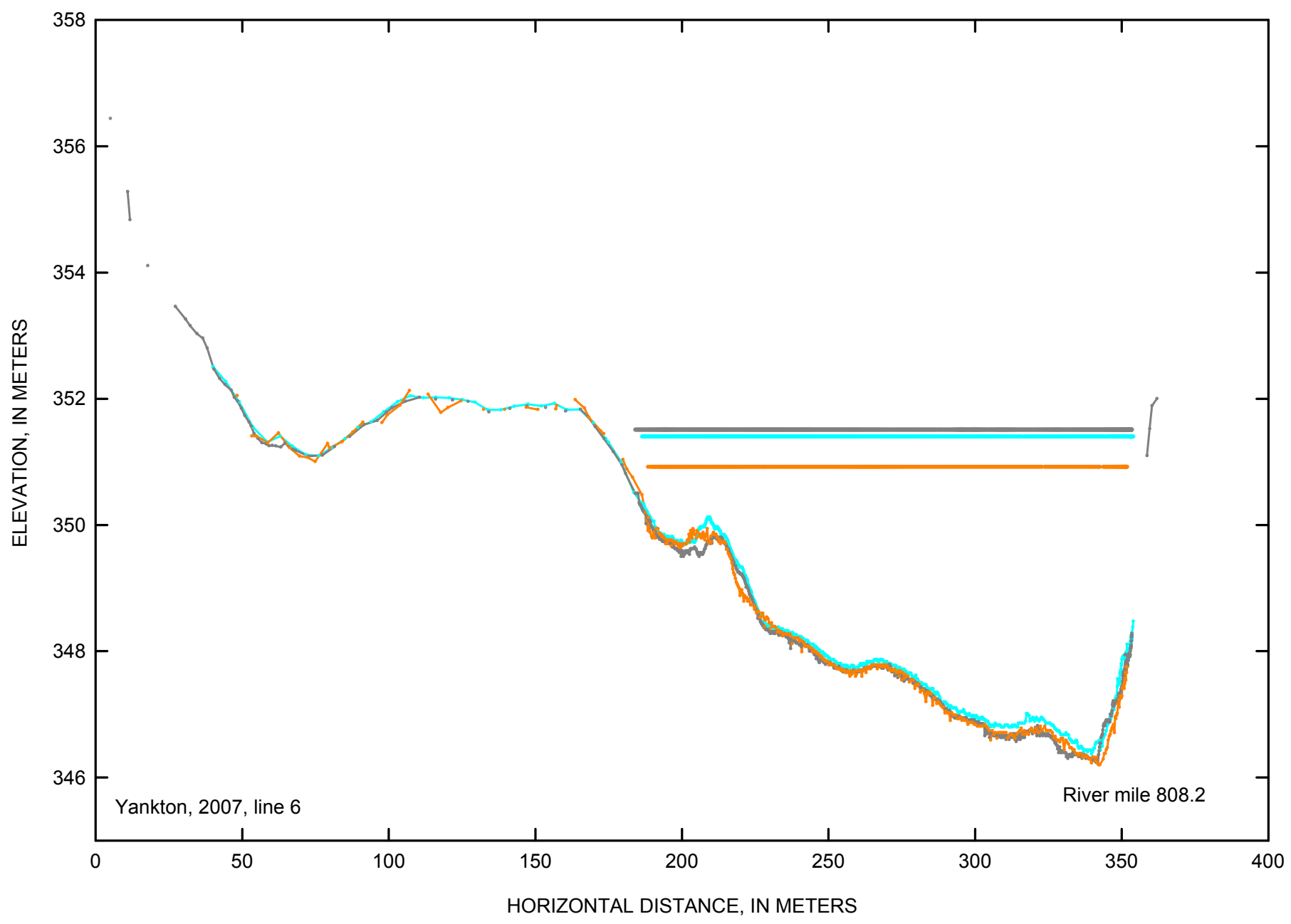

\section{EXPLANATION}

March 2007

June 2007

November 2007

Figure 1-4. Yankton cross-section line 6 at Missouri River mile 808.2 in 2007. 


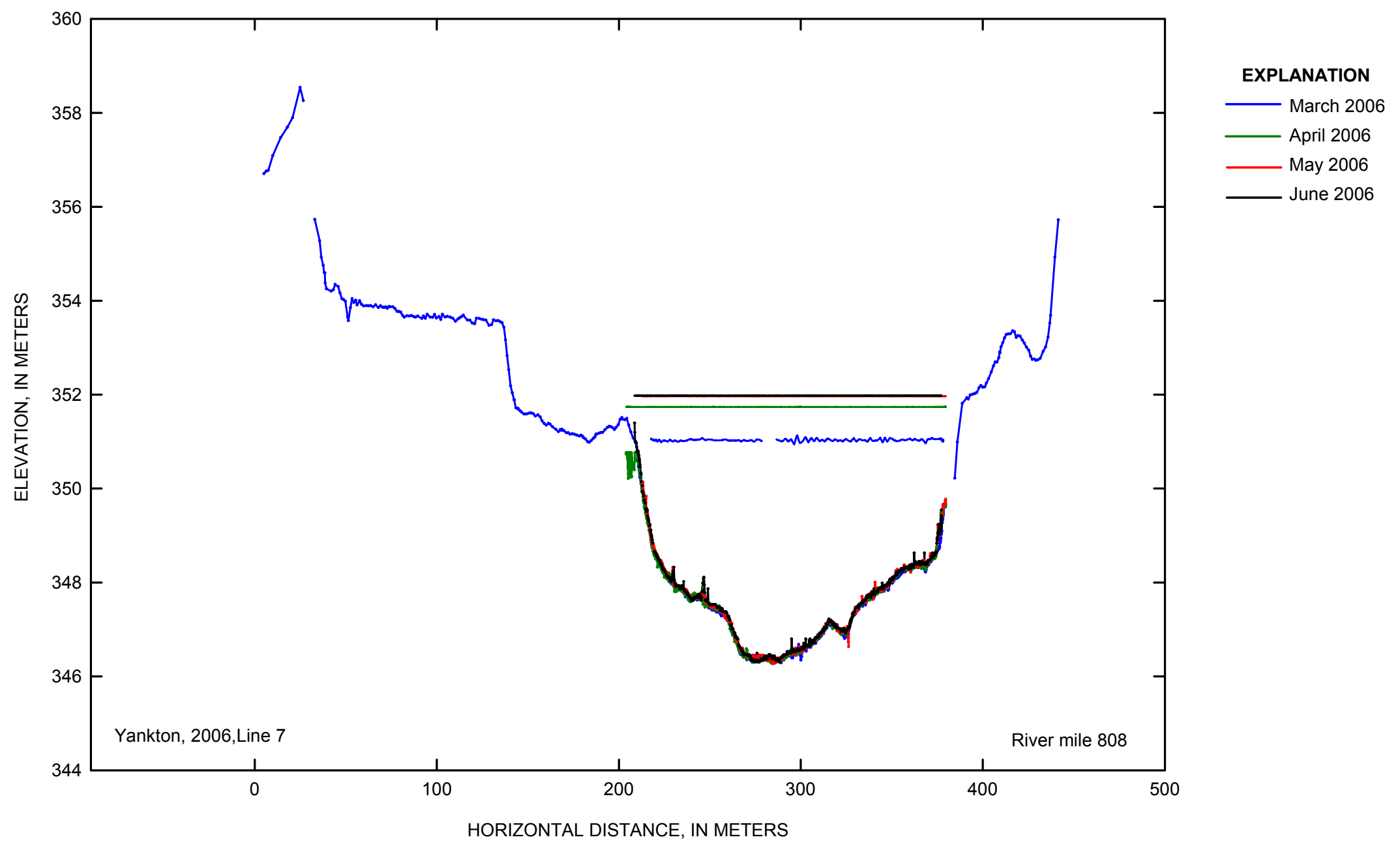

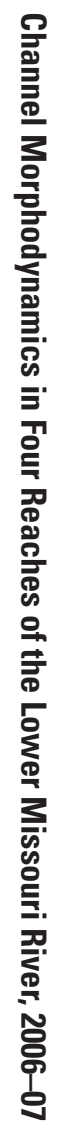

Figure 1-5. Yankton cross-section line 7 at Missouri River mile 808 in 2006. 


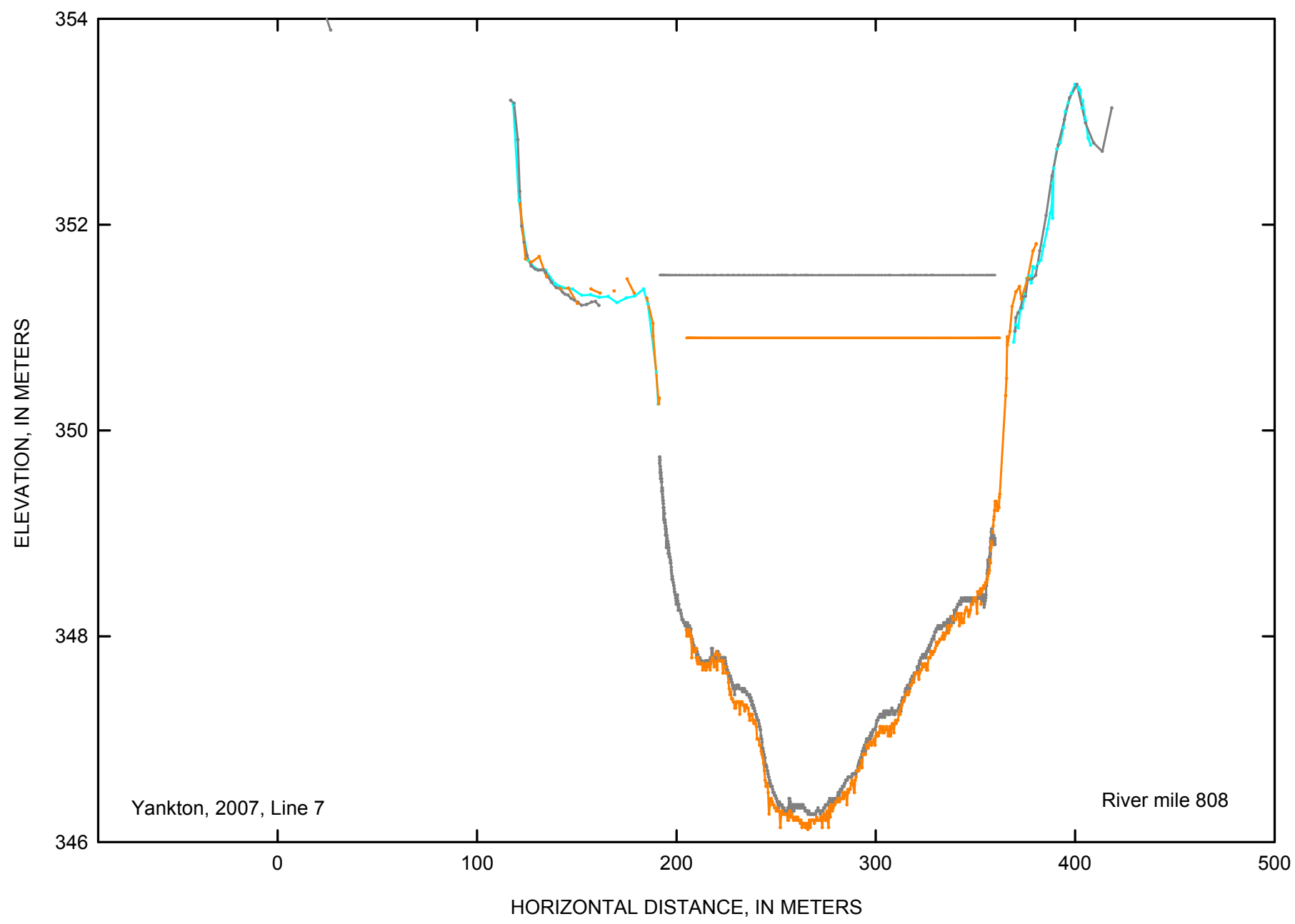

EXPLANATION

Figure 1-6. Yankton cross-section line 7 at Missouri River mile 808 in 2007. 


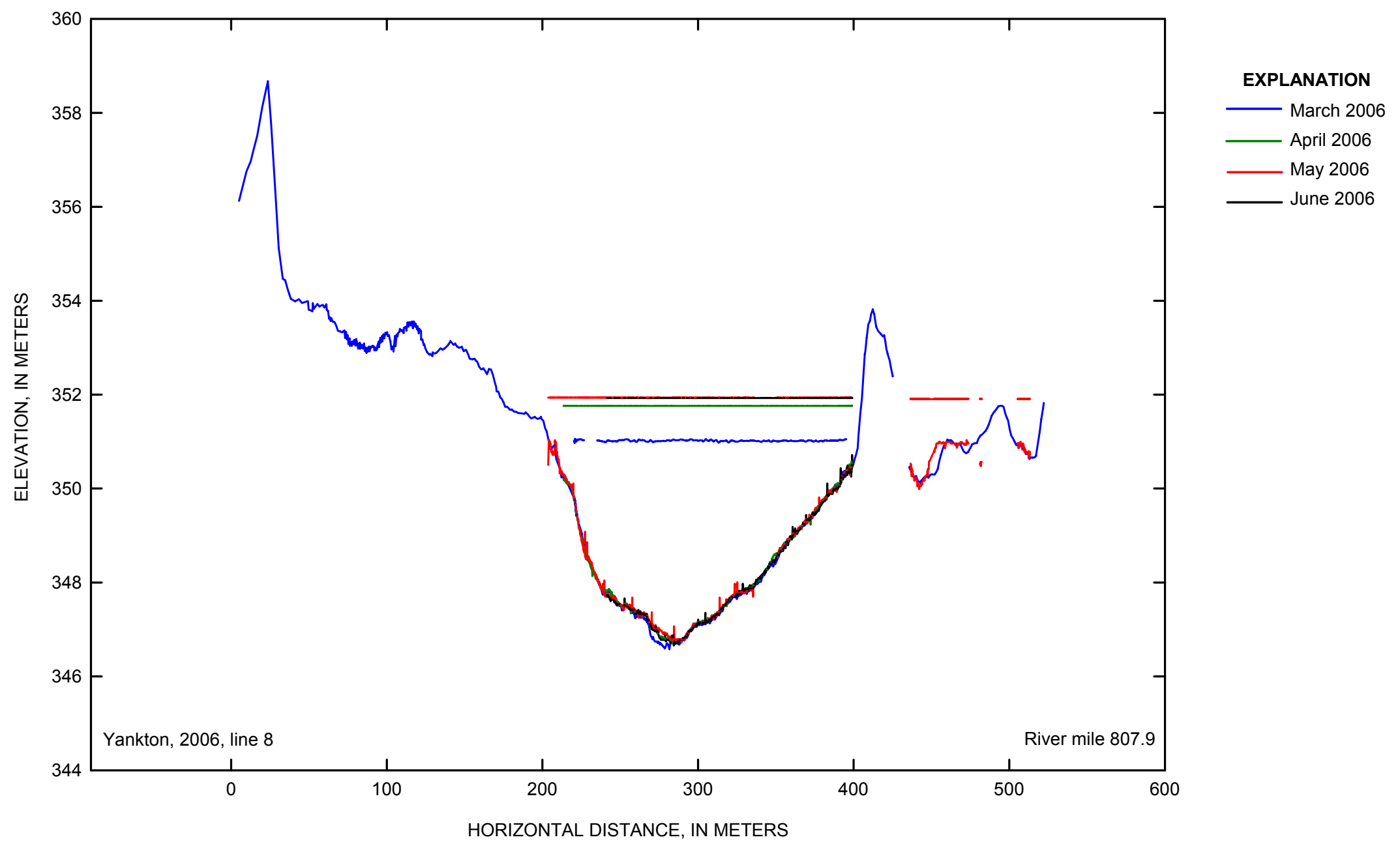

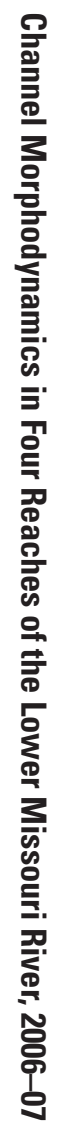

Figure 1-7. Yankton cross-section line 8 at Missouri River mile 807.9 in 2006. 


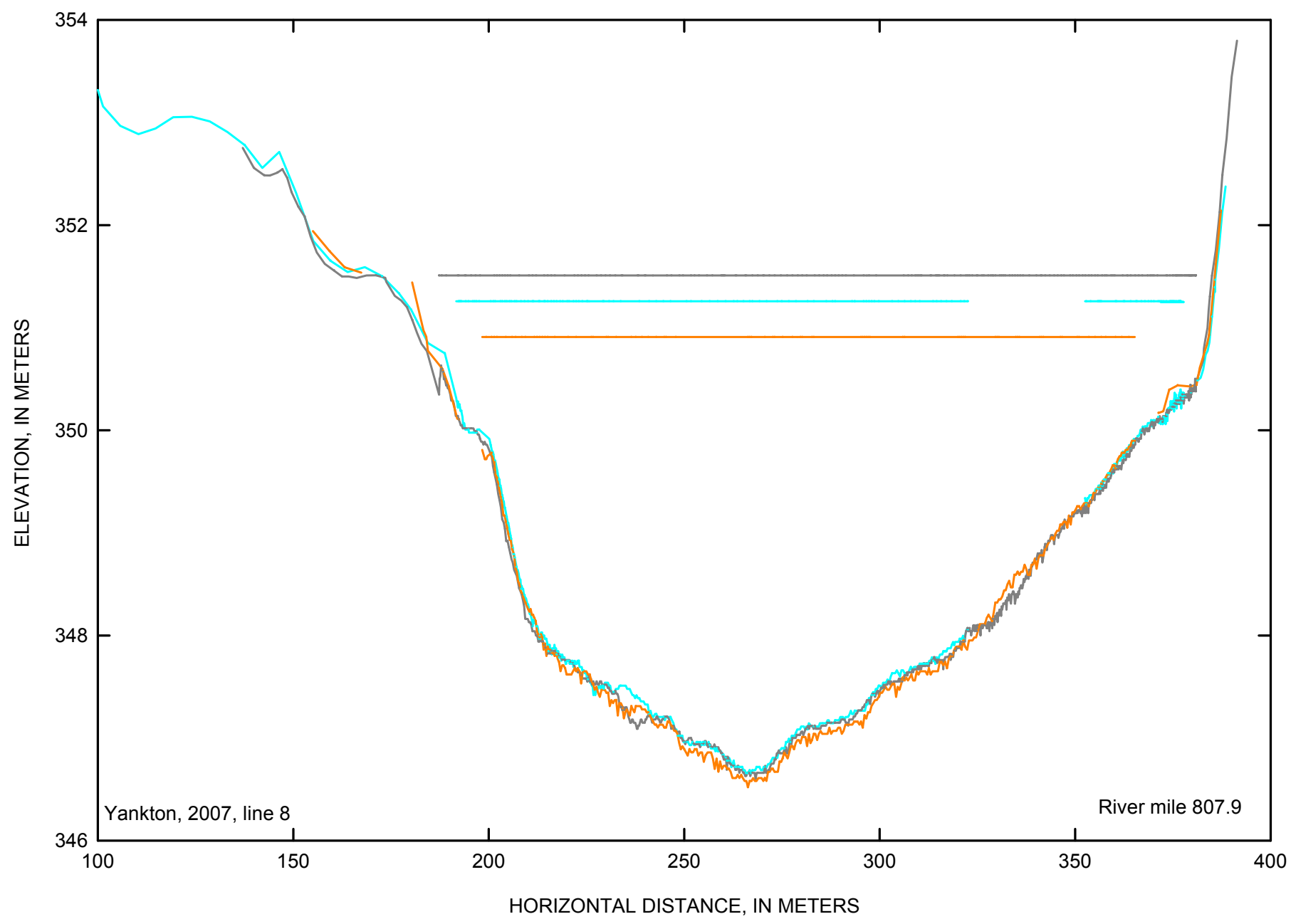

EXPLANATION

- March 2007

June 2007

November 2007

Figure 1-8. Yankton cross-section line 8 at Missouri River mile 807.9 in 2007. 


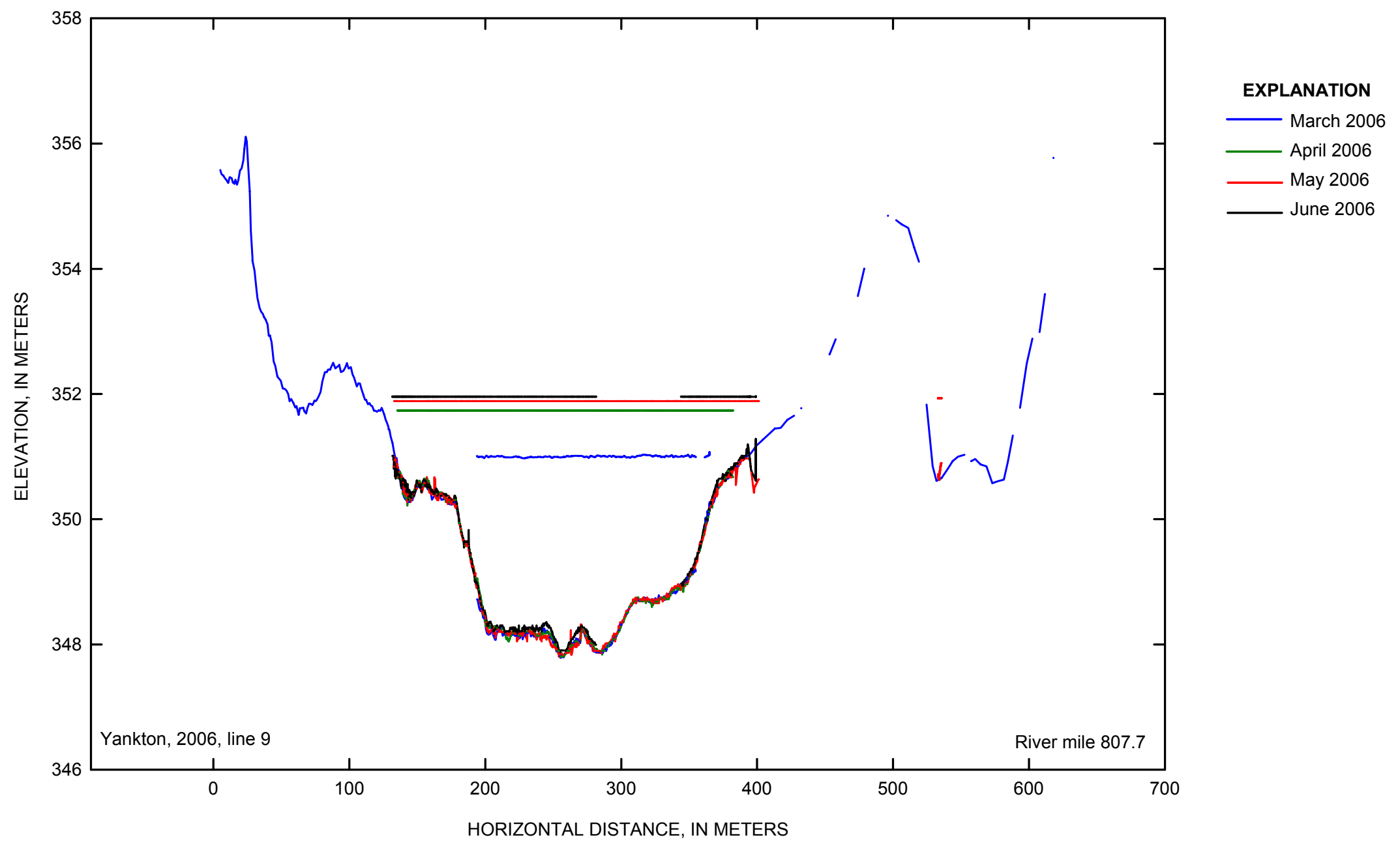

Figure 1-9. Yankton cross-section line 9 at Missouri River mile 807.7 in 2006. 


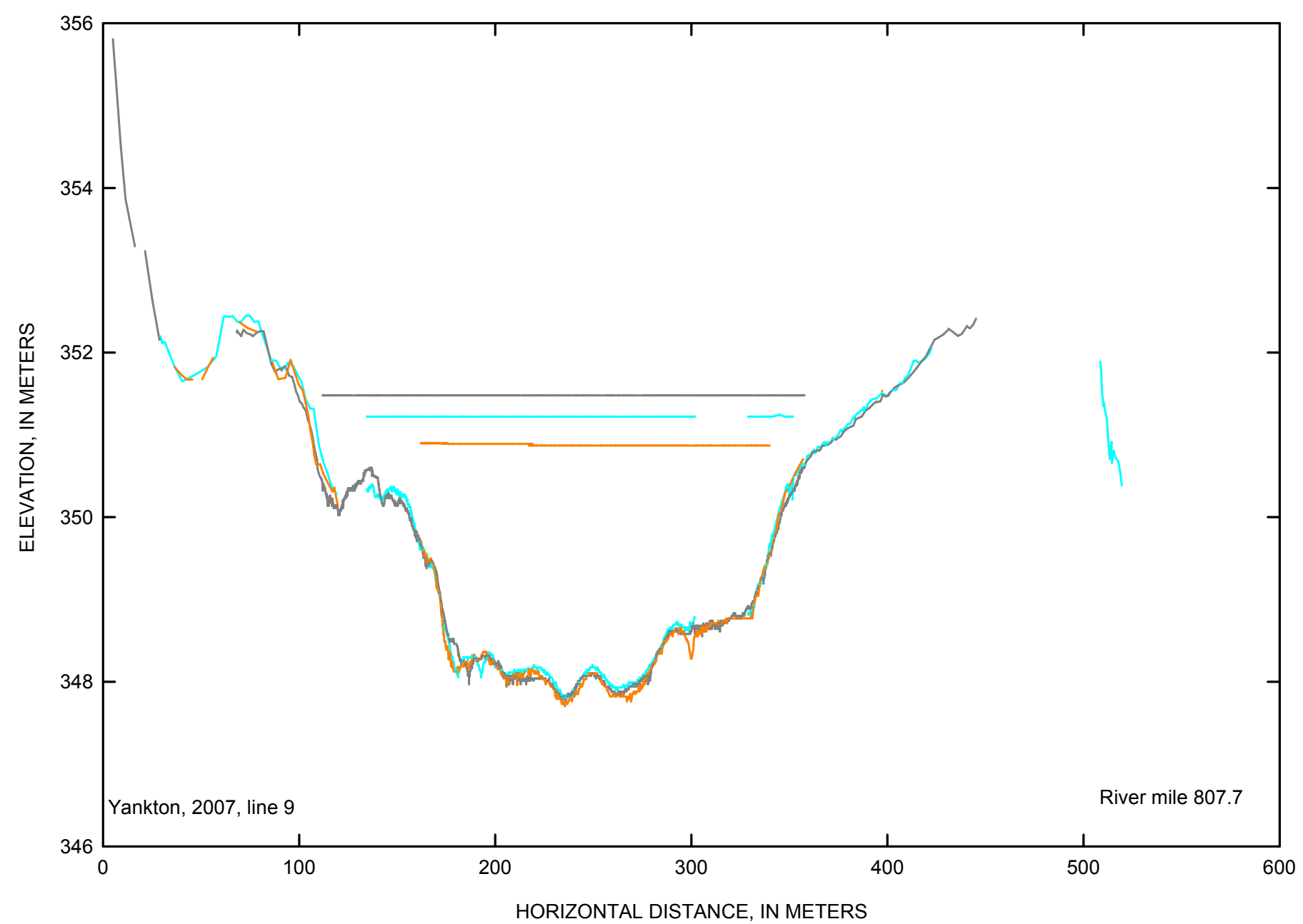

EXPLANATION

March 2007

June 2007

November 2007

Figure 1-10. Yankton cross-section line 9 at Missouri River mile 807.7 in 2007. 


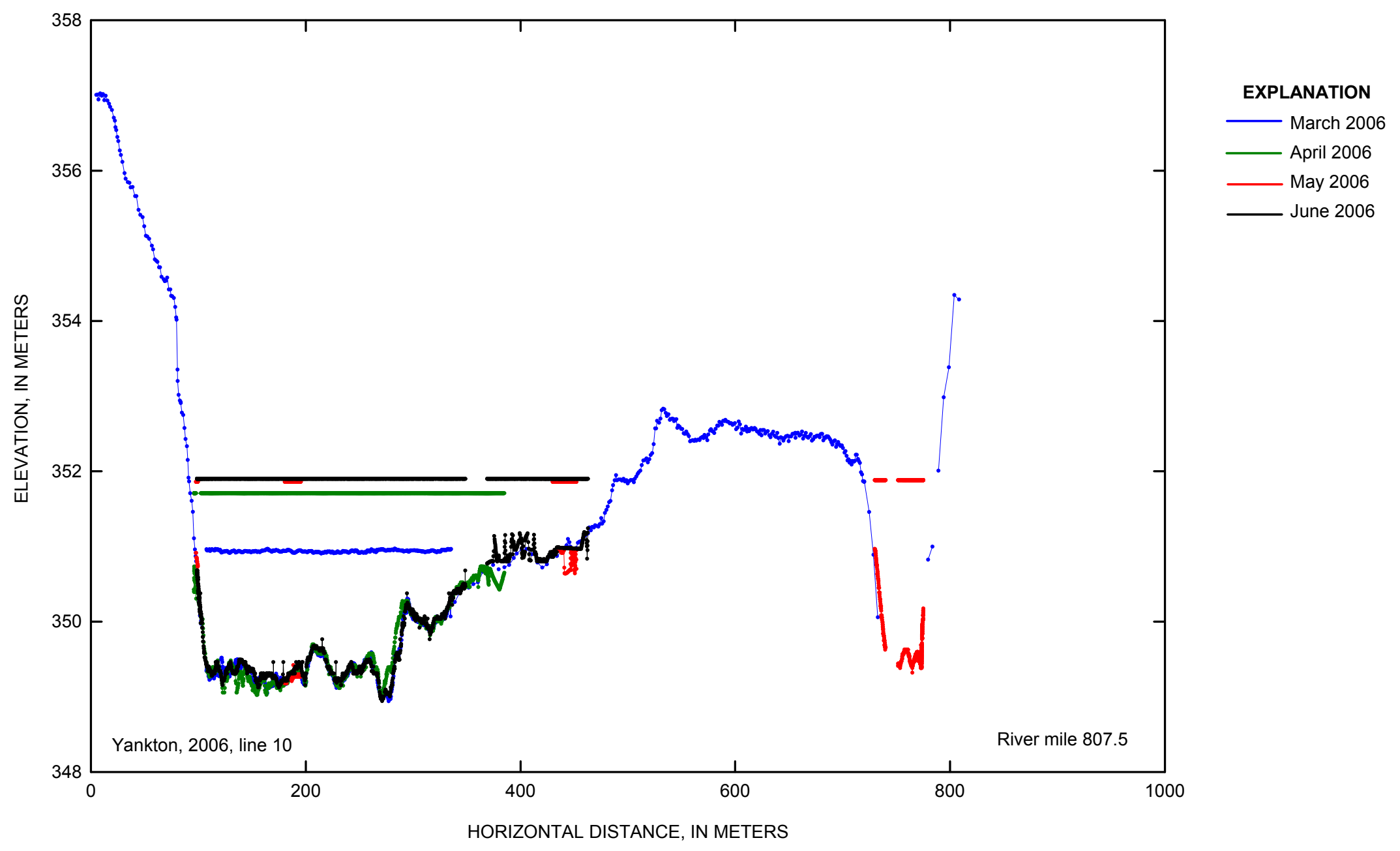

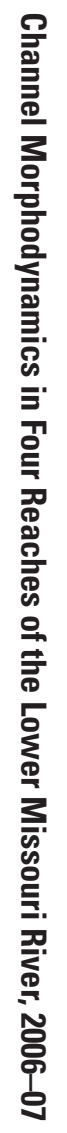

Figure 1-11. Yankton cross-section line 10 at Missouri River mile 807.5 in 2006. 


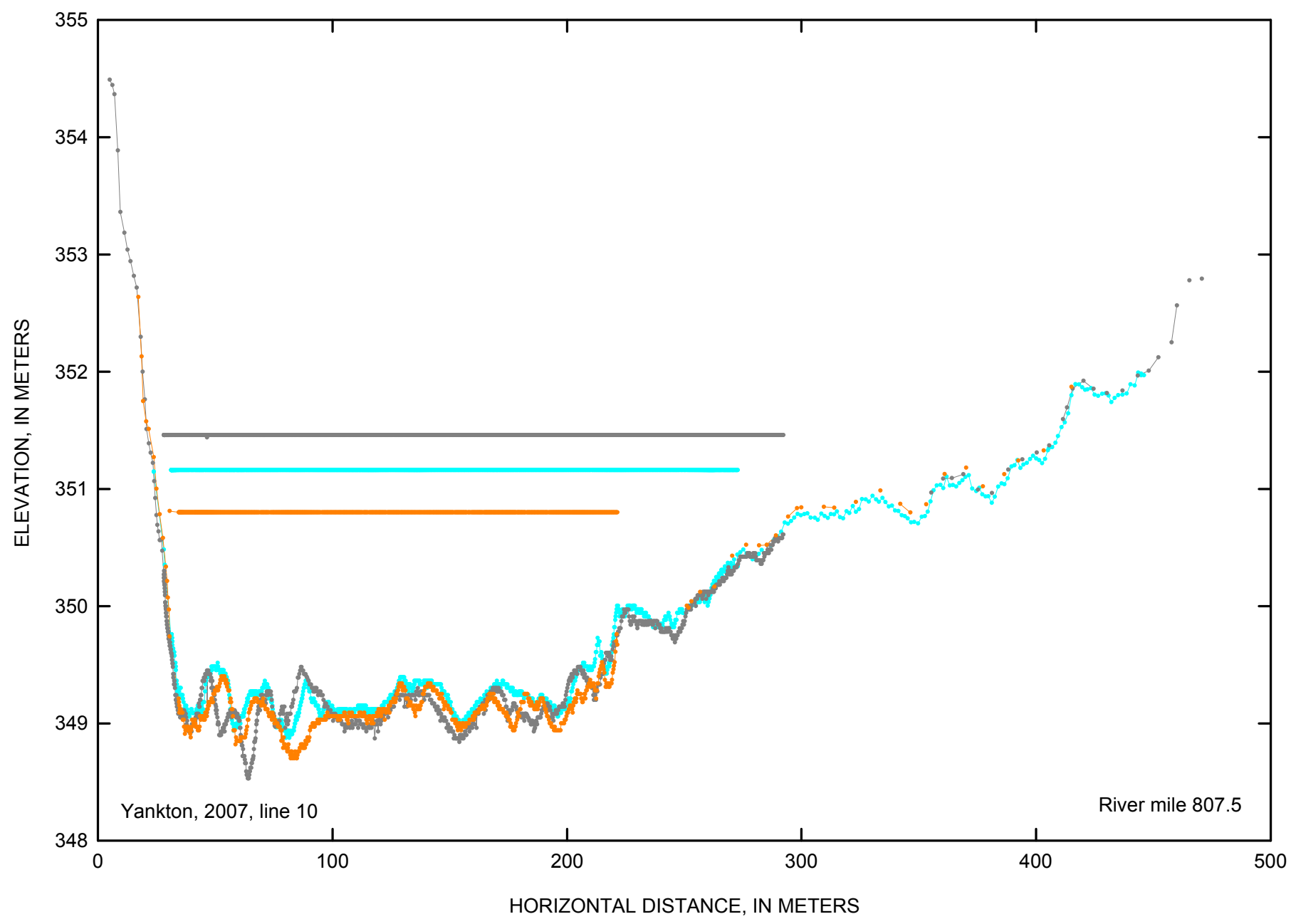

EXPLANATION

March 2007

June 2007

- November 2007

Figure 1-12. Yankton cross-section line 10 at Missouri River mile 807.5 in 2007. 


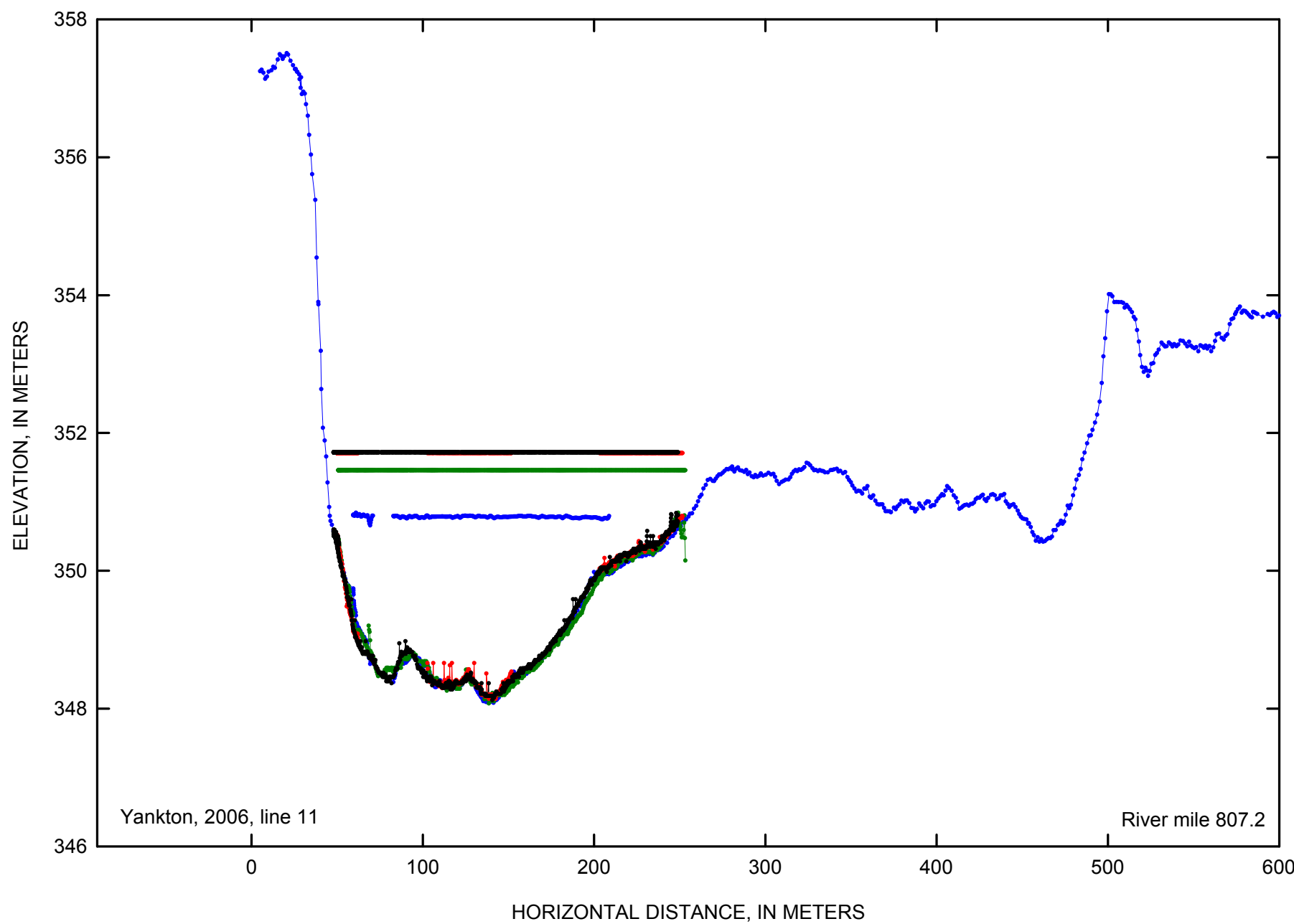

EXPLANATION

March 2006

April 2006

May 2006

June 2006

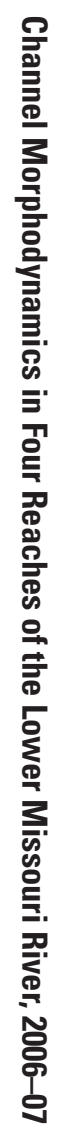

Figure 1-13. Yankton cross-section line 11 at Missouri River mile 807.2 in 2006. 


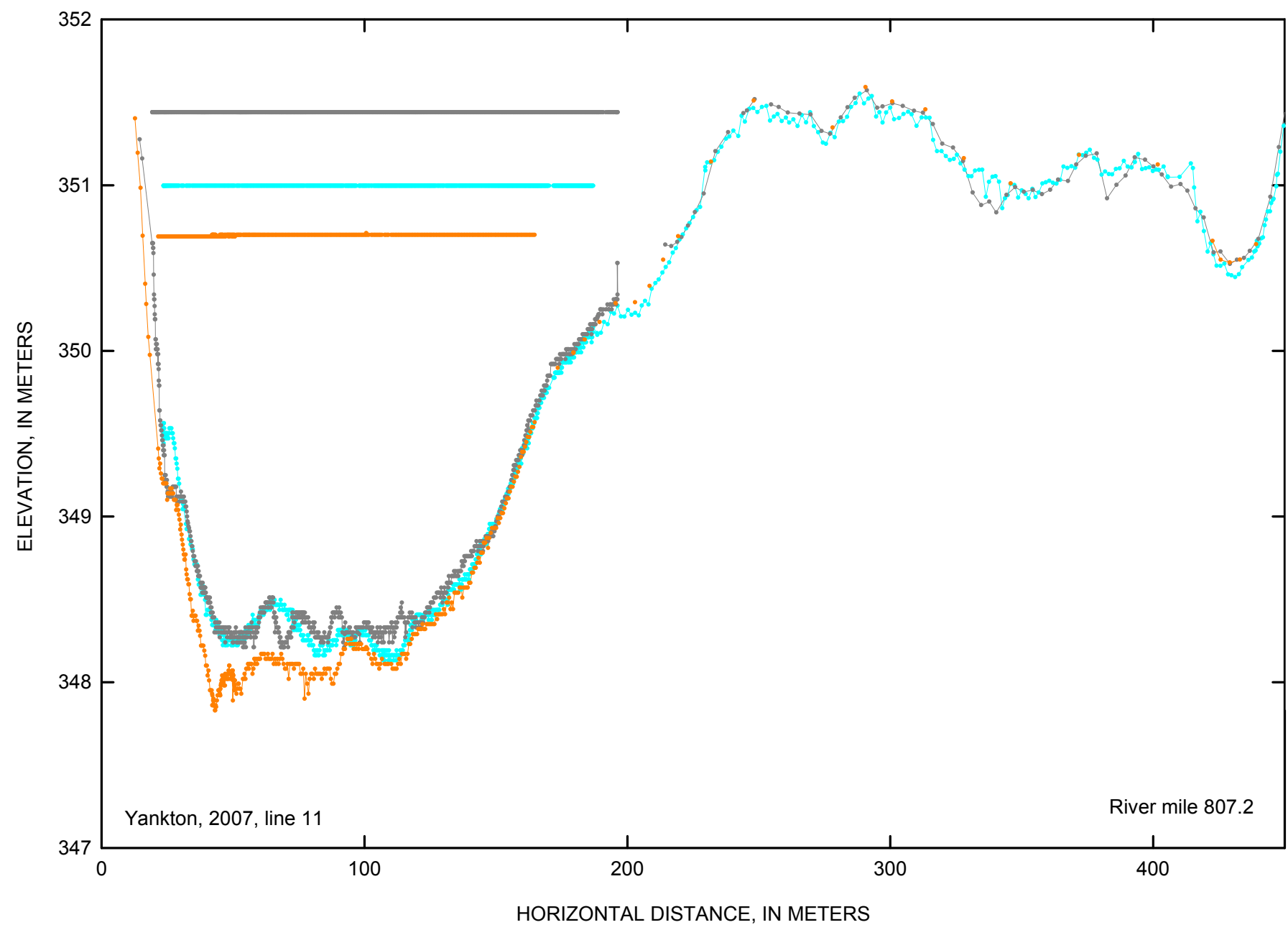

\section{EXPLANATION}

March 2007

June 2007

November 2007 


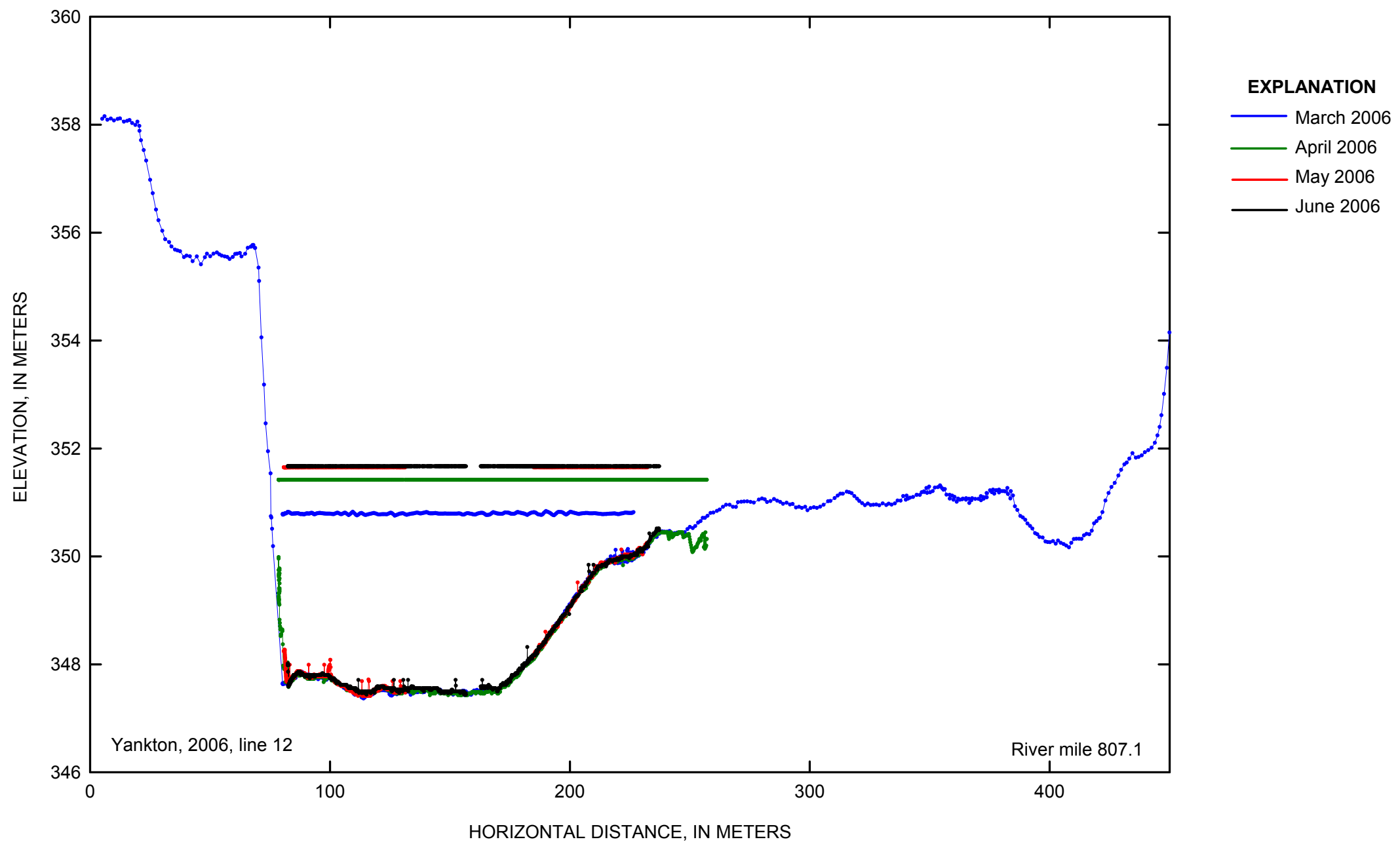

Figure 1-15. Yankton cross-section line 12 at Missouri River mile 807.1 in 2006. 


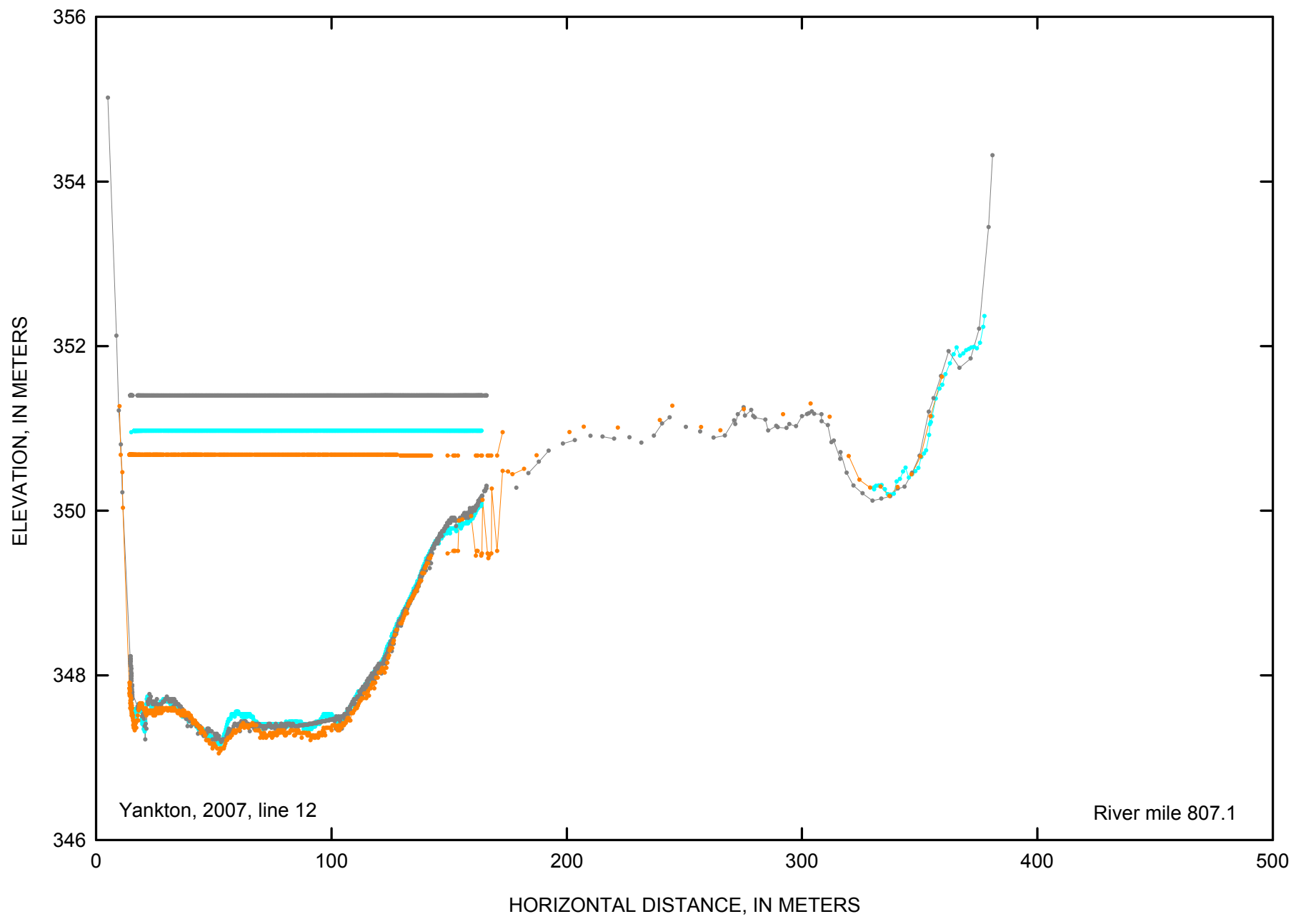

EXPLANATION

March 2007

June 2007

- November 2007

Figure 1-16. Yankton cross-section line 12 at Missouri River mile 807.1 in 2007. 


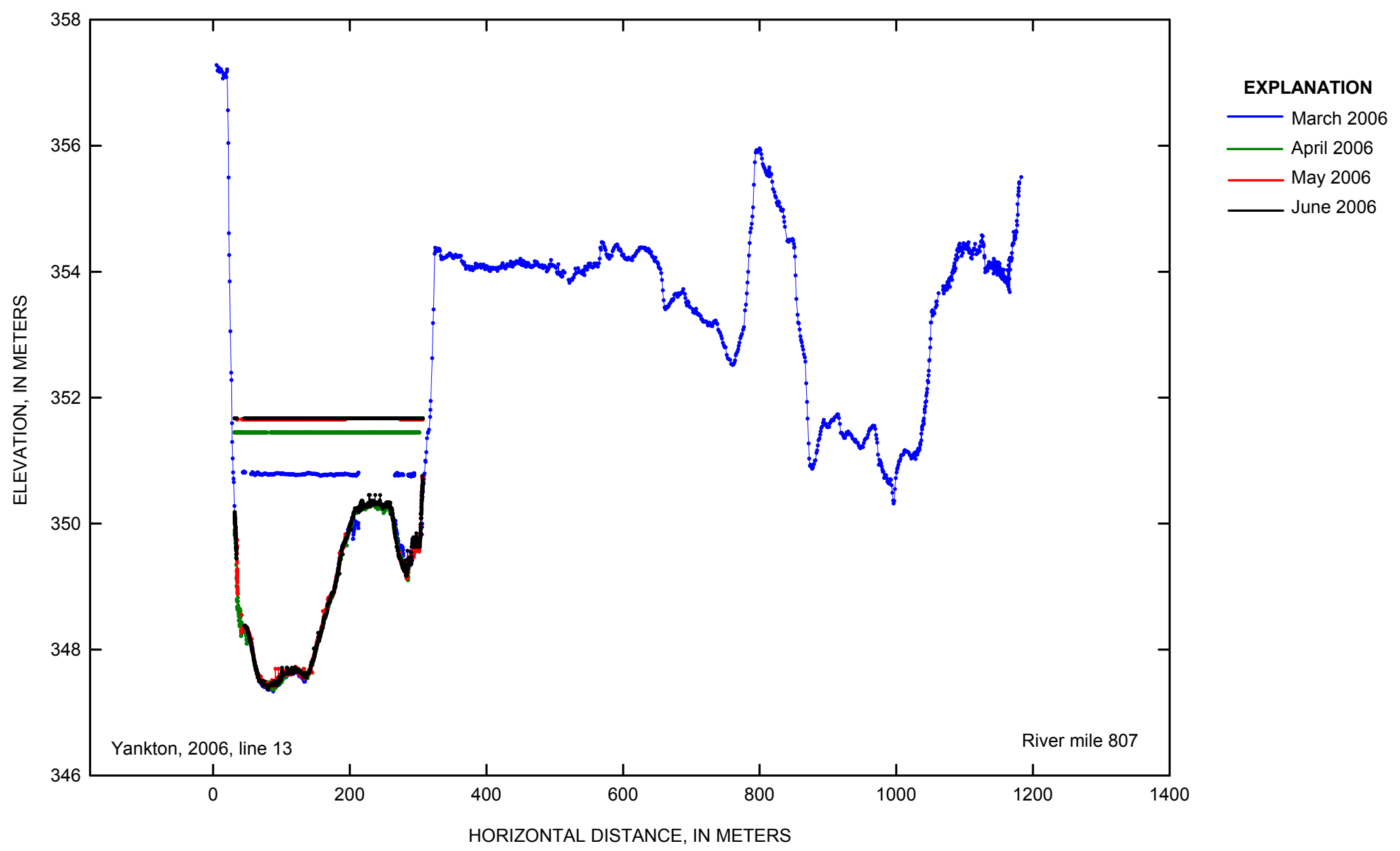

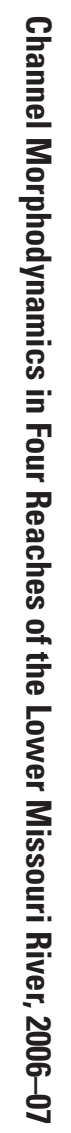

Figure 1-17. Yankton cross-section line 13 at Missouri River mile 807.0 in 2006. 


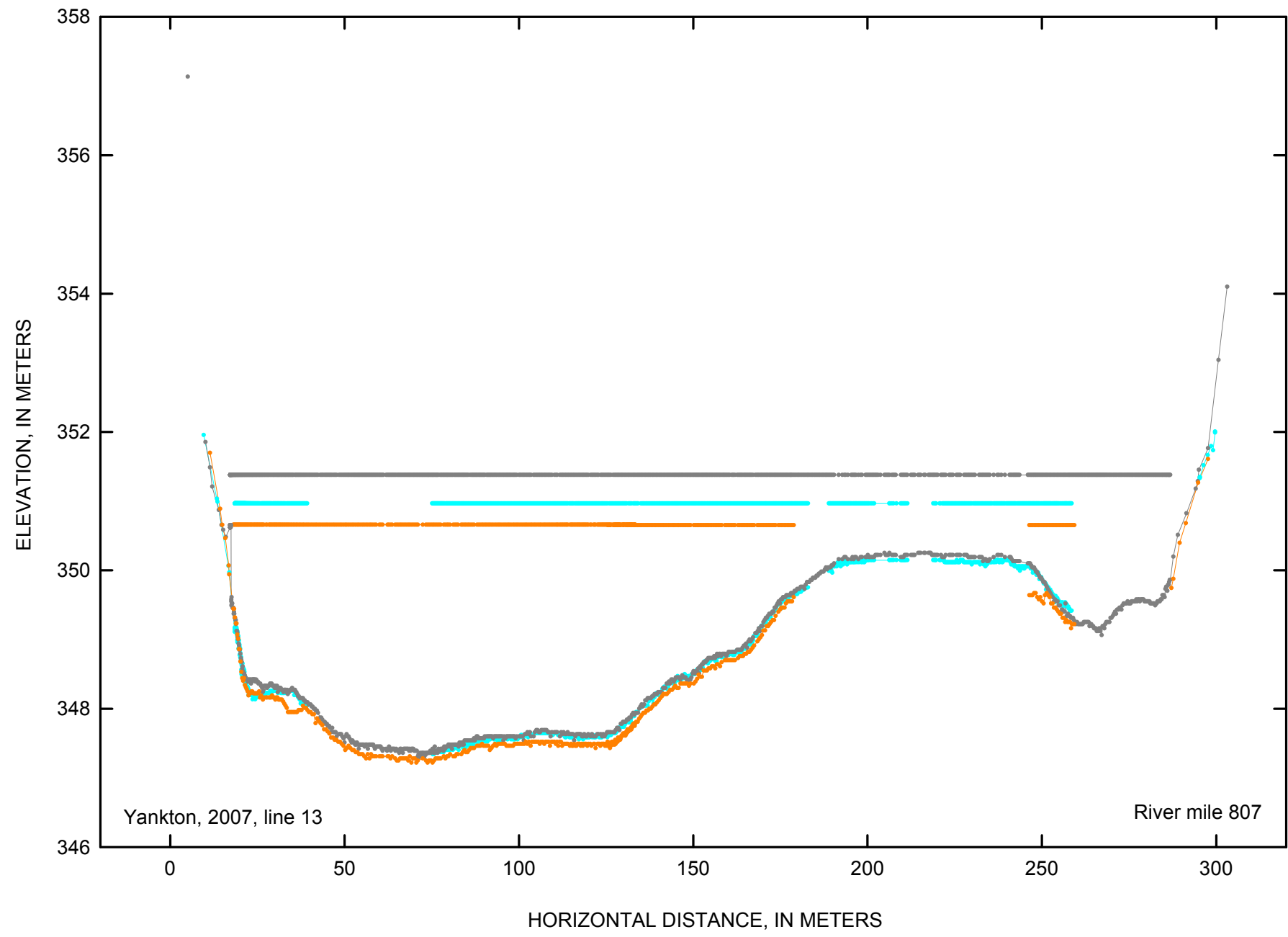

EXPLANATION

March 2007

June 2007

November 2007 


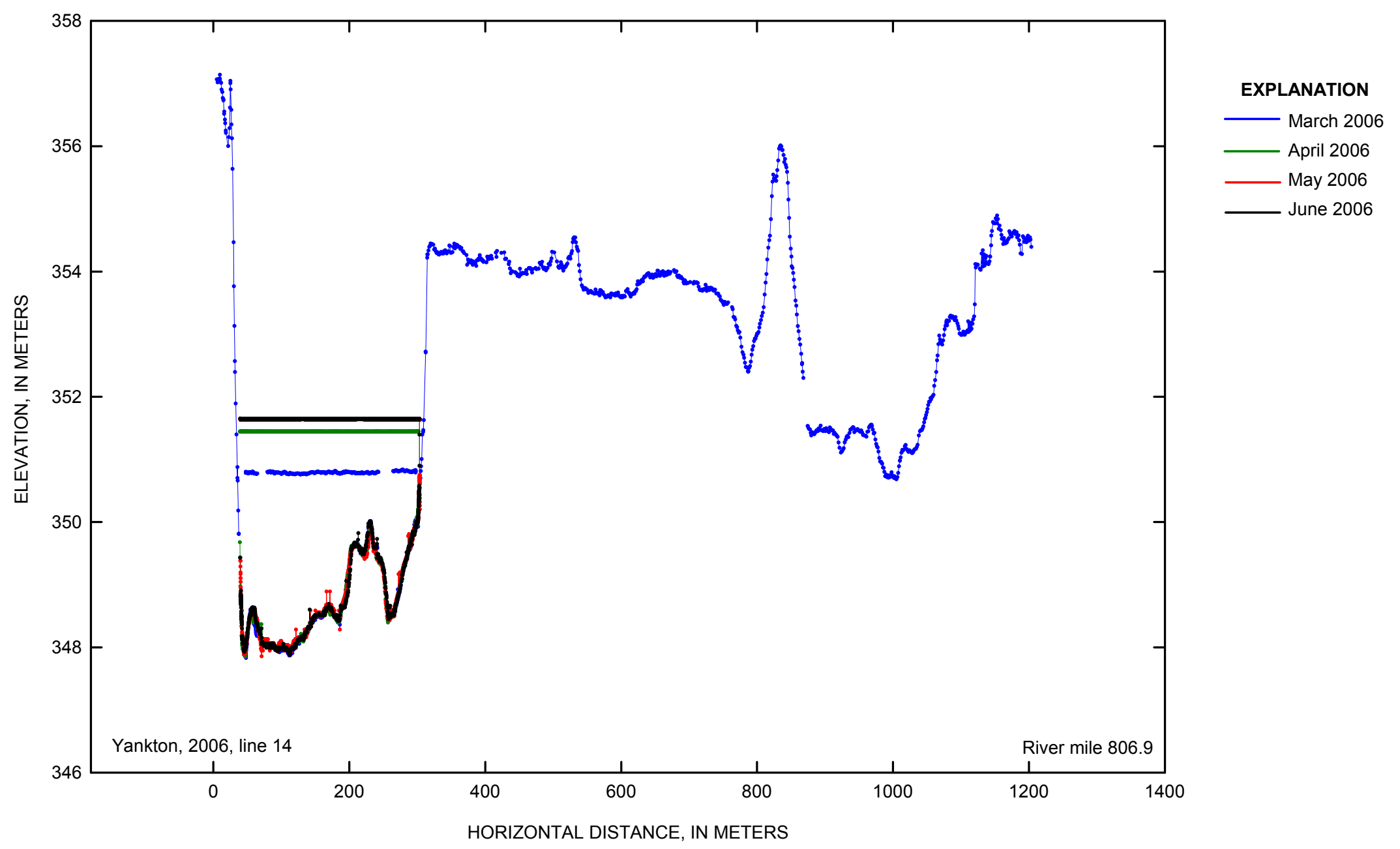

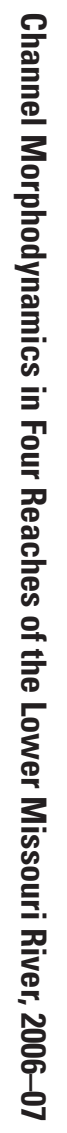

Figure 1-19. Yankton cross-section line 14 at Missouri River mile 806.9 in 2006. 


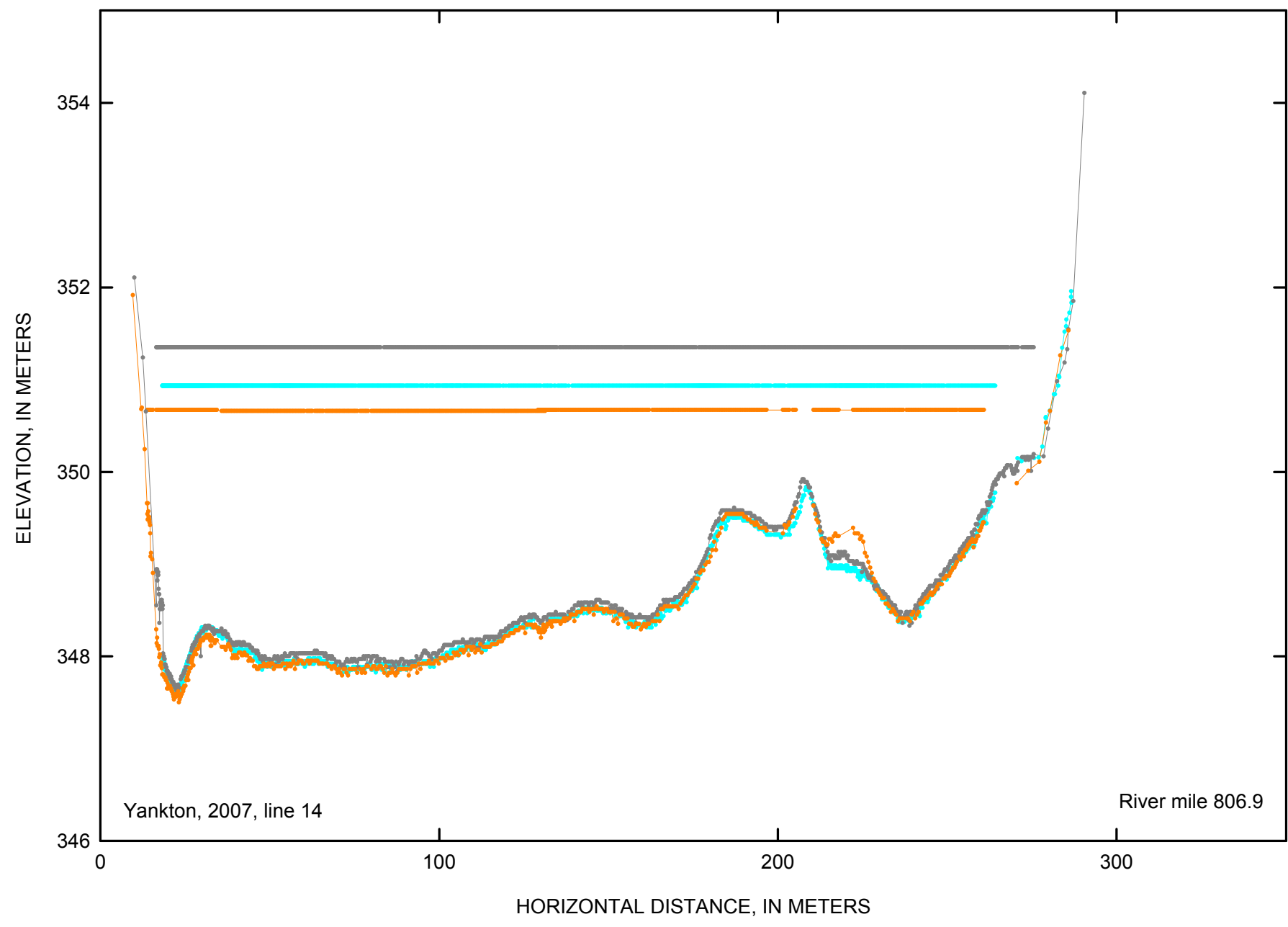

EXPLANATION

March 2007

une 2007

November 2007

Figure 1-20. Yankton cross-section line 14 at Missouri River mile 806.9 in 2007. 


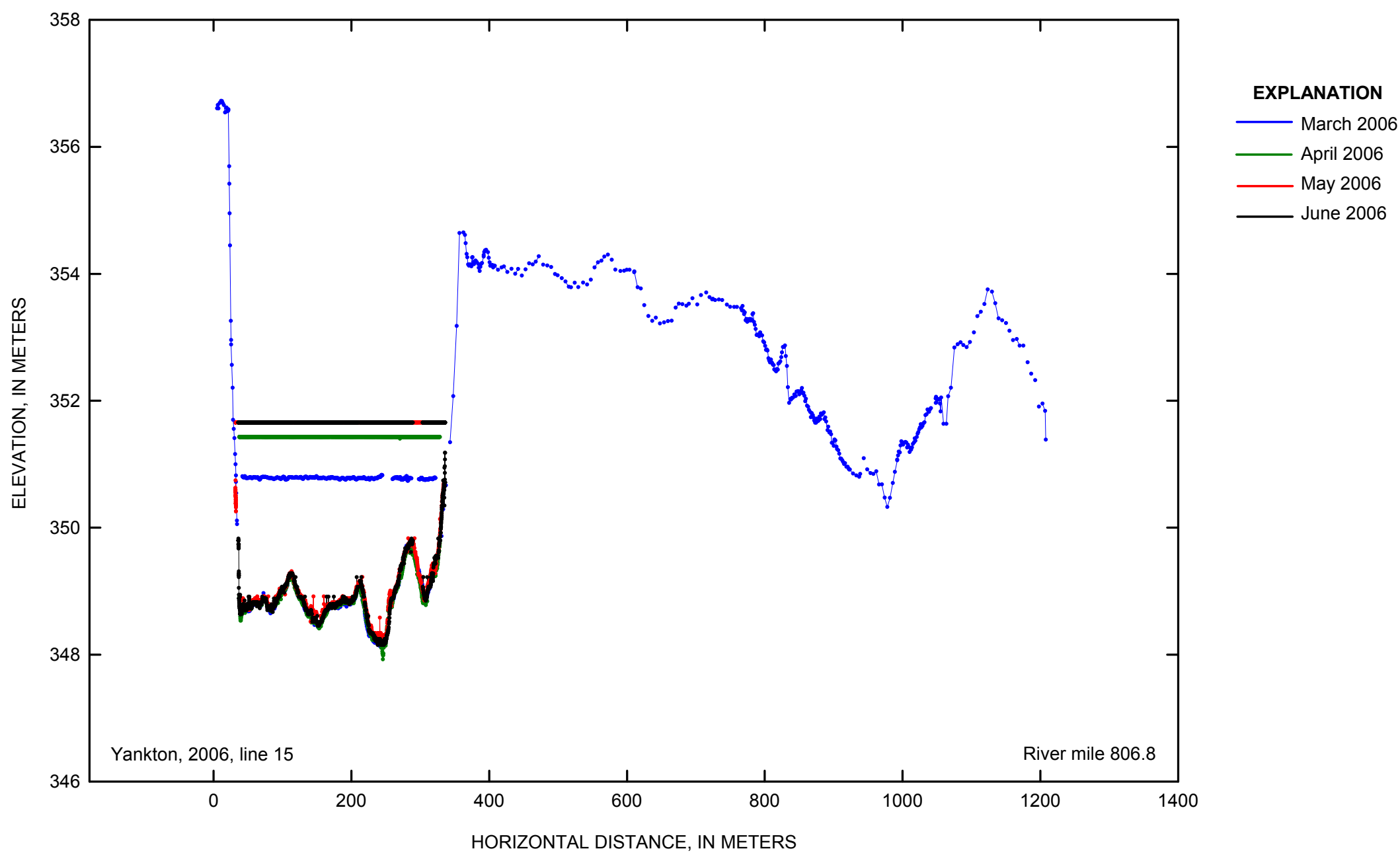

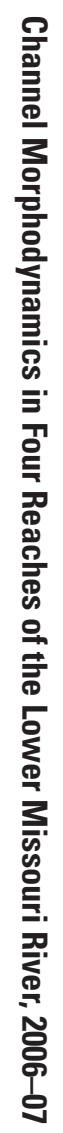

Figure 1-21. Yankton cross-section line 15 at Missouri River mile 806.8 in 2006. 


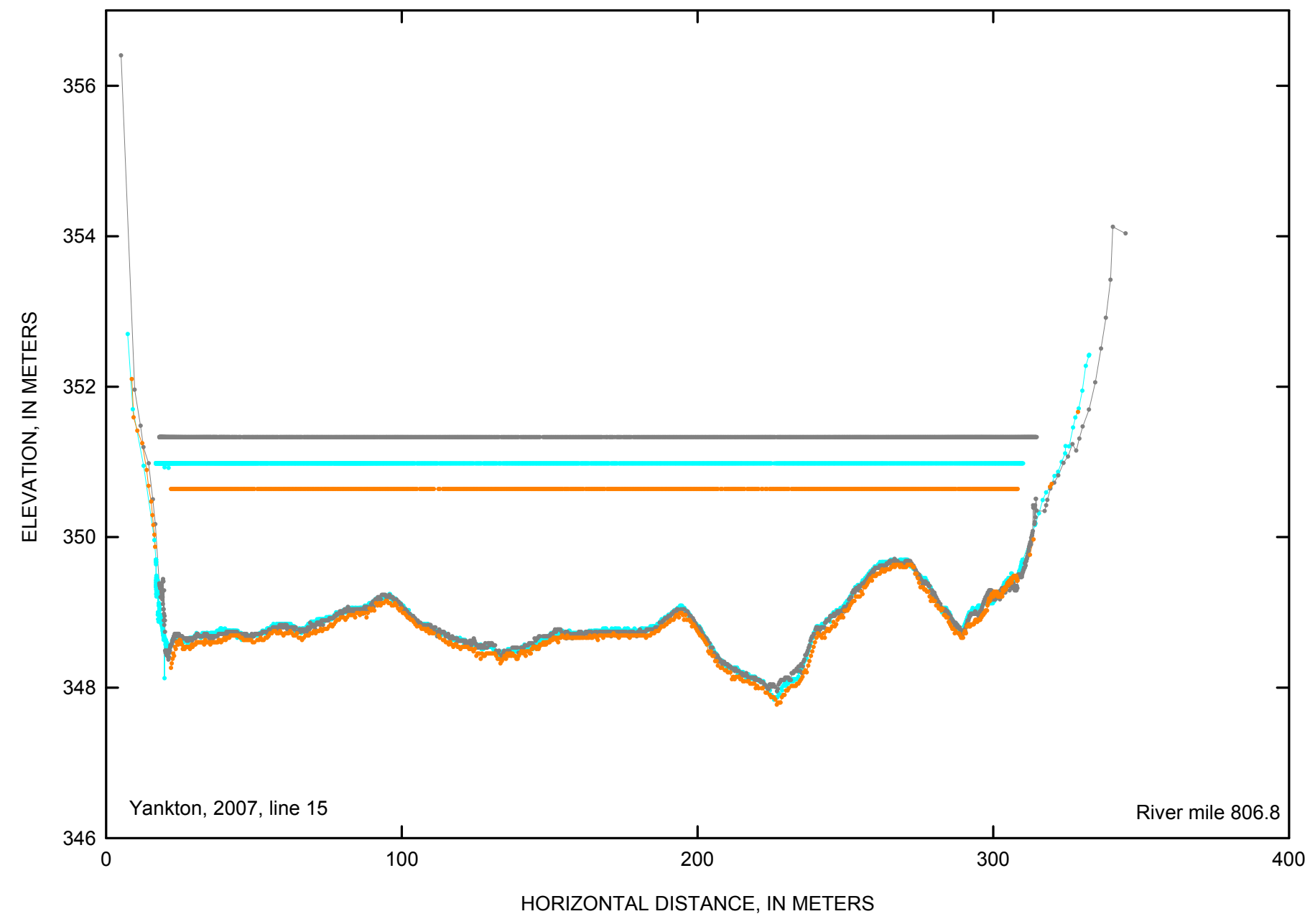

EXPLANATION

March 2007

June 2007

November 2007

Figure 1-22. Yankton cross-section line 15 at Missouri River mile 806.8 in 2007. 


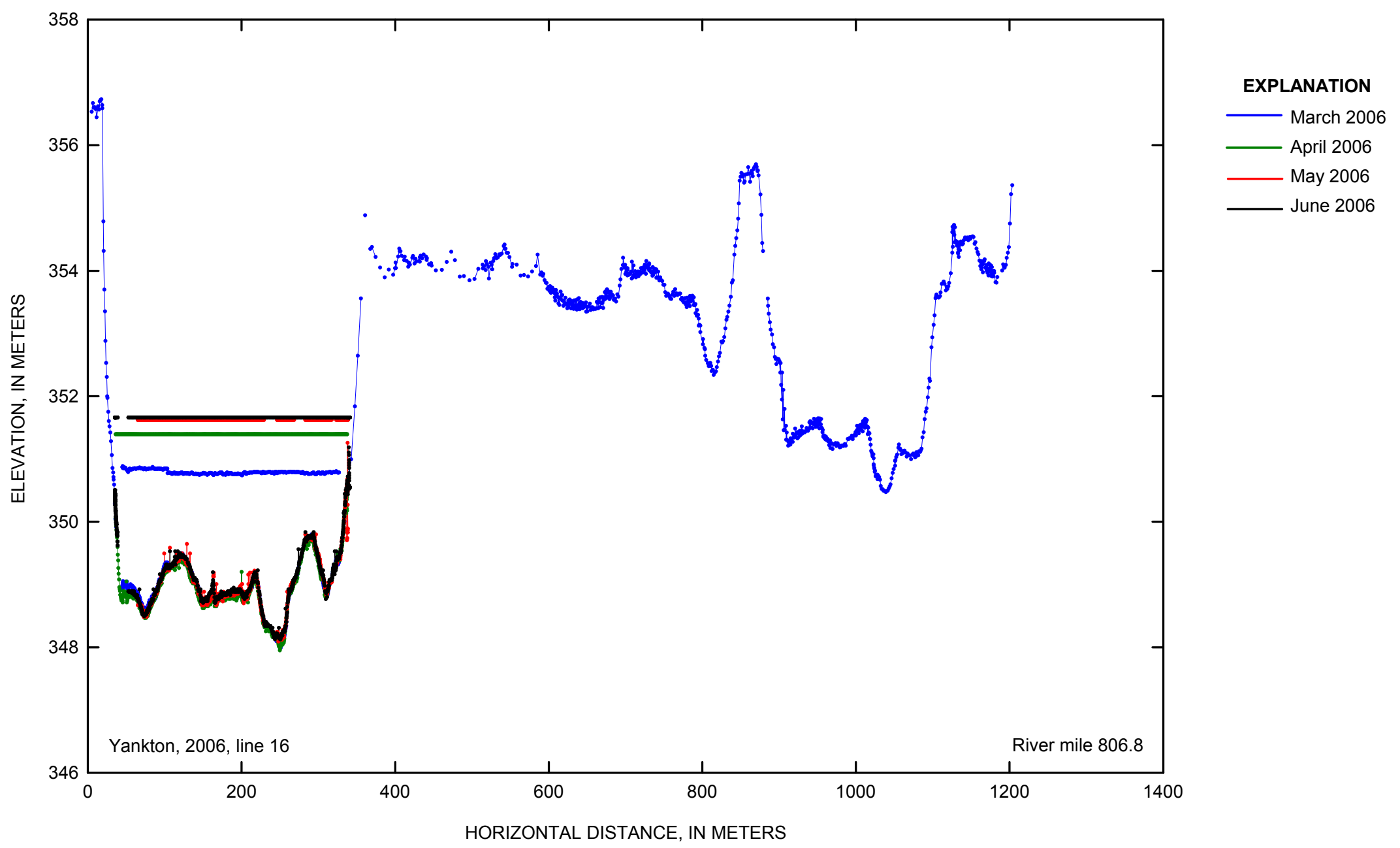

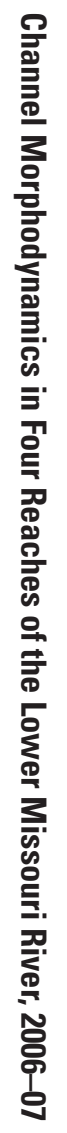

Figure 1-23. Yankton cross-section line 16 at Missouri River mile 806.8 in 2006. 


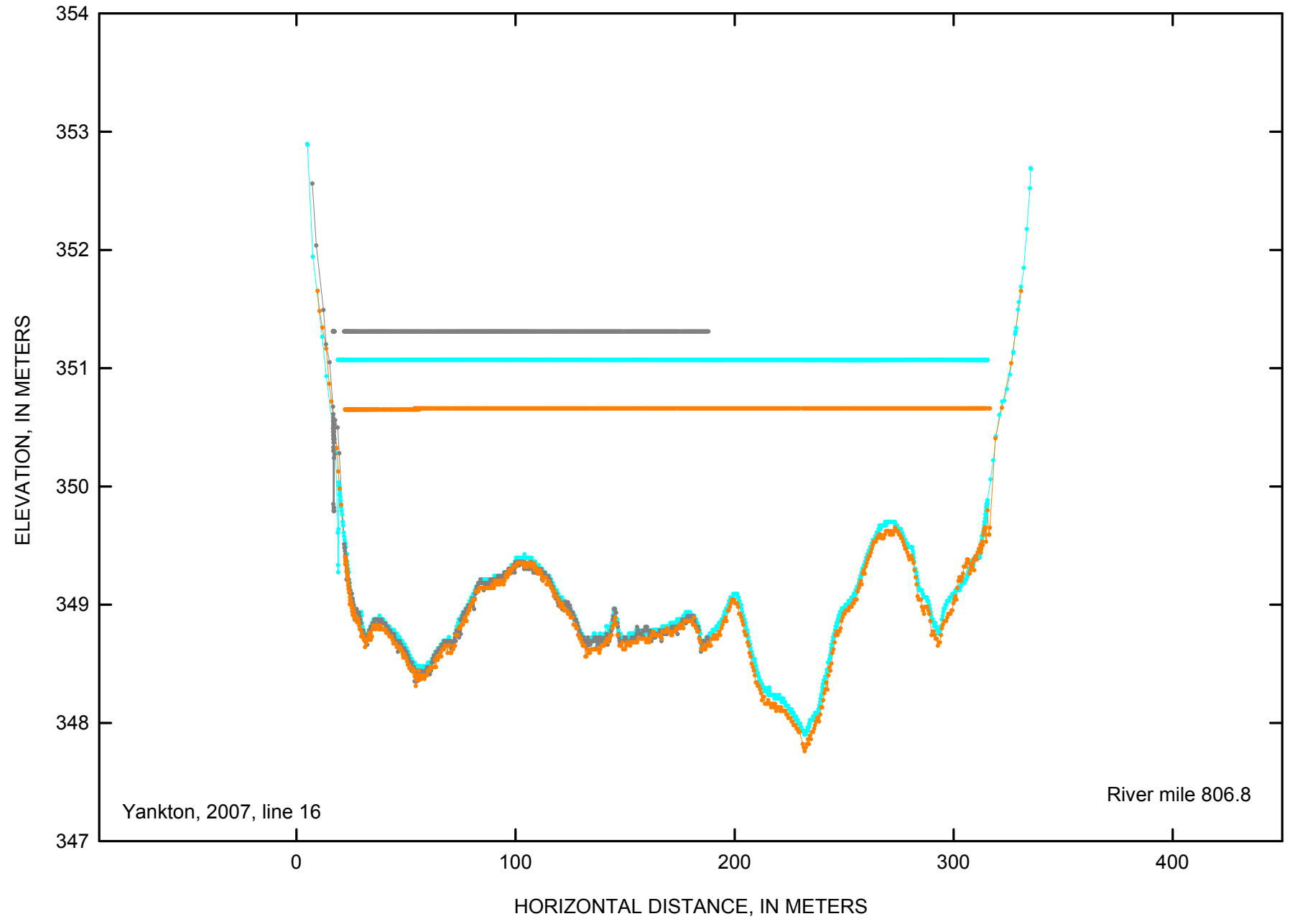

EXPLANATION

March 2007

June 2007

November 2007

Figure1-24. Yankton cross-section line 16 at Missouri River mile 806.8 in 2007. 


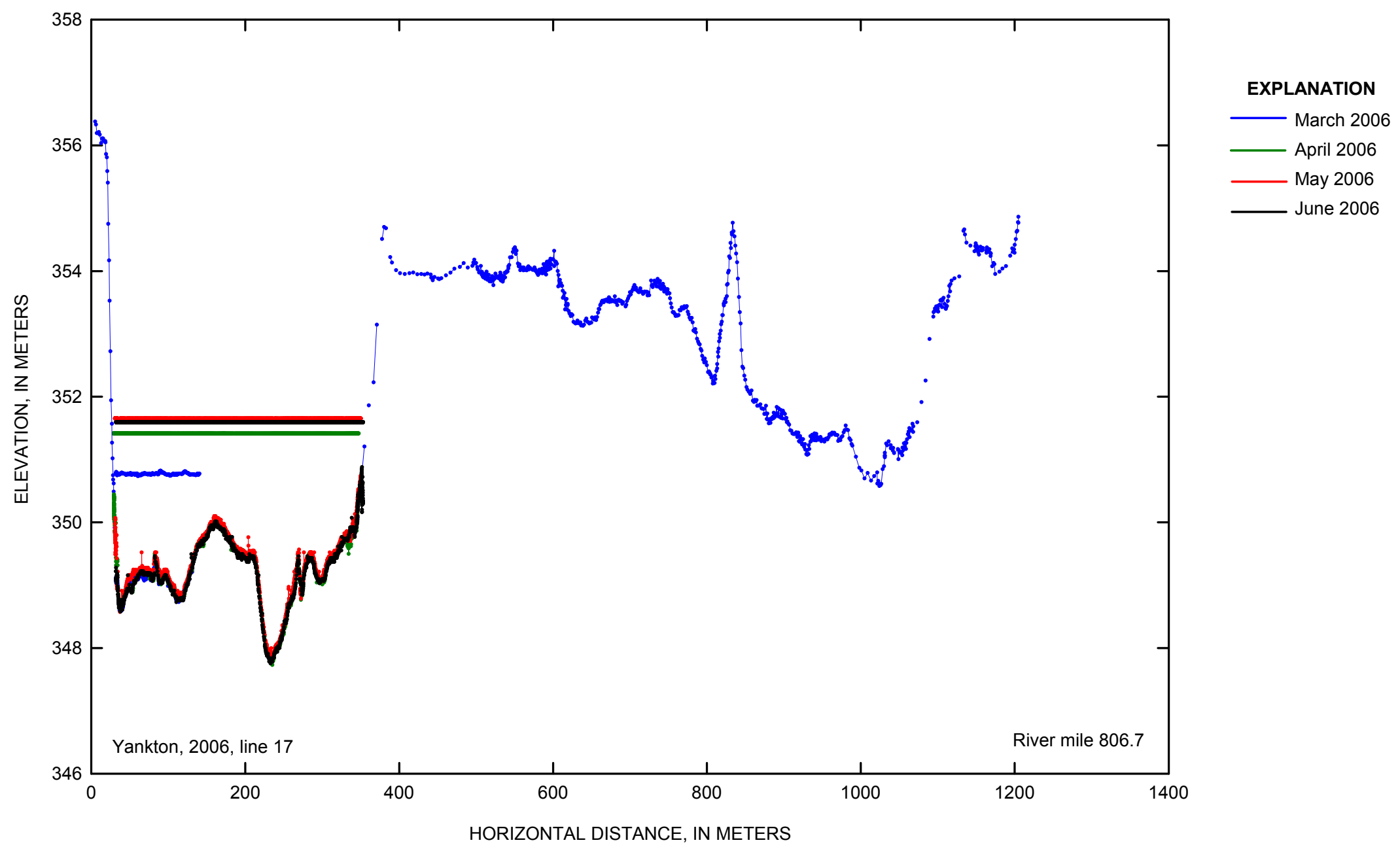

Figure 1-25. Yankton cross-section line 17 at Missouri River mile 806.7 in 2006. 


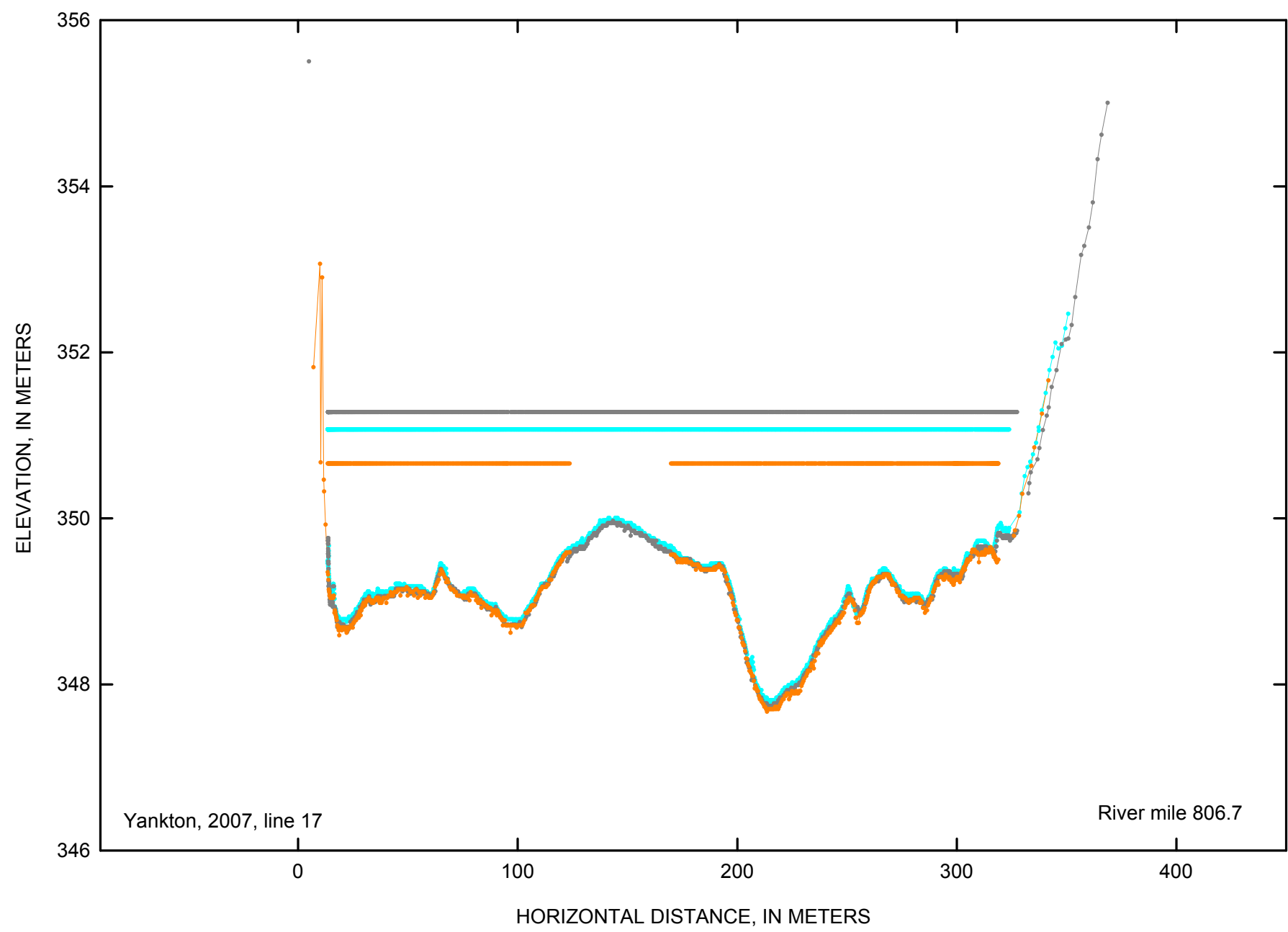

EXPLANATION

March 2007

une 2007

November 2007 


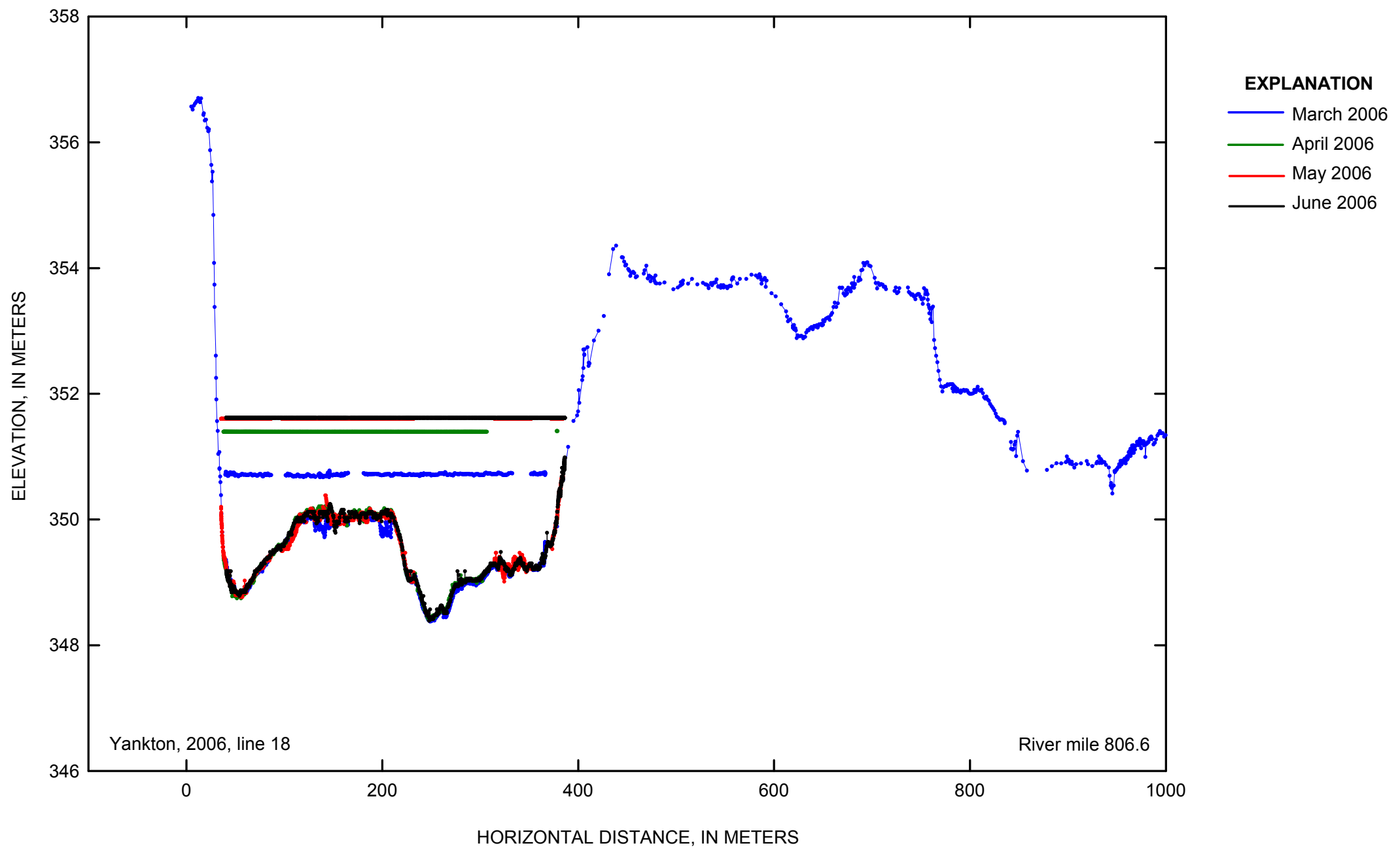

Figure 1-27. Yankton cross-section line 18 at Missouri River mile 806.6 in 2006. 


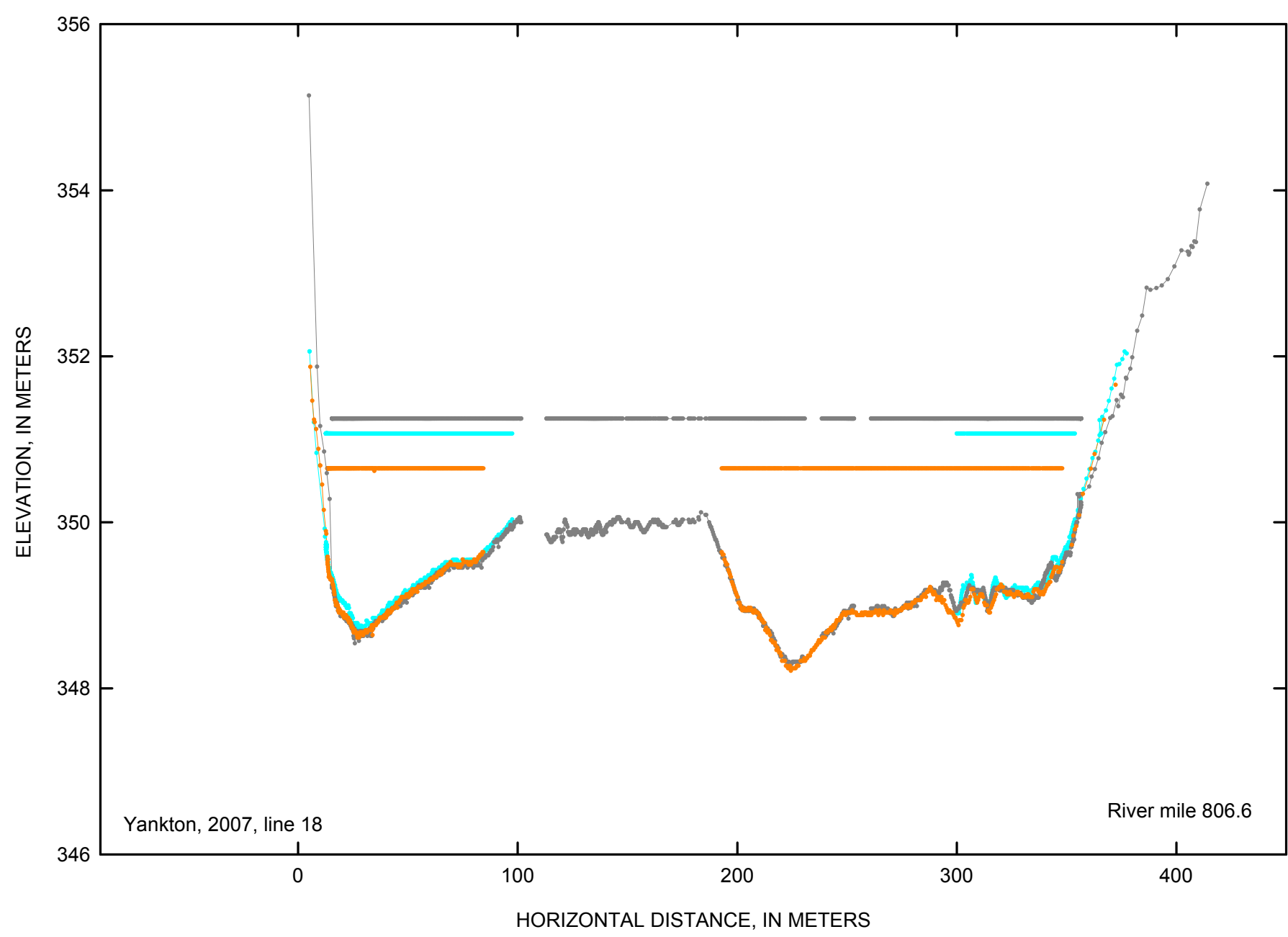

\section{EXPLANATION}

March 2007
June 2007
November 2007




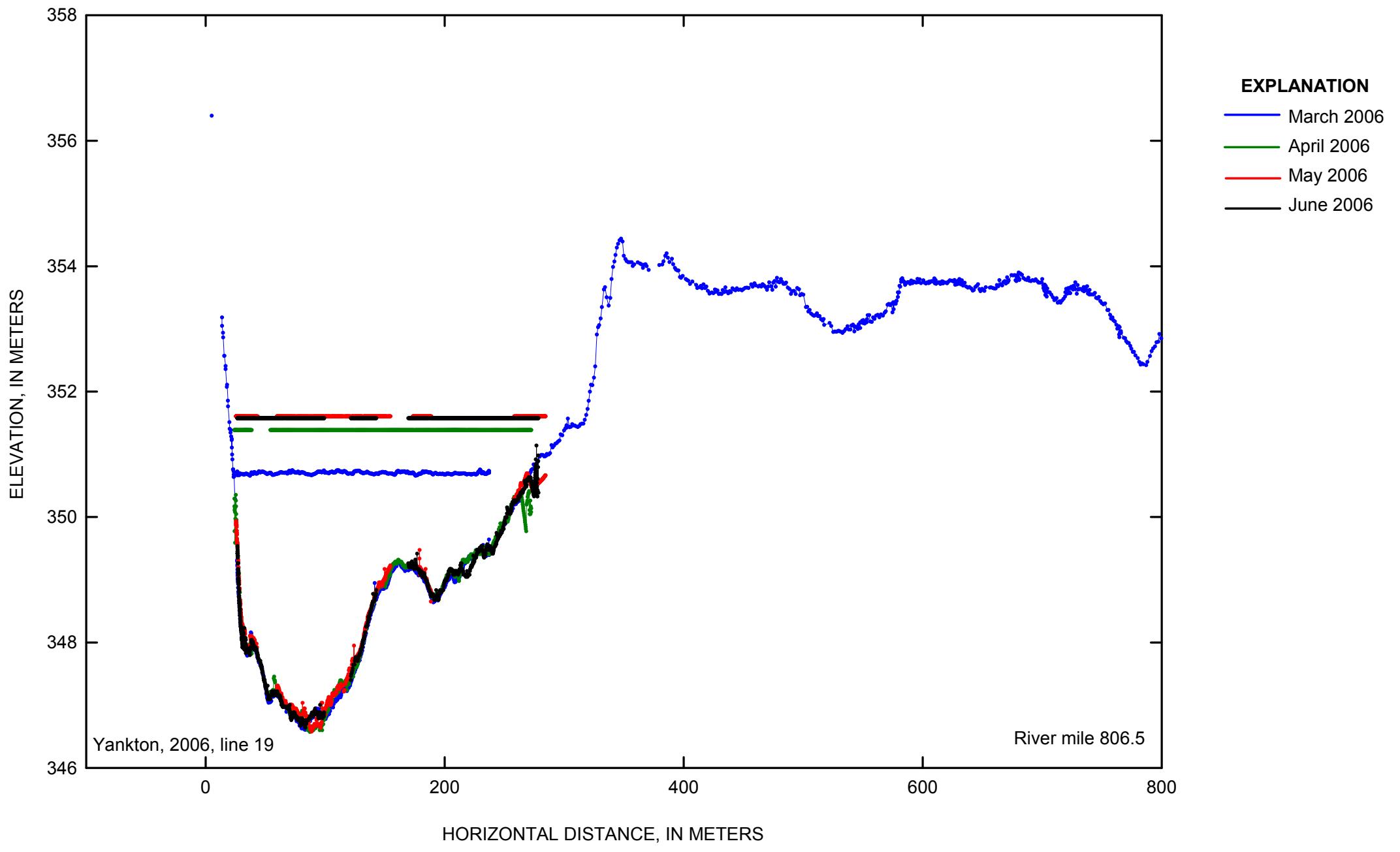

Figure 1-29. Yankton cross-section line 19 at Missouri River mile 806.5 in 2006. 


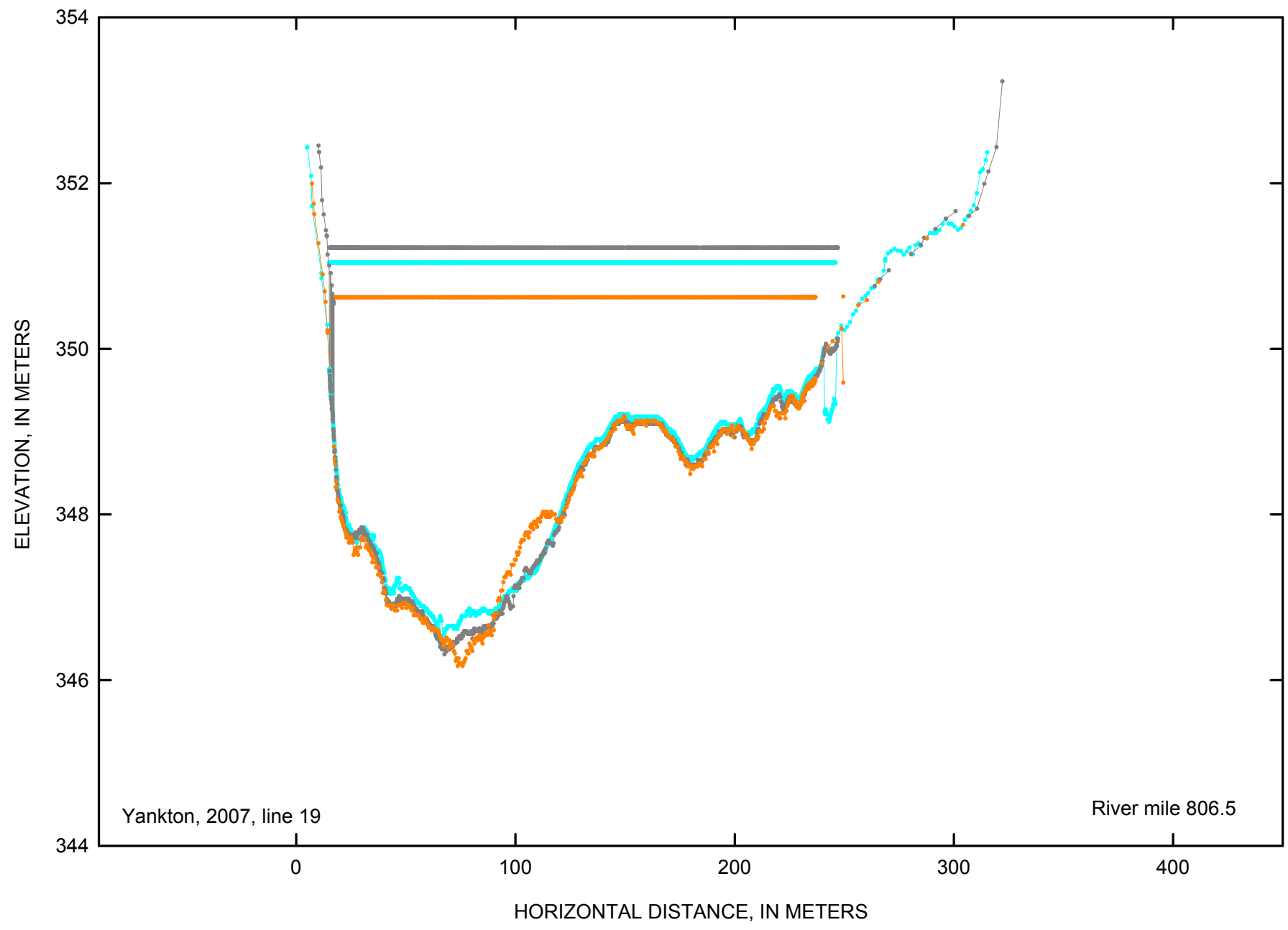

EXPLANATION

March 2007

June 2007

November 2007

Figure 1-30. Yankton cross-section line 19 at Missouri River mile 806.5 in 2007. 


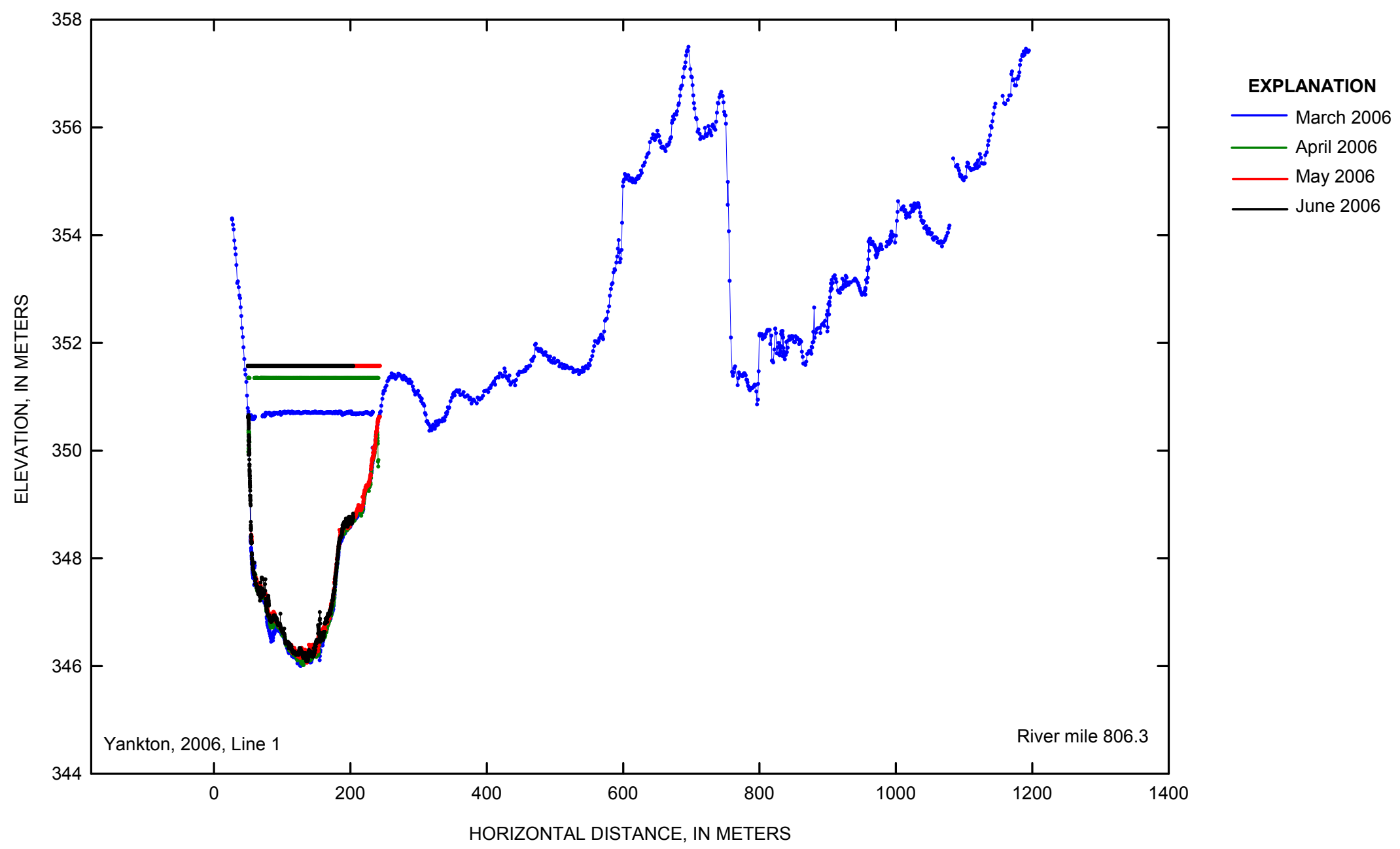

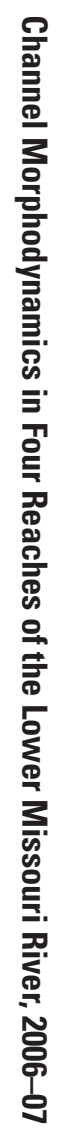

Figure 1-31. Yankton cross-section line 1 at Missouri River mile 806.3 in 2006. 


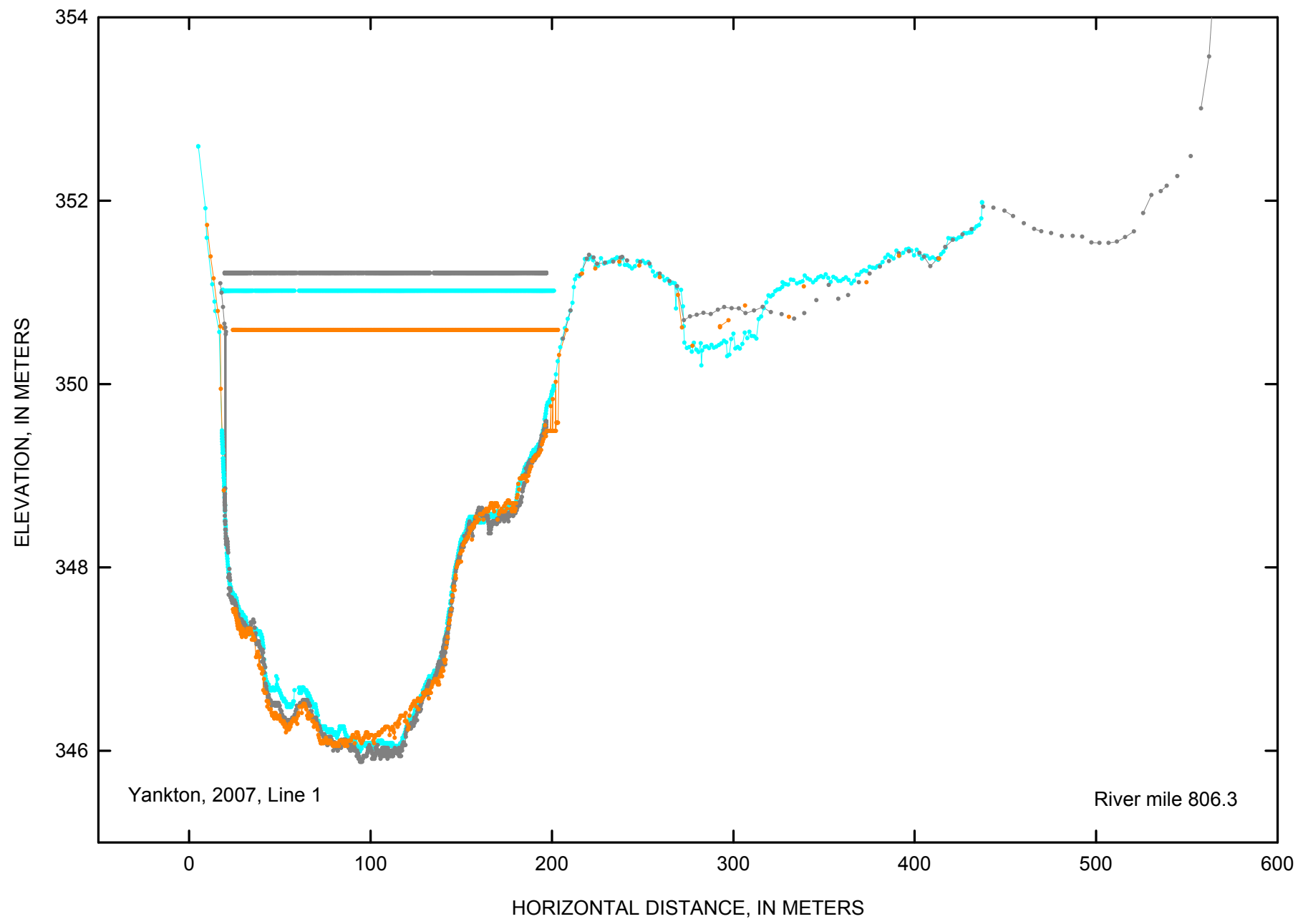

EXPLANATION

— March 2007

- June 2007

November 2007

Figure 1-32. Yankton cross-section line 1 at Missouri River mile 806.3 in 2007. 


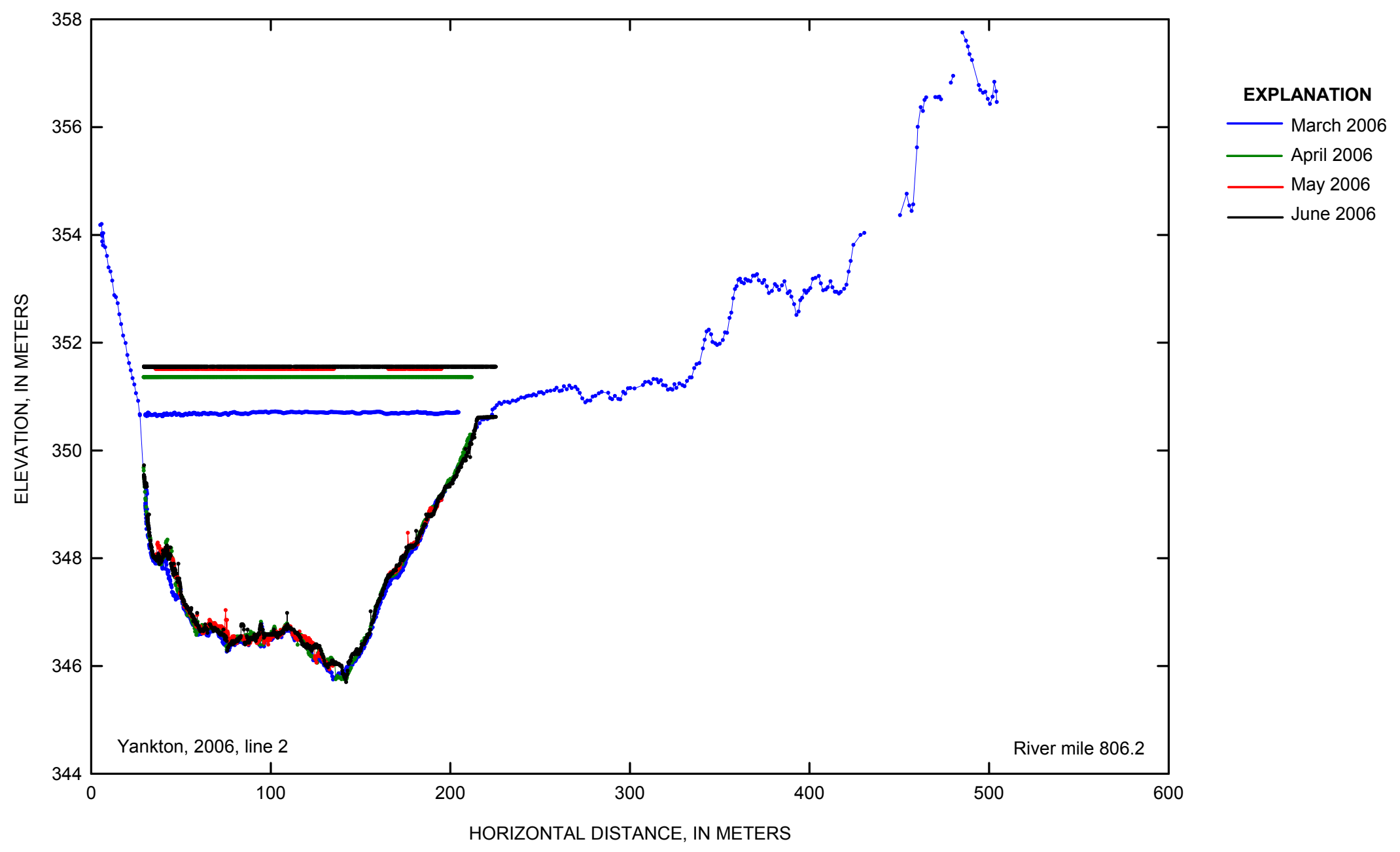

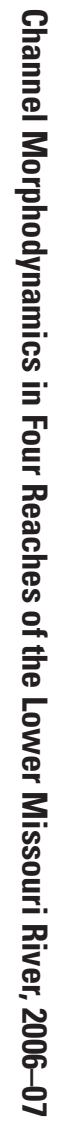

Figure 1-33. Yankton cross-section line 2 at Missouri River mile 806.2 in 2006. 


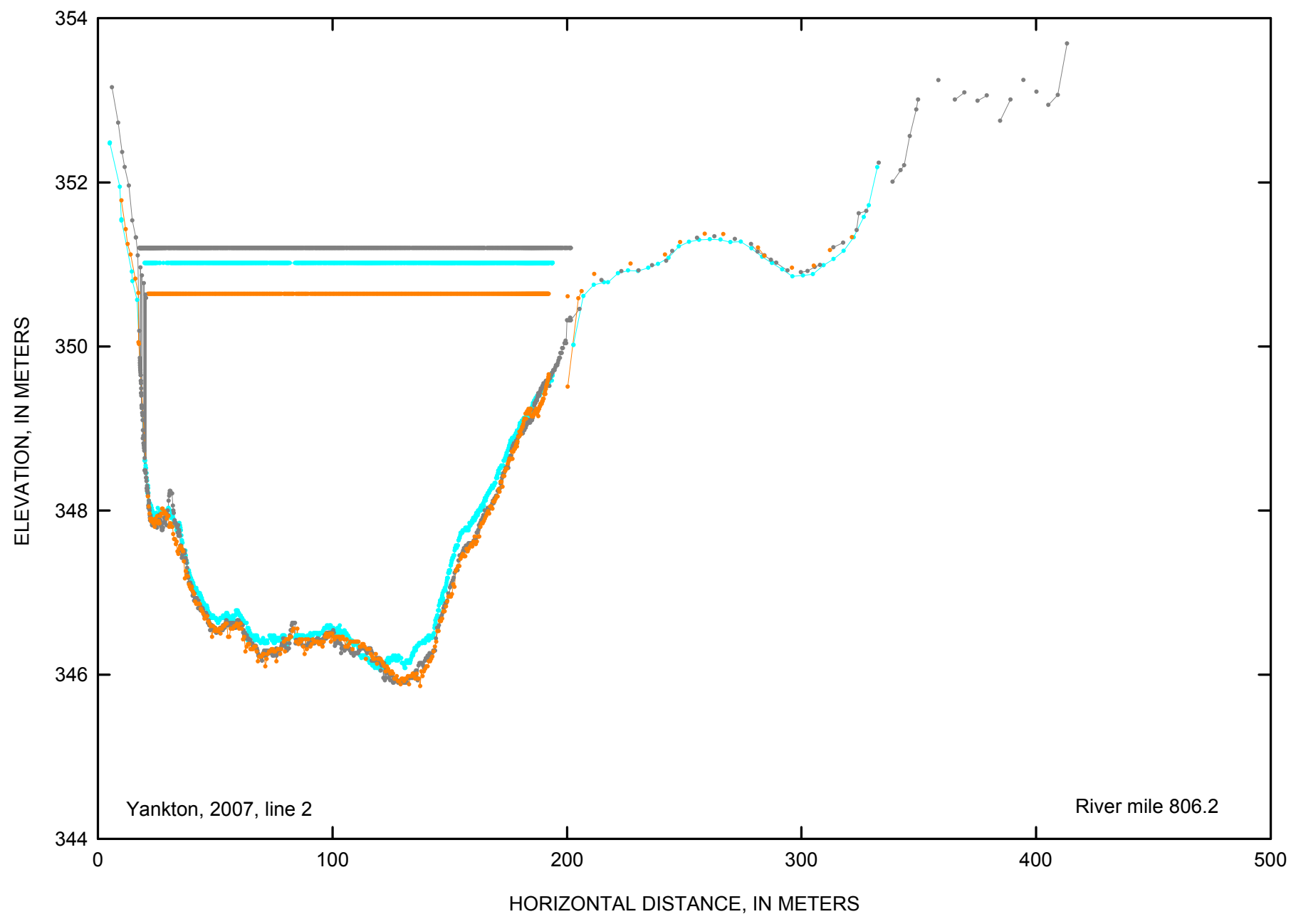

EXPLANATION

March 2007

— June 2007

November 2007

Figure 1-34. Yankton cross-section line 2 at Missouri River mile 806.2 in 2007. 


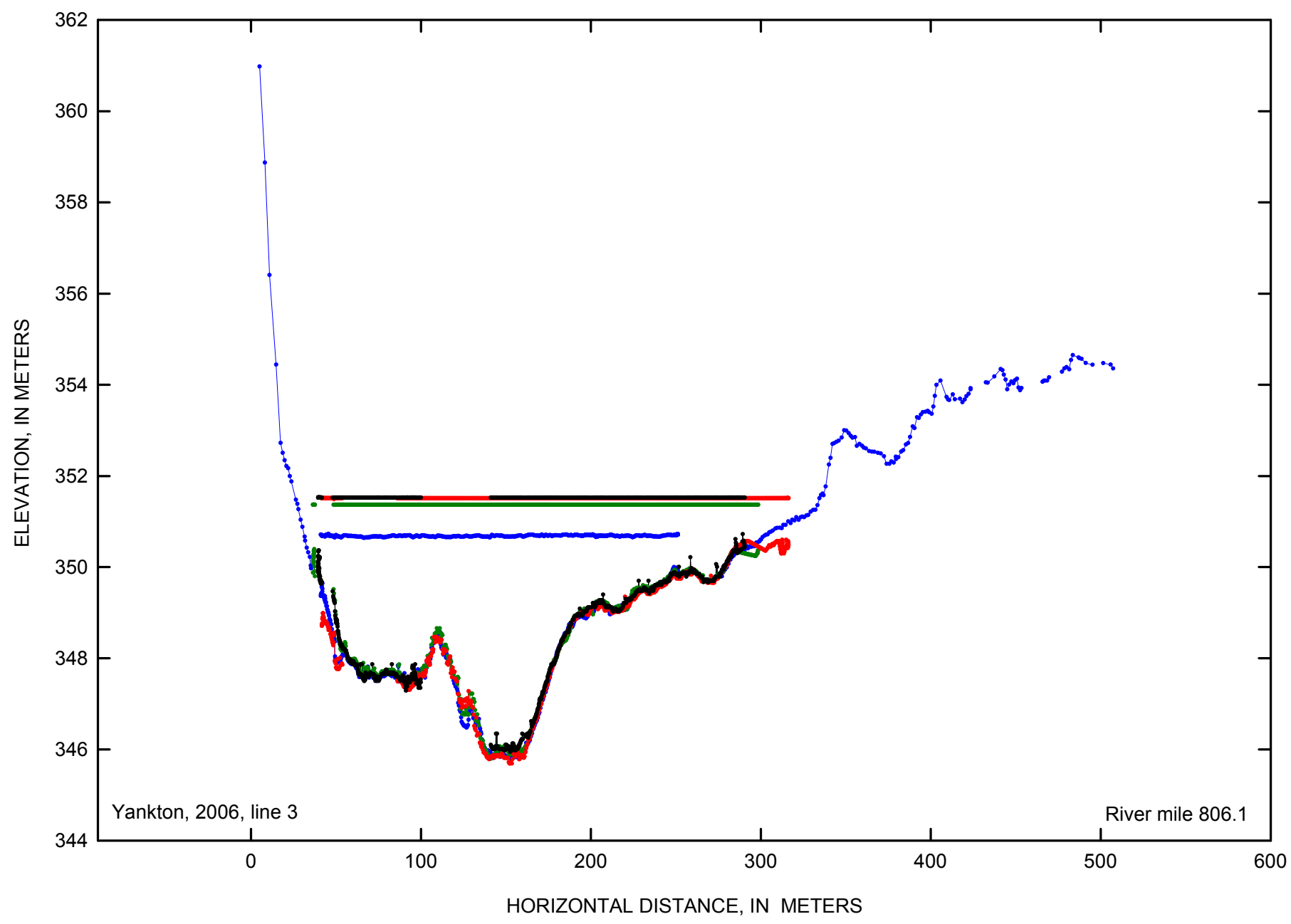

EXPLANATION

- March 2006

April 2006

May 2006

June 2006

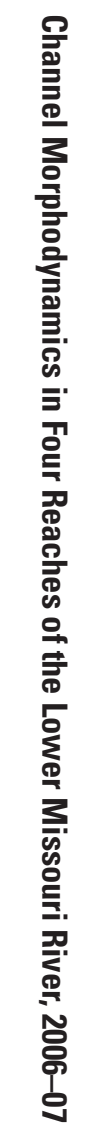

Figure 1-35. Yankton cross-section line 3 at Missouri River mile 806.1 in 2006. 


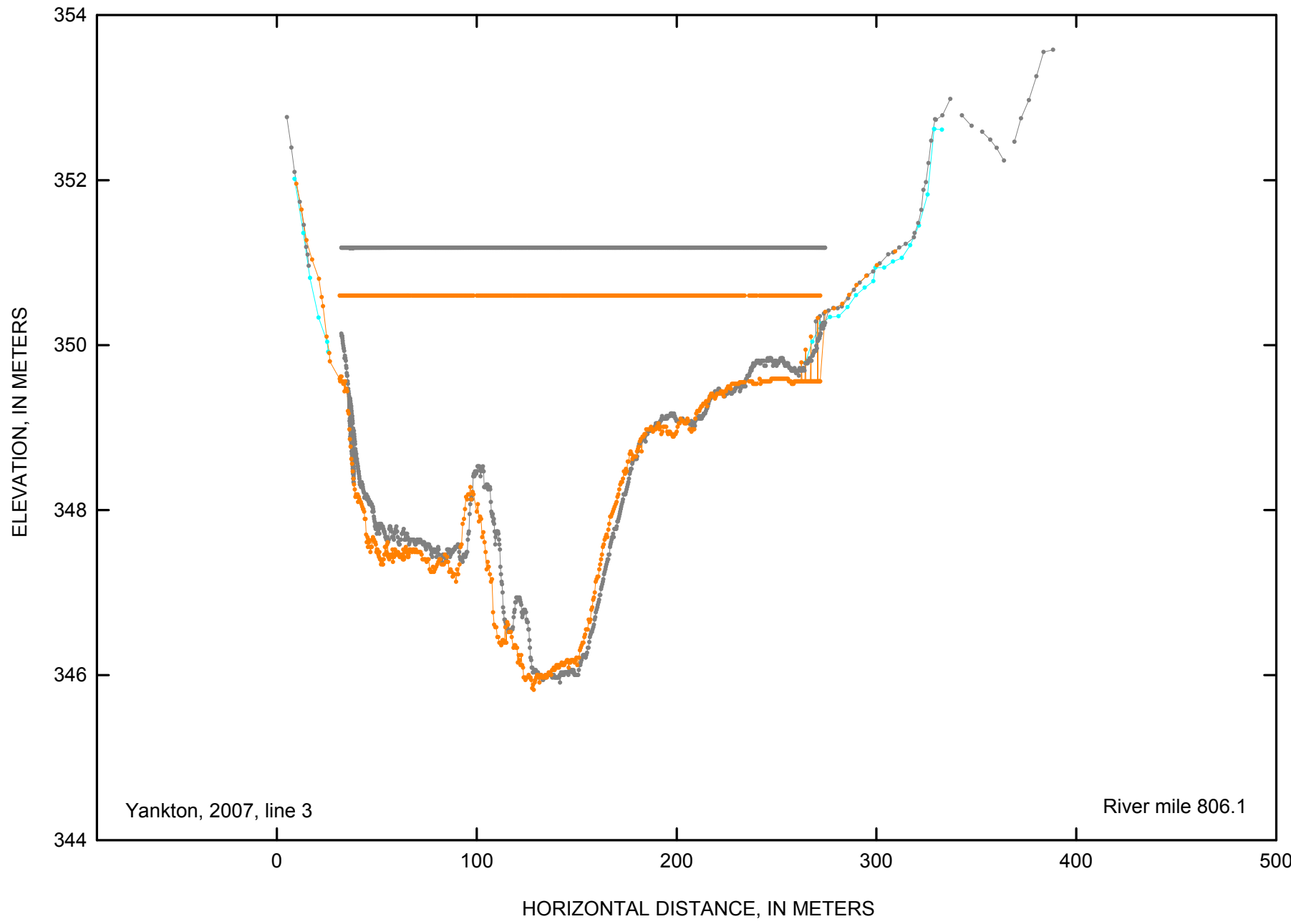

EXPLANATION

March 2007

June 2007

November 2007

Figure 1-36. Yankton cross-section line 3 at Missouri River mile 806.1 in 2007. 


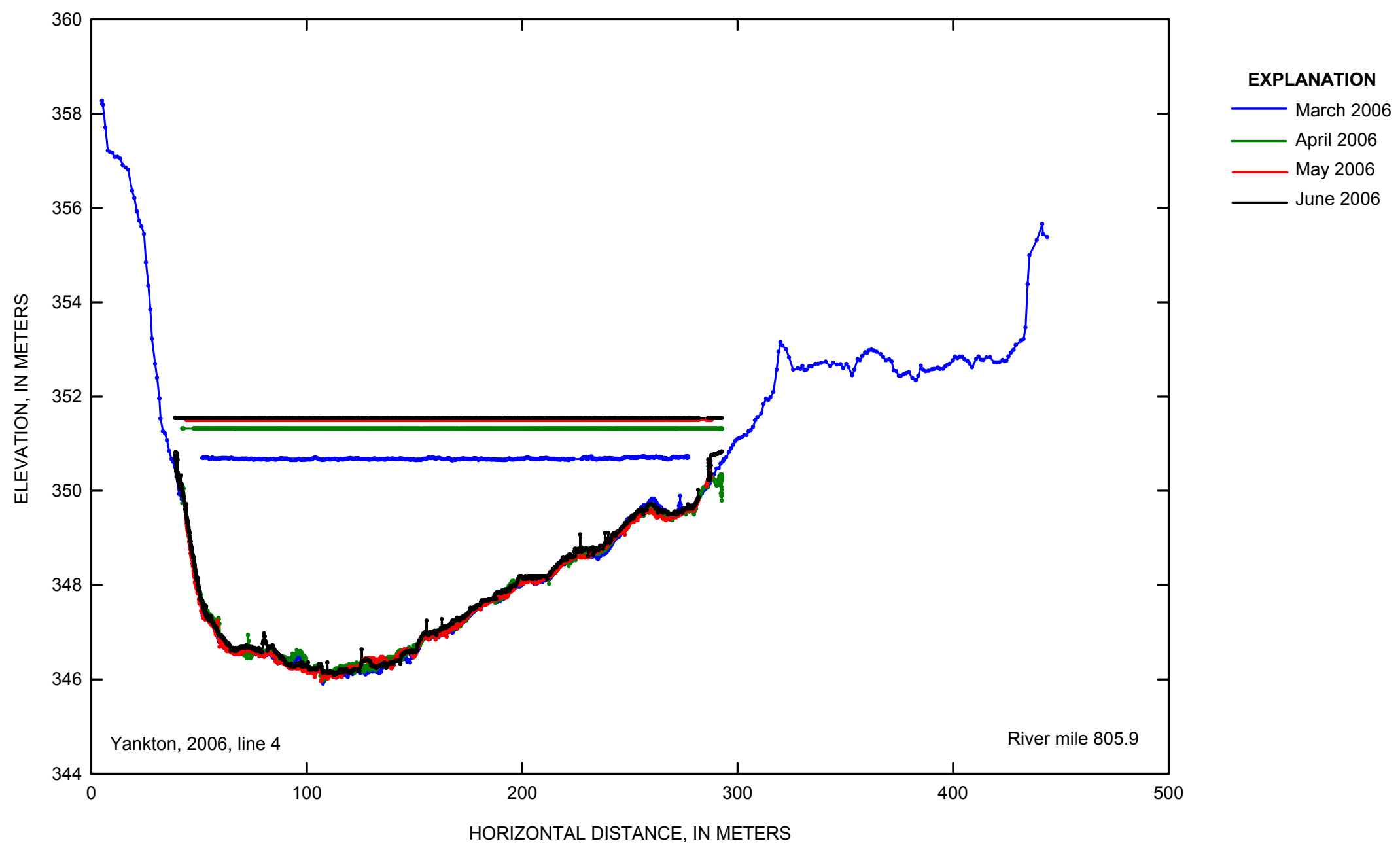

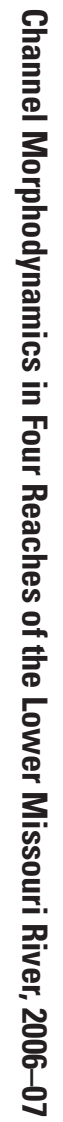

Figure 1-37. Yankton cross-section line 4 at Missouri River mile 805.9 in 2006. 


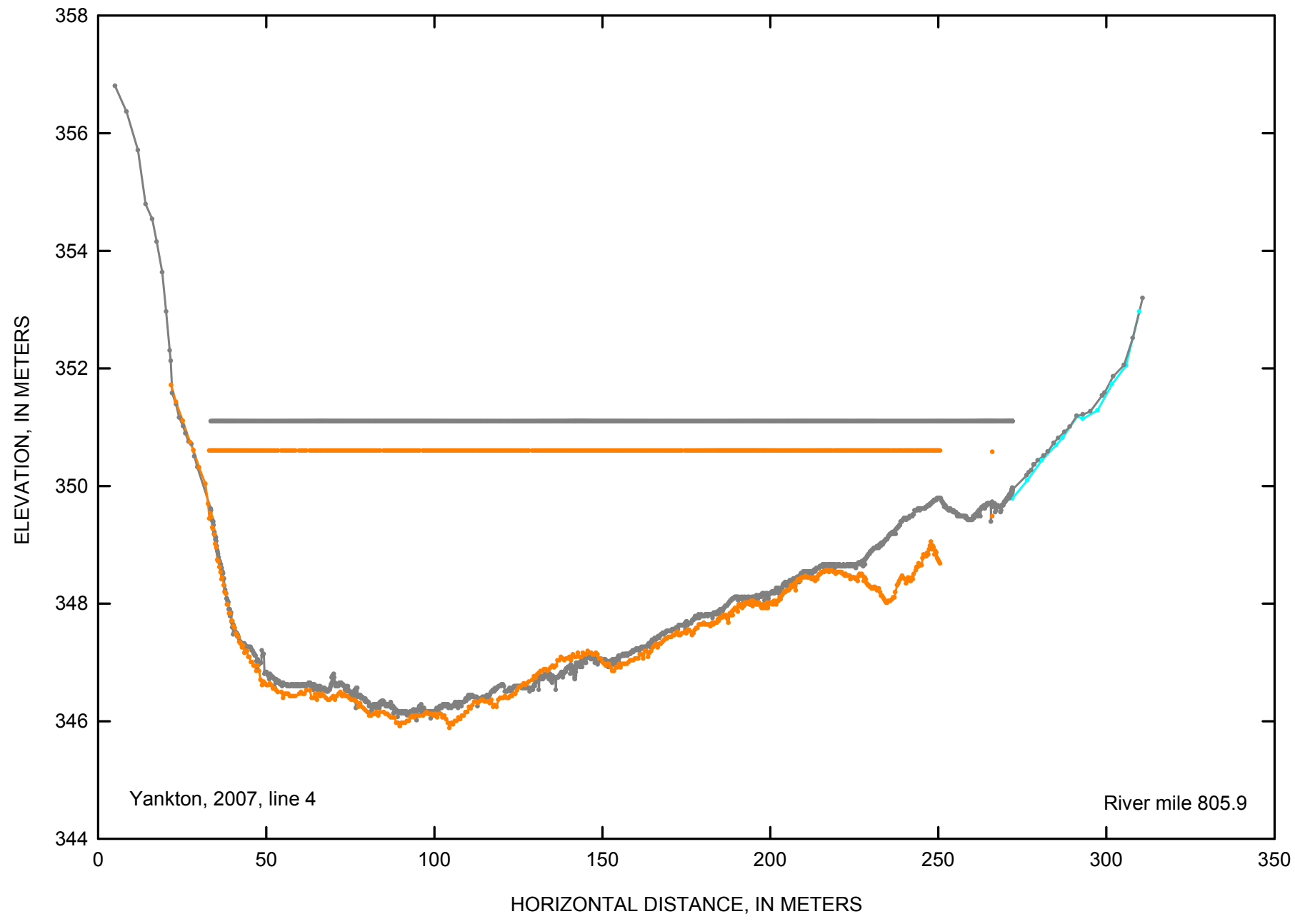

EXPLANATION

March 2007

June 2007

November 2007

Figure 1-38. Yankton cross-section line 4 at Missouri River mile 805.9 in 2007. 


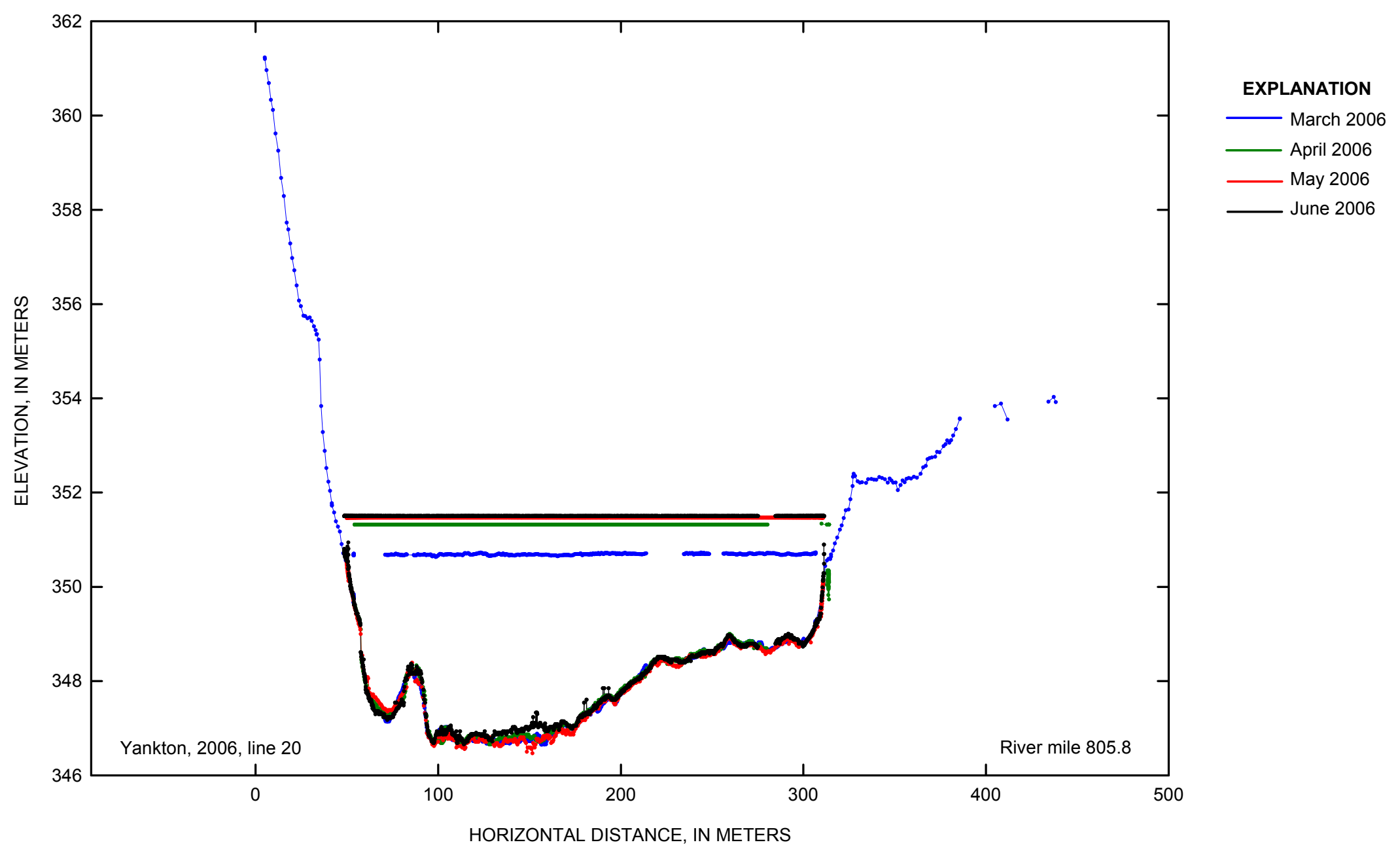

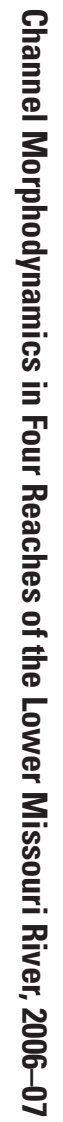

Figure 1-39. Yankton cross-section line 20 at Missouri River mile 805.8 in 2006. 


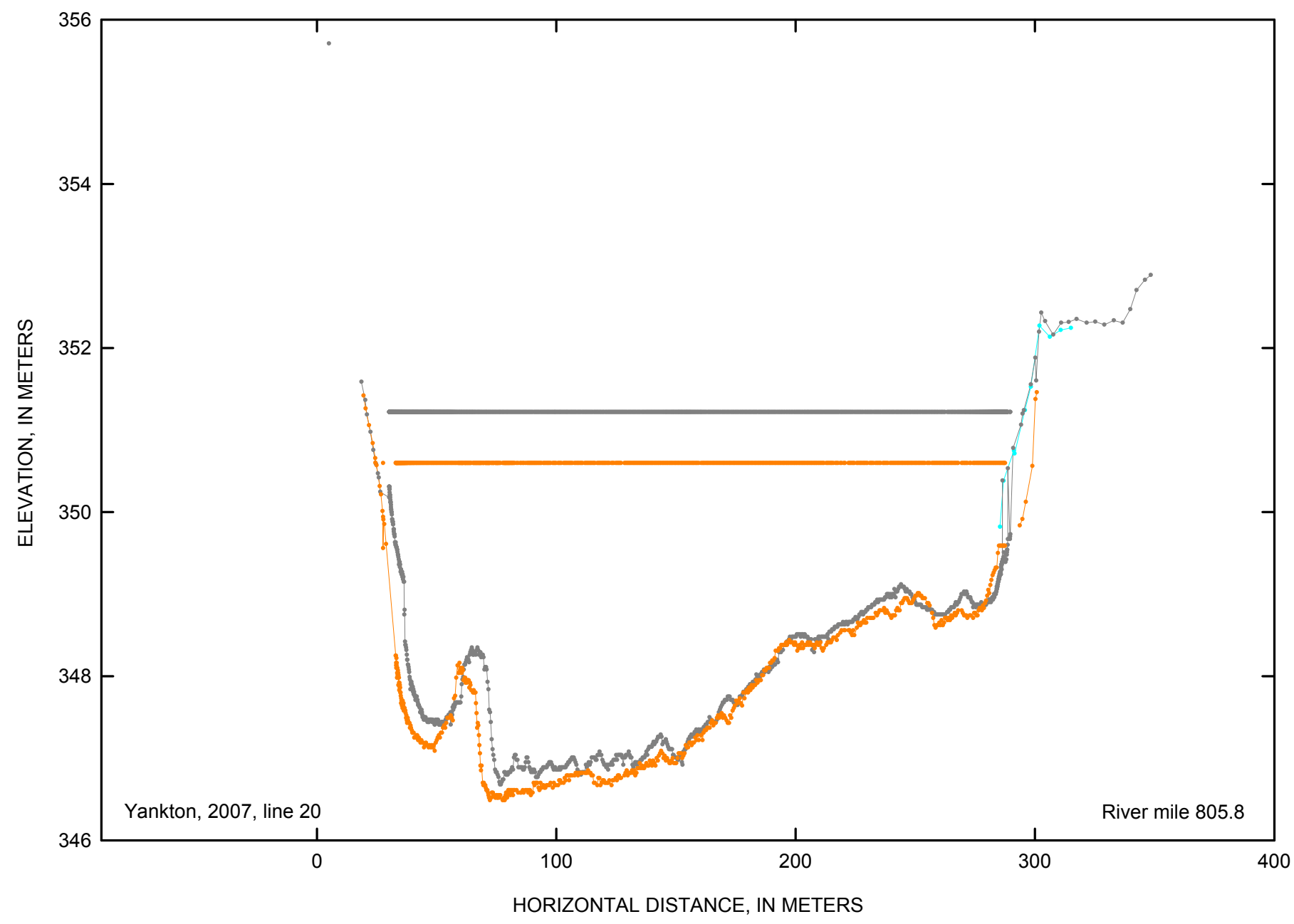

EXPLANATION

March 2007

- June 2007

November 2007

Figure 1-40. Yankton cross-section line 20 at Missouri River mile 805.8 in 2007. 


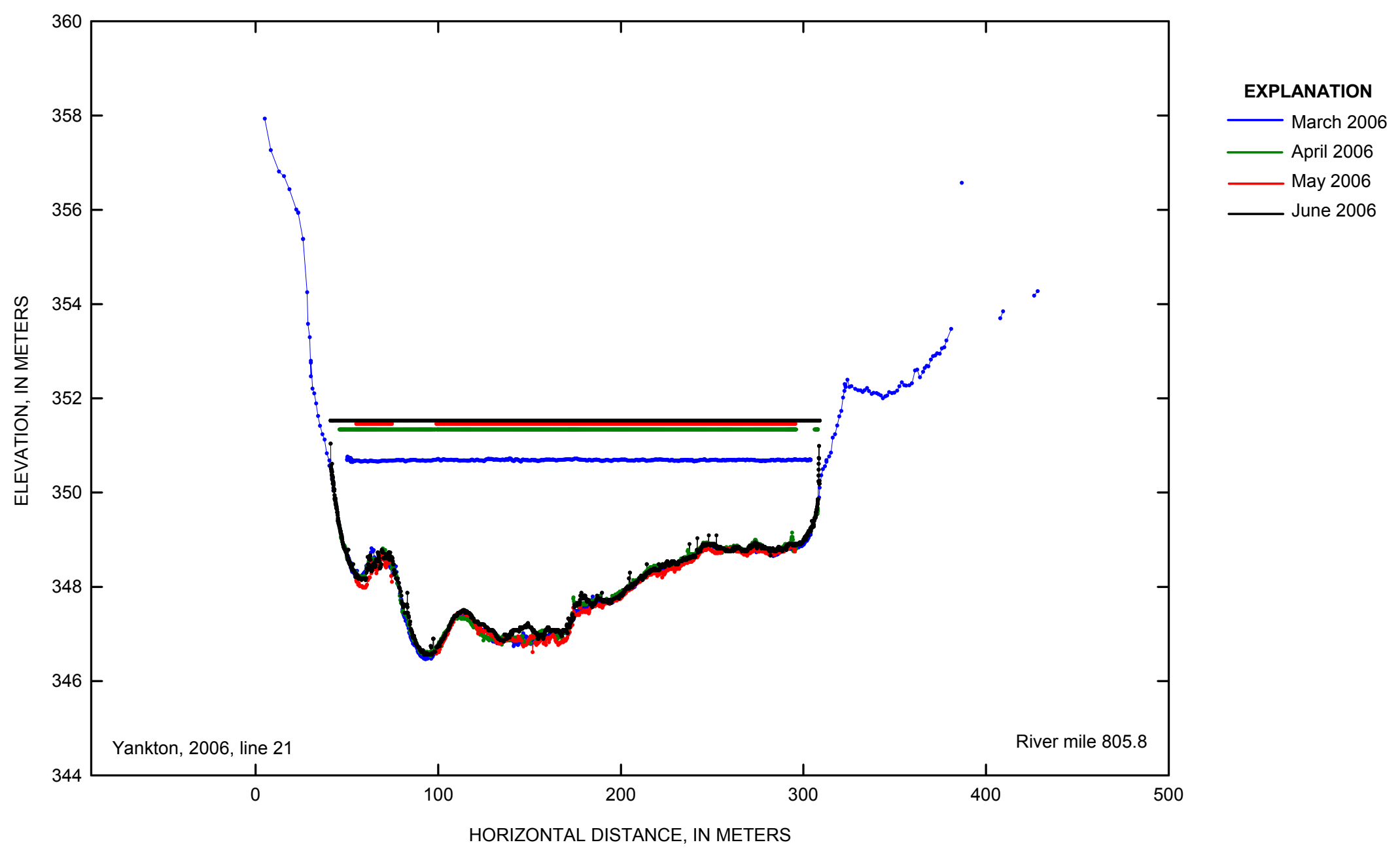

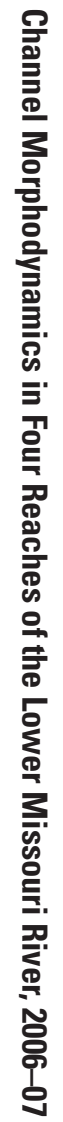

Figure 1-41. Yankton cross-section line 21 at Missouri River mile 805.8 in 2006. 


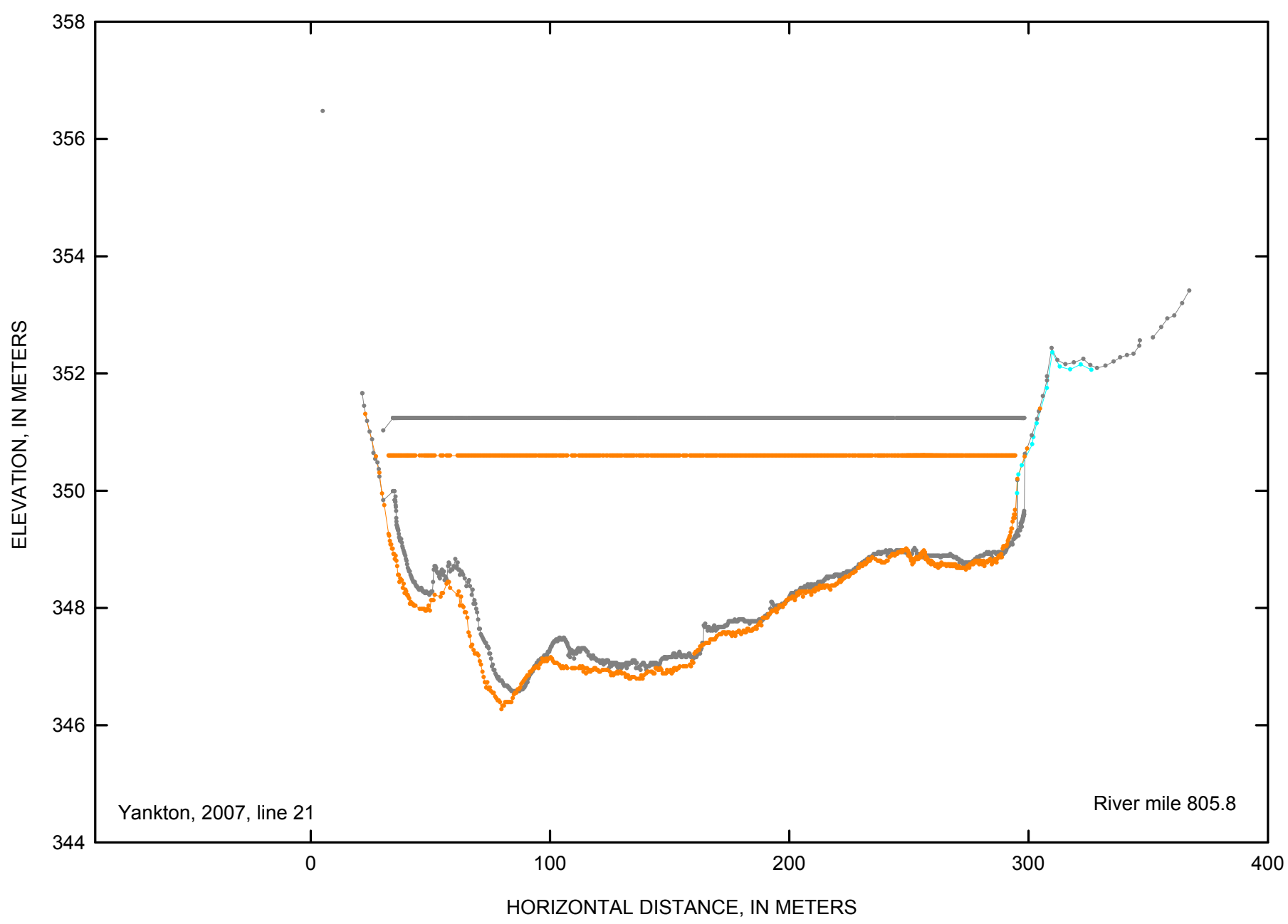

EXPLANATION

March 2007

June 2007

November 2007

Figure 1-42. Yankton cross-section line 21 at Missouri River mile 805.8 in 2007. 


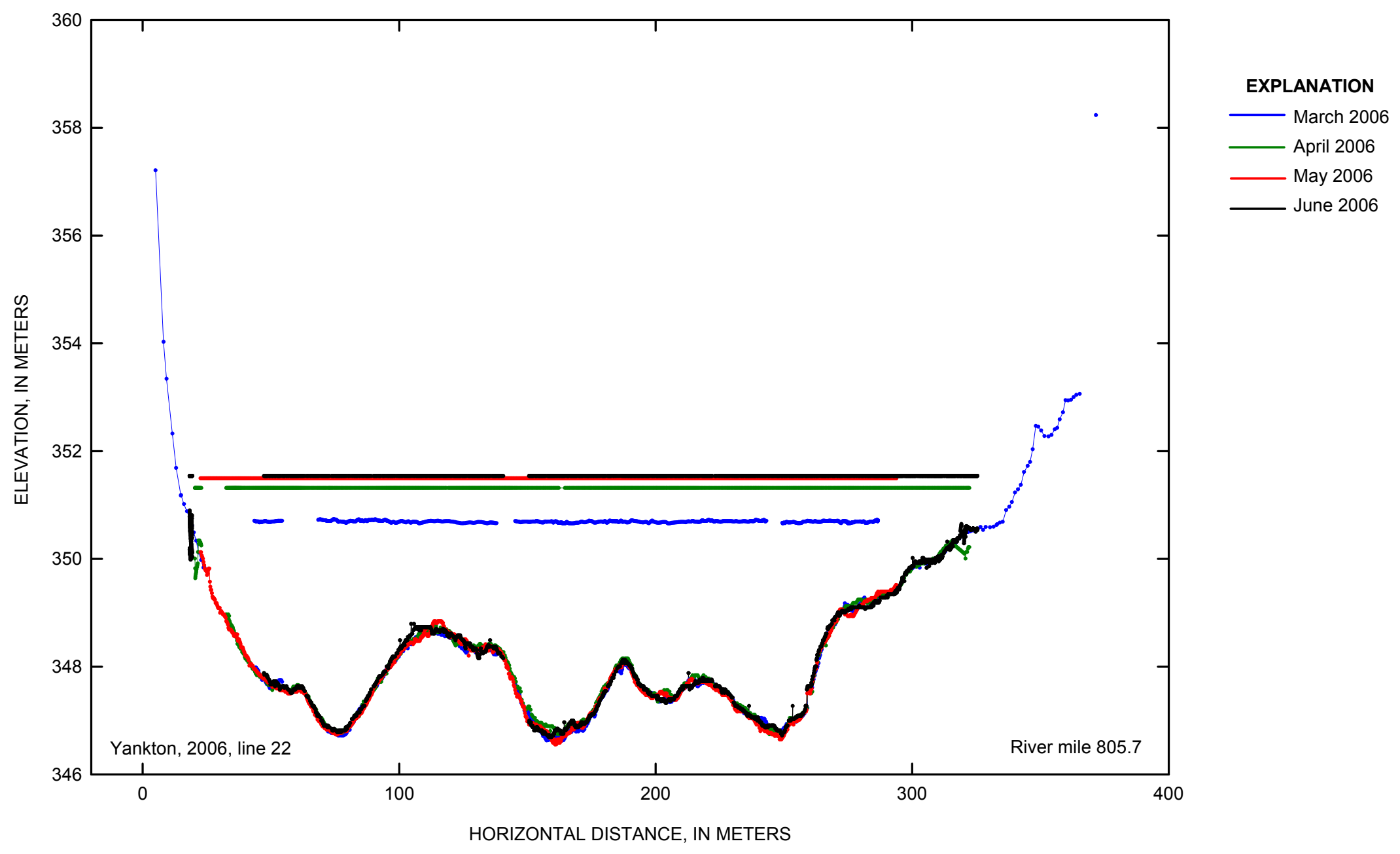

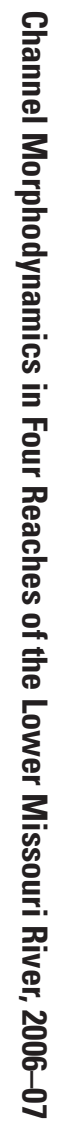

Figure 1-43. Yankton cross-section line 22 at Missouri River mile 805.7 in 2006. 


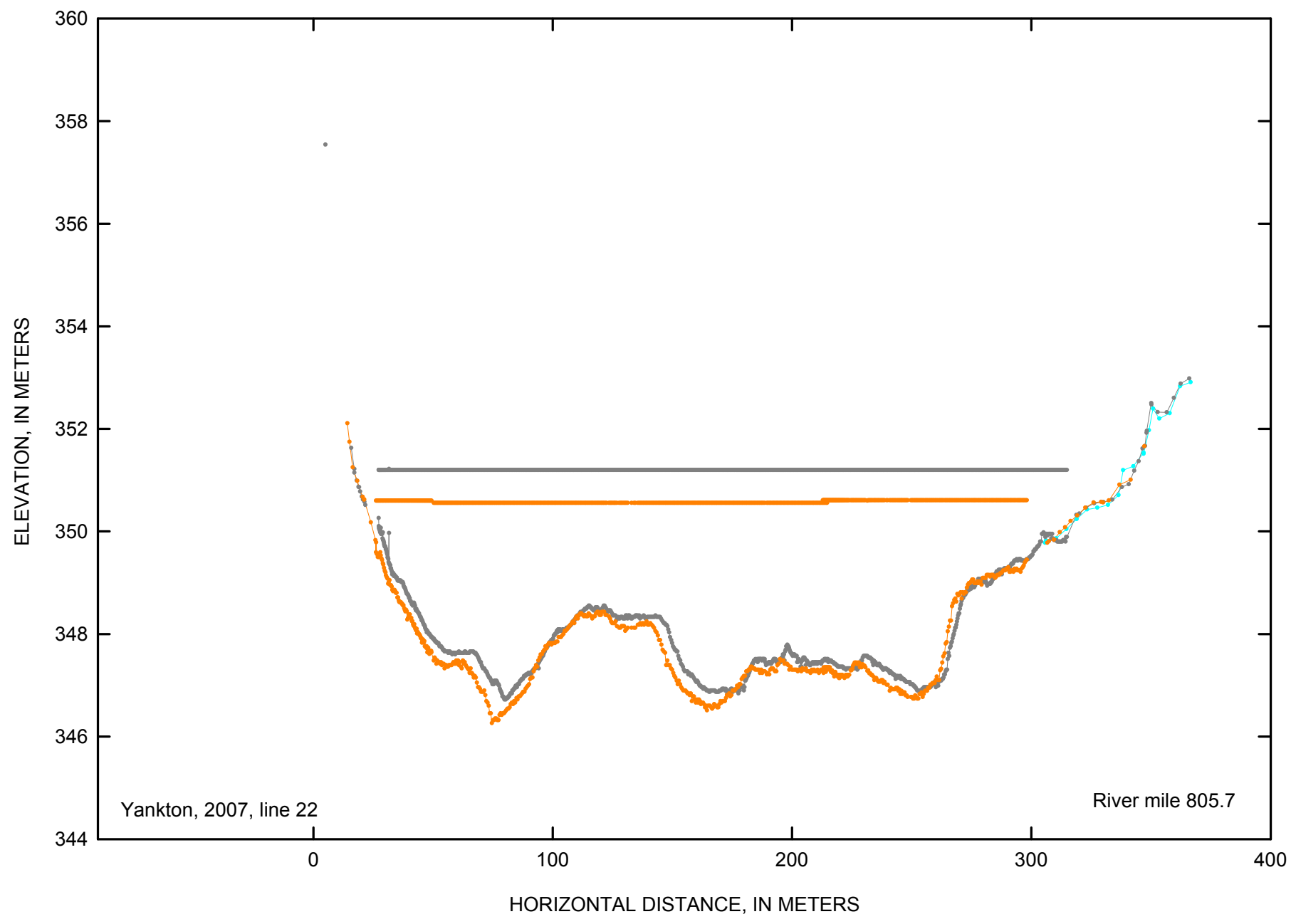

EXPLANATION

March 2007

June 2007

November 2007

Figure 1-44. Yankton cross-section line 22 at Missouri River mile 805.7 in 2007. 


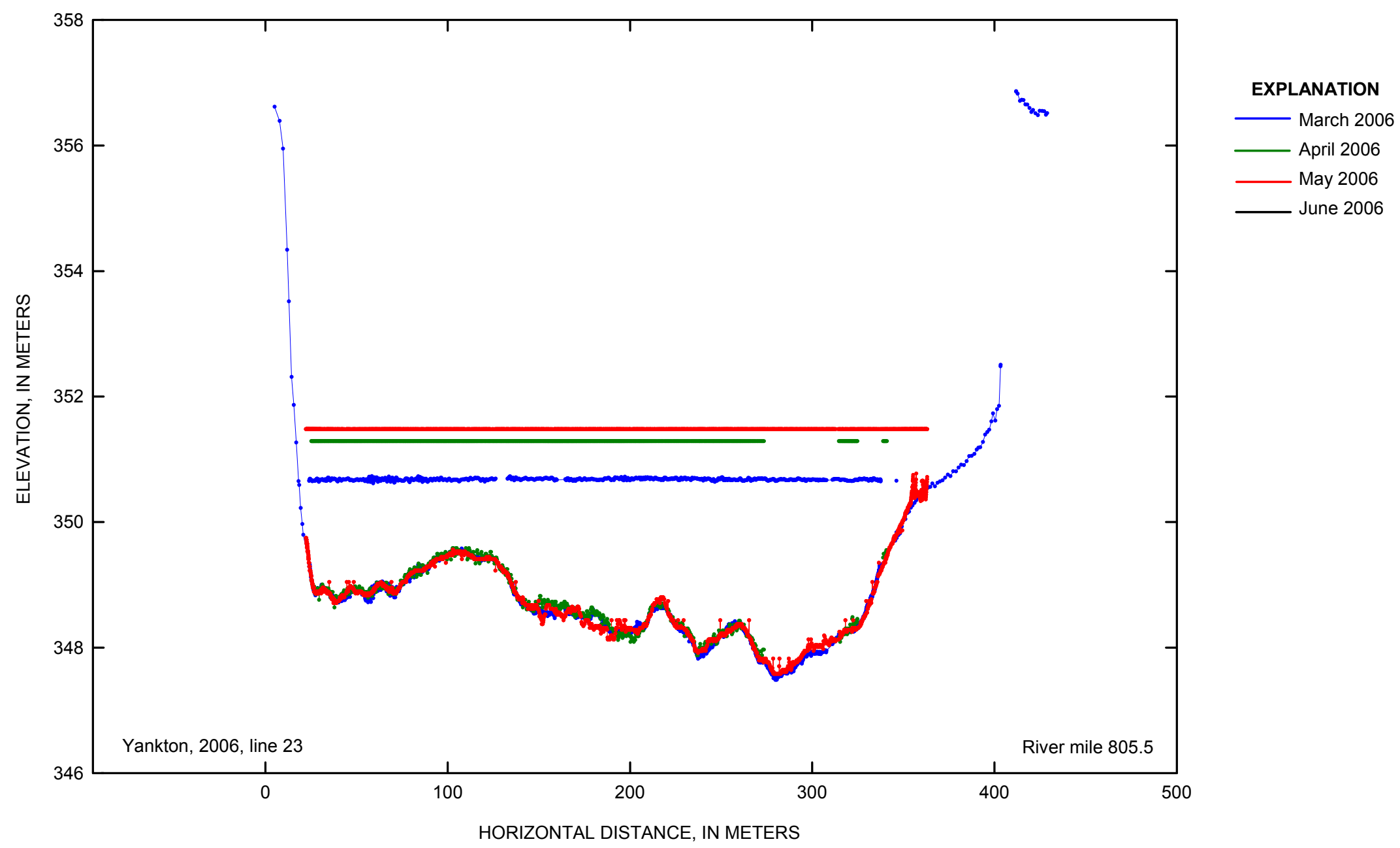

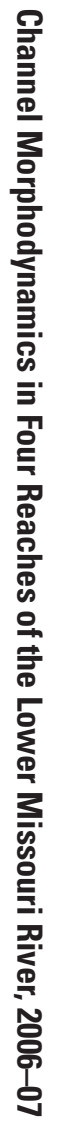

Figure 1-45. Yankton cross-section line 23 at Missouri River mile 805.5 in 2006. 


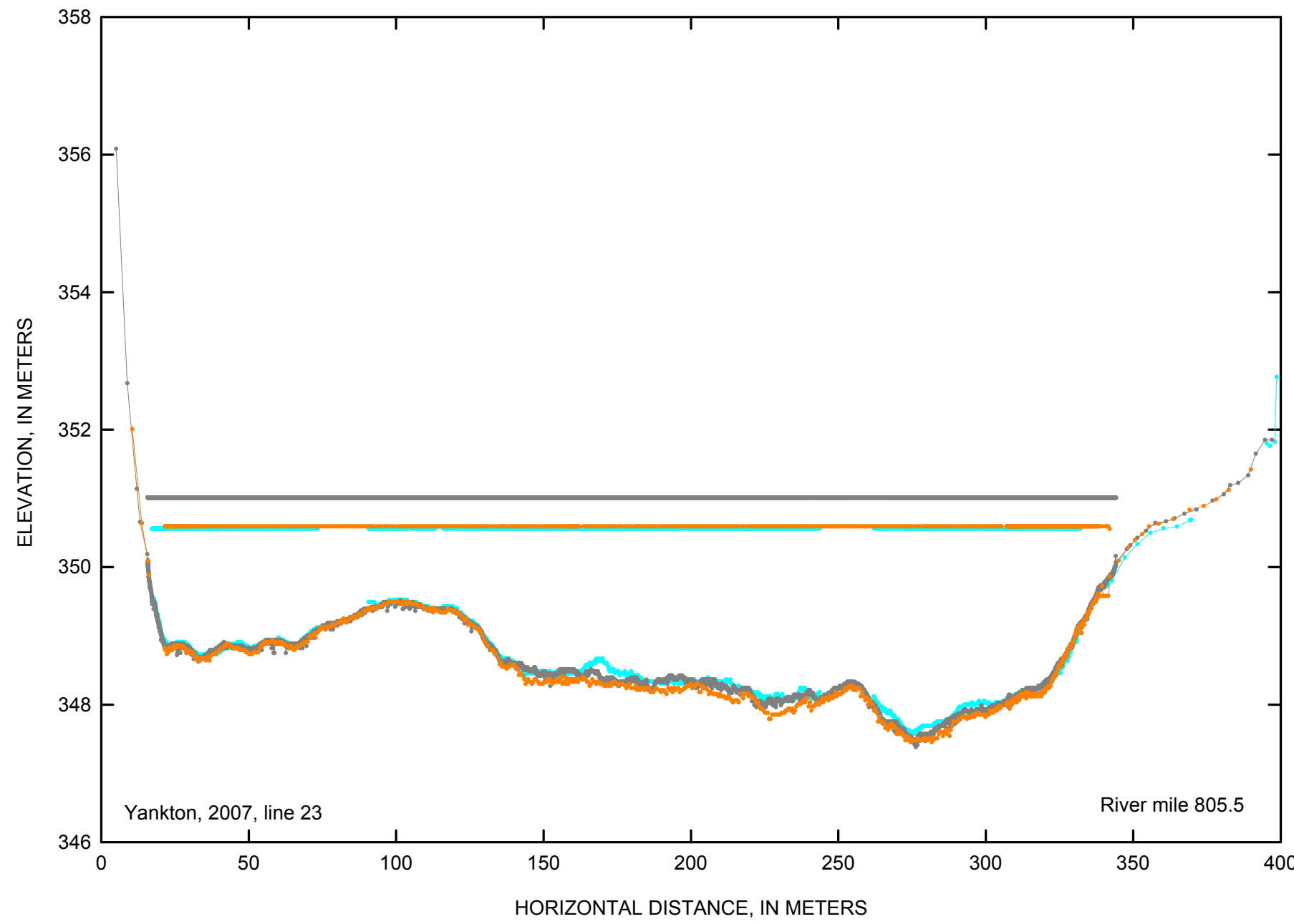

\section{EXPLANATION}

March 2007

June 2007

November 2007

Figure 1-46. Yankton cross-section line 23 at Missouri River mile 805.5 in 2007. 


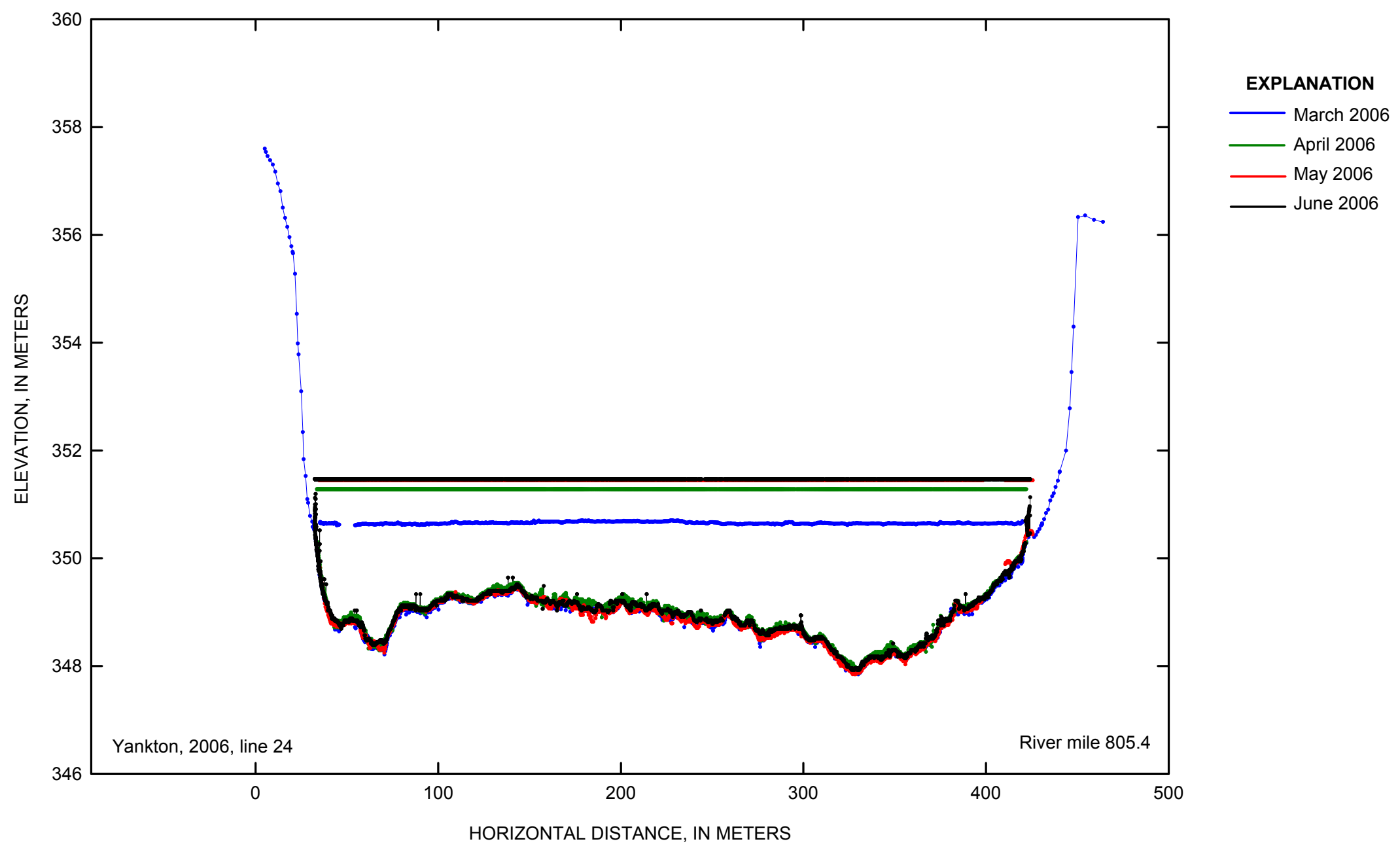

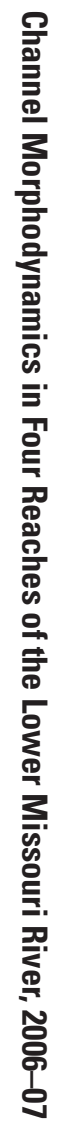

Figure 1-47. Yankton cross-section line 24 at Missouri River mile 805.4 in 2006. 


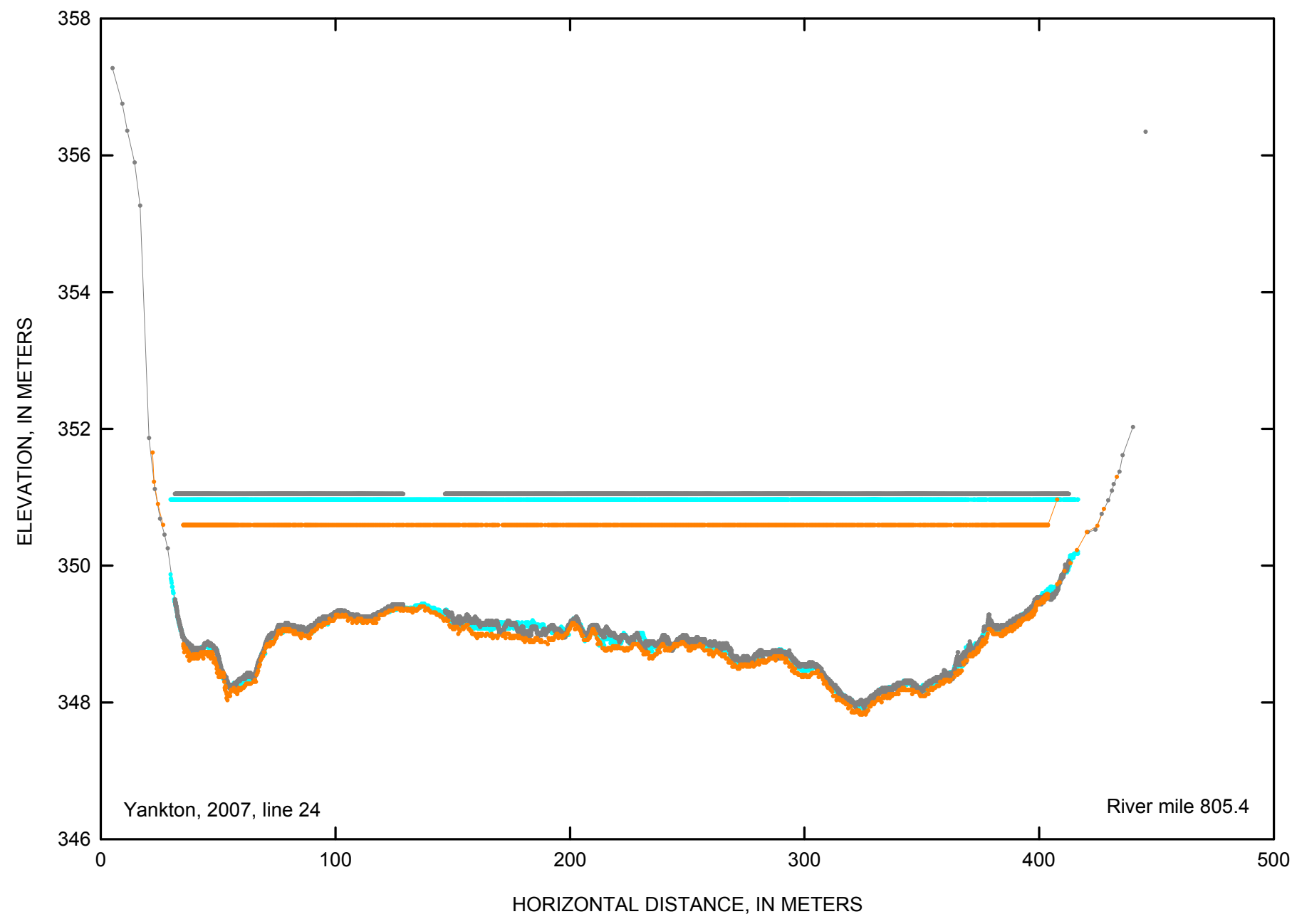

EXPLANATION

March 2007

June 2007

November 2007

Figure 1-48. Yankton cross-section line 24 at Missouri River mile 805.4 in 2007. 


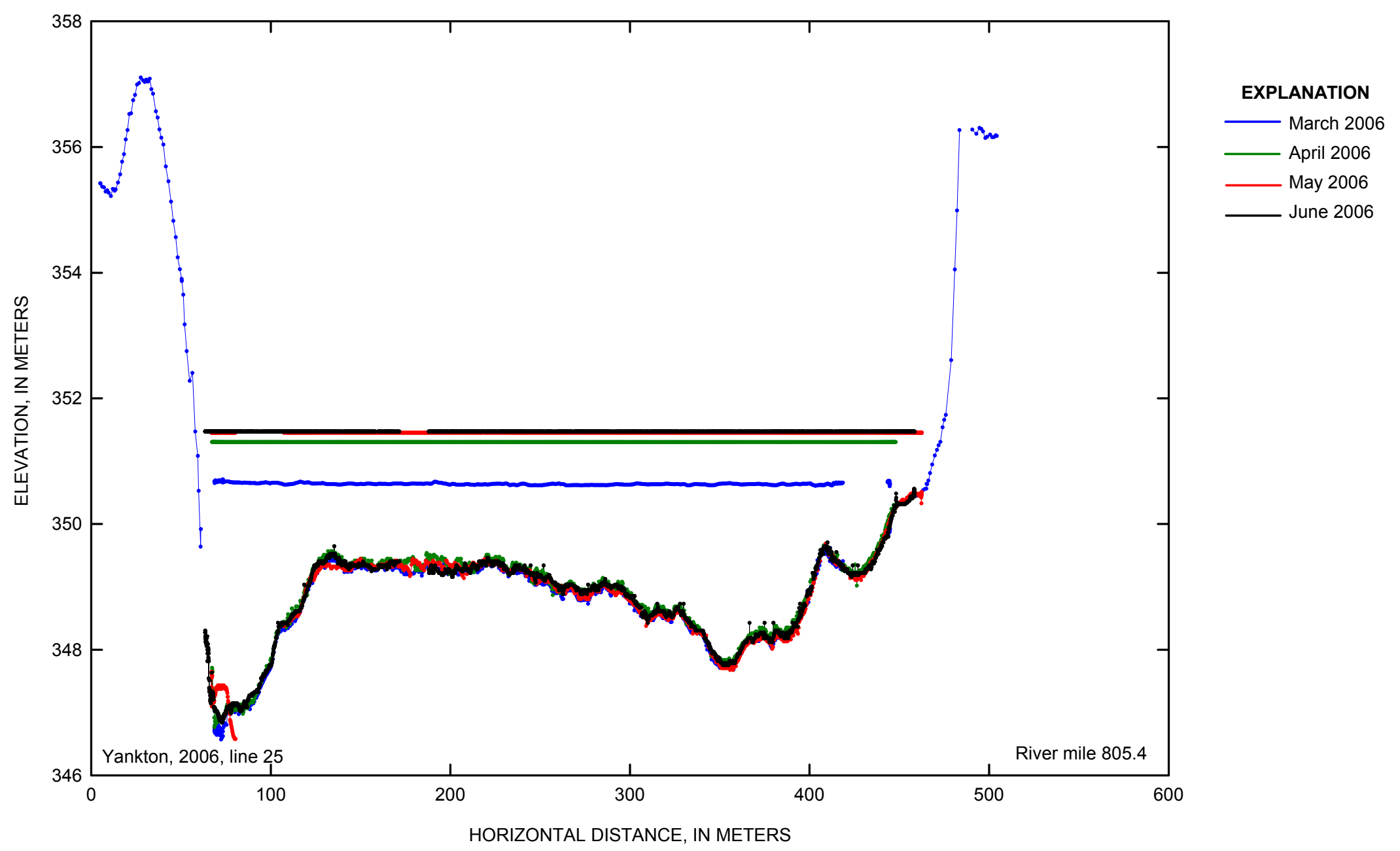

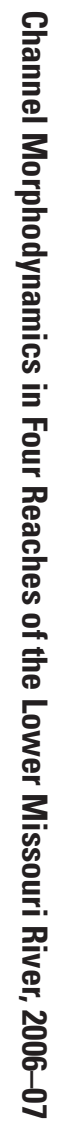

Figure 1-49. Yankton cross-section line 25 at Missouri River mile 805.4 in 2006. 


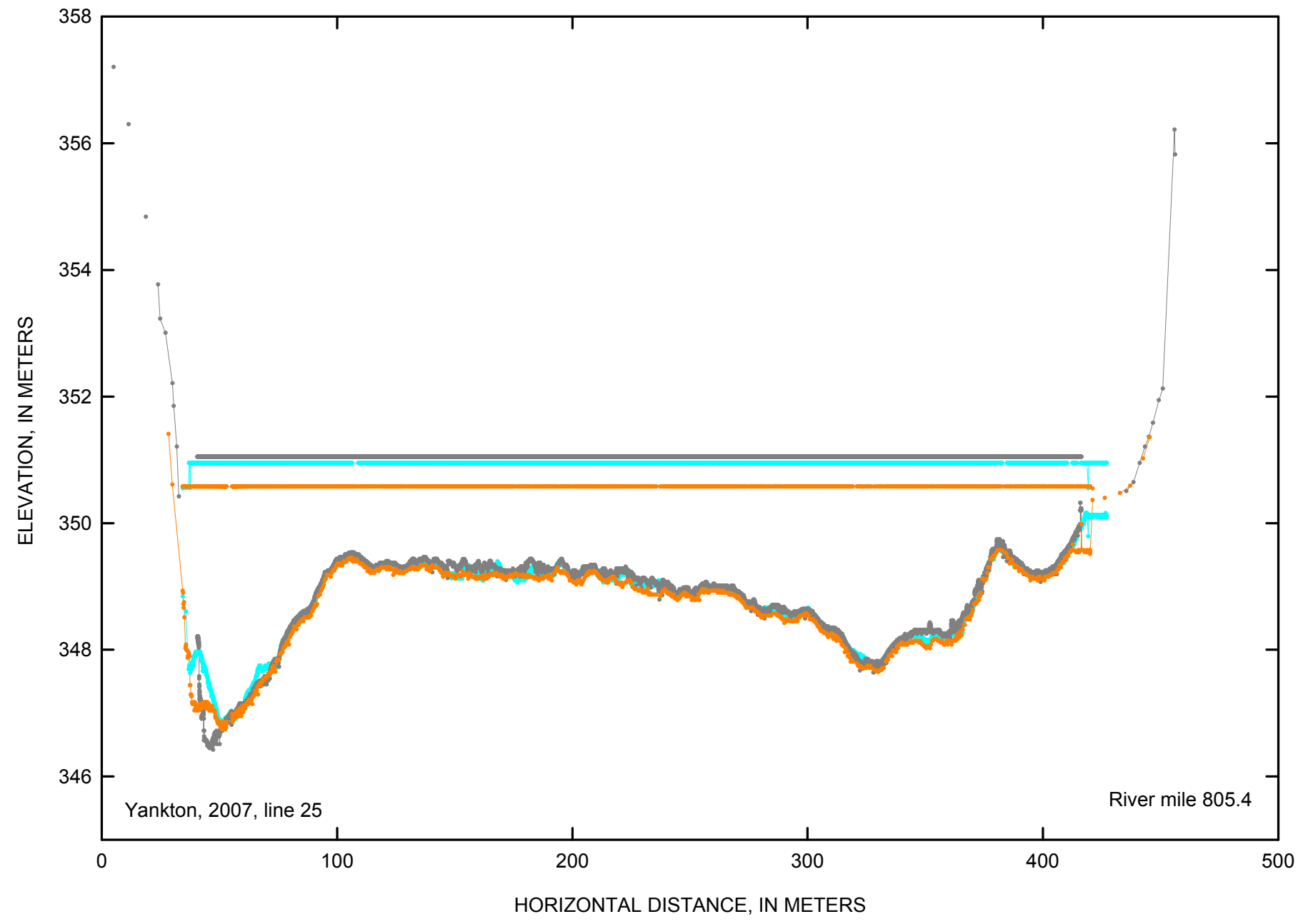

\section{EXPLANATION}

March 2007

June 2007

November 2007

Figure 1-50. Yankton cross-section line 25 at Missouri River mile 805.4 in 2007. 


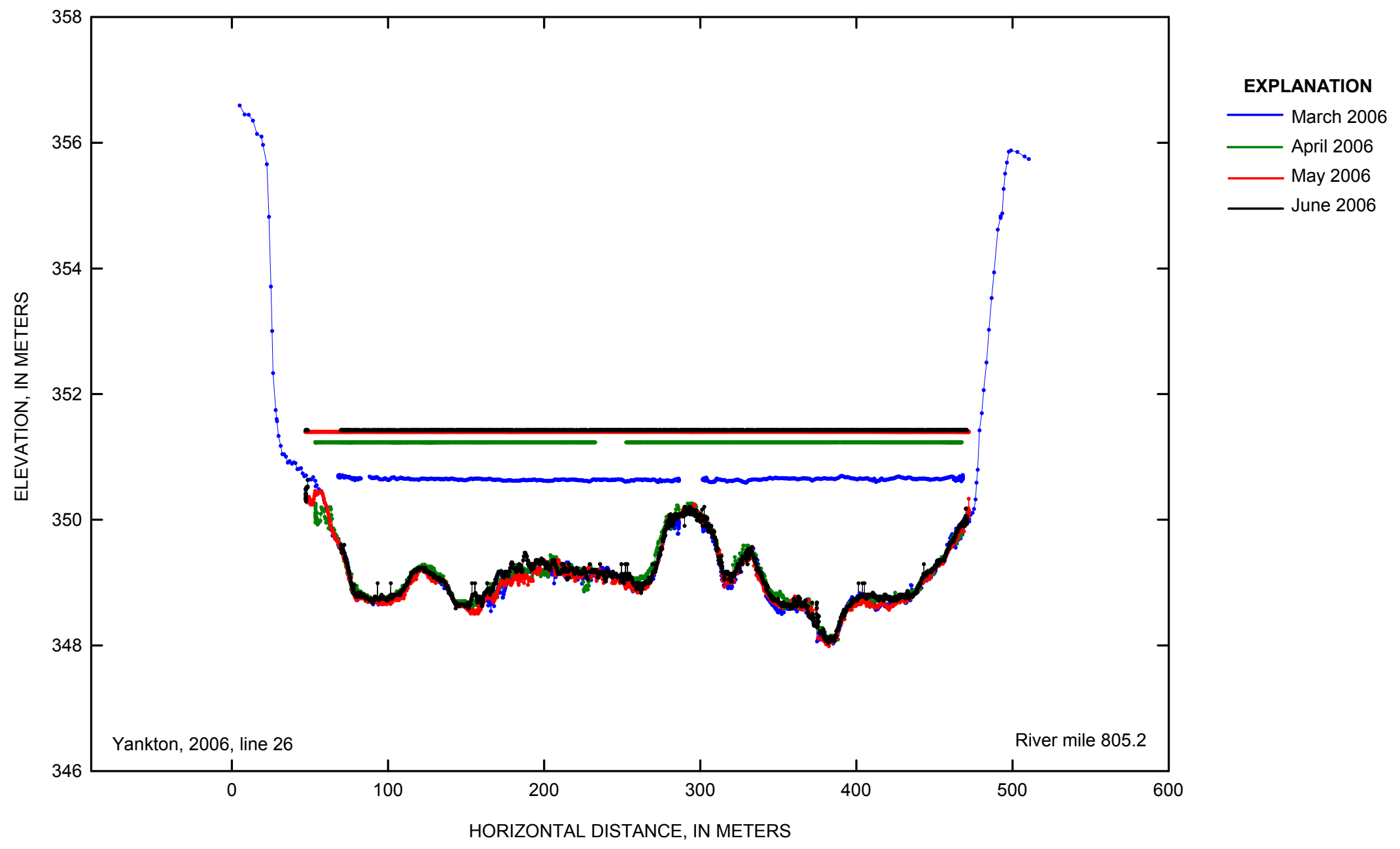

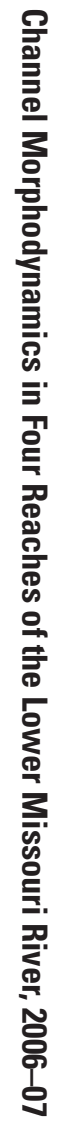

Figure 1-51. Yankton cross-section line 26 at Missouri River mile 805.2 in 2006. 


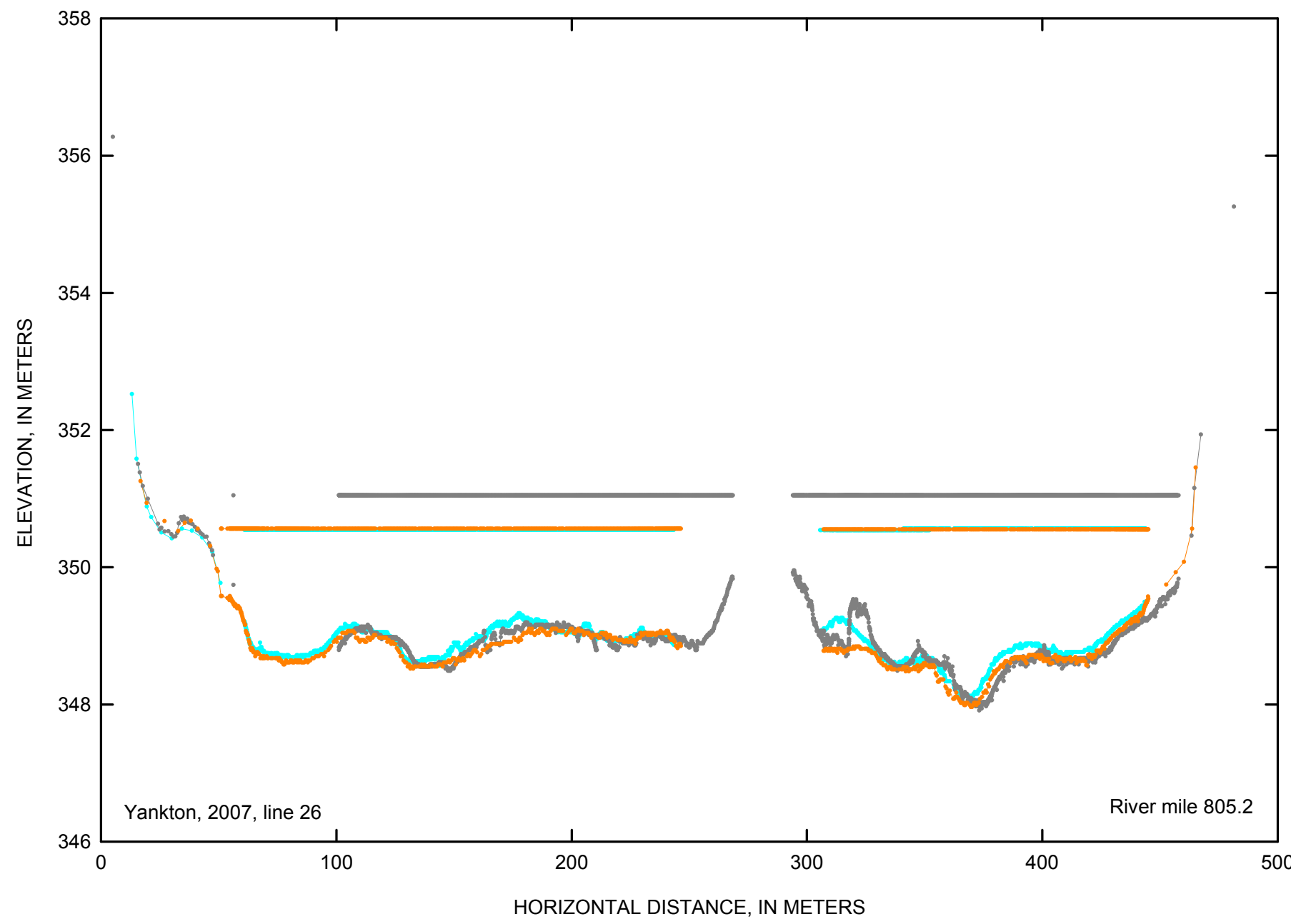

EXPLANATION

March 2007

June 2007

November 2007

Figure 1-52. Yankton cross-section line 26 at Missouri River mile 805.2 in 2007. 


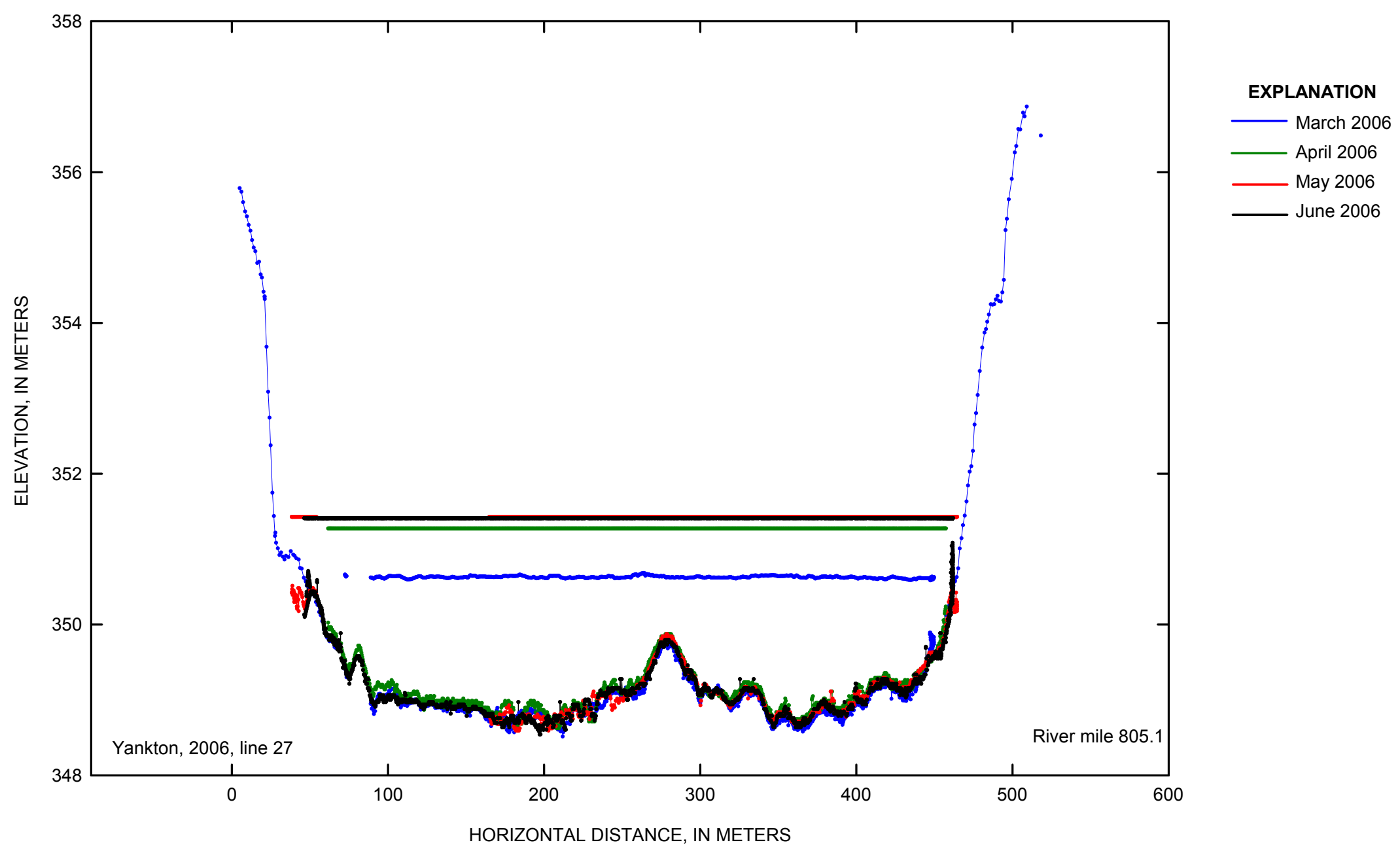

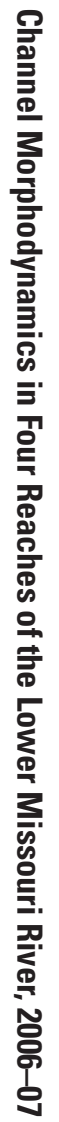

Figure 1-53. Yankton cross-section line 27 at Missouri River mile 805.1 in 2006. 


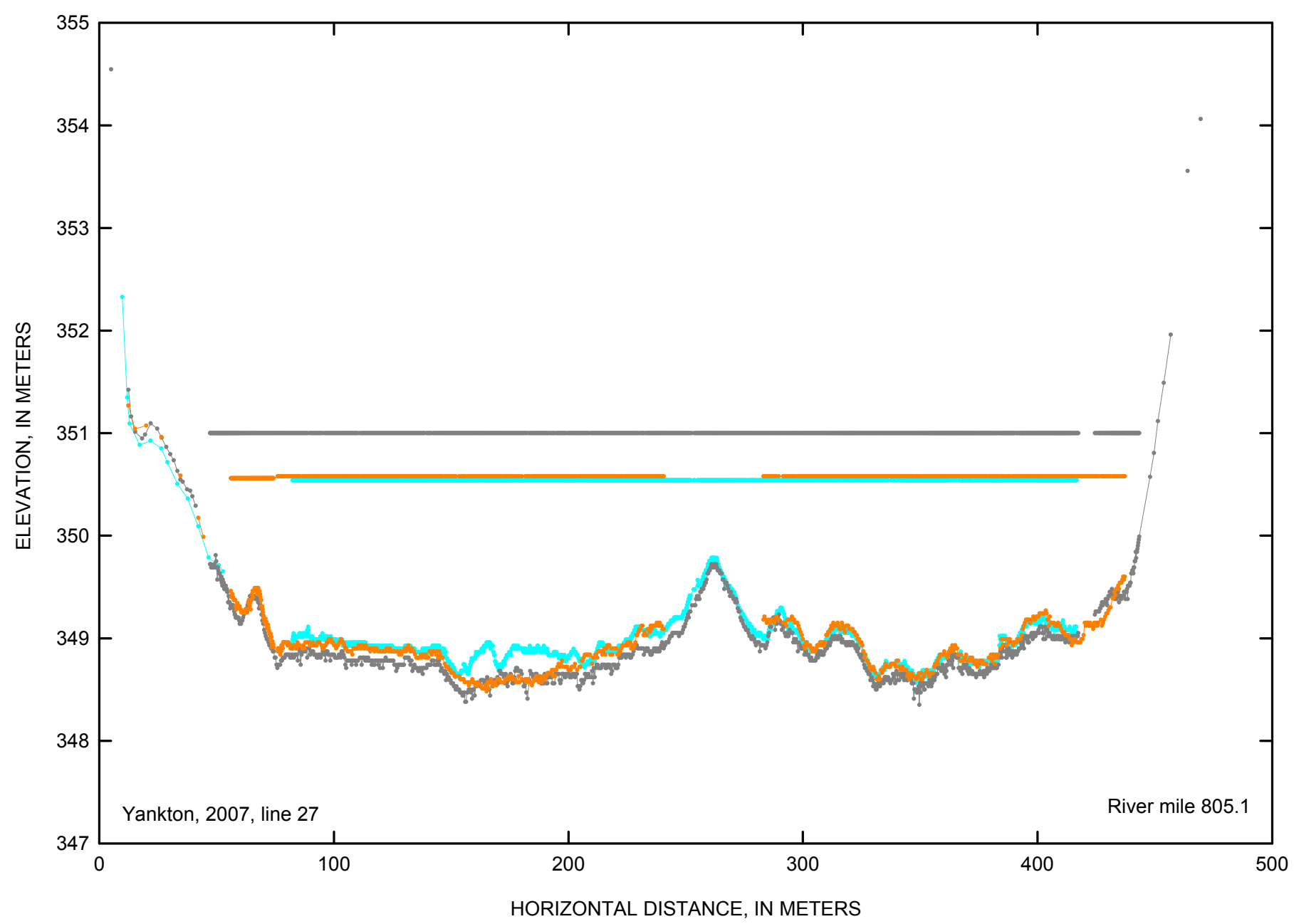

EXPLANATION

- March 2007

June 2007

- November 2007

Figure 1-54. Yankton cross-section line 27 at Missouri River mile 805.1 in 2007 


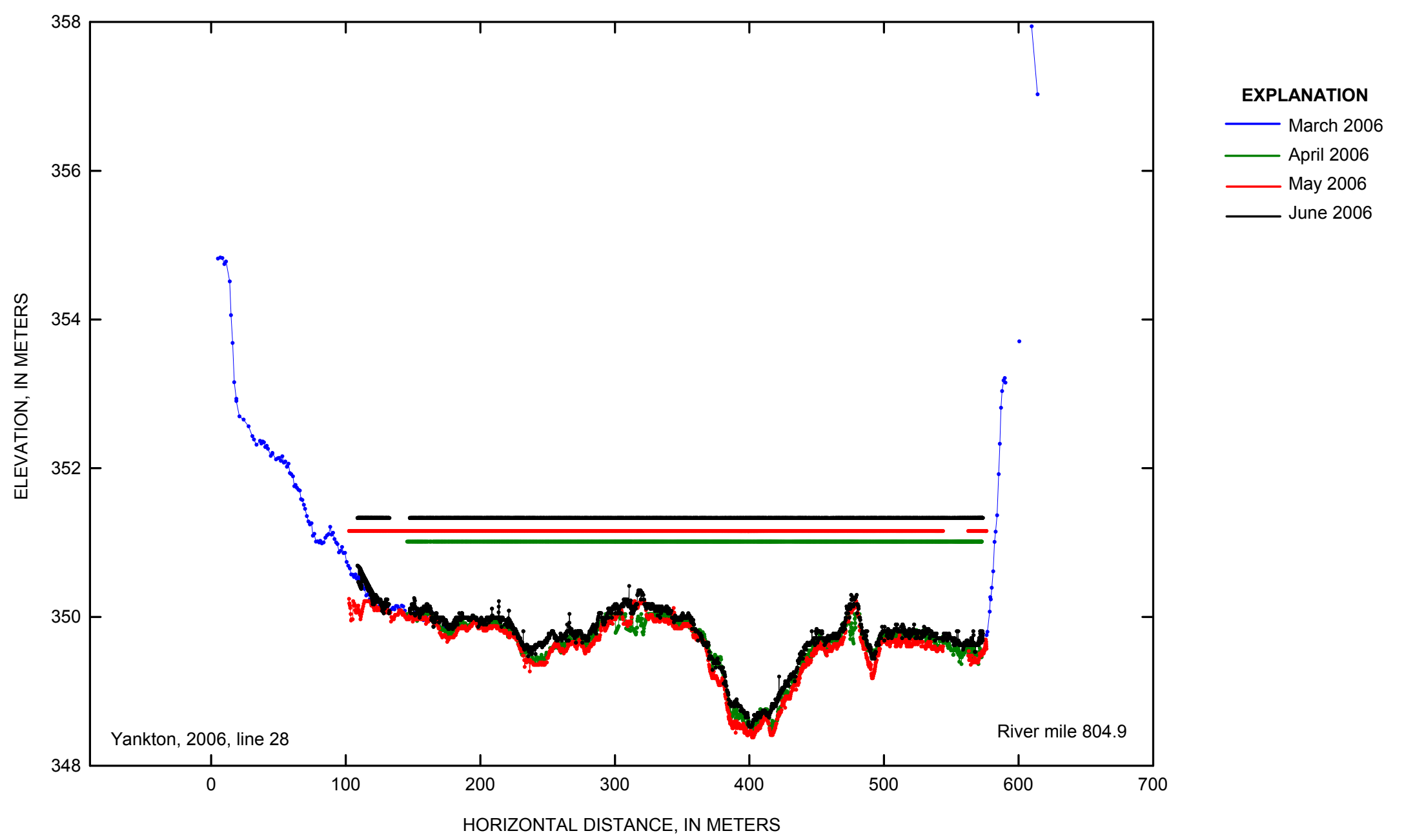

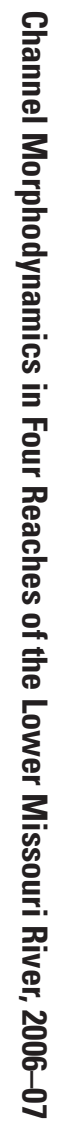

Figure 1-55. Yankton cross-section line 28 at Missouri River mile 804.9 in 2006. 


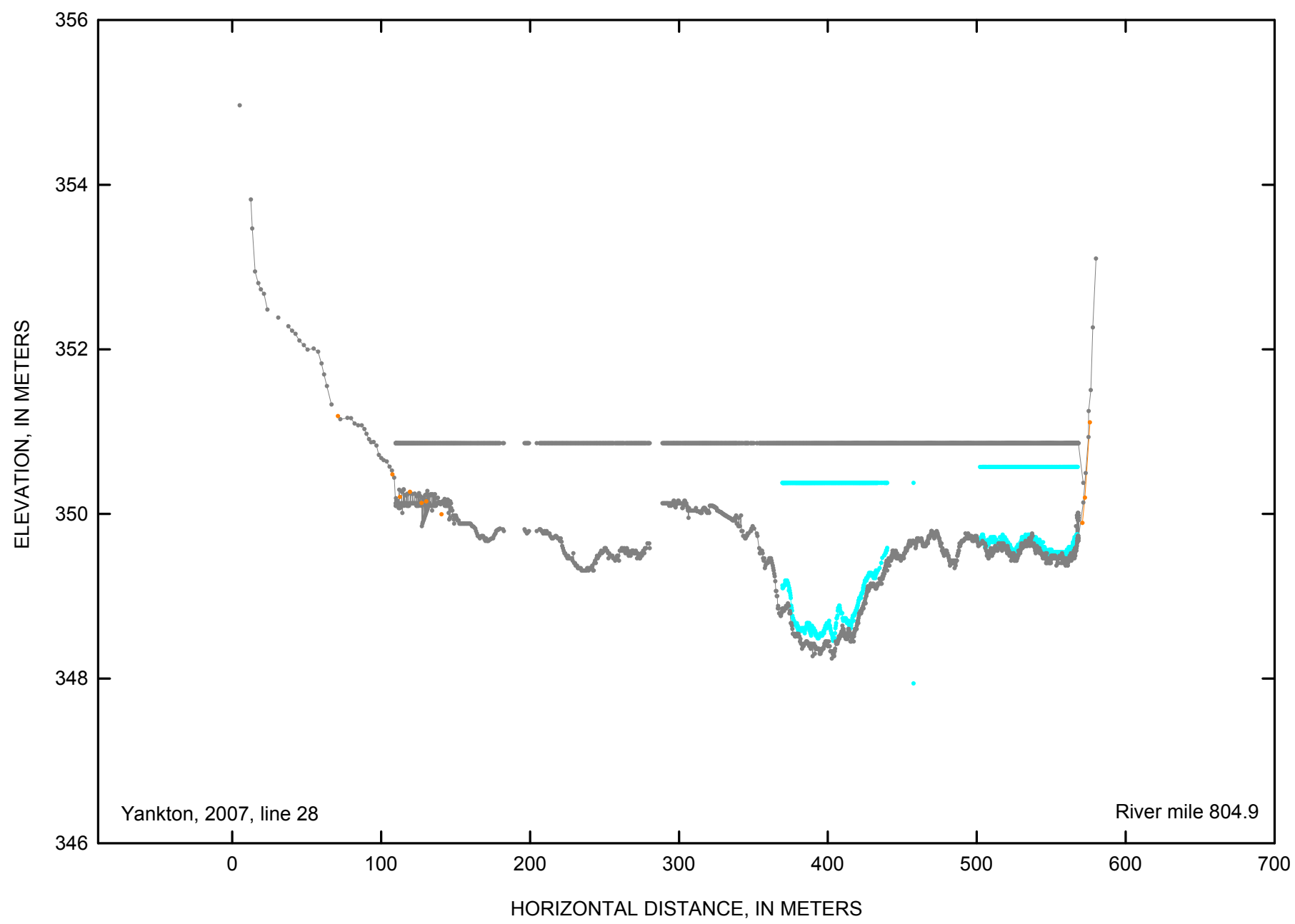

EXPLANATION

March 2007

June 2007

November 2007

Figure 1-56. Yankton cross-section line 28 at Missouri River mile 804.9 in 2007. 


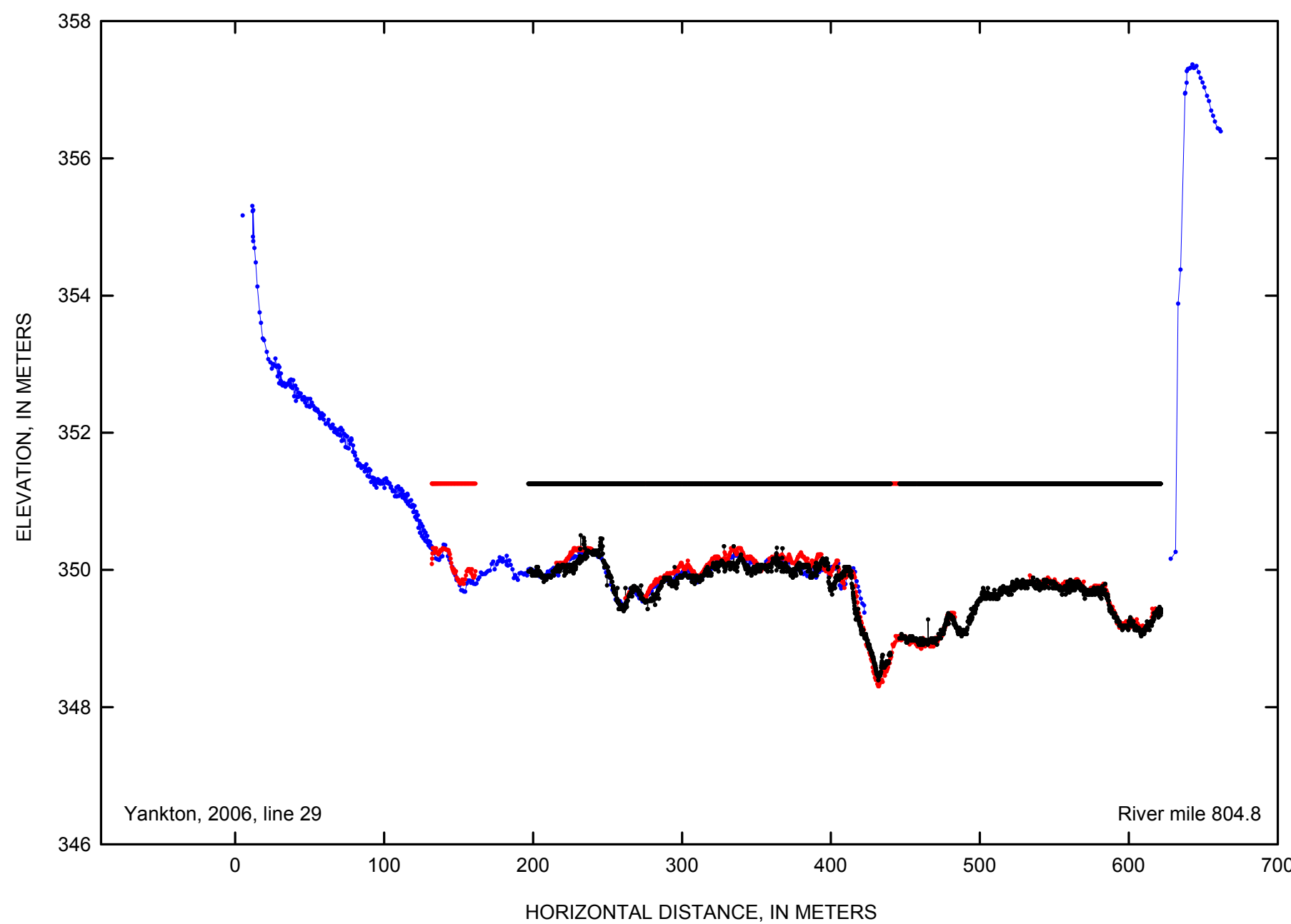

EXPLANATION

March 2006

April 2006

May 2006

June 2006

Figure 1-57. Yankton cross-section line 29 at Missouri River mile 804.8 in 2006. 


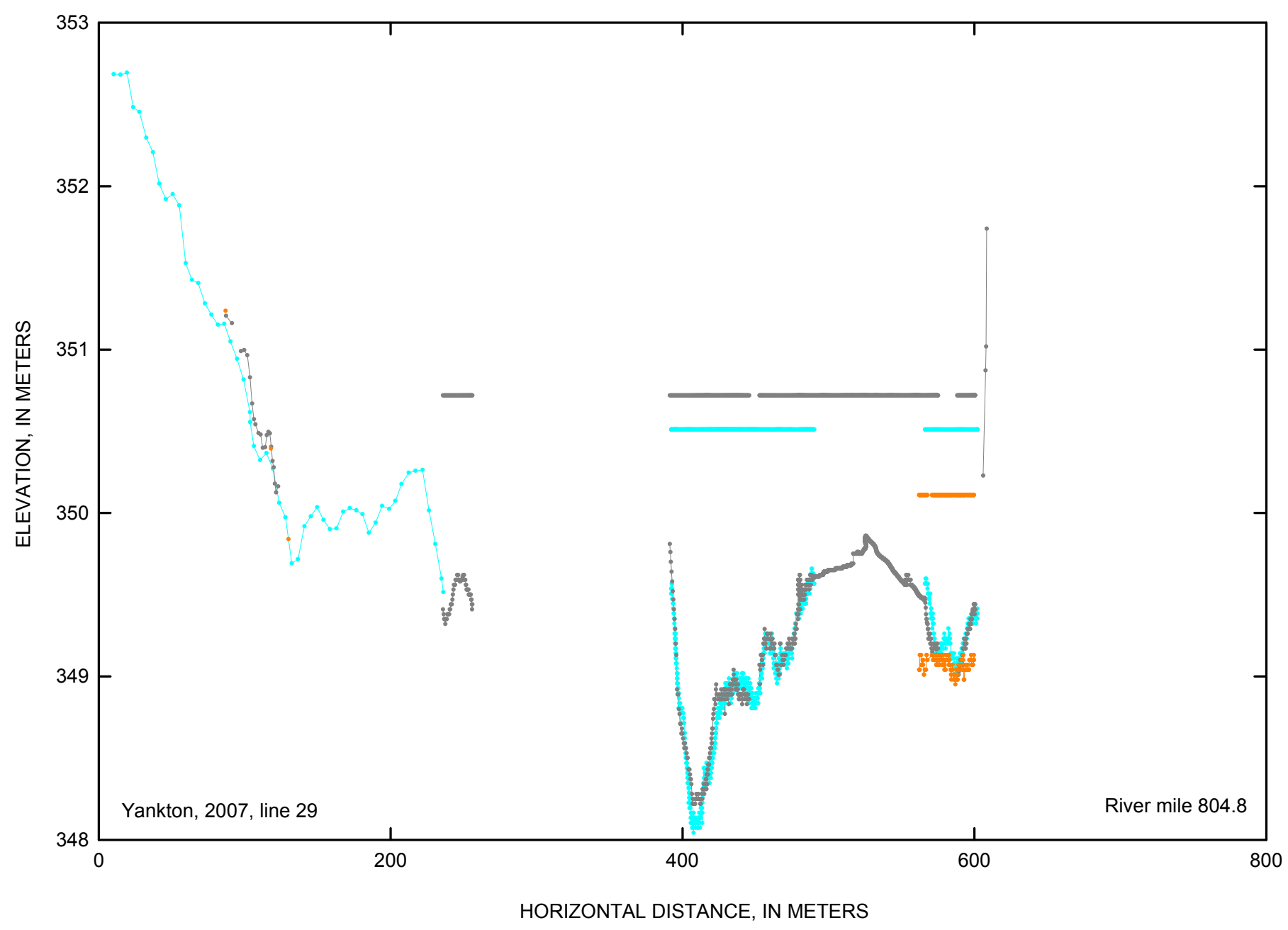

EXPLANATION

March 2007

June 2007

- November 2007

Figure 1-58. Yankton cross-section line 29 at Missouri River mile 804.8 in 2007. 


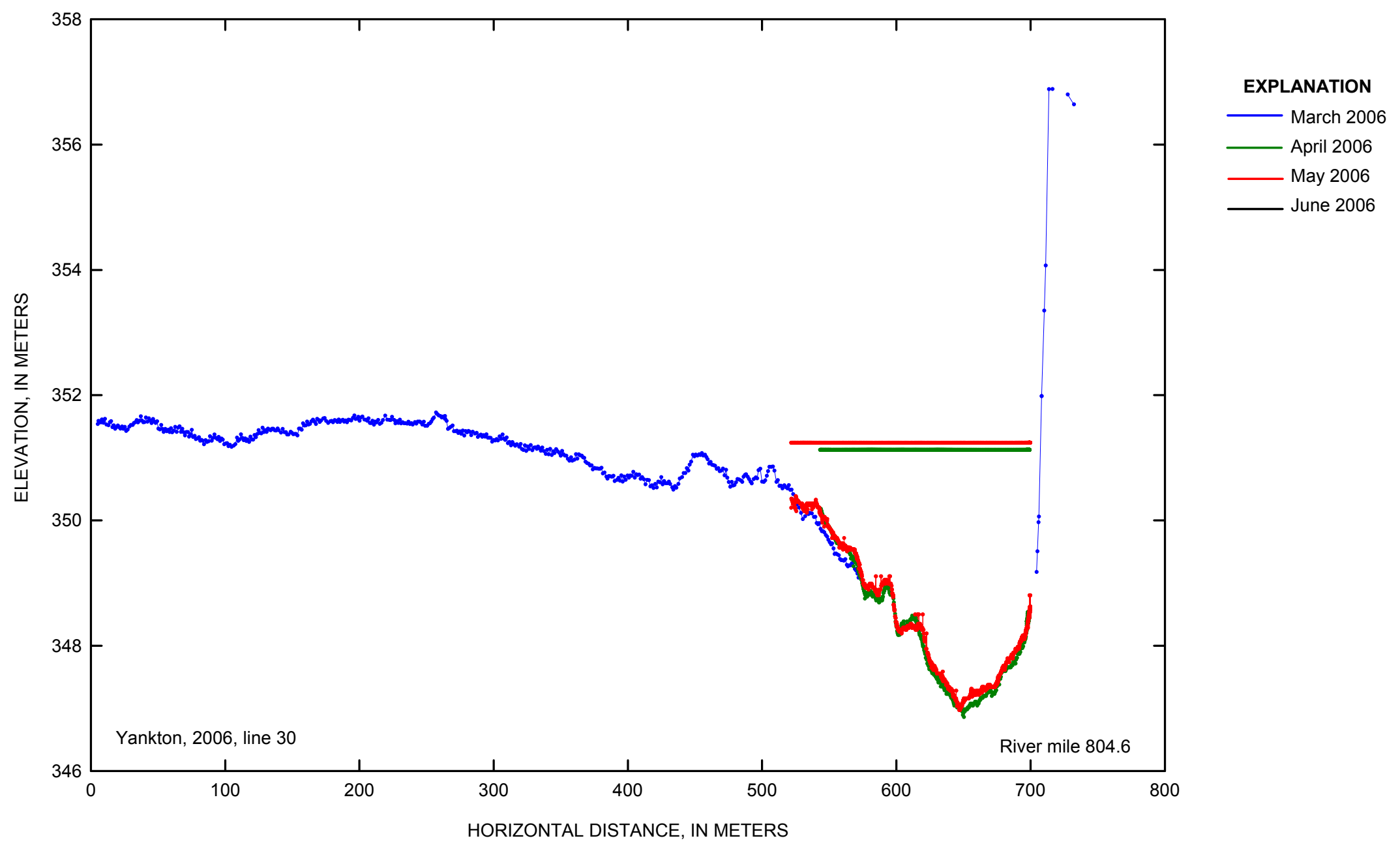

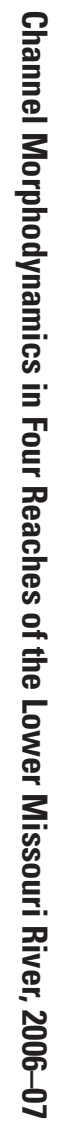

Figure 1-59. Yankton cross-section line 30 at Missouri River mile 804.6 in 2006. 


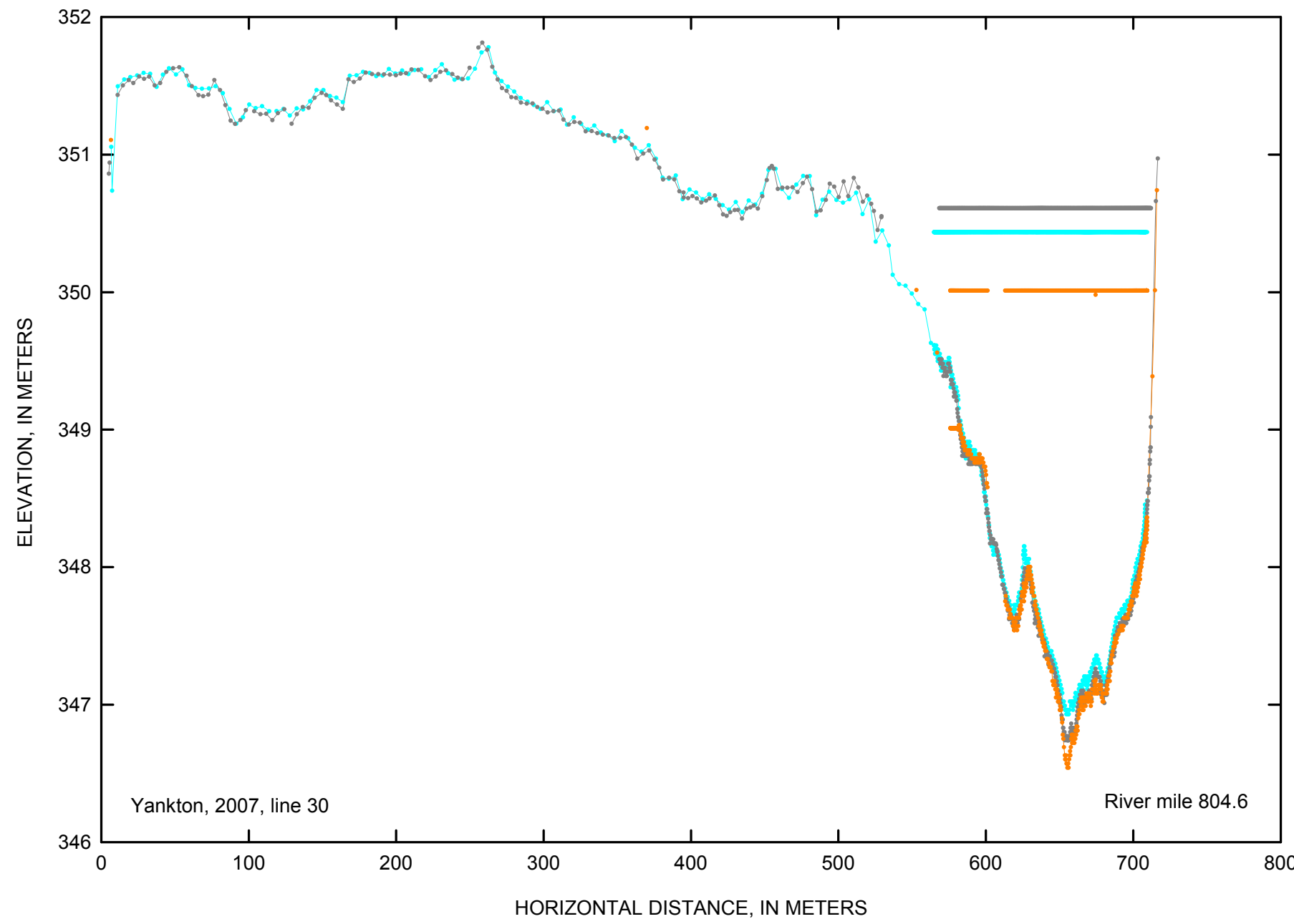

EXPLANATION

- March 2007

June 2007

November 2007

Figure 1-60. Yankton cross-section line 30 at Missouri River mile 804.6 in 2007. 



\section{Appendix 2. Kenslers Bend Reach Cross Sections}




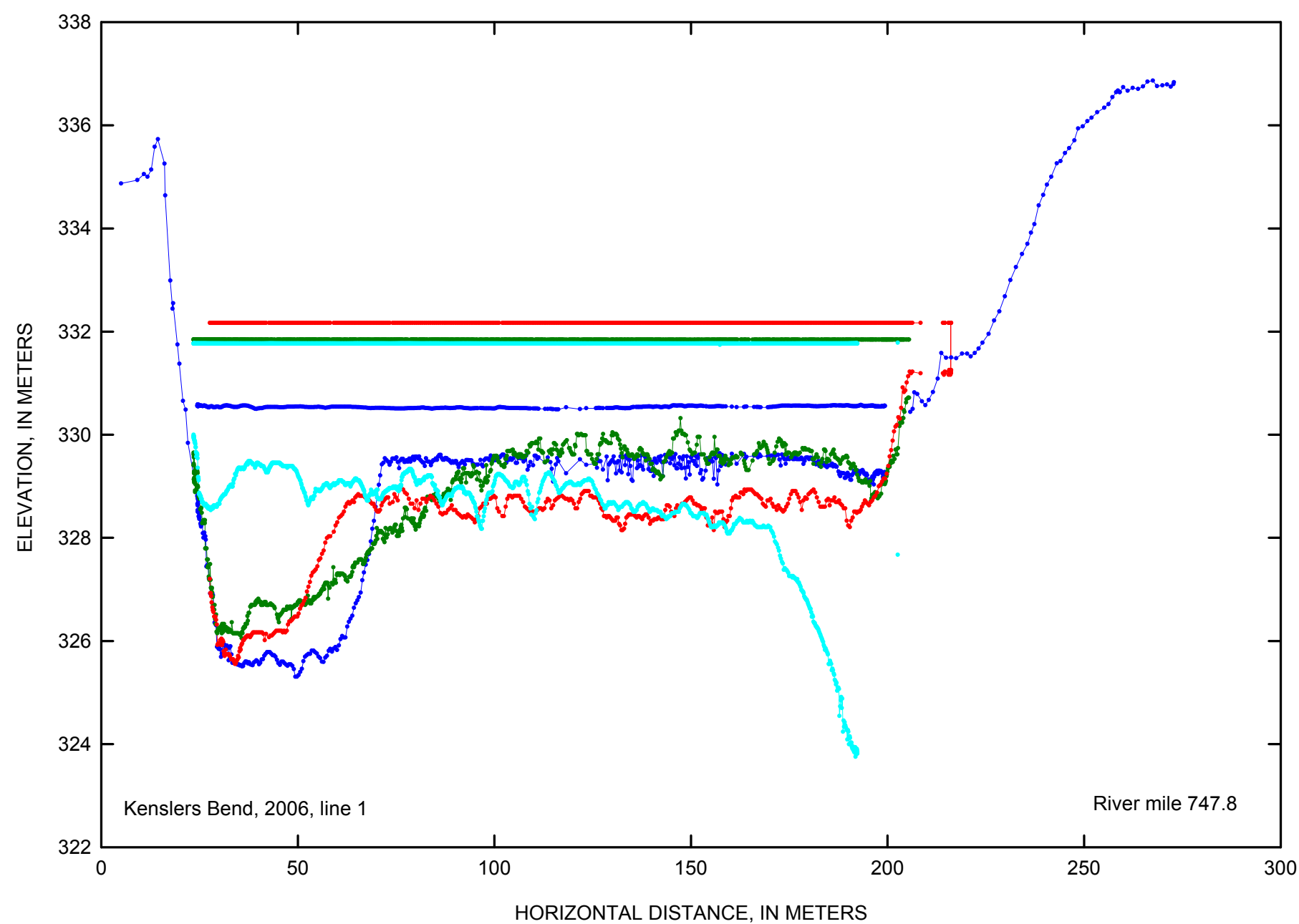

\section{EXPLANATION}

March 2006

March 2006 II

May 2006

June 2006

March 2007

Figure 2-1. Kenslers Bend cross-section line 1 at Missouri River mile 747.8 in 2006. 


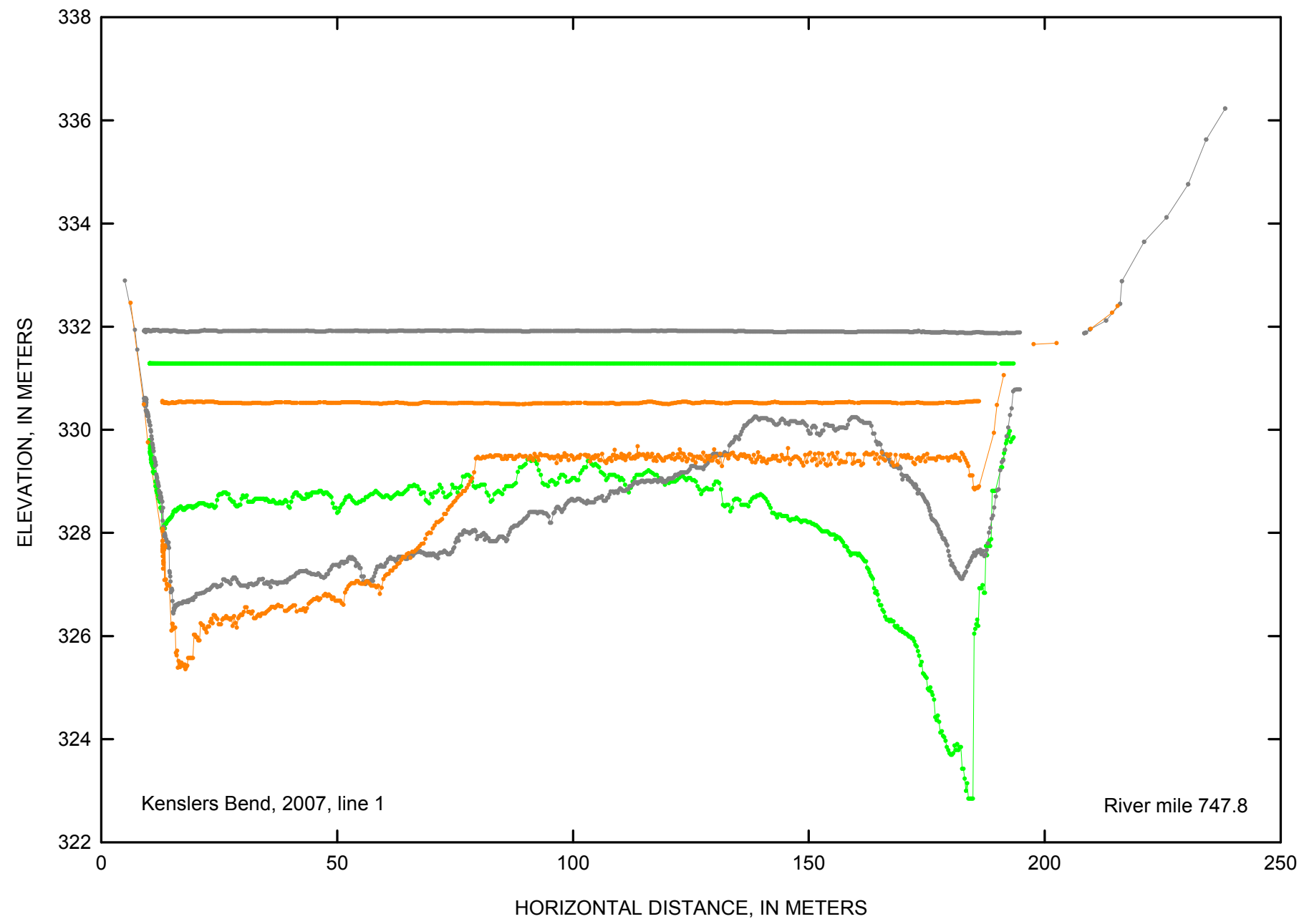

EXPLANATION

April 2007

July 2007

October 2007

Figure 2-2. Kenslers Bend cross-section line 1 at Missouri River mile 747.8 in 2007. 


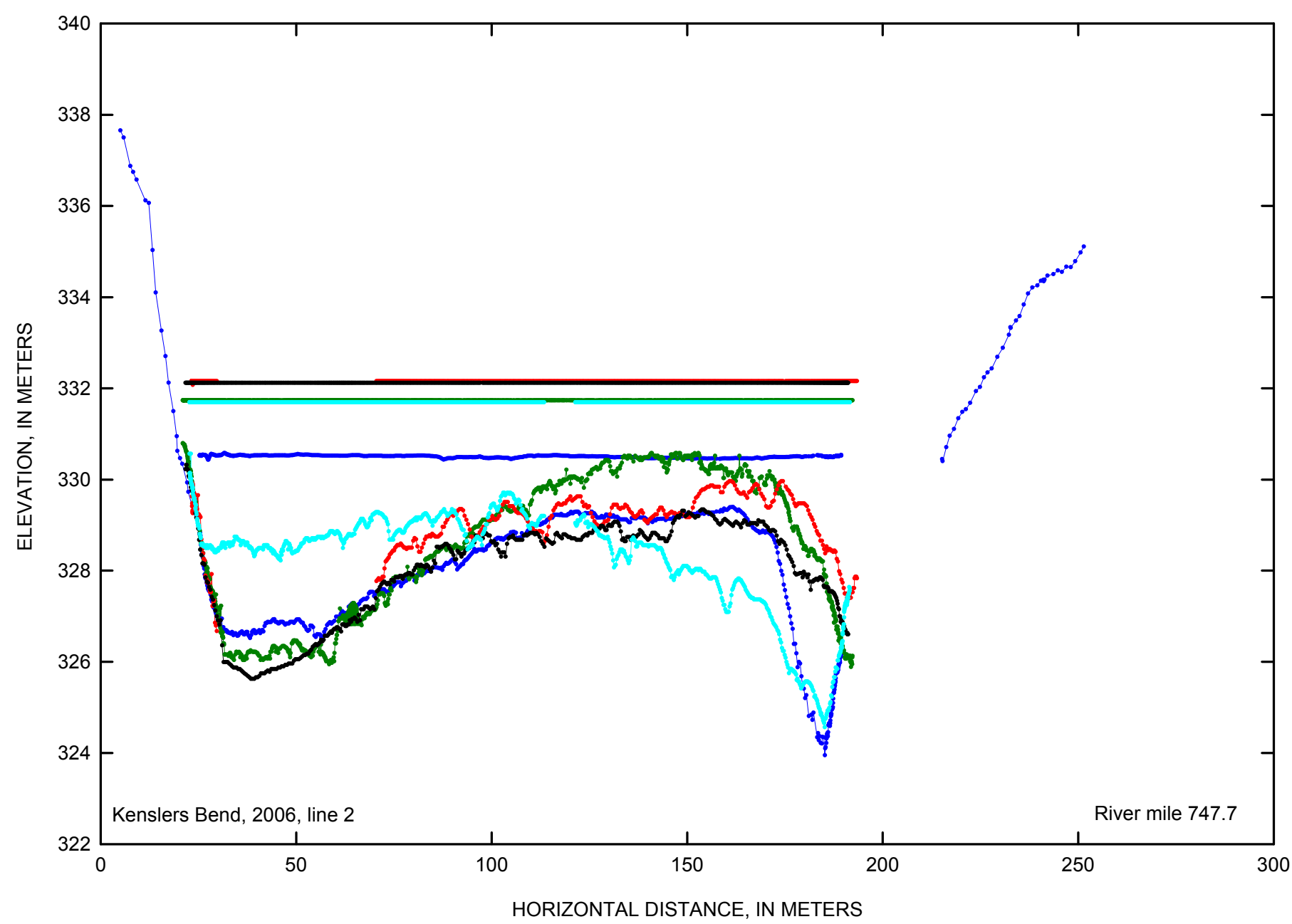

EXPLANATION

March 2006 I

March 2006 II

May 2006

June 2006

March 2007

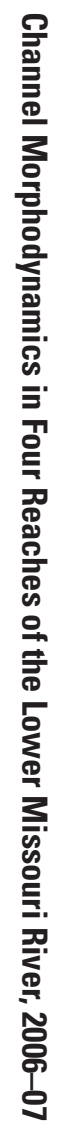

Figure 2-3. Kenslers Bend cross-section line 2 at Missouri River mile 747.7 in 2006. 


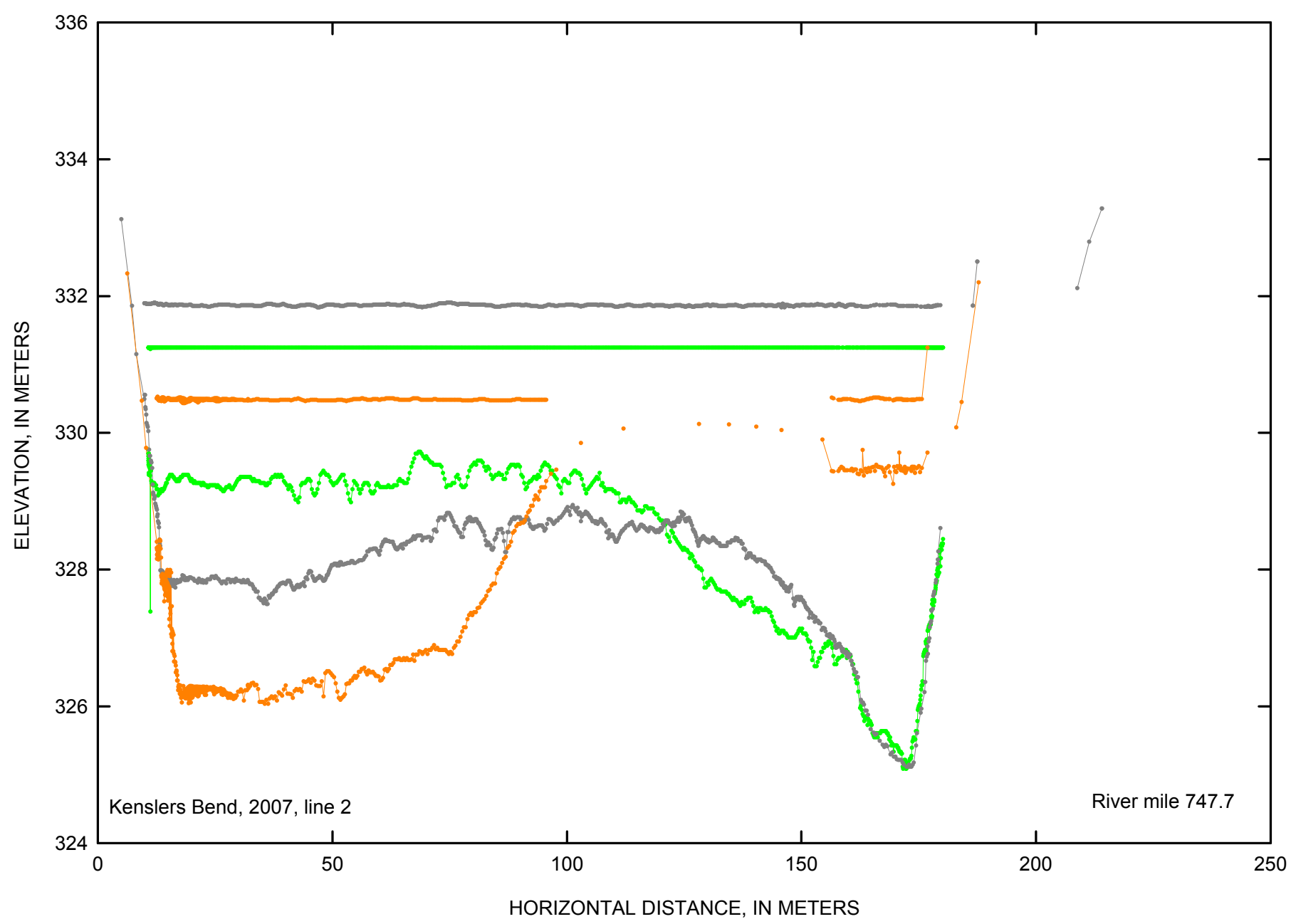

EXPLANATION

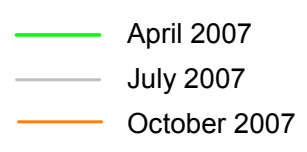

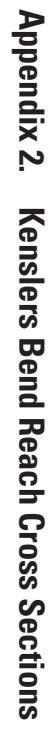




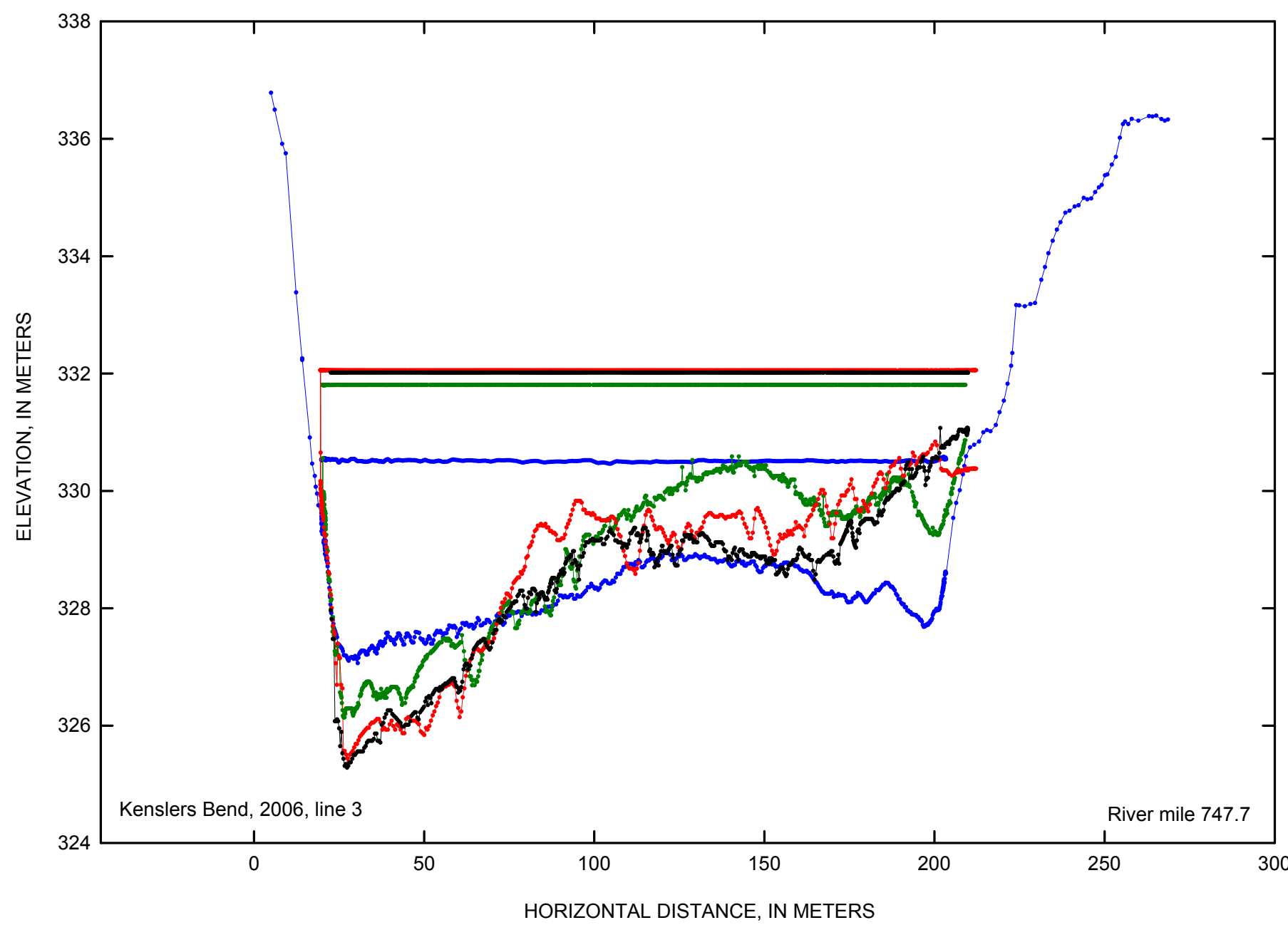

EXPLANATION

March 2006 I

March 2006 II

May 2006

June 2006

March 2007

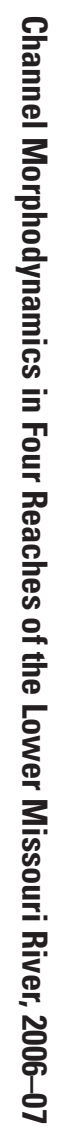

Figure 2-5. Kenslers Bend cross-section line 3 at Missouri River mile 747.7 in 2006. 


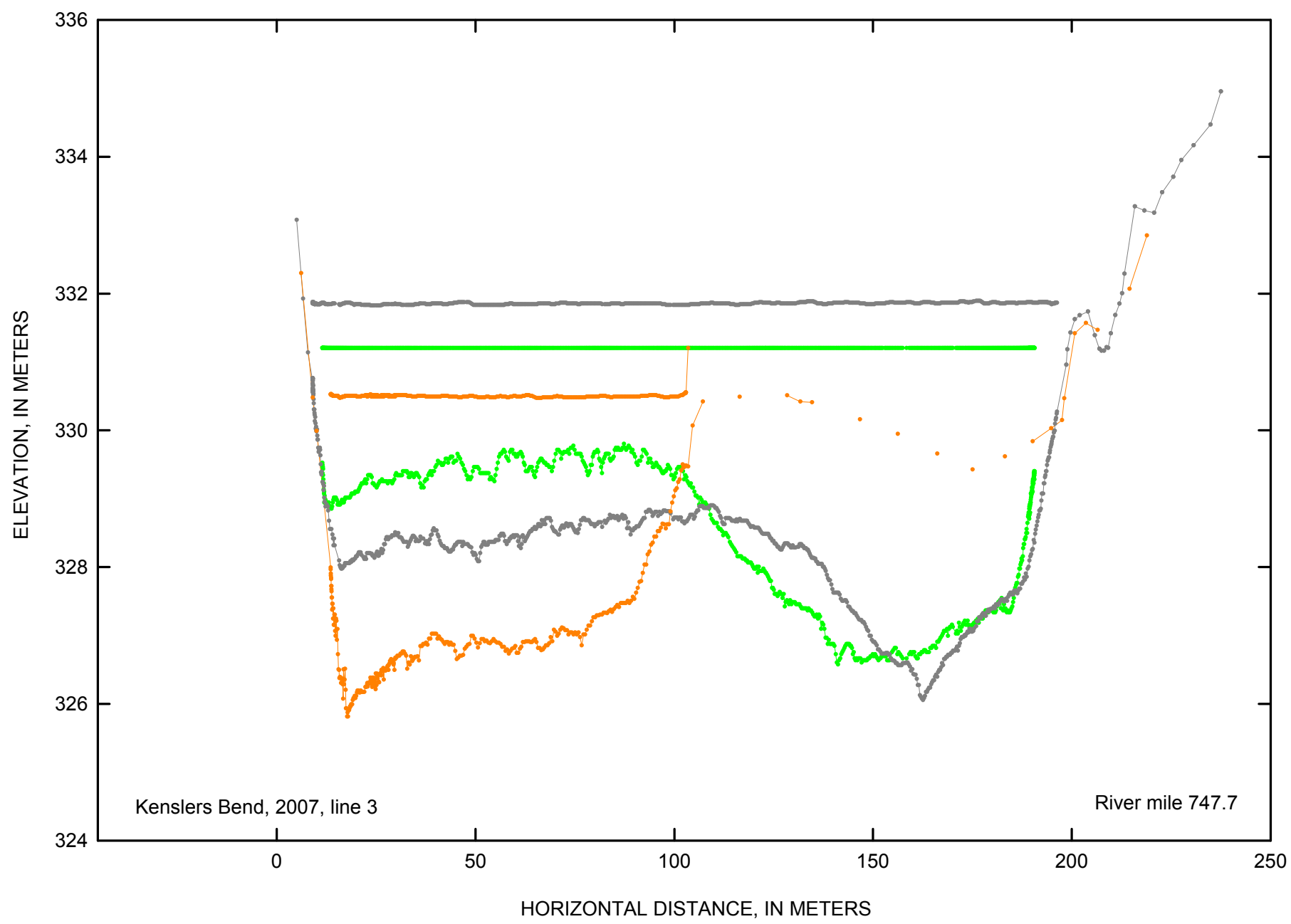

EXPLANATION

April 2007

July 2007

October 2007

Figure 2-6. Kenslers Bend cross-section line 3 at Missouri River mile 747.7 in 2007. 


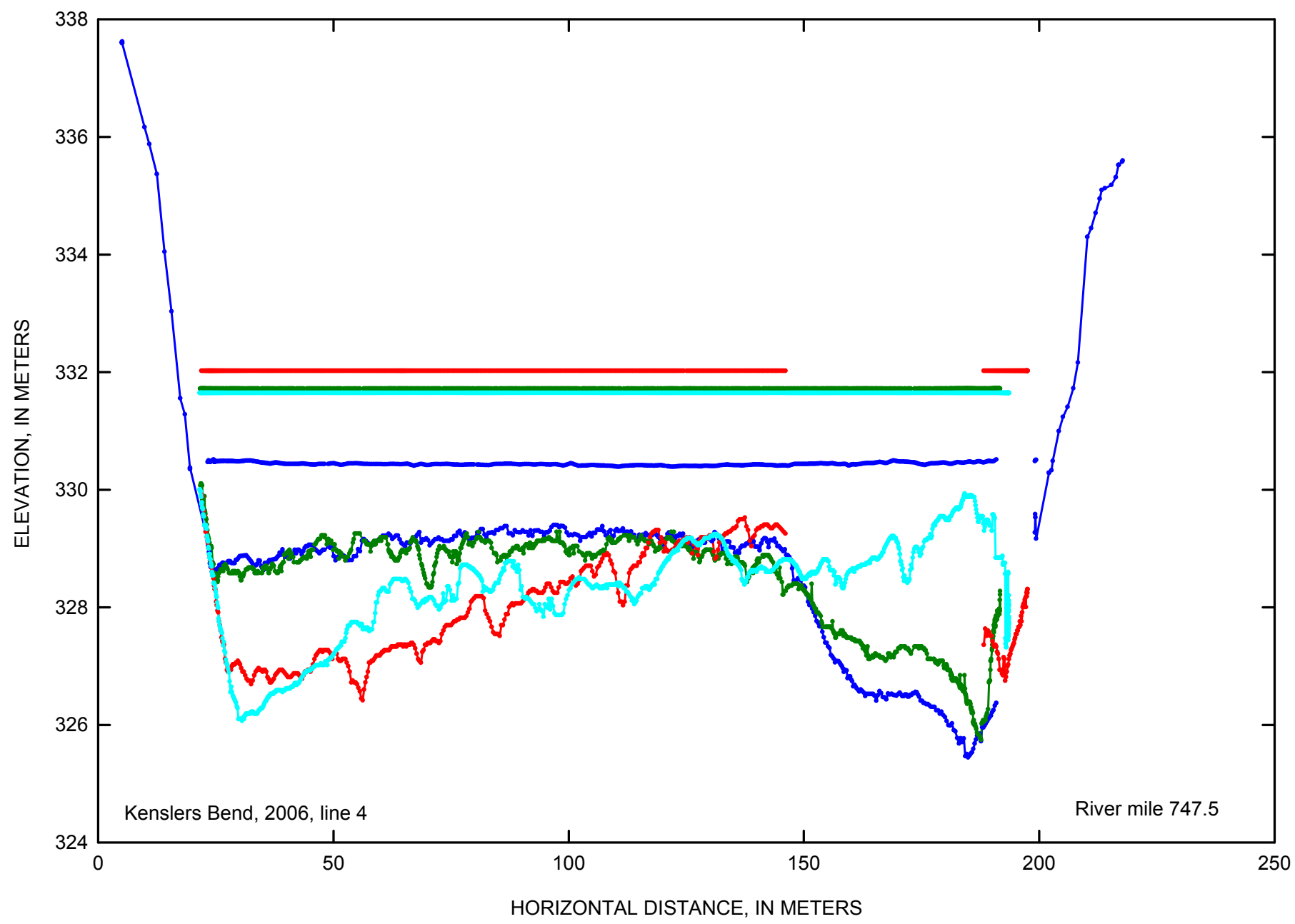

EXPLANATION

March 2006 I

March 2006 II

May 2006

June 2006

March 2007

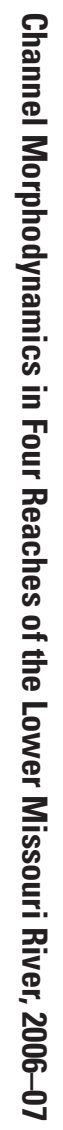

Figure 2-7. Kenslers Bend cross-section line 4 at Missouri River mile 747.5 in 2006. 


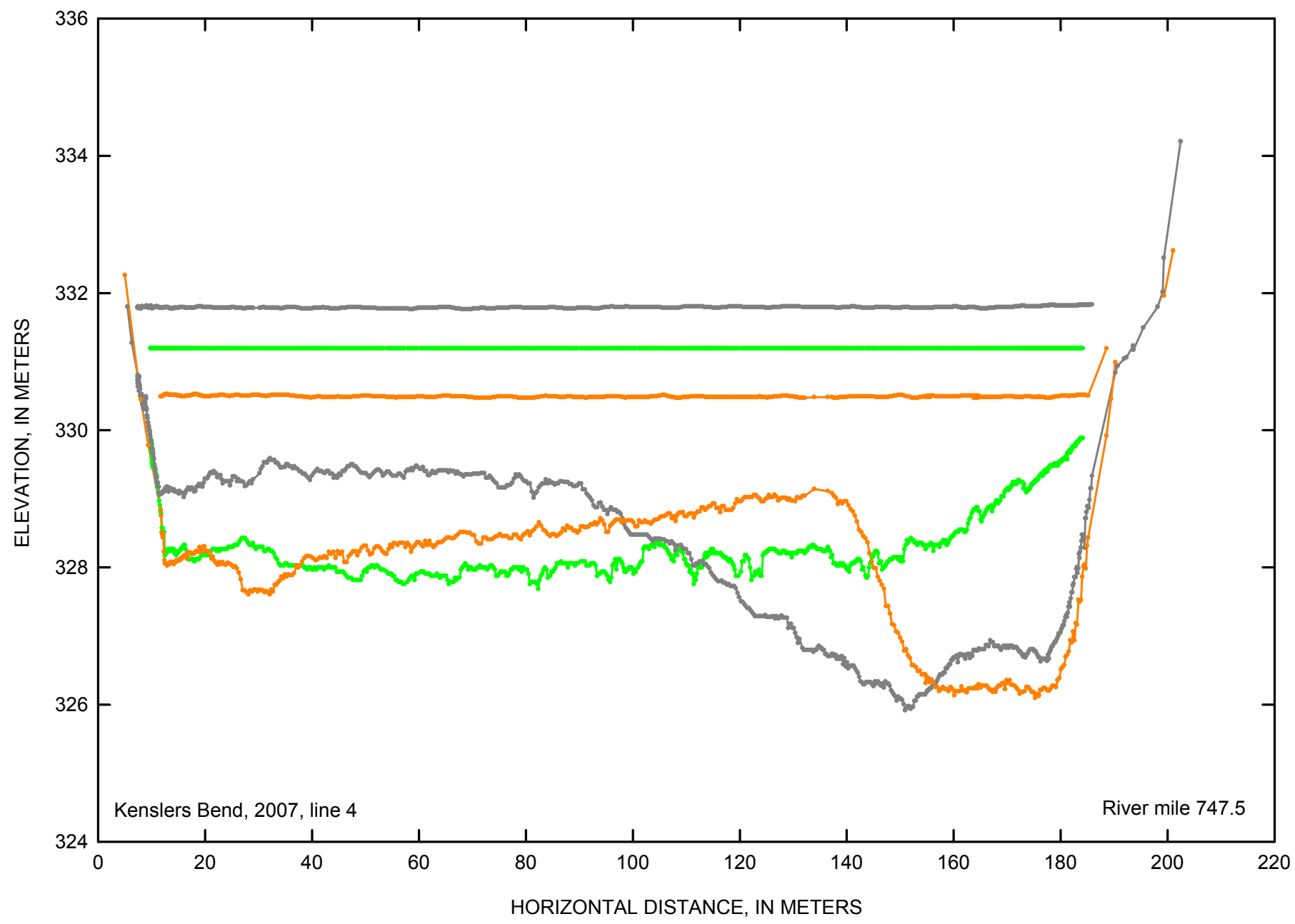

EXPLANATION

April 2007

July 2007

October 2007

Figure 2-8. Kenslers Bend cross-section line 4 at Missouri River mile 747.5 in 2007. 


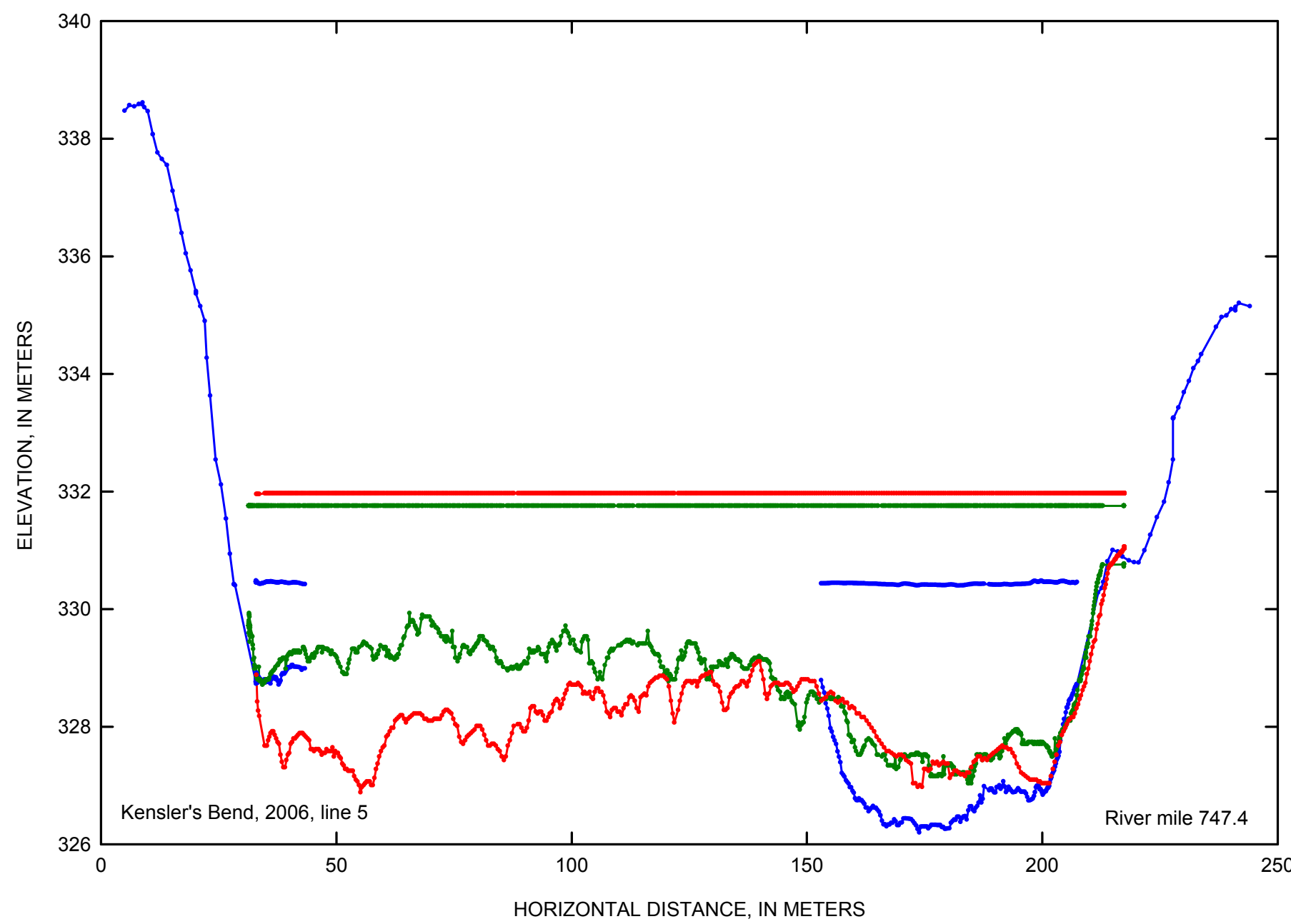

EXPLANATION

March 2006 I

March 2006 II

May 2006

June 2006

March 2007

Figure 2-9. Kenslers Bend cross-section line 5 at Missouri River mile 747.4 in 2006. 


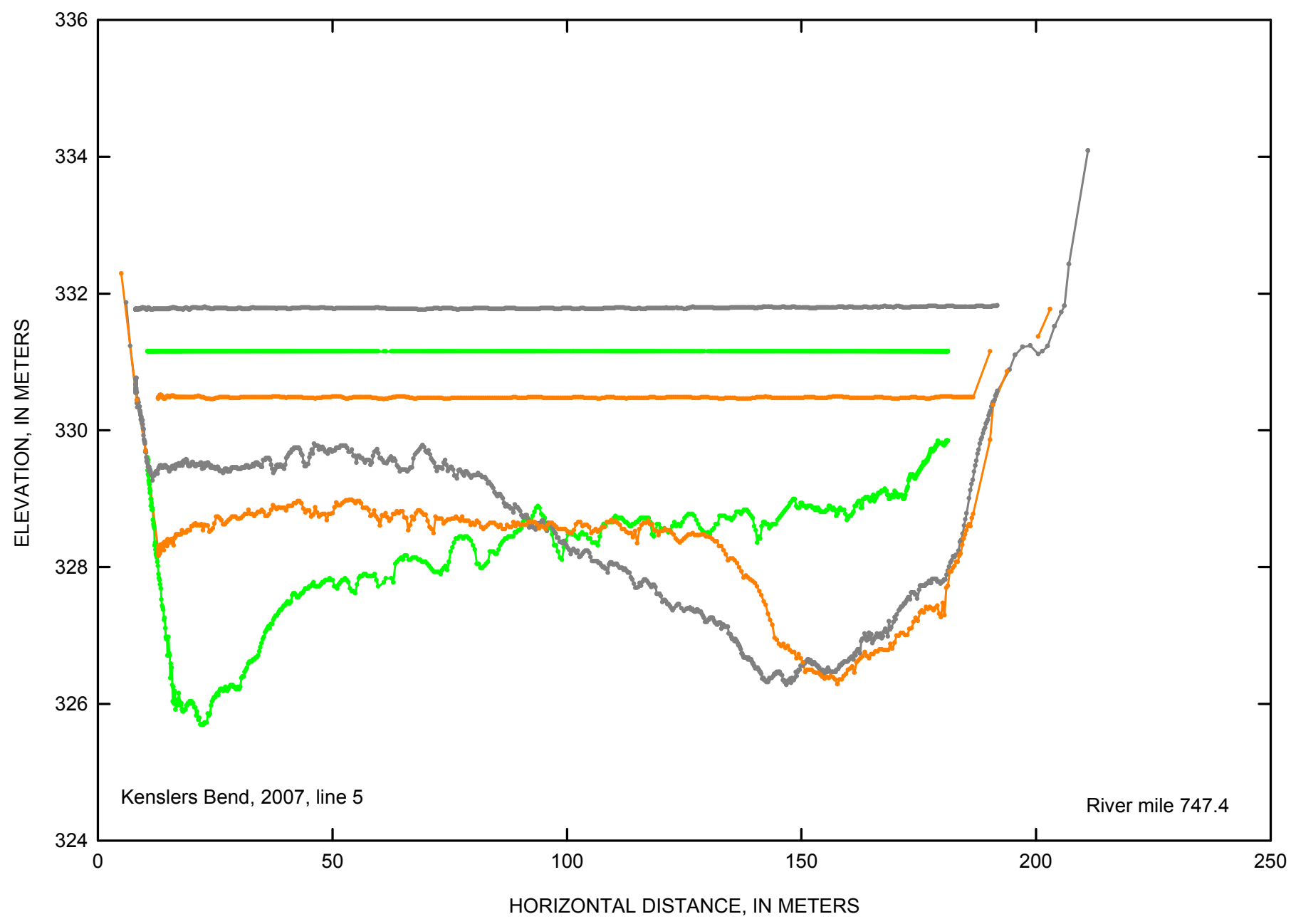

EXPLANATION

April 2007

July 2007

October 2007

Figure 2-10. Kenslers Bend cross-section line 5 at Missouri River mile 747.4 in 2007. 


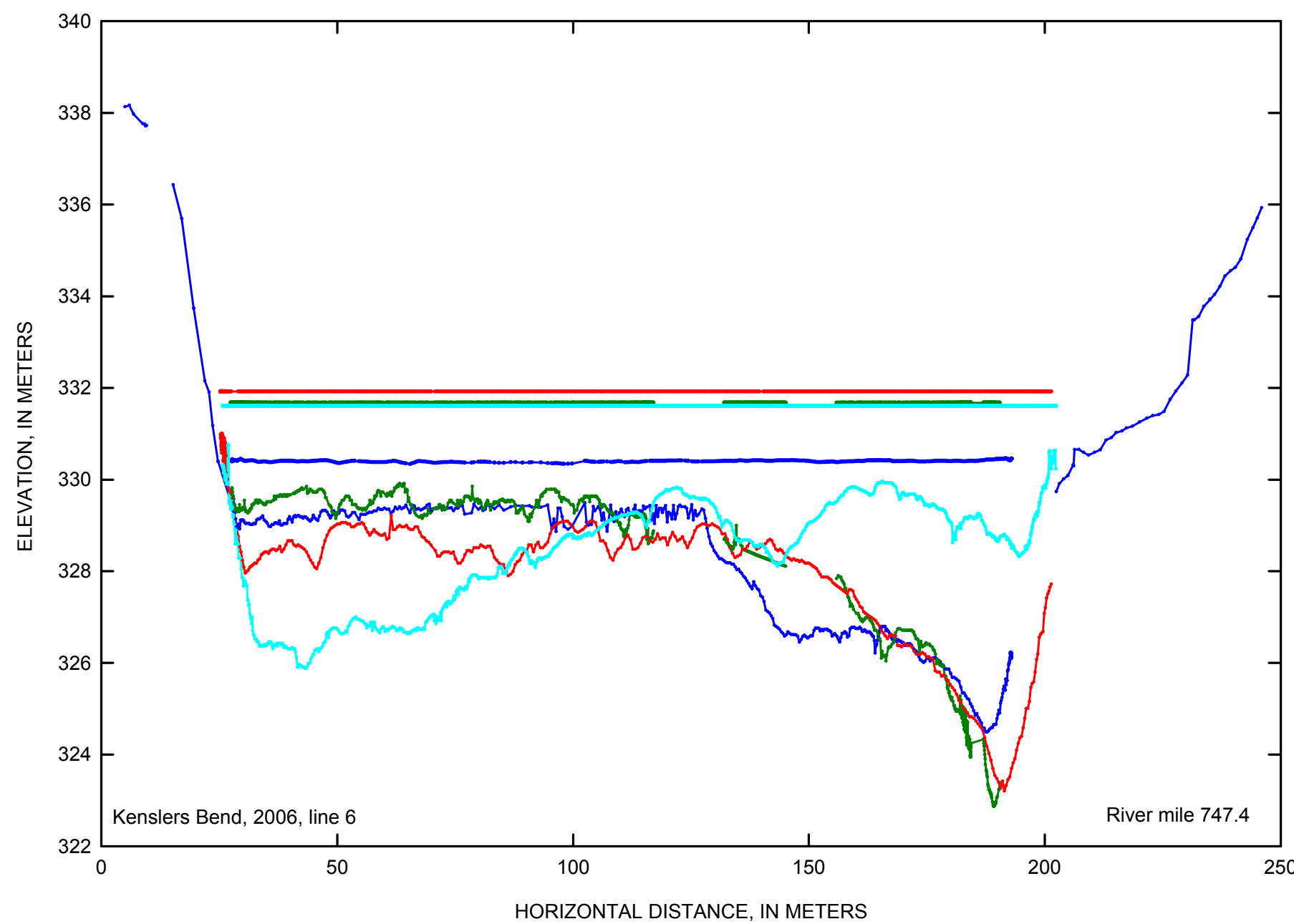

EXPLANATION

March 2006 I

March 2006 II

May 2006

June 2006

March 2007

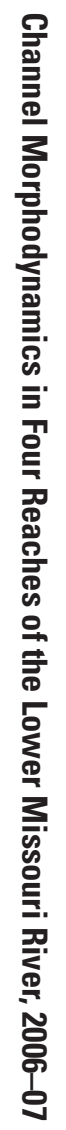

Figure 2-11. Kenslers Bend cross-section line 6 at Missouri River mile 747.4 in 2006. 


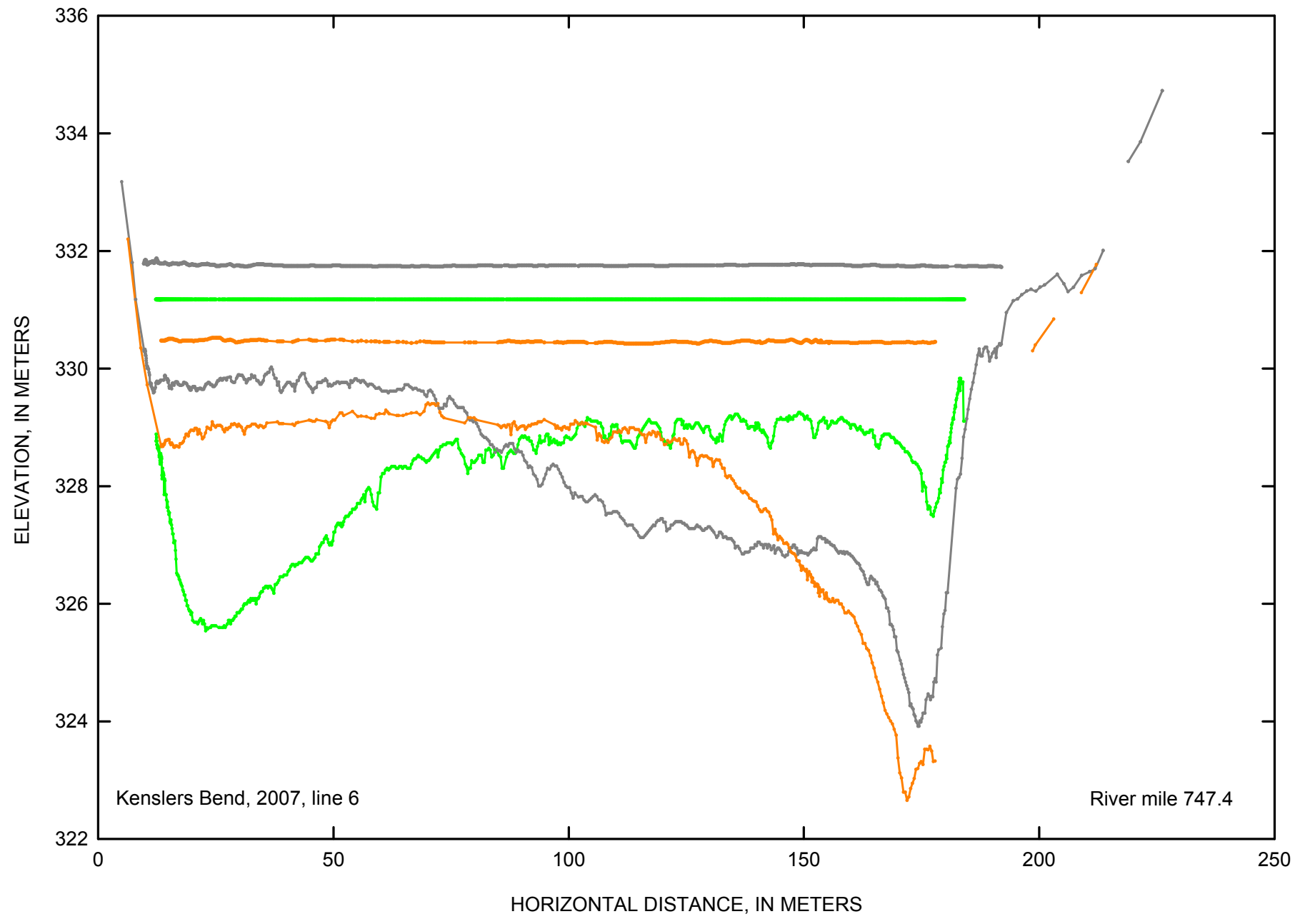

\section{EXPLANATION}

April 2007 July 2007

October 2007 


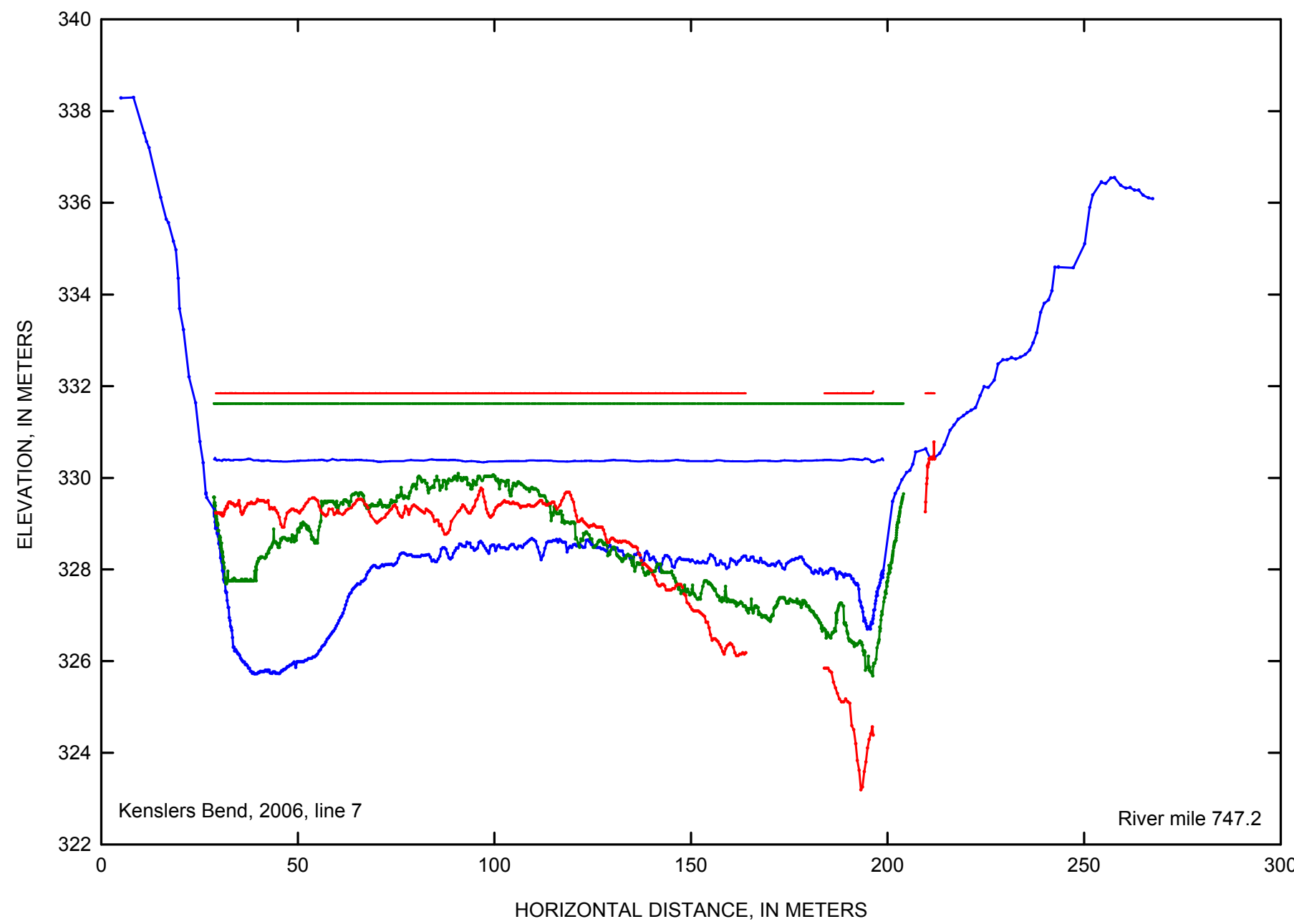

EXPLANATION

March 2006 I

- March 2006 II

- May 2006

June 2006

March 2007

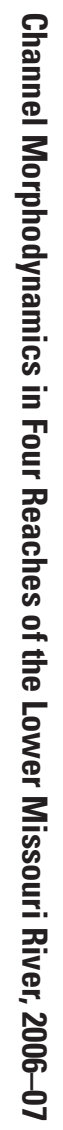

Figure 2-13. Kenslers Bend cross-section line 7 at Missouri River mile 747.2 in 2006. 


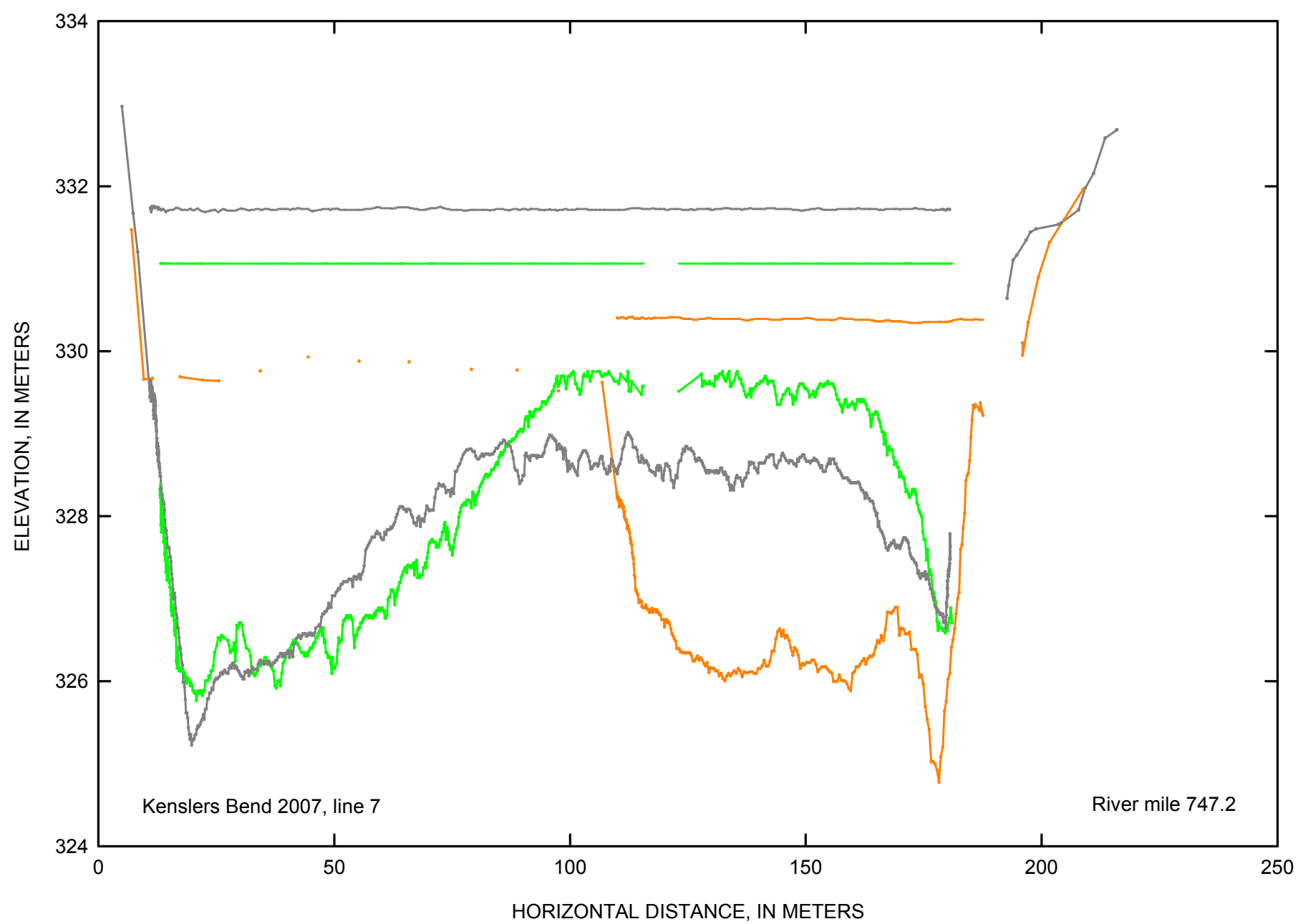

EXPLANATION

April 2007

July 2007

October 2007

Figure 2-14. Kenslers Bend cross-section line 7 at Missouri River mile 747.2 in 2007. 


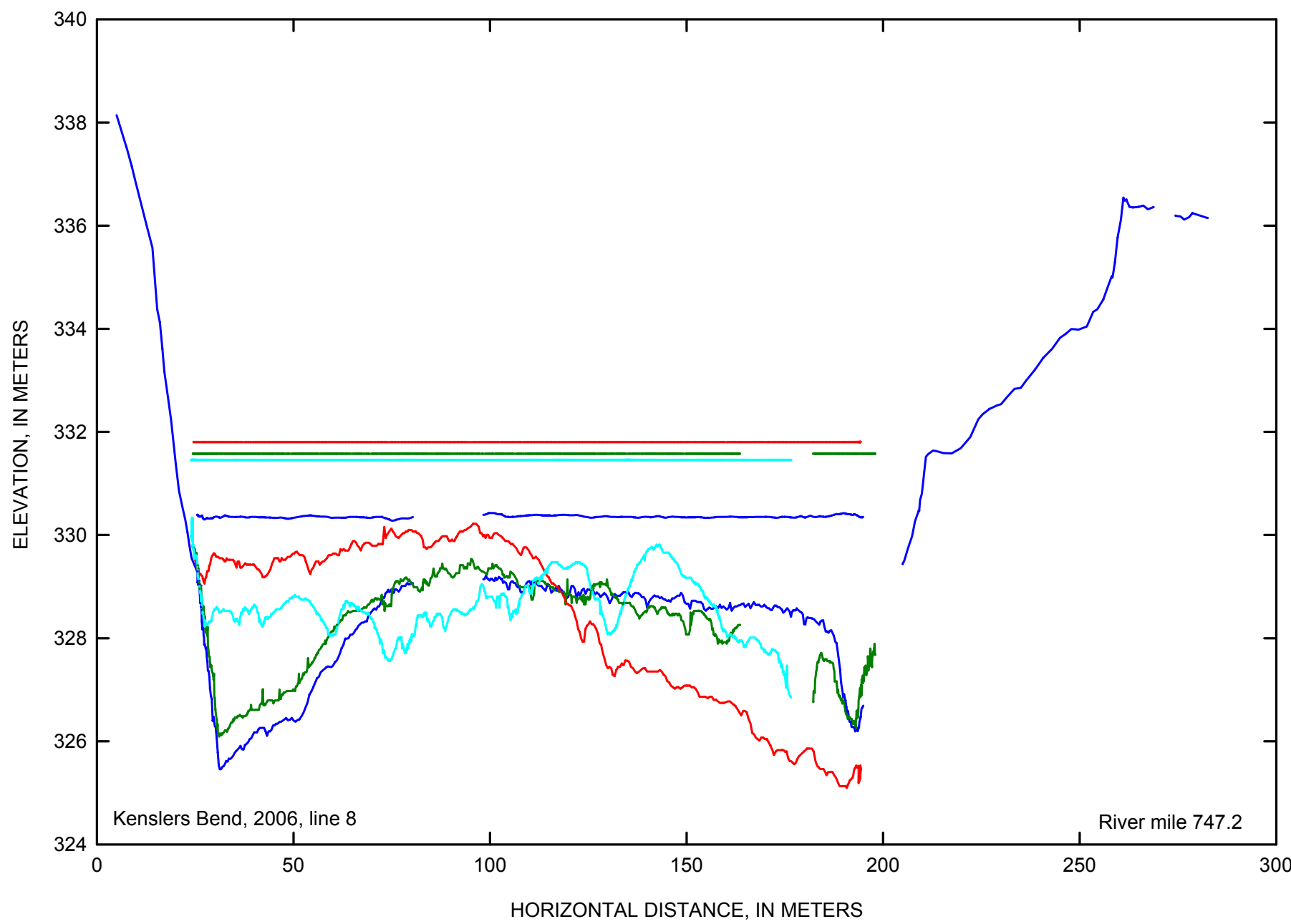

EXPLANATION

March 2006 I

March 2006 II

May 2006

June 2006

March 2007

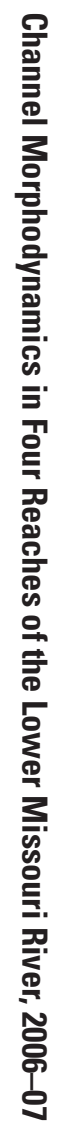

Figure 2-15. Kenslers Bend cross-section line 8 at Missouri River mile 747.2 in 2006. 


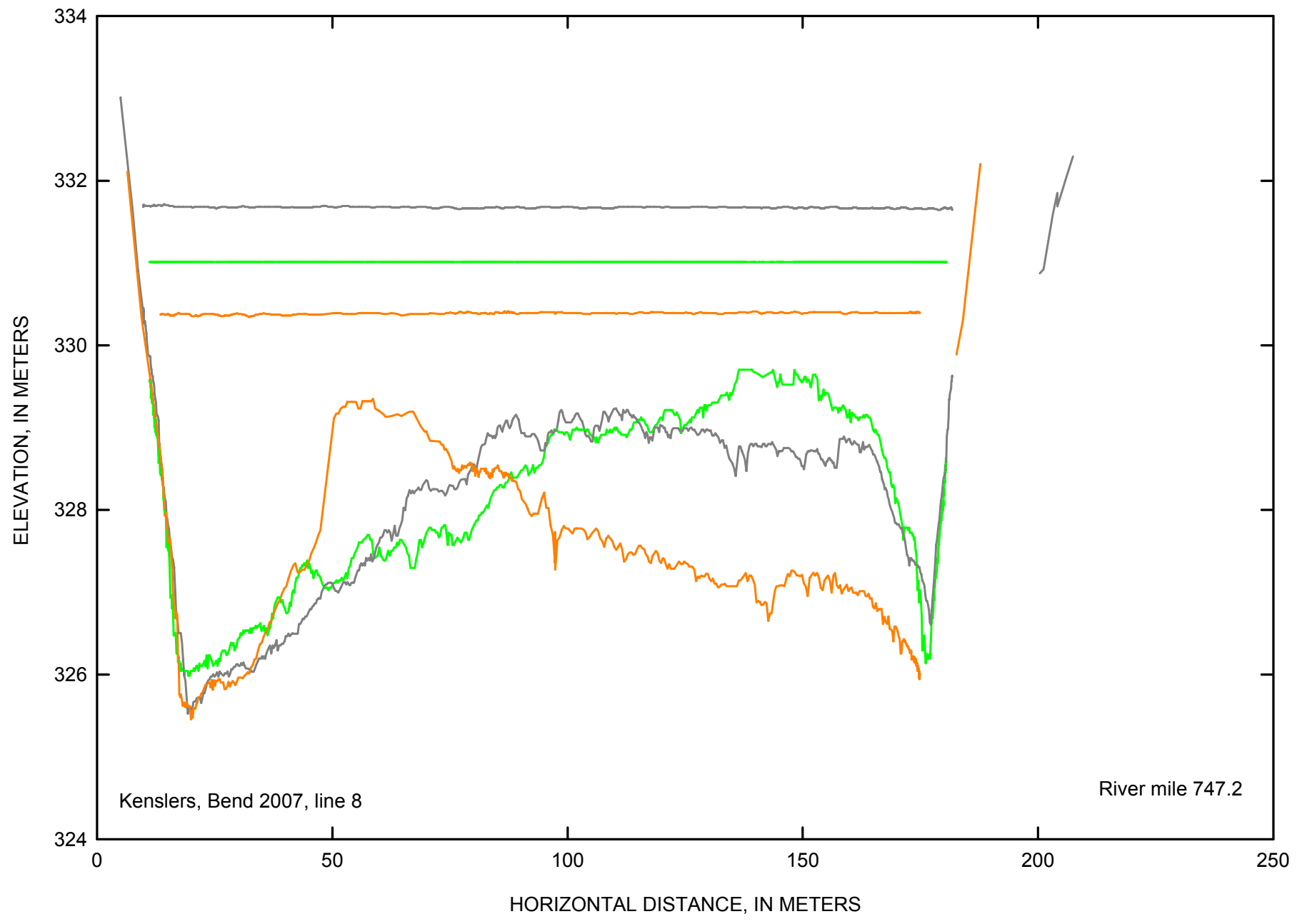

EXPLANATION

April 2007

October 2007

Figure 2-16. Kenslers Bend cross-section line 8 at Missouri River mile 747.2 in 2007. 


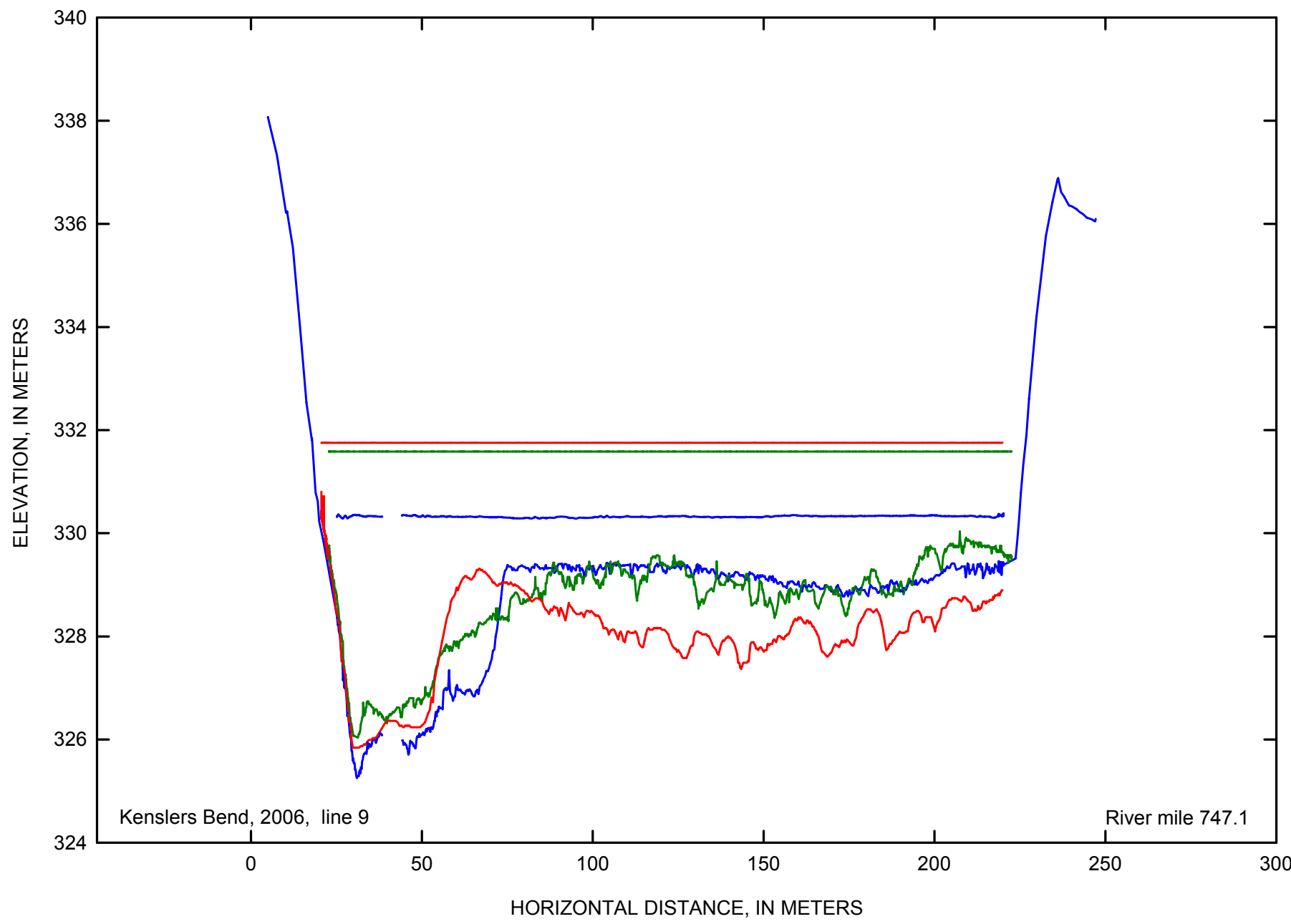

EXPLANATION

March 2006 I

March 2006 II

May 2006

June 2006

March 2007

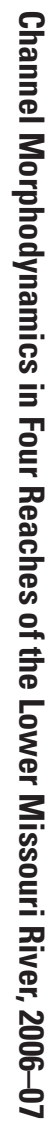

Figure 2-17. Kenslers Bend cross-section line 9 at Missouri River mile 747.1 in 2006. 


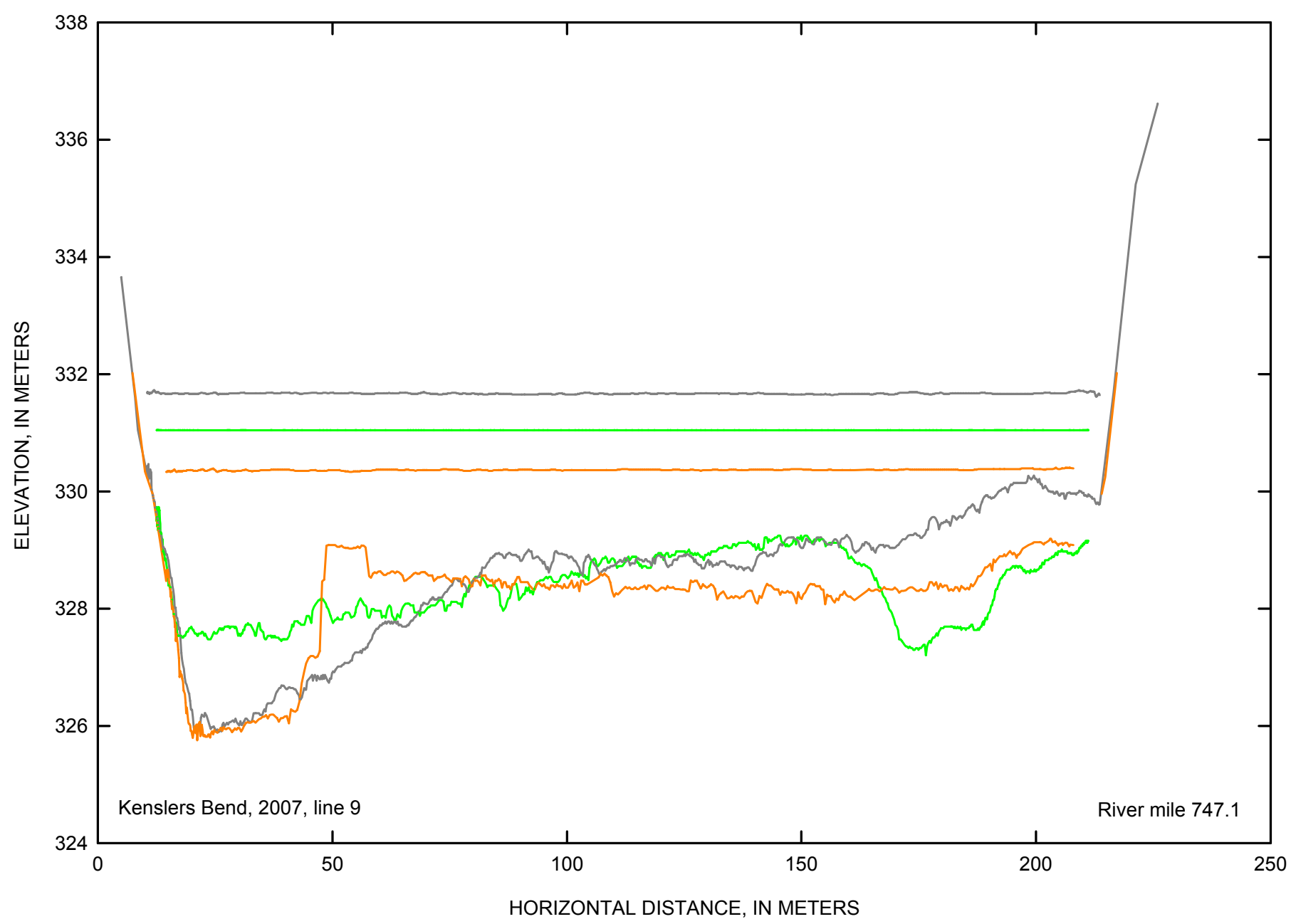

EXPLANATION

April 2007

July 2007

October 2007

Figure 2-18. Kenslers Bend cross-section line 9 at Missouri River mile 747.1 in 2007. 


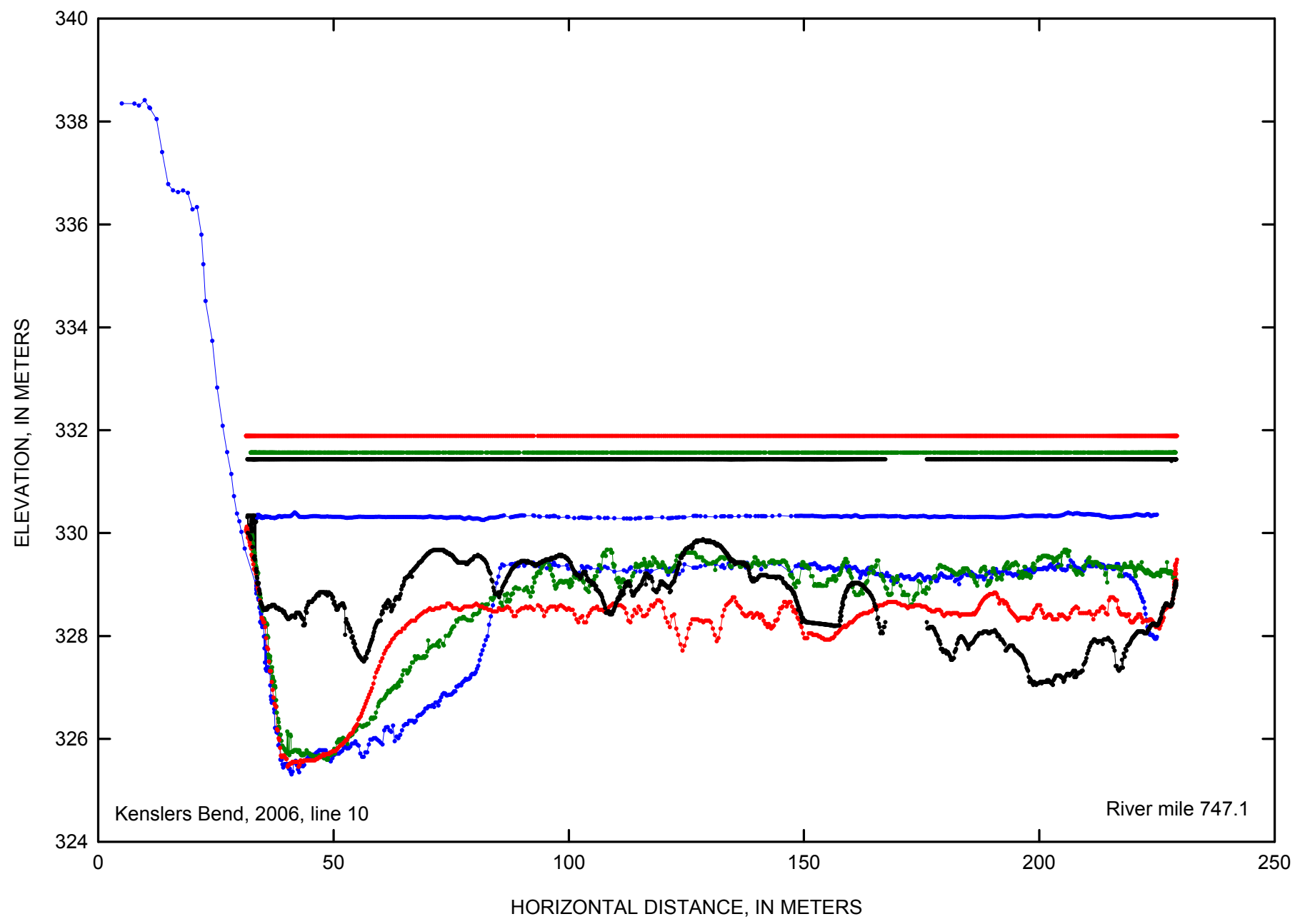

EXPLANATION

March 2006 I

March 2006 II

May 2006

June 2006

March 2007

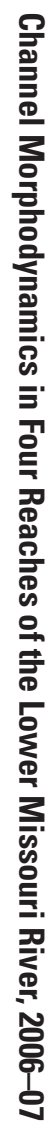

Figure 2-19. Kenslers Bend cross-section line 10 at Missouri River mile 747.1 in 2006. 


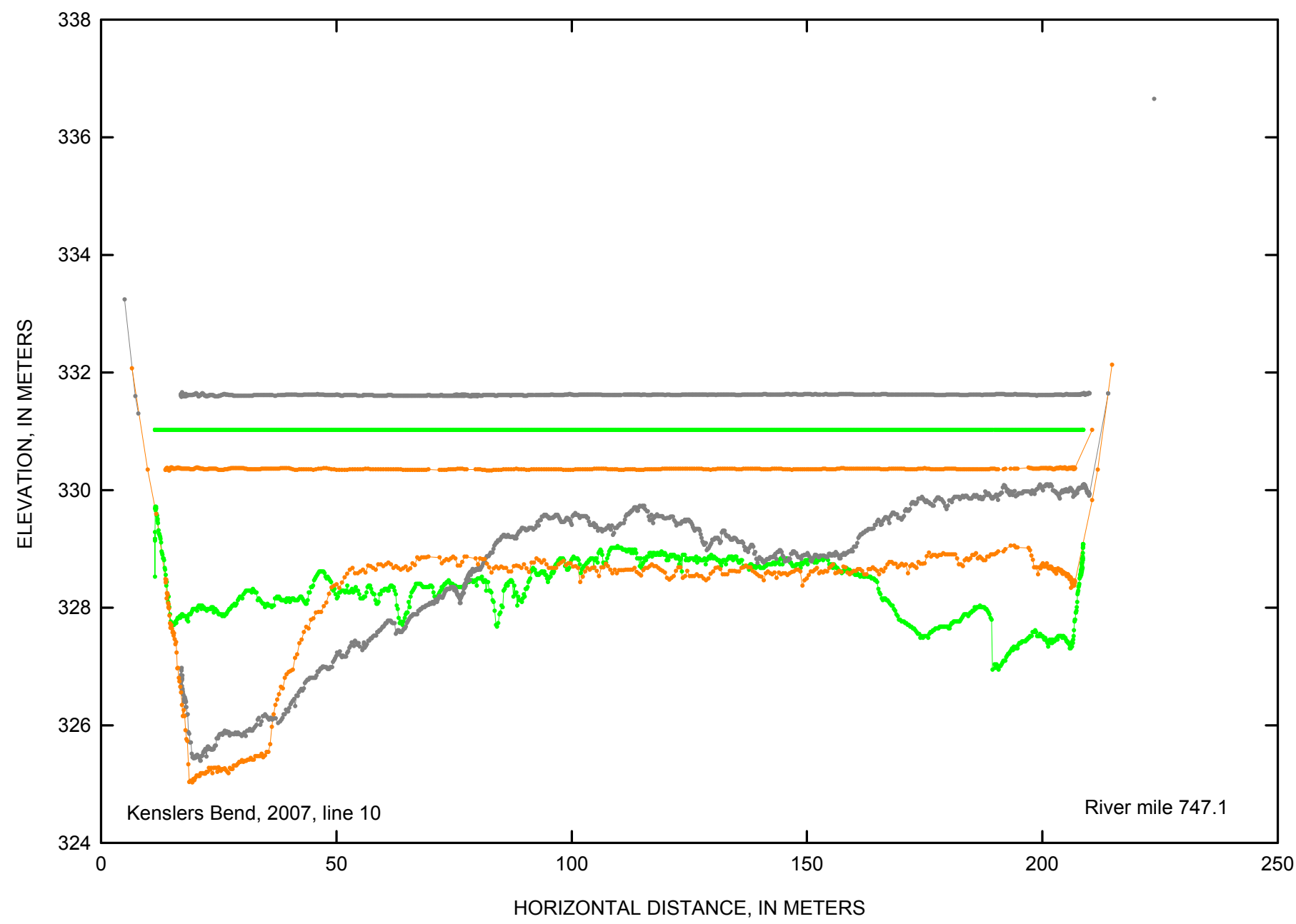

\section{EXPLANATION}

April 2007

July 2007

October 2007

Figure 2-20. Kenslers Bend cross-section line 10 at Missouri River mile 747.1 in 2007. 


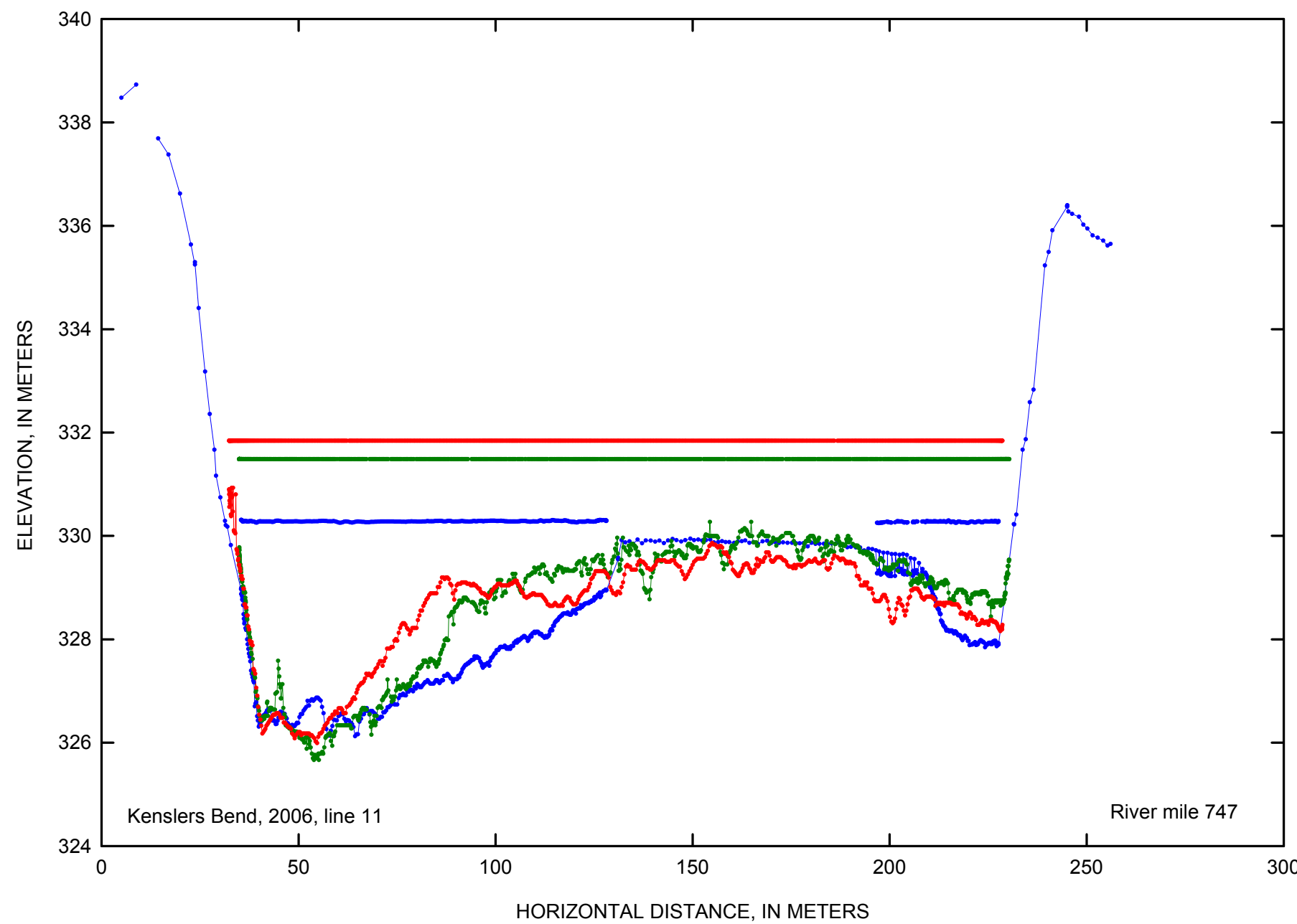

EXPLANATION

March 2006 I

March 2006 II

May 2006

June 2006

March 2007

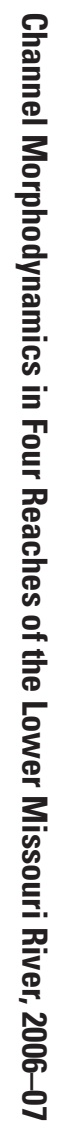

Figure 2-21. Kenslers Bend cross-section line 11 at Missouri River mile 747 in 2006. 


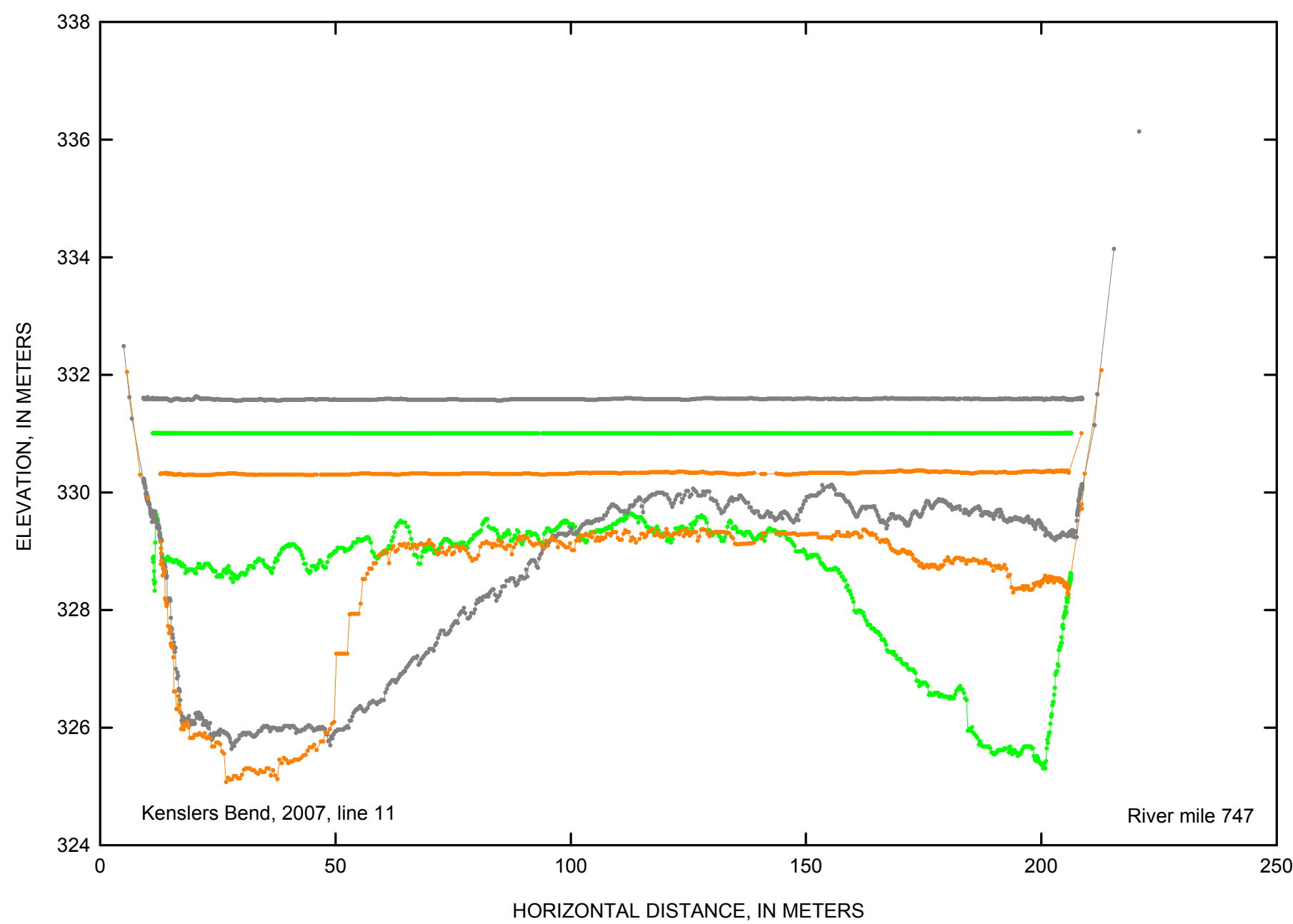

EXPLANATION

April 2007

July 2007

October 2007

Figure 2-22. Kenslers Bend cross-section line 11 at Missouri River mile 747 in 2007. 


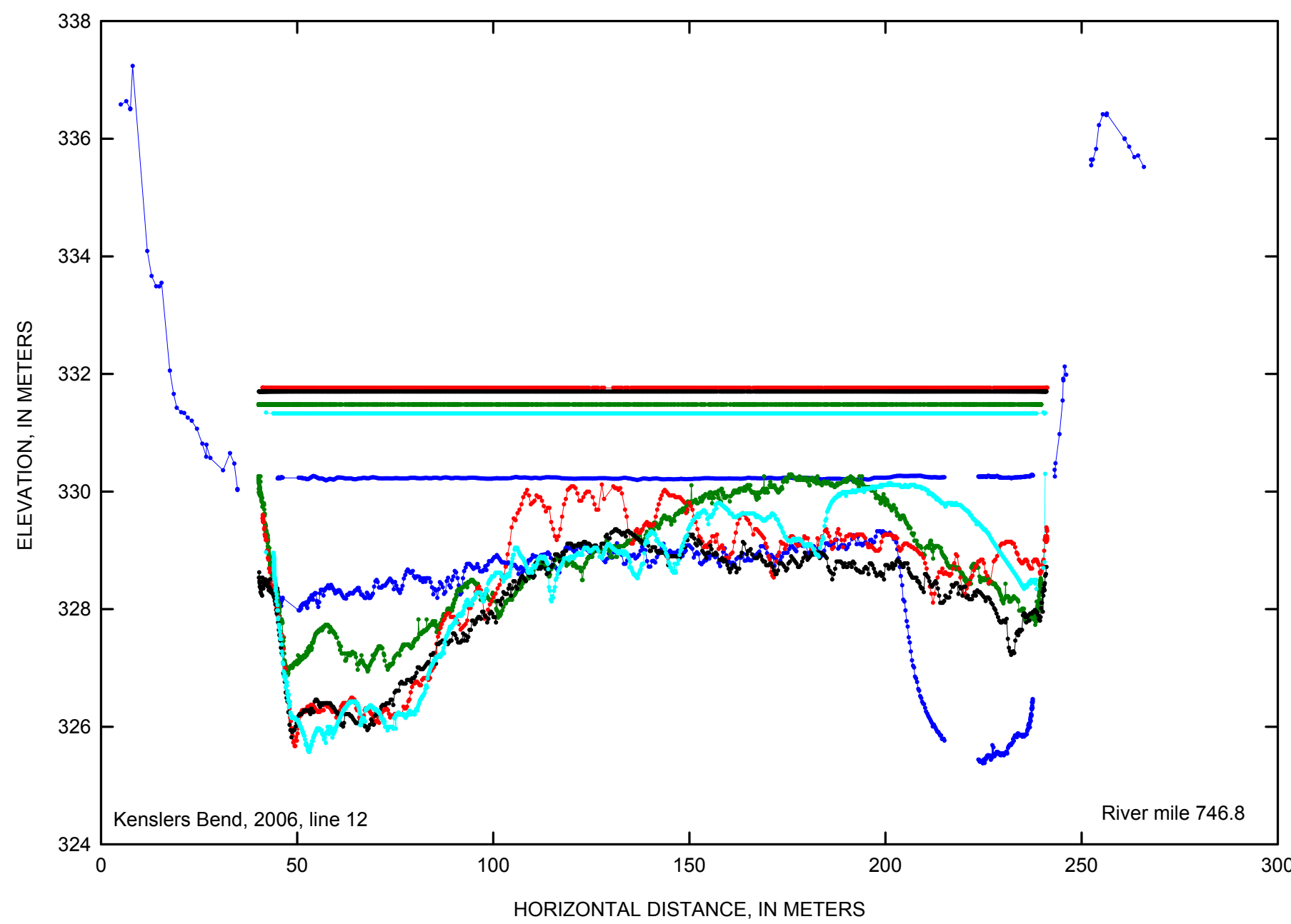

EXPLANATION

March 2006 I

March 2006 II

May 2006

June 2006

March 2007

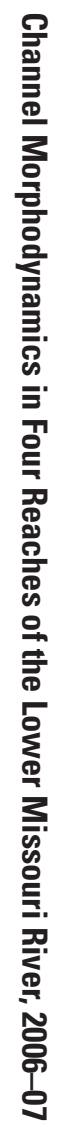

Figure 2-23. Kenslers Bend cross-section line 12 at Missouri River mile 746.8 in 2006. 


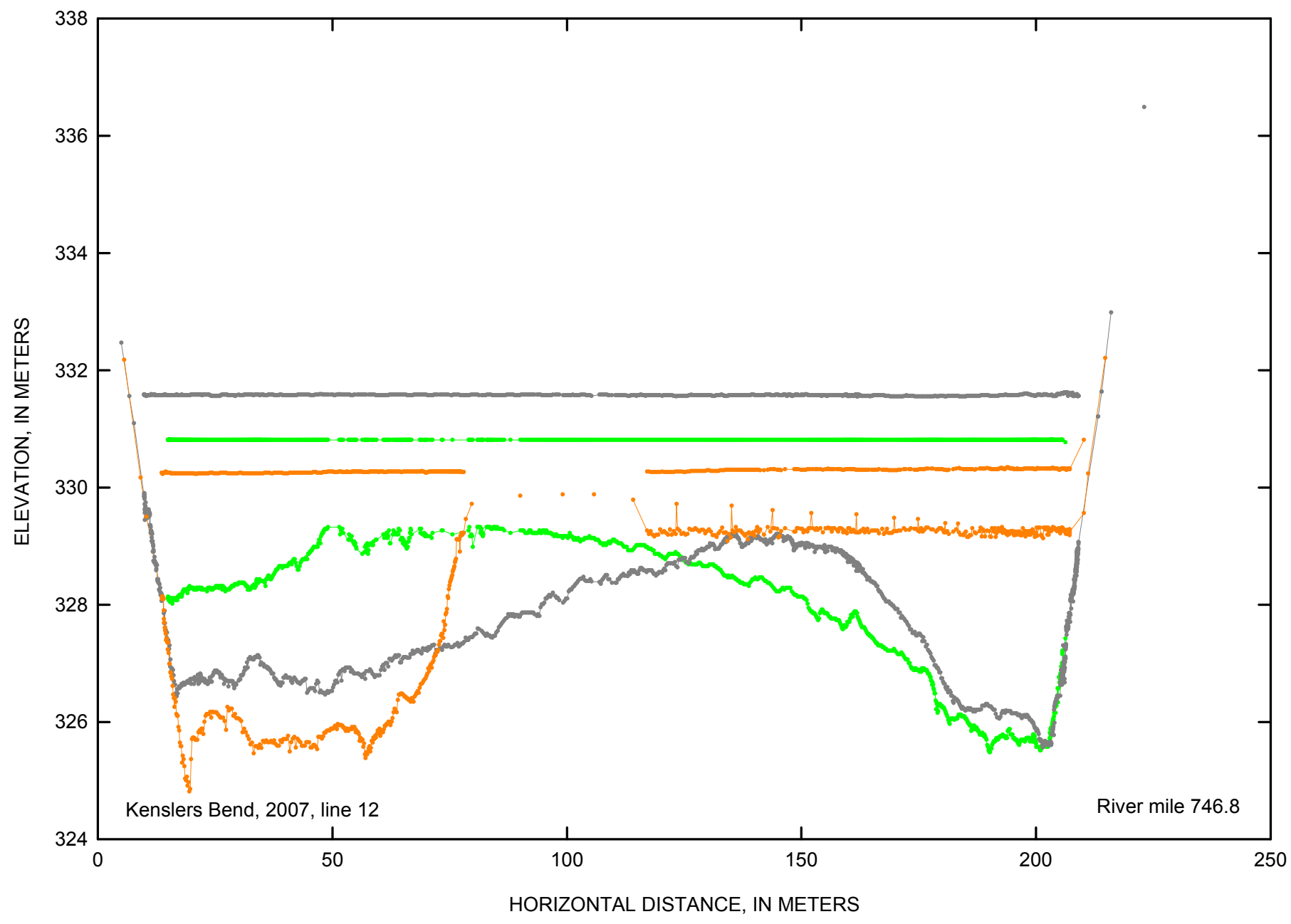

EXPLANATION

April 2007

July 2007

October 2007

Figure 2-24. Kenslers Bend cross-section line 12 at Missouri River mile 746.8 in 2007. 


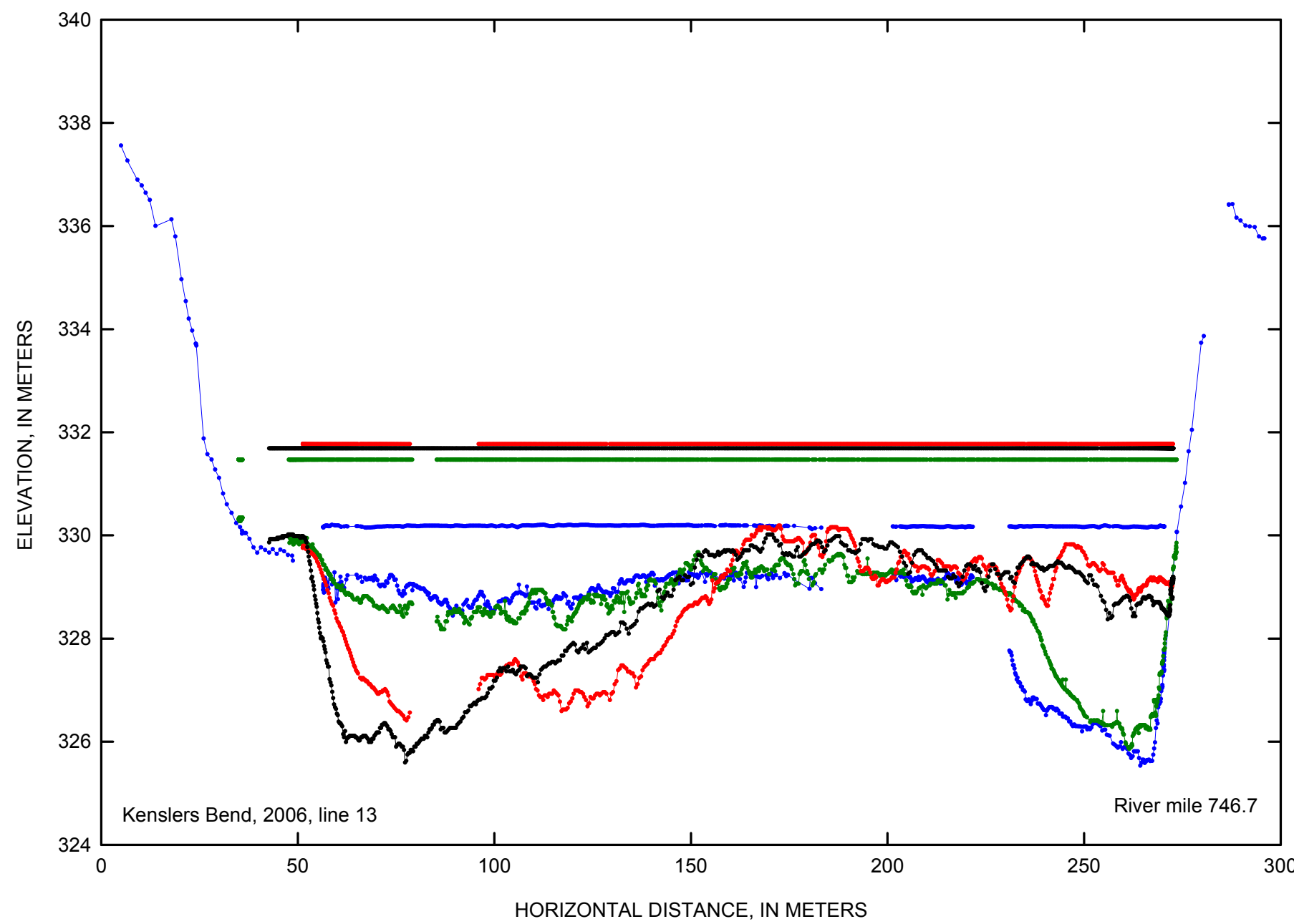

EXPLANATION

March 2006 I

March 2006 II

May 2006

June 2006

March 2007

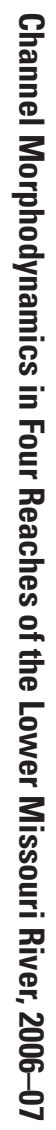

Figure 2-25. Kenslers Bend cross-section line 13 at Missouri River mile 746.7 in 2006. 


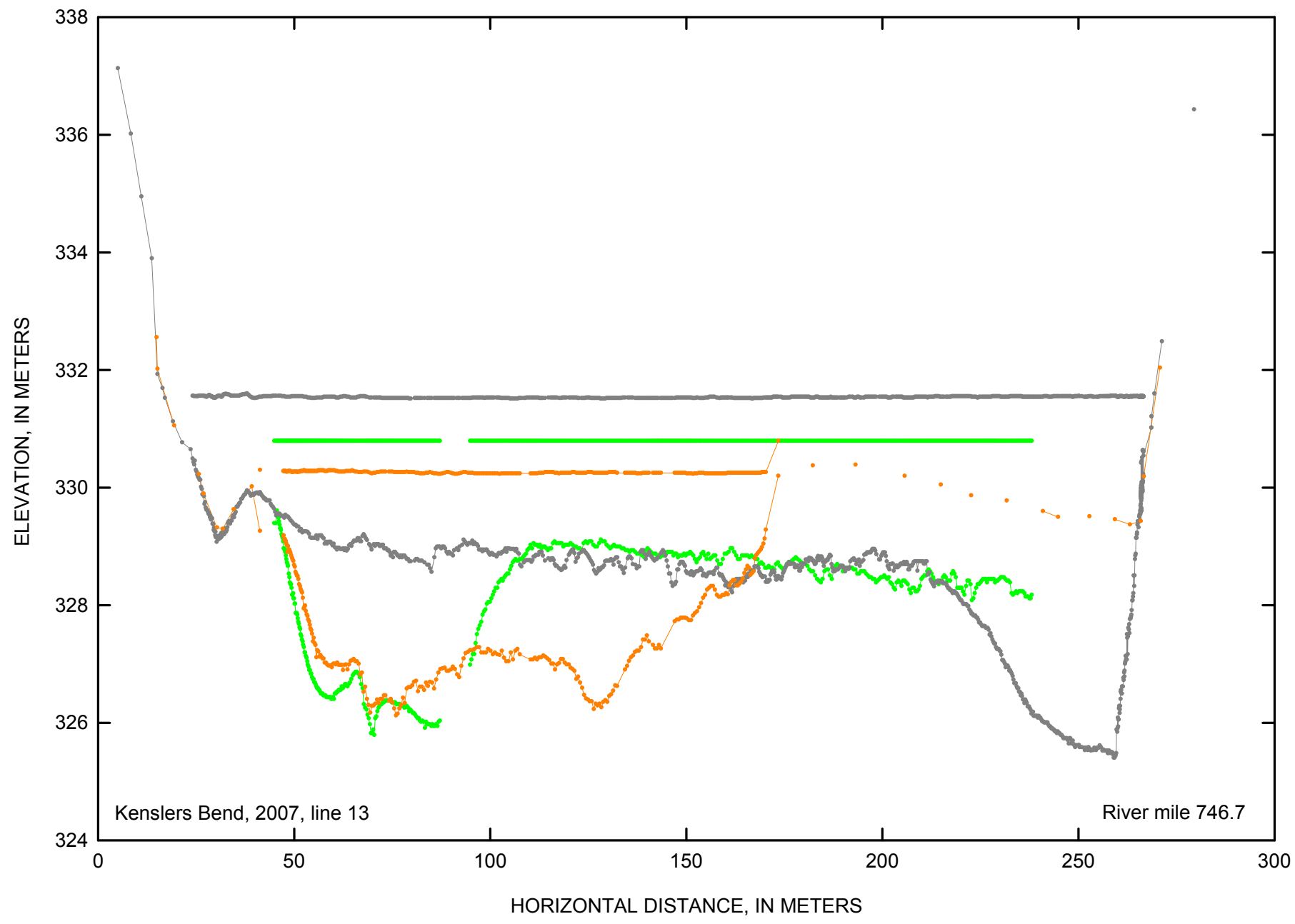

EXPLANATION

April 2007

July 2007

October 2007

Figure 2-26. Kenslers Bend cross-section line 13 at Missouri River mile 746.7 in 2007. 


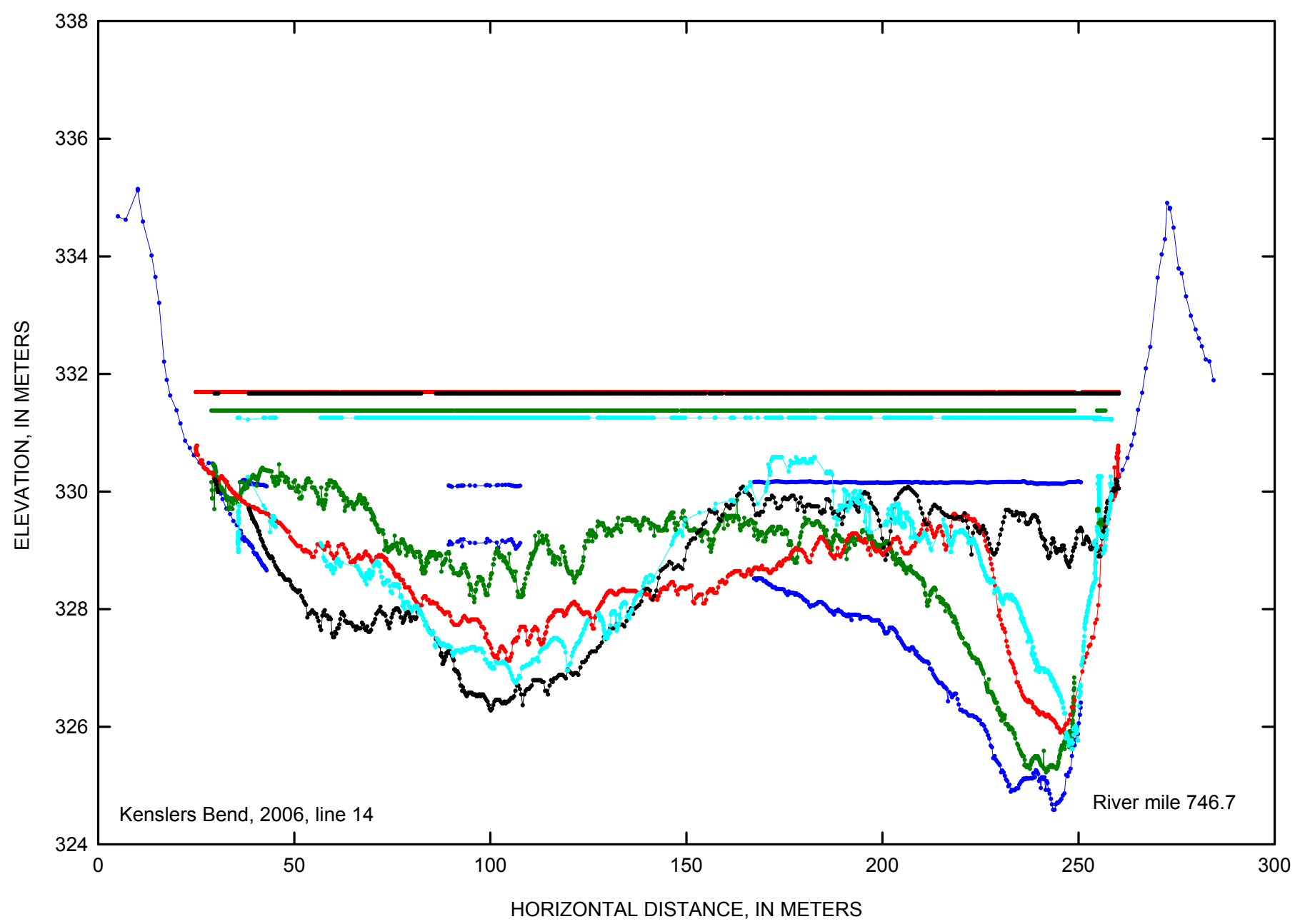

\section{EXPLANATION}

March 2006 I

March 2006 II

May 2006

June 2006

March 2007

Figure 2-27. Kenslers Bend cross-section line 14 at Missouri River mile 746.7 in 2006. 


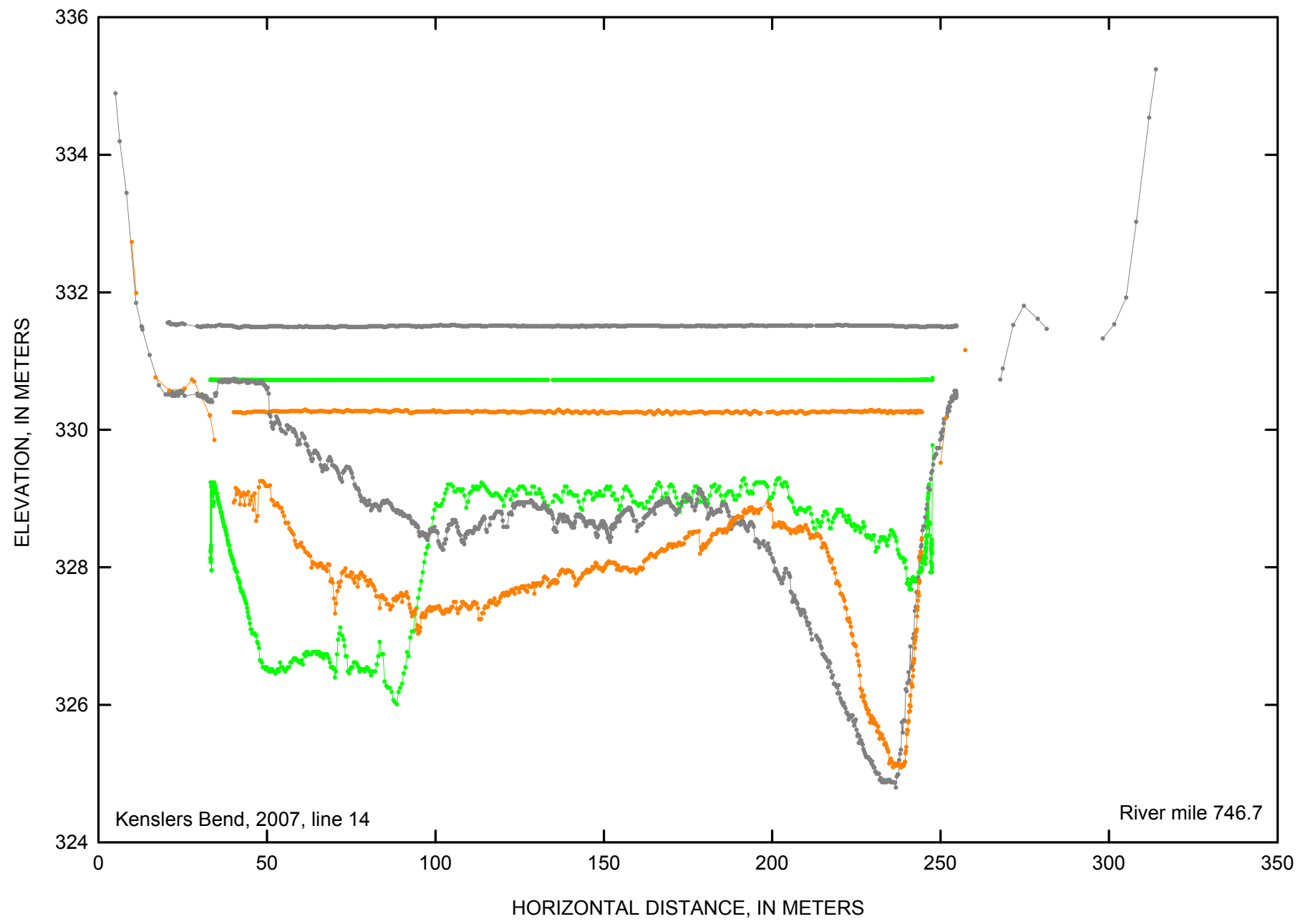

EXPLANATION

April 2007 July 2007

October 2007

Figure 2-28. Kenslers Bend cross-section line 14 at Missouri River mile 746.7 in 2007. 


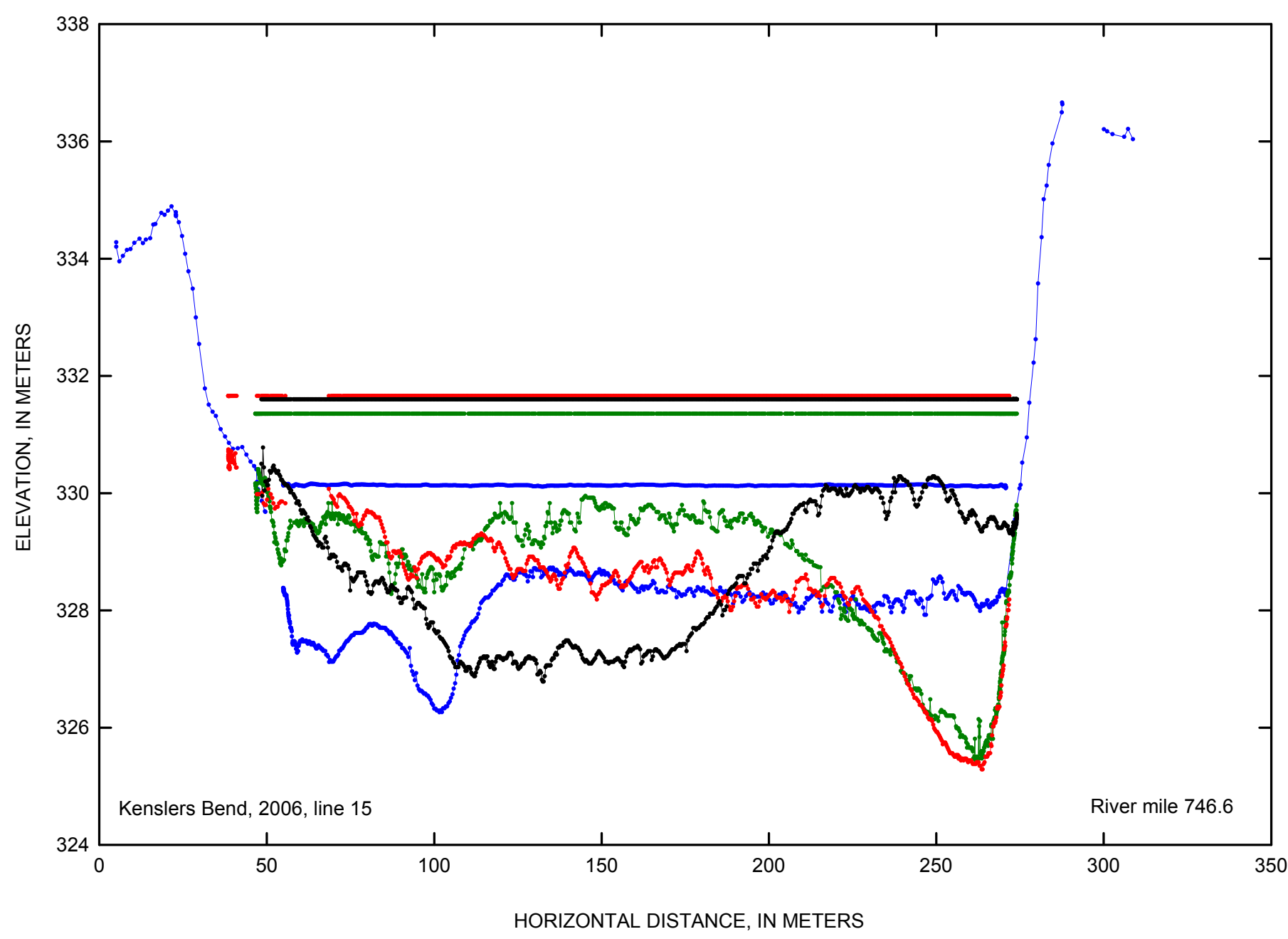

EXPLANATION

March 2006 I

March 2006 II

May 2006

June 2006

March 2007

Figure 2-29. Kenslers Bend cross-section line 15 at Missouri River mile 746.6 in 2006. 


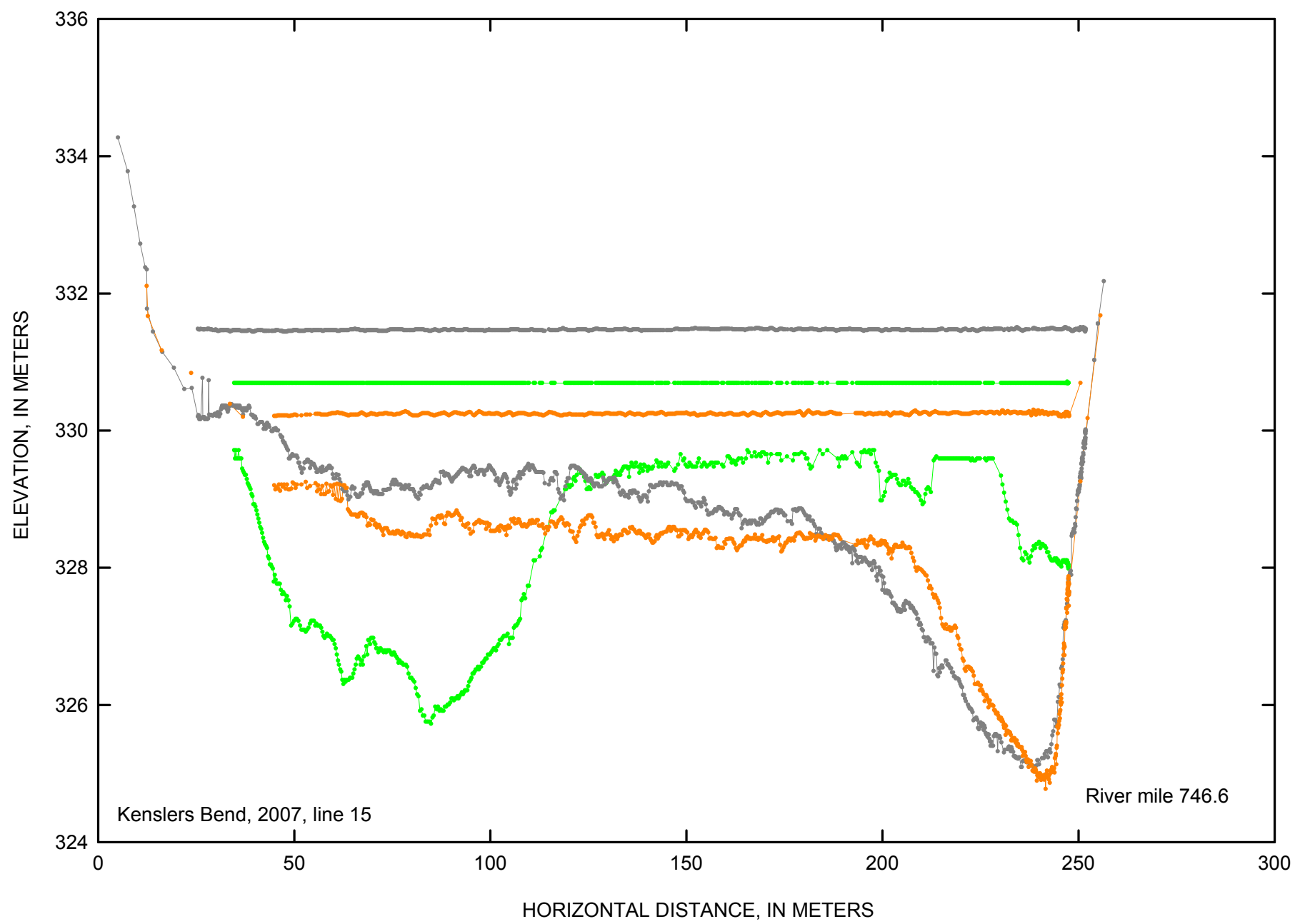

EXPLANATION

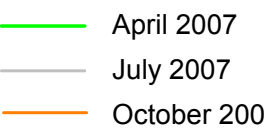




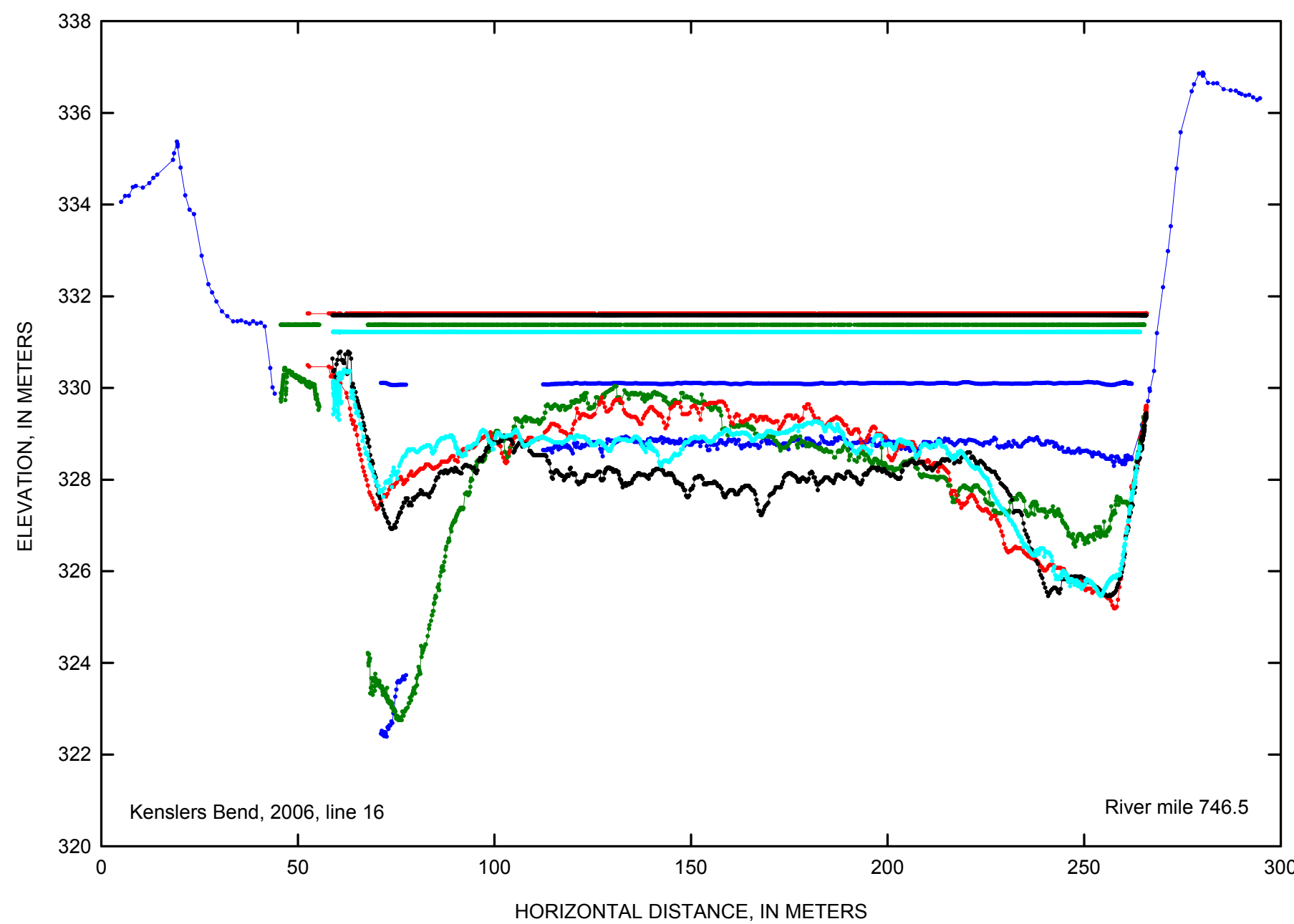

Figure 2-31. Kenslers Bend cross-section line 16 at Missouri River mile 746.5 in 2006. 


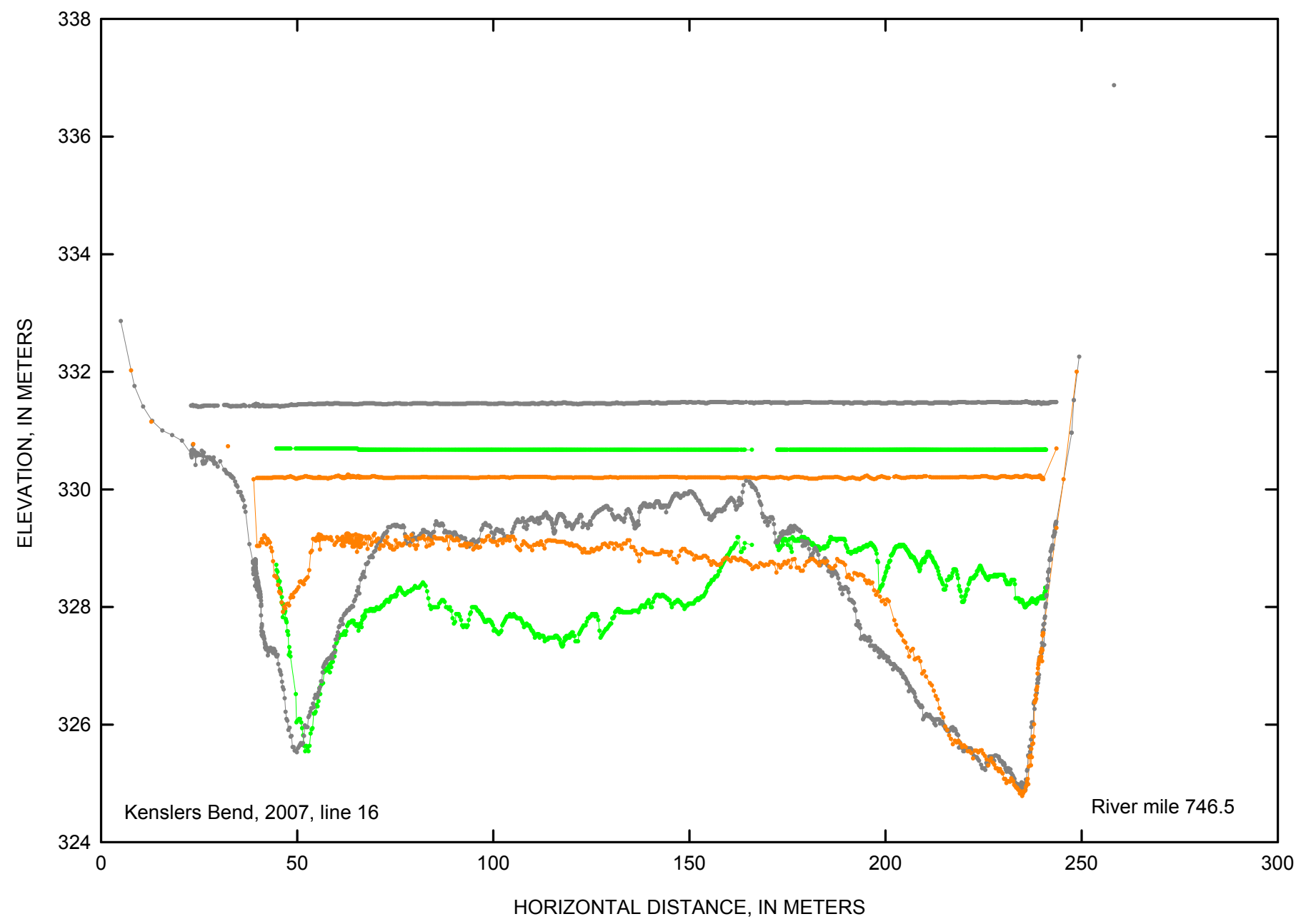

EXPLANATION

April 2007

July 2007

October 2007

Figure 2-32. Kenslers Bend cross-section line 16 at Missouri River mile 746.5 in 2007. 


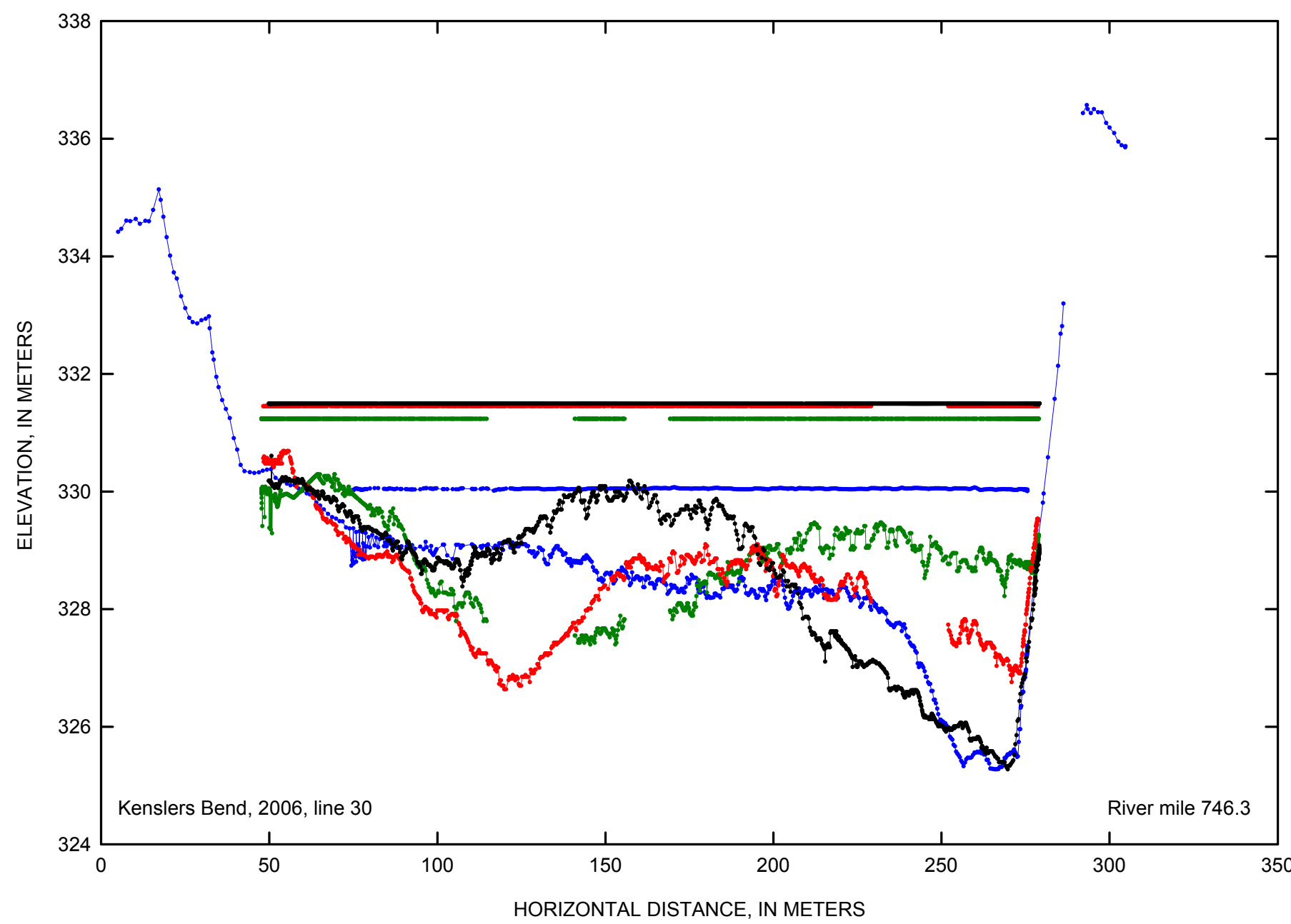

EXPLANATION

March 2006 I

March 2006 II

May 2006

June 2006

March 2007

Figure 2-33. Kenslers Bend cross-section line 30 at Missouri River mile 746.3 in 2006. 


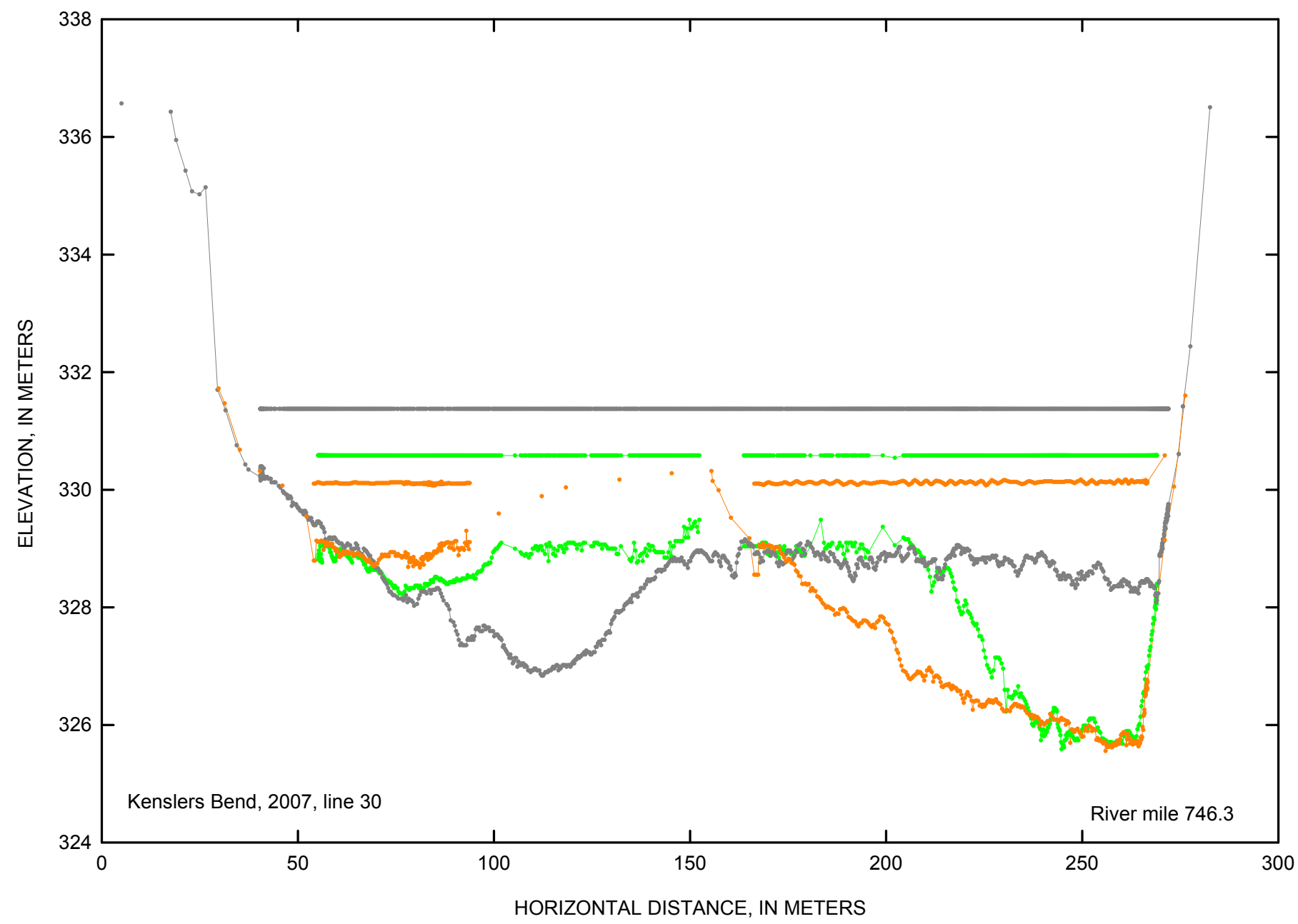

EXPLANATION

April 2007

July 2007

October 2007

Figure 2-34. Kenslers Bend cross-section line 30 at Missouri River mile 746.3 in 2007. 


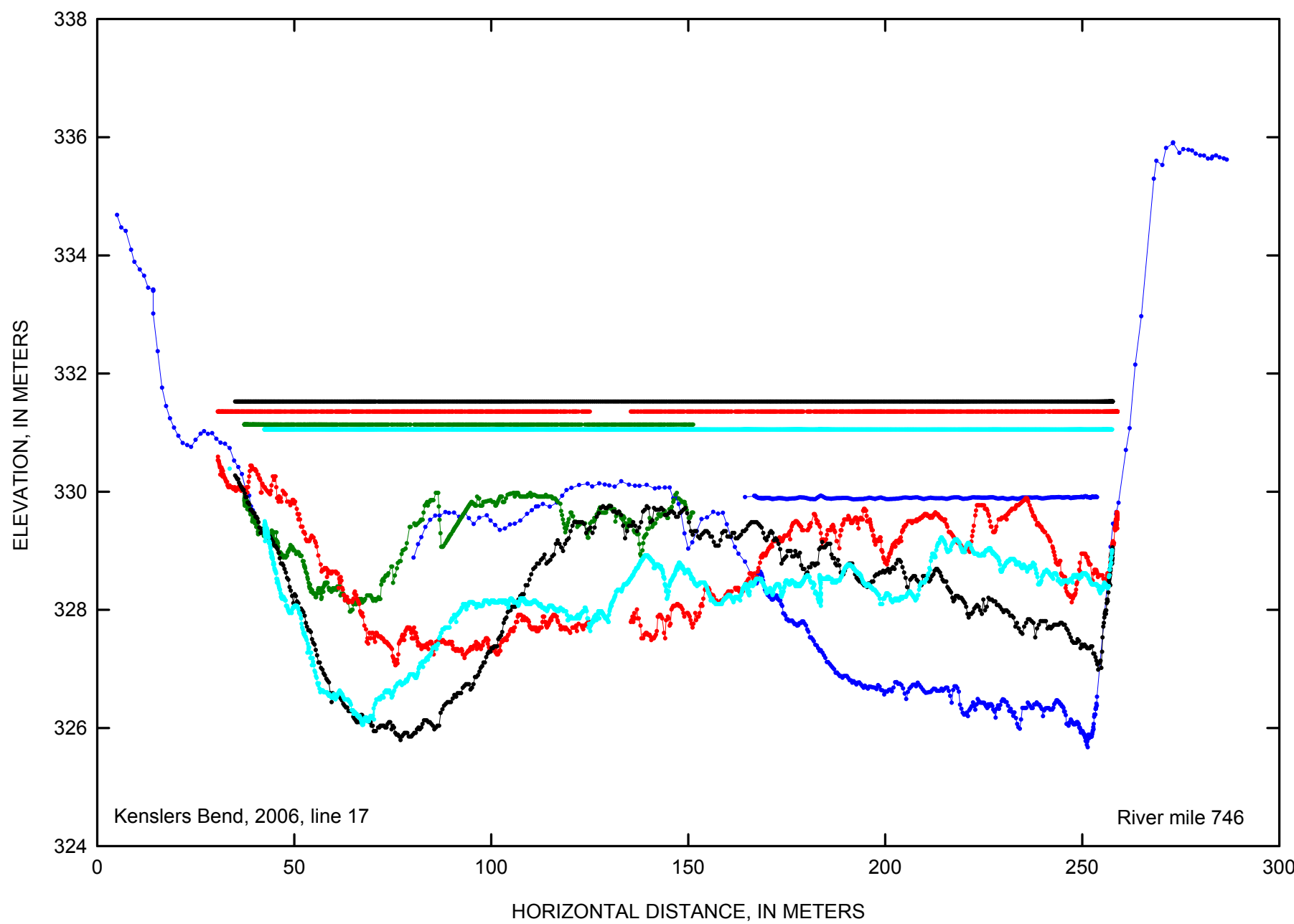

EXPLANATION

March 2006 I

March 2006 II

- May 2006

- June 2006

March 2007

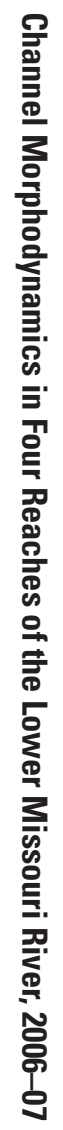

Figure 2-35. Kenslers Bend cross-section line 17 at Missouri River mile 746 in 2006. 


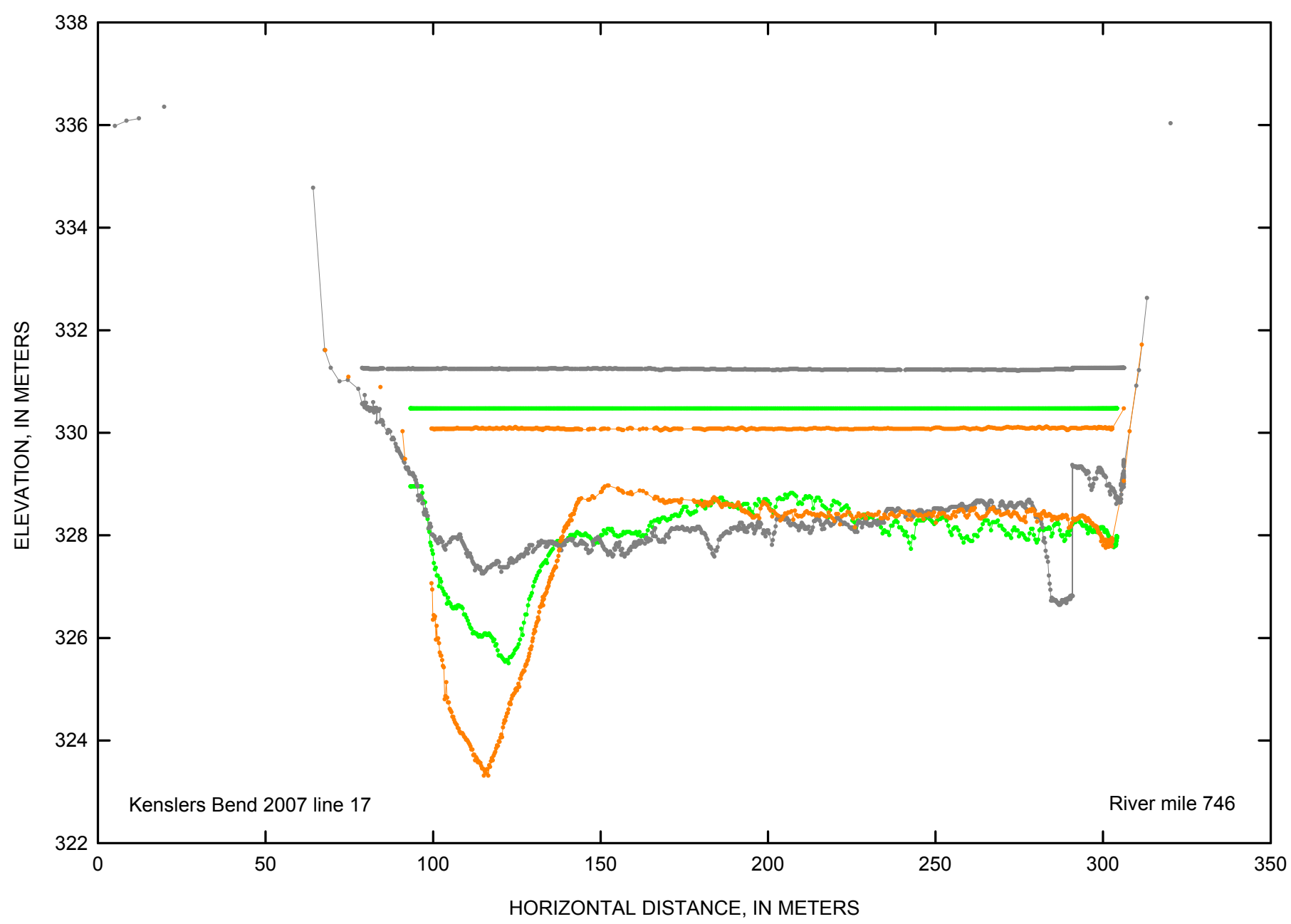

EXPLANATION

April 2007

July 2007

October 2007

Figure 2-36. Kenslers Bend cross-section line 17 at Missouri River mile 746 in 2007. 


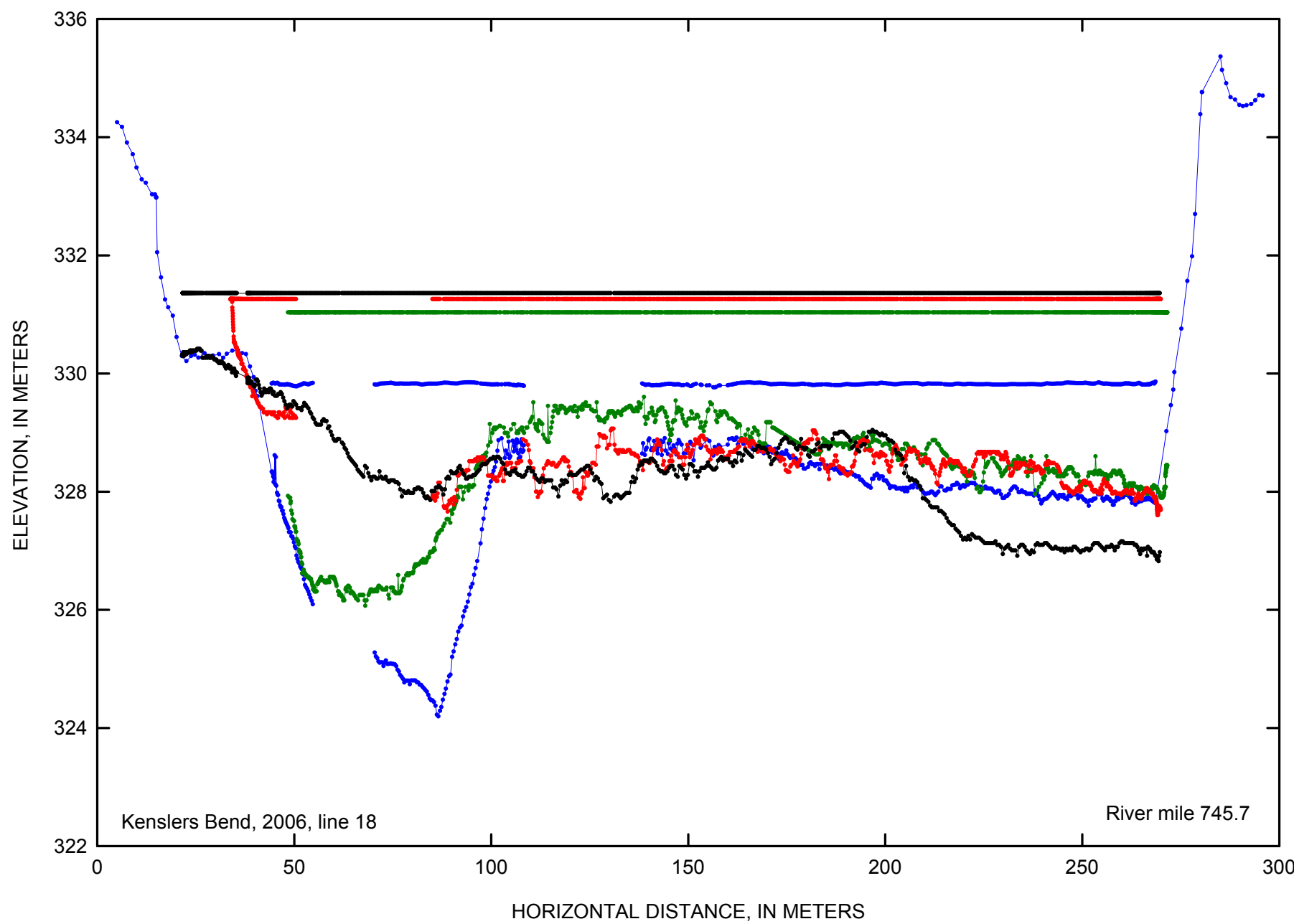

EXPLANATION

- March 2006 I

March 2006 II

May 2006

June 2006

March 2007

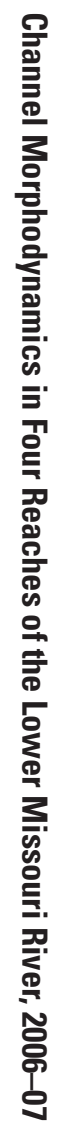

Figure 2-37. Kenslers Bend cross-section line 18 at Missouri River mile 745.7 in 2006. 


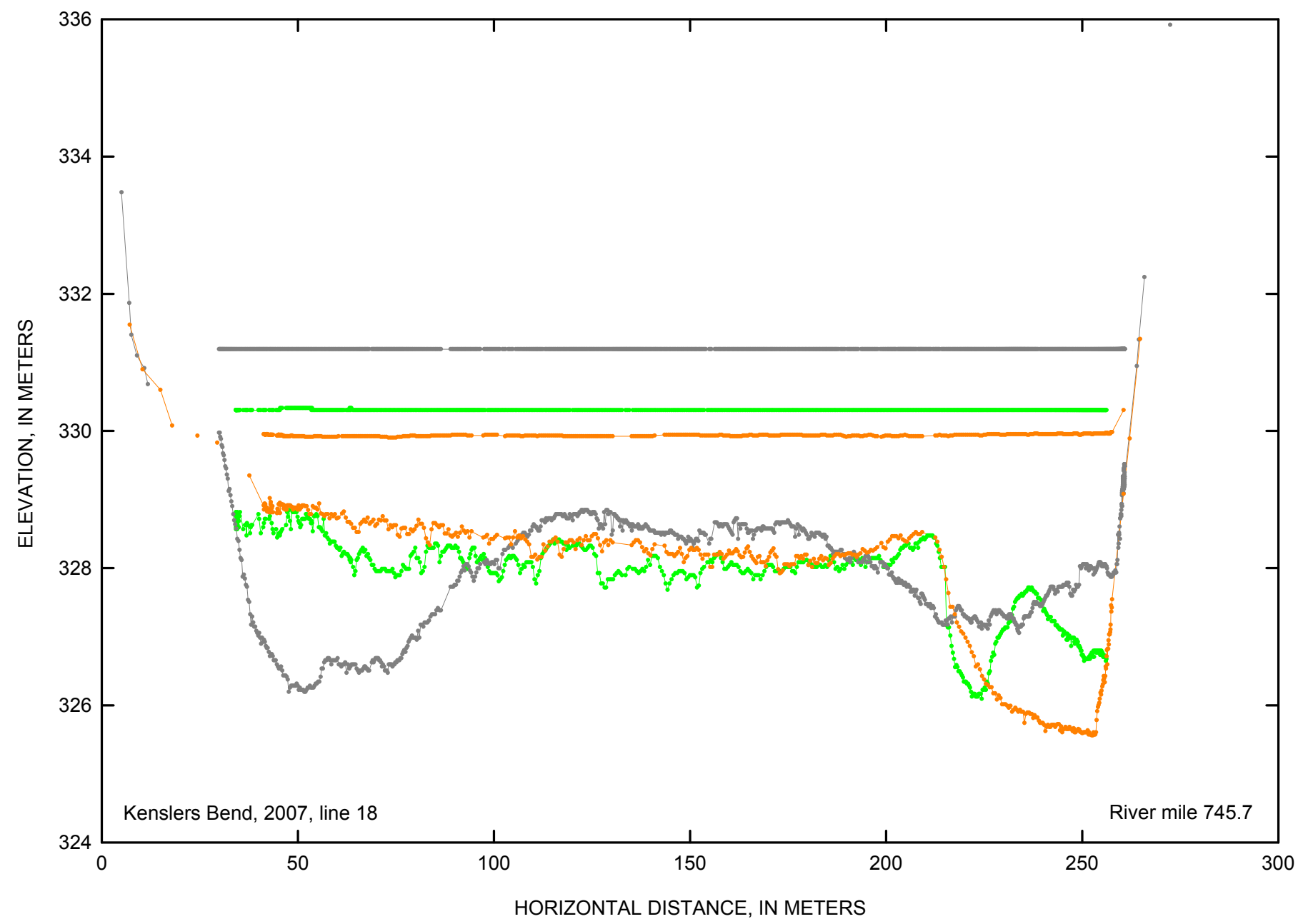

\section{EXPLANATION}

April 2007

July 2007

October 2007 


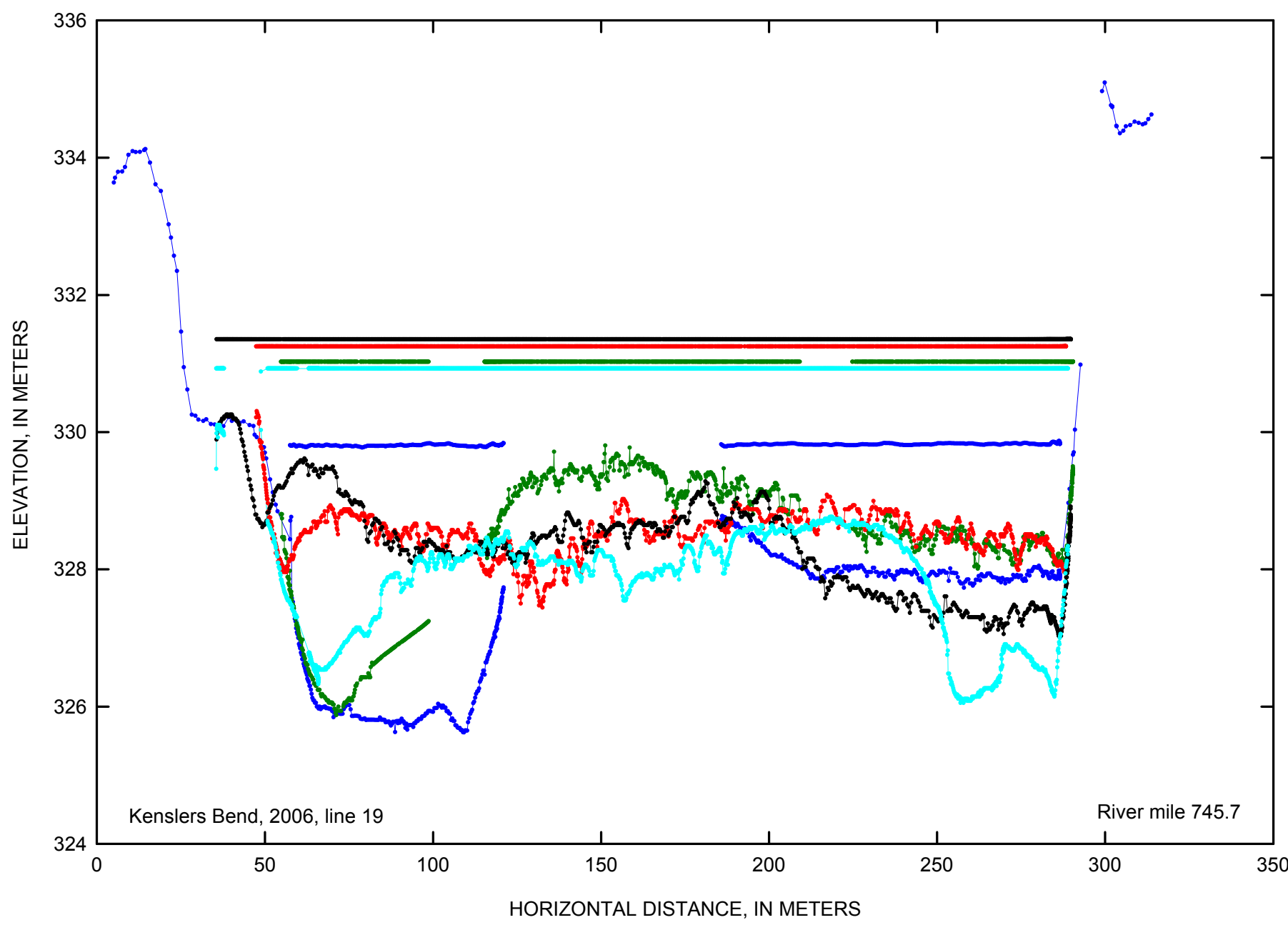

\section{EXPLANATION}

March 2006 I

March 2006

May 2006

June 2006

March 2007

Figure 2-39. Kenslers Bend cross-section line 19 at Missouri River mile 745.7 in 2006. 


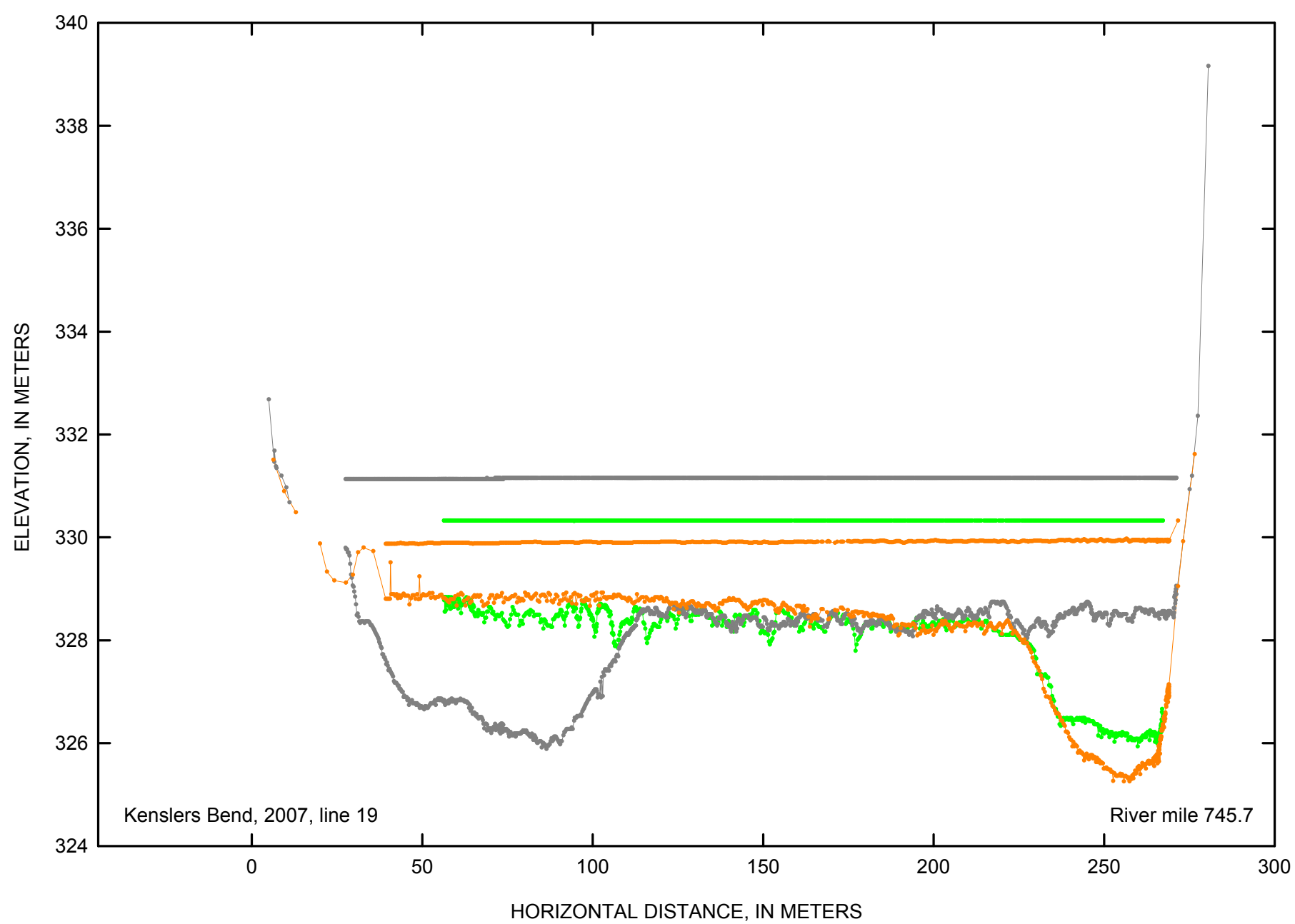

EXPLANATION

April 2007

July 2007

October 2007

Figure 2-40. Kenslers Bend cross-section line 19 at Missouri River mile 745.7 in 2007. 


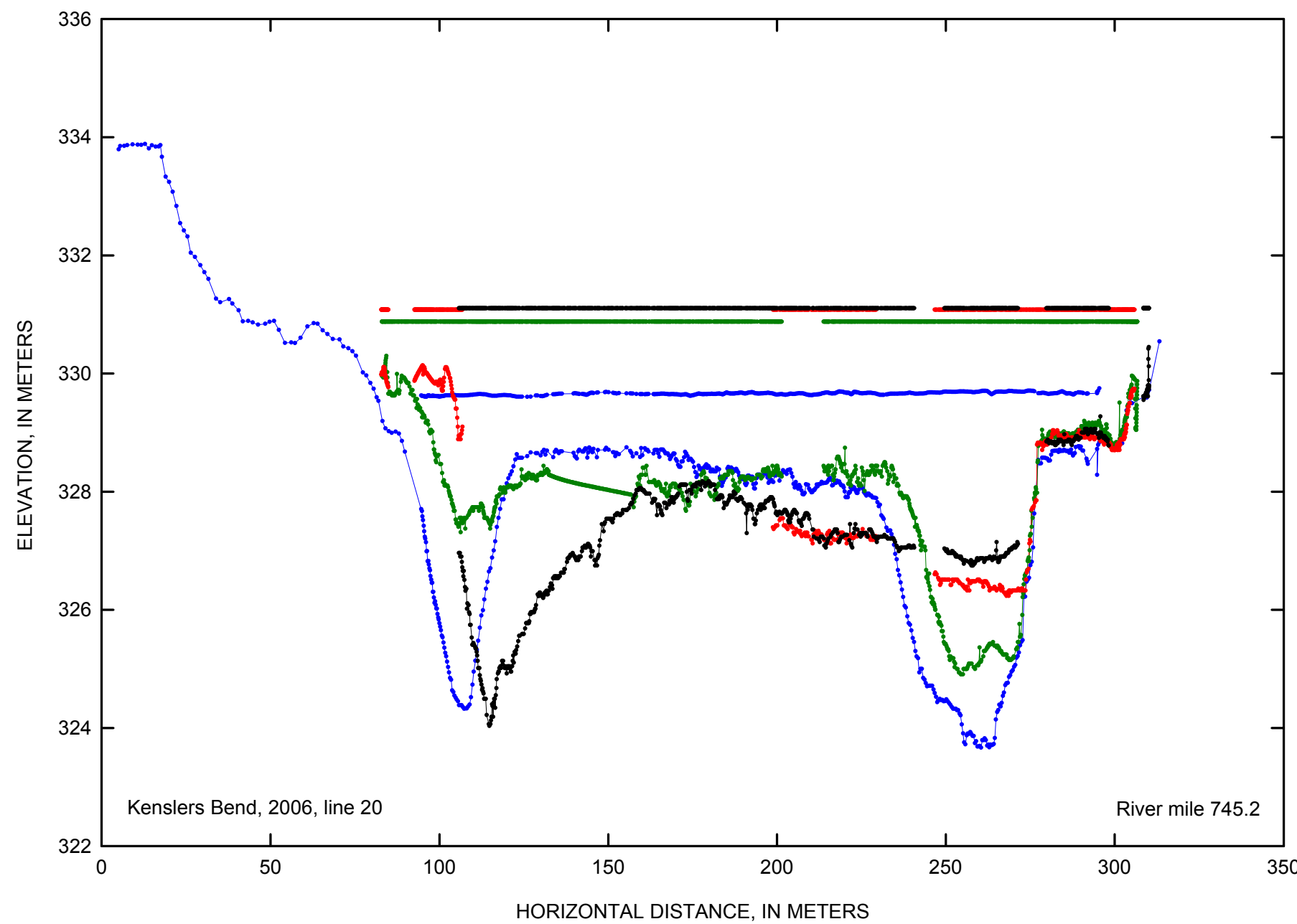

EXPLANATION

March 2006

March 2006 II

May 2006

June 2006

March 2007

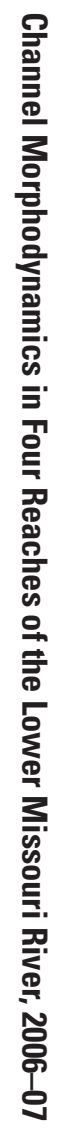

Figure 2-41. Kenslers Bend cross-section line 20 at Missouri River mile 745.2 in 2006. 


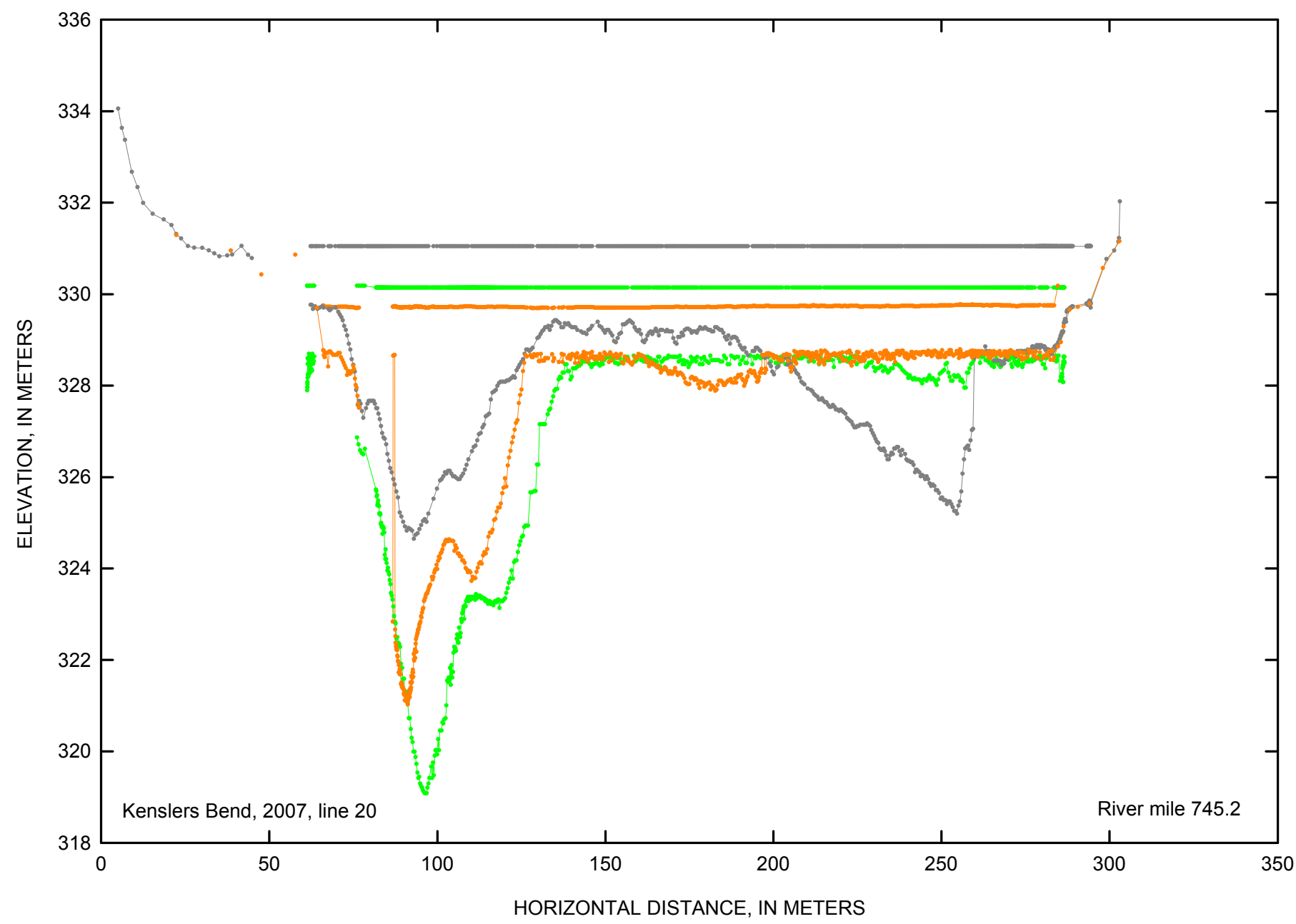

\section{EXPLANATION}

April 2007

July 2007

October 2007 


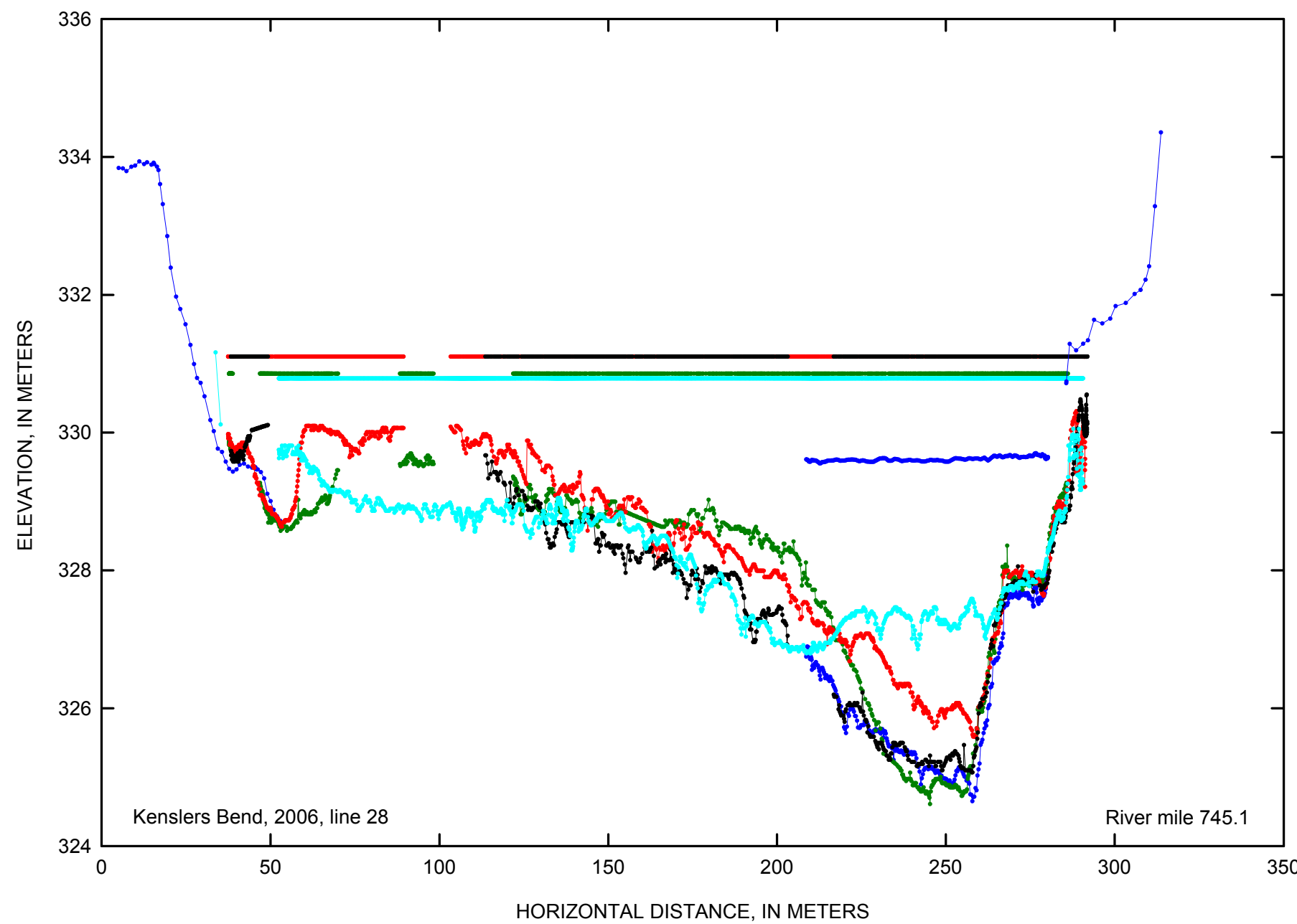

EXPLANATION

- March 2006 I

March 2006 II

May 2006

June 2006

March 2007

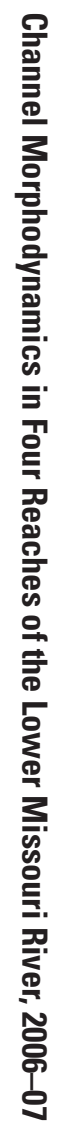

Figure 2-43. Kenslers Bend cross-section line 28 at Missouri River mile 745.1 in 2006. 


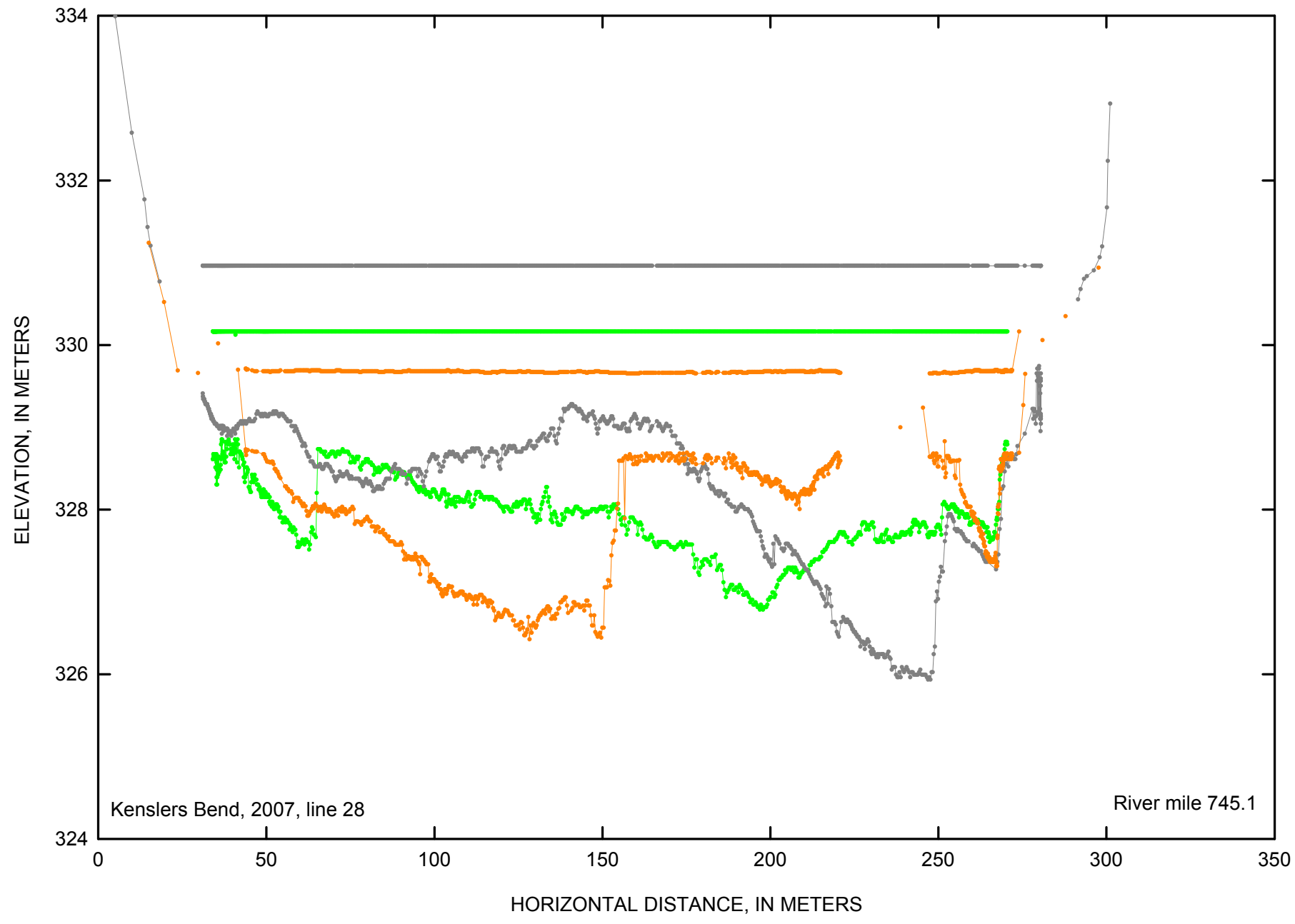

EXPLANATION

April 2007

July 2007

October 2007

Figure 2-44. Kenslers Bend cross-section line 28 at Missouri River mile 745.1 in 2007. 


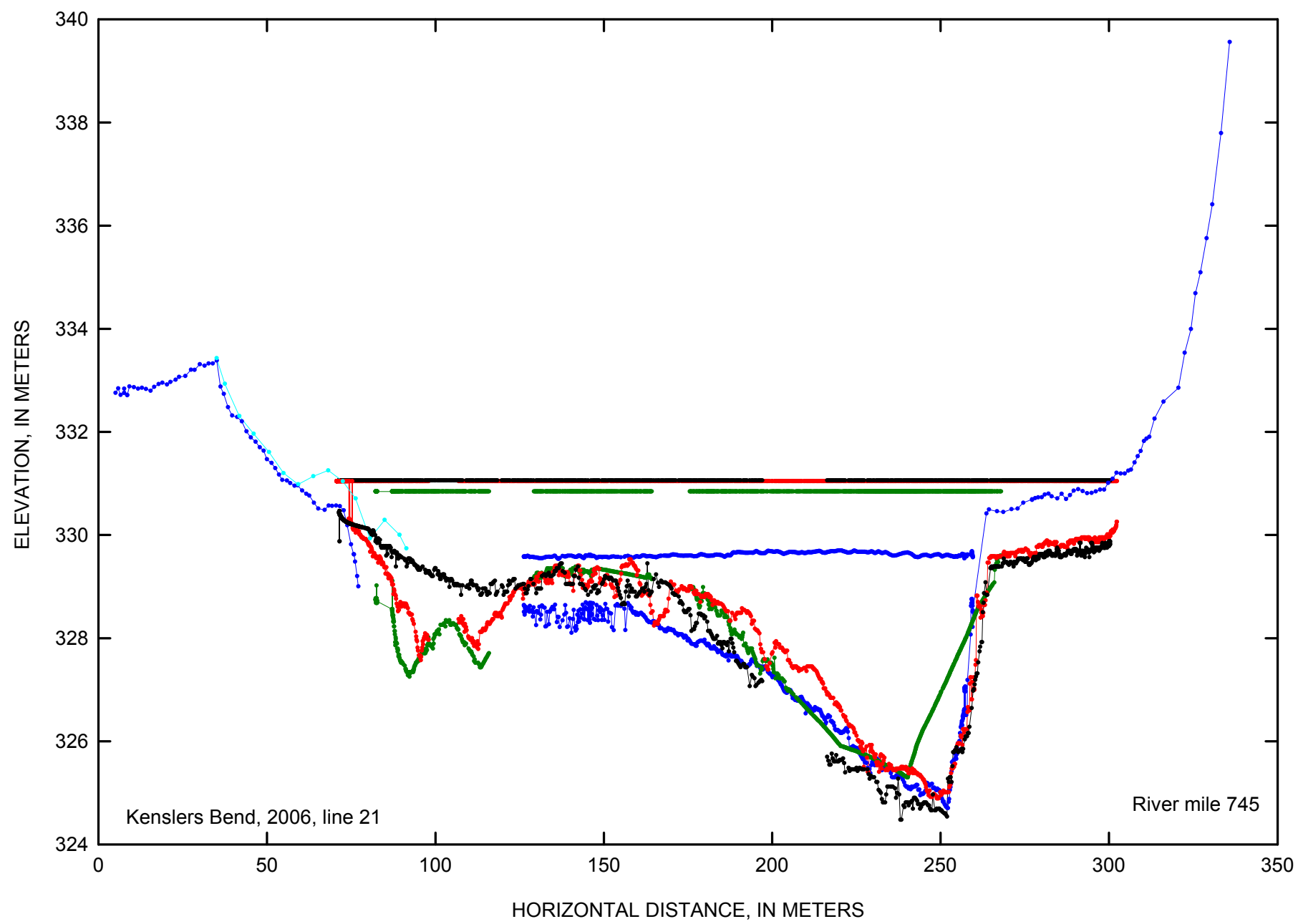

EXPLANATION

March 2006 I

March 2006 II

May 2006

June 2006

March 2007

Figure 2-45. Kenslers Bend cross-section line 21 at Missouri River mile 745 in 2006 


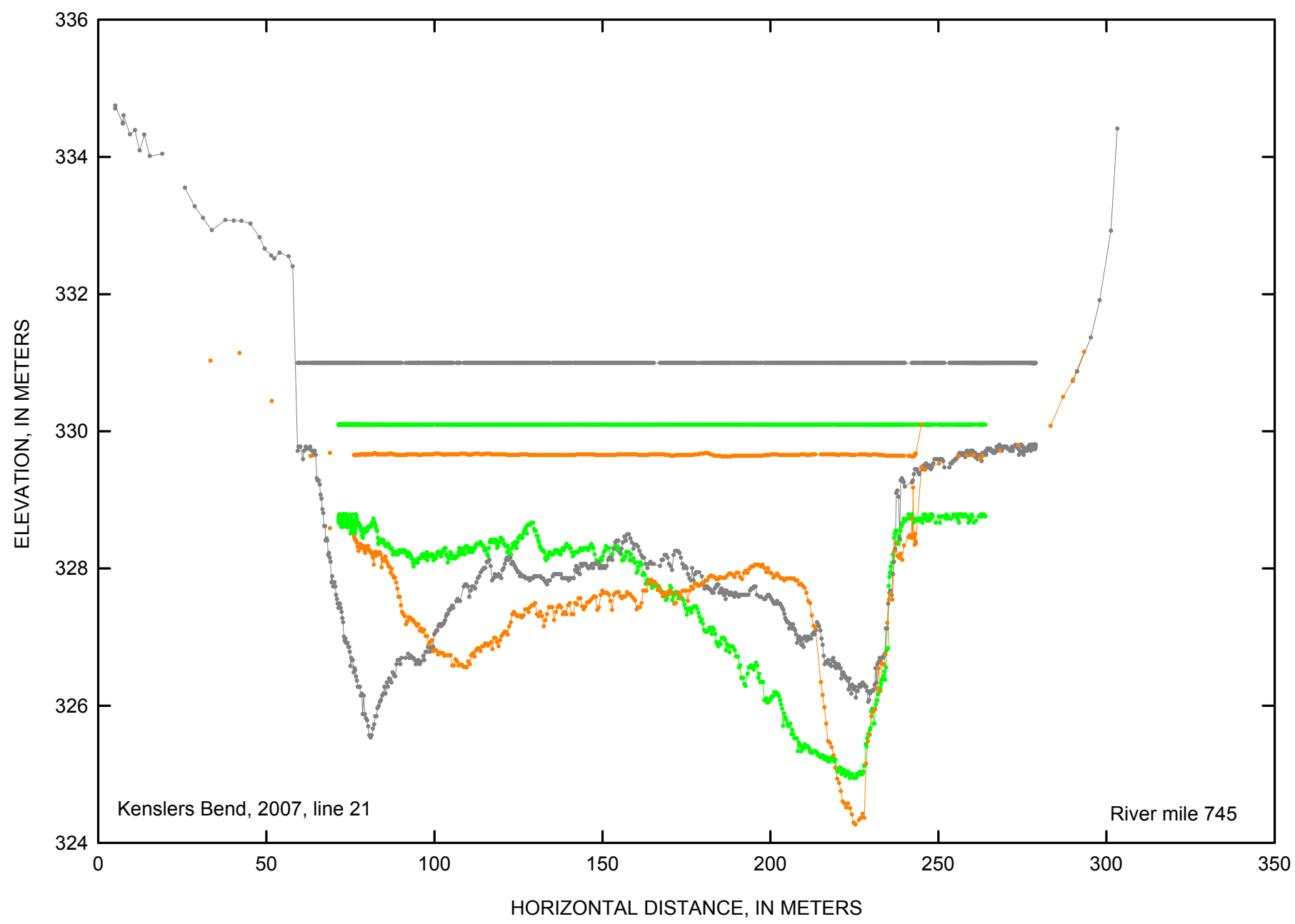

EXPLANATION

April 2007

July 2007

October 2007 


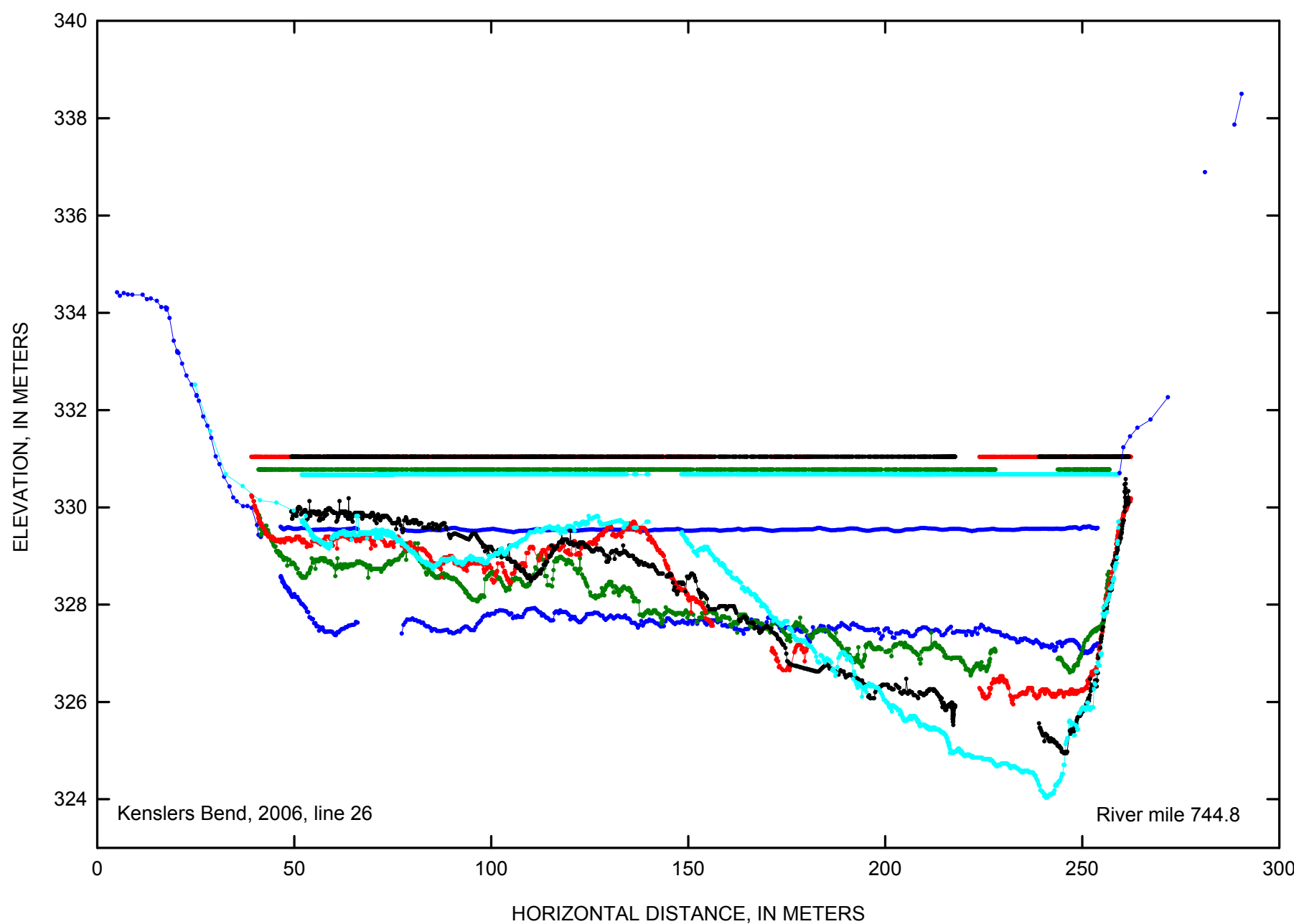

\section{EXPLANATION}

March 2006 I

- March 2006 II

- May 2006

- June 2006

March 2007

Figure 2-47. Kenslers Bend cross-section line 26 at Missouri River mile 744.8 in 2006. 


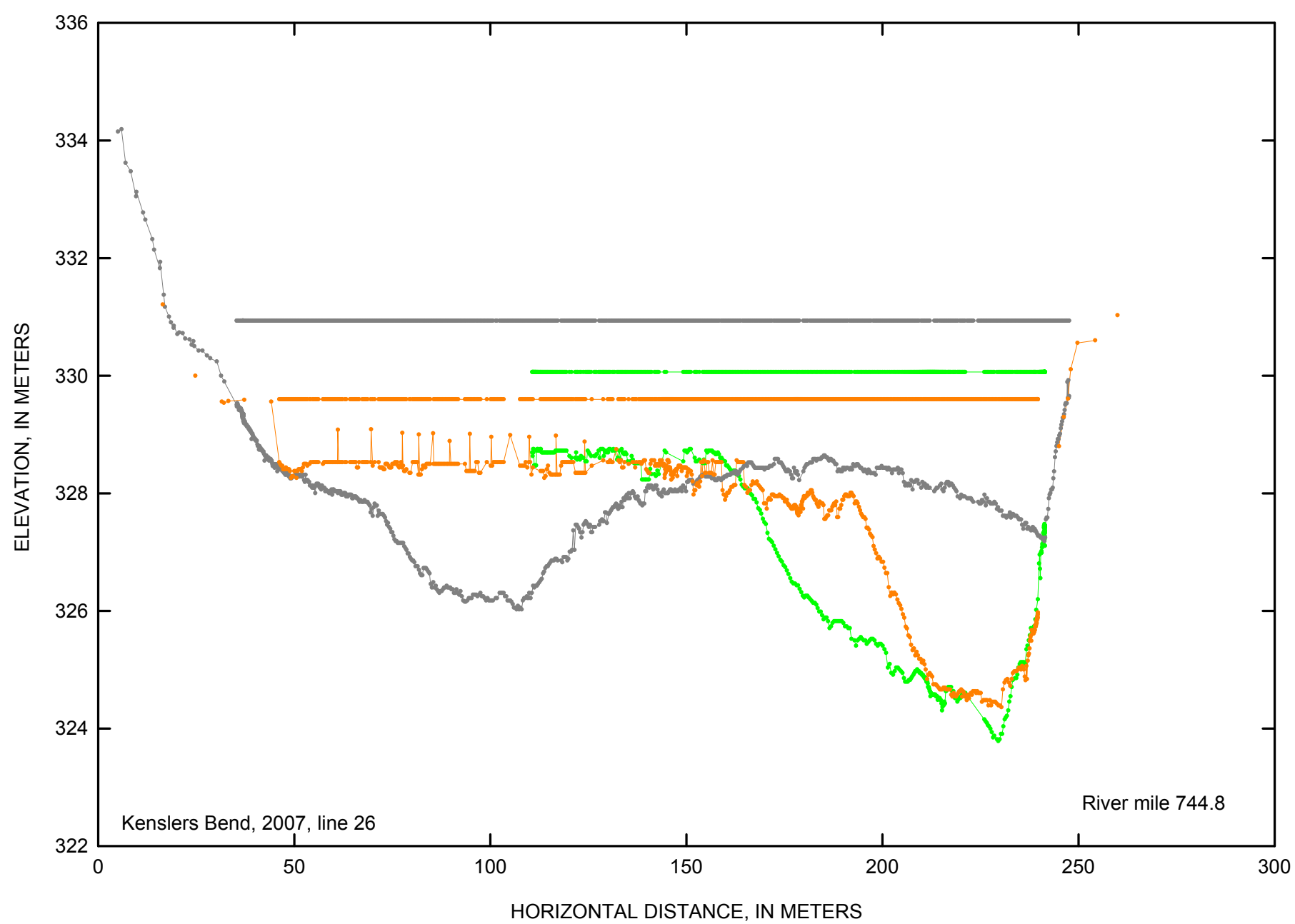

EXPLANATION

April 2007

July 2007

October 2007

Figure 2-48. Kenslers Bend cross-section line 26 at Missouri River mile 744.8 in 2007. 


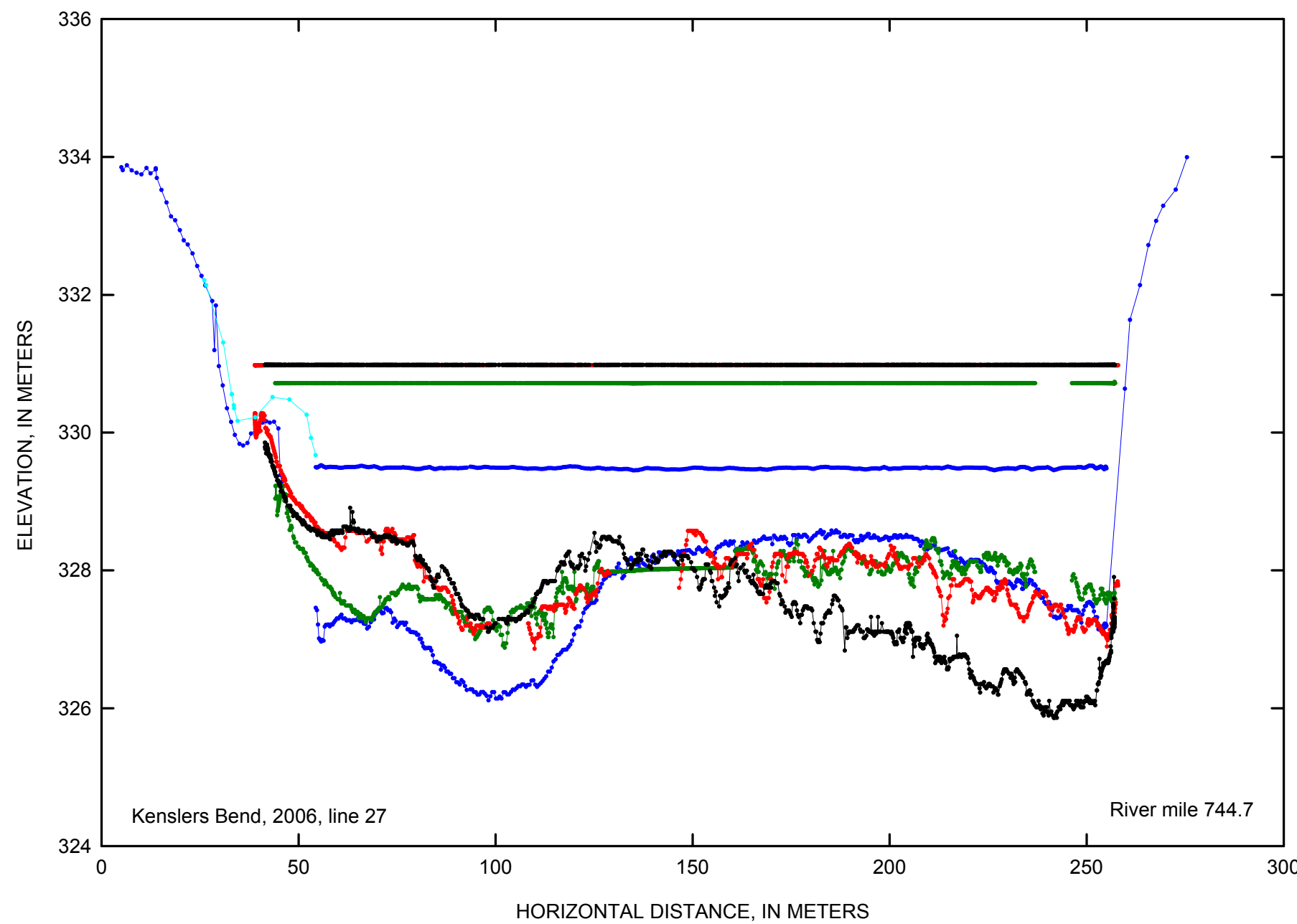

EXPLANATION

- March 2006 I

March 2006 II

May 2006

June 2006

March 2007

Figure 2-49. Kenslers Bend cross-section line 27 at Missouri River mile 744.7 in 2006. 


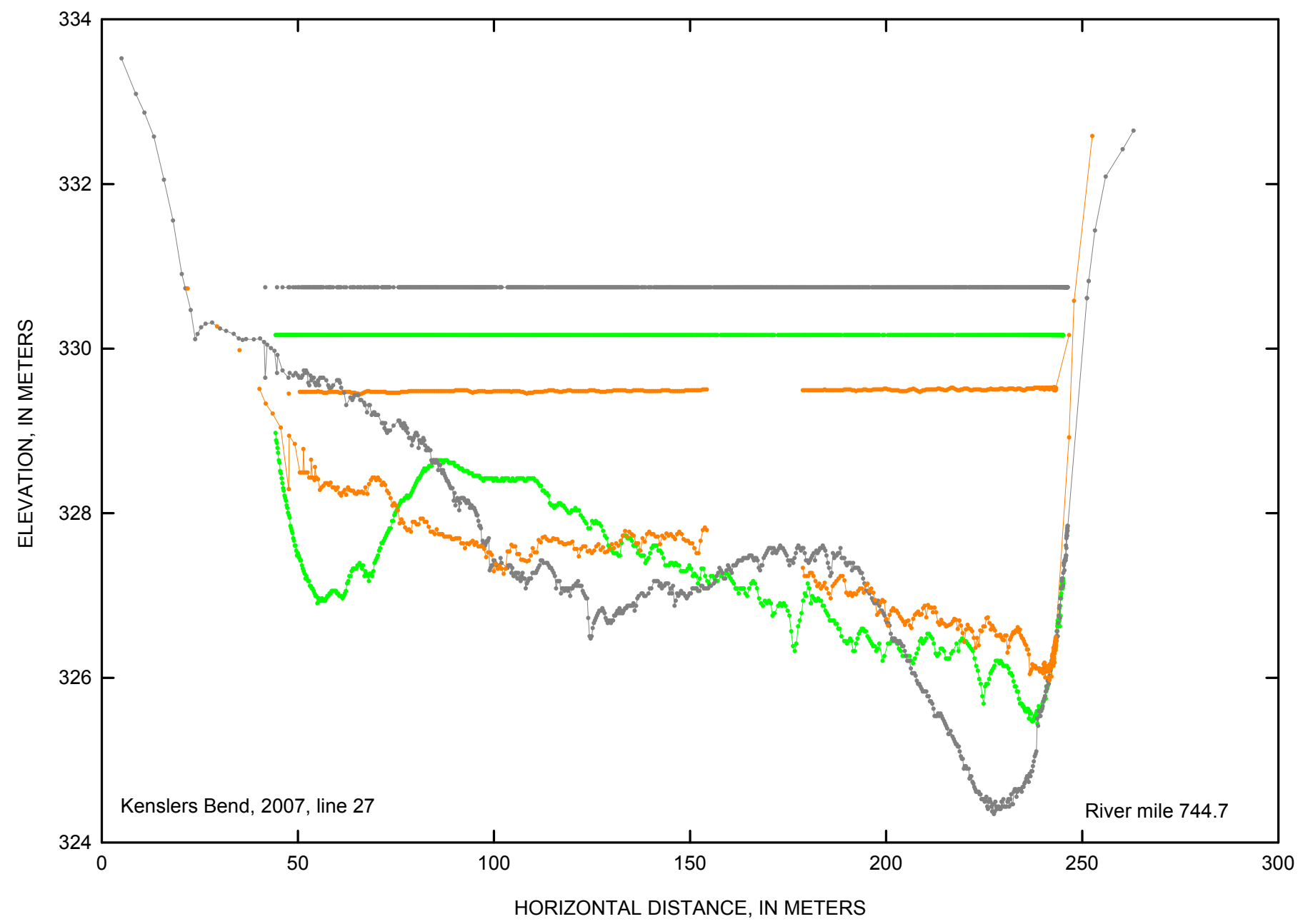

EXPLANATION

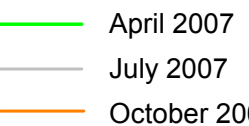




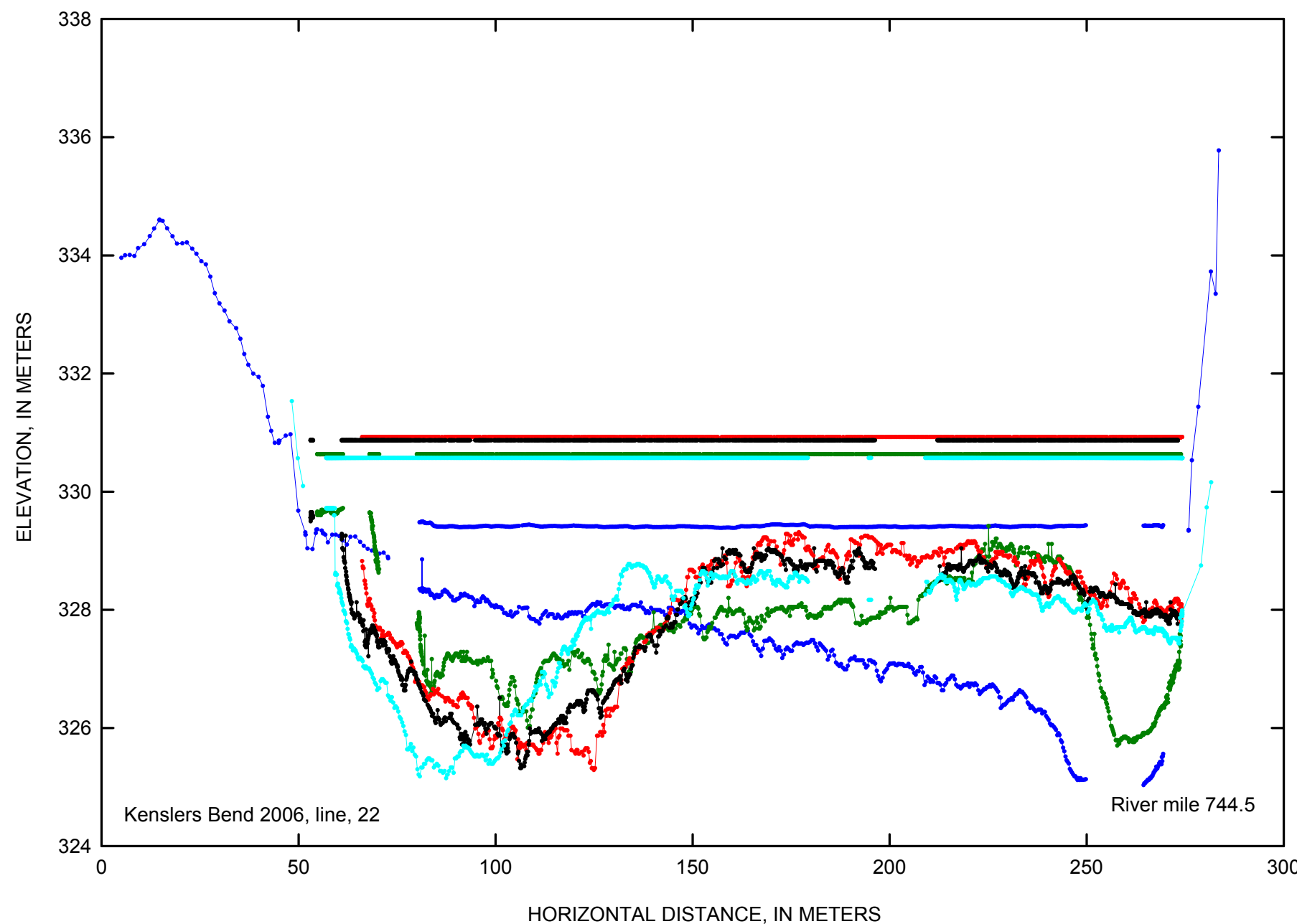

EXPLANATION

March 2006 I

March 2006 II

May 2006

June 2006

March 2007

Figure 2-51. Kenslers Bend cross-section line 22 at Missouri River mile 744.5 in 2006. 


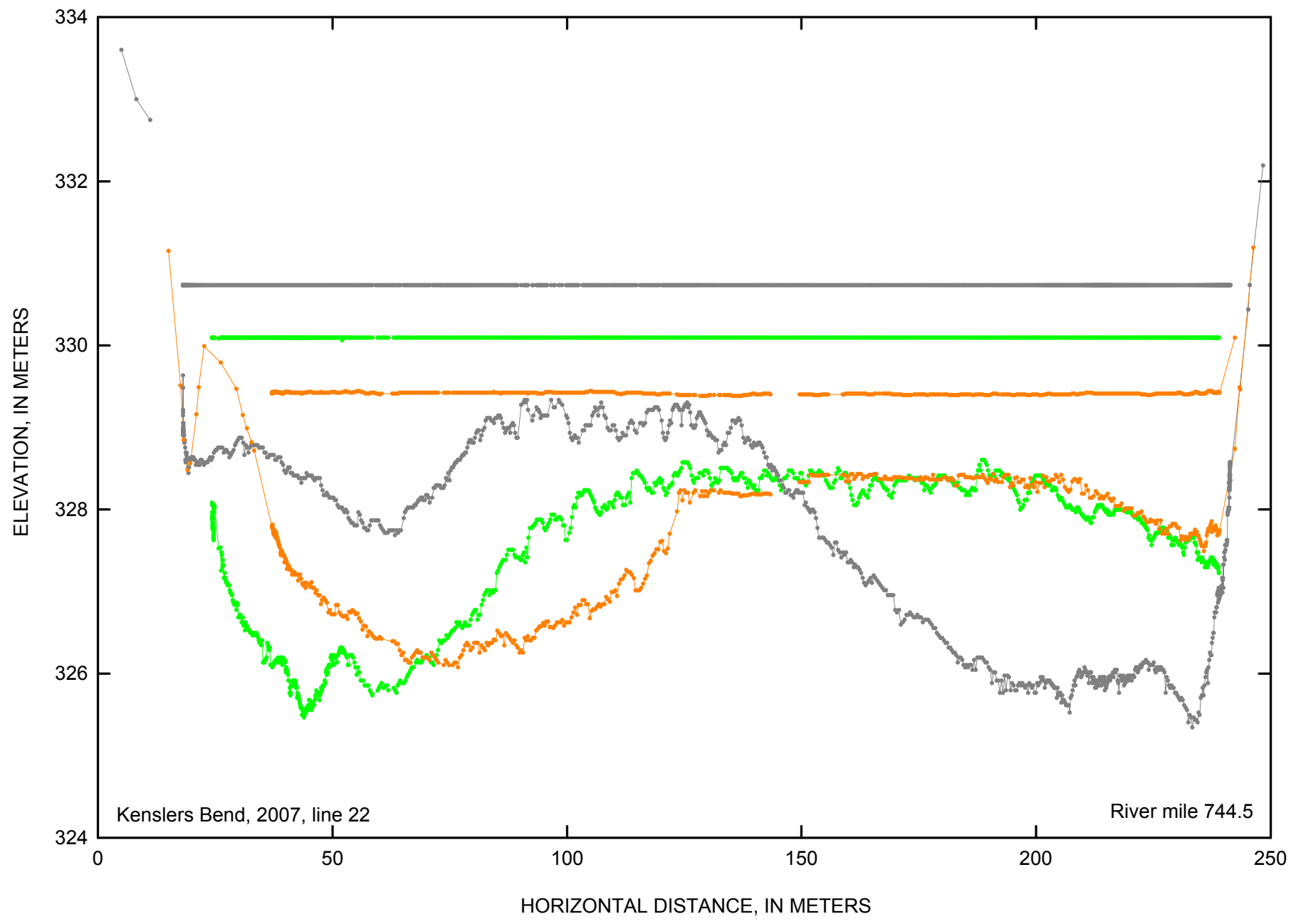

EXPLANATION

April 2007

July 2007

October 2007

Figure 2-52. Kenslers Bend cross-section line 22 at Missouri River mile 744.5 in 2007. 


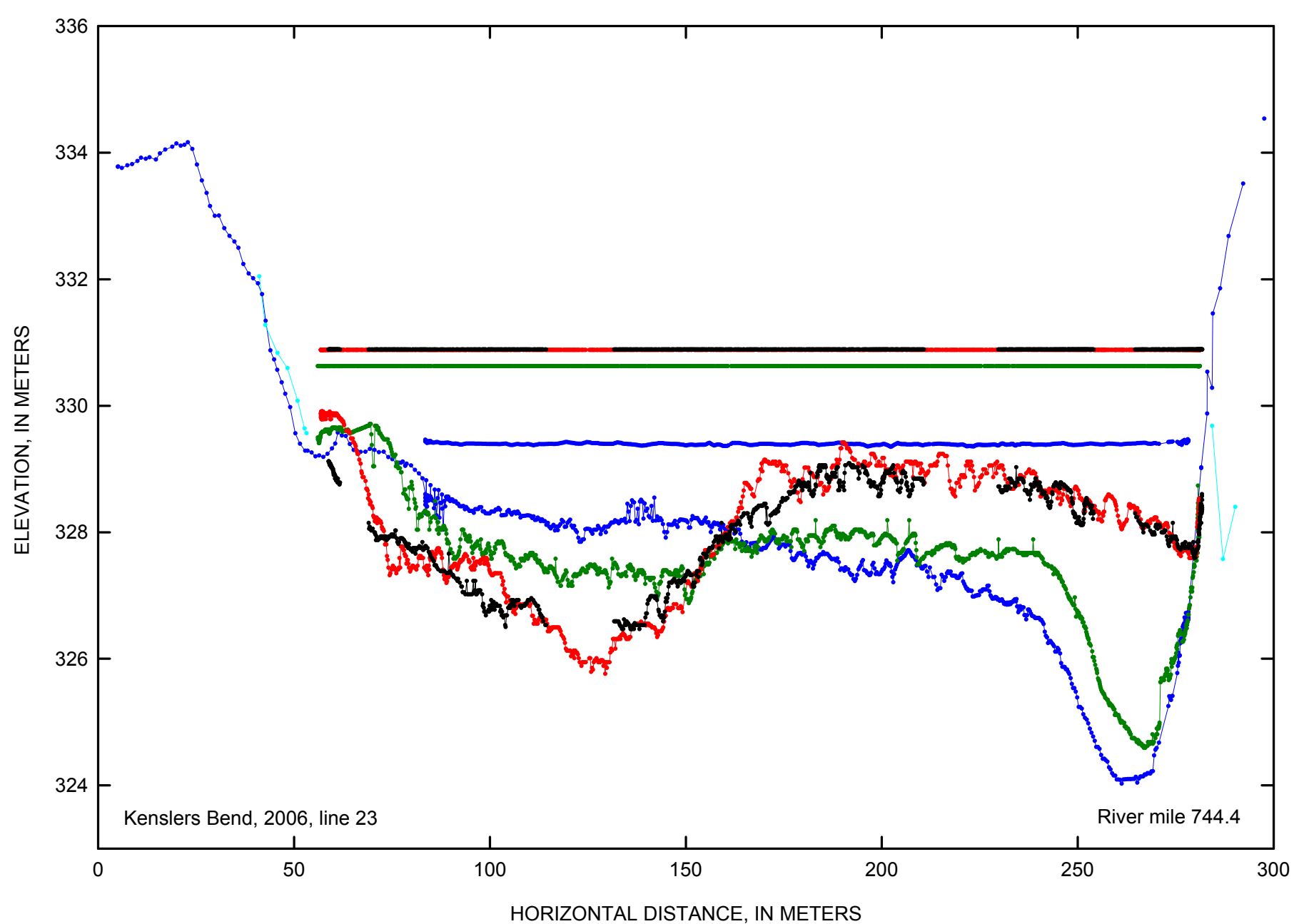

EXPLANATION

- March 2006 I

- March 2006 II

- May 2006

- June 2006

March 2007

Figure 2-53. Kenslers Bend cross-section line 23 at Missouri River mile 744.4 in 2006. 


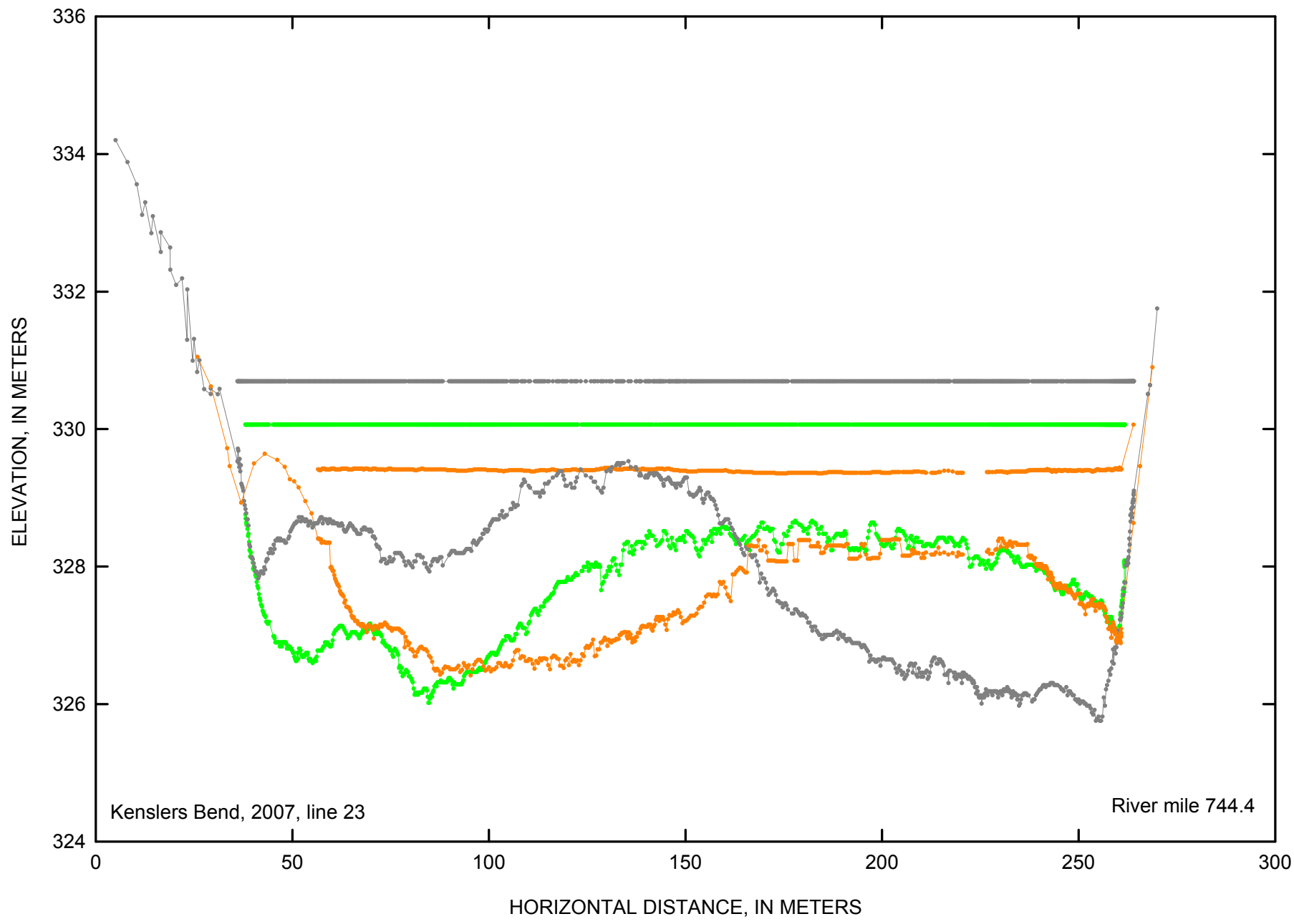

EXPLANATION

— April 2007

July 2007

October 2007

Figure 2-54. Kenslers Bend cross-section line 23 at Missouri River mile 744.4 in 2007. 


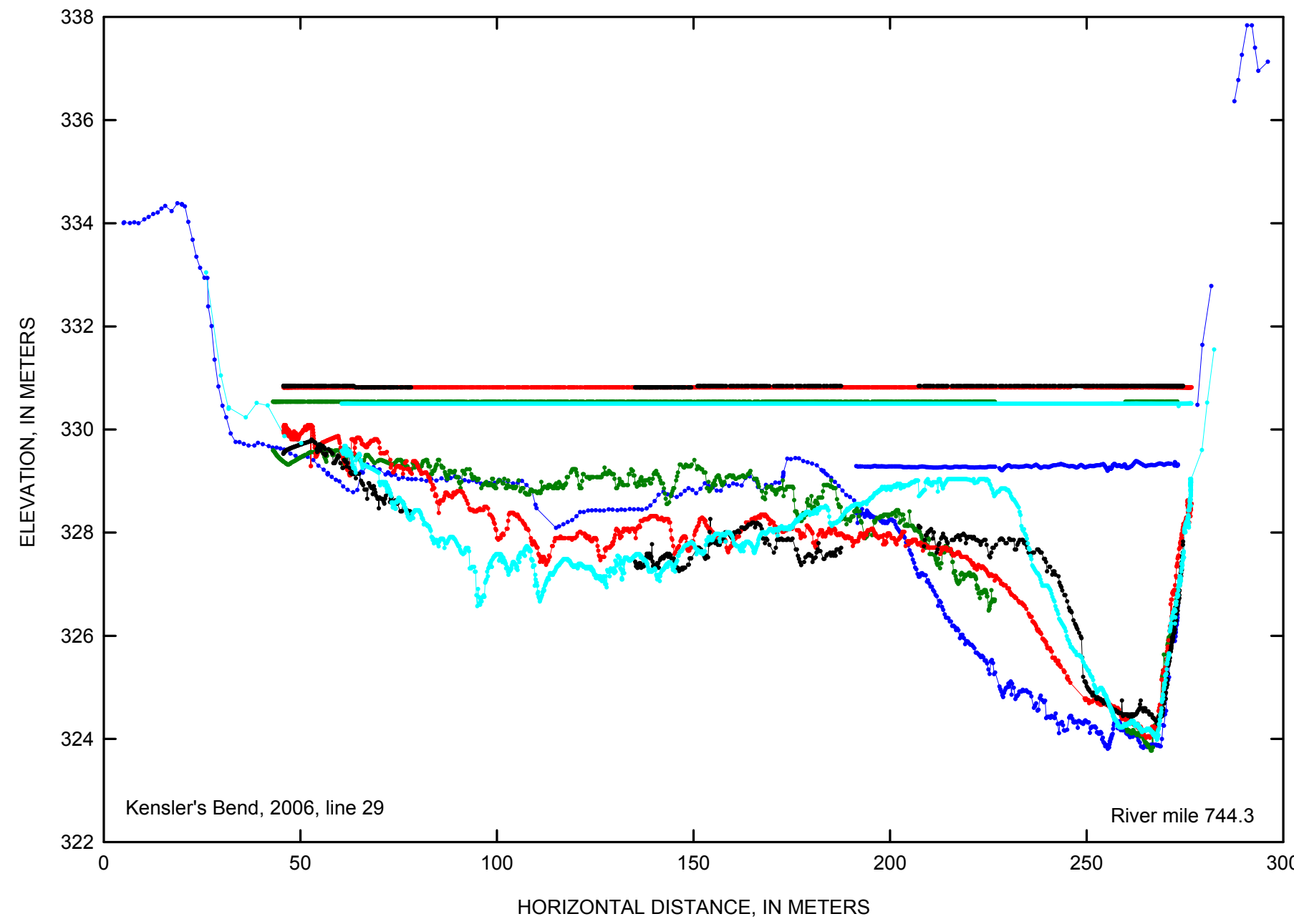

EXPLANATION

March 2006 I

March 2006 II

May 2006

June 2006

March 2007

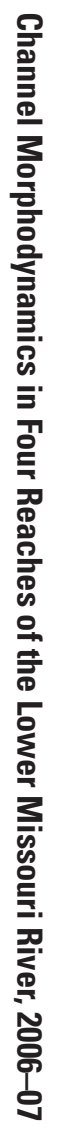

Figure 2-55. Kenslers Bend cross-section line 29 at Missouri River mile 744.3 in 2006. 


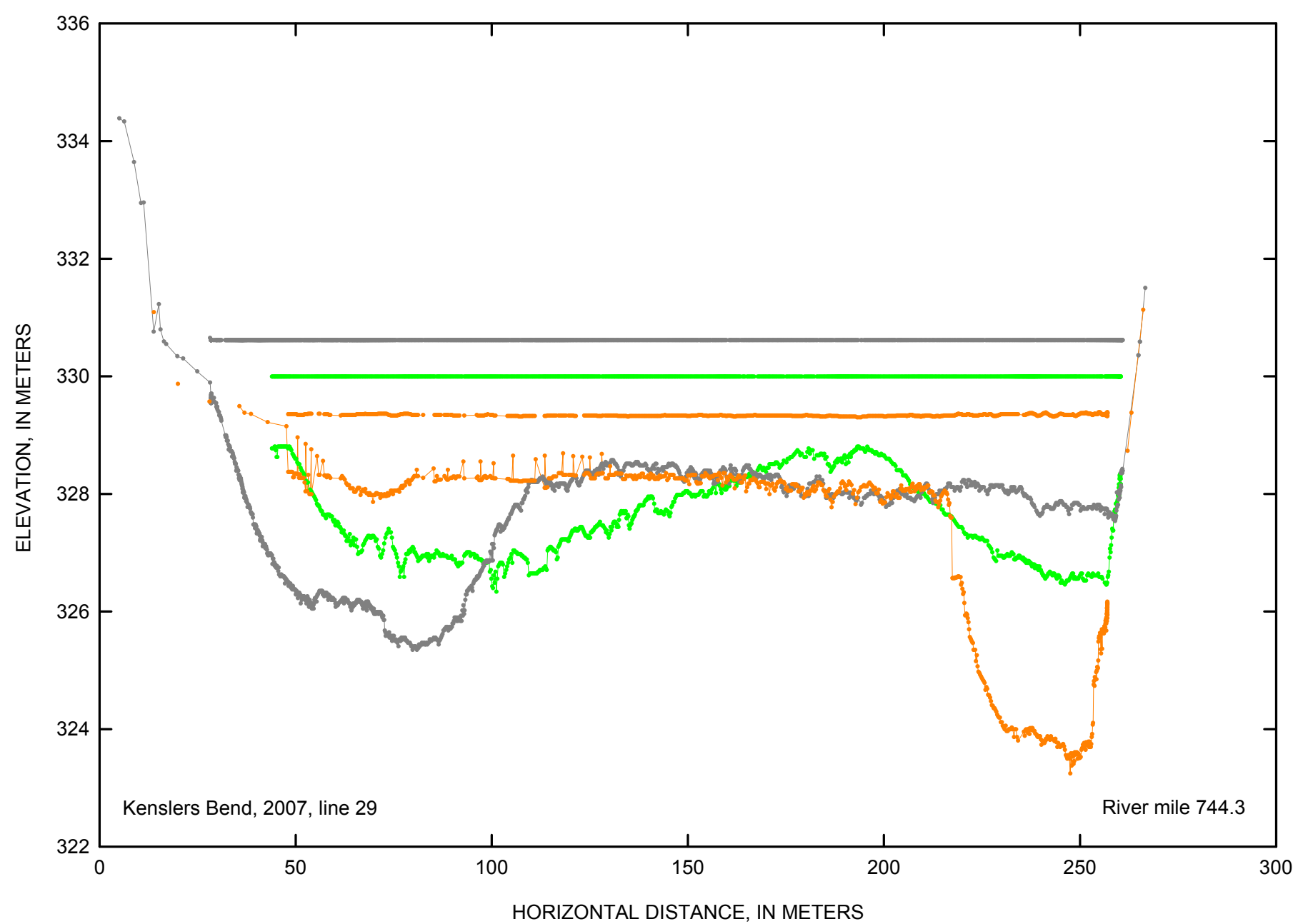

EXPLANATION

April 2007

July 2007

October 2007

Figure 2-56. Kenslers Bend cross-section line 29 at Missouri River mile 744.3 in 2007. 


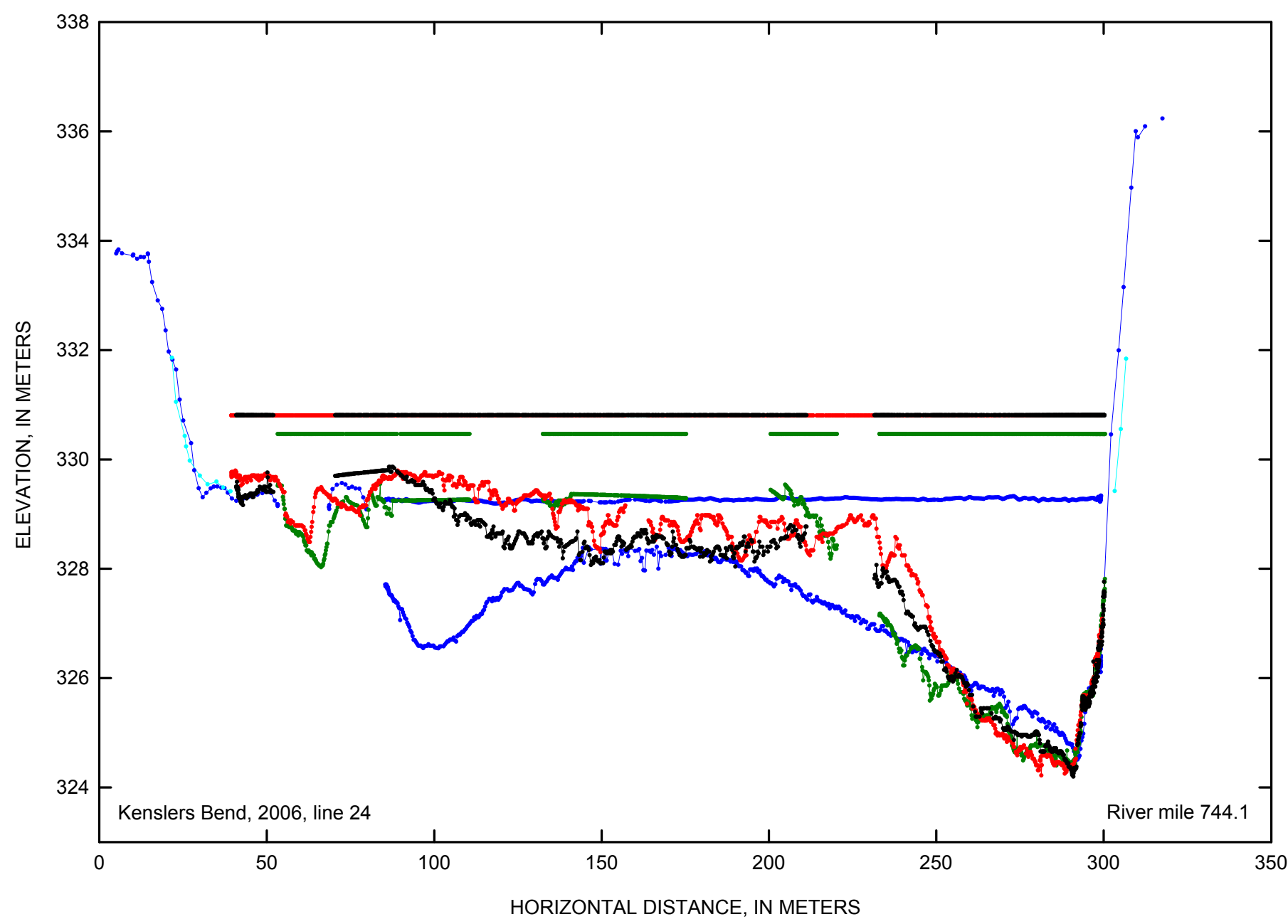

EXPLANATION

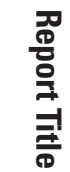

- March 2006 I

March 2006 II

May 2006

June 2006

March 2007

Figure 2-57. Kenslers Bend cross-section line 24 at Missouri River mile 744.1 in 2006. 


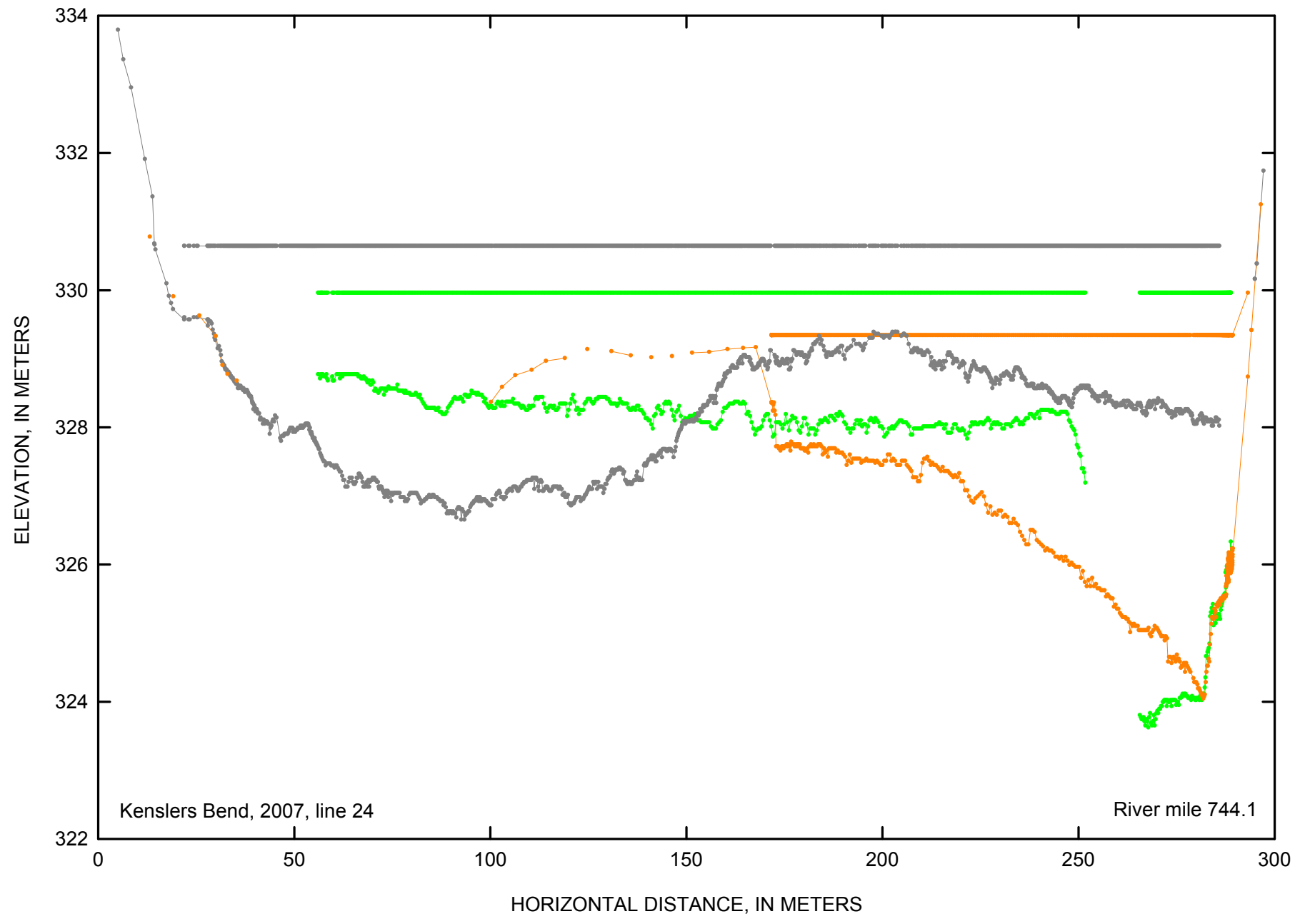

\section{EXPLANATION}

$\begin{array}{ll}\longrightarrow & \text { April } 2007 \\ & \text { July } 2007 \\ & \text { October } 2007\end{array}$

Figure 2-58. Kenslers Bend cross-section line 24 at Missouri River mile 744.1 in 2007. 


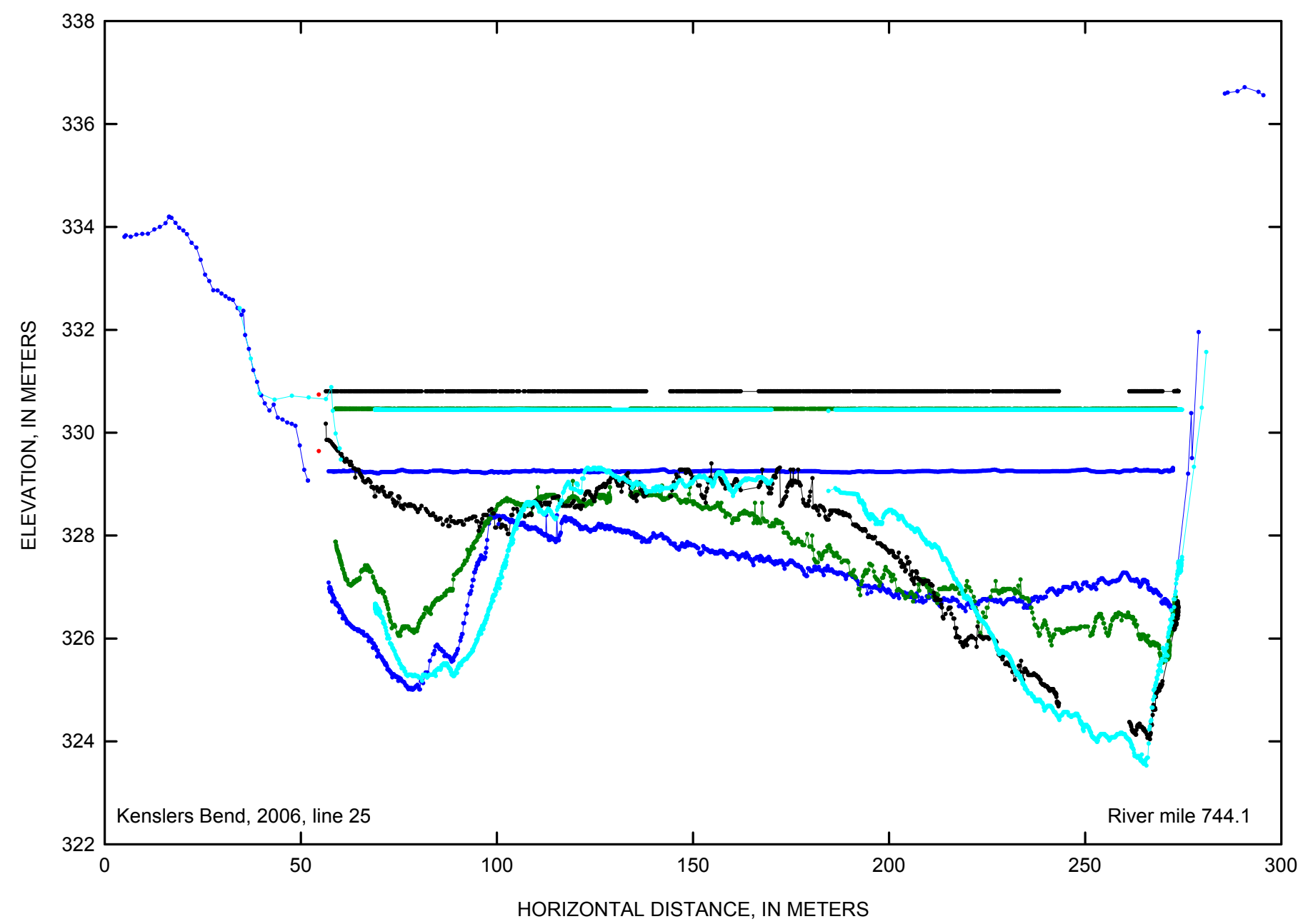

EXPLANATION

March 2006 I

March 2006 II

May 2006

June 2006

March 2007

Figure 2-59. Kenslers Bend cross-section line 25 at Missouri River mile 744.1 in 2006. 


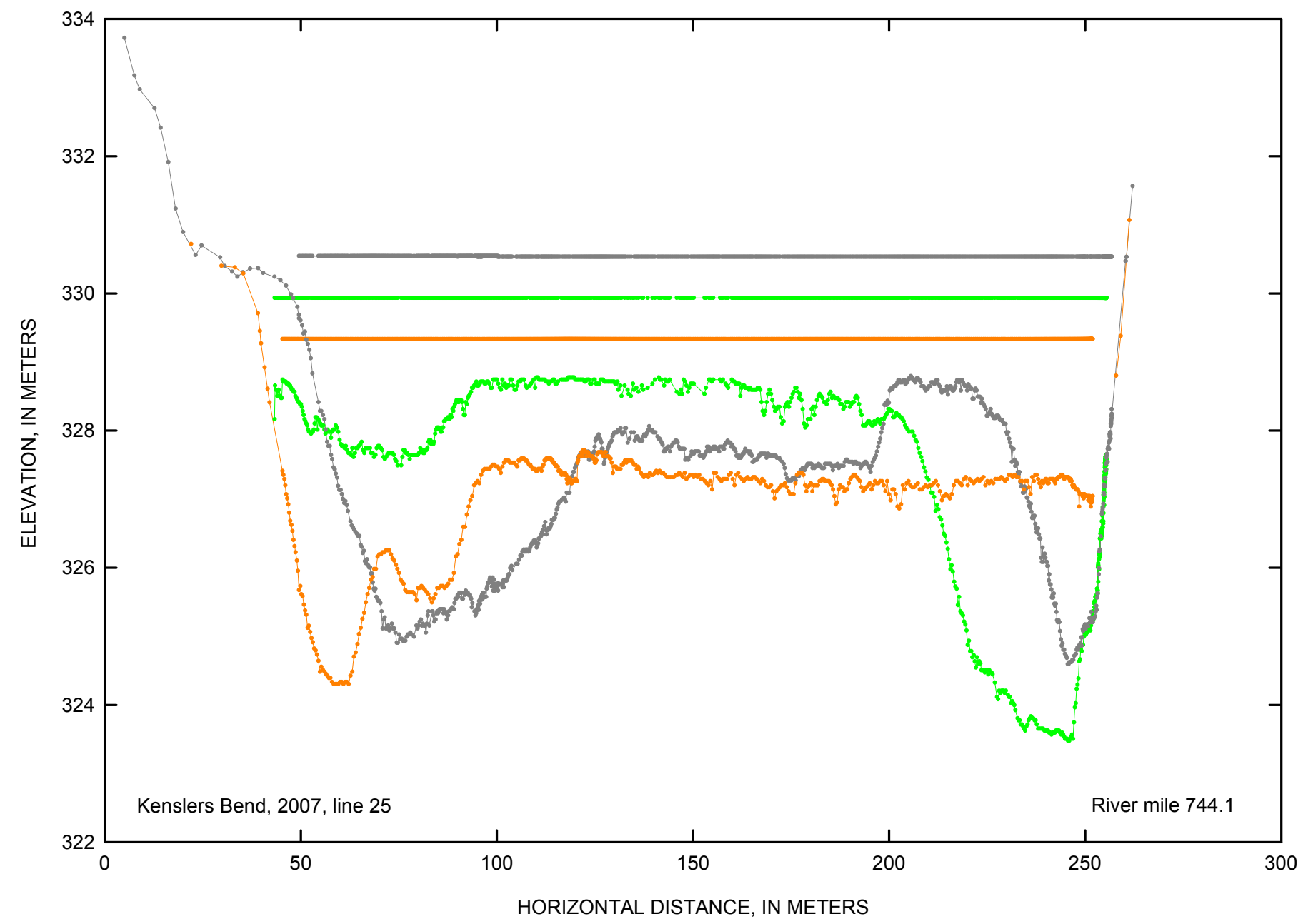

EXPLANATION

April 2007

July 2007

October 2007

Figure 2-60. Kenslers Bend cross-section line 25 at Missouri River mile 744.1 in 2007. 



\section{Appendix 3. Little Sioux Reach Cross Sections}




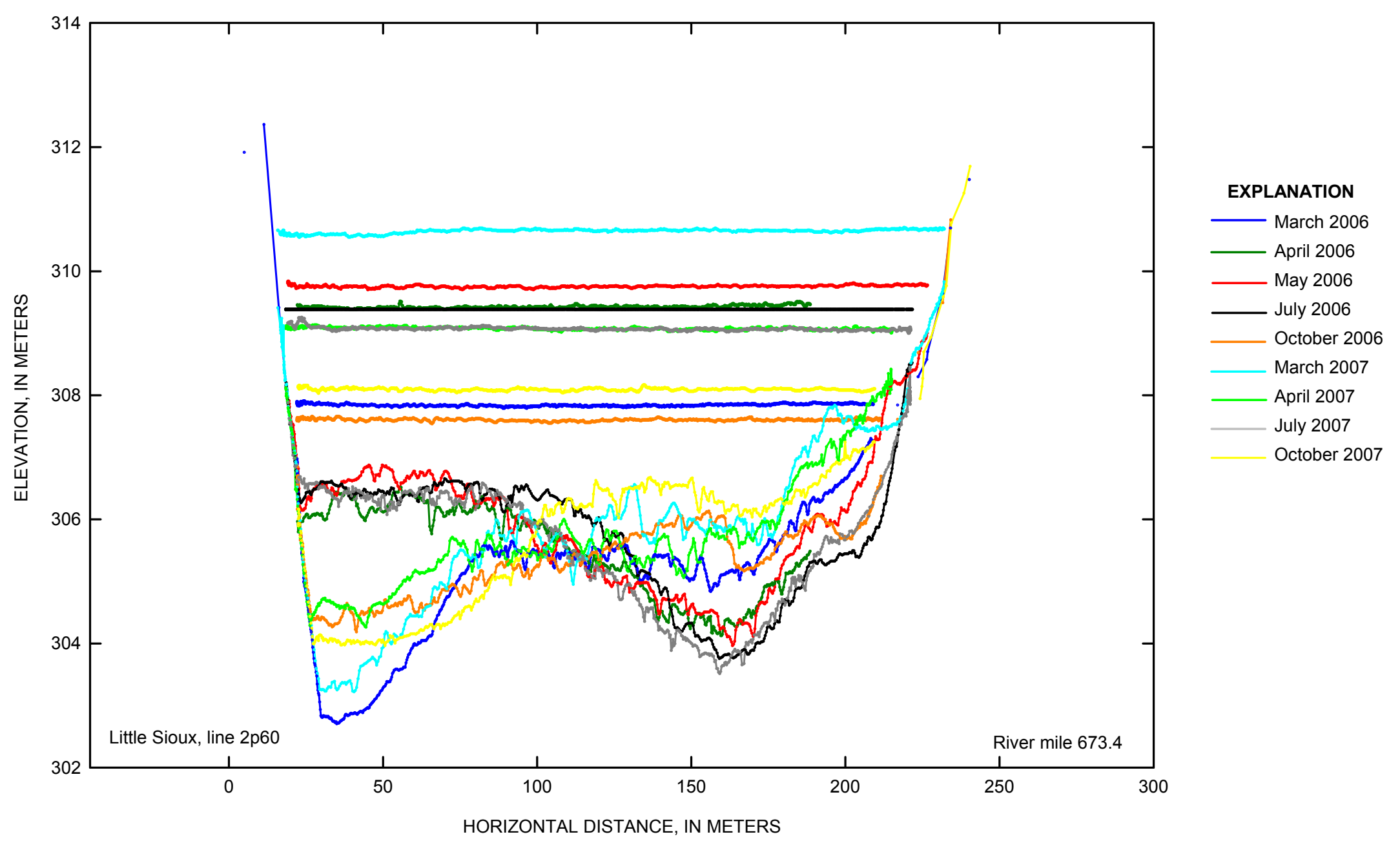

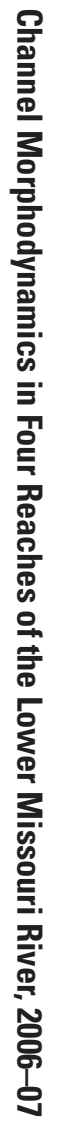

Figure 3-1. Little Sioux cross-section line $2 p 60$ at Missouri River mile 673.4 for 2006-07. 


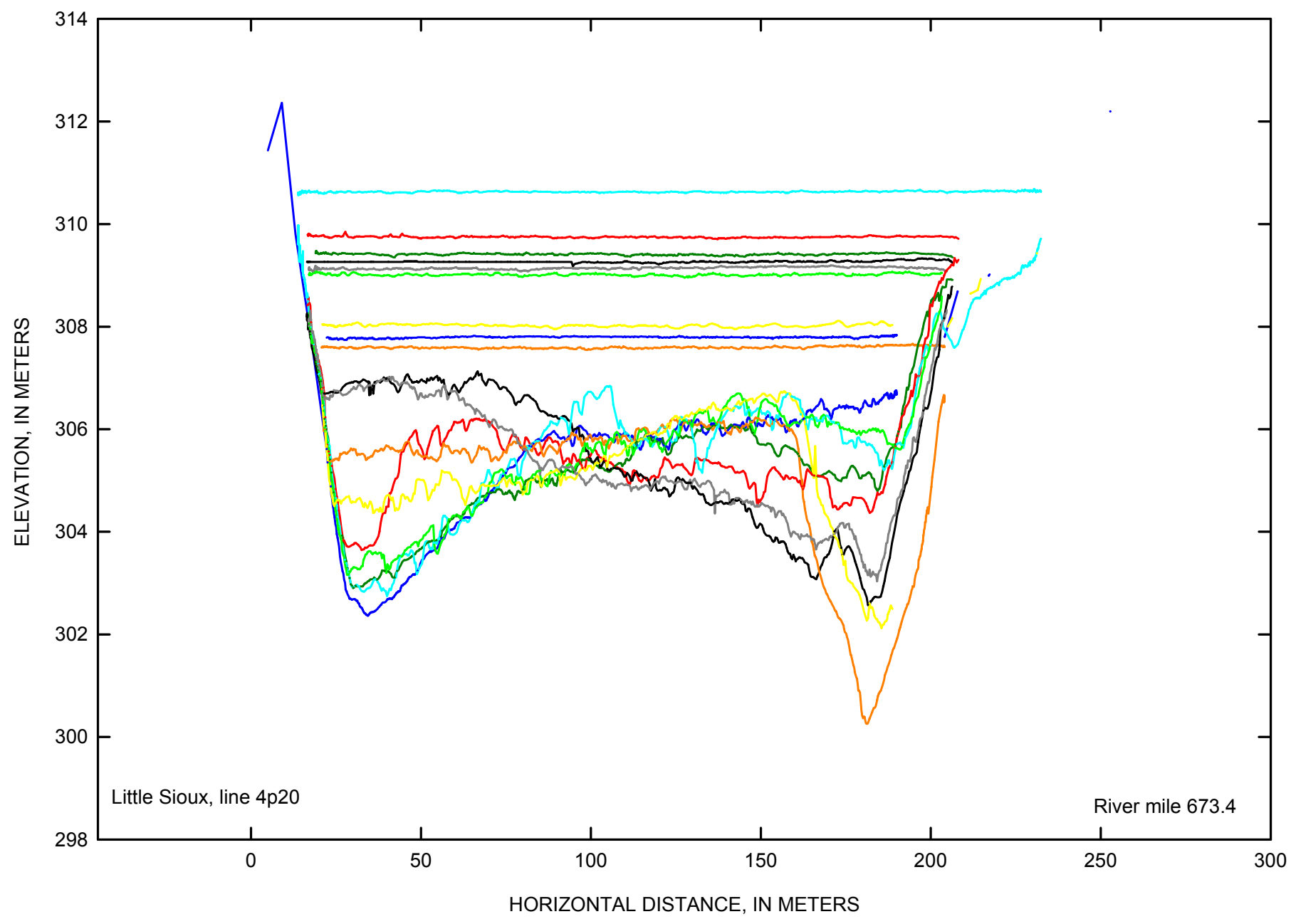

\begin{tabular}{c} 
EXPLANATION \\
\hline March 2006 \\
\hline April 2006 \\
\hline May 2006 \\
\hline July 2006 \\
October 2006 \\
March 2007 \\
April 2007 \\
July 2007 \\
October 2007
\end{tabular}

Figure 3-2. Little Sioux cross-section line 4p20 at Missouri River mile 673.4 for 2006-07. 


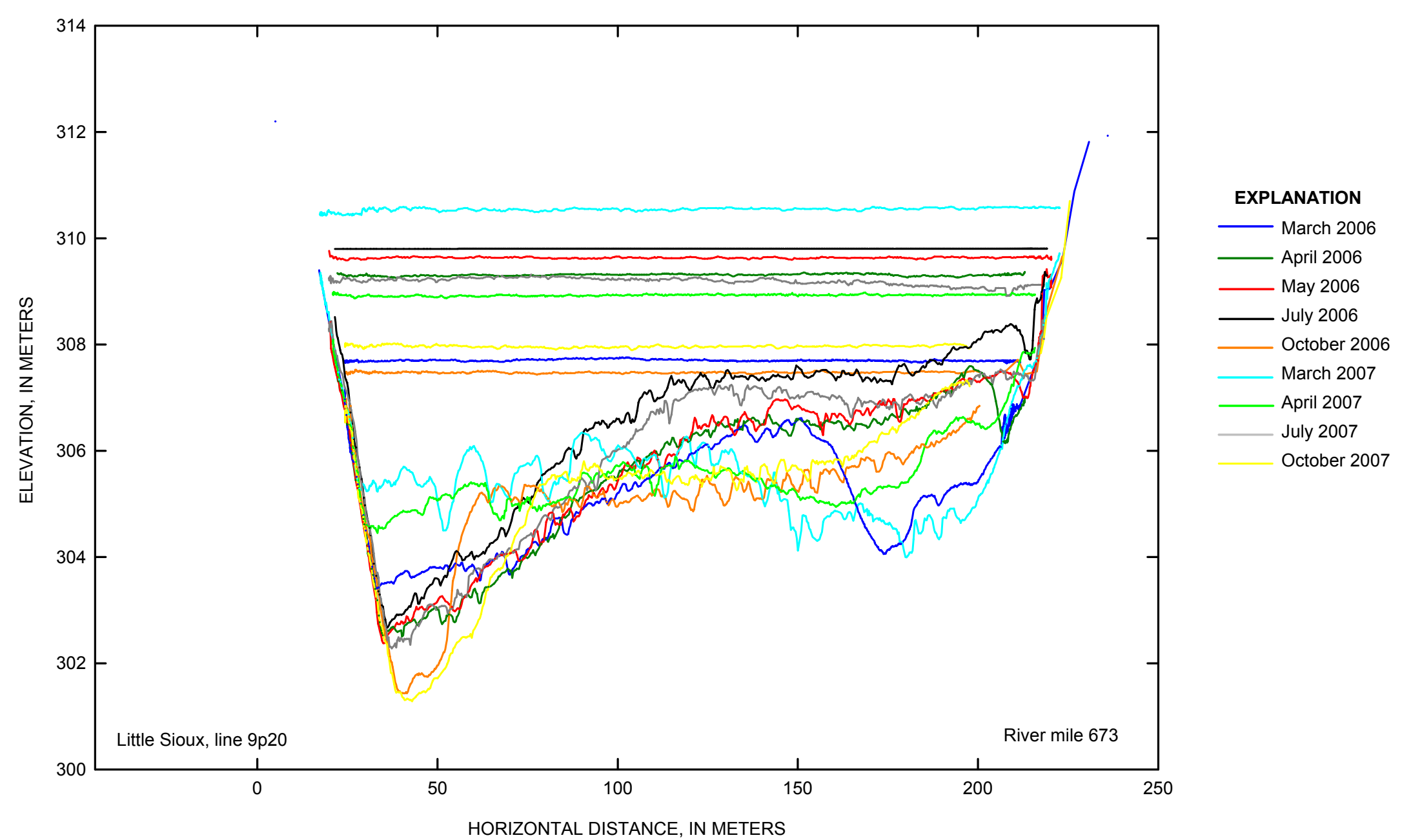

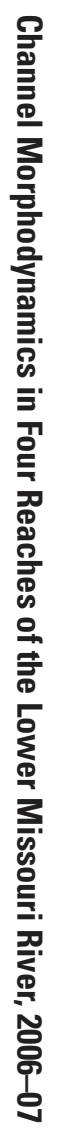

Figure 3-3. Little Sioux cross-section line 9p20 at Missouri River mile 673.0 for 2006-07. 


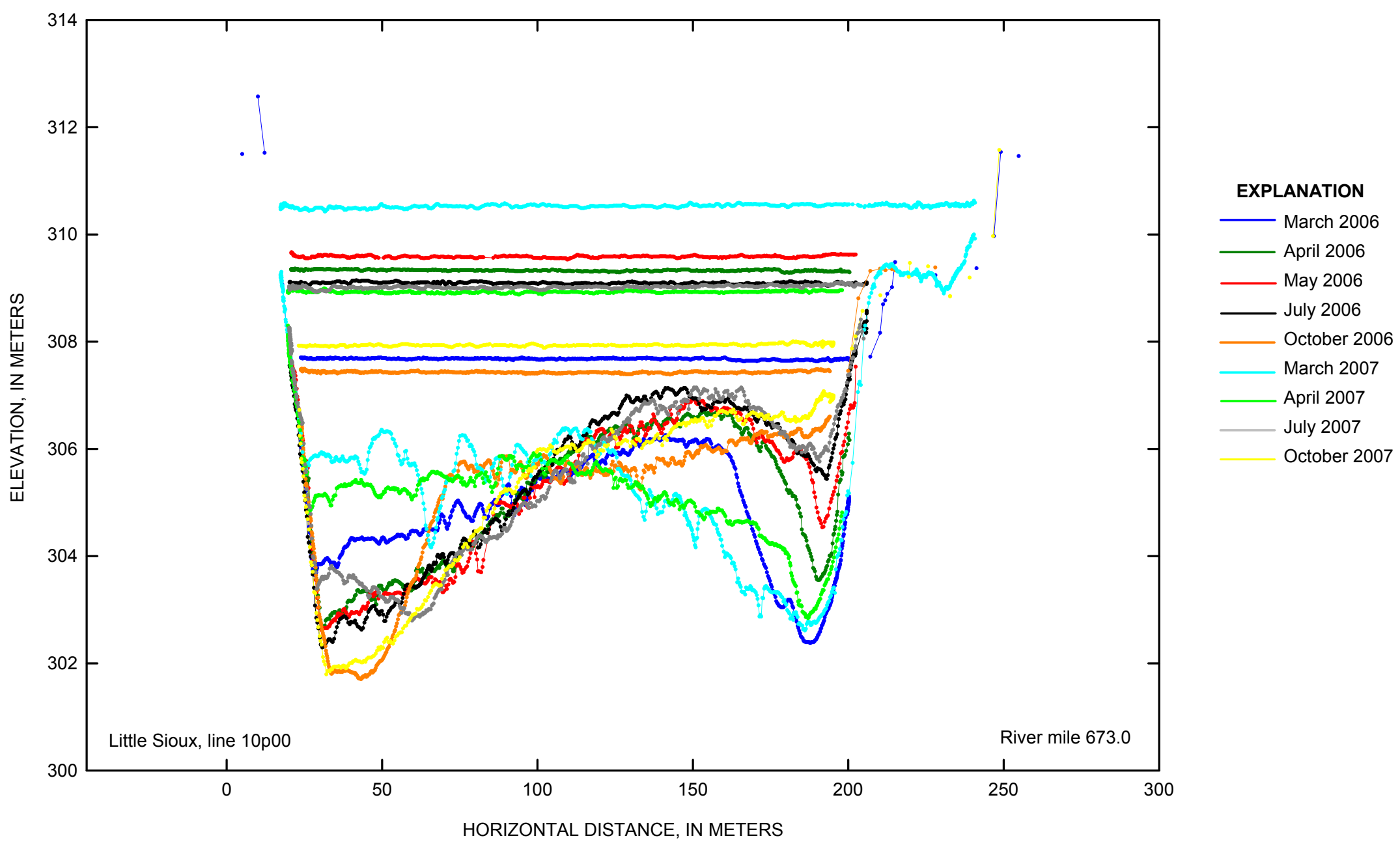

Figure 3-4. Little Sioux cross-section line 10p00 at Missouri River mile 673.0 for 2006-07. 


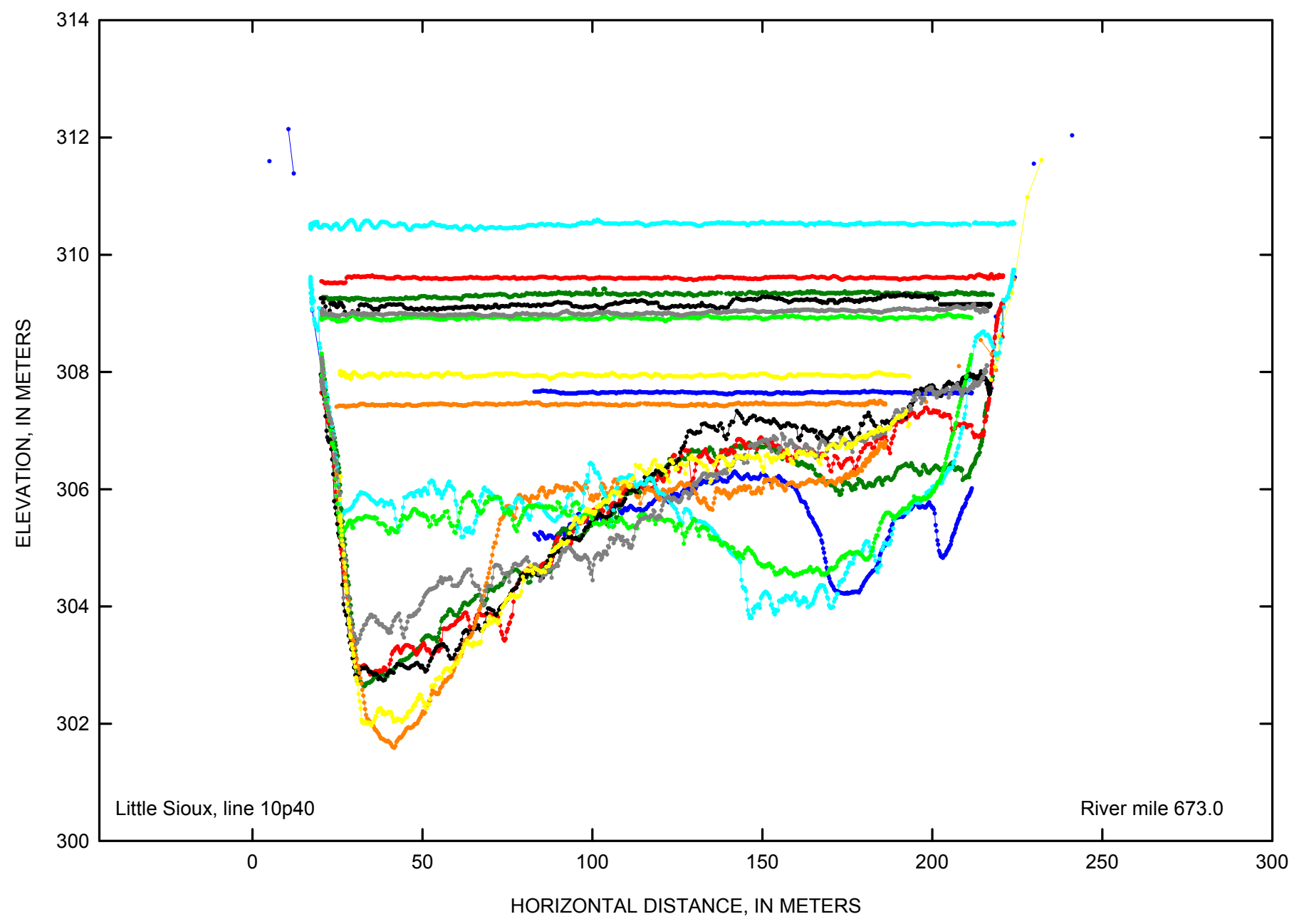

EXPLANATION

- March 2006

April 2006

May 2006

July 2006

October 2006

March 2007

April 2007

July 2007

October 2007

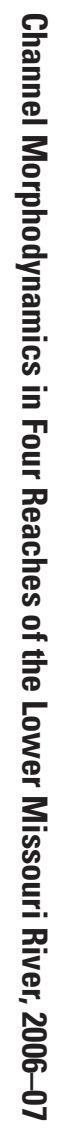

Figure 3-5. Little Sioux cross-section line 10p40 at Missouri River mile 673.0 for 2006-07. 


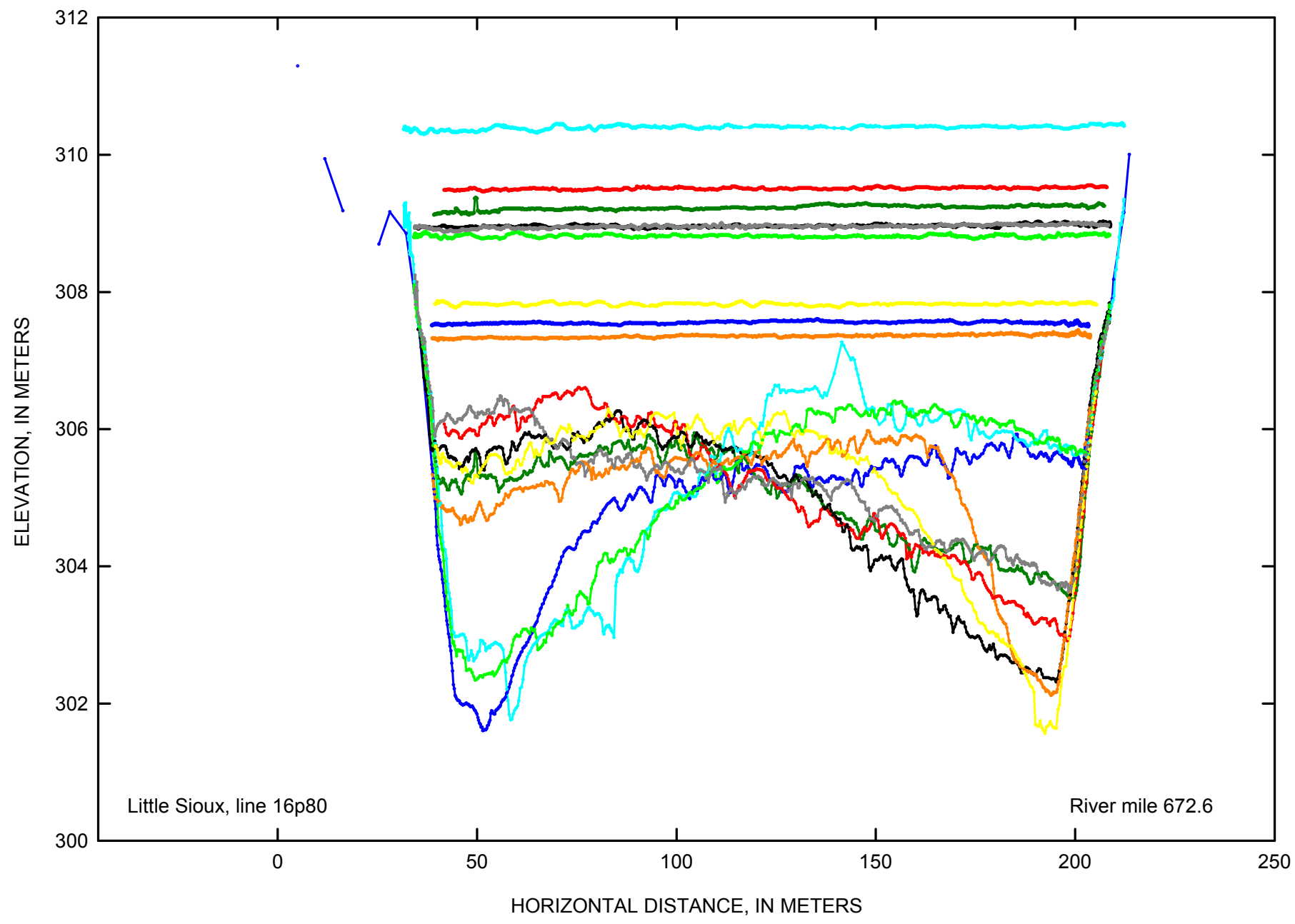

EXPLANATION

- March 2006

April 2006

May 2006

_ July 2006

October 2006

March 2007

April 2007

July 2007

October 2007

Figure 3-6. Little Sioux cross-section line 16p80 at Missouri River mile 672.6 for 2006-07. 


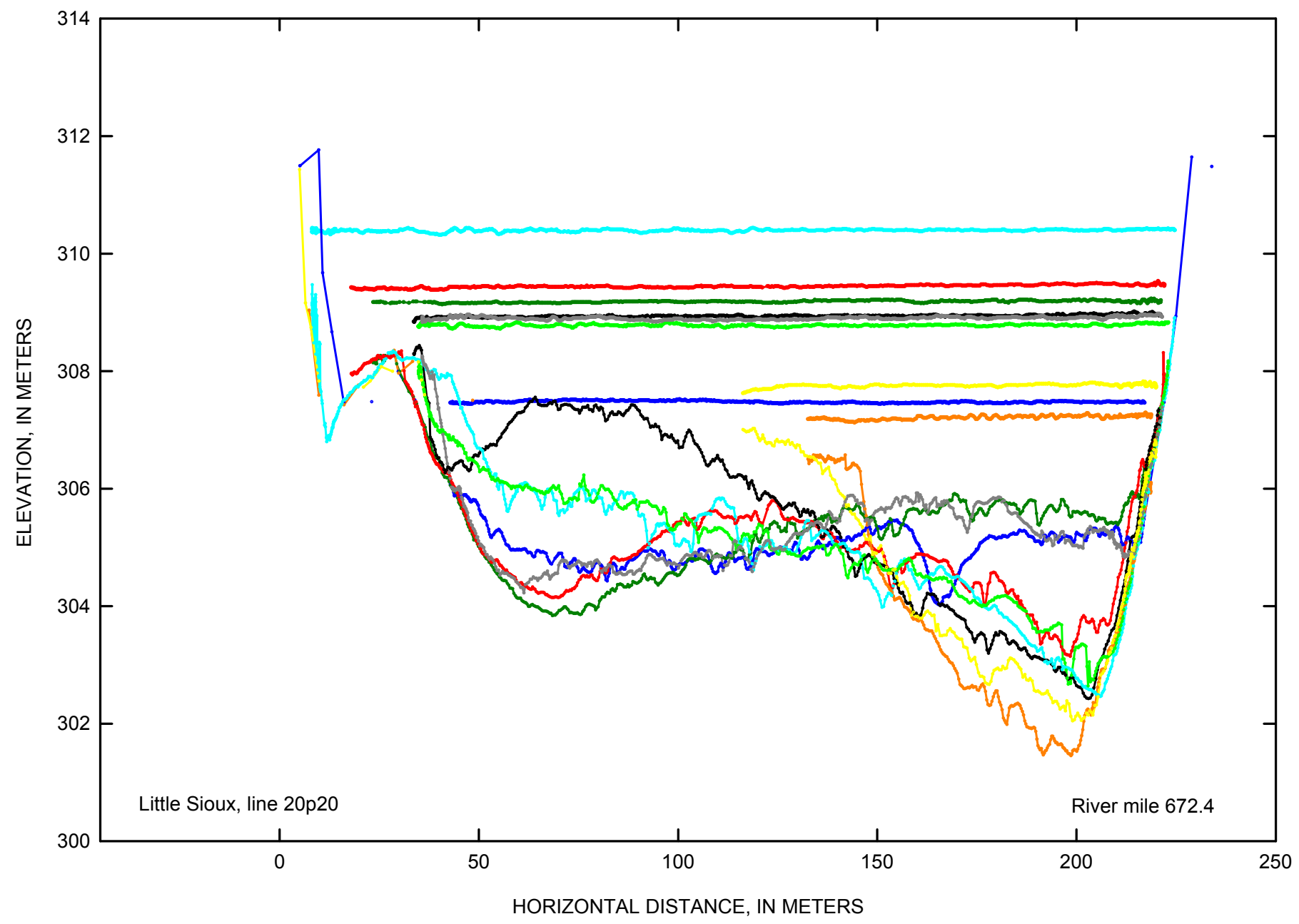

\section{EXPLANATION}

— March 2006

April 2006

May 2006

July 2006

October 2006

March 2007

April 2007

July 2007

October 2007

Figure 3-7. Little Sioux cross-section line 20p20 at Missouri River mile 672.4 for 2006-07. 


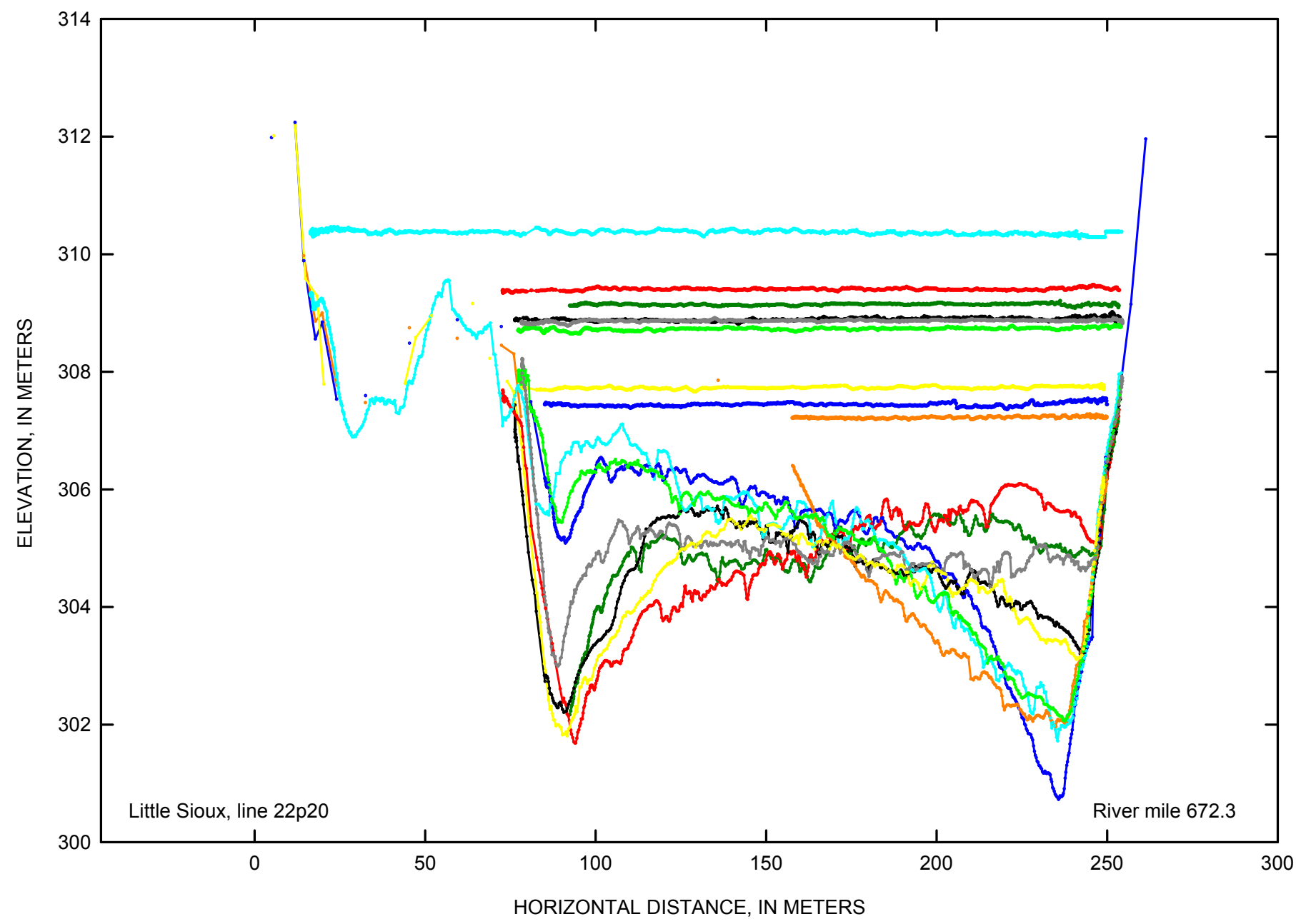

\begin{tabular}{c} 
EXPLANATION \\
\hline March 2006 \\
\hline April 2006 \\
\hline May 2006 \\
July 2006 \\
October 2006 \\
March 2007 \\
April 2007 \\
July 2007 \\
October 2007
\end{tabular}

Figure 3-8. Little Sioux cross-section line 22p20 at Missouri River mile 672.3 for 2006-07. 


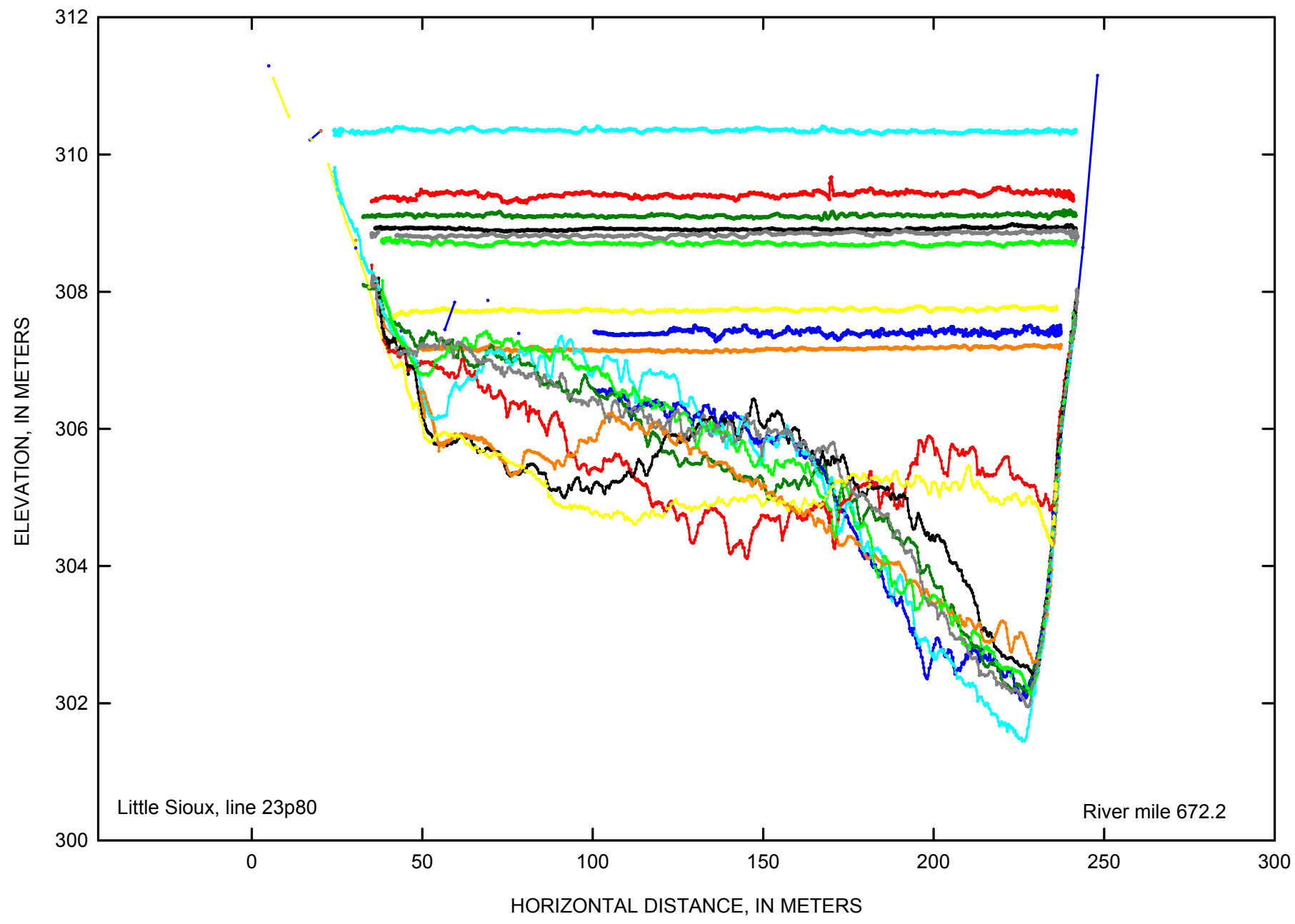

\section{EXPLANATION}

- March 2006

April 2006

May 2006

July 2006

October 2006

March 2007

April 2007

July 2007

October 2007

Figure 3-9. Little Sioux cross-section line 23p80 at Missouri River mile 672.2 for 2006-07. 


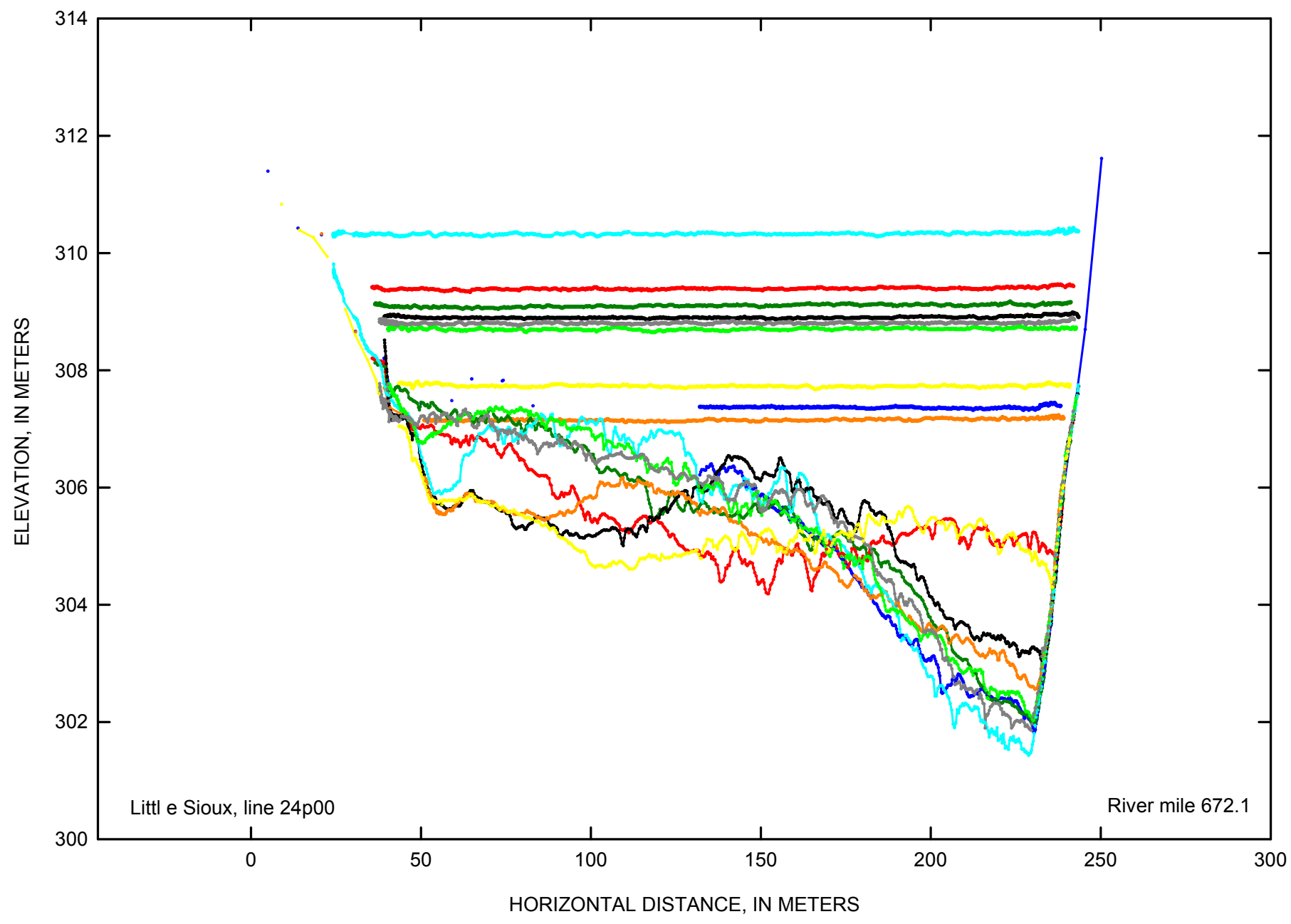

EXPLANATION

- March 2006

April 2006

May 2006

July 2006

October 2006

March 2007

April 2007

July 2007

October 2007

Figure 3-10. Little Sioux cross-section line 24p00 at Missouri River mile 672.1 for 2006-07. 


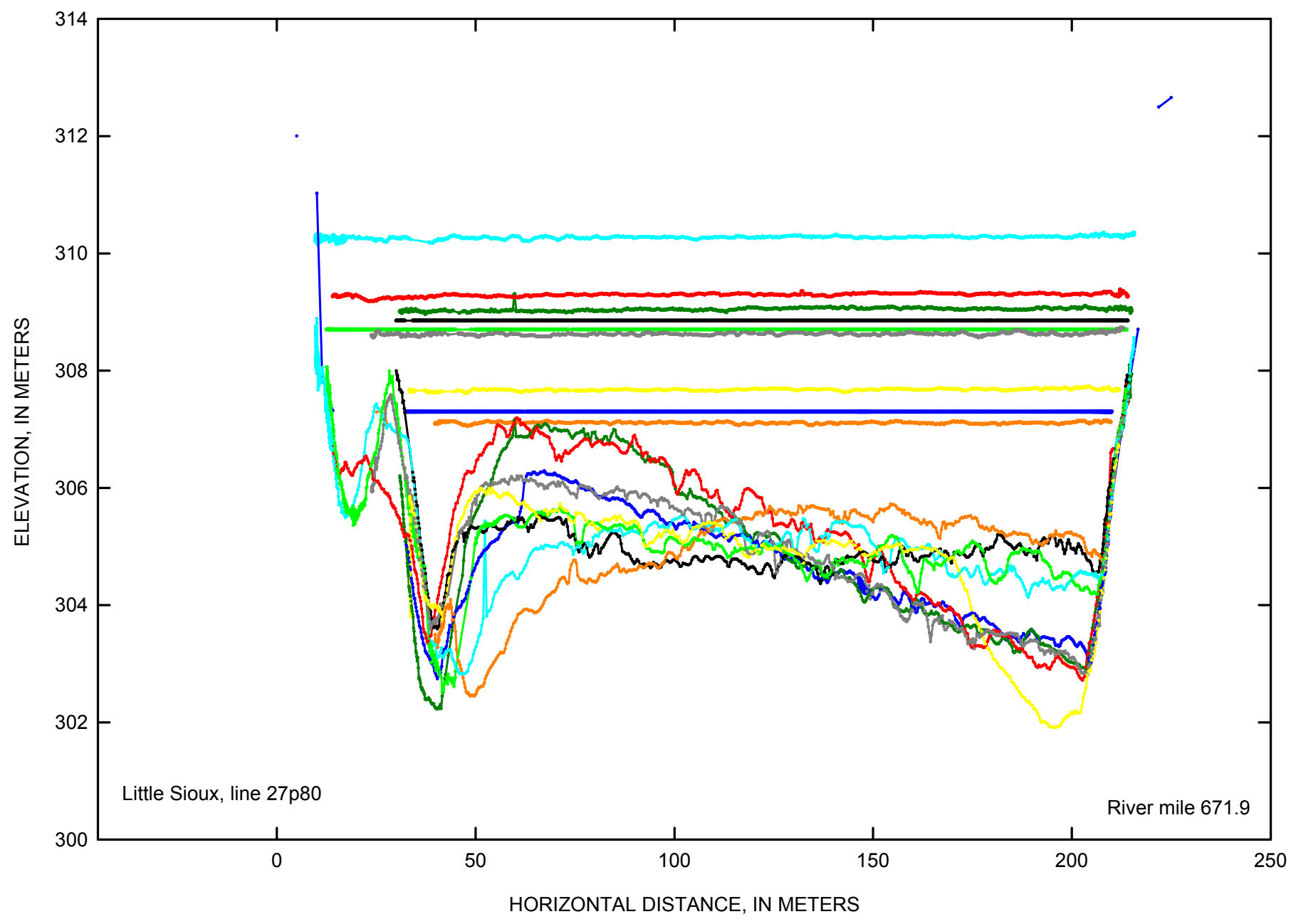

\section{EXPLANATION}

- March 2006

April 2006

May 2006

July 2006

October 2006

March 2007

April 2007

July 2007

October 2007

Figure 3-11. Little Sioux cross-section line 27p80 at Missouri River mile 671.9 for 2006-07. 


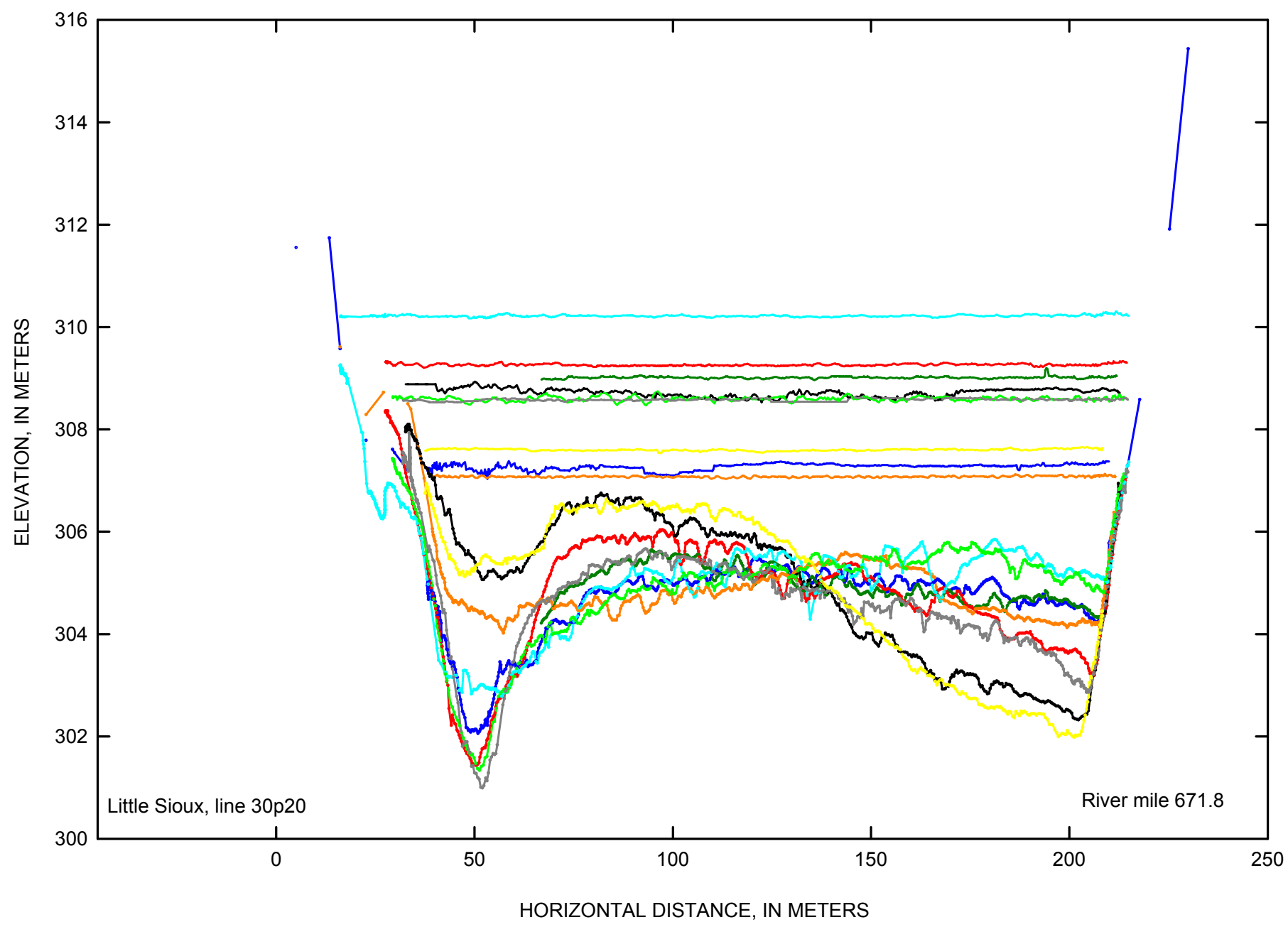

EXPLANATION

- March 2006

- April 2006

— May 2006

_ July 2006

October 2006

March 2007

- April 2007

July 2007

October 2007

Figure 3-12. Little Sioux cross-section line 30p20 at Missouri River mile 671.8 for 2006-07. 


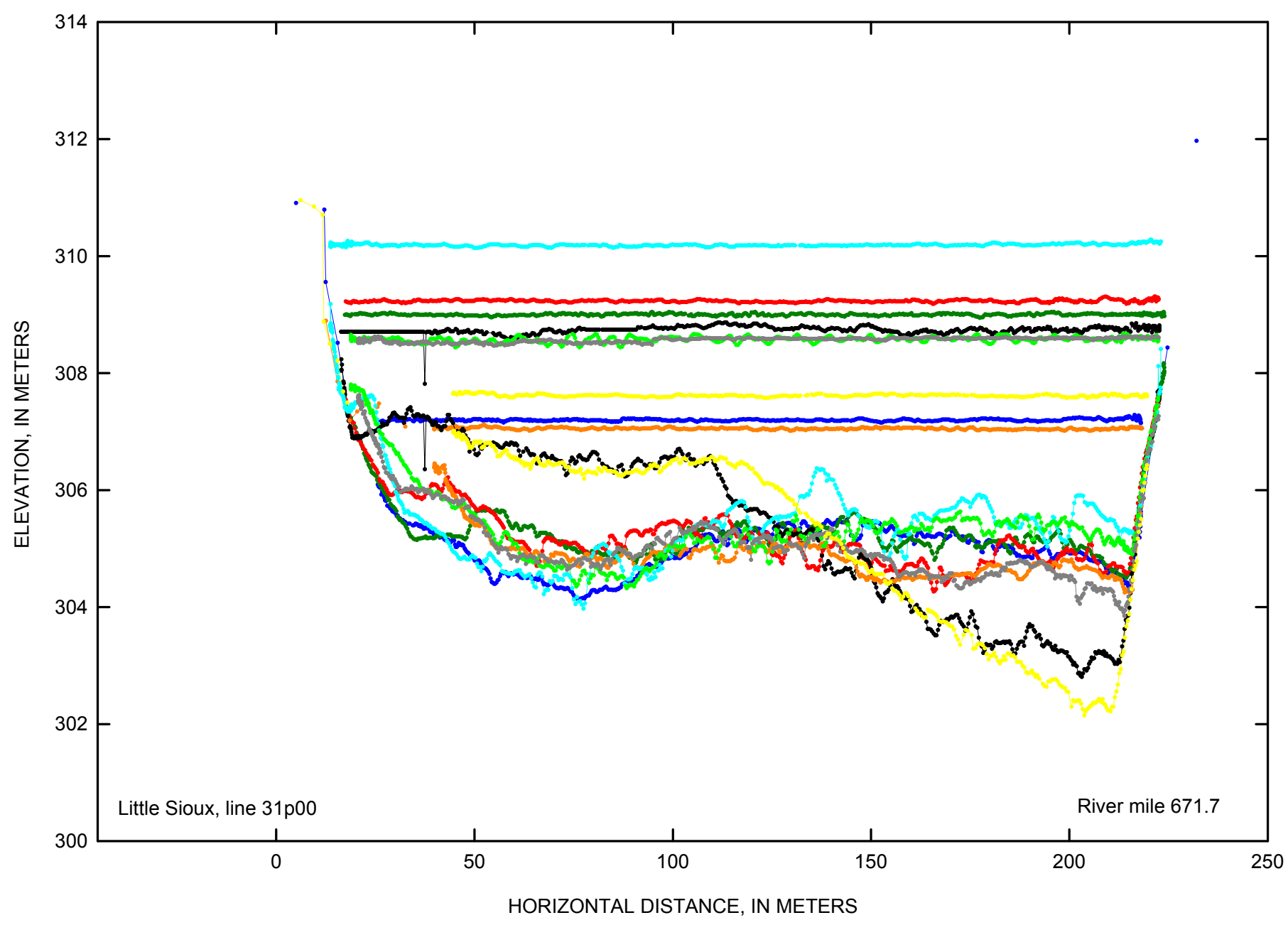

\section{EXPLANATION}

- March 2006

April 2006

May 2006

July 2006

October 2006

March 2007

April 2007

July 2007

October 2007

Figure 3-13. Little Sioux cross-section line 31p00 at Missouri River mile 671.7 for 2006-07. 


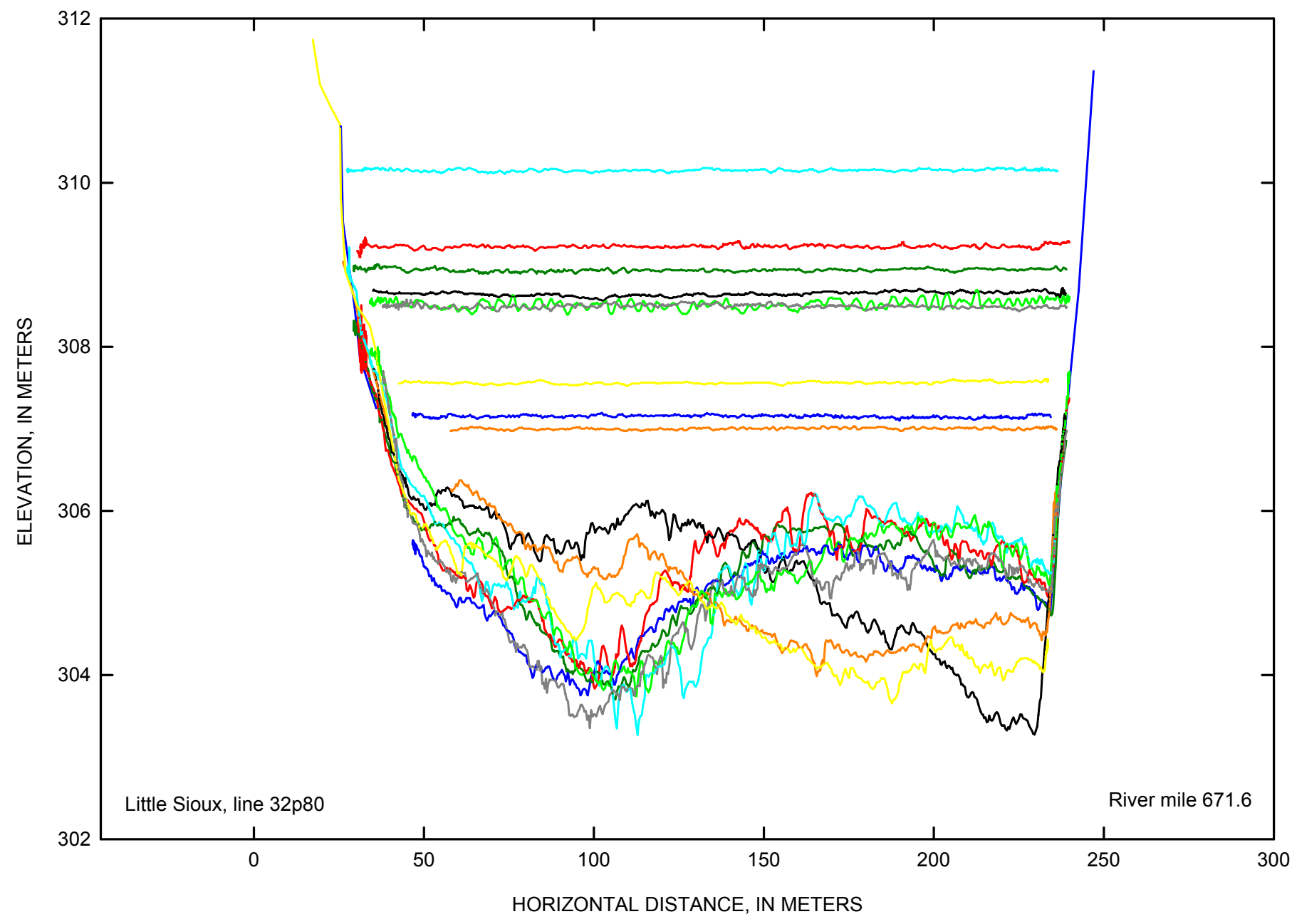

\section{EXPLANATION}

March 2006

April 2006

May 2006

July 2006

October 2006

March 2007

April 2007

July 2007

October 2007

Figure 3-14. Little Sioux cross-section line 32p80 at Missouri River mile 671.6 for 2006-07. 


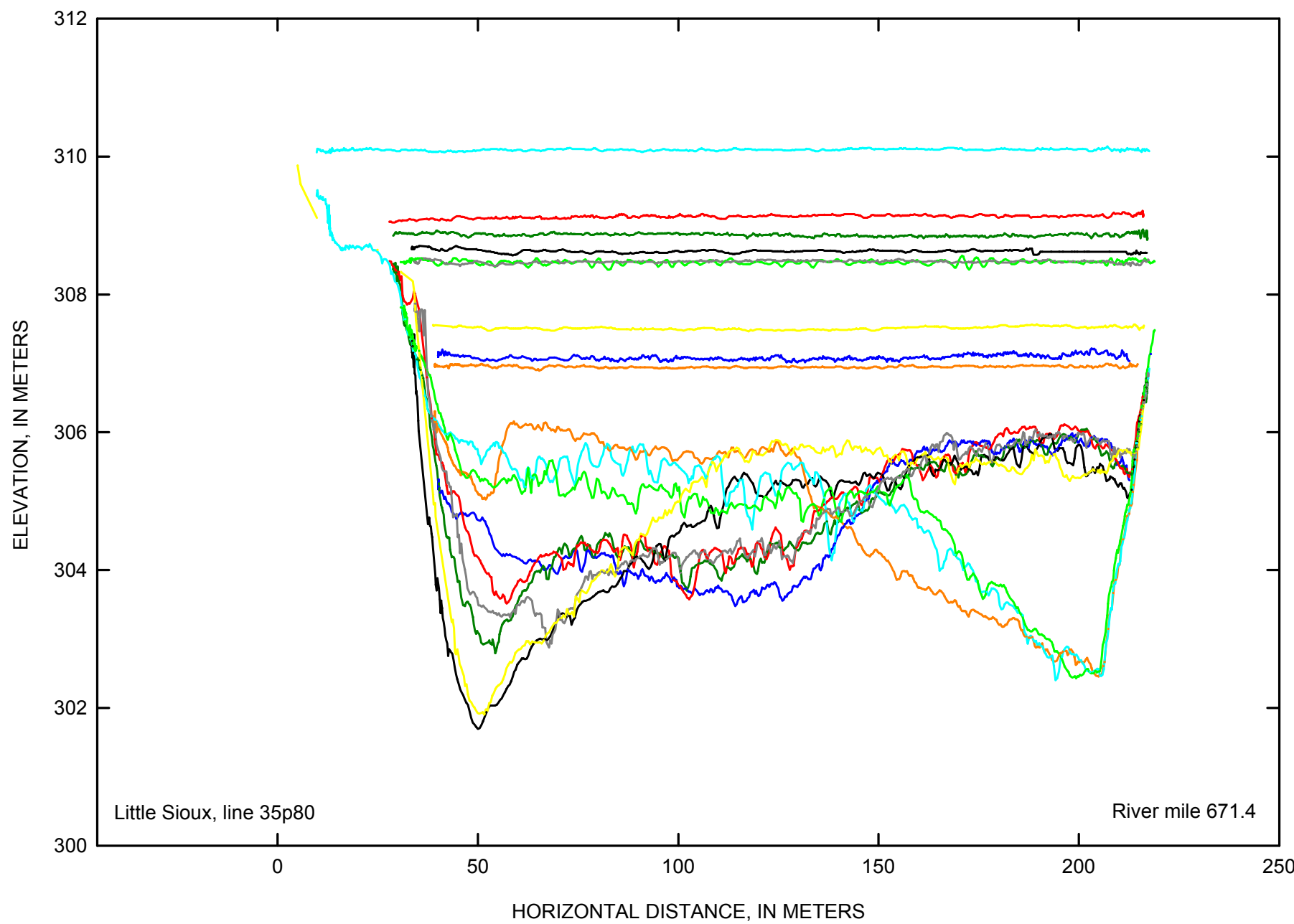

EXPLANATION

- March 2006

April 2006

- May 2006

- July 2006

October 2006

March 2007

April 2007

July 2007

October 2007

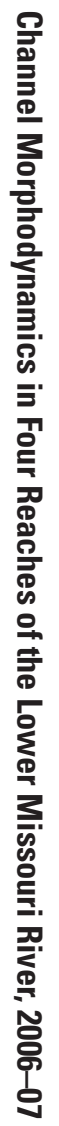

Figure 3-15. Little Sioux cross-section line 35p80 at Missouri River mile 671.4 for 2006-07. 


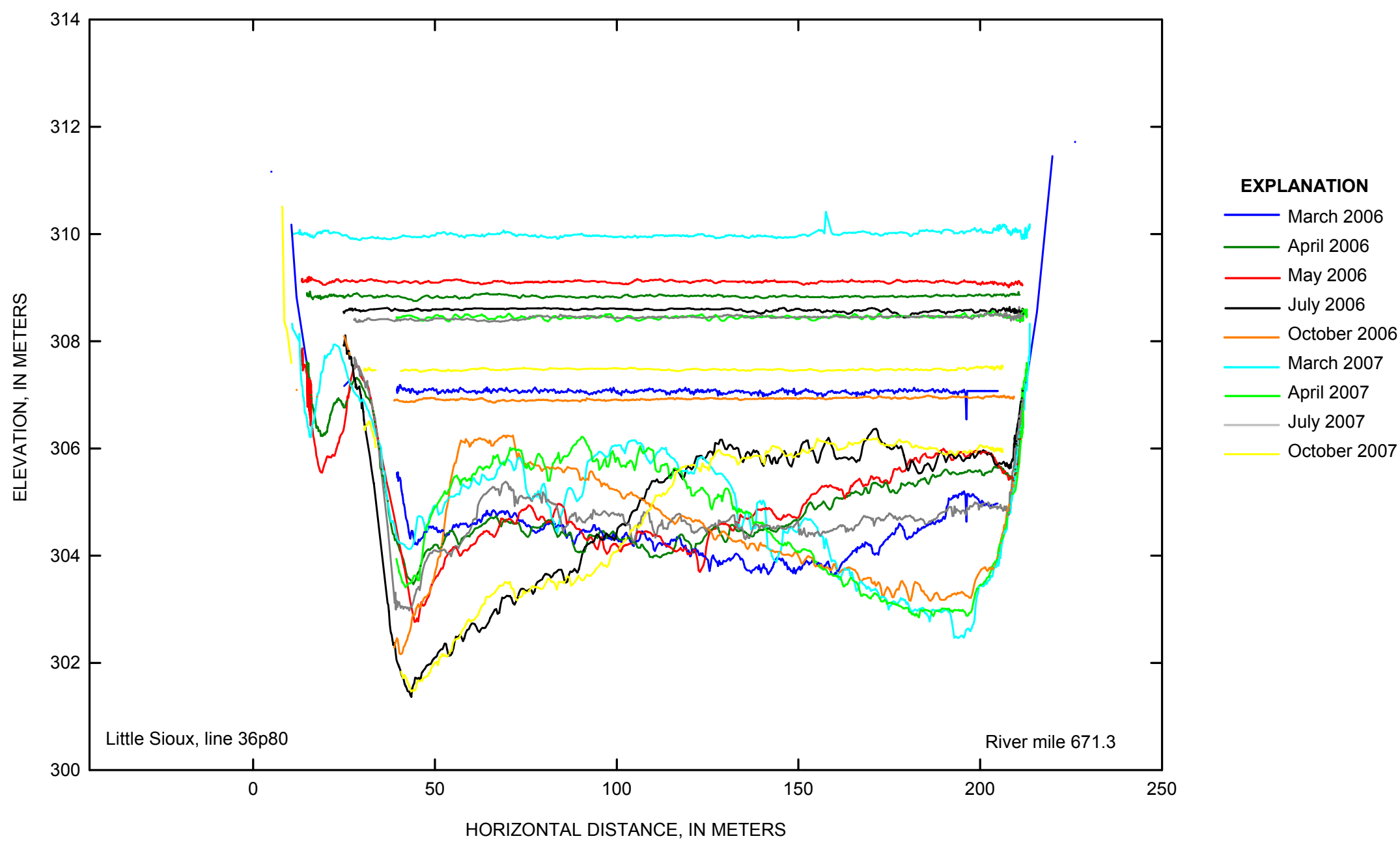

Figure 3-16. Little Sioux cross-section line 36p80 at Missouri River mile 671.3 for 2006-07. 


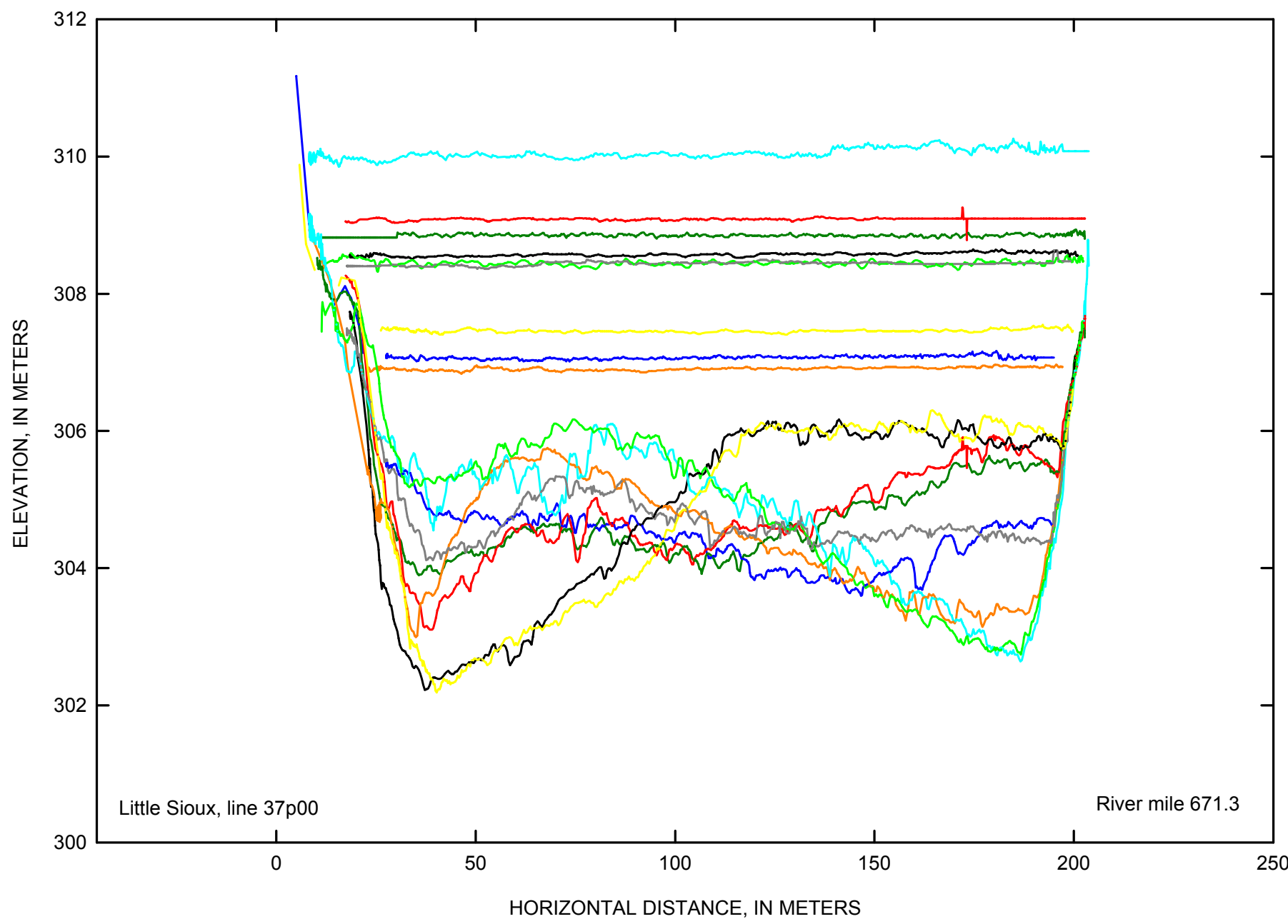

\section{EXPLANATION}

- March 2006

April 2006

May 2006

July 2006

October 2006

March 2007

April 2007

July 2007

October 2007

Figure 3-17. Little Sioux cross-section line 37p00 at Missouri River mile 671.3 for 2006-07. 


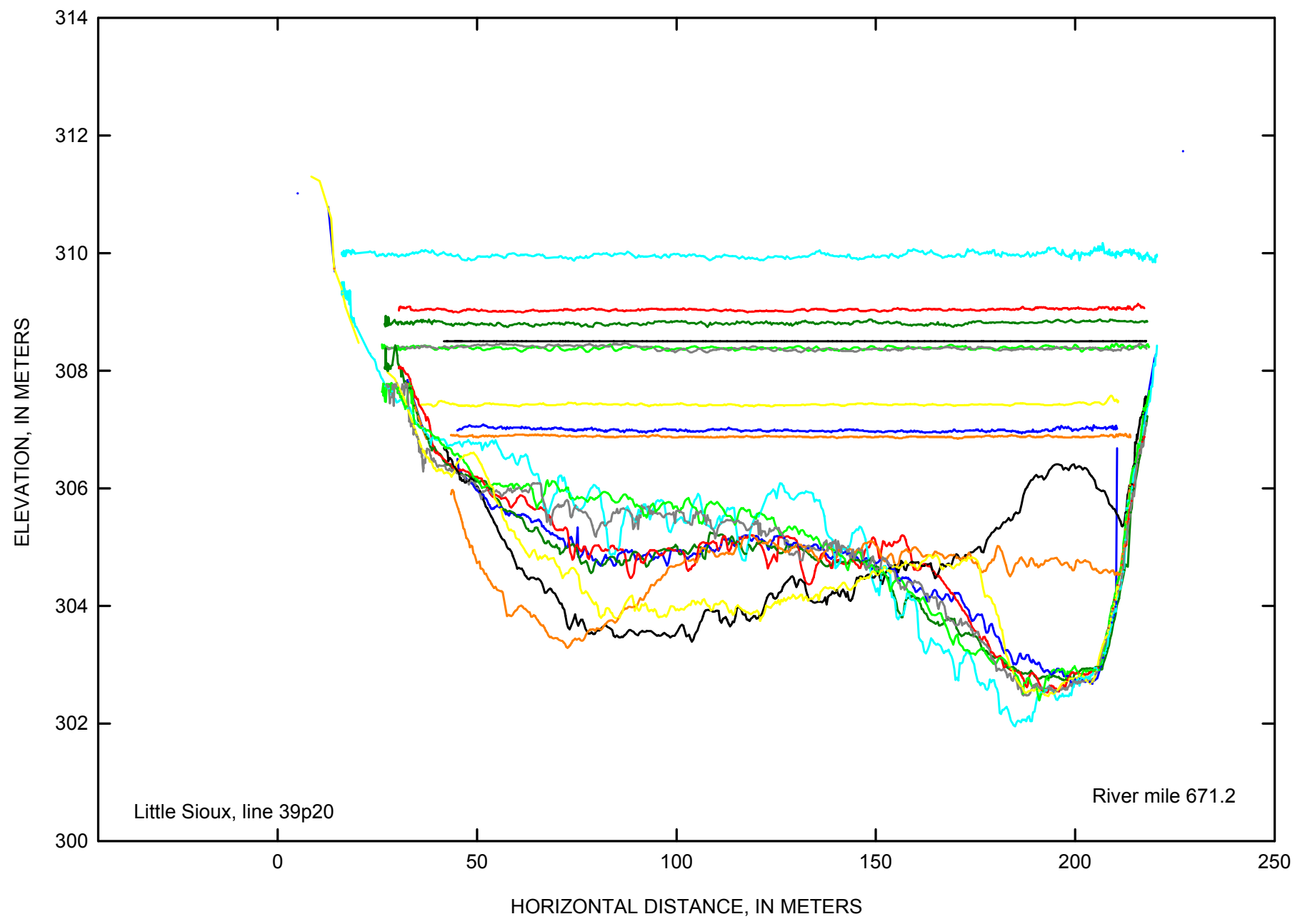

\section{EXPLANATION}

- March 2006

April 2006

May 2006

July 2006

October 2006

March 2007

April 2007

July 2007

October 2007

Figure 3-18. Little Sioux cross-section line 39p20 at Missouri River mile 671.2 for 2006-07. 


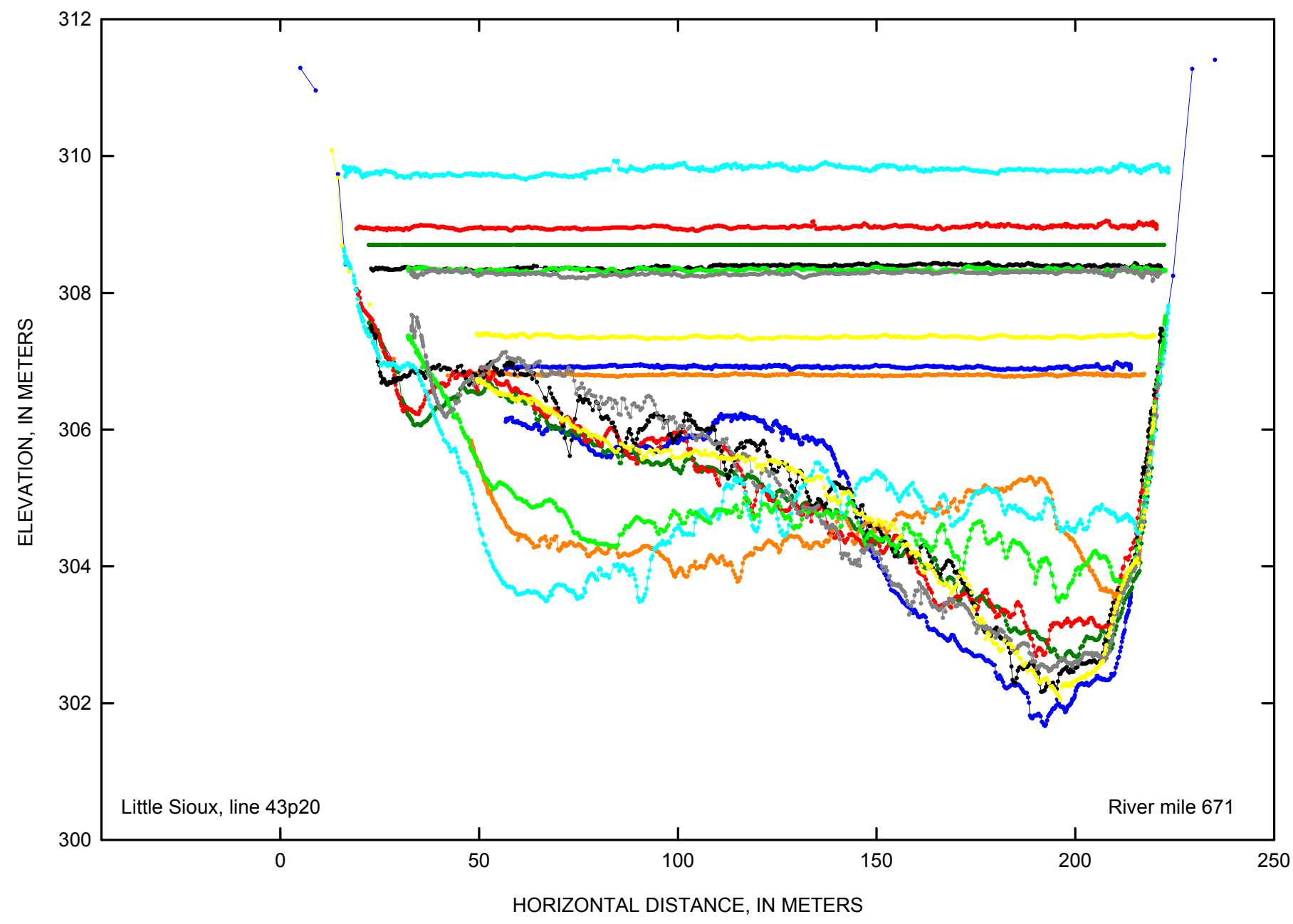

EXPLANATION

- March 2006

April 2006

May 2006

July 2006

October 2006

March 2007

April 2007

July 2007

October 2007

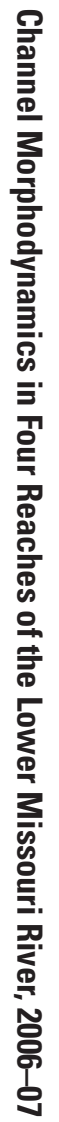

Figure 3-19. Little Sioux cross-section line 43p20 at Missouri River mile 671.0 for 2006-07. 


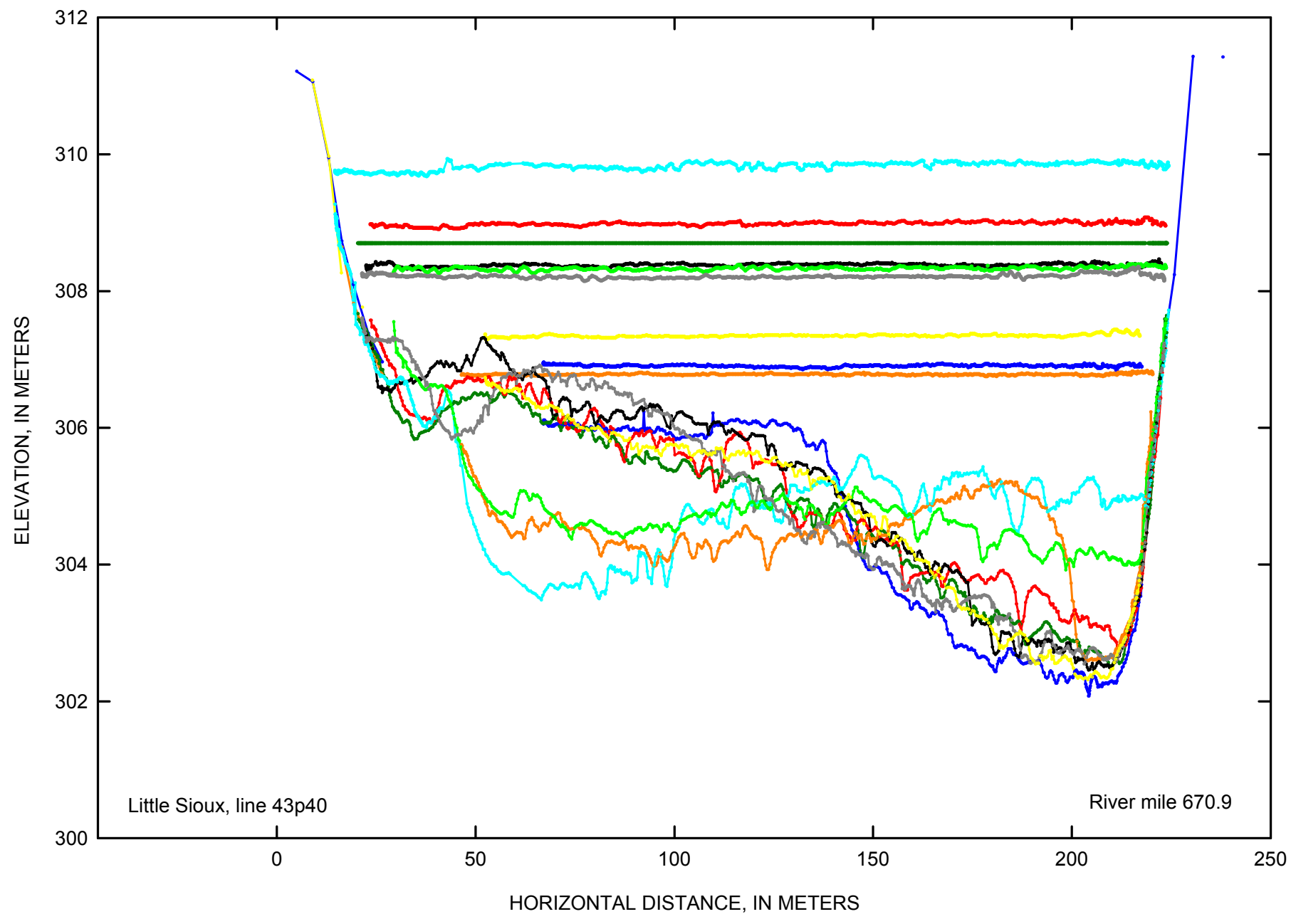

\section{EXPLANATION}

— March 2006

April 2006

May 2006

July 2006

October 2006

March 2007

April 2007

July 2007

October 2007

Figure 3-20. Little Sioux cross-section line 43p40 at Missouri River mile 670.9 for 2006-07. 


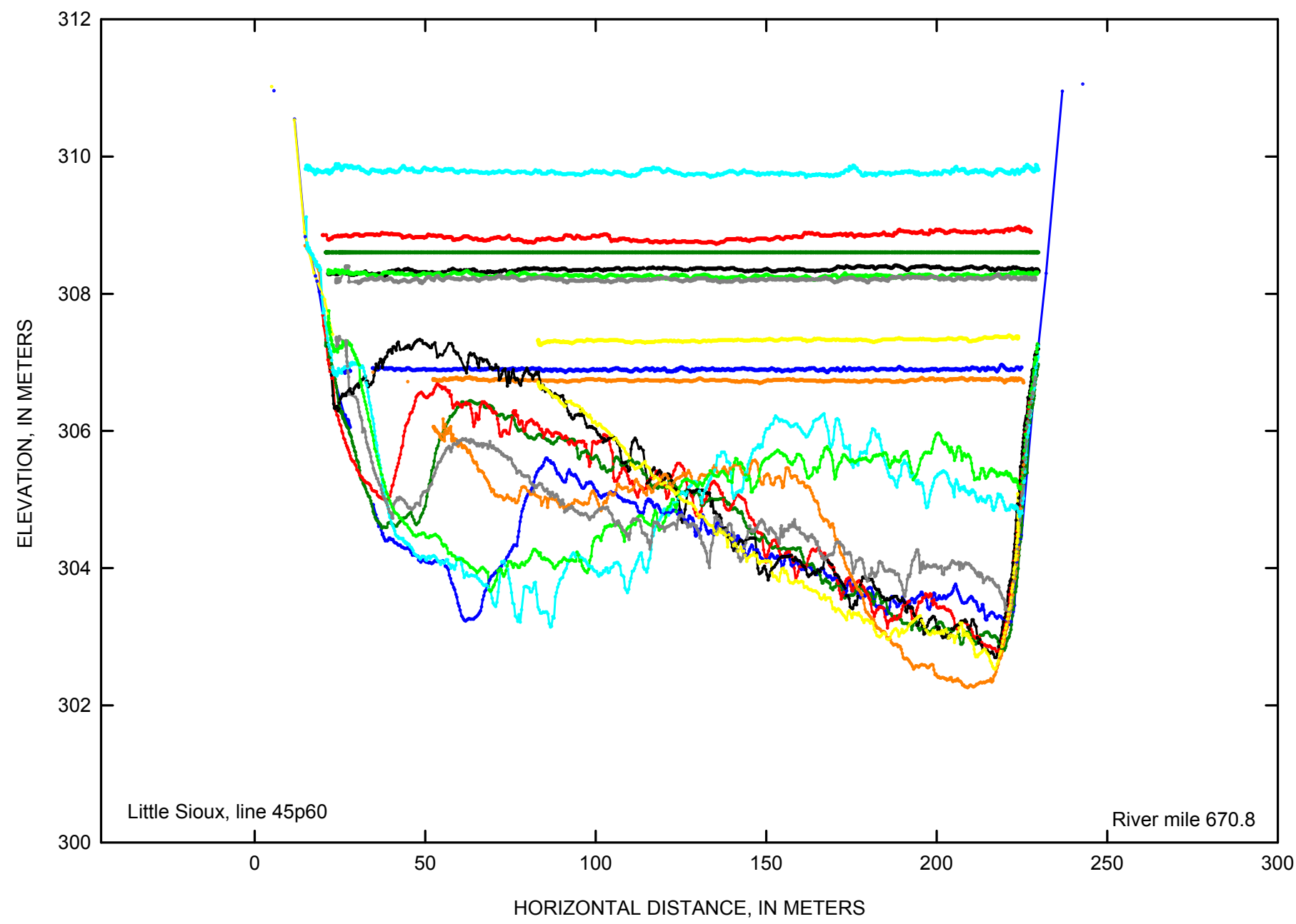

EXPLANATION

- March 2006

April 2006

- May 2006

- July 2006

October 2006

March 2007

- April 2007

July 2007

October 2007

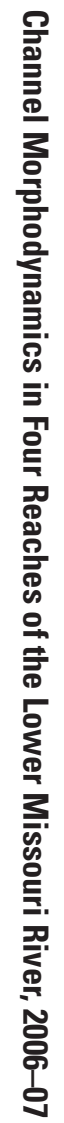

Figure 3-21. Little Sioux cross-section line 45p60 at Missouri River mile 670.8 for 2006-07. 


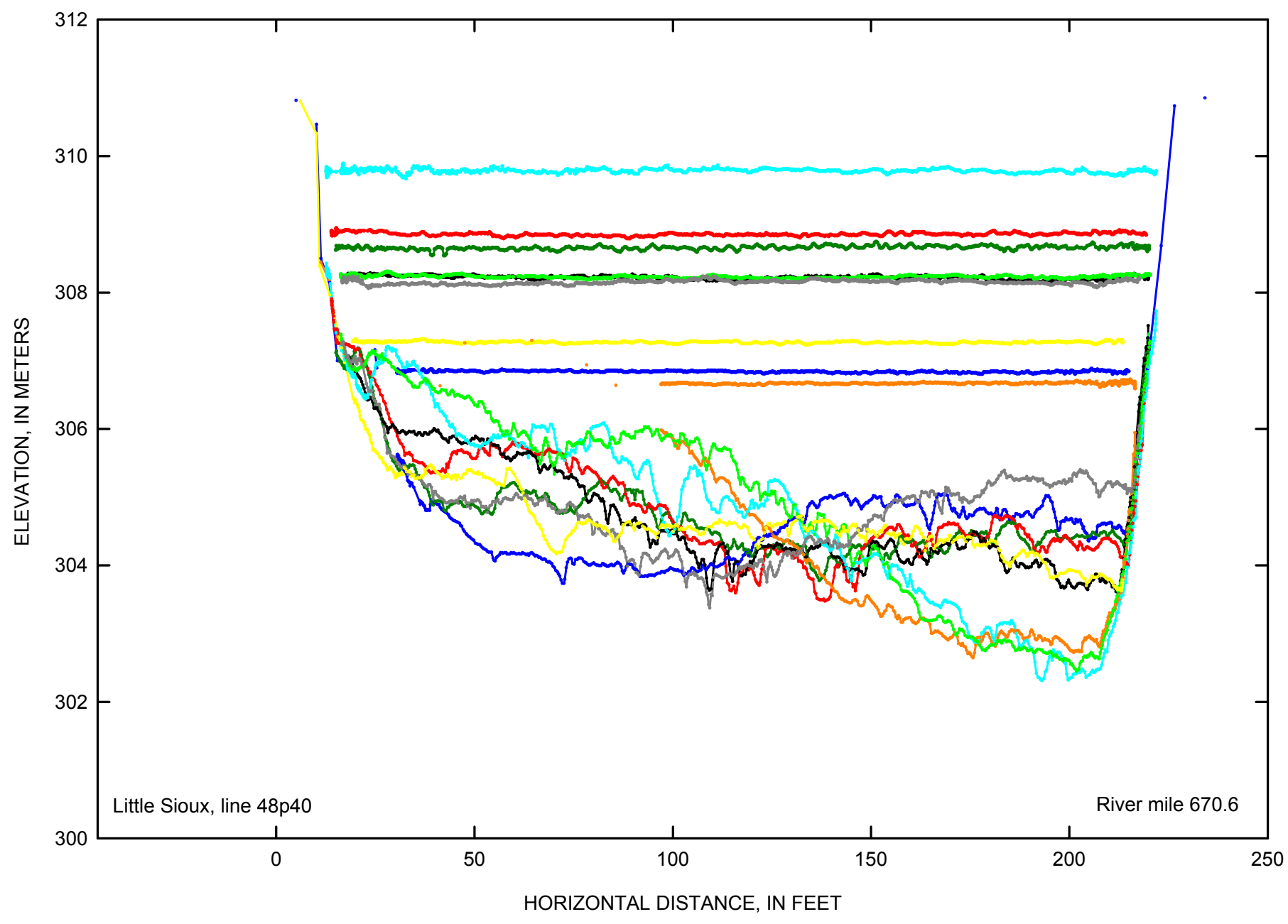

\begin{tabular}{l} 
EXPLANATION \\
\hline March 2006 \\
April 2006 \\
May 2006 \\
\hline July 2006 \\
October 2006 \\
March 2007 \\
April 2007 \\
July 2007 \\
October 2007
\end{tabular}

Figure 3-22. Little Sioux cross-section line 48p40 at Missouri River mile 670.6 for 2006-07. 


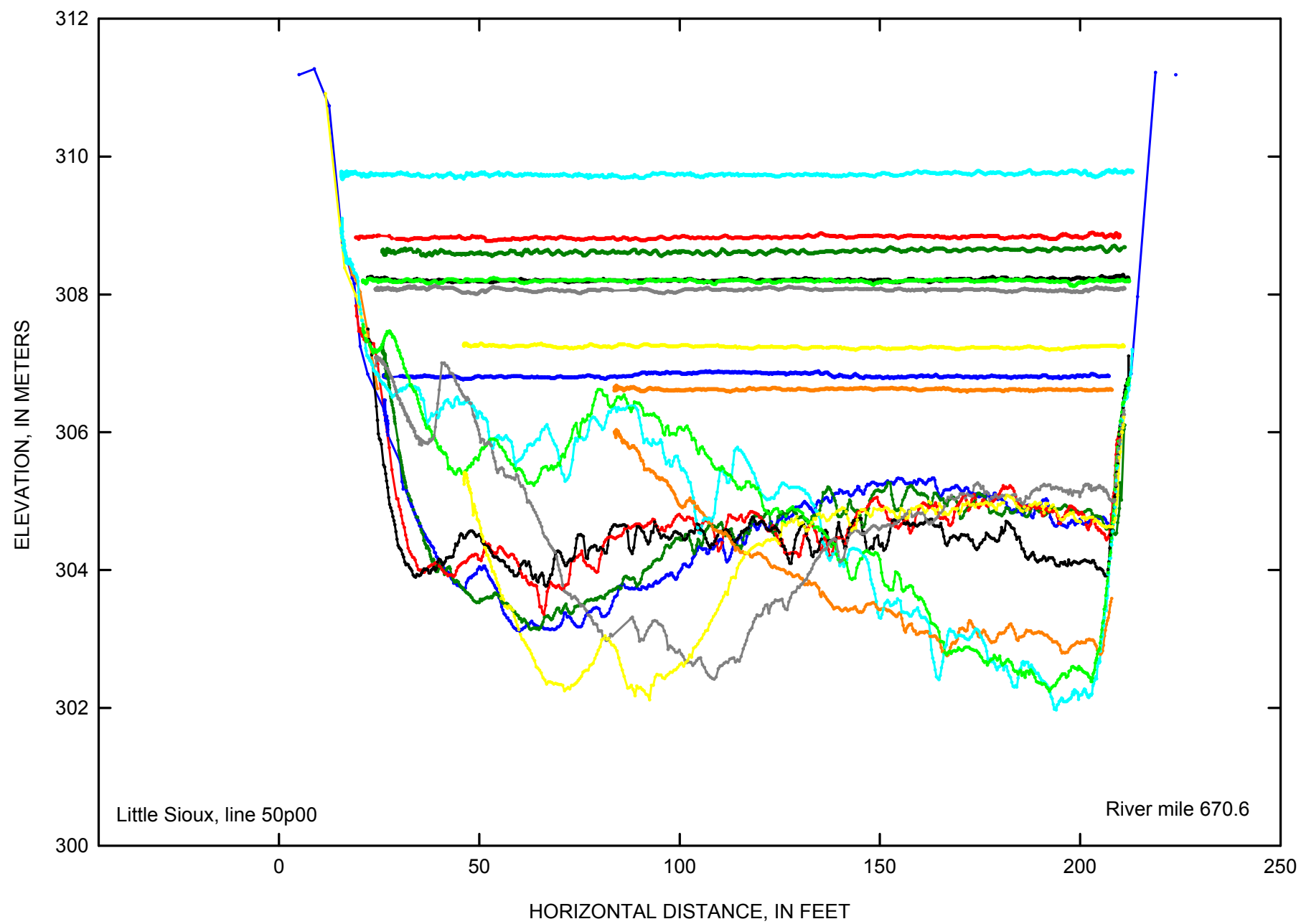

EXPLANATION

- March 2006

April 2006

May 2006

July 2006

October 2006

March 2007

April 2007

July 2007

October 2007

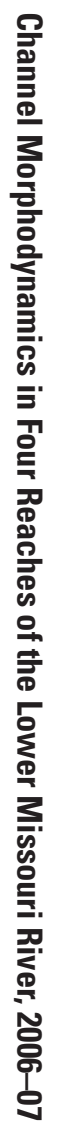

Figure 3-23. Little Sioux cross-section line 50p00 at Missouri River mile 670.6 for 2006-07. 


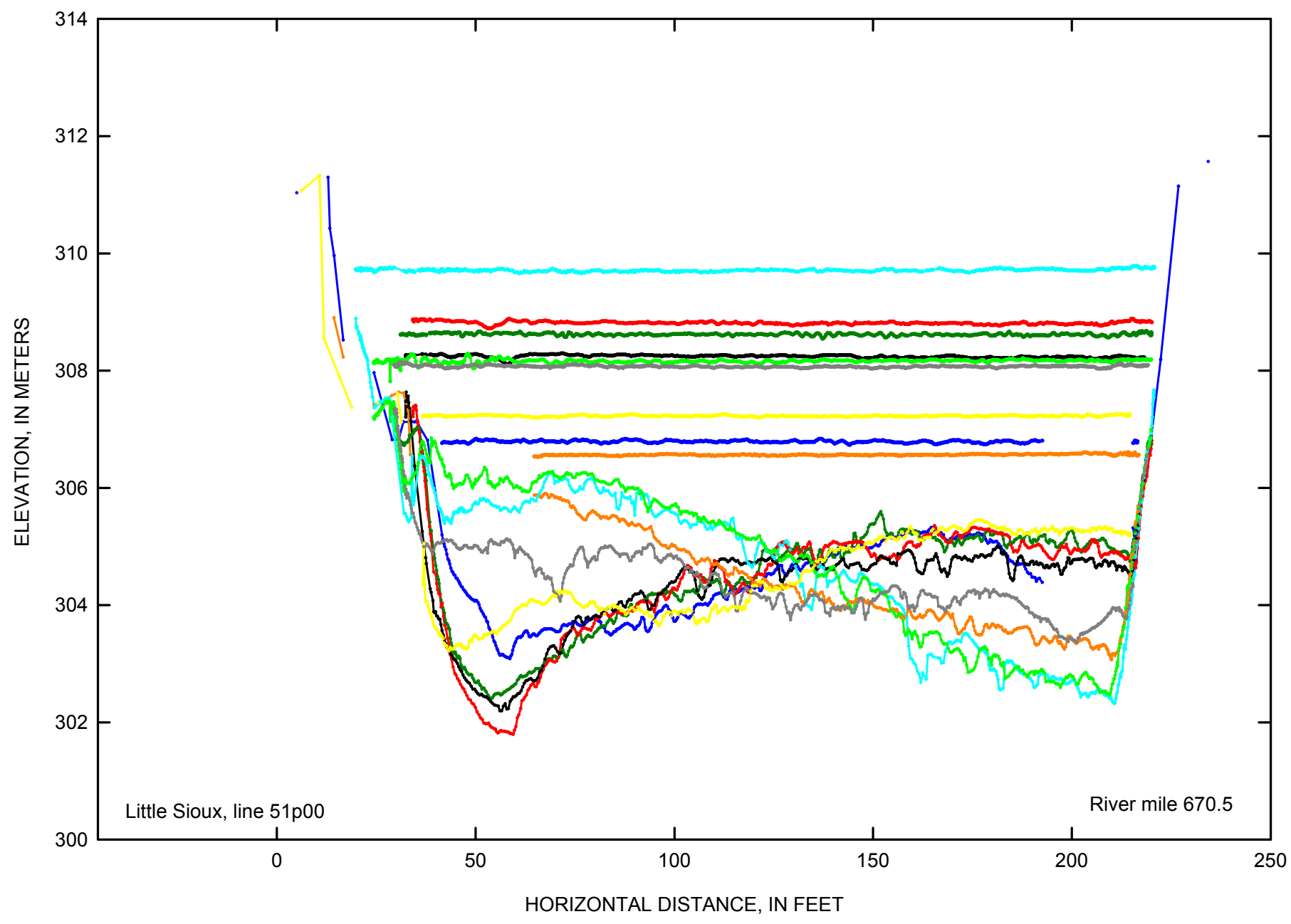

EXPLANATION

- March 2006

April 2006

May 2006

July 2006

October 2006

March 2007

April 2007

July 2007

October 2007

Figure 3-24. Little Sioux cross-section line 51p00 at Missouri River mile 670.5 for 2006-07. 


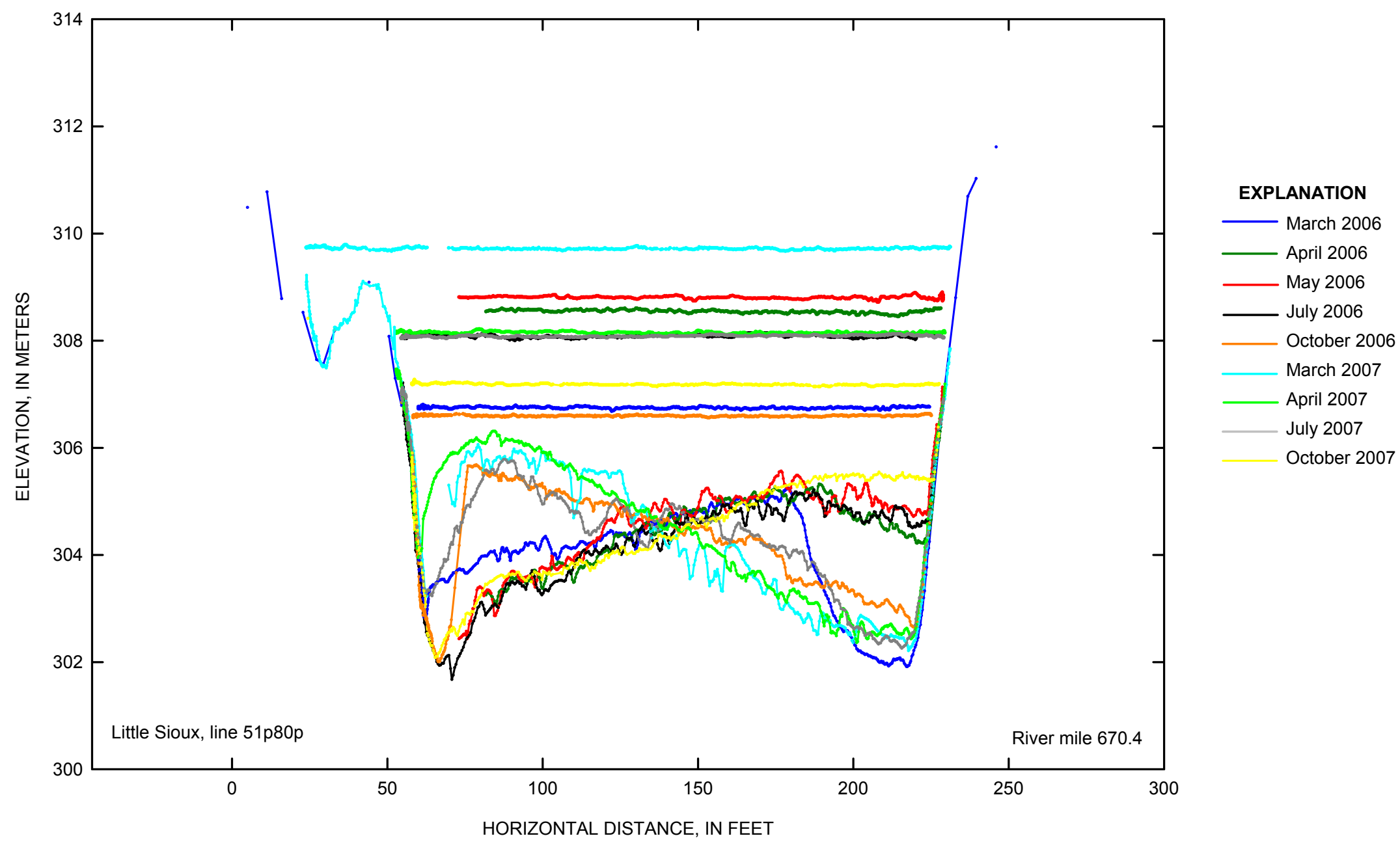

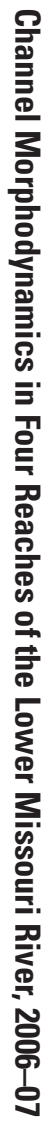

Figure 3-25. Little Sioux cross-section line 51p80 at Missouri River mile 670.4 for 2006-07. 


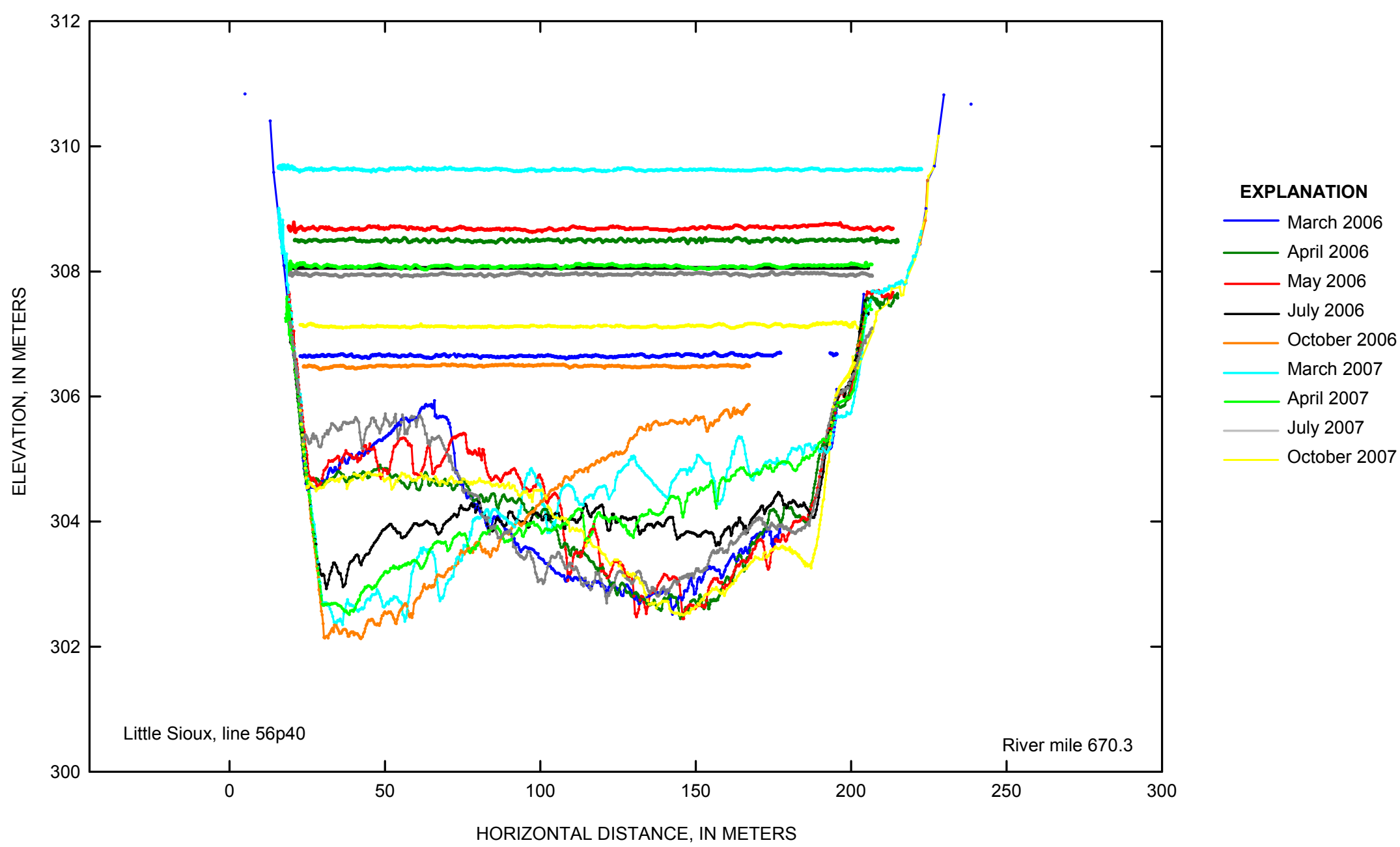

Figure 3-26. Little Sioux cross-section line $56 p 40$ at Missouri River mile 670.3 for 2006-07. 


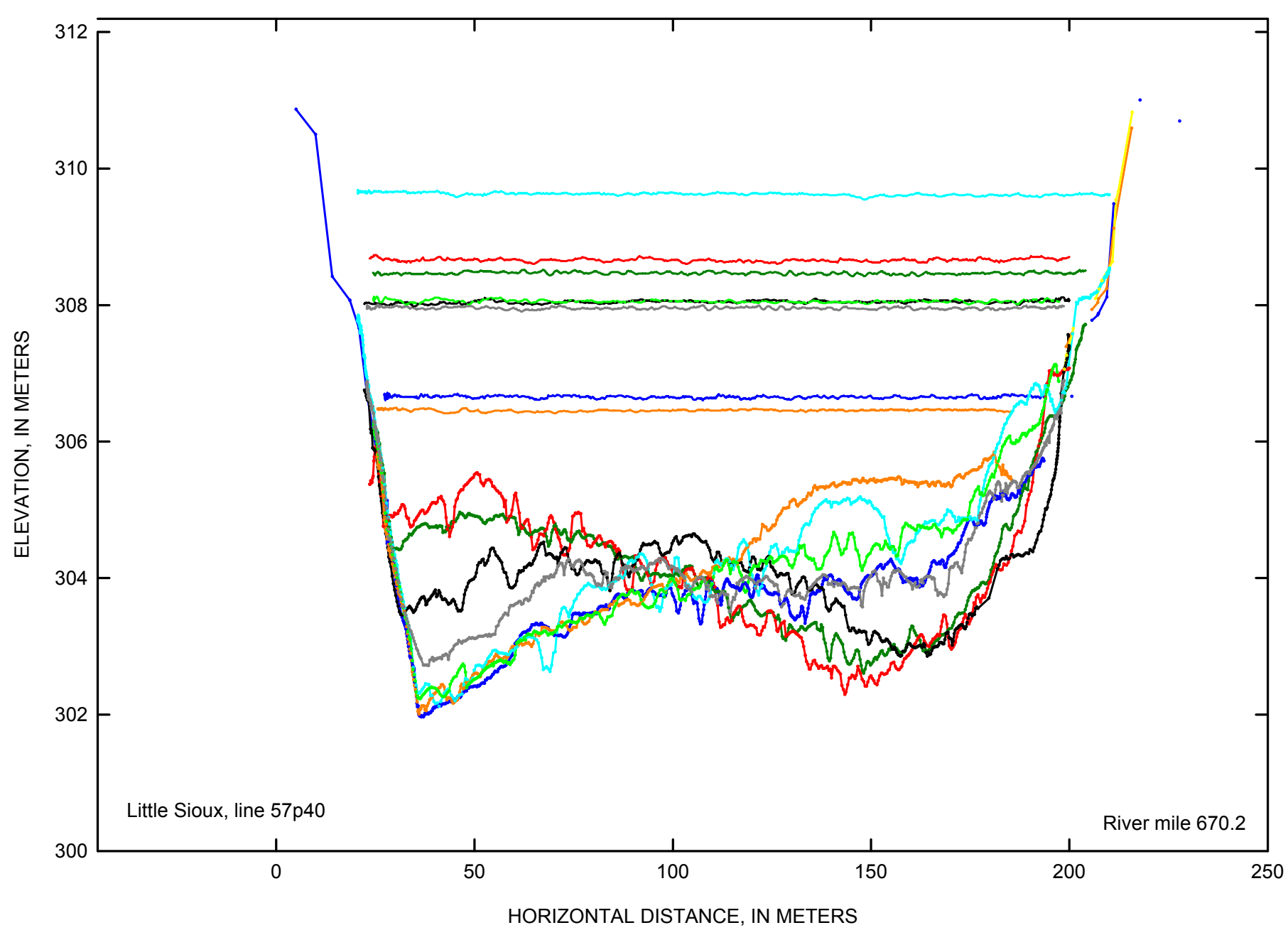

\section{EXPLANATION}

March 2006

April 2006

May 2006

July 2006

October 2006

March 2007

April 2007

July 2007

October 2007

Figure 3-27. Little Sioux cross-section line 57p40 at Missouri River mile 670.2 for 2006-07. 


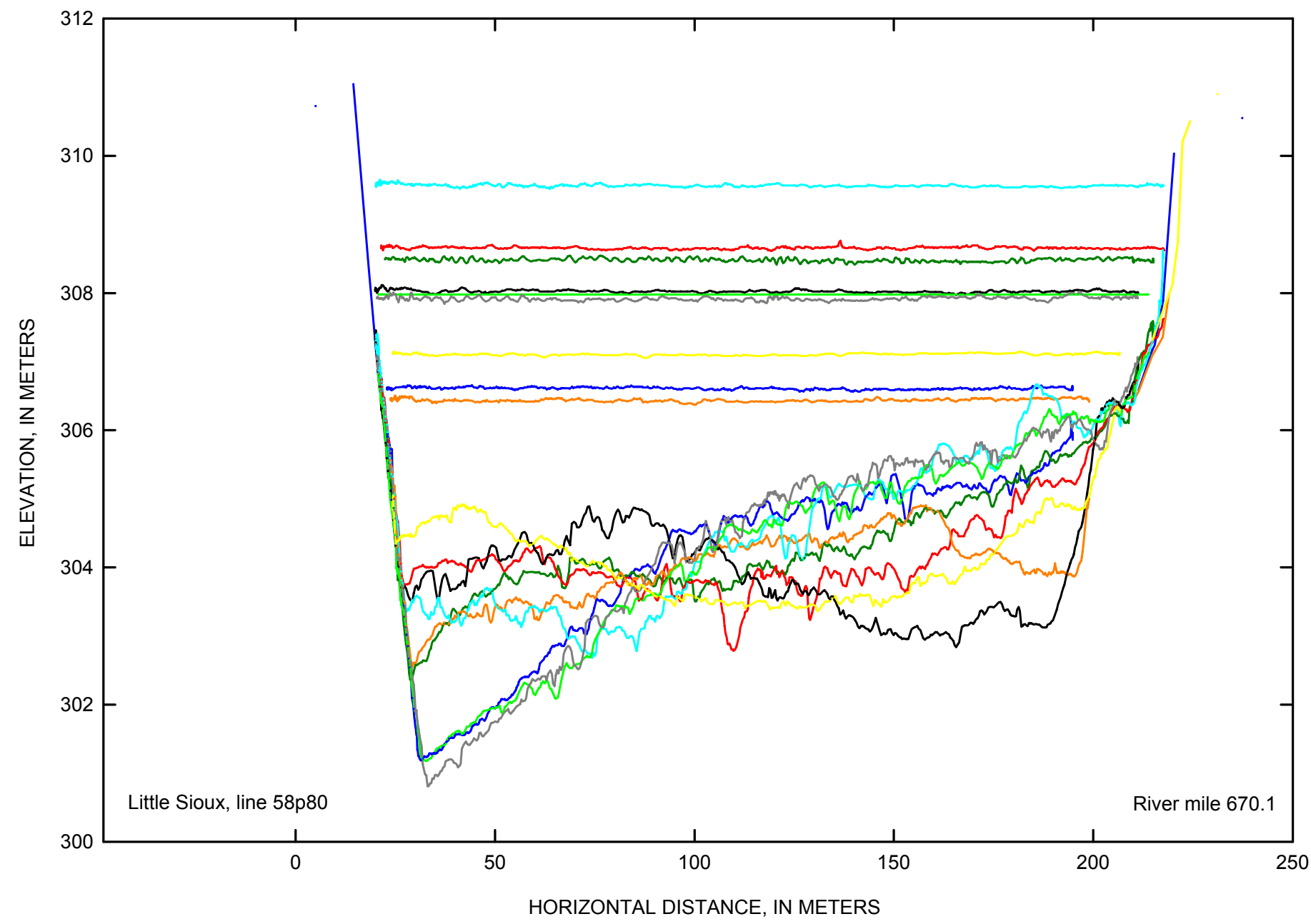

EXPLANATION

\begin{tabular}{l}
\hline March 2006 \\
\hline April 2006 \\
\hline May 2006 \\
July 2006 \\
October 2006 \\
\hline March 2007 \\
April 2007 \\
July 2007 \\
October 2007
\end{tabular}

Figure 3-28. Little Sioux cross-section line 58p80 at Missouri River mile 670.1 for 2006-07. 


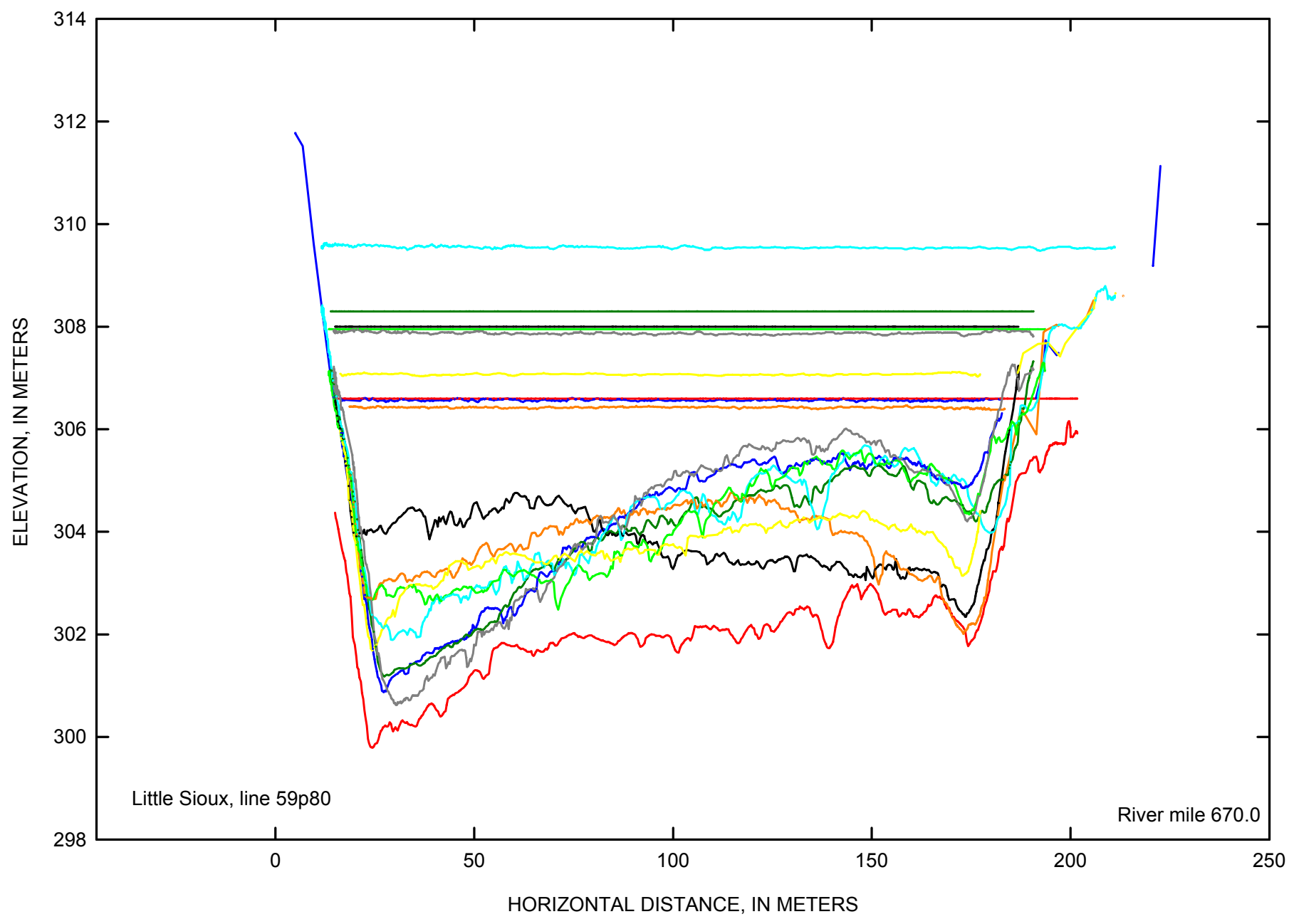

EXPLANATION

- March 2006

- April 2006

- May 2006

_ July 2006

October 2006

March 2007

April 2007

July 2007

October 2007

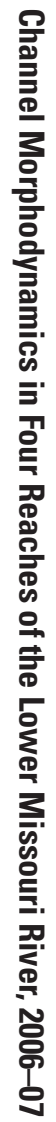

Figure 3-29. Little Sioux cross-section line 59p80 at Missouri River mile 670.0 for 2006-07. 


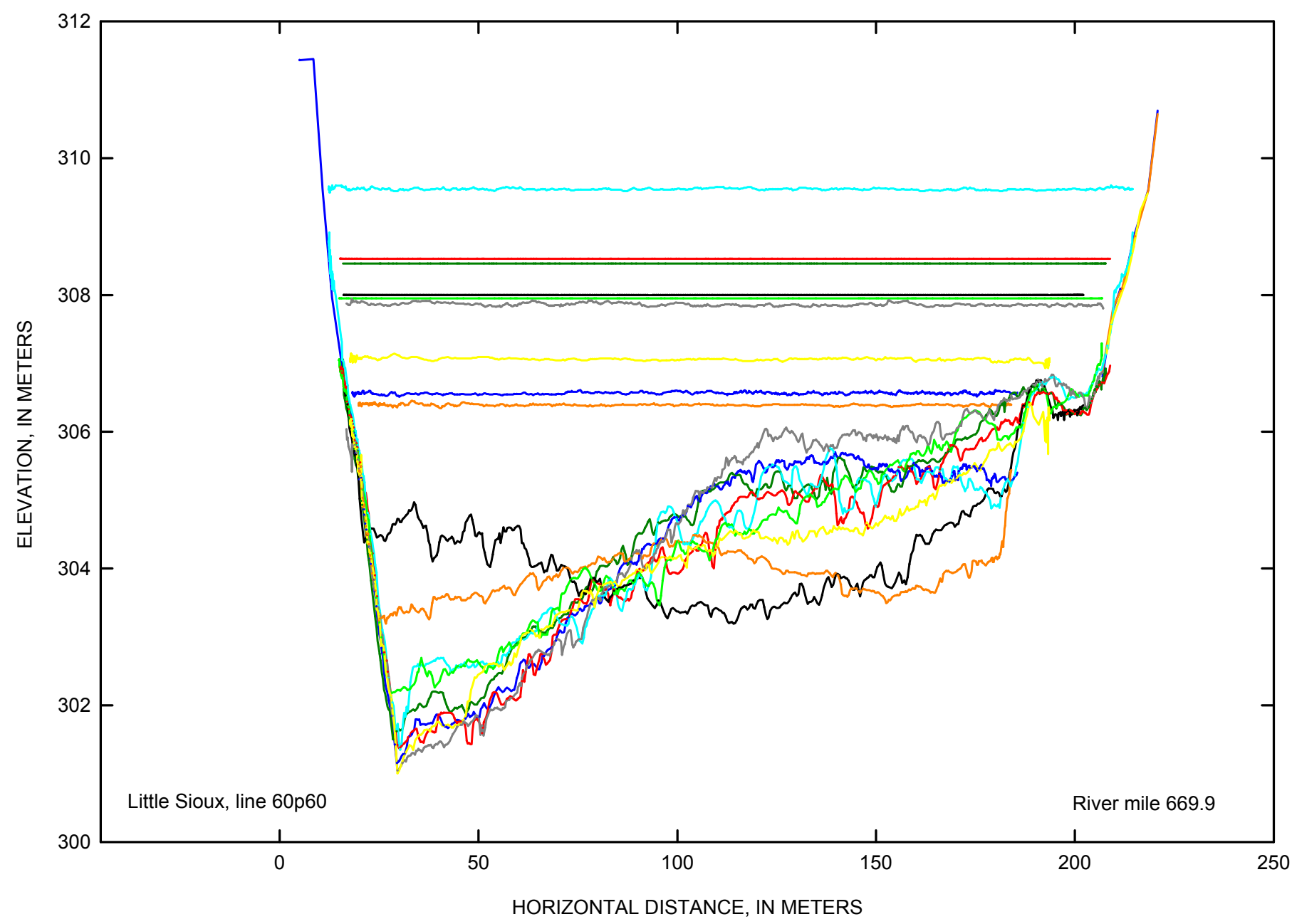

EXPLANATION

March 2006

- April 2006

May 2006

July 2006

October 2006

March 2007

April 2007

July 2007

October 2007

Figure 3-30. Little Sioux cross-section line 60p60 at Missouri River mile 669.9 for 2006-07. 


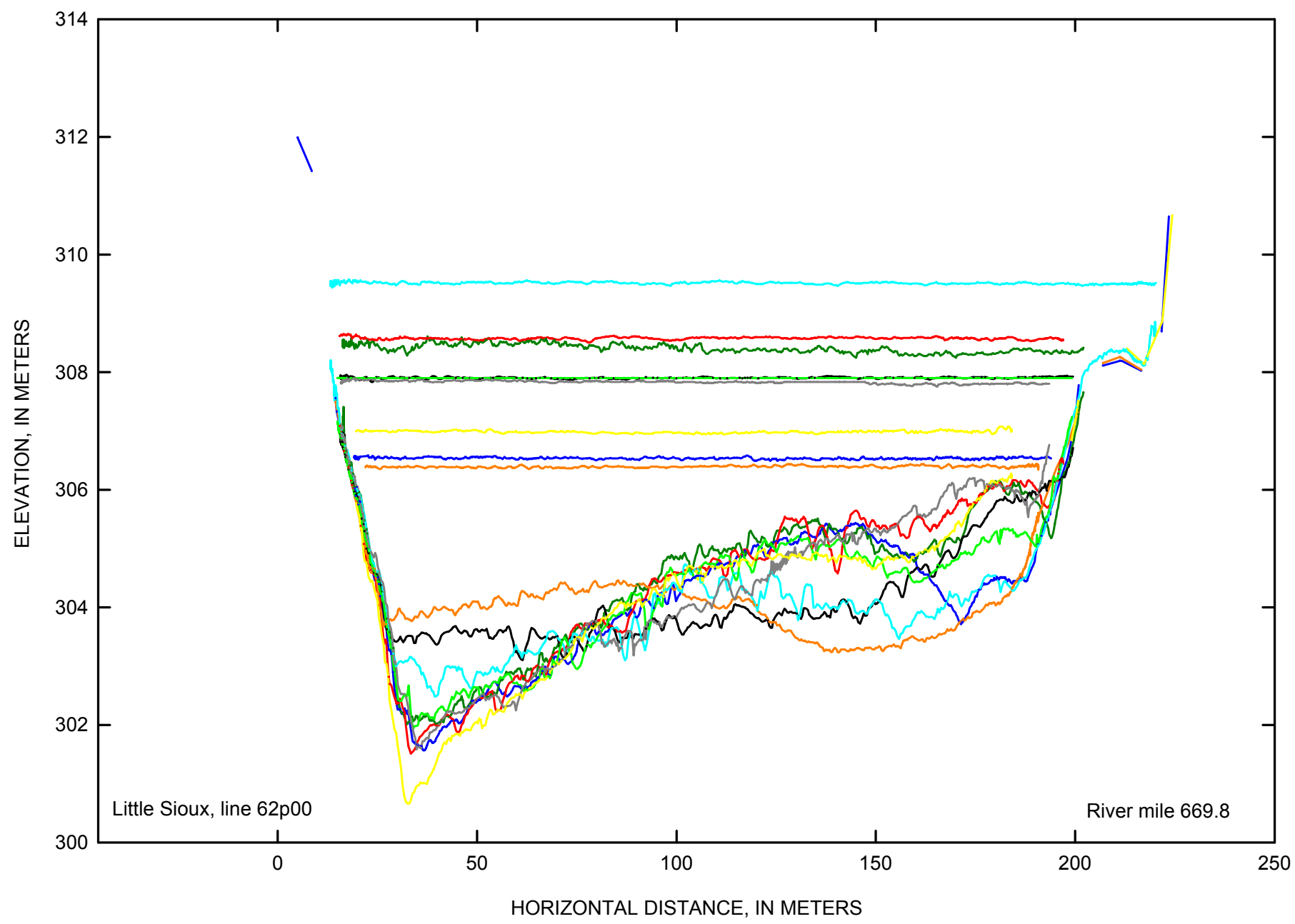

EXPLANATION

March 2006

April 2006

_ May 2006

July 2006

October 2006

March 2007

April 2007

July 2007

October 2007

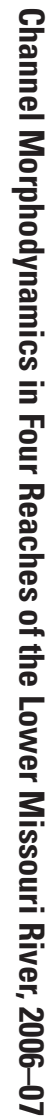

Figure 3-31. Little Sioux cross-section line $62 p 00$ at Missouri River mile 669.8 for 2006-07. 


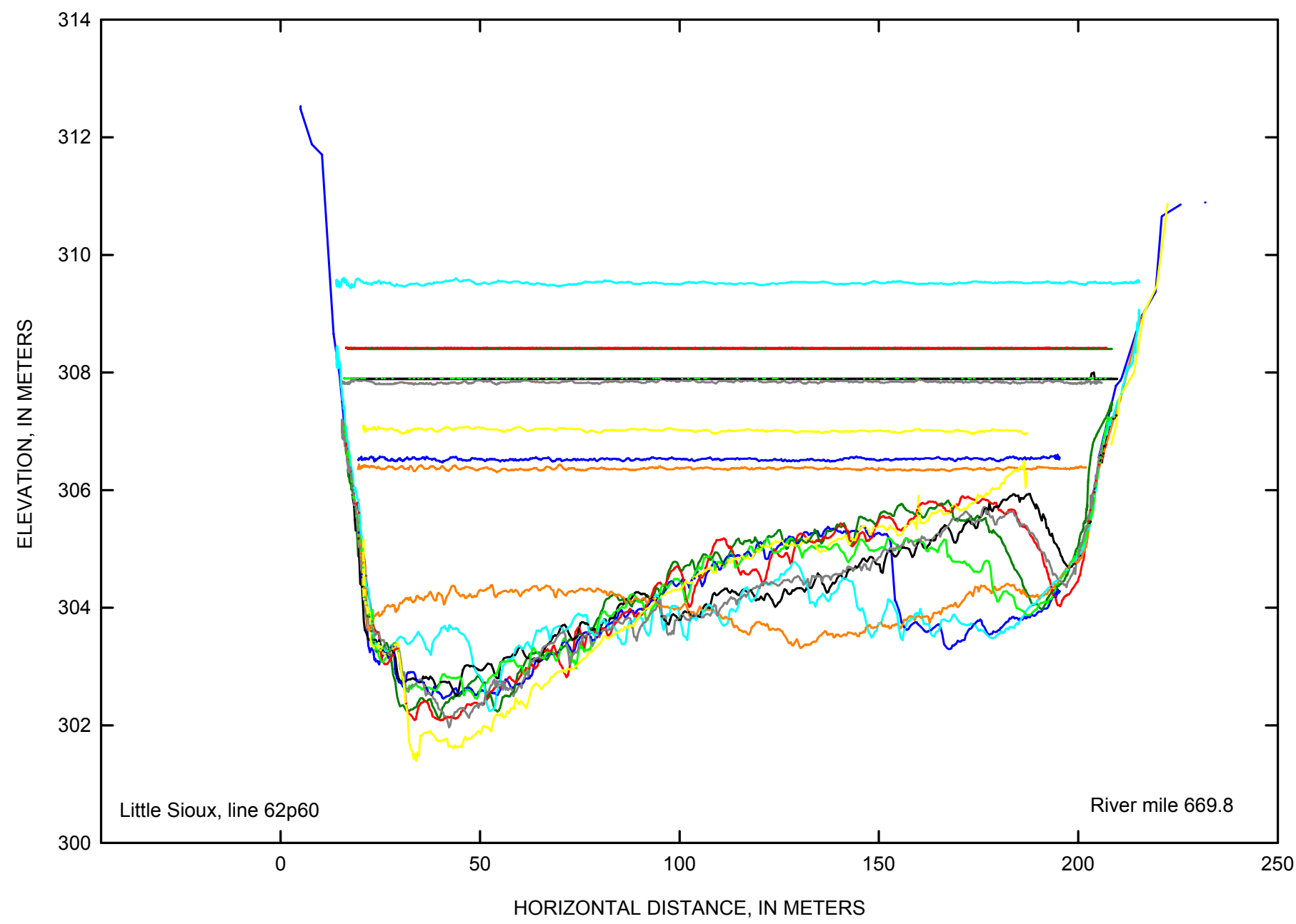

EXPLANATION

March 2006

April 2006

May 2006

July 2006

October 2006

March 2007

April 2007

July 2007

October 2007

Figure 3-32. ittle Sioux cross-section line 62 p60 at Missouri River mile 669.8 for 2006-07. 

Appendix 4. Miami Reach Cross Sections 


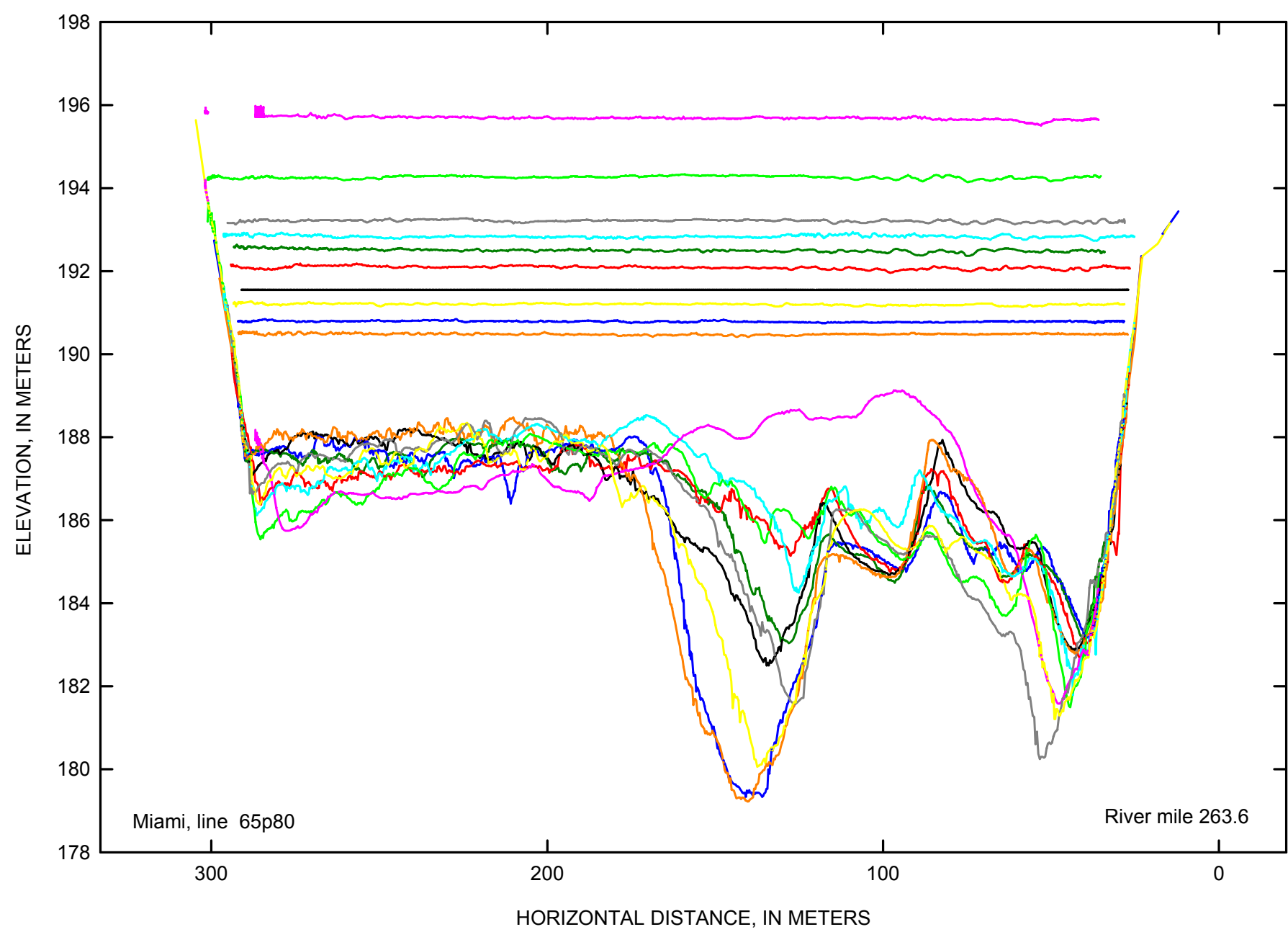

EXPLANATION

March 2006

April 2006

May 2006

July 2006

October 2006

March 2007

April 2007

May 2007

July 2007

October 2007

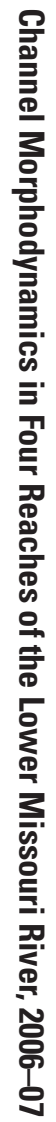

Figure 4-1. Miami cross-section line 65 p80 at Missouri River mile 263.6 for 2006-07. 


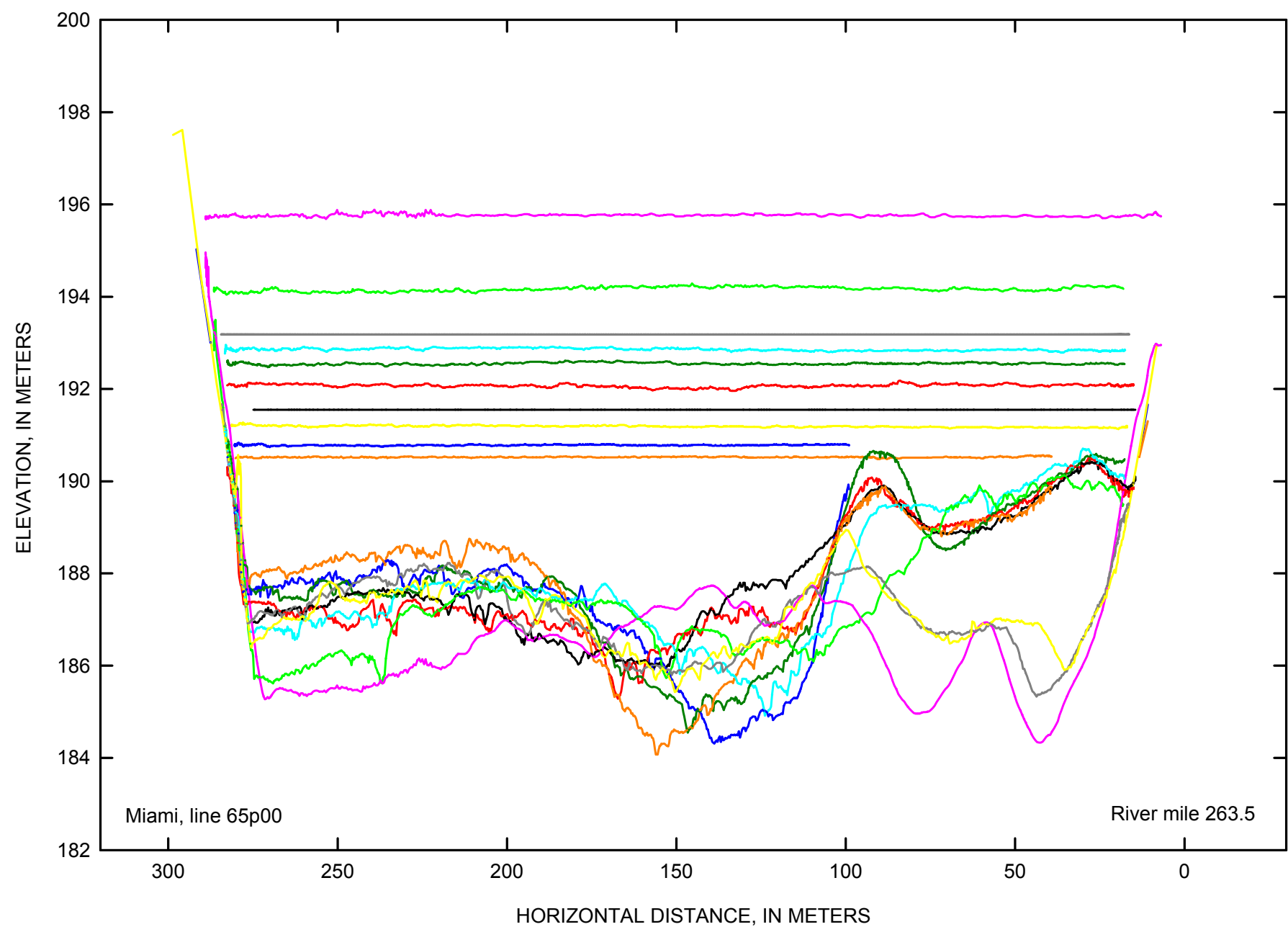

\section{EXPLANATION}

— March 2006

April 2006

May 2006

July 2006

October 2006

March 2007

April 2007

May 2007

July 2007

October 2007

Figure 4-2. Miami cross-section line 65p00 at Missouri River mile 263.5 for 2006-07. 


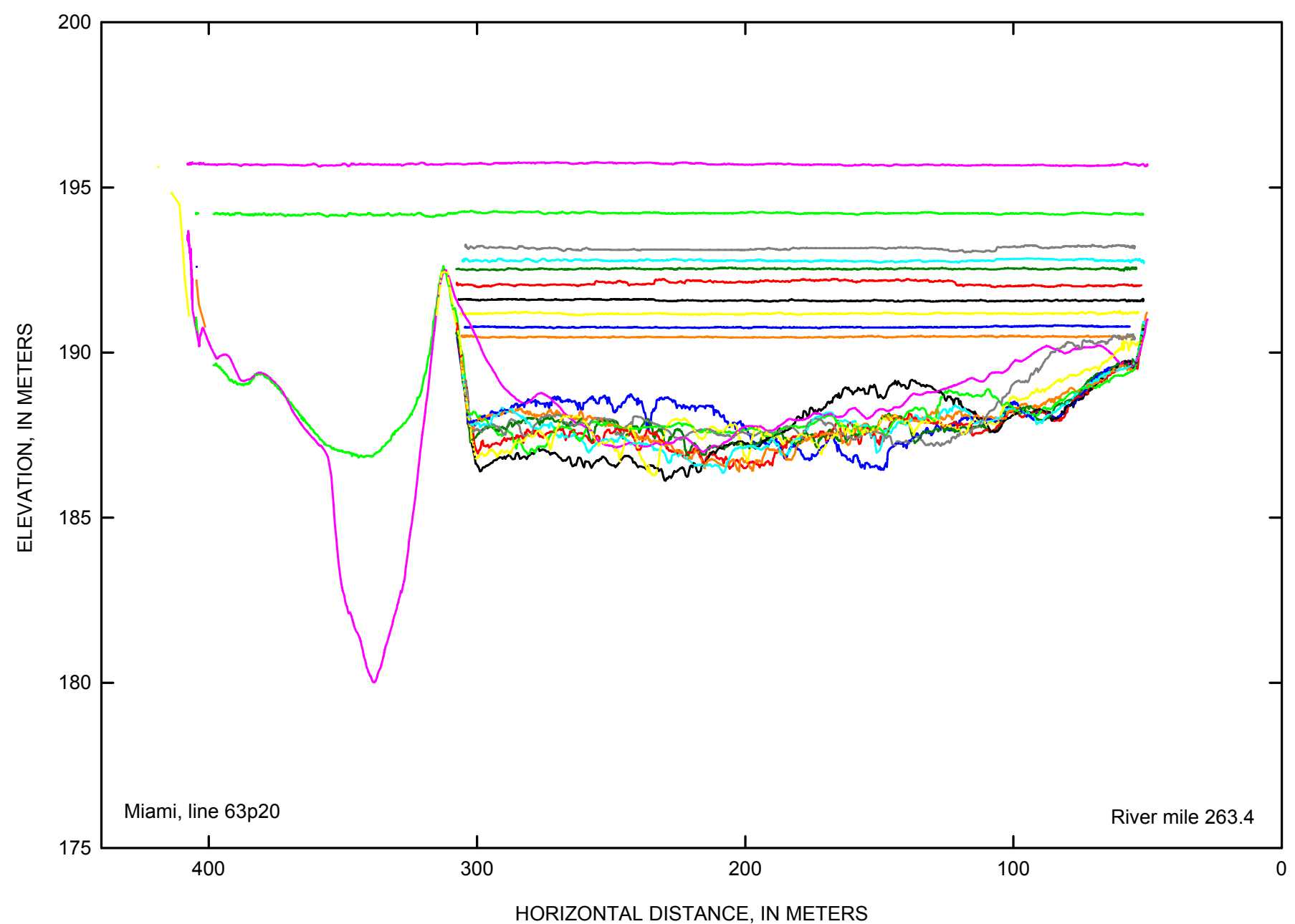

EXPLANATION

March 2006

April 2006

May 2006

July 2006

October 2006

March 2007

April 2007

May 2007

July 2007

October 2007

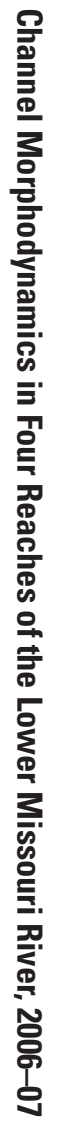

Figure 4-3. Miami cross-section line 63p20 at Missouri River mile 263.4 for 2006-07. 


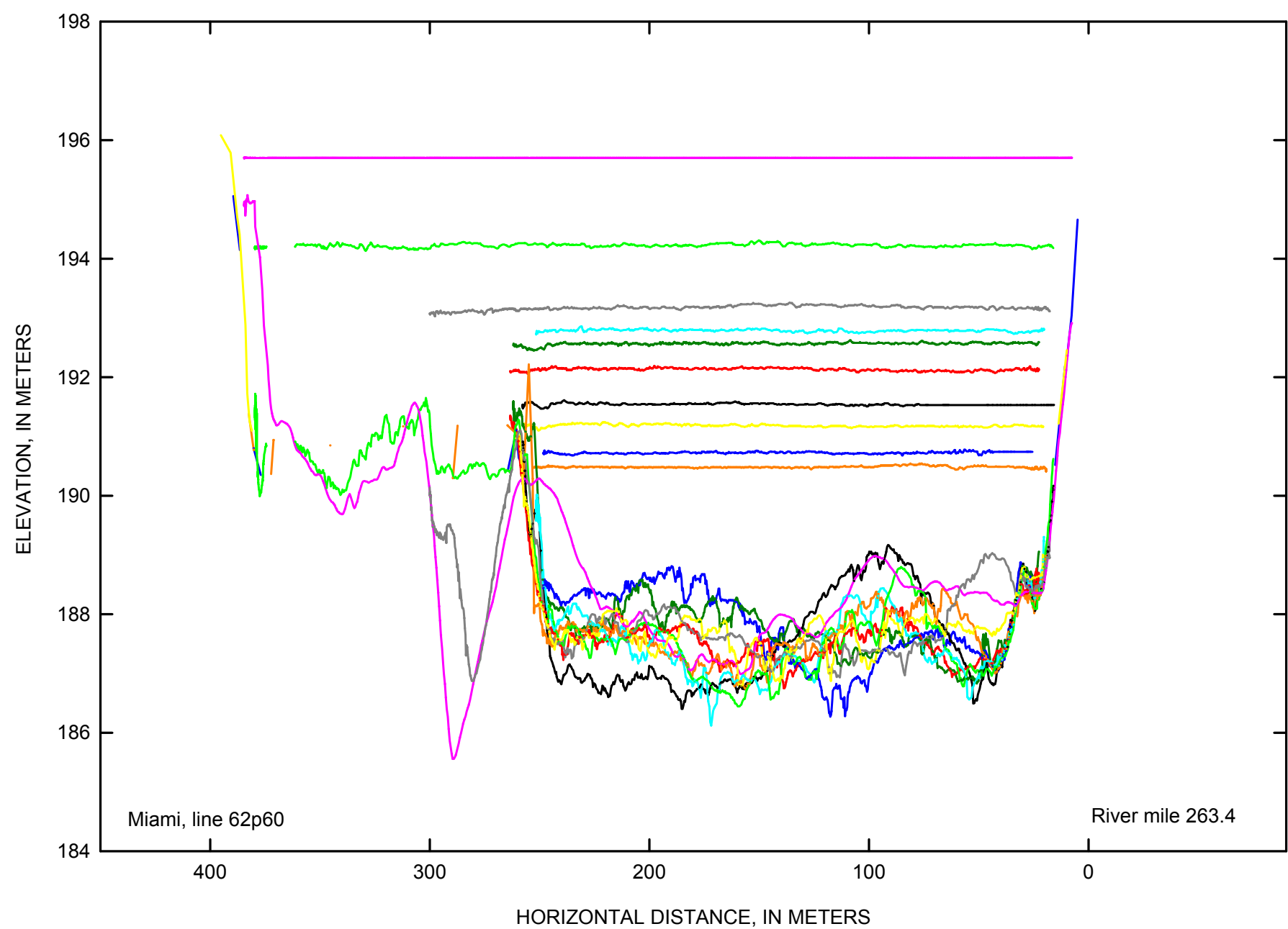

\section{EXPLANATION}

March 2006

April 2006

May 2006

— July 2006

October 2006

March 2007

April 2007

May 2007

July 2007

October 2007

Figure 4-4. Miami cross-section line 62p60 at Missouri River mile 263.4 for 2006-07. 


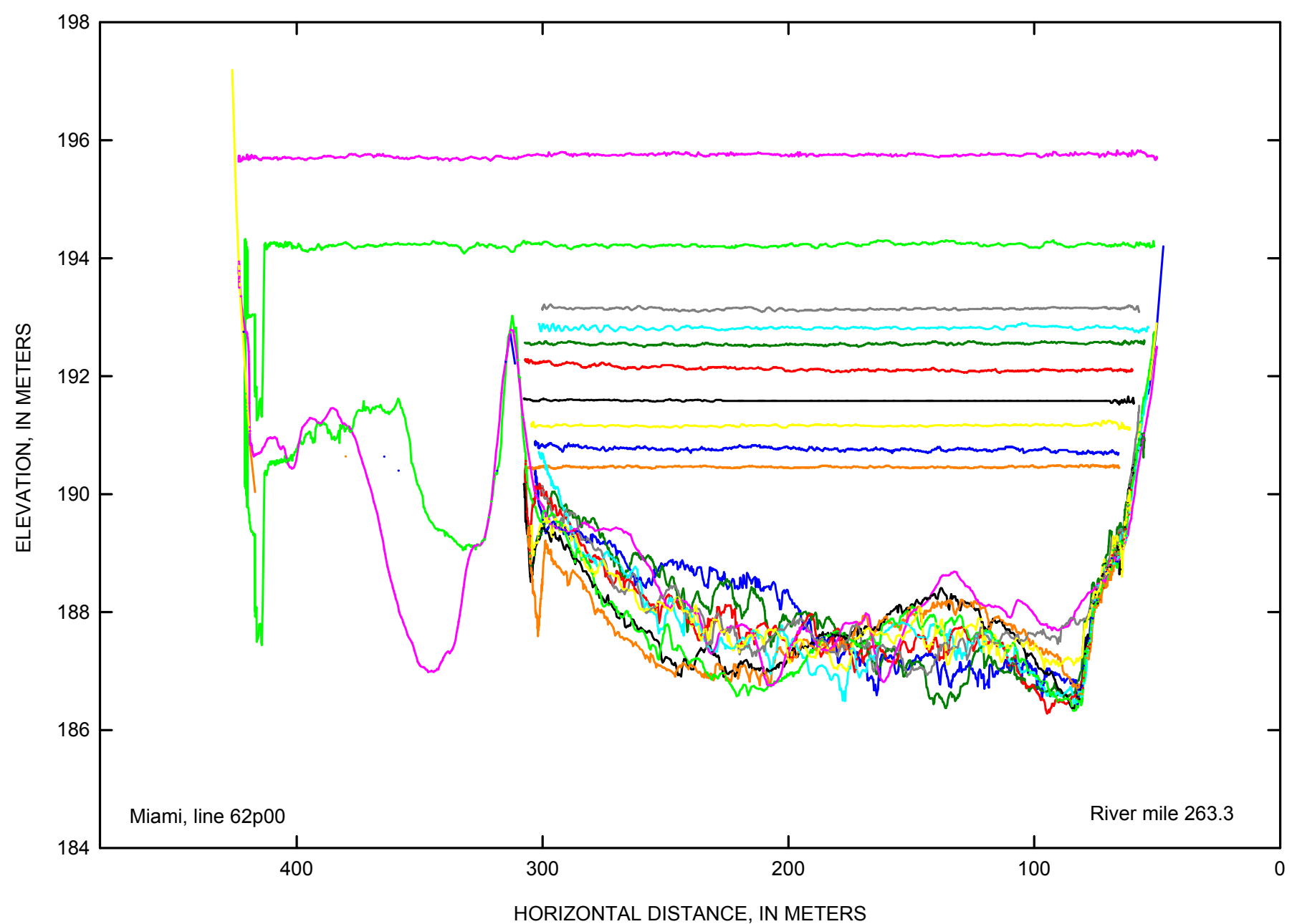

EXPLANATION

March 2006

April 2006

May 2006

July 2006

October 2006

March 2007

April 2007

May 2007

July 2007

October 2007

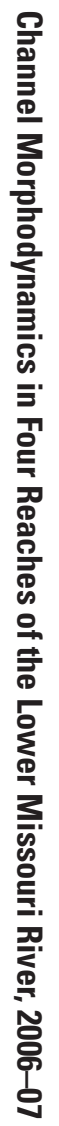

Figure 4-5. Miami cross-section line 62p00 at Missouri River mile 263.3 for 2006-07. 


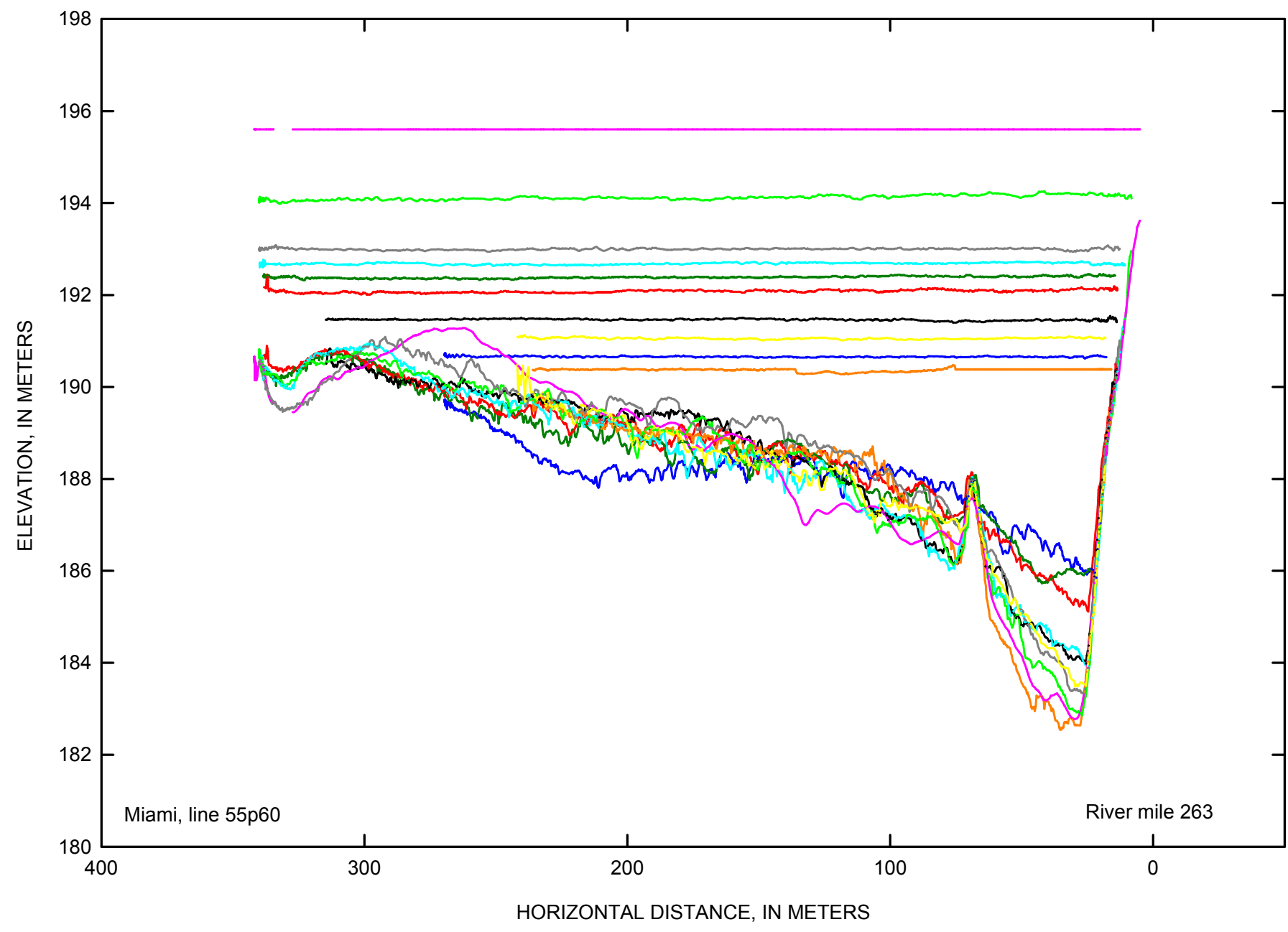

EXPLANATION

March 2006

April 2006

May 2006

July 2006

October 2006

March 2007

April 2007

May 2007

July 2007

October 2007

Figure 4-6. Miami cross-section line 55p60 at Missouri River mile 263 for 2006-07. 


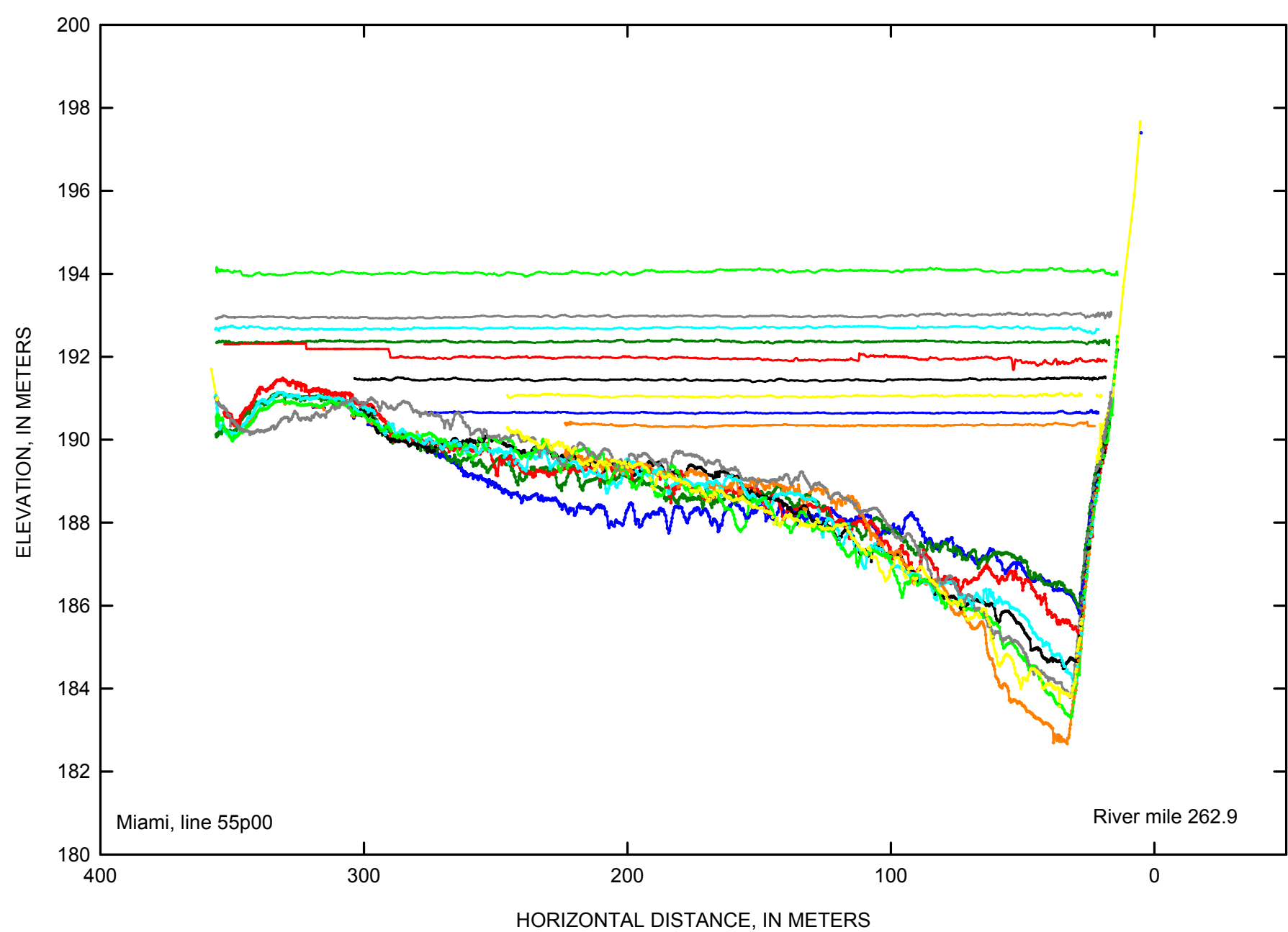

EXPLANATION

— March 2006

April 2006

May 2006

July 2006

October 2006

March 2007

April 2007

May 2007

July 2007

October 2007

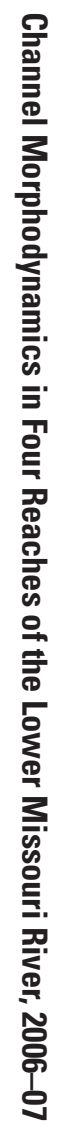

Figure 4-7. Miami cross-section line 55p00 at Missouri River mile 262.9 for 2006-07. 


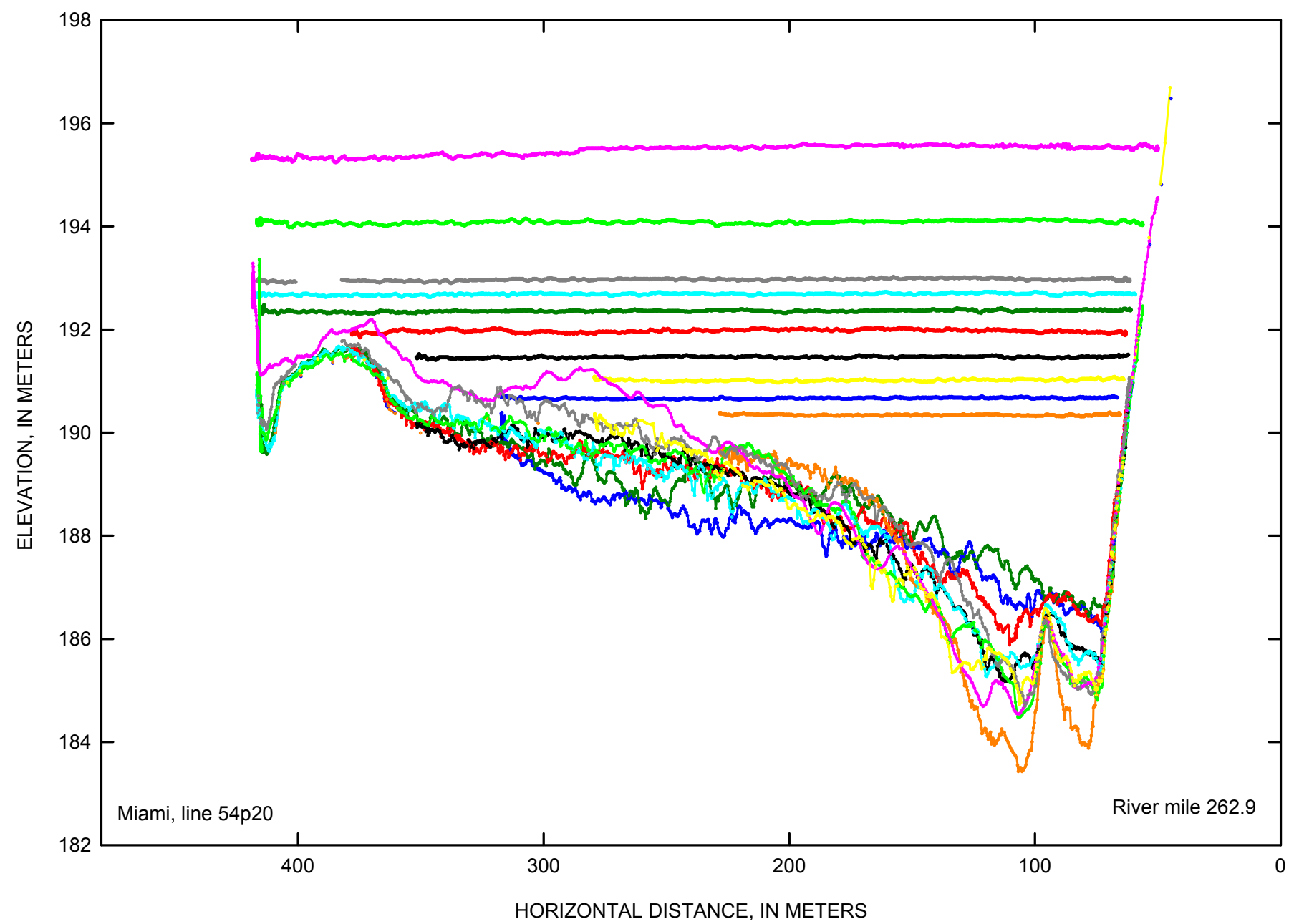

\section{EXPLANATION}

— March 2006

_ April 2006

- May 2006

— July 2006

October 2006

March 2007

April 2007

May 2007

July 2007

October 2007

Figure 4-8. Miami cross-section line 54p20 at Missouri River mile 262.9 for 2006-07. 


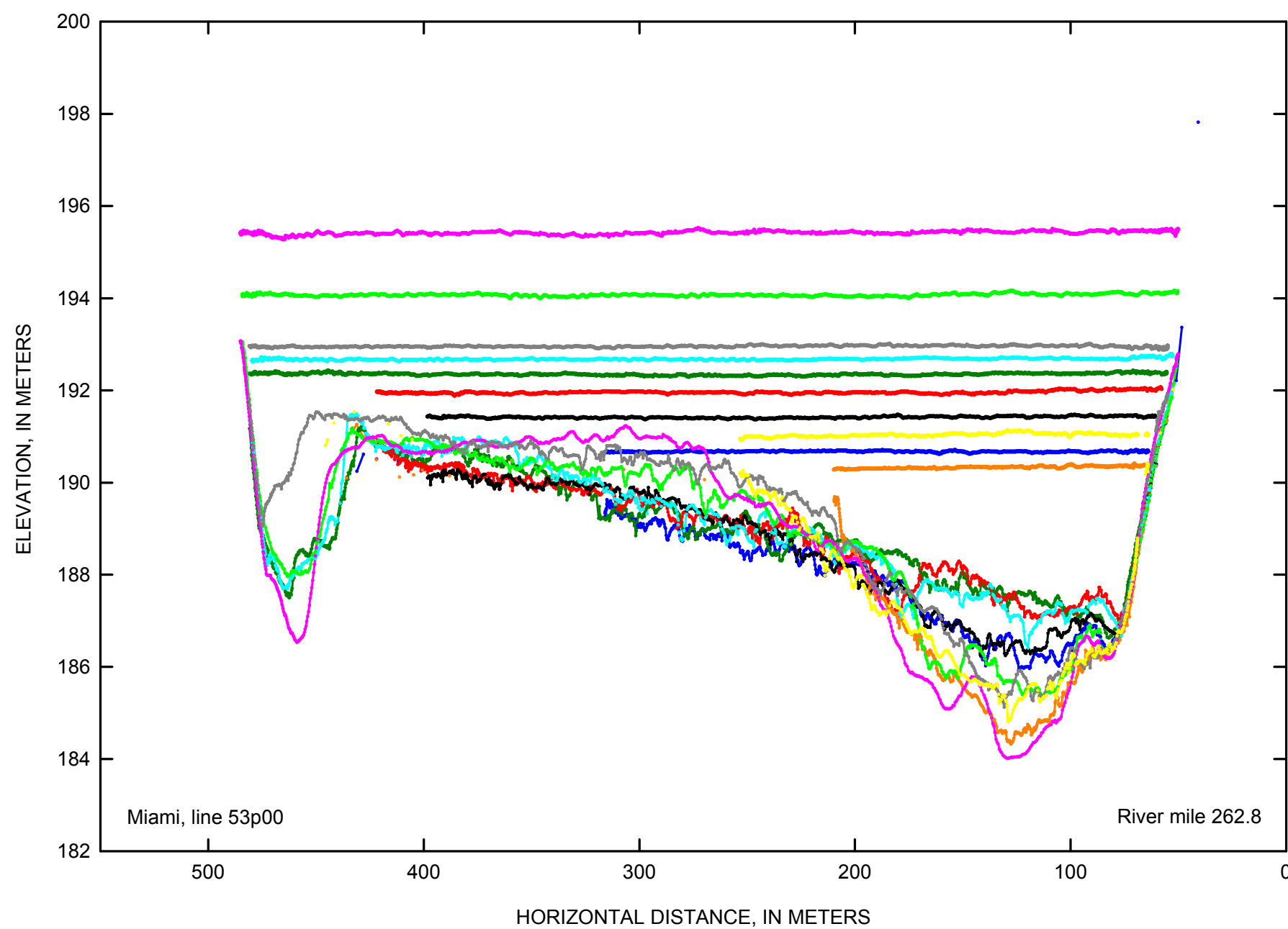

EXPLANATION

March 2006

April 2006

May 2006

July 2006

October 2006

March 2007

April 2007

May 2007

July 2007

October 2007

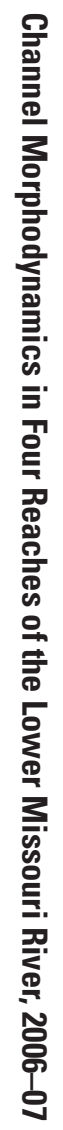

Figure 4-9. Miami cross-section line 53p00 at Missouri River mile 262.8 for 2006-07. 


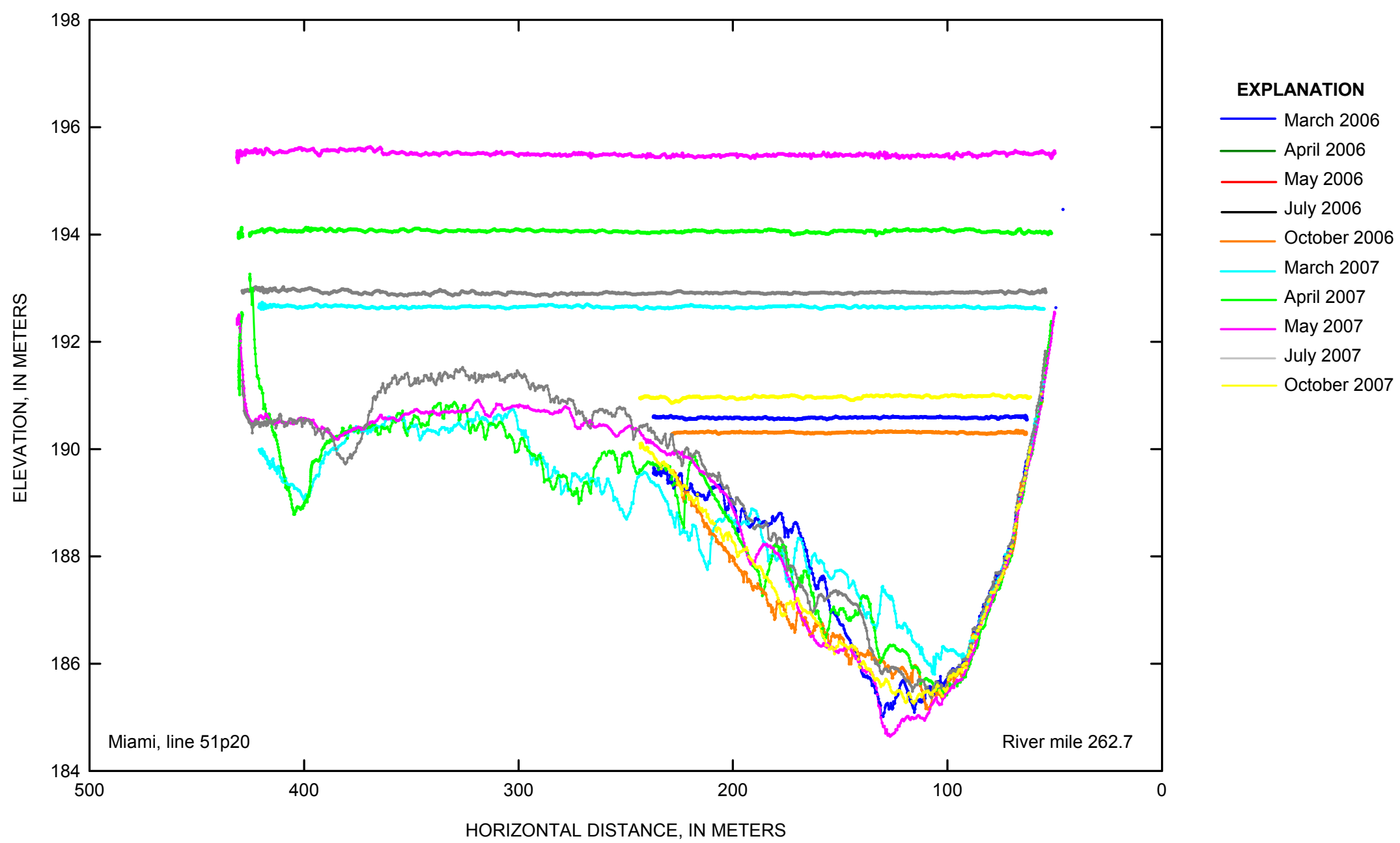

Figure 4-10. Miami cross-section line 51p20 at Missouri River mile 262.7 for 2006-07. 


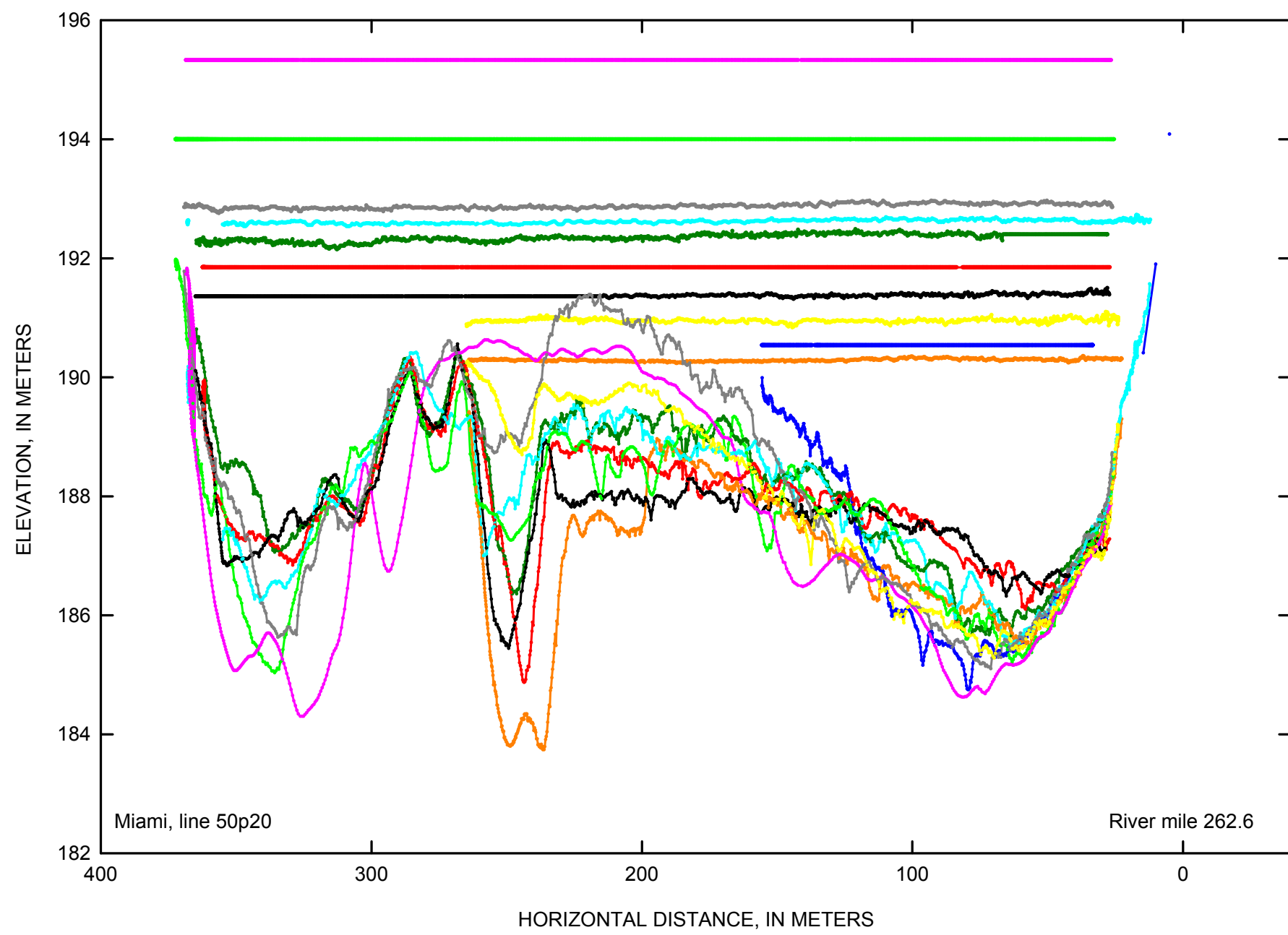

EXPLANATION

- March 2006

- April 2006

May 2006

July 2006

October 2006

March 2007

April 2007

May 2007

July 2007

October 2007

Figure 4-11. Miami cross-section line 50p20 at Missouri River mile 262.6 for 2006-07. 


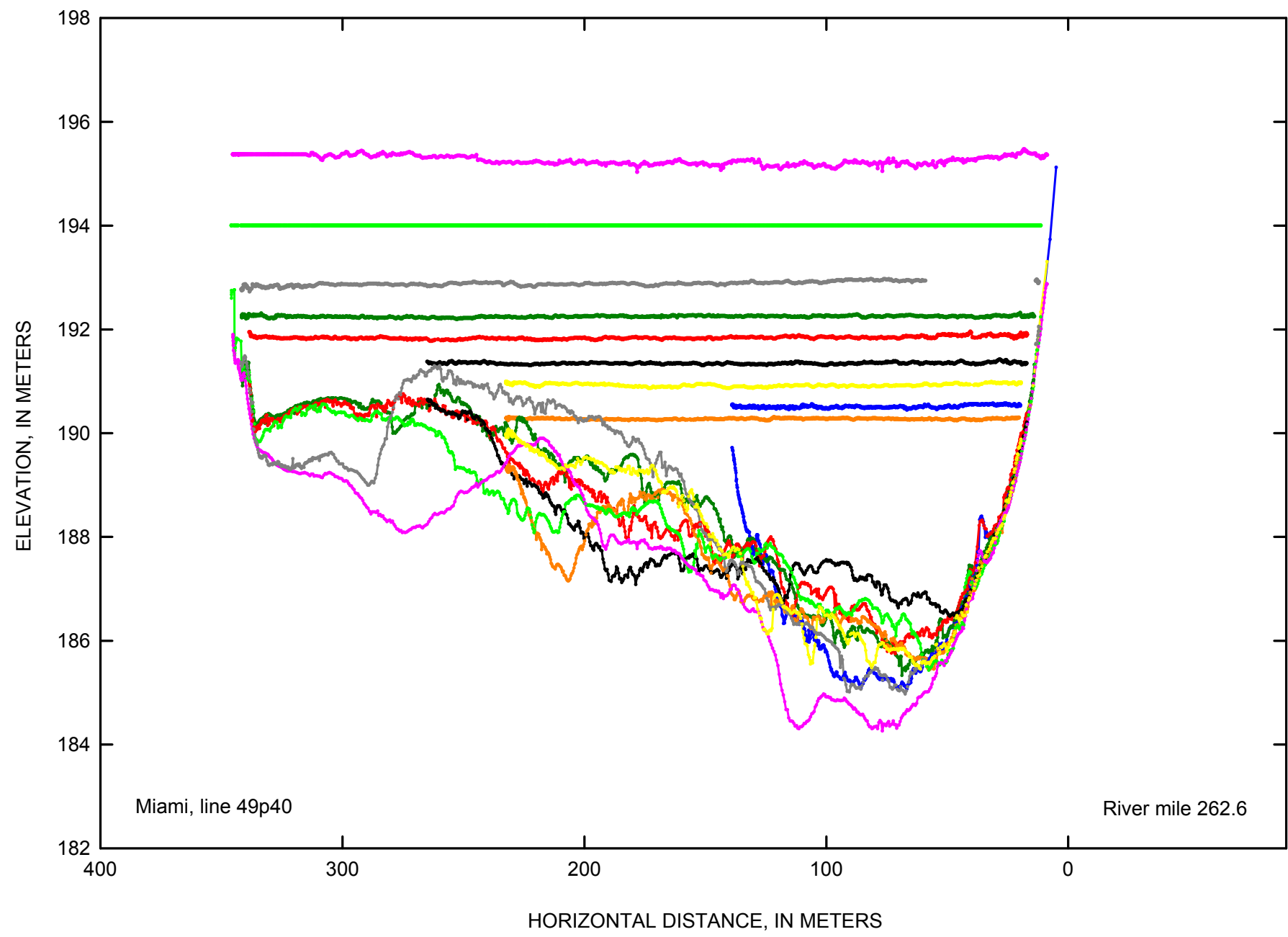

\section{EXPLANATION}

— March 2006

— April 2006

_ May 2006

— July 2006

October 2006

March 2007

April 2007

May 2007

July 2007

October 2007

Figure 4-12. Miami cross-section line 49p40 at Missouri River mile 262.6 for 2006-07. 


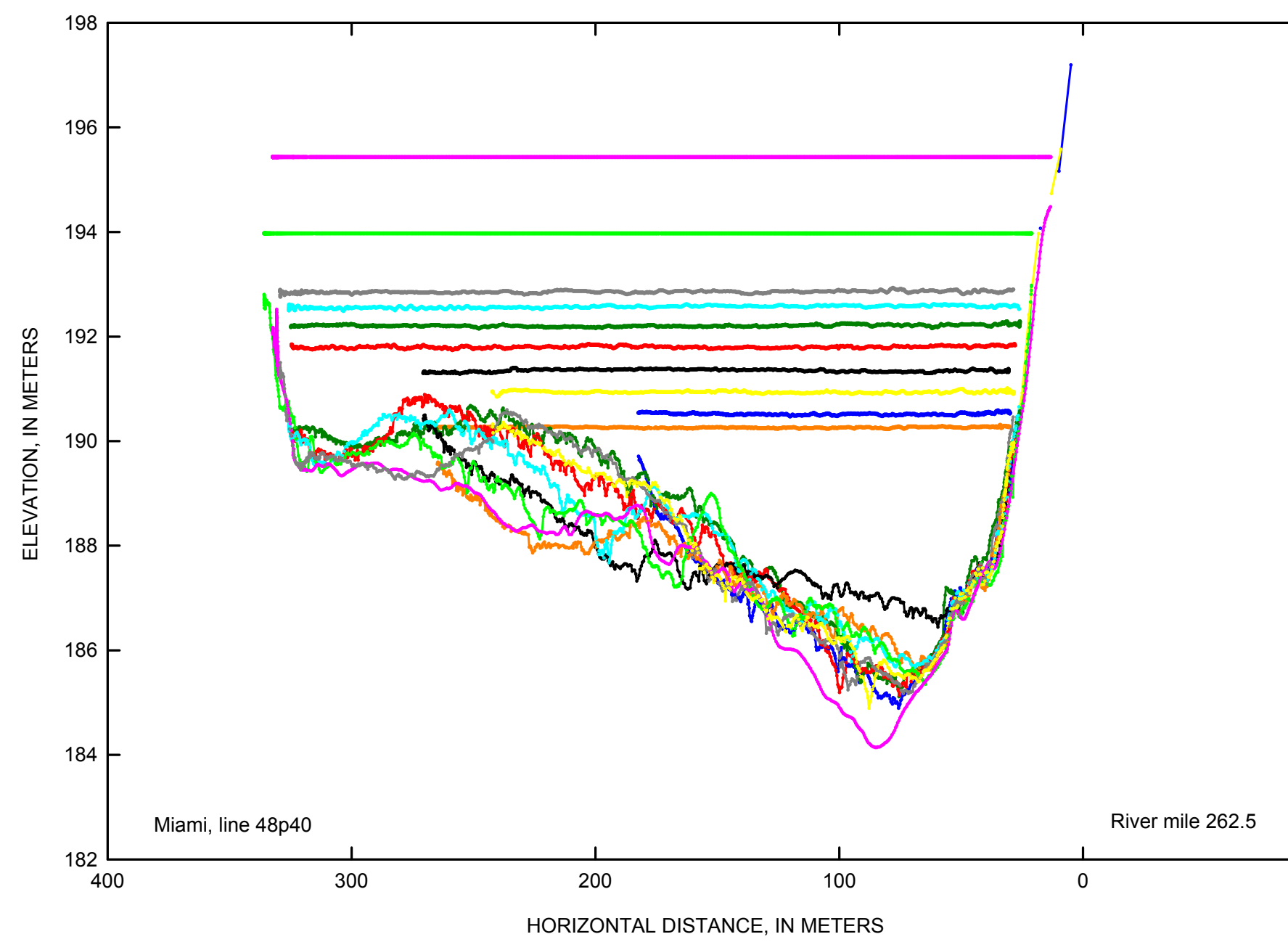

EXPLANATION

- March 2006

April 2006

May 2006

July 2006

October 2006

March 2007

April 2007

May 2007

July 2007

October 2007

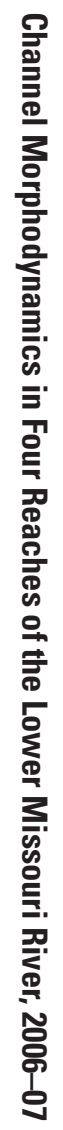

Figure 4-13. Miami cross-section line 48p40 at Missouri River mile 262.5 for 2006-07. 


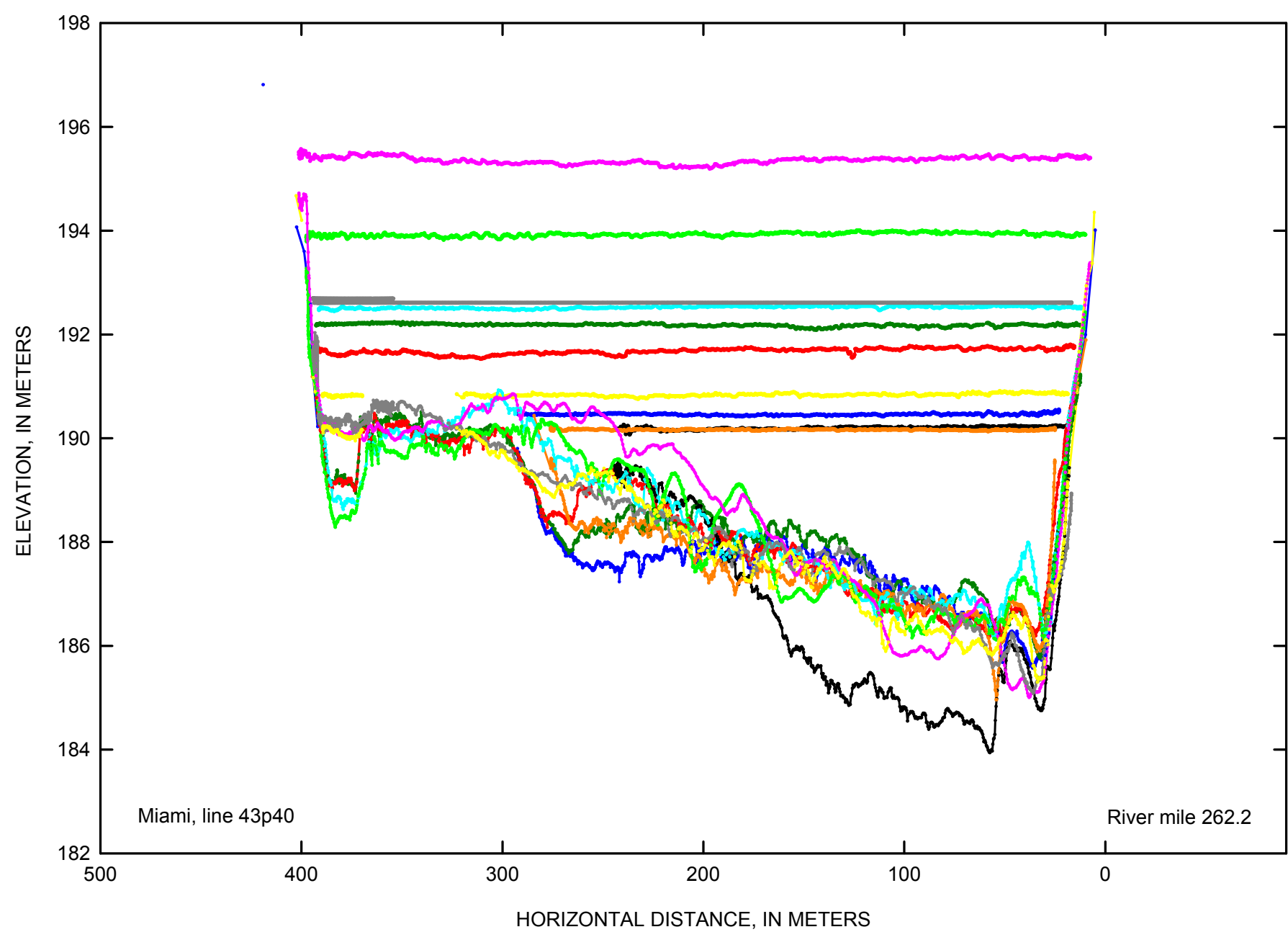

EXPLANATION

— March 2006

April 2006

- May 2006

July 2006

October 2006

March 2007

April 2007

May 2007

July 2007

October 2007

Figure 4-14. Miami cross-section line 43p40 at Missouri River mile 262.2 for 2006-07. 


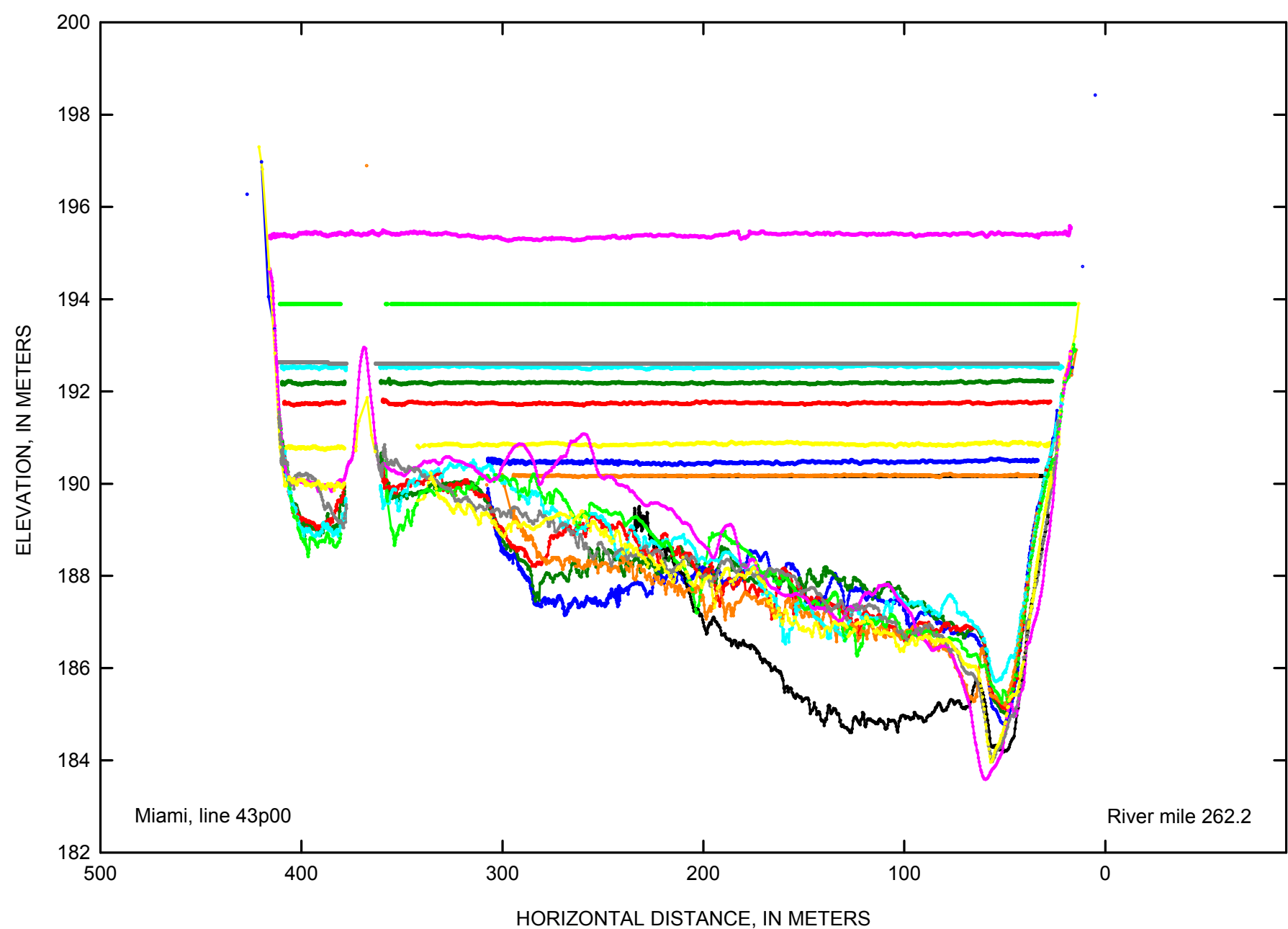

EXPLANATION

- March 2006

April 2006

May 2006

July 2006

October 2006

March 2007

April 2007

May 2007

July 2007

October 2007

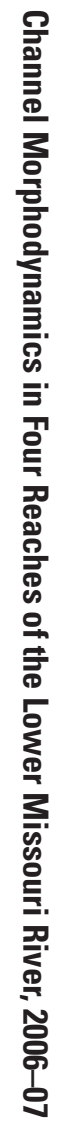

Figure 4-15. Miami cross-section line 43p00 at Missouri River mile 262.2 for 2006-07. 


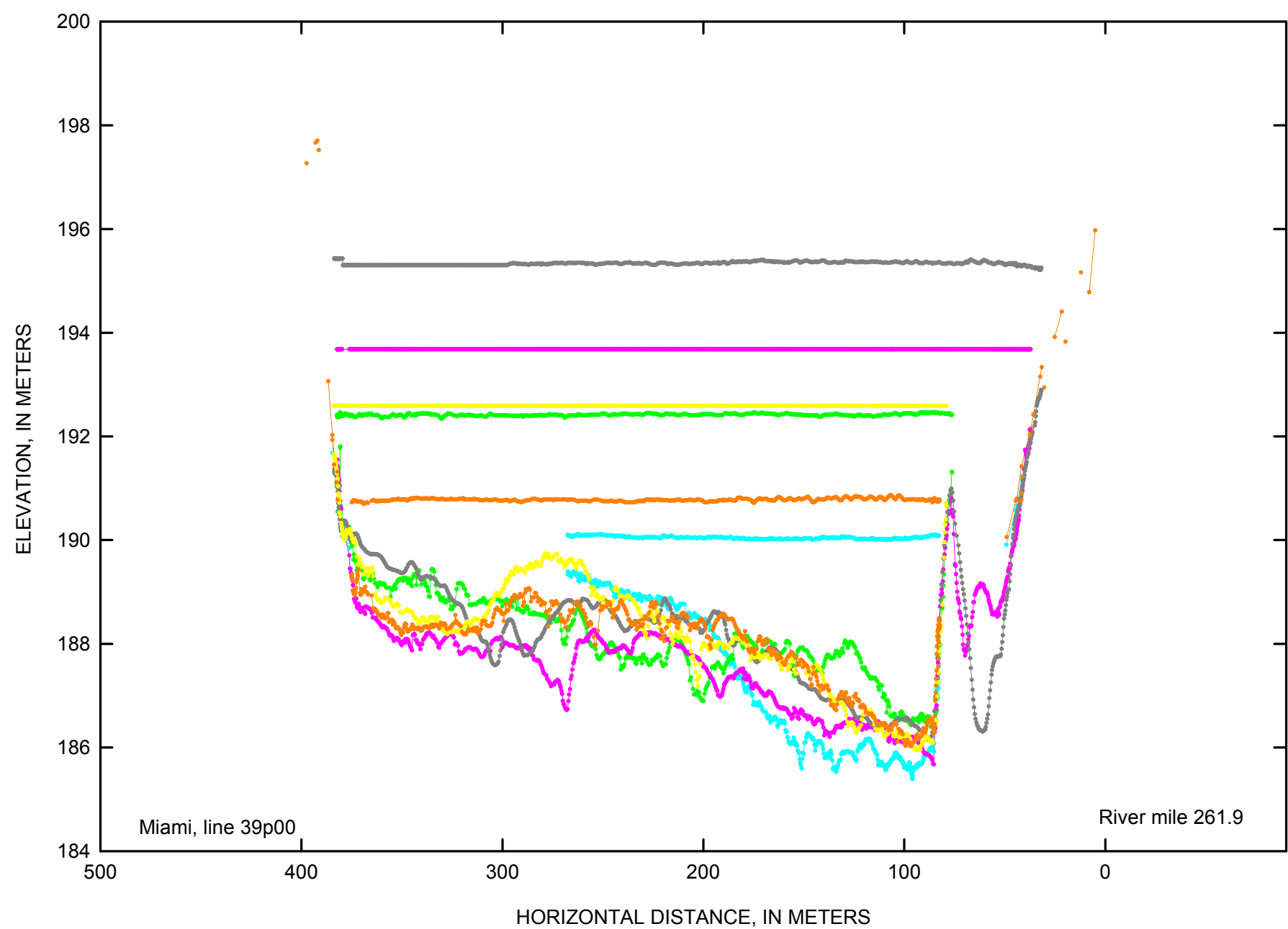

EXPLANATION

March 2006

April 2006

May 2006

_ July 2006

- October 2006

March 2007

April 2007

May 2007

July 2007

October 2007

Figure 4-16. Miami cross-section line 39p00 at Missouri River mile 261.9 for 2006-07. 


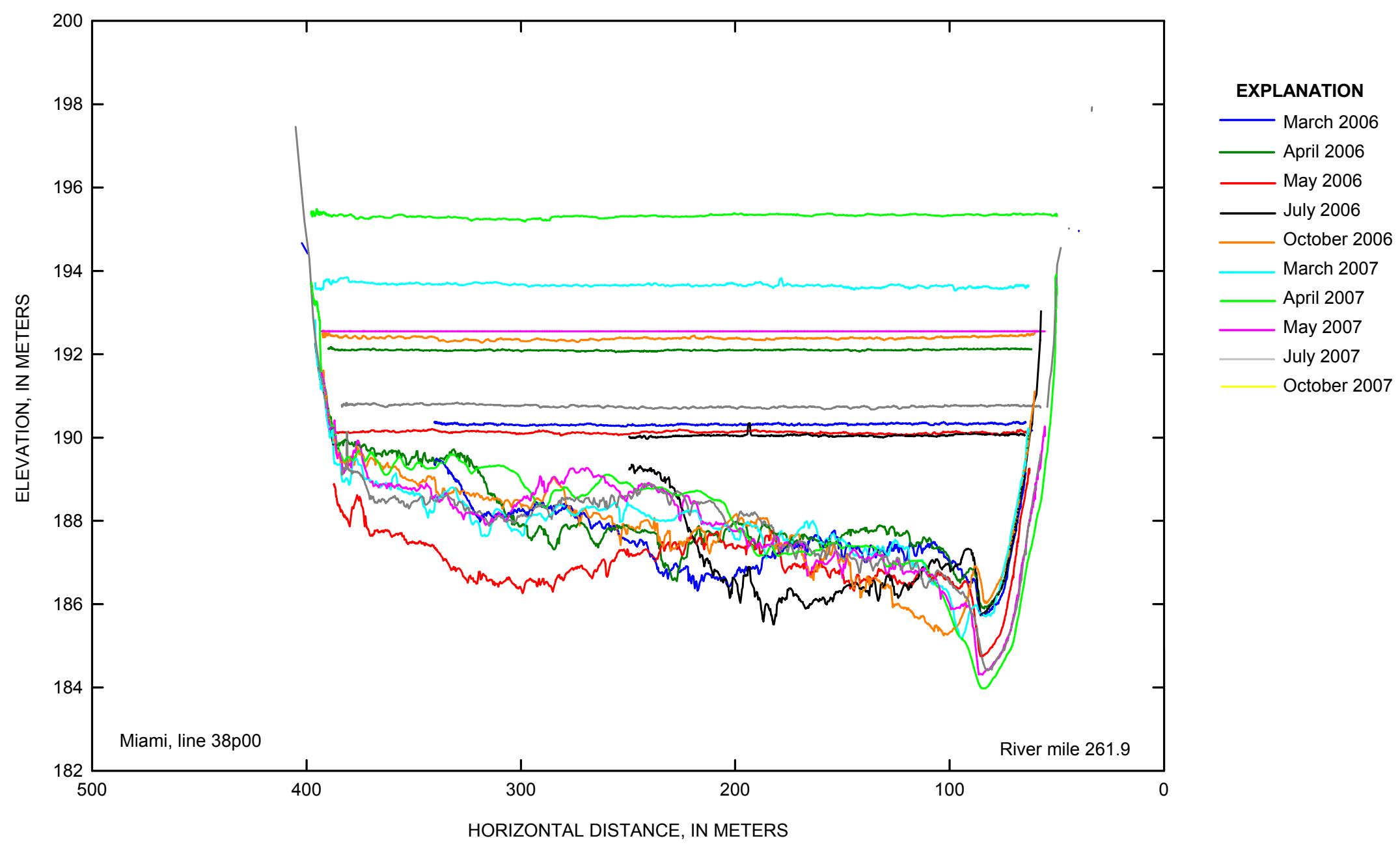

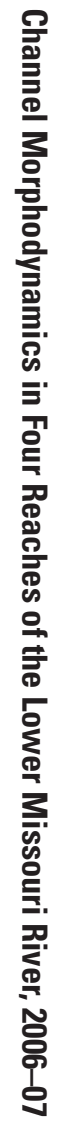

Figure 4-17. Miami cross-section line 38p00 at Missouri River mile 261.9 for 2006-07. 


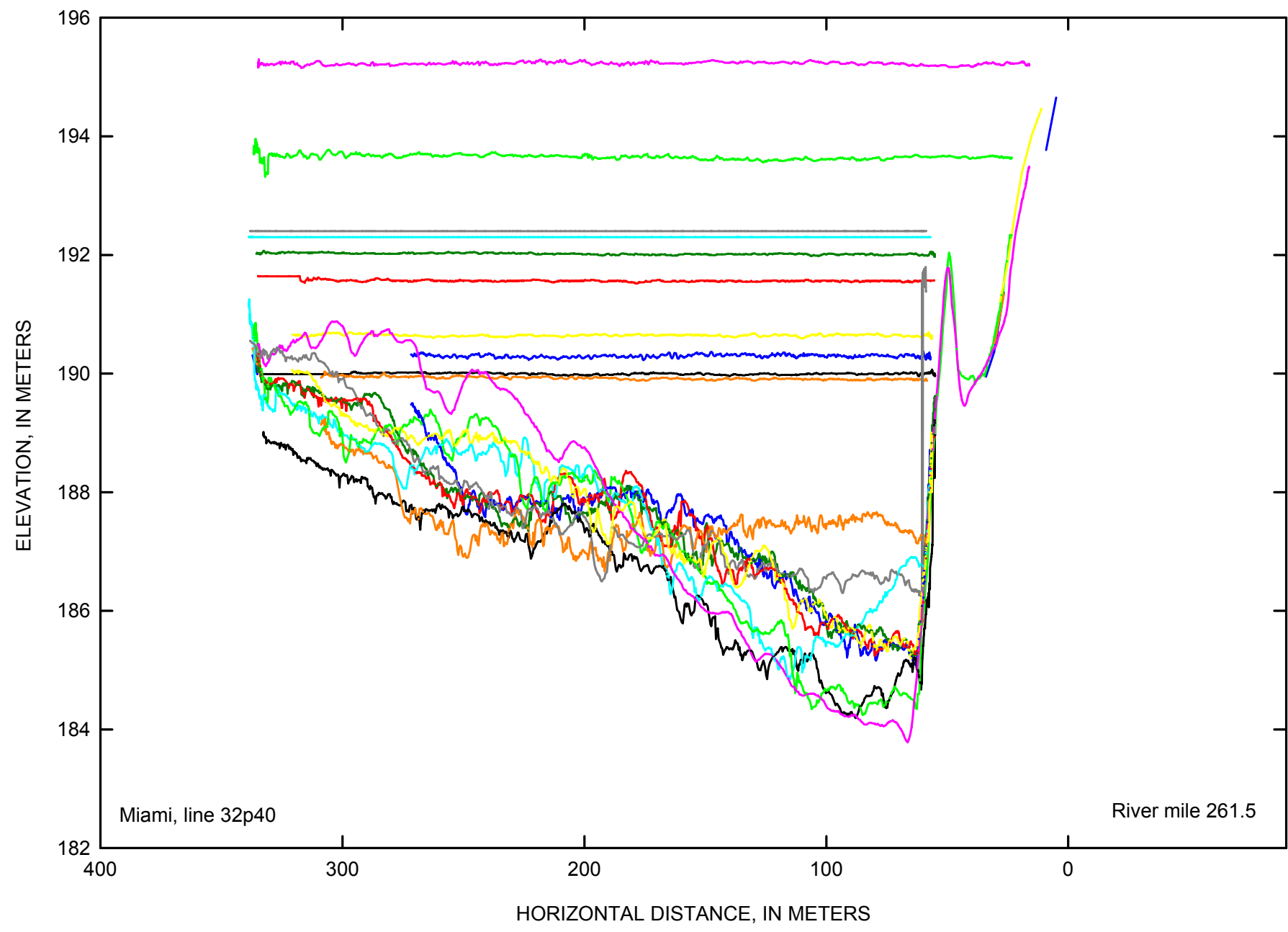

\section{EXPLANATION}

\begin{tabular}{c}
- March 2006 \\
\hline April 2006 \\
May 2006 \\
\hline July 2006 \\
October 2006 \\
March 2007 \\
\hline April 2007 \\
\hline May 2007 \\
July 2007 \\
October 2007
\end{tabular}

Figure 4-18. Miami cross-section line 32p40 at Missouri River mile 261.5 for 2006-07. 


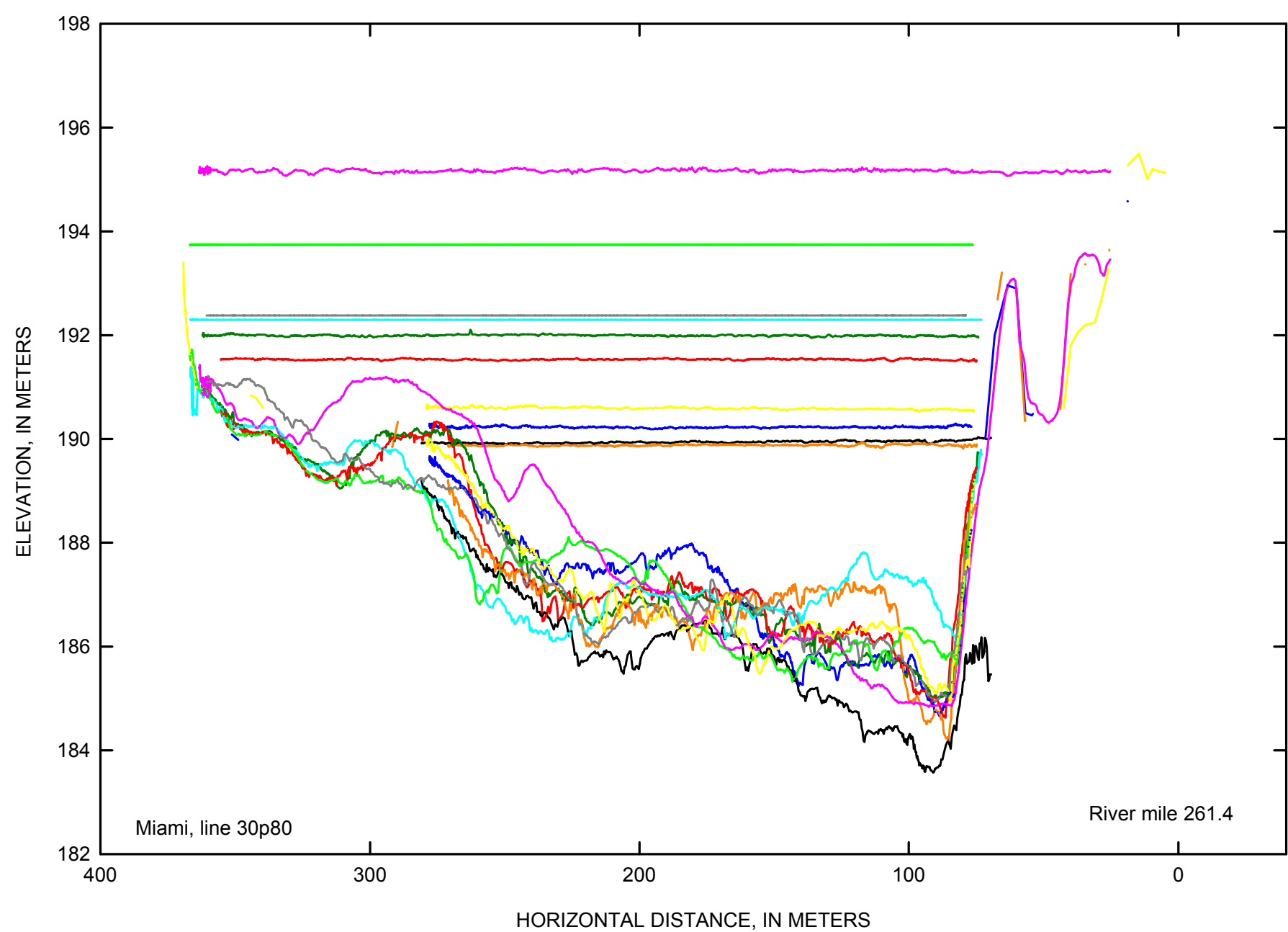

EXPLANATION

- March 2006

- April 2006

May 2006

July 2006

October 2006

March 2007

April 2007

May 2007

July 2007

October 2007

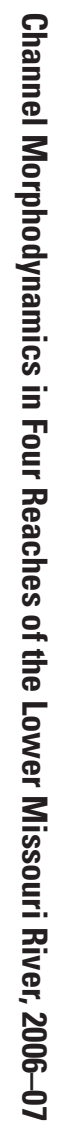

Figure 4-19. Miami cross-section line 30p80 at Missouri River mile 261.4 for 2006-07. 


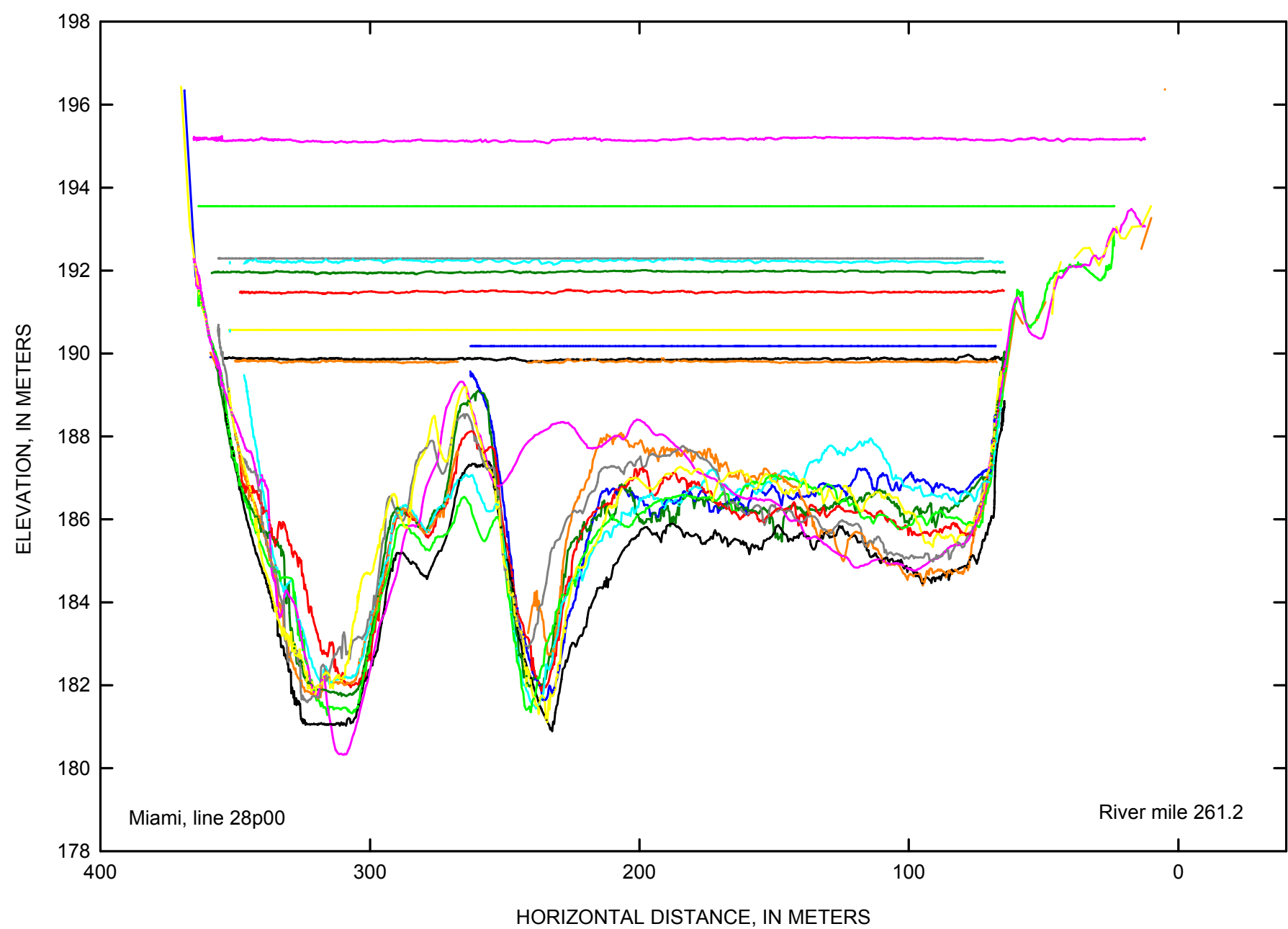

\section{EXPLANATION}

- March 2006

April 2006

May 2006

July 2006

October 2006

March 2007

April 2007

May 2007

July 2007

October 2007

Figure 4-20. Miami cross-section line 28p00 at Missouri River mile 261.2 for 2006-07. 


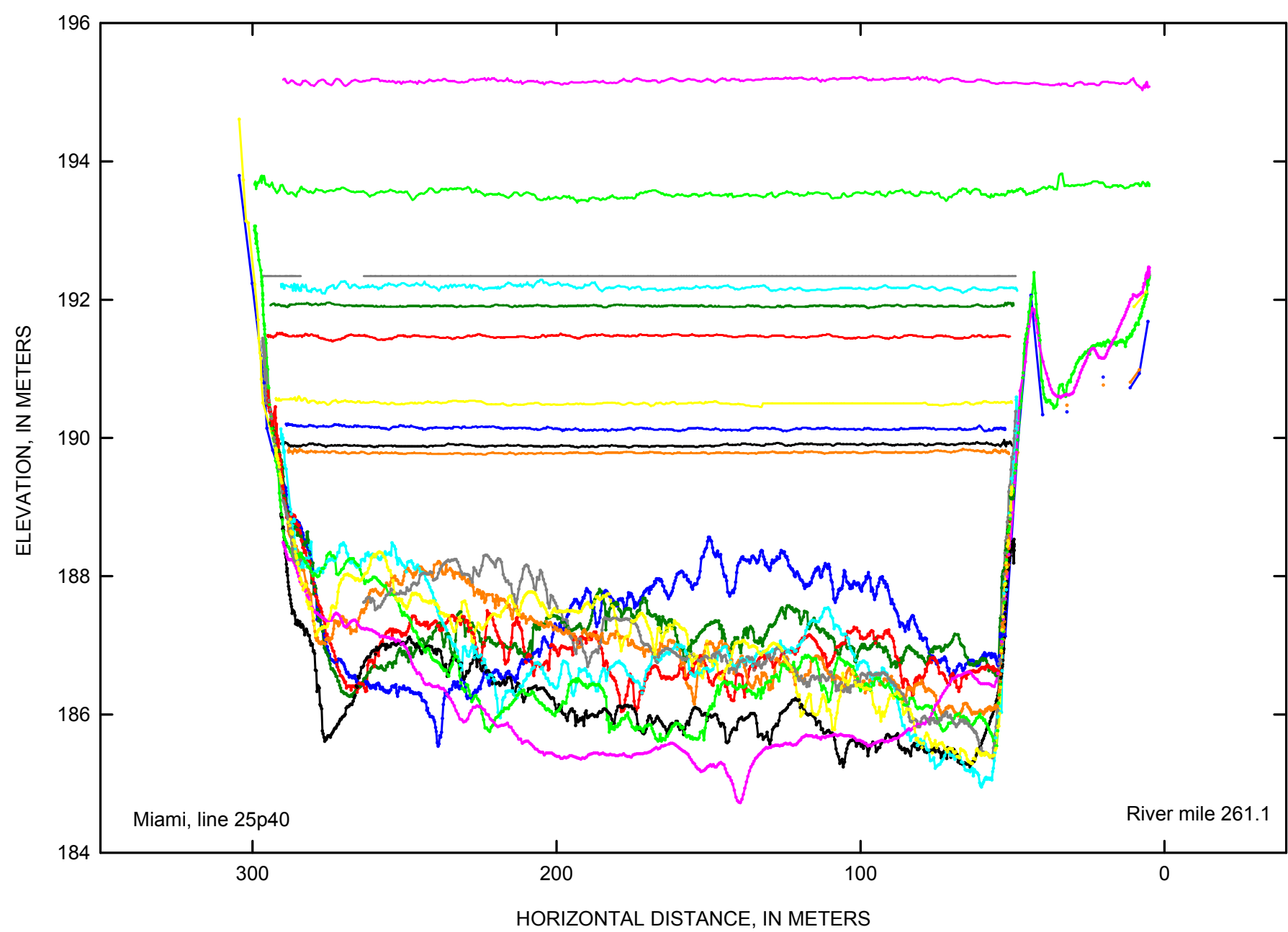

EXPLANATION

- March 2006

April 2006

May 2006

July 2006

October 2006

March 2007

April 2007

May 2007

July 2007

October 2007

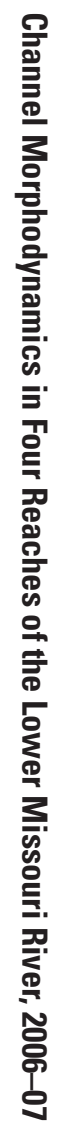

Figure 4-21. Miami cross-section line 25p40 at Missouri River mile 261.1 for 2006-07. 


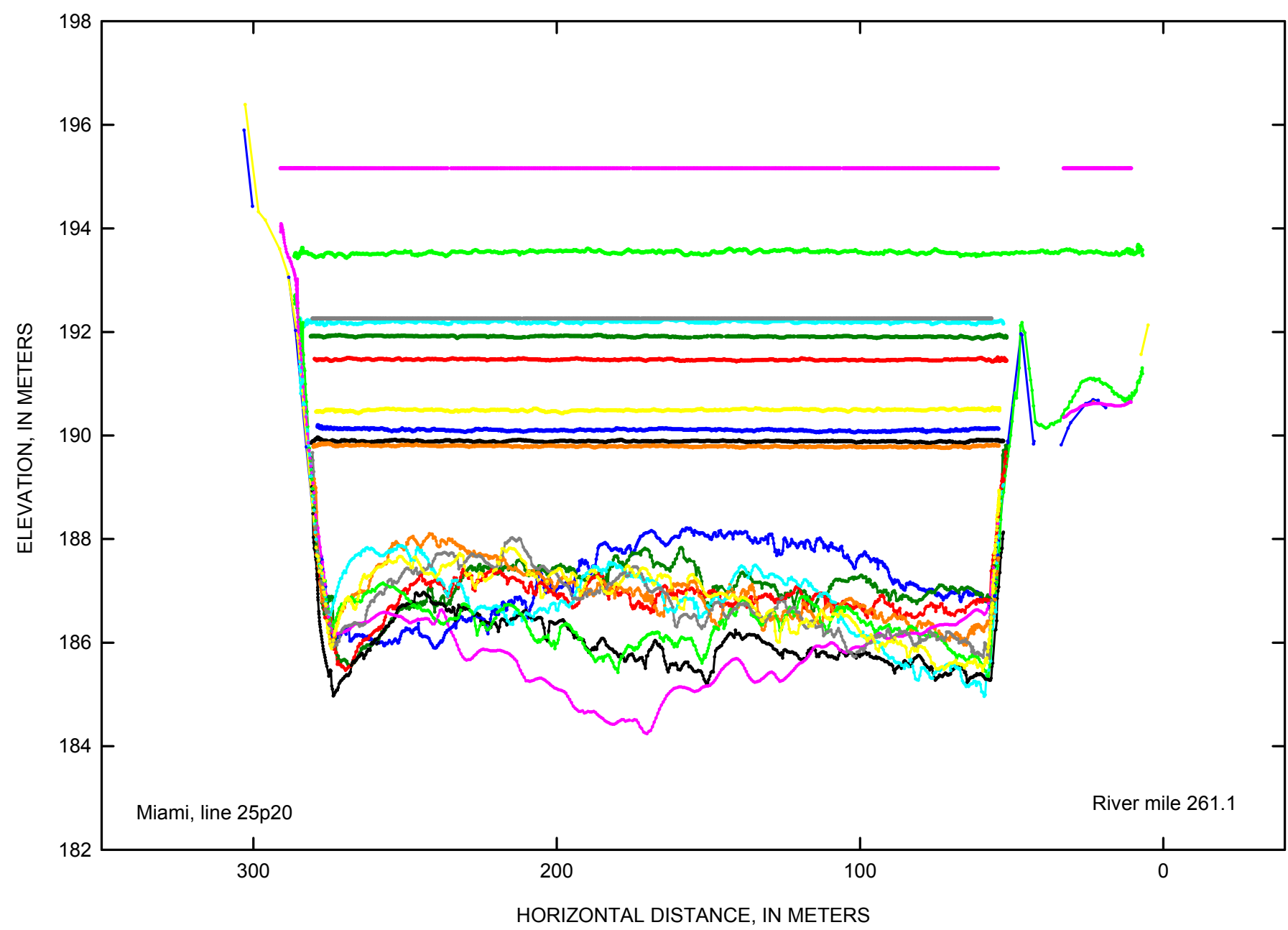

EXPLANATION

— March 2006

April 2006

May 2006

July 2006

October 2006

March 2007

April 2007

May 2007

July 2007

October 2007

Figure 4-22. Miami cross-section line 25p20 at Missouri River mile 261.1 for 2006-07. 


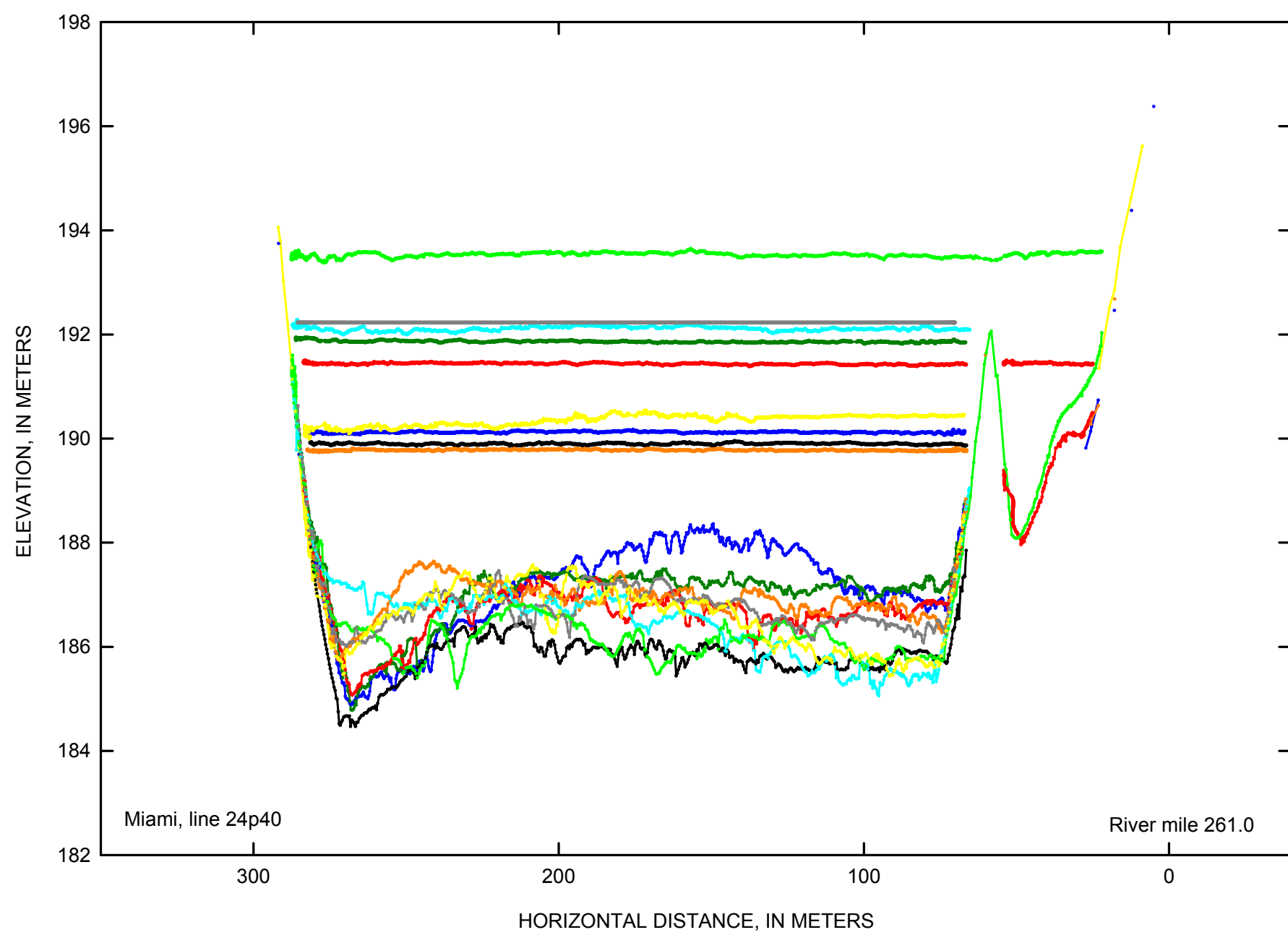

EXPLANATION

- March 2006

April 2006

May 2006

July 2006

October 2006

March 2007

April 2007

May 2007

July 2007

October 2007

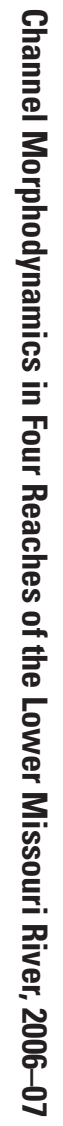

Figure 4-23. Miami cross-section line 24p40 at Missouri River mile 261 for 2006-07. 


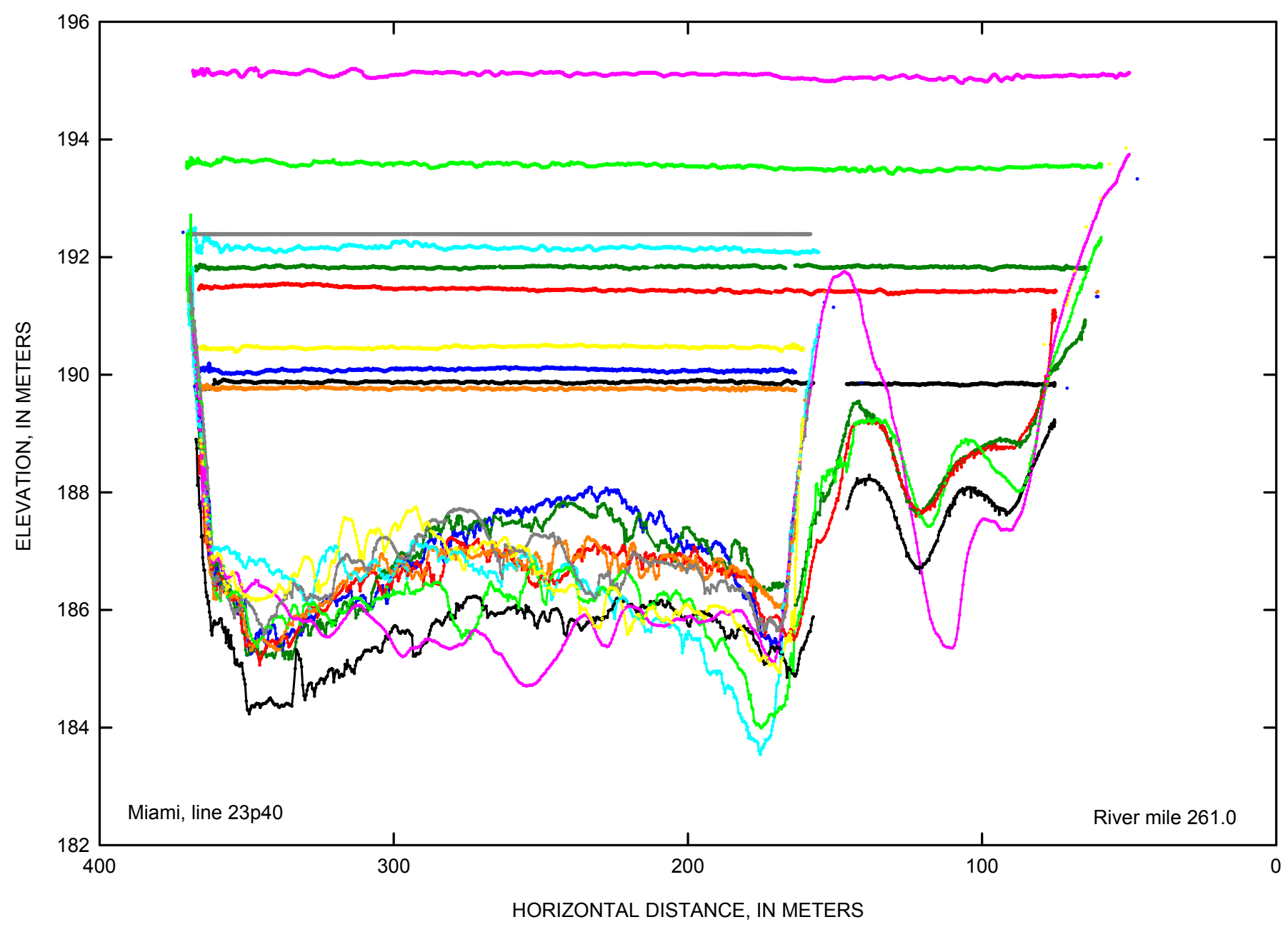

\section{EXPLANATION}

— March 2006

April 2006

May 2006

July 2006

October 2006

March 2007

April 2007

May 2007

July 2007

October 2007

Figure 4-24. Miami cross-section line 23p40 at Missouri River mile 261 for 2006-07. 


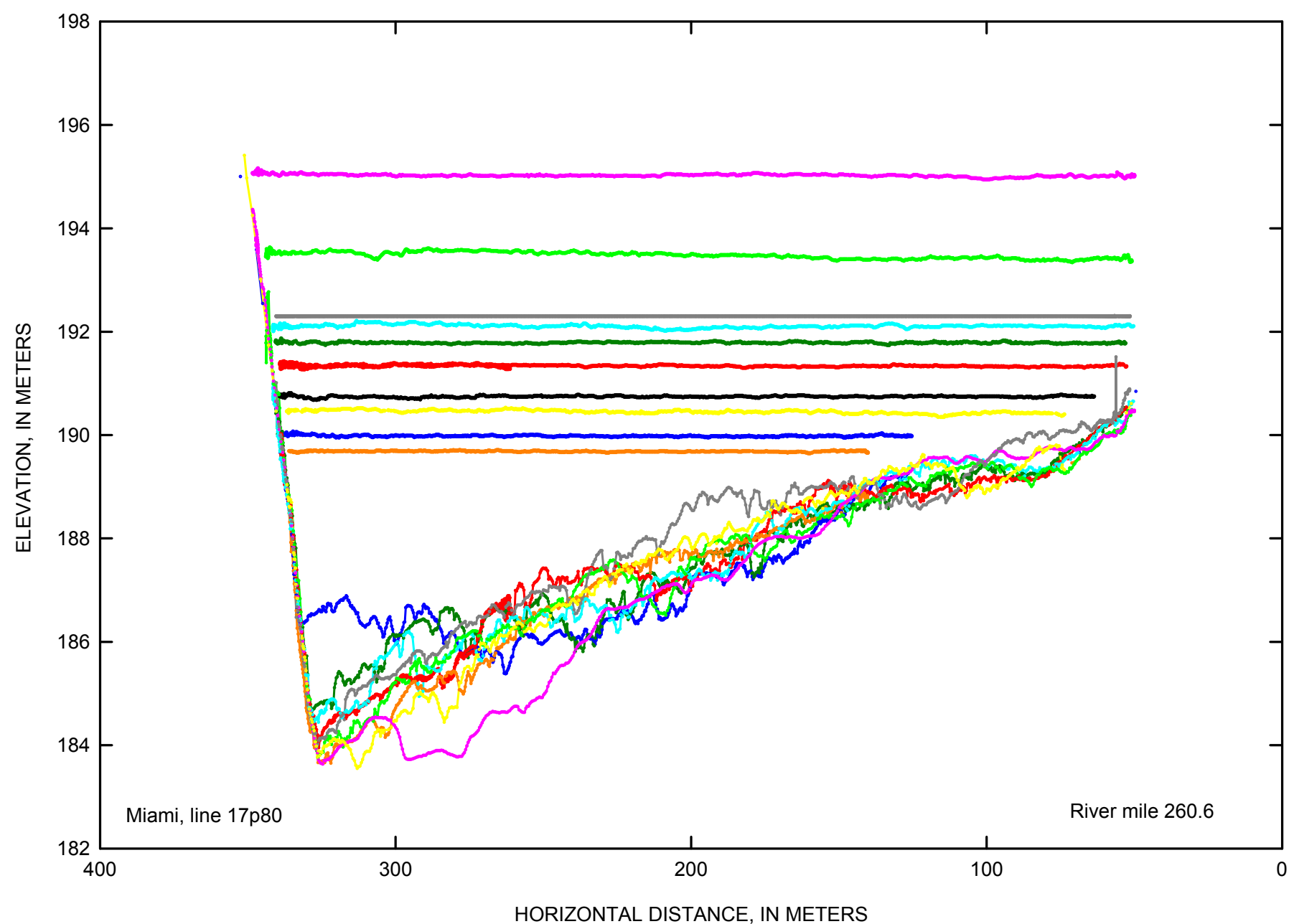

EXPLANATION

- March 2006

April 2006

May 2006

July 2006

October 2006

March 2007

April 2007

May 2007

July 2007

October 2007

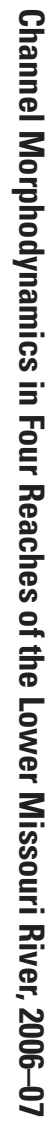

Figure 4-25. Miami cross-section line $17 p 80$ at Missouri River mile 260.6 for 2006-07. 


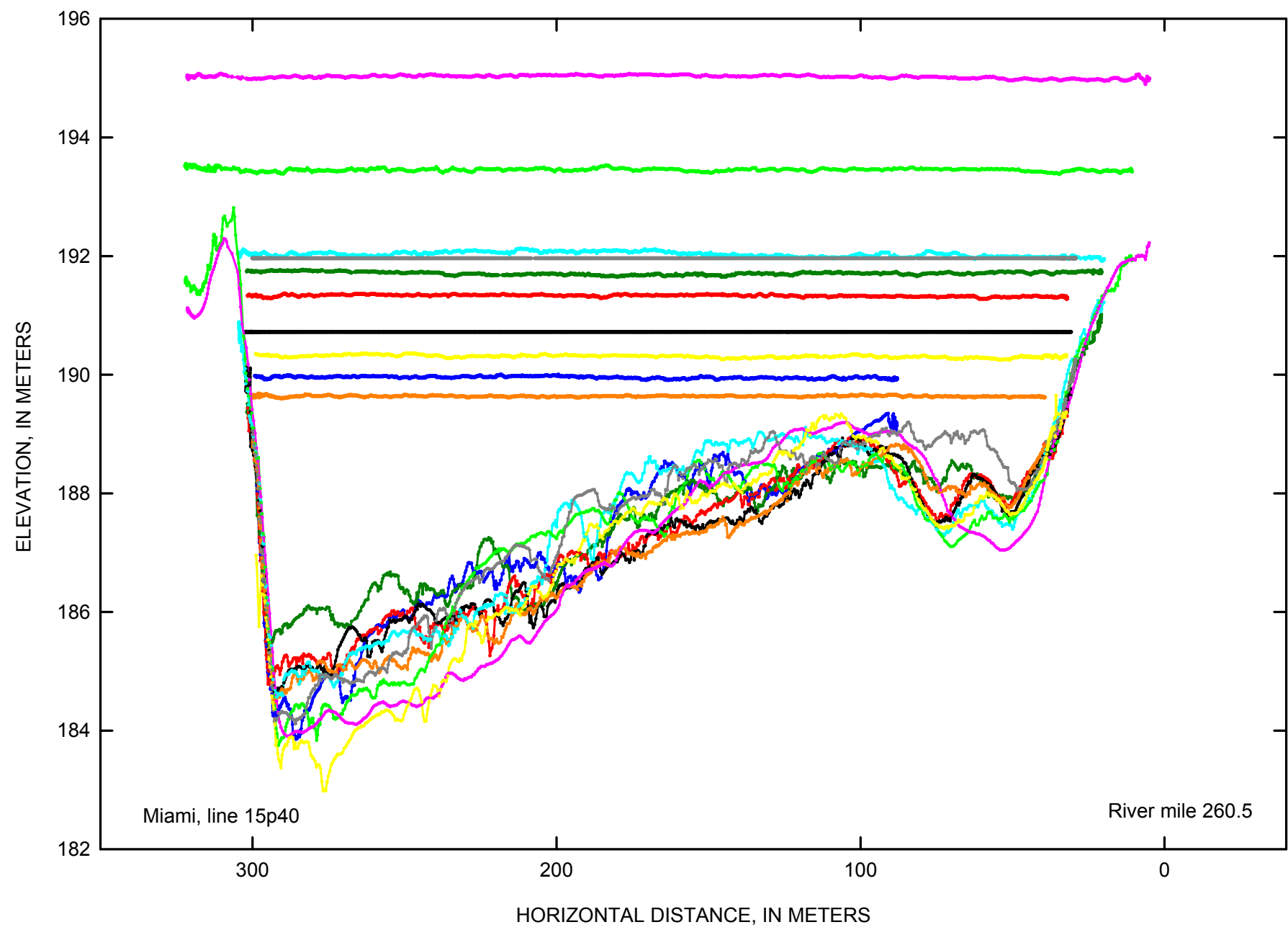

EXPLANATION

March 2006

April 2006

May 2006

July 2006

October 2006

March 2007

April 2007

May 2007

July 2007

October 2007

Figure 4-26. Miami cross-section line 15p40 at Missouri River mile 260.5 for 2006-07. 


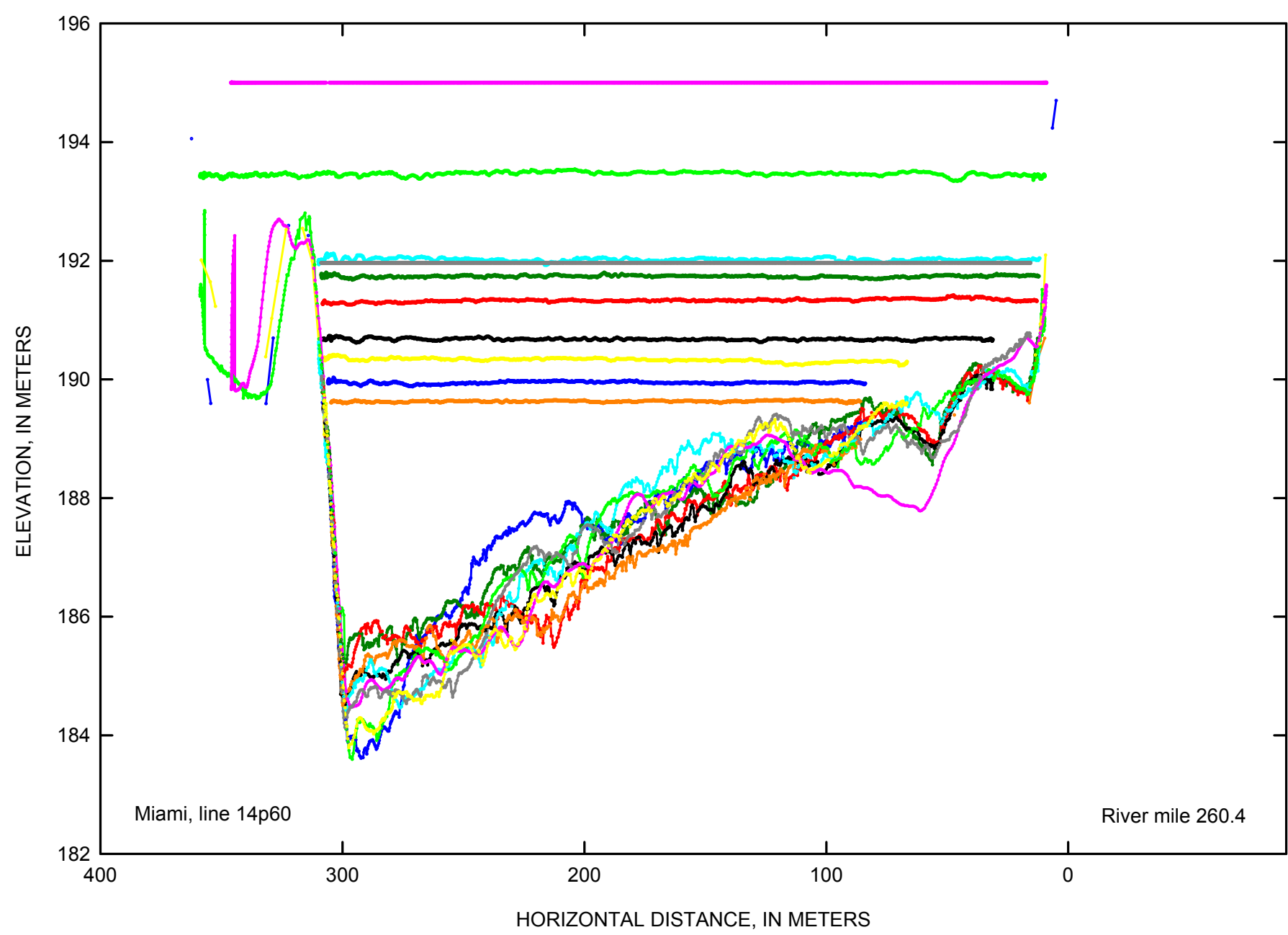

EXPLANATION

- March 2006

April 2006

May 2006

July 2006

October 2006

March 2007

April 2007

May 2007

July 2007

October 2007

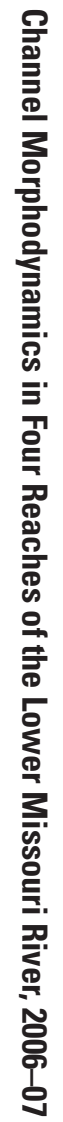

Figure 4-27. Miami cross-section line $14 p 60$ at Missouri River mile 260.4 for 2006-07. 


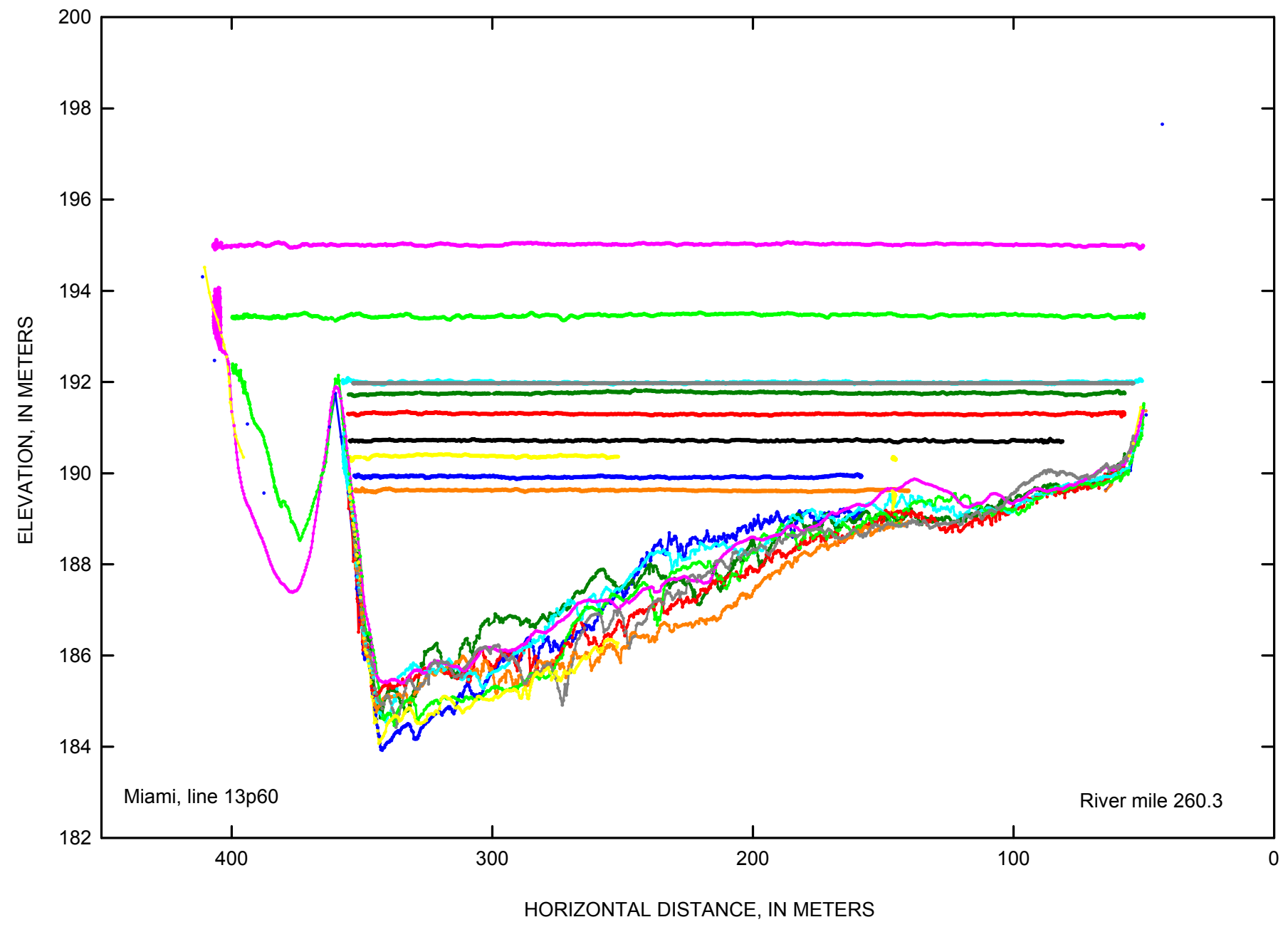

EXPLANATION

March 2006

— April 2006

May 2006

- July 2006

_ October 2006

March 2007

April 2007

May 2007

July 2007

October 2007

Figure 4-28. Miami cross-section line $13 p 60$ at Missouri River mile 260.3 for 2006-07. 


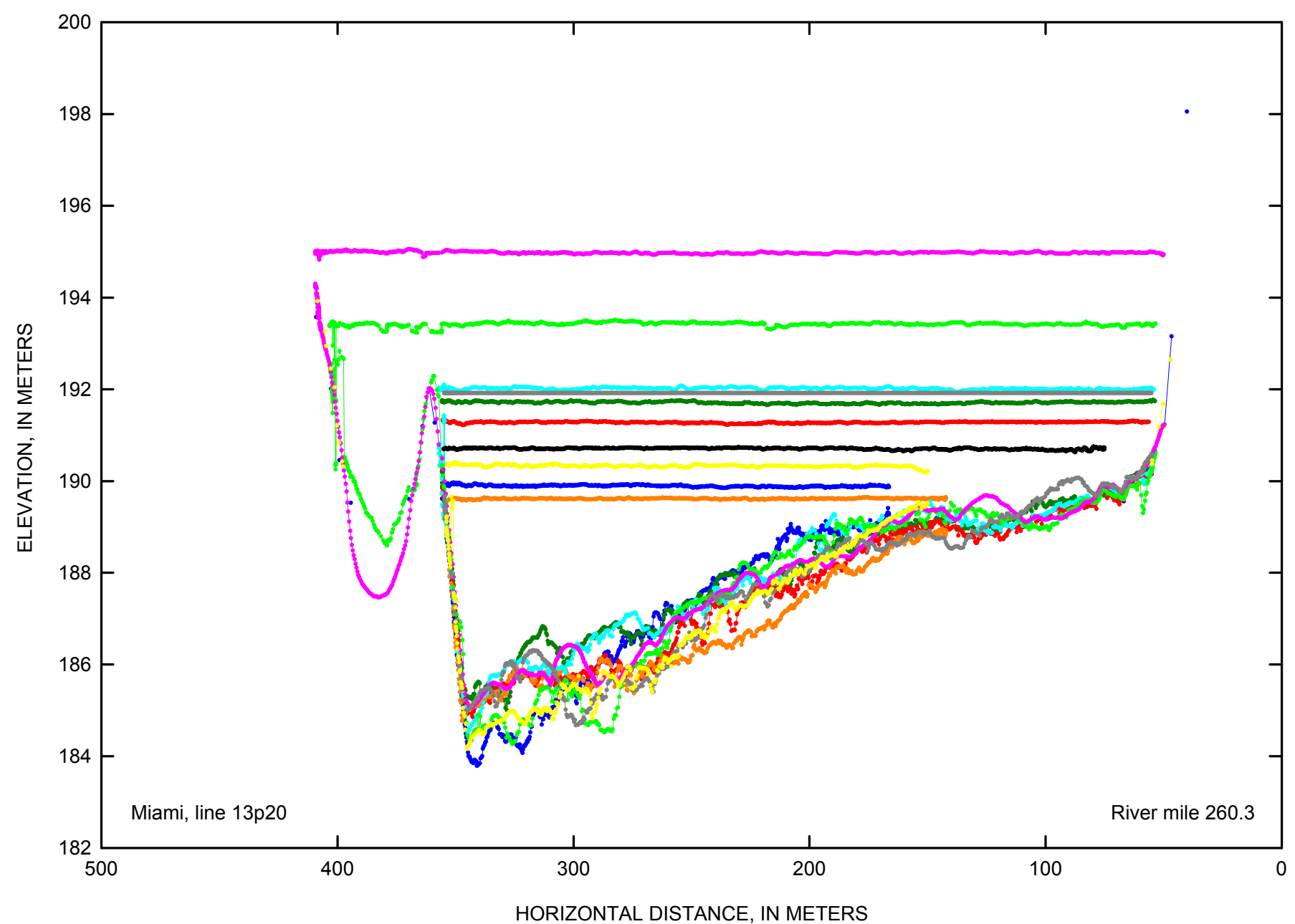

EXPLANATION

March 2006

April 2006

- May 2006

— July 2006

October 2006

March 2007

April 2007

May 2007

July 2007

October 2007

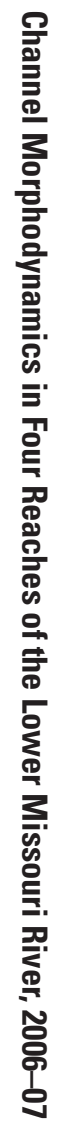

Figure 4-29. Miami cross-section line 13p20 at Missouri River mile 260.3 for 2006-07. 


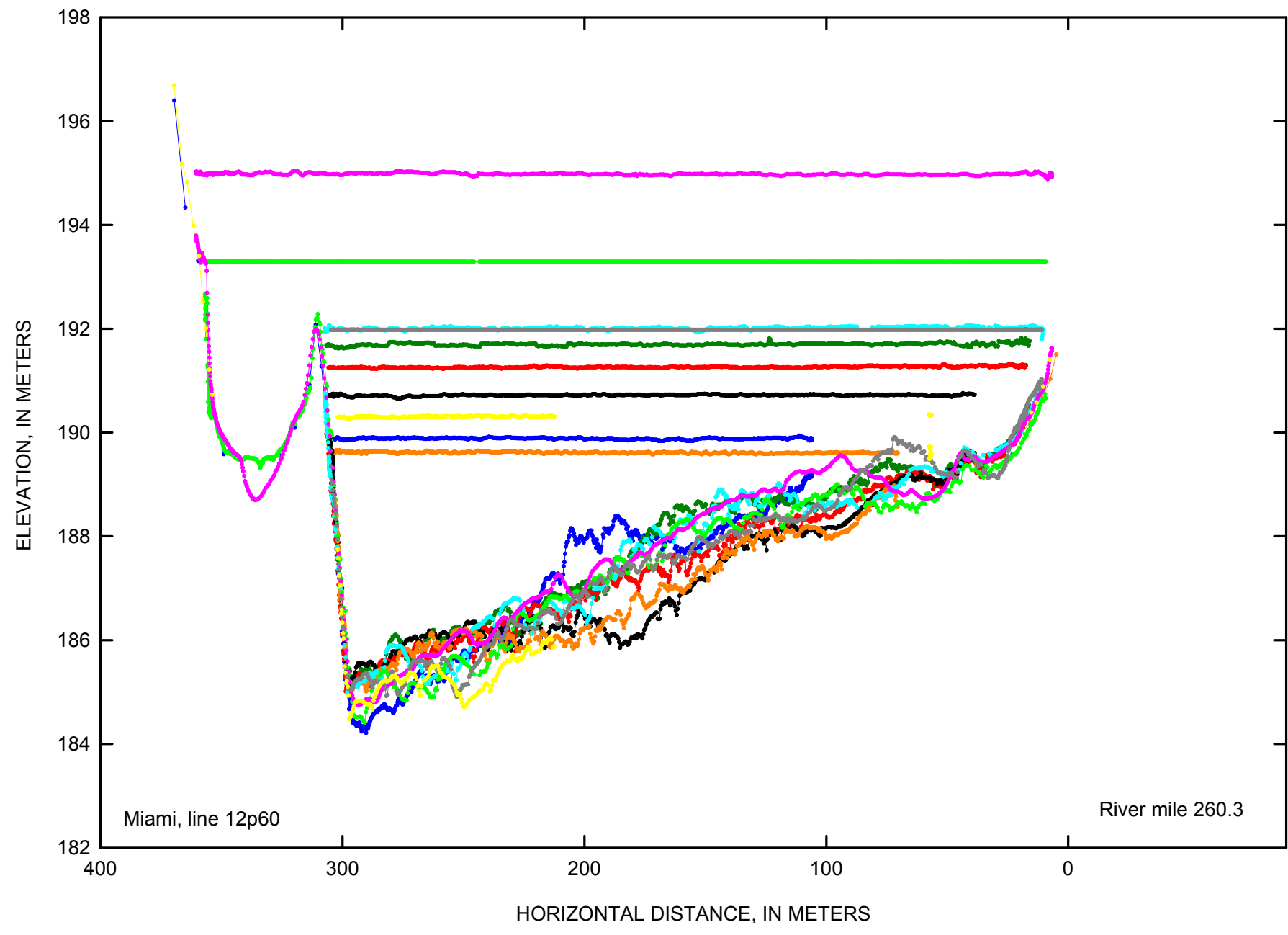

\section{EXPLANATION}

March 2006

April 2006

May 2006

July 2006

October 2006

March 2007

April 2007

May 2007

July 2007

October 2007

Figure 4-30. Miami cross-section line 12p60 at Missouri River mile 260.3 for 2006-07. 


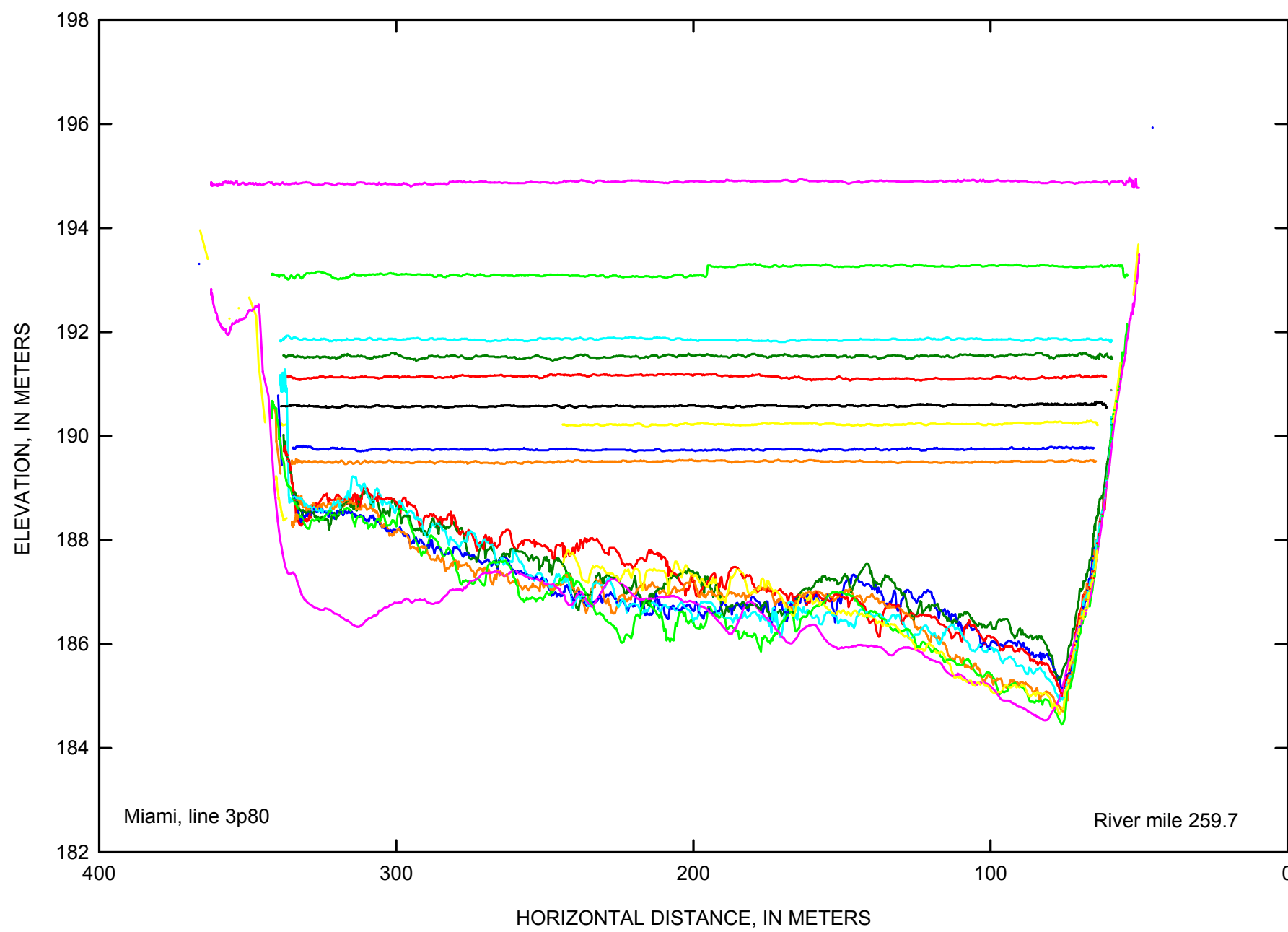

EXPLANATION

- March 2006

April 2006

May 2006

July 2006

October 2006

March 2007

April 2007

May 2007

July 2007

October 2007

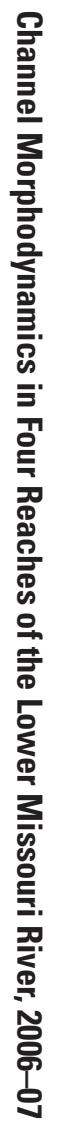

Figure 4-31. Miami cross-section line 3p80 at Missouri River mile 259.7 for 2006-07. 


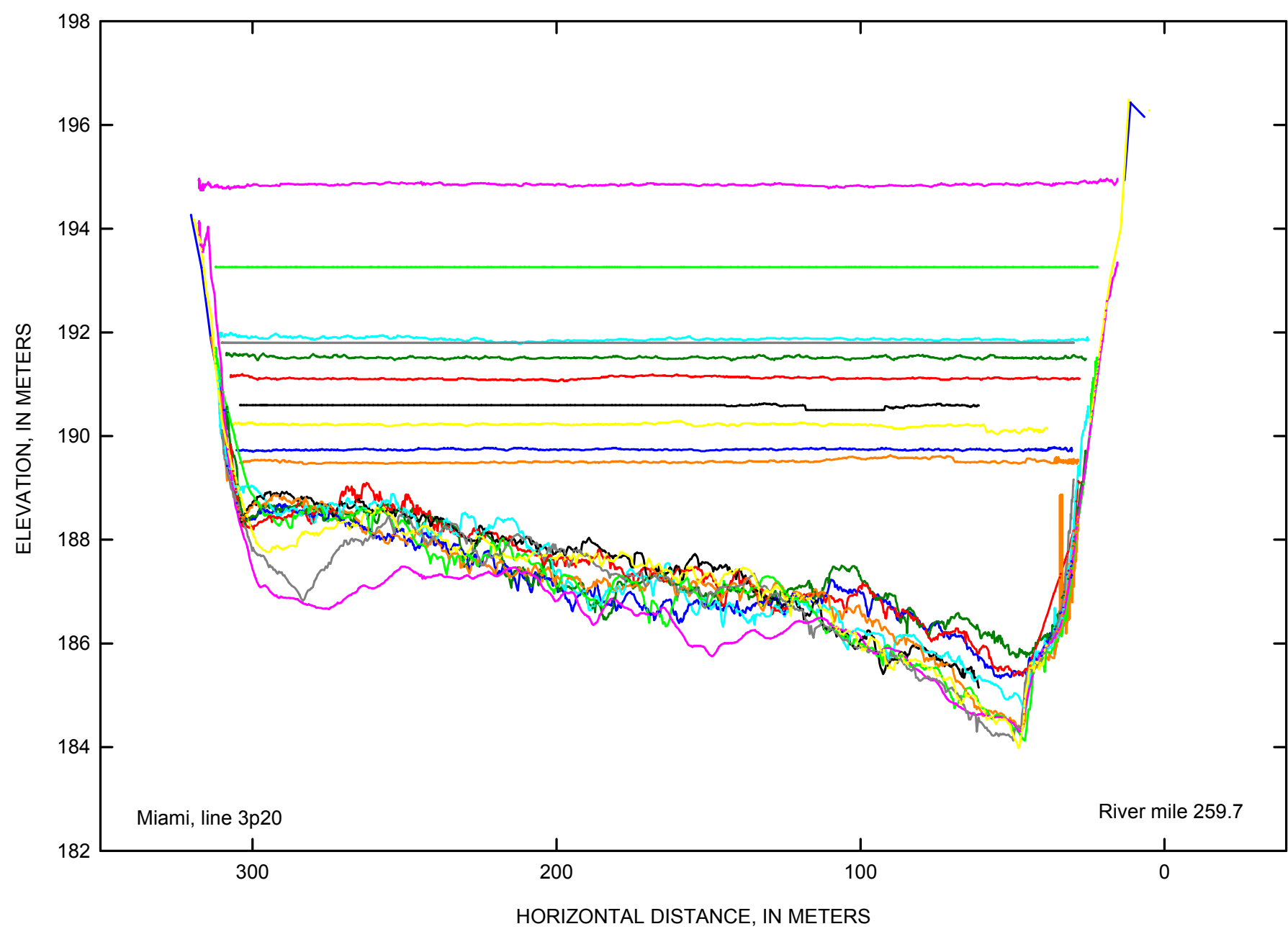

\section{EXPLANATION}

- March 2006

April 2006

May 2006

July 2006

October 2006

March 2007

April 2007

May 2007

July 2007

October 2007

Figure 4-32. Miami cross-section line 3p20 at Missouri River mile 259.7 for 2006-07. 


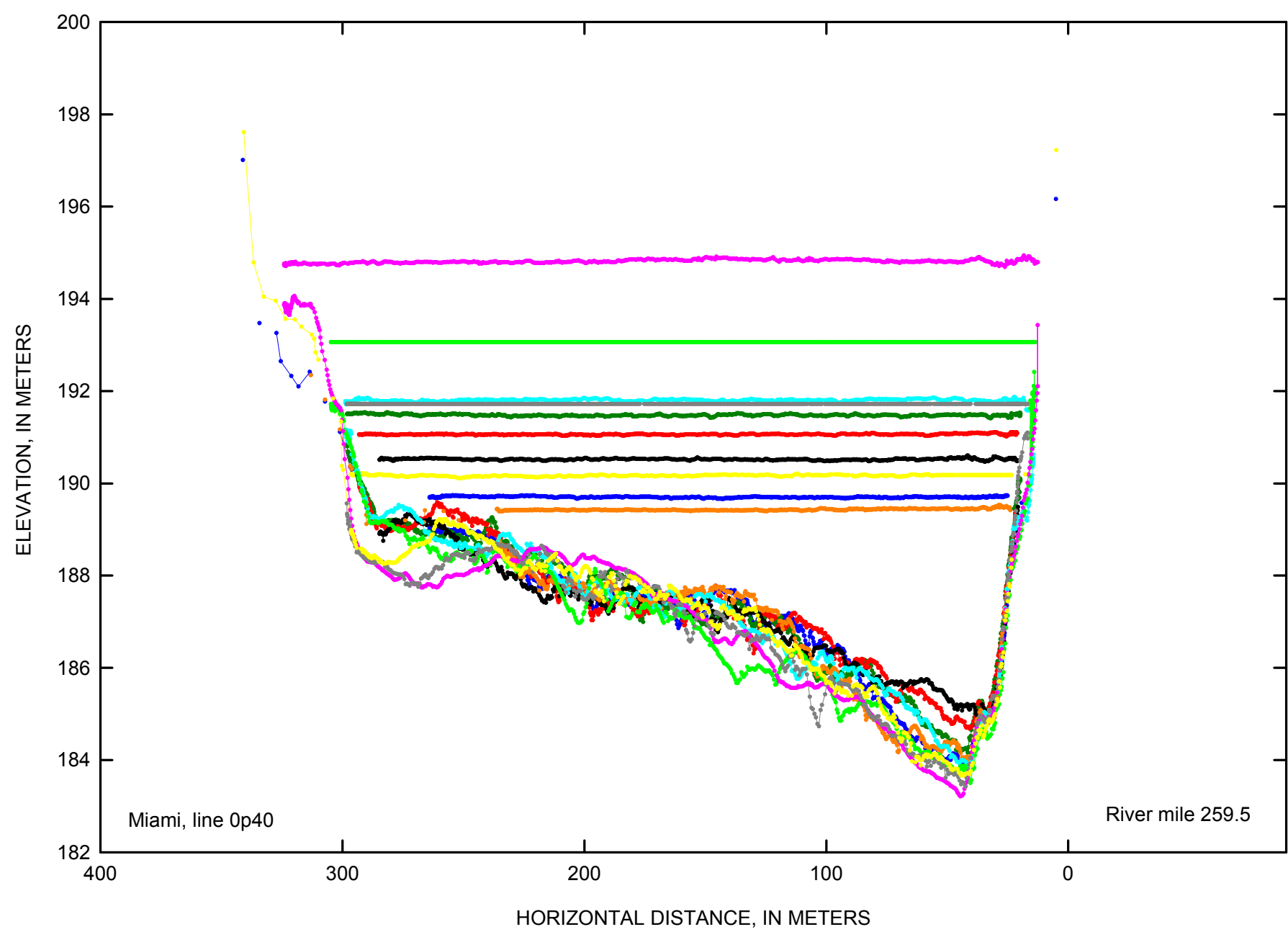

EXPLANATION

- March 2006

April 2006

May 2006

July 2006

October 2006

March 2007

April 2007

May 2007

July 2007

October 2007

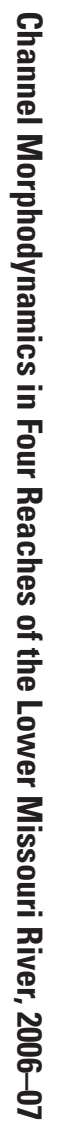

Figure 4-33. Miami cross-section line 0p40 at Missouri River mile 259.5 for 2006-07. 
Prepared by:

Rolla Publishing Service Center

1400 Independence Road

Rolla, M0 65401

For more information concerning this publication, contact:

Director

U.S. Geological Survey

Columbia Environmental Research Center

4200 New Haven Road

Columbia, M0 65201

(573) 875-5399

Or visit the Columbia Environmental Research Center website at: http://www.cerc.usgs.gov 




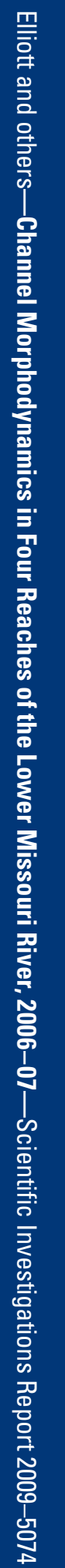

